\title{
Stefan Zweig
}

\section{A Bibliography}

Randolph Klawiter 
Stefan Zweig 
From 1949 to 2004, UNC Press and the UNC Department of Germanic \& Slavic Languages and Literatures published the UNC Studies in the Germanic Languages and Literatures series. Monographs, anthologies, and critical editions in the series covered an array of topics including medieval and modern literature, theater, linguistics, philology, onomastics, and the history of ideas. Through the generous support of the National Endowment for the Humanities and the Andrew W. Mellon Foundation, books in the series have been reissued in new paperback and open access digital editions. For a complete list of books visit www.uncpress.org. 


\section{Stefan Zweig}

\section{A Bibliography}

RANDOLPH J. KLAWITER

UNC Studies in the Germanic Languages and Literatures Number 50 
Copyright (C) 1965

This work is licensed under a Creative Commons CC BY-NC-ND license. To view a copy of the license, visit http://creativecommons. org/licenses.

Suggested citation: Klawiter, Randolph J. Stefan Zweig: A Bibliography. Chapel Hill: University of North Carolina Press, 1965. DOI: https:// doi.org/10.5149/978146965766o_Klawiter

Library of Congress Cataloging-in-Publication Data

Names: Klawiter, Randolph J.

Title: Stefan Zweig : A bibliography / by Randolph J. Klawiter.

Other titles: University of North Carolina Studies in the Germanic Languages and Literatures ; no. 50.

Description: Chapel Hill : University of North Carolina Press, [1965] Series: University of North Carolina Studies in the Germanic Languages and Literatures.

Identifiers: LCCN 65003222 | ISBN 978-1-4696-5765-3 (pbk: alk. paper) | ISBN 978-1-4696-5766-o (ebook)

Subjects: Zweig, Stefan, 1881-1942 — Bibliography.

Classification: LCC PD25 .N6 NO. 50| DCC 016/.838912 




\section{PREFACE}

To compile a completely comprehensive bibliography is a task as futile as it is seemingly vain. No matter how hard one tries to gather in all the pertinent information, let him but look at one more book and without fail there he will find another indispensable entry for his already "completed" list. More than once the bibliographer is sorely tempted to doubt the efficacy of his protracted labors and to end them all with a slight touch of sulfur. But here as elsewhere "conscience doth make cowards of us all," and we carry on towards our goal, as arbitrary as it may seem to be.

From such scholarly pangs of conscience I too have suffered more than once, knowing that I couldn't possibly do justice to my undertaking and yet fearing that were I not at least to try, the project might never be attempted. Which would be worse - to make an effort and fall short of the desired goal or make no effort at all, waiting for the day when someone more competent should have the time and desire to do that which I could but hope to accomplish in part? It is to be hoped that my choice of the former alternative may nevertheless prove beneficial to those interested in the works of Stefan Zweig for their own sake or those engaged in future Zweig scholarship.

Admitting without reserve the incompleteness of the following bibliography and taking upon myself the onus of any factual as well as all typographical errors, I nevertheless take great pleasure in acknowledging my sincere debt of gratitude to the many people without whose efforts I would most assuredly have been able to accomplish little: in sequence of time I must first mention Dr. F. X. Braun of the University of Michigan under whose guidance I wrote my doctoral dissertation on Stefan Zweig and to whom I owe the idea of assembling the present bibliography; to Mrs. Friderike M. Zweig my thanks must surely prove inadequate to the amount of encouragement and friendship that she has shown me; for much material that I would never have otherwise seen and for his ever ready assistance in so many instances I wish to thank Dr. Harry Zohn of Brandeis University; for permission to compare my efforts with his unpublished American Zweig Bibliography I likewise thank Dr. Thomas L. Broadbent of the University of Utah; for information on 
recent articles about Zweig appearing in foreign periodicals as well as for copies of her own lectures my appreciation is extended to Mrs. Mimi Grossberg of New York City; to the many friends of Stefan Zweig in Germany, Austria, France, Italy, Holland, Japan and India who so willingly gave of their time to correspond with me concerning problems that arose with respect to articles and translations of Zweig's works in these respective languages my gratitude is without limit; for many enjoyable hours spent together searching library stacks I tip my hat to my friends Michael C. Downs and Ralph Kent, students at the University of Notre Dame, who likewise shared the joys and sorrows of the several proofreadings of the typescript, a dubious honor shared by many other friends and most of my family; for the financial support at every step of the way as well as the unflagging moral and morale support I take special pleasure in paying herewith in some small manner the enormous debt I owe to my parents; for the many long hours spent behind her relentless typewriter with an equally relentless script of undecipherable languages before her I thank Mrs. M. Webber, the most perfect secretary any language department could ever hope to acquire; and to my wife I also owe more than I can here express, not only for her willingness to shoulder all the domestic responsibilities while I wiled away the fruitful hours among the weeds and flowers of literary scholarship but perhaps even more am I indebted to her sense of practicality and self-discipline, both of which effectively made me realize that the business at hand was a task to be accomplished and not a life-long dedication. It would also indeed be remiss on my part were I to fail to mention the many services rendered to me by the library staffs of the Universities of Notre Dame, Michigan, and Chicago without whose ready assistance the information needed from far and near would never have been made available. Lastly I might erect a type of prefatory altar to the forgotten god upon which is to be placed anyone whom I have inadvertently forgotten to mention or whom perhaps I have never met, but to whom nevertheless my debt of gratitude is rendered with the utmost sincerity.

University of Notre Dame

Randolph J. Klawiter

Notre Dame, Indiana June, I964. 


\section{TABLE OF CONTENTS}

Preface. . . . . . . . vii

Abbreviations. . . . . . . . xi

Introduction . . . . . . . xxvi

Collected Works. . . . . . . . I

Poetry in Book Form . . . . . . 2

Single Poems . . . . . . . 2

Dramas ........ . 4

Prose Fiction . . . . . . . . 6

Essays . . . . . . . . . . 24

Biographical Studies . . . . . . 28

Amerigo Vespuccio . . . . . 28

Balzac ... . . . . . 29

Calvin ........ 3I

Marceline Desbordes-Valmore . 32

Erasmus von Rotterdam . . . . 32

Joseph Fouché . . . . . . 33

Magellan ...... . 36

Marie Antoinette . . . . . 38

Maria Stuart . . . . . . . 39

Frans Masereel . . . . . 4 4I

Romain Rolland. . . . . . . 4I

Emile Verhaeren . . . . . 42

Paul Verlaine . . . . . . 43

Baumeister der Welt . . . . . 43

Drei Dichter ihres Lebens . . . 43
Drei Meister . . . . . 4 45

Der Kampf mit dem Dämon . . 47

Die Heilung durch den Geist . . 48

Sternstunden der Menschheit . . 49

Brasilien . . . . . . . 52

Die Welt von Gestern . . . . . 53

Correspondence in Book-form. . . 55

Letters to or from Zweig in Books 55

Letters to or from Zweig in Periodicals . . . . . . . 56

Excerpts from Zweig's Works appearing in Periodicals, Newspapers and Books . . . . . . 57

Articles and Lectures by Zweig . . 6r

Translations by Zweig . . . . . . 69

Forewords and Epilogues by Zweig $7 \mathrm{I}$

Book Reviews by Zweig . . . . . 74

Articles and Book Reviews of Works by Zweig . . . . . . 77

Poetry . . . . . . . . 77

Dramas . . . . . . . 78

Fiction. . . . . . . 8 $8 \mathrm{I}$

Essays . . . . . . . . 86

Amerigo Vespuccio . . . . . . 87

Balzac . . . . . . . . 87

Calvin . . . . . . . . 88

Marceline Desbordes-Valmore . 89 
Erasmus von Rotterdam . . . . 89

Joseph Fouché . . . . . . . 90

Magellan . . . . . . . 92

Marie Antoinette . . . . . 93

Maria Stuart . . . . . . 95

Romain Rolland. . . . . . . . 96

Emile Verhaeren . . . . . . 97

Baumeister der Welt . . . . . . 98

Drei Dichter ihres Lebens . . . 99

Drei Meister . . . . . . . IO०

Der Kampf mit dem Dämon . . IOI

Die Heilung durch den Geist . . IO2

Sternstunden der Menschheit . . I04

Brasilien ......... Ios

Die Welt von Gestern . . . . . Io6

Stefan Zweig - Friderike Zweig:

Correspondence . . . . . . . 107

Richard Strauss - Stefan Zweig:

Correspondence . . . . . . . . 107

Review of Books with Forewords by Zweig . . . . . . . . . I08

Review of Works about Zweig. . . I09

Books and Articles about and References to Zweig in Various Works I IO

Czechoslovakian ...... I Io

Dutch .......... I IO

English ......... . Iro
French . . . . . . . . IIS

German . . . . . . . II

Hebrew . . . . . . . . I 28

Hindi . . . . . . . I 28

Italian . . . . . . . . . I 29

Japanese . . . . . . . . . I29

Norwegian . . . . . . . I29

Polish . . . . . . . . . . I 29

Portuguese . . . . . . . . . I 29

Russian . . . . . . . I30

Spanish . . . . . . 130

Ukranian . . . . . . . . I3I

Yiddish ........ I I I

Literary and Biographical Personalia I3 I

Unpublished Masters' Theses and Doctoral Dissertations . . . . . I34

General Reference Works . . . I35

Histories of Literature . . . . . I38

Miscellany . . . . . . . 14 I

German Manuscripts in Zweig's Manuscript Collection . . . . I42

Articles by Zweig Concerning His Manuscript Collection and Articles about Zweig as a Manuscript Collector . . . . . . . . . 146

Notes . . . . . . . . . . I49

Index ......... I53 


\section{ABBREVIATIONS}

I. GENERAL

$\begin{array}{ll}\text { a. } & \text { year (Cf. also J. = year) } \\ \text { cf. } & \begin{array}{l}\text { compare, see } \\ \text { col. }\end{array} \\ \text { diss. } & \text { dissertation } \\ \text { ed(s). } & \text { editor(s), edition(s), edited by } \\ \text { enl. } & \text { enlarged } \\ \text { esp. } & \text { especially } \\ \text { f.m. } & \text { frequently mentioned } \\ \text { fr. } & \text { foreword, foreword by } \\ \text { intro. } & \text { introduction, introduction by } \\ \text { Lit. Beil. } & \text { literary supplement (Literarische Beilage) } \\ \text { m. } & \text { mentioned, scattered references } \\ \text { n.d. } & \text { no date given } \\ \text { N.F. } & \text { new series (Neue Folge) } \\ \text { n.l. } & \text { no place given } \\ \text { n.p. } & \text { no publisher given } \\ \text { opp. } & \text { opposite } \\ \text { p. } & \text { page } \\ \text { pp. } & \text { pages } \\ \text { pref. } & \text { preface, preface by } \\ \text { rev. } & \text { review, reviewed by, revised } \\ \text { sel. } & \text { selected by } \\ \text { ser. } & \text { series } \\ \text { tr. } & \text { translator, translation, translated by } \\ \text { Tsd. } & \text { thousand } \\ \text { vol. } & \text { volume } \\ & \end{array}$

2. GERMAN ABBREVIATIONS IN THE APPENDIX

B1. sheet

d. the

e. a

f. for

Frhrn v. Baroness of (Freiherrin von)

Gr. format, size

$\mathrm{Ms}(\mathrm{s})$. manuscript(s)

S. page(s)

Slg. collection

Tl. part

u. and

u.a. and others

usw. and so forth, etc. 
Balzac

Begegnungen

Brasilien

Calvin

Drei Dichter

Drei Meister

Durch

Erasmus

Europäisches Erbe

Fahrten

Fouché

Heilung durch den

Geist

Kampf mit dem

Dämon

Magellan

Marceline Desbordes-

Valmore

Marie Antoinette

Rolland

Sternstunden

Verhaeren

Verlaine

WvG

Zeit und Welt

4. MONOGRAPHS

ALA

Antlitz

Blätter

Brod
Balzac. Der Roman seines Lebens. Stockholm: Bermann-Fischer, 1946 (Or later eds. as indicated).

Begegnungen mit Menscben, Bücbern, Städten. Wien-Leipzig-Zürich: Herbert Reichner, I937 (Pagination quoted from the r955 ed., Berlin-Frankfurt am Main: S. Fischer).

Brasilien, Land der Zukunft. Stockholm: Bermann-Fischer, I94I. Castellio gegen Calvin oder ein Gewissen gegen die Gewalt. Wien: Herbert Reichner, 1936.

Drei Dichter ibres Lebens: Casanova - Stendbal-Tolstoi. Leipzig: Insel, r928.

Drei Meister: Balzac - Dickens - Dostojewski. Leipzig: Insel, I920 (Or later eds. as indicated).

Durch Zeiten und Welten. Graz-Wien: Stiasny, I96r.

Triumph und Tragik des Erasmus von Rotterdam. Wien - Leipzig Zürich: Herbert Reichner, I934 (Or later eds. as indicated).

Europäisches Erbe. Frankfurt am Main: Fischer, 1960.

Fabrten, Landscbaften und Städte. Leipzig - Wien - Zürich: E. P. Tal, r9r9.

Joseph Fouché. Bildnis eines politischen Menschen. Leipzig: Insel, 1929 (Or later eds. as indicated).

Die Heilung durch den Geist: Franz Anton Mesmer - Mary Baker Eddy-Sigmund Freud. Leipzig: Insel, I 93 I (Or later eds. as indicated). Der Kampf mit dem Dämon: Hölderlin - Kleist - Nietzsche. Leipzig: Insel, r925.

Magellan. Der Mann und seine Tat. Wien - Leipzig - Zürich: Herbert Reichner, 1938 (Or later eds. as indicated).

Marceline Desbordes-Valmore. Das Lebensbild einer Dicbterin. Leipzig: Insel, r920 (Or later eds. as indicated).

Marie Antoinette. Bildnis eines mittleren Cbarakters. Leipzig: Insel, 1932 (Or later eds. as indicated).

Romain Rolland. Der Mann und das Werk. Frankfurt am Main: Ruetten and Loening, I921.

Sternstunden der Menschbeit. Fünf historische Miniaturen. Leipzig: Insel, 1927 (Or the later expanded $Z$ wolf bistoriscbe Miniaturen. Stockholm: Bermann-Fischer, I945 (Or later eds. as indicated)). Erinnerungen an Emile Verbaeren. Wien: Christoph Reissers Söhne, I 9 I 7 (Or later eds. as indicated).

Paul Verlaine. Eine Monographie. Berlin - Leipzig: Schuster and Loeffler, I904.

Die Welt von Gestern. Erinnerungen eines Europäers. Stockholm: Bermann-Fischer, r944 (Or later eds. as indicated).

Zeit und Welt. Gesammelte Aufsätze und Vorträge 1904-1940. Stockholm: Bermann-Fischer, I 943 (Pagination quoted from the 1946 ed.).

Anthologie des Lyriques allemands contemporains depuis Nietzsche. Ed. Henri Guilbeaux. Paris: E. Figuière and Cie, I9r3.

Gustav Settler. Vom Antlitz junger Menschen. Acbt Radierungen. Grenchen (die Schweiz): Verlag Gute Graphik, 1956. Blätter der Internationalen Stefan Zweig Gesellschaft. Wien. Max Brod. Festscbrift zum fuinfzigsten Geburtstag. Ed. Felix Weltsch. Mährisch-Ostrau, I934. 
Carossa

Ceterum

CGP

Crumbs

CurBio

DdG

DeB

Diktate

DuZ

EdD

Europäer

Francé

Franz Dichter

Franz Lyrik

Frau

Geistesleben

Gewalt

Herz

Herzl

HLAS: $194 \mathrm{I}$

Hofmannsthal

Hommage

Journeys

Juden

Karussell

Kippenberg

Kraus

Leben-Werk
Buch des Dankes fir Hans Carossa. Ed. Albrecht Schaeffer.

Emanuel Bin Gorion. Ceterum Recenso. Kritische Aufsätze und Reden.

Tübingen: Alexander Fischer, 1929.

Contemporary German Poetry. Tr. and ed. Jethro Bithell. LondonNew York: Walter Scott, I909.

M. Burrell. Crumbs are also Bread. Toronto: Macmillan, I934.

Current Biography. Who's News and Why. 1942. New York: H. W. Wilson, 1942 .

Dichter der Gegenwart. Vol. IV. Ed. Toni Meder. Bamberg-Wiesbaden: Bayerische Verlagsanstalt, I955.

Der ewige Brunnen. Ein Volksbuch deutscher Dicbtung. 2. ed. München:

Beck, I959.

Diktate aus dem deutschen Scbrifttum. Ed.Willi Hopff, Hans Thiel and Wilhelm Reininghaus. 8. ed. Frankfurt am Main - Berlin Bonn: Mantz Diesterweg, n.d.

Dicbter unserer Zeit. Vol. II. Ed. Hans Göttling. Bamberg: Buchners, I954.

Erzäblungen deutscher Dicbter. Vol. III. Ed. Wilhelm Helmich and Paul Nentwig. n.1.: Georg Westermann, I958.

Der grosse Europäer Stefan Zweig. Ed. Hanns Arens. München: Kindler, 1956.

R. H. Francé. Festscbrift zu seinem fünfzigsten Geburtstag. Heilbronn: Seifert, I924.

Französische Dichter des XIX. und XX. Jabrbunderts in deutscher Übertragung. Sel. and Intro. Otto Heuschele. Bühl in Baden: Roland, 1948.

Französische Lyrik aus acbt Jabrbunderten. Ed. Franz von Rexroth. Saarbrücken: Saar Verlag, 1946.

Die Frau von Morgen, wie wir sie winschen (Von Max Brod, Arnolt Bronnen, Axel Eggebrecht, Stefan Zweig et al.). Ed. Friedrich M. Huebner. Leipzig: Seemann, 1929.

Deutsches Geistesleben der Gegenwart. Ed. Otto Koischwitz. New York: Knopf, I928 (School ed.).

Gewalt und Gewaltlosigkeit. Handbuch des aktiven Pazifismus. Ed. Franz Kobler. Zürich: n.p., I928.

Das Herz Europas. Österreichisches Vortragsbuch. Wien: n.p., 1935 .

Theodor Herzl. A Memorial. Ed. Meyer W. Weisgal. New York: The New Palestine, I929.

Handbook of Latin American Studies: 1941. Ed. Miron Burgin. Cambridge (Mass.): n.p., I941.

Hugo von Hofmannstbal. Der Dicbter im Spiegel der Freunde. Ed. Helmut A. Fiechtner. 2. ed. Bern - München: Francke, 1963.

Hommage à Romain Rolland. Ed. Charles Baudoin. Genève: Les Editions du Mont Blanc, 1945 .

Journeys in Time. Ed. B. R. Niles. New York: Coward-McCann, I946.

Von Juden in München. Ein Gedenkbuch. Ed. Hans Lamm. München: Ner-Tamid Verlag, I958.

Das Karussell. Geschichten aus dieser Zeit. Vol. III. 2. ed. Ed. Walter Urbanek. Bamberg: Buchners, I962.

Navigare necesse est. Festscbrift für Anton Kippenberg. Ed. P. Jessen. Leipzig: Insel, 1924.

Rundfrage über Karl Kraus. Ed. L. von Ficker. Innsbruck: n.p., I9I 7. Stefan Zweig. Sein Leben - sein Werk. Ed. Hanns Arens. Esslingen: Bechtle, I949. 
Librarians

Life-Work

Liliencron

Lyrik

Mann-Werk

Masereel

Moissi

Negen Vuurmakers

PeR

Ramuz

Schmidtbonn

Schmitz

Schorer

Schweitzer

Spectrum

Spiegelungen

SZ

SZG

Tag

Wedekind

Weltdichtung

$\mathrm{Zdz}$
Of, By and For Librarians. Further Contributions to Library Literature Ed. J. D. Marshall. New York: Shoe String Press, 1960.

Stefan Zweig. A Tribute to His Life and Work. Ed. Hanns Arens. Tr. from the German. London: n.p., I 95 I.

Osterreichischer Dicbter. Zum sechzigsten Geburtstag von Detlev von Liliencron. Ed. Adolf Donath. Wien: Carl Konegen, I904.

Lyrik des Abendlands. Ed. Georg Britting. München: Hanser, 1948. Erwin Rieger. Stefan Zweig. Der Mann und das Werk. Berlin: Spaeth, I 928.

Frans Masereel (Mit Beiträgen von Stefan Zweig, Pierre Vorms, Gerhard Pommeranz-Liedtke und einer Bibliographie von Hanns Conon von der Gabelentz). Dresden: Verlag der Kunst, 1959.

Moissi. Der Mensch und Kinstler in Worten und Bildern. Ed. Hans Böhm. Berlin: Eigenbrödler, 1927.

Negen Vuurmakers voor bet Kampvuur. Utrecht: Vrijzinnig Christelijke Jeugd Centrale, 1946.

M. Robertazzi. Poesia e Realtà. Modena: Guandi, I934.

Hommage à Ramuz, zum sechzigsten Geburtstag. Lausanne: Porchet and $\mathrm{Cie}, \mathrm{I} 938$.

Chor um Wilbelm S. Schmidtbonn. Zum sosten Geburtstag, 6. Feb. 1927. Ed. Herbert Saekel. Stuttgart - Berlin - Leipzig: Deutsche Verlagsanstalt, 1926.

Oskar A. H. Scbmitz zum fünfzigsten Geburtstage. München: Georg Müller, I923.

Henri Delarue and Paul F. Geisendorf. Calvin, Stefan Zweig et $M$. Jean Schorer. Genève: Librairie de l'Université - Georg and Cie, 1949.

Albert Scbweitzer, Genie der Menscblicbkeit. Frankfurt am MainHamburg: Fischer, I955 (Fischer Bücherei, No. 83).

Spectrum. Modern German Thought in Science, Literature, Pbilosopby and Art. Ed. W. P. Lehmann, Helmut Rehder and Hans Beyer. New York: Holt, Rinehart and Winston, 1964.

Stefan Zweig. Spiegelungen einer schöpferischen Persönlichkeit. Ed. Erich Fitzbauer. Wien: Bergland, 1959 (Erste Sonderpublikation der Stefan Zweig Gesellschaft).

Friderike Maria Zweig. Stefan Zweig, wie ich ibn erlebte. Stockholm New York: Neuer Verlag, 1947.

Paul Zech. Stefan Zweig. Eine Gedenkscbrift. Buenos Aires: Quadriga, I943.

Der Tag bricht an (Neue Gedichte von Waldemar Bonsels, Felix Braun, Hermann Hesse, Stefan Zweig et. al.). Ed. Carl Seelig. Dortmund: Der Garten Eden, I921.

Das Wedekindbuch. Ed. Joachim Friedenthal. München - Leipzig: n.p., r9I4.

Lyrische Weltdicbtung in deutscher U'bertragung aus sieben Jabrbunderten. Ed. Julius Petersen and Erich Trunz. Berlin: Juncker and Dünnhaupt, 1933.

Zeichen der Zeit. Ein deutsches Lesebuch in vier Bänden. Vol. IV. Ed. Walther Killy. Frankfurt am Main - Hamburg: Fischer, 196I (This work also appeared as one volume in Fischer, 1962).

5. NEWSPAPERS AND PERIODICALS

A

AAP
America (New York)

Atti della Accademia Pontaniana (Napoli) 
AB

$\mathrm{Abl}$

Äbl

$\mathrm{Abz}$

AdP

ADV

AfdW

AH

AHR

AIR

AJPH

$\mathrm{AL}$

AM

AMoz

An

APL

APzt

AR

ARsch

As

Ate

$\mathrm{Au}$

AUC

Auf

$\mathrm{Au}(\mathrm{NY})$

AWJD

$A Z$

AZJ

BA

$\mathrm{Bb}$

BBB

BBC

BBM

BBSF

BBZ

Bd

BdBh

BdTG

BET

BF

$\mathrm{Bg}$

$\mathrm{Bib}$

BibS

BIG

Bkg

Bks

Bl

$\mathrm{BM}$

$\mathrm{Bm}$

BMB

$\mathrm{BMCN}$

BN

Bng

Atlantic Bookshelf (Boston)

Abendland (Köln)

Ärzteblatt (Bremen)

Arbeiterzeitung (Wien)

Almanach der Psychoanalyse (Wien)

Almanach des deutschen Volkstheaters (Leipzig - Wien - Zürich)

Alles für die Welt (Baden-Baden)

The American Hebrew (New York)

American Historical Review (New York)

Adam. International Review (London)

American Journal of Public Health (Boston)

Art Libre (Bruxelles)

Atlantic Monthly (Boston)

Acta Mozartiana. Mitteilungen der deutschen Mozart Gesellschaft (Kassel-Wilhelmshöhe)

Athenaeum (London)

Les Annales Politiques et Littéraires (Paris)

Augsburger Postzeitung (Augsburg)

American Review (New York)

Autographen Rundschau

Asia (Concord, N.H.)

Atenea (Concepción de Chile)

Aufbau (Berlin)

Anales de la Universidad de Chile (Santiago de Chile)

Aufstieg (Wiesbaden)

Aufbau (New York)

Allgemeine Wochenzeitung der Juden in Deutschland (Düsseldorf)

Allgemeine Zeitung (München)

Allgemeine Zeitung des Judentums (Leipzig - Berlin; Superseded in 1922 by the Central Verein Zeitung)

Books Abroad (Norman, Oklahoma)

Blaubuch (Berlin)

Baden-Badener Bühnenblatt (Baden-Baden)

Berliner Börsen Courrier (Berlin)

Blätter der Bücherstube am Museum (Wiesbaden)

Blätter der bayerischen Staatsoper. Festspiele 1962. Tagesprogramm. Ed. Rudolf Hartmann.

Berliner Börsen Zeitung (Berlin)

Bund (Bern)

Börsenblatt für den deutschen Buchhandel (Leipzig)

Blätter des deutschen Theaters in Göttingen (Göttingen)

Boston Evening Transcript (Boston)

Bibliografia Fascista (Roma)

Bergstadt (Breslau)

Der Bibliothekar (Leipzig)

Biblioteca Selecta (Panama)

Bayrisch-israelitische Gemeindezeitung (München)

Boekengids (Antwerpen)

Boekenschouw (Amsterdam)

Booklist (Chicago)

Biochemische Monatsblätter (Leipzig)

Bookman (New York)

Bulletin Bibliographique et Pédagogique du Musée Belge(Louvain)

Book of the Month Club News (New York)

Baseler Nachrichten (Basel)

Die Besinnung (Nürnberg) 


Br
Bre
BrsZ
Brü
BS
BT
Btr
BU
BüB
Büg
Büh
BuI
BüK
BuL
BV
BW
BWk
BZ
C
Car
CC
CdS
CDT
CF
CH
CHF
Chm
Chr
Cht
ChW
CJR
ClW
CM
Cmy
Coe
Colq
Com
ConR
ConW
CorS
COS
CR
CV
CVZ
CW
Cwl
D
DA
DAnq
DAZ
DB
DBa
DBei
DBib
DBK

Brunsviga

Brecha (San José, Costa Rica)

Breslauer Zeitung (Breslau)

Brücke. Blatt für Heimatvertriebene (München)

Best Sellers (Scranton, $\mathrm{Pa}$.)

Berliner Tageblatt (Berlin)

Boston Transcript (Boston)

Bibliothèque Universelle et Revue de Genève (Genève)

Bücherei und Bildungspflege (Leipzig-Stettin)

Büchergilde (Frankfurt am Main)

Die Bücherhalle (Leipzig)

Berichte und Informationen (Salzburg)

Bücher-Kommentare (Stuttgart-Berlin)

Buch und Leben (Stuttgart)

Bogens Verden (København)

Bühne und Welt (Berlin-Leipzig-Wien)

Book Week (Chicago)

Berliner Zeitung (Berlin)

Colliers (New York)

Carrefour (Paris)

Christian Century (Chicago)

Cahiers du Sud (Marseille)

Chicago Daily Tribune (Chicago)

Canadian Forum (Toronto)

Current History (Chicago)

Current History and Forum (New York)

Churchman (London)

Christendom (Chicago)

Chantecler

Die christliche Welt (Marburg)

Contemporary Jewish Record (New York)

The Classical Weekly (New York)

Civilta Moderna (Firenze)

Commentary (Chicago)

Coenabium (Lugano)

Colloquium (Berlin)

Commune (Paris)

Contemporary Review (London)

Congress Weekly (New York)

Corriere della Sera (Milano)

Cleveland Open Shelf (Cleveland, Ohio)

Chicago Review (Chicago)

Caritas. Zeitschrift des schweizerischen Caritasverbandes (Luzern)

Central Verein Zeitung (Berlin; Cf. AZJ above)

Catholic World (New York)

Commonweal (New York)

Demain (Genève-Paris)

Dresdener Anzeiger (Dresden)

Das Antiquariat (Wien)

Deutsche Allgemeine Zeitung (Berlin)

Die Bühnenkritik (Augsburg)

Die Barke (Frankfurt am Main)

Deutsche Beiträge (München)

Deutsche Bibliographie (Frankfurt am Main)

Deutscher Bibliophilen Kalender (Wien; Later became Jahrbuch deutscher Bibliophilen) 


\begin{tabular}{|c|c|}
\hline DBP & Deutsche Blätter in Polen (Posen) \\
\hline $\mathrm{DBu}$ & Das deutsche Buch (Leipzig) \\
\hline DD & Deutsche Dichtung (Berlin) \\
\hline DDi & Die Dichtung (Berlin-München) \\
\hline DDr & Das deutsche Drama (Berlin) \\
\hline DeG & De Gids (Amsterdam) \\
\hline DeuE & Deutschlands Erneuerung (München) \\
\hline DeuR & Deutsche Rundschau (Berlin) \\
\hline DeuV & Deutsches Volkstum (Hamburg) \\
\hline DF & Die Fackel (Wien) \\
\hline $\mathrm{DFa}$ & Der Fackelreiter (Hamburg-Bergedorf) \\
\hline DFR & Deutsch-französische Rundschau (Paris) \\
\hline $\mathrm{DFu}$ & Der Funke (Leipzig) \\
\hline DG & Die Getreuen (Hamburg) \\
\hline $\mathrm{DgD}$ & Das ganze Deutschland (Detmold) \\
\hline DGT & Das goldene Tor (Lahr) \\
\hline DH & Die Horen (Berlin) \\
\hline $\mathrm{Di}$ & Dial (New York) \\
\hline DIZ & Deutsche Internierten Zeitung (Bern) \\
\hline DJZ & Das junge Zentrum \\
\hline DK & Die Kultur (Stuttgart) \\
\hline $\mathrm{DKw}$ & Der Kunstwanderer (Berlin) \\
\hline DL & Die Literatur (Berlin; Before 1923 called Das literarische Echo) \\
\hline DIDÖ & Das literarische Deutsch-Österreich (Wien) \\
\hline DLE & $\begin{array}{l}\text { Das literarische Echo (Berlin; After Vol. XXV (1923) renamed } \\
\text { Die Literatur, see above) }\end{array}$ \\
\hline DLe & Die Lebenden (Görlitz) \\
\hline DLW & Die literarische Welt (Berlin) \\
\hline DLZ & Düsseldörfer Lokal-Zeitung (Düsseldorf) \\
\hline DLz & Der Lesezirkel (Zürich) \\
\hline DLZg & Deutsche literarische Zeitung (Berlin) \\
\hline $\mathrm{DM}$ & Die Masken (Düsseldorf) \\
\hline DMfC & Deutsche Monatshefte für Chile \\
\hline DMo & Der Monat (München) \\
\hline DMW & Deutsche medizinische Wochenschrift (Leipzig) \\
\hline DN & Die Nation (Berlin) \\
\hline DNE & Das neue Europa (Zürich) \\
\hline DNG & De nieuwe Gids (Den Haag) \\
\hline DNN & Dresdener Neueste Nachrichten (Dresden) \\
\hline DNS & Die neueren Sprachen (Marburg) \\
\hline DNSch & Die neue Schau (Kassel) \\
\hline $\mathrm{DNZ}$ & Die neue Zeit (New York-Leipzig) \\
\hline DNZg & Die neue Zeitung (München) \\
\hline Do & Donauland (Wien) \\
\hline DoA & Dorem Afrike (Johannesburg) \\
\hline DoiB & Doitsu Bungaku (Tokyo) \\
\hline DPbl & Deutsches Pfarrerblatt (Stuttgart) \\
\hline DPr & Die Presse (Wien) \\
\hline DR & Deutsche Republik (Berlin-Frankfurt am Main) \\
\hline $\mathrm{DRa}$ & Die Rampe (Hamburg) \\
\hline DRg & Der Ring (Berlin) \\
\hline DRZ & Deutsche Reichs-Zeitung (Bonn) \\
\hline DS & Die Schrift (Brünn) \\
\hline DSa & Die Sammlung (Göttingen) \\
\hline DSch & Die Schaubühne (Berlin) \\
\hline DSL & Die schöne Literatur (Leipzig; After I930 called Neue Literatur) \\
\hline
\end{tabular}




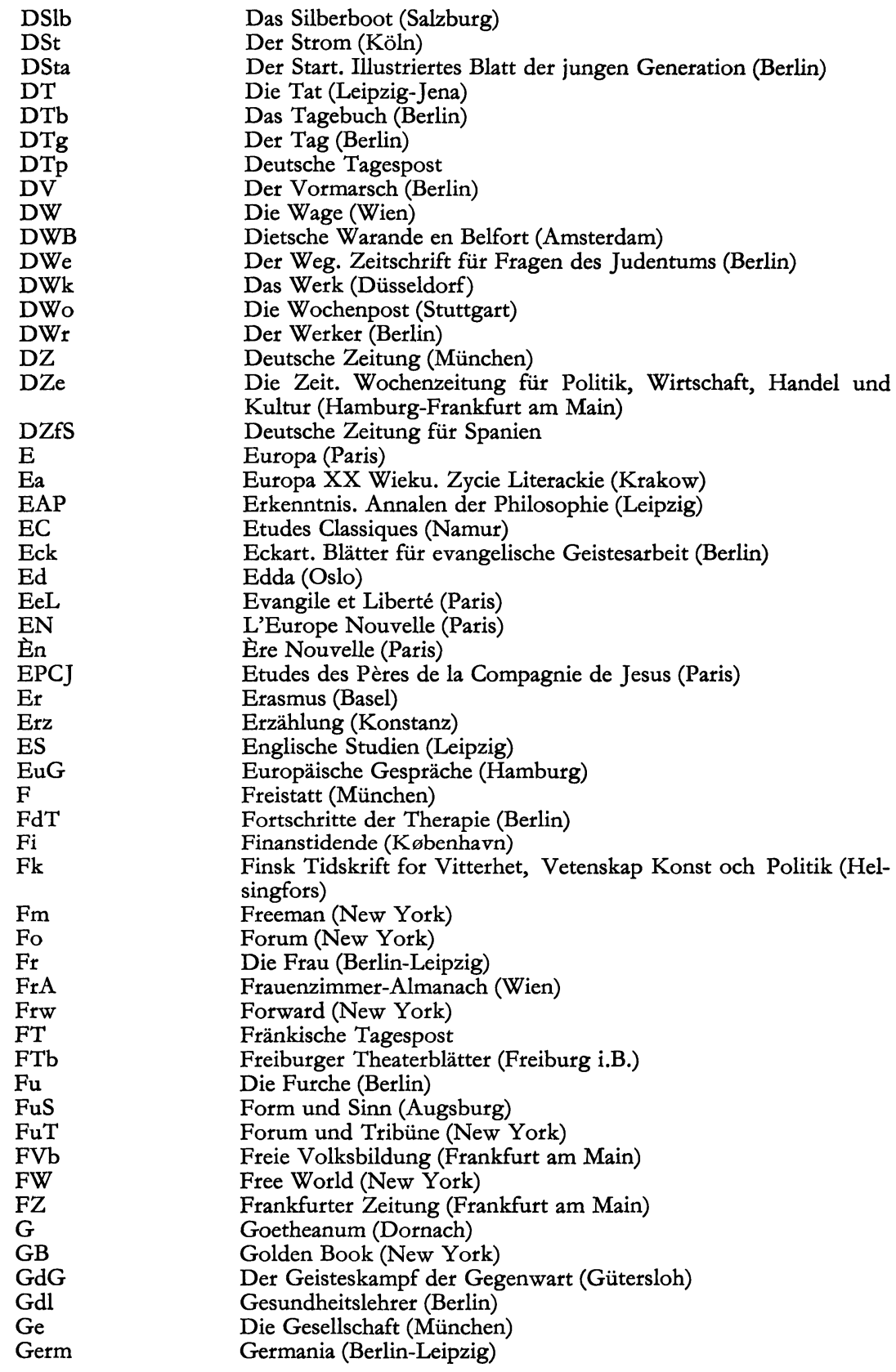




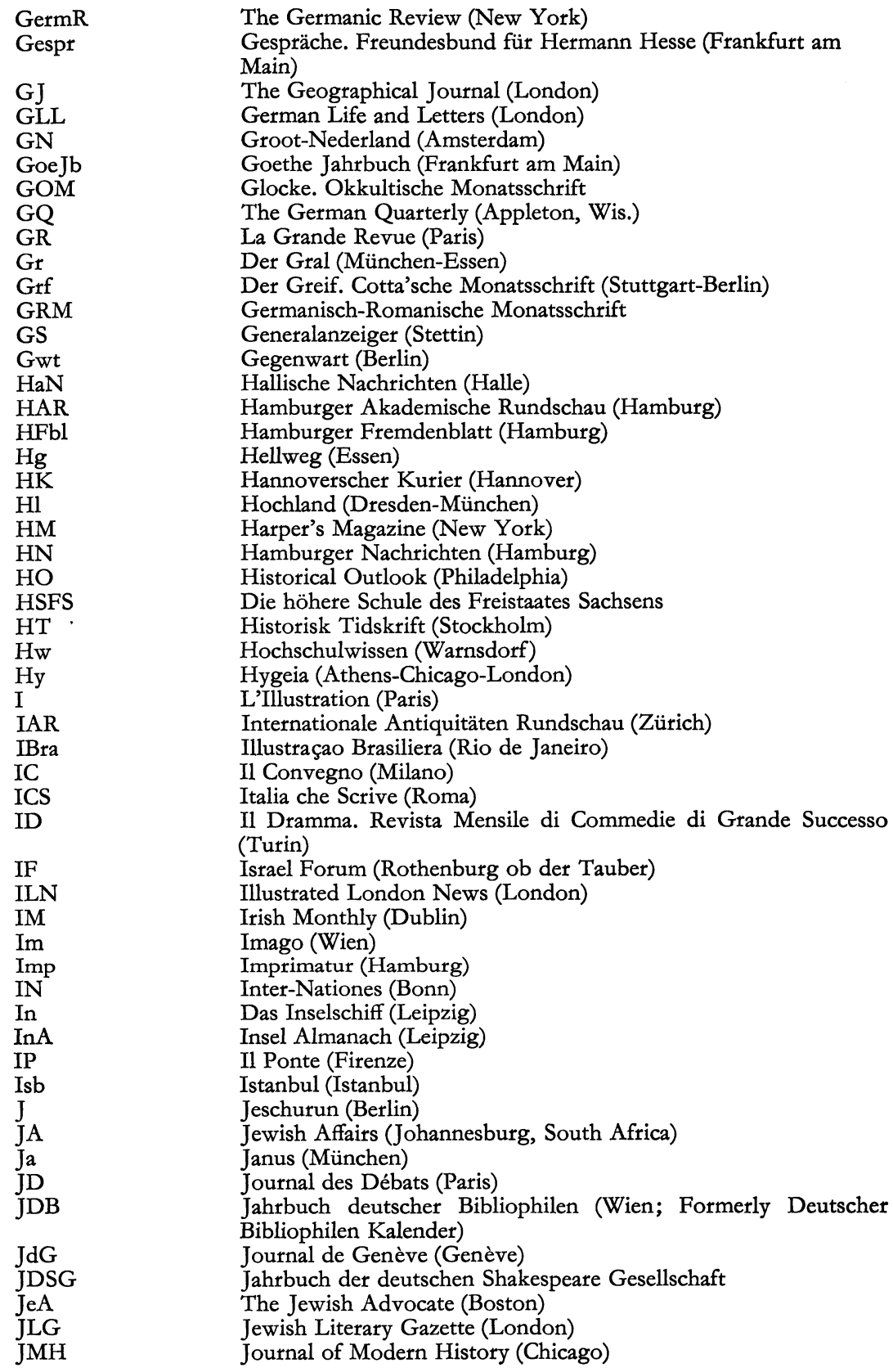




\begin{tabular}{|c|c|}
\hline JNMD & Journal of Nervous and Mental Diseases (Richmond, Va.) \\
\hline & Jüdische Rundschau (Berlin) \\
\hline JSp & $\begin{array}{l}\text { Jewish Spectator (New York) } \\
\text { Jïdischer Almanach (Berlin) }\end{array}$ \\
\hline JüGbl & $\begin{array}{l}\text { Judischer Almanach (Berlin) } \\
\text { Das jüdische Gemeindeblatt für die britische Zone (Düsseldorf) }\end{array}$ \\
\hline JulR & The Julliard Review (New York) \\
\hline $\mathrm{K}$ & Kirkus (New York) \\
\hline $\mathrm{KAZ}$ & Königsberger Allgemeine Zeitung (Königsberg) \\
\hline $\mathrm{KD}$ & Katholischer Digest (Aschaffenburg) \\
\hline KdöL & Die Kritik des öffentlichen Lebens (Berlin) \\
\hline $\mathrm{KHZ}$ & Königsberger Hartung'sche Zeitung (Königsberg) \\
\hline KNN & Kasseler Neueste Nachrichten (Kassel) \\
\hline KöT & Kölner Tageblatt (Köln) \\
\hline $\mathrm{KP}$ & Kasseler Post (Kassel) \\
\hline $\mathrm{Kr}$ & Der Kreis (Berlin) \\
\hline $\mathrm{Krl}$ & Kristall (Hamburg) \\
\hline $\mathrm{KrZ}$ & Karlsruher Zeitung (Karlsruhe) \\
\hline KS & Kirjath Sepher (Jerusalem) \\
\hline $\mathrm{KT}$ & Kasseler Tageblatt (Kassel) \\
\hline $\mathrm{Kt}$ & Der Kunstwart (München) \\
\hline KVZ & Kölnische Volks-Zeitung (Köln) \\
\hline $\mathrm{KZ}$ & Kölner Zeitung (Köln) \\
\hline $\mathrm{L}$ & Leonardo (Firenze) \\
\hline LA & Living Age (Boston) \\
\hline LAM & L'Approdo Musicale (Roma) \\
\hline LB & Literarische Blätter (Genf) \\
\hline LC & Le Carmel (Zürich) \\
\hline LCFD & Literarisches Centralblatt für Deutschland (Leipzig) \\
\hline LCP & Les Cahiers Protestants \\
\hline LCQ & The Lutheran Church Quarterly (Gettysburg, Pa.) \\
\hline LD & Literary Digest (New York) \\
\hline LF & La Flamberge (Mons, Belgium) \\
\hline LGRP & $\begin{array}{l}\text { Literaturblatt für germanische und romanische Philologie (Heil- } \\
\text { bronn-Leipzig) }\end{array}$ \\
\hline LH & Literarischer Handweiser (Regensburg-Münster) \\
\hline LI & Il Libro Italiano (Roma) \\
\hline Lib & Librarians (Northwestern University Library, Evanston, Ill.) \\
\hline LJ & Library Journal (New York) \\
\hline LJZ & $\begin{array}{l}\text { Leipziger jüdische Zeitung (Leipzig; Later Leipziger jüdisches } \\
\text { Familienblatt) }\end{array}$ \\
\hline LLM & Les Langues Modernes (Paris) \\
\hline LM & The London Mercury (London) \\
\hline LMF & Le Monde Français (Paris-Montreal) \\
\hline LNE & La Nouvelle Equipe (Bruxelles) \\
\hline LNL & Les Nouvelles Littéraires (Paris) \\
\hline LNN & Leipziger Neueste Nachrichten (Leipzig) \\
\hline LNR & La Nouvelle Relève (Montreal, Canada) \\
\hline LP & La Libre Pensée (Lausanne-Paris) \\
\hline LT & London Times (London) \\
\hline LTbl & Leipziger Tageblatt (Leipzig) \\
\hline LW & Literarische Wochenschrift (Weimar) \\
\hline M & $\begin{array}{l}\text { Der Merker. Österreichische Zeitschrift für Musik und Theater } \\
\text { (Wien) }\end{array}$ \\
\hline MA & Musical America (New York) \\
\hline $\mathrm{MaL}$ & Music and Letters (London) \\
\hline März & März (Leipzig) \\
\hline
\end{tabular}




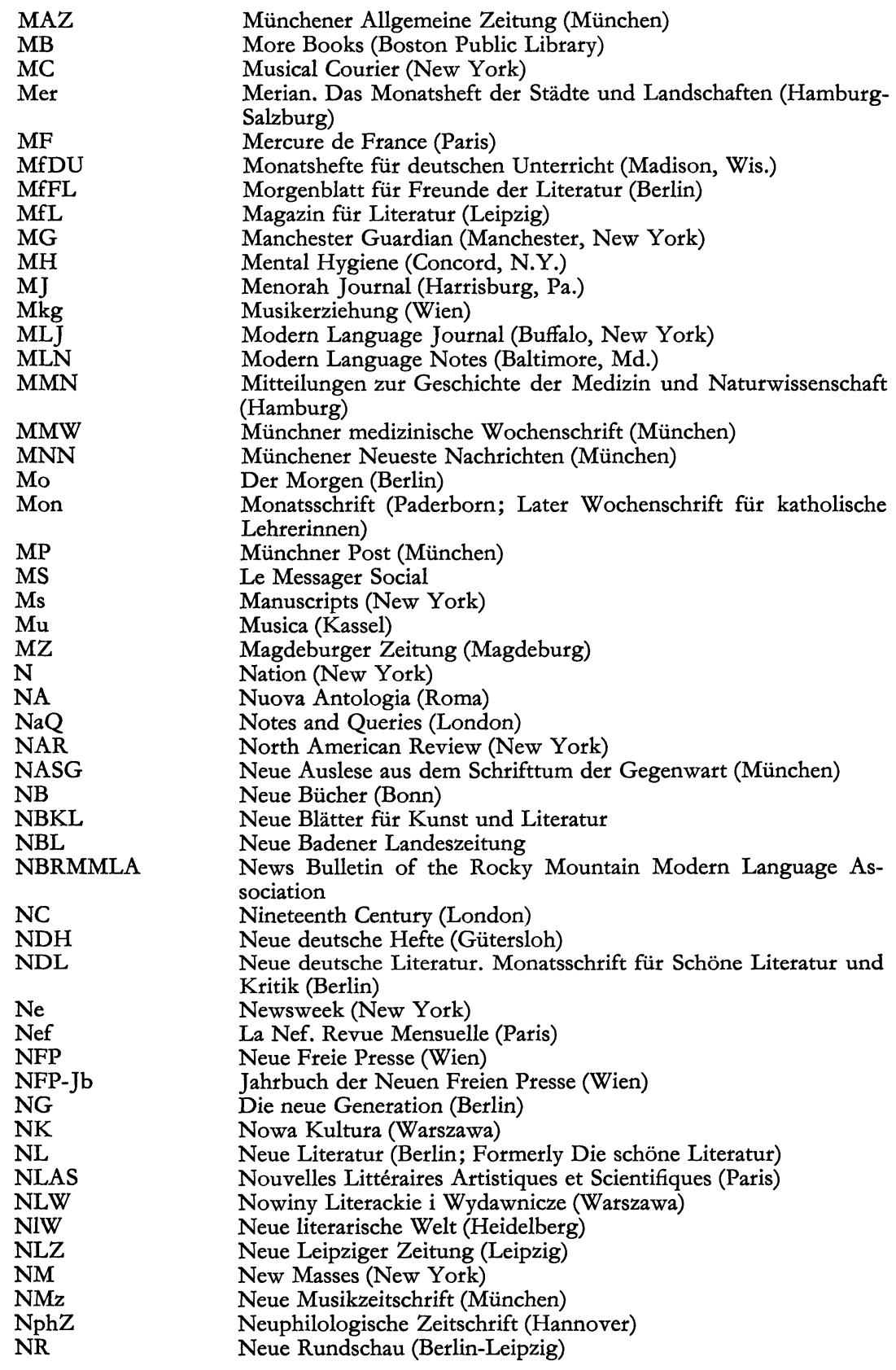




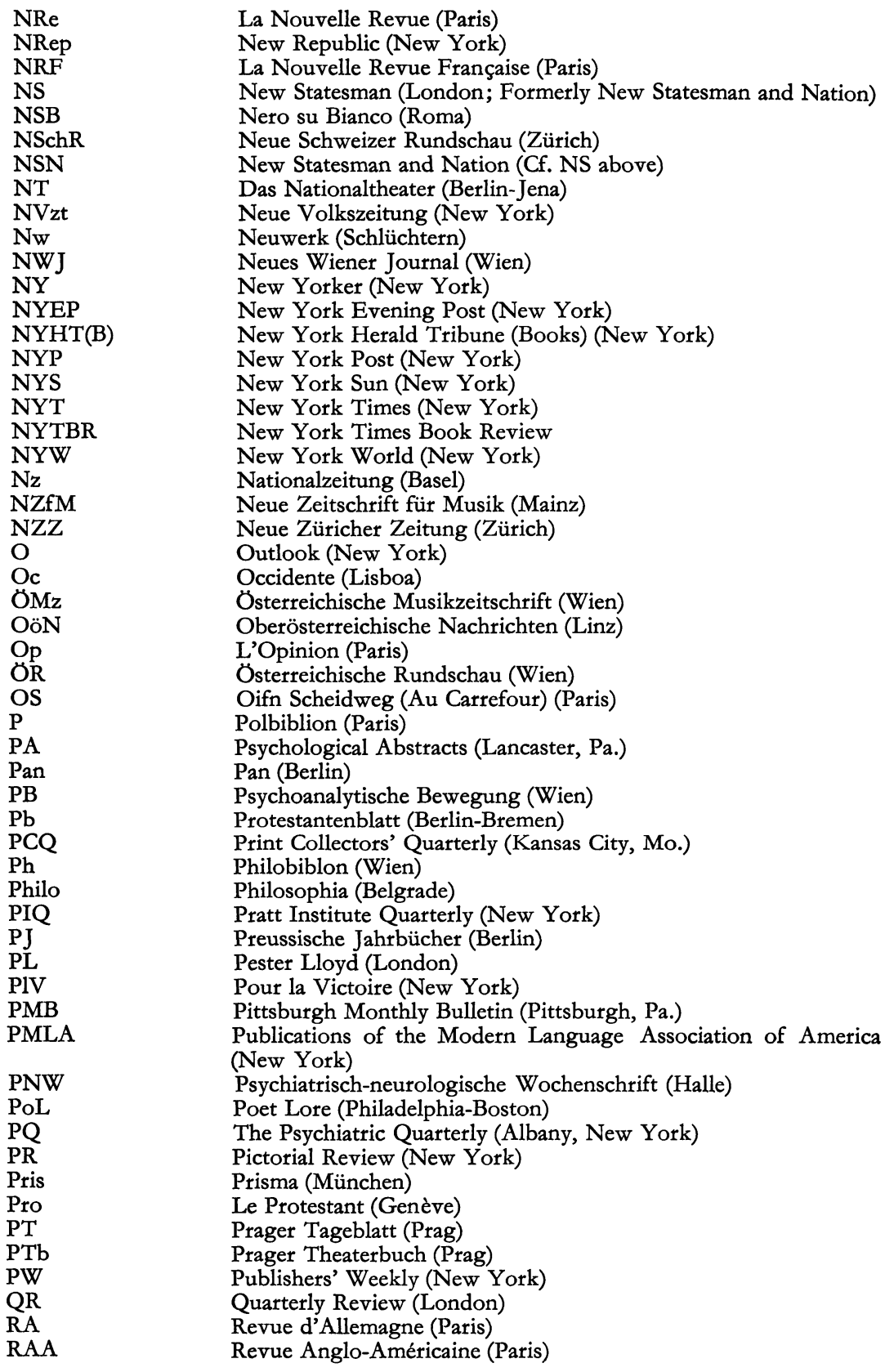




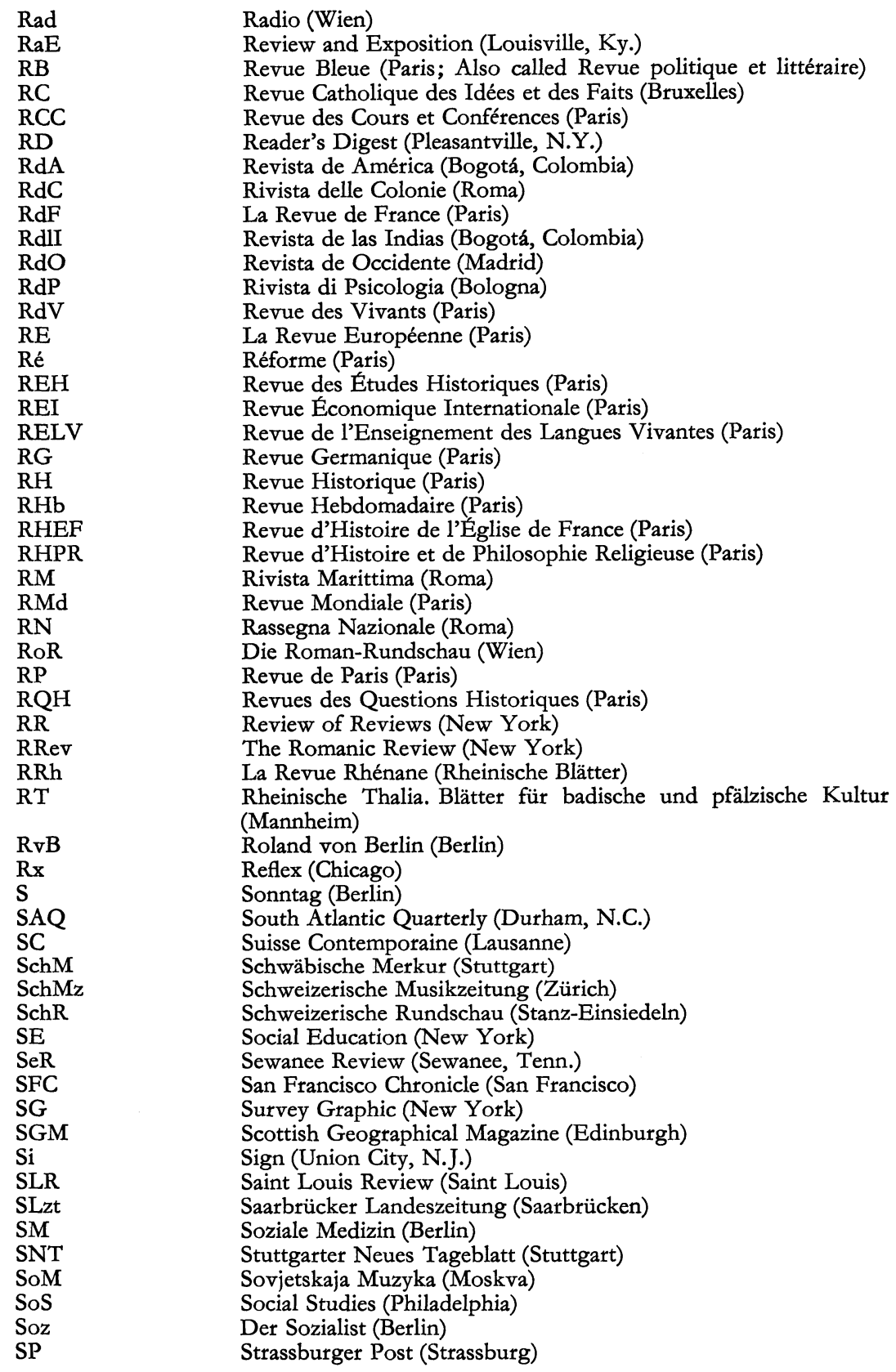




Sp
SR
SRL
SS
SSA
SSR
ST
Sy
T
Tab
TAM
Tb
Th
TL
TR
Tr
TrG
Ts
Two
U
Ud
UfL
Um
Un
Uns
V
Vbü
VdF
Ven
Ver
VFZM
Vin
VK
VP
VQR
VuV
VV
VZ
W
WaM
WAZ
Wbü
WdA
WE
WHC
Wi
WiaL
WidZ
WieZ
WL
WLB
WM
WR
Wst
Wt

Spectator (London)

Springfield Republican (Springfield, Mass.)

Saturday Review of Literature (New York)

Sächsische Schulzeitung

Social Science Abstracts (Menasha, Wis.)

The Social Service Review (Chicago)

Salzburger Tagblatt (Salzburg)

Survey (New York)

Time. The Weekly Magazine (New York)

Tablet (London-Boston-Brooklyn)

Theater Arts Monthly (New York)

Tagebuch (Berlin)

Thought (New York)

Trybuna Ludu (Warszawa)

Tägliche Rundschau (Berlin)

Travel (New York)

Tribune de Genève (Genève)

Le Temps (Paris)

Twórczość (Warszawa)

Universidad (Zaragoza)

Universidad (Mexico City)

Ugeskrift for Laeger (København)

Die Umschau (Frankfurt am Main)

Universum (Leipzig)

Universitas (Stuttgart)

Vorwärts (New York-Leipzig)

Volksbühne (Hamburg)

La Voix de France (New York)

Vendredi (Paris)

Veritas (Buenos Aires)

Vom Fels zum Meer (Berlin)

Vinduet (Oslo)

Velhagens und Klasings Monatshefte (Leipzig)

$\mathrm{La}$ Vie Protestante

Virginia Quarterly Review (Charlottesville, Va.)

Verbannte und Verbrannte (Berlin)

Vivos Voco (Leipzig)

Vossische Zeitung (Berlin)

Weltwoche (Zürich)

Welt am Montag. Wiener Wochenzeitung (Wien)

Wiener Allgemeine Zeitung (Wien)

Weltbühne (Berlin)

Welt der Arbeit (Köln; Continuation of Der Bund, Nos. 33445747)

Welt Echo. Aktuelles aus aller Welt (Innsbruck)

Woman's Home Companion (Springfield, Ohio)

Witiko (Kassel)

Wiadomości Literackie (Warszawa)

Wort in der Zeit (Graz)

Wiener Zeitung (Wien)

Wissen und Leben (Zürich)

Wisconsin Library Bulletin (Madison, Wis.)

Westermanns Monatshefte (Braunschweig)

Wissenschaftliche Rundschau (Leipzig)

Weltstimmen (Stuttgart)

Die Wirtschaft (Prag) 


$\begin{array}{ll}\text { WU } & \text { World Unity (New York) } \\ \text { WuW } & \text { Welt und Wort (Tübingen) } \\ \text { WW } & \text { Wirkendes Wort (Düsseldorf) } \\ \text { W-Z } & \text { Weser - Zeitung (Bremen) } \\ \text { WZV } & \text { Weg zur Vollendung (Darmstadt) } \\ \text { Ybk } & \text { Yearbook of the Leo Baeck Institute of Jews from Germany } \\ \text { YCD } & \text { (London) } \\ & \text { Yearbook of the Cemetery Department. Jewish Workmen's } \\ \text { YR } & \text { Circle (New York) } \\ \text { Z } & \text { Yale Review (New Haven, Conn.) } \\ \text { ZB } & \text { Die Zukunft (Berlin) } \\ \text { ZD } & \text { Zeitschrift für Bücherfreunde (Bielefeld-Leipzig) } \\ \text { Ze } & \text { Zeitschrift für Deutschkunde (Leipzig) } \\ \text { ZFEU } & \text { Die Zeit (Wien) } \\ \text { ZfG } & \text { Zeitschrift für französischen und englischen Unterricht (Berlin) } \\ \text { ZP } & \text { Zeitschrift für Geopolitik (Darmstadt) }\end{array}$




\title{
INTRODUCTION
}

\author{
STEFAN ZWEIG - BIOGRAPHICAL SKETCH \\ "Paix adviendra et malheur cessera. \\ Mais entre deux que mal l'on souffrera!"1
}

On February 22, 1942, Stefan Zweig and his second wife, Lotte Altmann Zweig, committed suicide. To those who loved him the news was a terrible blow; to those who considered him to be a stalwart ally against the madness of the times, his death came almost as a willful desertion of a common cause. But in the minds of all the same question was evident: why? Indeed, his was not a solitary fate - one need but mention Tucholsky, Toller, Hasenclever, E. Weiss, Friedell many had preceded him, others were to follow him and yet for some reason his fate does seem unique. Kurt Böttcher would see in Zweig a singular, representative figure who realized in his person:

... jenes Charakteristische also für eine Gruppe von humanistischen bürgerlichen Schriftstellern mit gleicher oder ähnlicher gesellschaftlicher und geistiger Position sowie menschlicher Beschaffenheit. ${ }^{2}$

Undoubtedly there is some partial justification for this view but it would seem to fall far short of explaining the individual magnetism and creative spirit which was Zweig's. If he must be a symbol, in justice to him, the symbol should be that of a life dedicated to international understanding and not merely that of a certain social-intellectual reserve.

Zweig was born on the 28th of November, I88I, into a wealthy Jewish family, which, having made a fortune in the textile industry in Czechoslovakia, removed to Vienna about the middle of the nineteenth century. Stefan's father, Moritz Zweig, married an ItalianGerman girl whose father was an international banker of great repute, having done business even for the Vatican. ${ }^{3}$ In keeping with customs of the time, Stefan and his older brother were cared for almost entirely by nurses and governesses. This obvious lack of motherly affection and the many restrictions placed on children, in keeping with social decorum, often arrested the growing spirits of the younger generation. 
His mother was a very vivacious woman, but one of very fixed, often obstinate ideas, a trait which Stefan seems to have inherited, along with an unbending resentment of his systematized life as a child. ${ }^{4}$

The family vacations were usually spent dashing from one spa to another amid baggage far in excess, noise, and a regular domestic cortege. As a result, when older, Zweig never took anything with him when he traveled, leaving for anywhere on the spur of the moment. 5

At the age of six Zweig started his school career - a long, dull struggle in dark rooms with bored teachers, whose lives did not correspond with their contemporary society. Sports and dating were still unheard of, their stead being filled with cultural pursuits: literaryphilosophical debates in cafés, extensive reading, regular attendance at opera premières or theatrical performances in Vienna's Burgtheater. ${ }^{6}$

The turn of the century saw an almost revolutionary change in attitude and mode of expression in European Letters. As the onesided, aggressive, tendentious Naturalism had thirty years previously supplanted the historic Epigonentum of the $1860 \mathrm{~s}$ and $1870 \mathrm{~s}$, so in its turn was Naturalism supplanted by a vogue of Impressionism Expressionism. French, Belgian, Norwegian painting and literature were everywhere considered to be of leading significance; Debussy and Strauss dominated the field of music as did Nietzsche the realm of philosophy. Rimbaud, Verlaine, Zola, Hauptmann, Rilke, Dostoyevs$\mathrm{ky}$ - such were the dominant figures of the age, names which today seem enwrapped in the sobering effects of distance, but which at that time were names of a younger generation struggling to attain new forms of expression to correspond to their new forms of thought. This new literary movement was amply represented in the "Jung Wien" school which boasted such members as Schnitzler, Hermann Bahr and the young Hofmannsthal, who at nineteen had become the hero of his generation through an essay on art and his exquisitely mature poems. ${ }^{7}$

Having graduated from the Gymnasium in 1900, Zweig enrolled immediately at the University of Vienna. He attended very few classes but read everything of modern literature that he could find. Fearing little success, he nevertheless sent an article to the editor of the leading Viennese newspaper, Die Neue Freie Presse, Theodor Herzl, 8 requesting its publication in Herzl's Feuilleton section which normally contained articles on art, music, the theater and literature, contributed only by well known writers. When his article was accepted and appeared on the first page it seemed to Zweig that a dream had been realized. By nature modest, Zweig was embarrassed by the prestige and popularity that he had attained, in his own eyes prematurely. To escape unwelcome celebrity as well as to gain more personal freedom, Zweig transferred to the University of Berlin 
(1902), where in a milieu of international intellectualism he formed friendships and ties with artists of other nations which lasted throughout his life (e.g. Ellen Key, Giobanni Cena, Georg Brandes, Peter Hille, Rudolf Steiner). ${ }^{9}$

Through his contacts with local journals in Berlin Zweig was soon introduced into a club known as "Die Kommenden" which consisted of individuals from all social strata, nationality and profession, all united through a common bond: their Bohemian life. The club resembled the literary-café groups which Zweig had frequented in Vienna but how far intellectually superior were the debates, how far more perfected the artistry in Berlin! Zweig actually felt embarrassed in their midst, his self-confidence diminished considerably. He soon realized how childish, how deprived of knowledge of real life his works were. A novel begun in Vienna was soon filed in the stove, its successor appearing only 37 years later. The greater part of the time Zweig spent observing and studying all types of people, from the best to the worst. This psychological curiosity lasted throughout his entire life and by many is considered to be his outstanding characteristic. ${ }^{10}$

By 1910 Zweig could boast of two volumes of verse (Silberne Saiten (I902) and Die früben Kränze (1906)), a volume of four Novellen (Die Liebe der Erika Ewald (1904)), and a drama, Tersites (1907) - all of which were begun under the influence of the new psychological literature predominating in Austria, wherein aestheticism joined forces with irrationalism and cosmic mysticism to produce a neo-romanticism characterized by melodiousness of sound and hues. ${ }^{11}$ Realizing his own inadequacies and short-comings, however, Zweig all but completely abandoned his own writings and dedicated himself almost exclusively to translating foreign works into German: some poems of Baudelaire and Verlaine, a drama by Charles van Lerberghe, a novel by Camille Lemmonier and by rigro the complete works of Emile Verhaeren. Though translation is difficult, demanding a precise knowledge of the nuances of one's own as well as the foreign language, the effort expended is commensurately rewarding in its formative effects.

Und wenn ich heute einen jungen Schriftsteller beraten sollte, der noch
seines Weges ungewiss ist, würde ich ihn zu bestimmen suchen, zuerst einem
grösseren Werke als Darsteller oder Ubbertragender zu dienen. In allem auf-
opfernden Dienen ist für einen Beginnenden mehr Sicherheit als im eigenen
Schaffen, und nichts, was jemals hingebungsvoll geleistet, ist vergebens
getan. ${ }^{12}$

The summer of 1902 Zweig spent in Belgium, where through the mediation of the sculptor van der Stappen, he met Emile Verhaeren for the first time. The two became extremely close friends, a friend- 
ship which meant much more to Zweig than companionship, for from Verhaeren Zweig acquired that very quality which was so painfully missing in his own works - a love of and direct contact with daily life and an awareness that work and responsibility are moral values of the international credo. It is at this period that his former Viennese bourgeois concepts began to dissolve, but to be sure literature still remained for him "eine Steigerungsform der Existenz." Until the outbreak of the First World War in I9I4 (and Verhaeren's death in I9I6) Zweig spent a few weeks each summer with his beloved friend at the latter's summer residence, Caillou-qui-bique. ${ }^{13}$

The year 1904 was spent in Vienna with furious months of hard work in order to obtain his doctorate and thus fulfill a promise which he had made to his parents. He chose a discussion of Taine for his thesis and within a few months received his Degree with honorable mention. Thereafter he was free to live as he saw fit and, driven by a desire to learn everything possible in the shortest possible time, he chose Paris as his port of call. ${ }^{14} \mathrm{He}$ took up residence at the small Hotel Beaujolais in the Palais Royal in the immediate vicinity of the Bibliothèque Nationale and the Louvre, directly across from the former house of Marceline Desbordes-Valmore. ${ }^{15}$ During this first extended stay in Paris he visited every nook and cranny of the city, one might say he literally breathed in the spirit of the French people. Through Verhaeren he met the leading artists of the new Impressionism - Bazalgette, Duhamel, Valéry, Rodin, ${ }^{16}$ Renoir - and was accepted among them as one in a family circle. In no way and by no one was he regarded as a foreigner. That his love for France and especially Paris was so sincere and expansive is but natural under such circumstances. ${ }^{17}$

Zweig's first visit to England (1905) made a rather unfavorable impression upon him. ${ }^{18} \mathrm{He}$ missed the laughing gaiety and charm of the French. The English were far too reserved and, not understanding English too well, Zweig couldn't follow their clipped conversations which seemed to revolve incessantly around sports and politics. Most of his time was spent in the British Museum, studying the writings and drawings of William Blake or translating English works into German. He did, however, manage to purchase Blake's etching "King John" to add to his treasured collection of autographs. ${ }^{19}$

Between I905 and the outbreak of the war in I914 Zweig occupied himself wholly in traveling around the world and translating foreign works (especially Verhaeren) into German. In addition to his works previously mentioned, he published another volume of Novellen: Erstes Erlebnis. Vier Geschichten aus Kinderland (I I I I) and two dramas: Das Haus am Meer (1912) and Der verwandelte Komödiant (1913). He was horrified by the misery and poverty of the masses in India which he visited in I9I2. The climate made him uncomfortable and the 
countryside grew monotonous. Even the riches of the temples seemed more strange than awesome to him. ${ }^{20}$ His journey was not a complete loss to him, however, for he utilized every moment possible in studying the people - on the streets, in cabarets, in opium dens and in absorbing the cults and doctrines of the "Land of Mystery."21

The first visit to America (I9I2) was an event of somewhat mixed reactions. Zweig was greatly impressed by the beauties of the Hudson Valley and the unheard of tolerance toward other races and religions. New York, too, was fascinating for a time. ${ }^{22}$ In Philadelphia he saw some of his works on display, a fact which greatly flattered his ego and did much to enhance his disposition toward the New World. Before returning to his small apartment in Vienna, Kochgasse 8, which was to be his home until his removal to Salzburg, Zweig visited the final construction on the Panama Canal, ${ }^{23}$ the impressions of which he crystalized in his essay: "Die Stunde zwischen zwei Ozeanen."24

When World War I broke out Zweig was working on his literaryhistorical essay about Dostoyevsky. He had planned a trip to Russia to acquaint himself personally with the Russian people and atmosphere but the outbreak of hostilities put an immediate end to his projected trip. ${ }^{25}$ After the War such conflicting reports were received about Russia that he couldn't make up his mind to go there. It wasn't until I928 that he finally visited Russia and then as an invited delegate to celebrate the Tolstoy centenary. ${ }^{26} \mathrm{He}$ closely observed the enthusiasm of the masses, the educational system, and the technological advances; he was greatly impressed. The journey was not without its somber note, however, in spite of the cordial reception given him. Just before leaving the country he found a note in his pocket, written in French, warning him not to be deceived by appearances, all was not as progressive as one might suppose, freedom was being sacrificed to technology and the people were slowly being imprisoned in their own homeland. ${ }^{27}$

Friendships were always a decisive influence on Zweig - two in particular, with Verhaeren and Romain Rolland. As mentioned above, Zweig first met Verhaeren in 1902 through the mediation of the Belgian sculptor van der Stappen. Between 1902 and I9 10 Zweig translated Verhaeren's complete works and even arranged a lecture tour in Germany for him, having previously aided in the staging of some of Verhaeren's lyric-dramas, though to be sure not without some misgiving concerning their reception by the public - an anxiety, however, which proved to be totally unfounded. The two friends saw each other for the last time in the spring of 1914 , the war preventing Zweig from visiting Verhaeren in Belgium and, long before the armistice was signed, Verhaeren was dead, having been run over by a train in I9I6. One of the greatest sadnesses in Zweig's life was 
a direct result of Verhaeren, who in I9Is published his La Belgique Sanglante, a work in which he poured out all the penned up hatred in his heart for Germany and its people who had wrought such havoc on Europe and occupied his beloved Belgium. To Zweig such scathing bitterness, such unadulterated hatred seemed a desertion of the cause of international brotherhood which Zweig had first experienced so poignantly through Verhaeren. Though disappointed that even Verhaeren should succumb to the mass hatred so rampant in Europe, Zweig could not help but love him dearly to the end, as his tribute "Erinnerungen an Emile Verhaeren" so eloquently testifies. ${ }^{28}$

The other great friendship that contributed so much to Zweig's mental and emotional development was that with Romain Rolland. ${ }^{29}$ While in Paris (I9I3), just by chance Zweig read Rolland's "L'Aube" in the periodical Cabiers de la Quinzaine. Inquiring about the author, Verhaeren informed him that he was primarily known as a musicologist through his essay on Beethoven. A meeting of the two was arranged by Verhaeren and from their first encounter they both were aware of the harmony of mind and spirit which they shared - a harmony which served as the basis for an intimate, brotherly friendship which was to endure some forty years.

In August, I9I4, Rolland published his essay Au-dessus de la mêlée in which he sought to combat the hatred and fanaticism of the intellectuals from whom alone a spiritual fraternity could flow. ${ }^{30}$ Shortly thereafter (September I9, I9I4) Zweig published his "Abschiedsbrief an die Freunde im Ausland" in the Berliner Tageblatt wherein he bid a temporary farewell to his foreign friends, promising not to forget them and begging them to work with him again after the war to reconstruct European culture. ${ }^{31}$ Most of Zweig's German friends began to avoid him, feeling him to be unpatriotic and Zweig himself would have wanted to be classed as a conscientious objector. $\mathrm{He}$ lacked, however, the strength to face the consequences of such flagrant anti-national sentiment and soon took a position in the War Archives. Though isolated in his native Vienna, Zweig was not "alone" for very long - soon letters from Rolland began to reach him, urging him to aid in organizing a congress of intellectuals to meet in Switzerland, whose primary aim would be to work for peace and international understanding. Neither Zweig nor Rolland found much support, the time was still premature, the invigorating effects of the night before hadn't had quite enough time to transform into the nausea of the morning after. ${ }^{32}$

The war was another decisive factor in Zweig's life. He fought the war-mania with all the powers at his command and in his effort to oppose the unreasonableness of sheer force he raised the tragic defeat of the conquered to a poetic principle. ${ }^{33}$ His opposition to the war was based primarily on intellectual, humane grounds, not on an 
understanding of the economic causes which had aided the explosion at Serajevo. Zweig saw his task in the propagation of anti-war thought (a position in which he was greatly encouraged by Rolland), a task which he fulfilled in his essays, his prose works and above all in his drama Jeremias. Early in the war he wrote reviews of such antimilitary works as Le Feu (1916) by Henri Barbusse ${ }^{34}$ and the Die Waffen nieder (Novel, 2 volumes, I 889) of Bertha von Suttner, the founder of the Pacifist Movement who had persuaded Alfred Nobel to establish his Peace Prize. Zweig's later Novellen "Der Zwang" (1920), "Episode am Genfersee" (1918) and "Buchmendl" (1929) are stirring protests against the senselessness and barbarism of war.

During the Spring of I9 Is Zweig was sent to Galicia to collect Russian posters for the State Archives. The dirt, filth and suffering that surrounded him on every side revolted him and he decided to combat such conditions directly and vigorously. ${ }^{35}$ The immediate outcome of his decision was the drama Jeremias, a symbolic impassioned plea for peace which, though clothed in the biblical garb of the prophet Jeremiah, sought to expose the crimes of warmongers and the tragic consequences of their wars. He represented the suffering of the people and attempted to point out a spiritual-moral path for the future - an unbroken brotherhood of all peoples. The drama first appeared in 1917 during the Easter season and almost overnight 20,000 copies were sold. The director of the Züricher Theater proposed to produce the play and in February, I91 8, Jeremias enjoyed an overwhelmingly successful première with Zweig attending the performance. ${ }^{36}$

While in Switzerland Zweig met and worked again with most of the influential, anti-war intellectuals of Europe who had emigrated to neutral territory: Romain Rolland, Masereel, Leonhard Frank, Franz Werfel, among others. It was a period of furious activity for all of them, all with but one objective: peace. ${ }^{37}$ Zweig lectured and wrote article after article for the periodicals La Feuille and Demain. ${ }^{38}$ An article he wrote in 1917 entitled "Das Herz Europas" describes in terms of admiration the selflessness and devotion of Rolland in his voluntary services in the Red Cross agency for the repatriation and solace of war prisoners. Though written primarily in honor of Rolland, his idealism and self-sacrifice can easily be applied to the group as a whole. ${ }^{39}$ With the signing of the armistice in I9I 8 the group as such broke up, each returning to his respective country with but one thought in mind - to rebuild Europe as a united whole and to establish international brotherhood and peace. ${ }^{40}$

In I91 2 Zweig had met a certain Frau Friderike von Winternitz (née Burger, born I882), the wife of Felix von Winternitz and the mother of two small daughters. In the course of two years the two fell in love and Friderike left her husband. They moved near to one 
another at Baden, Austria, and then together to Rodaun in I9r6. In 1920 their life together was legalized by a new Austrian law which allowed divorced Catholics to remarry. From the end of World War I to their divorce on December 25, 1938, the two were inseparable companions and help-mates, of one mind and body. ${ }^{51}$ In 1917 , while in Salzburg, Stefan saw a vacant house, an abandoned hunting lodge, on the Kapuzinerberg, just above the city. The site provided everything one could desire - quietude, gardened landscape, a beautiful view, everything - and miracle of miracles, it was for sale. Stefan decided immediately in favor of buying it and his wife completed negotiations for its purchase. ${ }^{42}$ With the signing of the armistice Zweig decided to return immediately to Austria, in spite of the misery and deplorable conditions the war had left in its wake. The house on the Kapuzinerberg was almost in ruins and, with the scarcity of building materials, it was months before the house was really habitable. ${ }^{43}$ The years Zweig spent in Salzburg (1919-1934) were the happiest and most productive of his life. The city itself was an ideal setting - a romantic blend of German Gothic and Baroque with Italian Renaissance, picturesque natural surroundings, within easy reach of the leading European capitals, and, for a few years at least, a harbor of quiet and rest. ${ }^{44}$ It was only then that the Salzburg Festival grew to monstrous proportions, and during the Mozart Season his home became the gathering place of the world's great: Max Reinhardt, Thomas Mann, Franz Werfel, Emil Ludwig, Arturo Toscanini, ${ }^{45}$ Bruno Walter, ${ }^{46}$ Richard Strauss, Albert Einstein, Duhamel, Ravel, Hermann Bahr, ${ }^{47}$ Albert Schweitzer, ${ }^{48}$ Gustav Mahler ${ }^{49}$ and of course Rolland and Masereel, ${ }^{50}$ to mention but a few. ${ }^{51} \mathrm{Had}$ he been able to foresee the future importance of the city with the resultant loss of his own seclusion Zweig undoubtedly would never have purchased the house. For a time Zweig almost buried himself in Salzburg except for a few side excursions to Italy and France. The signs of rising Fascism in Italy and Germany and the assassination of Walter Rathenau were disturbing, but on the whole life was rather normal.52 By 1933 he had published over so books, Novellen and essays and was asked by the Insel Verlag, a distinguished publishing company, to be allowed to publish all his works. His writings were soon translated into almost every European language and Chinese, with introductions by such famous authors as Maxim Gorki and Romain Rolland. It was estimated by a committee of the League of Nations that by 1930 he was the most translated author in the world. ${ }^{53}$ It was during this period that Zweig's most famous works appeared, among them Drei Meister (1920), the Novellen, Der Zwang and Angst (both I920), Romain Rolland (1921), the Novellen collection, Amok (1922), Der Kampf mit dem Dämon (1925), Verwirrung der Gefüble (1927), Sternstunden der Menschbeit (1927), Drei Dichter ibres Lebens (1928), 
Joseph Fouché (1929), Die Heilung durch den Geist (1931) and Marie Antoinette (1932). ${ }^{54}$ In November, I93 I, Zweig celebrated his 5oth birthday, a birthday which was remembered throughout the world, for from every corner of the globe best wishes and congratulations poured into Salzburg, feelings which were perhaps most fittingly summed up in an article of praise and affection written for the Neue Rundschau by Otto Zarek. ${ }^{55}$ The following year Zweig was approached by Richard Strauss who asked him to write the libretto to an opera he had planned, Die scbweigsame Frau. Zweig gladly accepted, not only because of the literary pleasure such a task would provide but in part flattered that Strauss had asked him to assume a position which had been the exclusive prerogative of Austria's literary esthete, Hugo von Hofmannsthal, until his death in $1929 .{ }^{56}$

Zweig was not only internationally famous and revered but equally loved and respected by the people of Salzburg with whom he would spend a few evenings a week in "his café," reading the newspapers and listening to the radio (having banned such disturbing elements from his house). All in all life was very pleasant and then January, I933, Hitler seized power in Germany!

Life changed almost over night. With the burning of the Reichstag the signal for an all out persecution of the Jews began. Not only were their personal liberties abrogated and their human dignity degraded but their works proscribed almost in toto. A film based on Zweig's "Brennendes Geheimnis" was suppressed, the opera, Die scbweigsame Frau, was banned after only one performance and his works were publicly burned and forbidden either to be sold, read or kept in a public or private collection. Zweig was not alone, however, for this "literary exile" from Germany was shared equally by the majority of the best writers of the German language (Th. Mann, Brecht, Brod, Kafka, Remarque, Schnitzler, Beer-Hofmann, Freud, etc., etc., etc.), but one which completely annihilated German literature from I933 until the collapse of the "Tausendjähriges Reich" twelve years later. ${ }^{57}$

In order to watch the progress of things at a distance, Zweig went to London via Switzerland (October ,1933). At that time he had no thought of permanently leaving Salzburg. ${ }^{.8}$ The calmness of the life in England had a soothing effect on him and soon he was at work again. The result of this short sojurn was his biography of Mary Stuart. ${ }^{59}$ By January, 1934, he was back in Vienna, but it was only too obvious that the situation on the continent was in no sense getting better. On the contrary, even his former friends and acquaintances in Salzburg avoided him. ${ }^{60}$ It was at this time that Zweig decided to leave Austria and set up housekeeping in London. Not wishing to leave her aging mother-in-law alone, Mrs. Zweig remained in Austria. Realizing, however, that Zweig could not carry on his research alone, 
Mrs. Zweig procured for him a young Jewish secretary, Miss Lotte Altmann, who five years later became the second Mrs. Zweig. ${ }^{61}$

A Congress of the International Pen-Club was held in 1936 in Buenos Aires to which Zweig was invited as a guest speaker. Hoping to escape his fears as to the future of Europe, fears and pessimistic premonitions which insisted on invading his thoughts and his works, he willingly accepted the proffered invitation. The warm reception given him and the beauty of South America did lighten his mind, enough so that he was able to see some hope for the future. ${ }^{62}$ In his works on Brazil he praised her for her warm welcome, her glorious past and her burning hope for the future. ${ }^{63}$ His return to England (via a lecture tour of the U.S.A.) ${ }^{64}$ found the international situation even more sinister. He flew to Vienna for the last time to visit his mother and to give most of his library and autograph collection away most of his materials being given either to the Viennese National Library or the National Library at Jerusalem. What property he did not give away or send out of the country was immediately confiscated when Hitler annexed Austria.

Two of Zweig's biographies written during the years 1933-1939 are worthy of particular note for they both reflect his thought and in a veiled manner Zweig himself - they are his Triumph und Tragik des Erasmus von Rotterdam (1934) and Castellio gegen Calvin (1936). Both works seem to preach the same doctrine of humanism and tolerance but whereas Erasmus foreshadows the defeat of the individual, Castellio attempts to portray an example of resistance against brutal suppression. The former, a rather accurate portrait of Zweig as he was, the latter of him as he wished he could have been. Both, however, represent the struggle of individual freedom against force, of the one against the overpowering many, a struggle in which might seems to triumph. This triumph, however, is mere illusion, for the "ideal" lives on indefinitely. Man is at his best, is most noble only when he willingly sacrifices his own selfish ambitions to serve as an instrument of this ideal, as a tireless warrior in defense of peace and tolerance, a champion of the humanities and the spirit. ${ }^{65}$

The outbreak of World War II caused a complete change in Zweig's life. The house which he purchased in Bath became a regular home for refugees, among them Zweig's beloved friend Sigmund Freud, who, being forced to leave his native Austria, had come to England to accept a Chair of Honor which the British Royal Society of Science had extended to him. ${ }^{66}$ During the Spring of 1940 Zweig lectured in Paris where he had gone primarily to see his first wife, desperately in need of the spiritual strength and mental equilibrium which she alone seemed to possess and from whom alone he could draw the courage needed to continue. Upon his return to England he found another letter from South America requesting that he undertake a second 
lecture tour. He gladly accepted. The tour was a triumph beyond all expectation. Until January, 194I, he remained in Brazil and then moved to New York to be near the facilities of the Yale University library and be nearFriderike, who alone could furnish him with much of the material he needed for his autobiography which was already in preparation. To be able to see her daily, he rented a house in Ossining, New York, where he remained until August, 194I. The summer, however, proved too strenuous for both Stefan and his second wife who was failing rapidly. Fearing that Lotte couldn't survive another winter in the North, they returned to Brazil again, where they rented a house, a miniature replica of Zweig's Salzburg retreat on the Kapuzinerberg, in Petropolis, a small city not far distant from Rio de Janeiro. ${ }^{67}$ That Fall Zweig finished his autobiography, all but completed his monumental study of Balzac ${ }^{68}$ and wrote his last Novelle, "Schachnovelle," written in somewhat the same spirit as his Castellio, a masterpiece in the genre and by many considered to be the finest Novelle he ever wrote. On the morning of February 23, I 942, Zweig and his wife were found in their bedroom, lying side by side, two empty glasses on the night stand. Before freely ending his life Zweig wrote one last message to those whom he loved and was now leaving, a message of thanks to the country of Brazil for the obvious affection which it had tendered him, and a prayer of hope that those who still fought on would soon see the dawn of victory and peace. He, however, could no longer wait, his strength was gone, his usefulness ended here. Assuredly though he would wait for them beyond. ${ }^{69}$

To discuss the suicide of a human being and to attempt to judge its full import is tantamount to treading upon ground to which none but the Divine has right. Therefore, the comments which follow seek neither to condone nor to condemn Zweig's death, but rather to explain its causes. Although we can not read the thoughts of another and consequently can never attain absolute surety that our judgments are correct, we can, and should, seek to understand the most obvious reasons which would drive a man to will calmly and rationally the destruction of that life force within him, which by instinctive nature demands its own preservation.

Comparatively speaking Zweig had little reason to end his own life. Brazil was a quiet, beautiful country which had offered him not only refuge but love. He had sufficient time (perhaps too much?) to work on his "Balzac" manuscript and to revive his interest in Montaigne. He had been driven from his home, that is true, but his lot was for the most part far better than that of the hundreds of thousands who lost everything in their flight from the advancing Nazi hordes or those thousands who didn't escape in time. Zweig at least had some money, reputation, friends and to a great extent personal liberty. Viewed strictly externally, Zweig's position was better than most. But even 
here all was not what it seemed - his work on Brazil, although generally acclaimed, was most unfavorably criticized in Nazi controlled newspapers and Zweig himself was openly flaunted by Nazi agitators. The country was quiet and restful, but unfortunately too much so. Zweig was accustomed to activity and people and suddenly there was neither. Although the requisite time for working on his manuscripts was provided in excess, he was deprived access to primary source materials and even believed his beloved "Balzac" manuscript lost during the Blitz over England. ${ }^{70}$ It should also be remembered that his whole life had been spent in luxury and security, now almost everything was swept away. ${ }^{71}$ Through predisposition he desired a private, well ordered life and now he was forced to flee around the world, exposed to conditions which, though normal to most people, were disastrous to him. Too, he was in no sense of the word a business-minded individual. Any decision concerning financial or legal transactions had been left up to his first wife, and now he was left totally to his own devices. ${ }^{72}$

As bearing upon his suicide as these exterior circumstances might be, the very nature of his character preponderates in explaining his final act of resignation. As Zweig grew older the attacks of morbid depression and pessimism to which he was subject became increasingly more frequent and severe. Toward the end of his life he felt himself hunted, homeless and persecuted as a Jew. As a Humanist he saw only terror and force unleashed throughout the world. Even his mother tongue was denied him - he saw his final works appear only in translation, and with whom was he to converse in German, except his wife? To be forced to think and express oneself in a foreign language at his age seemed too great a strain (the language of Brazil, his last refuge, is Portuguese, a language Zweig did not speak). He was just plain tired. Impatient by nature, living far from his home and his friends (both of which constituted the core of his life), lacking sufficient strength to control his basically passionate nature and seeing nothing but destruction and hatred in a world in which he had endeavored to propagate peace and brotherhood, Zweig's resistance was broken. He was literally driven into the next world ahead of his friends for whom he was too impatient to wait.

Since 1942 a great many articles have appeared attempting to interpret and/or explain Zweig's death. Some commentators see in his suicide an act of martyrdom, a protest against Nazi barbarism. ${ }^{73}$ Others consider it an act of treason and cowardice, springing from personal despair, a decided betrayal of those millions who looked to him for comfort and guidance. In his article "Réflexions sur le suicide de Stefan Zweig" Paul Beaulieu contends that such censure of contemporary intellectuals supports the view which forbids suicide on moral grounds. ${ }^{74}$ According to this view we are all bound one to 
another and all to God. Thus our freedom is limited by our obligations. In the realm of theory this is true but in practical application it fails to take into consideration subjective elements which usually outweigh speculative arguments. ${ }^{75}$

As a Jew Zweig was not reared in the Christian tradition of "love thy neighbor as thyself." His God was more probably the deistic God of the Enlightenment and not the personal God of Christianity. The kenotic being worshipped in Christ by His followers had little or no significance for him. Zweig's humanism was based on intellectual principles of mutual understanding, not on Christian principles of mutual love. Even the desired ends differ - Zweig's humanism would establish cultural refinement and international peace as its final goal; the goal of Christian Humanism being an eternal and direct union with God, affected through love.

As a man Zweig was physically more delicate than most human beings, his sensibilities more refined. By nature somewhat egocentric Zweig was controlled by his ideas and ideals. Once these had been destroyed his life lost its meaning. His temperament demanded immediate contact with friends, perceptible signs of affection. The abstract concept of devotion given by the mysterious "masses" could hardly be considered a substitute.

As an individual Zweig repeatedly asserted that he was no hero he didn't even want to be one. His natural inclination toward suicide can be gleaned through a psychological interpretation of his Novellen where the tendency toward resignation and death is usually a prime factor. His desire for personal freedom became almost an obsession with him, it was literally the highest good on earth. This meant that he was bound first to himself, to himself he must therefore be true. Zweig lived for and served an "idea." Individuals he loved and aided wherever and whenever possible, but the concept "masses" meant far less to him directly than the concept "humanity." Zweig's greatness flows from his psychological mastery and penetrating insight into the human soul, but this gift was likewise the source of his weakness. A realist in the realm of the psyche, Zweig remained to the end an idealist, an utopian in the macrocosm of political-social problems. ${ }^{76}$ Zweig's Humanism is truly admirable, but he was actually driven to his death, broken by the world he loved.

... seduit par Montaigne au point de s'identifier avec sa pensée, il a abandonné la vie victime lointaine de la guerre qu'il avait cru pouvoir fuir. ${ }^{77}$ 


\title{
PRIMARY WORKS
}

\section{Editions and Translations Thereof}

\author{
I. COLLECTED WORKS
}

GERMAN

I. Ausgewäblte Werke (Teilsamml.). Vol. I/II. Düsseldorf: Deutscher Bücherbund, I960.

ITALIAN

2. Opere Scelte. Vol. I/II. Tr. Lavinia Mazzucchetti. Milano: Mondadori, I96r.

PORTUGUESE

3. Obras Completas. Vol. I/XX. Tr. Aurélio Pinheiro et al. Rio de Janeiro: Delta, I953.

\section{RUSSIAN}

4. Izbrannye Proizvedenïa. Vol. I/II. Tr. P. Bernštejn et al. Moskva: Goslitizdat, 1956; (Reprinted 1957).

5. - Kiev: Goslitizdat Ukrainy, 1957.

6. - Kujbysev: Kn. izd., i957.

7. - Minsk: Goslitizdat BSSR, 1960.

8. Sobranie Sochineniy Stefana Tsveyga. Avtorizovannoe izdanie s predisloviem M. Gor'kogo i kritiko-biograficeskim ocerkom Richarda Spechta (Collected Works of Stefan Zweig. Authorized edition with foreword by M. Gorki and a critical biographical essay by Richard Specht). Leningrad: Vremya, I928-1930.

I. Zhguchaya Tayna. Pervye Perezhivanija (Brennendes Gebeimnis. Erstes Erlebnis).

II. Amok. Novelly (Amok. Novellen).

III. Smjatenie Chuvsto (Verwirrung der Gefiuble).

Iv. Nezrimaja Kollekcija ( Die unsichtbare Sammlung et al.).

v. Rokovyemgnovenija (Sternstunden der Menscbbeit et al.).

vi. Tri Pevca svoej Zhizni (Drei Dichter ibres Lebens).

vir. Tri Mastera (Drei Meister).

virr. Marselina Debord-Val'more (Marceline Desbordes-Valmore).

Ix. Zhosef Fushe (Joseph Fouché).

x. Bor'ba s Bequmiem (Kampf mit dem Dämon).

9. Moskva: Izdatel'stvo Pravda, I 963.

I. Novelly ("V Sumerkakh" ("Geschichte in der Dämmerung"), "Guvernantka" "Die Gouvernante"), "Zhguchaya Tayna" ("Brennendes Geheimnis"), "Amok" (“Amok"), "Strakh" (“Angst”)).

II. Neterpenie Serdtsa (Ungeduld des Herzens); Legendy (Legenden). 
III. Zvezdnye Chasy Chelovechestva. Istoricheskie Miniatyury (Sternstunden der Menschbeit); Maggelan (Magellan); Amerigo (Amerigo).

Iv. Mariya Styuart (Maria Stuart); Zhosef Fushe (Joseph Fouché).

v. Bal'zak (Balzac).

vi. Frants Anton Mesmer (Franz Anton Mesmer); Gel'derlin (Hölderlin); Genrich fon Kleyst (Heinrich von Kleist); Stendal' (Stendhal); Marselina Debord Val'mor (Marceline Desbordes-Valmore); Dikkens (Dickens).

vir. Romen Rollan (Romain Rolland); Vospominaniya ob Emile Verkbarne (Erinnerungen an Emile Verbaeren); Vstrechi s Liud'mi, Gorodami, Knigami (Begegnungen mit Menschen, Städten und Bücbern).

\section{SPANISH}

ı. Obras Completas. Vol. I/II. Estudio crítico por Carlos Soldevila. Barcelona: Juventud, 1952, I 953. (Contains: Vol. I: "La Piedad Peligrosa (Impaciencia del Corazón)" (Ungeduld des Herzens), "Calidoscopio" (Kaleidoskop), "Conocimiento Casual de un Oficio" ("Unvermutete Bekanntschaft mit einem Handwerk"), "Leporella" ("Leporella"), "Miedo" ("Angst"), “Ardiente Secreto" ("Brennendes Geheimnis"), "Novela Veraniega" ("Sommernovellette"), "La Institutriz" ("Die Gouvernante"), "Buchmendel" ("Buchmendel"), "El Refugio" ("Episode am Genfer See"), "La Colección Invisible" ("Die unsichtbare Sammlung"), "Noche Fantástica" "(Phantastische Nacht"), "La Calle del Claro de Luna" ("Die Mondscheingasse"), "Sendas Equívocas" ("Verwirrung der Gefühle"), "Carta de una Desconocida" ("Brief einer Unbekannten"), "El Jugado de Ajedrez" ("Schachnovelle"), "Una Carta" ("Vierundzwanzig Stunden aus dem Leben einer Frau"?), "El Candelabro Enterrado" ("Der begrabene Leuchter"), "Los Ojos del Hermano Eterno" ("Die Augen des ewigen Bruders"), "Jeremias"(Jeremias). Vol. II: "Maria Antonieta" (Marie Antoinette), "Tres Maestros: Balzac, Dickens, Dostoiewski" (Drei Meister), "Magallanes" (Magellan), "Americo Vespucio" (Amerigo), "Tres Poetas de su Vida: Casanova, Stendhal, Tolstoi" (Drei Dichter ibres Lebens)).

II. Obras de Stefan Zweig. Vol. I/II. Barcelona: José Janés; Madrid: Ediciones Castilla (s.a.), r958. "Colección Maestros de Hoy" (Contains: "Stefan Zweig," por Friderike Maria Zweig. Tr. Alfredo Cahn; "Americo Vespucio" (Amerigo). Tr. Alfredo Cahn; "Tres Poetas de su Vida" (Drei Dicbter) and "La Lucha contra et Demonio" (Kampf mit dem Dämon). Tr. Joaquin Verdaguer; "La Curación por Espíritu" (Die Heilung durch den Geist). Tr. Francisco Payarols; "Balzac" (Balzac). Tr. Aristides Gamboa).

\section{POETRY IN BOOK FORM}

12. Ausgewählte Gedicbte. Leipzig: Insel, 193 I (Insel-Bücherei, No. 422, I93 I: I.-10. Tsd.; I934: Ir.-20. Tsd.)

13. - Wiesbaden: Insel, 1950. (Insel-Bücherei, No. I74, 1950: 21.-29. Tsd.; 1953: 30.-39. Tsd.).

14. Die früben Kränze. Leipzig: Insel, 1906 (1906: 1.-2. Tsd.; 1917: 3.-4. Tsd.; 1920: 5.-6. Tsd.).

15. Die gesammelten Gedicbte. Leipzig: Insel, I924.

16. Silberne Saiten. Berlin-Leipzig: Schuster und Loeffler, I gor.

\section{SINGLE POEMS}

17. “Abendklänge," Ge, I6.J., II: 4 (1900), 236.

I8. "Abendwolken," $D D, \mathrm{XXXI}$ (Oct., I901 - Mar., I902), 286. 
19. "Abschied," $D D, \operatorname{XXX}$ (Apr.-Sept., r901), 174.

20. "Am Abend," $D D$, XXVI (Apr.-Sept., I 899), I 47.

21. "Bäume im Frühling," Tag, p. I 20.

22. "Ballade von einem Traum," NR, XXXIV: 2 (1923), 716-722 (Reprinted in Europäer, pp. I57-162).

23. "Der Bildner (Meudon, Haus Rodin, I913)," In, V : x (1924), 42-46 (cf. Gesammelte Gedicbte, pp. 87-9I ; Begegnungen, pp. 74-77; In, XIII : I (Christmas, I93I), I5-16).

24. "Eine blaue Flamme," $D D, \mathrm{XXXI}$ (Oct., I901 - Mar., I902), I8I.

25. "Bruges" (English tr. of "Brügge"), $C G P$, p. r9i (French tr. cf. $A L A$, pp. 392-395).

26. "Brügge," $O R$, VIII : 94-95 (Aug.-Oct., 1906), 1 26.

27. "The Dark Butterfly" (English tr. of "Der dunkle Falter), CGP, p. I90.

28. "Deutsche Stadt (Konstanz), "ÖR, VIII : 94-95 (Aug.-Oct., 1906), I 28.

29. "Die Dinge, die die Abende erzählen...," (from "Die Lieder des Abends" in Die fruiben Kränze), from Paul Zech's review of this book as quoted in DLE, I 7. J.: 3 (Nov. I, I914), I7I-172.

30. "Der Dirigent. In memoriam Gustav Mahlers," In $A$ (1923), 95-99 (Reprinted in Begegnungen, pp. I 23- I 26).

3I. "Dostojewski" (French tr. of "Der Märtyrer"), $A L A$, pp. 396-405.

32. "Dunkle Sehnsucht," Spiegelungen, p. 22.

33. "Ein dunkler Weg," DD, XXXI (Oct., 1901 - Mar., 1902), 263.

34. "Das Einleitungsgedicht zu dem neuen Novellenbande: 'Verwirrung der Gefühle,," In, VII: 4 (1926), 277.

35. "Einsamkeit," $D D$, XXIX (Oct., I900 - Mar., I901), 57.

36. "Erkenntnis," $D D, \operatorname{XXX}$ (Apr.-Sept., I901), I94.

37. "Erstes Ahnen," Ge, I6.J., II : 3 (I900), I 82.

38. "Die Frage," $Z$, XLVII (June I 8, 1904), 456.

39. "Das fremde Lächeln," OR, V : 57 (Nov., r905 - Jan., 1906), 22.

40. "Frühlingssegen," $D D$, XXVIII (Apr.-Sept., I900), 262.

41. "Gefangen," $D D$, XXVII (Oct., I 899 - Mar., I900), I35.

42. "Hand in Hand," Spiegelungen, p. 22.

43. "Die Hände," Liliencron, p. 79.

44. "Herbst," Europäer, pp. I I-I 2.

45. "Herbstmorgen," DD, XXVII (Oct., I 899 - Mar., I900), I59.

46. "Herbstsonette," In $A$ (I9I I), 63-64 (Reprinted in Tag, pp. I 2O-I $2 \mathrm{I}$ ).

47. "Hymnus an die Reise," In, XIII : I (Christmas, I93 I), I 2-13.

48. "Im Feld," $D D$, XXIX (Oct., I 900 - Mar., I90r), 288.

49. "Im Glück," $D D$, XXVII (Oct., I 899 - Mar., I 1900), 88.

50. "In den Tag hinein," DD, XXVIII (Apr.-Sept., I900), 28.

51. "In der Kirche," DD, XXVI (Apr.-Sept., I 899), 267.

52. "In der Sternennacht," $D D$, XXIX (Oct., I 900 - Mar., I901), 93.

53. "Indischer Spruch," Tag, p. II9.

54. "Kinder im Dorfe," DD, XXXI (Oct., 1901 - Mar., 1902), 233.

55. "Das Leben," $D D, \mathrm{XXX}$ (Apr.-Sept., I90I), I I I.

56. "Liebeslied," DD, XXVI (Apr.-Sept., I 899), 77 (Reprinted in Tag, pp. I I 9-1 20).

57. "Lied des Einsiedlers," In, XIII : I (Christmas, I93 I), I4-1 5 .

58. "Linder schwebt der Stunden Reigen," $S Z G$, p. 43 (Reprinted in Europäer, p. 33; Leben-Werk, p. I 1о; cf. below "Der Sechzigjährige dankt").

59. "Das Mädchen," DD, XXX (Apr.-Sept., 1901), 71 .

6o. "Der Märtyrer" (Poem about Dostojewski, Dec. 22, I 849), In $A$ (1913), 26-34.

6oa. "Matkowskys Othello (Geschrieben bei der Nachricht seines Todes I909), $A D V$ (1920).

6r. "Meine Brust...," $D D$, XXV (Oct., I 898 - Mar., I899), 258.

62. "Meine Saat," $D D$, XXXII (Apr.-Sept., I902), I 22.

63. "Morgenlicht," $D D$, XXVII (Oct., I 899 - Mar., 1900), 234.

64. "Die Mutter," $D D$, XXIX (Oct., I900 - Mar., I90I), I45. 
65. "Die Nacht der Gnaden," $Z$, XLIV (Sept. I 2, 1903), 424-426.

66. "Nacht im Gebirge," $D D$, XXIX (Oct., I900 - Mar., I90I), I68.

67. "Neue Fülle," In, XIII : I (Christmas, I93 I), I I.

68. "Rauher Frühling," $D D$, XXIX (Oct., I900 - Mar., I90I), $24 \mathrm{I}$.

69. "Reifes Glück," $D D, \mathrm{XXX}$ (Apr. - Sept., I901), 86.

70. "Regentage," $D D$, XXIX (Oct., I900 - Mar., I901), I7.

71. "Rosenknospen," $D D, \mathrm{XXV}$ (Oct., 1898 - Mar., I899), 123.

72. "Die Sängerin," In $A$ (1914), 165-I69.

73. "Der Sechzigjährige dankt" (Zweig's last poem), Europäer, p. 33 (Photocopy of same opposite p. 337; cf. "Linder schwebt der Stunden Reigen").

74. "Singede Fontäne," In $A$ (1912), 103-105.

75. "Sommernächte am Komersee," $O R$, VIII : 94-95 (Aug.-Oct., 1906), 27.

76. "Sommerwende," $D D, \operatorname{XXX}$ (Apr.-Sept., I901), 218.

77. "Sonnenaufgang in Venedig," $O R$, VIII : 94-95 (Aug.-Oct., I906), I 26.

78. "Spätsommer," DD, XXVII (Oct., I 899 - Mar., I900), I 8.

79. "Spinoza," Spiegelungen, p. 23.

80. "Sternengedanke," $D D$, XXIX (Oct., I900 - Mar., 1901), 264.

81. "Stille Insel (Bretagne)," $O R$, VIII : 94-95 (Aug.-Oct., 1 906), I 27.

82. "Stimmen im Walde," $D D$, XXVIII (Apr.-Sept., 1900), 2 I 2.

83. "Der Sucher," $Z$, XLVI (Jan. 23, I904), I 46-147.

84. "Thautropfen," $D D$, XXVI (Apr.-Sept., I 899), I64.

85. "Ile Tranquille (Bretagne)," $A L A$, pp. 395-396.

86. "Ein Traum," $D D$, XXXI (Oct., I 901 - Mar., 1902), I09.

87. "Der verlorene Himmel. Eine Elegie," In $A$ (1917), I 5 2-I 55.

88. "Der Verschmähte," $D D$, XXXI (Oct., I 901 - Mar., I902), I 35.

89. "Verse in ein Stammbuch" (Poem dedicated to Anton Kippenberg on his 6oth birthday), Europäer, p. I 28 (Cf. Leben-Werk, p. 70).

90. "Weihnacht," Spiegelungen, p. 23.

91. "Werden," $D D$, XXXI (Oct., I90I - Mar., I902), I65.

92. "Winterabend im Zimmer," $D D$, XXVIII (Apr.-Sept., 1900), 52 (Reprinted in Spiegelungen, p. 22).

93. "Die Zärtlichkeiten," DeB, p. 90.

\section{DRAMAS}

94. Die Flucht zu Gott (Ein Epilog zu Leo Tolstois unvollendetem Drama Das Licht scheinet in der Finsternis). Berlin: Bloch, 1927.

95. - (Bühnenmanuskript). NT, I.J. : 2 (Dec., 1928), 34-55. (Premiere: Vereinigtes Städtisches Theater Kiel, Sept. 5, 1928).

96. Das Haus am Meer. Ein Schauspiel in zwei Teilen. Leipzig: Insel, I9I 2 (Premiere: Hofburgtheater Wien, Oct. 26, I91 2).

97. Jeremias. Eine dramatische Dichtung in neun Bildern. Leipzig: Insel, 1917 (Uraufführung: Stadttheater Zürich, Feb. 27, I918).

I9I 7 - 2. ed.

$1918-3 .$,

I9I9 - 4. " (9.-13 Tsd)

$1920-5 ., \quad$ (14.-18. Tsd)

1922 - 6. ”( (19.-2I. Tsd)

1923 - 7. " (22.-25. Tsd)

I 928 - 8. ” (26.-28. Tsd)

98. - Stockholm: Bermann-Fischer, I939; Amsterdam: de Lange, I939.

99. Jeremias. Eine dramatische Dichtung in sieben Bildern. Ed. L. Wery. Zwolle: W. E. J. Tjeenk Willink, I940 (Neue deutsche Bibliothek, No. 23).

I00. Dutch. Als de Scbaduwen Lengen (Fragment uit Jeremias). Tr. J. Haantjes. Utrecht: Vrijzinnig Christelijke Jeugdcentrale, 1938. 
Iо1. Jeremias. Treurspel in negen Deelen. Tr. Josef Boon.Antwerpen: P. Vink, 1934. I02. English. "The Everlasting Road" (Adaptation from Jeremiab). A Golden Treasury of Jewish Literature (Sec. "Plays of a Changing World"). Sel. and ed. Leo W. Schwarz. New York-Toronto: Farrare, Rinehart, 1937, pp. 41 I-426.

103. Jeremiah. A Drama in Nine Scenes. Tr. Eden and Cedar Paul. New York: Seltzer, 1922; new ed. New York: Viking, 1929; London: Allen and Unwin, 1929 (Preface by author in both 1929 editions).

104. - New York: Viking, 939 (A new preface by the author to mark the first American production by the New York Theater Guild, Feb. 3, 1939).

I05. French. Jérémie. Tr. Louis Charles Boudouin. Paris: Rieder, I 929 (Judaīsme, No. I0).

Io6. Hebrew. Yirmiyabu. Tr. Avigdor Ha-meiri. Jerusalem: Dept. de la Jeunesse de l'Organisation Sioniste Mondiale, 1950 (This edition contains a critical essay about the play by the translator).

107. Polish. Jeremiasz. Poemat Dramatyczny w Dziewieciu Obrazach. Tr. Melanja Wassermann. Warszawa: Hoesick, I926.

I08. Portuguese. Jeremias. Tr. Cándido de Carvalho. Porto (Portugal): Livro Civilização, I 957 (4th ed.).

I09. Spanish. Jeremias. Tr. Alfredo Cahn. Barcelona: Hispano-Americana de Ediciones, 1945 (Col. Cumbre).

I Iо. - Tr. Alfredo Cahn. Buenos Aires: Espasa-Calpe, 1956.

I I I. Yiddish. Jirmijabu. Dr'am'atis'e Diktung 'in nein Bild'er. Tr. Br'aq'azs. W'ars'e: Tur'em, r929.

I 1 2. Das Lamm des Armen. Leipzig: Insel, 1929 (Simultaneous premiere Lobetheater Breslau, Städtisches Schauspielhaus Hannover, Stadttheater Lübeck, Neues Deutsches Theater Prag, Mar. I5, 1930).

I13. French. Un Caprice de Bonaparte. Pièce en trois Actes. Tr. Alzir Hella. Paris: Grasset, 1952.

I14. Spanish. El Cordero de Pobre. Tragi-Comedia en tres Actos. Tr. Alfredo Cahn. Buenos Aires: Editorial Claridad, I942.

I 1 5. Legende eines Lebens. Ein Kammerspiel in drei Aufzügen. Leipzig: Insel, I919 (1923-3.-4. Tsd.) (Premiere: Deutsches Schauspielhaus Hamburg, Dec. 25, I918).

I16. Russian. Legenda odnoj Zhizni. P'esa v trech Dejstvijach. Foreword by A. G. Hornfeld. Tr. I. B. Mandelstam. Moskva-Petrograd: Gosudarstvennoe Izdatel'stvo, I 923.

I 1. Quiproquo. Komödie. Wien: n.p., 1928 (Written in conjunction with Alexander LernetHolenia, under the pseudonym Clemens Neydisser).

II 8. Die scbweigsame Frau. Komische Oper in drei Aufzügen frei nach Ben Jonson. Berlin: Adolph Fürstner, I935 (piano-vocal score Richard Strauss, Op. 80).

I 19. English. The Silent Woman. A Comic Opera in Three Acts. Tr. Herbert Bedford. New York: Program Publishing, n.d.

120. Italian. La Donna Silenziosa. Opera Comica in tres Atti. Tr. Ottone Schanzer. Milano: Sonzogno, 1936 .

I 21 . Tersites. Trauerspiel in drei Aufzügen. Leipzig: Insel, 1907 (1919, 2. rev. ed.) (Simultaneous premiere: Hoftheater Kassel and Dresden, Nov. 26, 1908).

122. Der verwandelte Komödiant. Ein Spiel aus dem deutschen Rokoko. Berlin: Bloch, 1912.

I23. - Leipzig: Insel, I9I3 (1920, 2. ed.; 1923 - Reclam Bibliothek, No. 6374; reprinted I 926, 1928) (Premiere: Lobetheater Breslau, May 5, I 9 I 2).

124. Swedish. Komedianten. Ett Spel fran Tyska Rokokon. Tr. Anders de Wahl. Stockholm: Albert Bonniers, I919.

125. Volpone. Eine lieblose Komödie in drei Akten (nach Ben Jonson frei bearbeitet von Stefan Zweig). Berlin: Bloch, 1925 (Stage manuscript; Premiere: Burgtheater Wien, Nov. II, I926).

126. - Potsdam: Kiepenheuer, 1926 (1927, 2. ed., 6.-8. Tsd.).

127. - Frankfurt am Main: Fischer, I950.

I 28. - Leipzig: Insel, 1959 (2. ed., Reclams Universal-Bibliothek, No. 8359). 
129. English. Volpone. A Loveless Comedy in Three Acts (by Ben Jonson, freely adapted by Stefan Zweig). Tr. Ruth Langner. London: Allen and Unwin, 1928.

130. - New York: Viking, 1928 (Reprinted I956; Premiere of the English version in New York in the Guild Theatre, April 9, 1928).

I3I. - Twenty Best European Plays on the American Stage. New York: Crown Publishing Co., 1957, pp. 40I-442.

132. French. Volpone. Comédie en cinq Actes (d'après Ben Jonson par Jules Romains et Stefan Zweig). Paris: Arthème Fayard et Cie, I929.

I33. - Théâtre de Jules Romains. Vol. 5. Paris: Librairie Gallimard, I929.

I34. - Paris: Paris-Théâtre, I955 (Paris-Théâtre, No. 95).

I35. Spanish. Volpone. Comedia en cinco Actos (de Ben Jonson para Jules Romain y Stefan Zweig). Tr. Artemio Precioso and Rafael Sánchez Guerra. Madrid: Editorial Colón, I930.

\section{PROSE FICTION}

136. Amok. Novellen einer Leidenschaft. Leipzig: Insel, 1922 (Contains "Der Amokläufer," "Die Frau und die Landschaft," "Phantastische Nacht," "Brief einer Unbekannten," "Die Mondscheingasse").

I923 - 2. ed. (II.-2I. Tsd.)

1924 - 3.,$(22 .-32$. Tsd.)

1925 - 4. ”(33.-45. Tsd.)

1927 - 5. " $"(46 .-50$. Tsd.)

1928 - 6. "( $\%$ I. -60 . Tsd.)

$1929-7 . "$ (6r. -65 . Tsd.)

1930 - 8. ",$(66 .-70$. Tsd.)

1931 - 9. ” (71.-74. Tsd.)

From here on in the series "2-Mark-5o-Bücher"

193 I - I. ed. (75.-I 25 . Tsd.)

I 93 I - 2. " (126.-I 50. Tsd.)

137. - Stockholm: Bermann-Fischer, 1946 (r.-10. Tsd.);

Frankfurt am Main: Fischer, 1950 (1r.-20. Tsd.);

Frankfurt am Main: Fischer, I956 (21.-40. Tsd.).

(Contains: "Der Amokläufer," "Brief einer Unbekannten," "Vierundzwanzig Stunden aus dem Leben einer Frau," "Buchmendel," "Episode am Genfer See," "Phantastische Nacht," "Verwirrung der Gefühle," "Die unsichtbare Sammlung").

138. Angst. Novelle. Berlin: H. S. Hermann, 1920 (Der kleine Roman. Illustrierte Wochenschrift, No. I9).

139. - Leipzig: Reclam, 1925 (Shortened version; Epilogue by Erwin H. Rainalter; Reclams Universal Bibliothek, No. 6540; Reprinted: 1927, 1928, 1929, 1930, 1931).

140. - Stuttgart: Reclam, 947 (Epilogue by Alexandra Carola Grisson; Reprinted 1954).

I4I. - Wien: Humbolt, 1947 (Epilogue by Gottfried Ippisch; Kleine Hand-Bibliothek, No. 104).

142. Die Augen des ewigen Bruders. Eine Legende. Leipzig: Insel, 1922

(Insel-Bücherei, No. 349).

I 922 - I. ed. (I.-1O. Tsd.)

I 924 - 2., (Ir.-20. Tsd.)

I $925-3 ., \quad(21 .-30$. Tsd.)

I 926 - 4. " (31.-40. Tsd.)

1927 - 5." (41.-6o. Tsd.)

1928 - 6.” (6r.-80. Tsd.) 


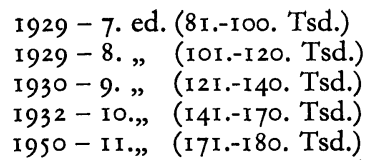

143. - Wiesbaden: Insel, I950 (Insel-Bücherei, No. 349).

I950 - I 2. ed. (181.-190. Tsd.)

1952 - I3." (191.-200. Tsd.)

1953 - I4." " (20I-210. Tsd.)

1954-15." (211.-220. Tsd.)

1955 - 16." (221.-240. Tsd.)

1956 - 17." (241.-255. Tsd.)

I958 - I8. ” (256.-265. Tsd.)

1959 - 19. " (266.-283. Tsd.)

144. - Frankfurt am Main: Insel, I96r (Insel-Bücherei, No. 349). 1961 - 20. ed. (284.-303. Tsd.).

145. - Wien: 39. Avalun-Druck, I924 (250 copies signed by author).

146. - Ed. J. G. Steersma. Amsterdam: Meulenhoff, I939 (Meulenhoffs Sammlung deutscher Schriftsteller, No. 75 I; Intro. J. G. Steersma; Includes notes and vocabulary).

1948 - 2. ed.

I 95 I - 3. "

I $955-4 \cdot$,

147. - Wie sie es sehen. (Sel. and ed., Harry Zohn et al.) New York: Henry Holt, I952, pp. I3I-I7I (School edition).

148. Ausgewäblte Novellen. Stockholm: Bermann-Fischer, I946 (1.-5. ed.; Contains: "Amok," "Brief einer Unbekannten," "Vierundzwanzig Stunden aus dem Leben einer Frau," "Buchmendel," "Episode am Genfer See," "Phantatische Nacht," "Die Mondscheingasse," "Verwirrung der Gefühle," "Leporella," "Die unsichtbare Sammlung").

149. Ausgewählte Prosa. Ed. W. Kuiper. Amsterdam: Meulenhoff, I952 (2. ed.; Meulenhoffs Sammlung deutscher Schriftsteller, No. 93).

I50. Der begrabene Leucbter. Wien: Reichner, reprinted 1937 (Illustrations by Berthold Wolpe; soo numbered copies; Frankfurt am Main: Fischer, 1963).

I5 I. Brennendes Geheimnis. Leipzig: Insel, I9I3 (Insel-Bücherei, No. 22).

I9I6 - 2. ed.

$1917-3 \cdot$,

I9I 8 - 4."

I $918-5$. ,

1922 - 6." ( $46 .-55$. Tsd.)

$1924-7 . ",(56 .-65$. Tsd. $)$

1926 - 8." ( 66.- 75. Tsd.)

1927 - 9." ( $76 .-90$. Tsd.)

1928 - 10." ( 91 1.-1 10. Tsd.)

I929 - II. " (III.-I20. Tsd.)

1930 - I2. ” ( ( $21 .-140$. Tsd.)

I931-13." (I4I.-150. Tsd.)

1932 - I4." (I

I52. - Ed. Eva C." Wunderlich. New York: Farrar, Rinehart, I938 (Intro. and vocabulary Eva C. Wunderlich).

I 53. Brennendes Geheimnis und andere Erzäblungen. Berlin: Fischer, I954 (Contains: "Brennendes Geheimnis," "Die Gouvernante," "Die Frau und die Landschaft," "Die Mondscheingasse," "Unerwartete Bekanntschaft mit einem Handwerk," "Leporella," "Untergang eines Herzens," "Angst").

154. Brennendes Gebeimnis und andere Erzäblungen. Frankfurt am Main: Büchergilde Gutenberg, 1957 (For members only). 
I55. "Brief einer Unbekannten," Deutsche Dicbterbandschriften. Ed. Hanns Martin Elster. Dresden: Lehmannsche Verlagsbuchhandlung, 1922 (Contains facsimile of the second manuscript and an autobiography of the author).

I 56. "Buchmendel" (Die Novelle wurde dem Bergischen Bibliophilen-Abend als erste Jahresgabe vom Verfasser zur Veröffentlichung überlassen. Sie erschien von der Officina Serpentis in der Walbaum-Fraktur gedruckt im Jahre 1930).

157. Episode am Genfer See (der Fliichtling). Leipzig: Insel, I927.

158. - Aus unserer Zeit. Ed. I. and C. Loran and Leland R. Phelps. New York: Norton, 1956 (School edition).

159. - Bul, 10 (Oct., I962), 7-10.

I60. - $\operatorname{In} A$ (1920), I70-179.

161. - Krl, III : i8 (1960) (With a drawing by Erich Behrend).

162. Erstes Erlebnis. Vier Geschichten aus Kinderland. Leipzig: Insel, I9 I (Contains: "Geschichte in der Dämmerung," "Die Gouvernante," "Brennendes Geheimnis," "Sommernovellette").

I 917 - 2. ed.

1919 - 3."

I 920 - 4." ( 8.-II. Tsd.)

1922 - 5." (12.-15. Tsd.)

1923 - 6. " (I6.-19. Tsd.)

From here on subtitle "Die Kette. Ein Novellenkreis. Der erste Ring":

1925 - 7. ed. (20.-22. Tsd.)

1926 - 8., (23.-27. Tsd.)

1927 - 9." " (28.-32. Tsd.)

1928 - I0." (33.-40. Tsd.)

I 930 - II. " (4I.-46. Tsd.)

163. Fragment einer Novelle. Wien: Verlag der Internationalen Stefan Zweig Gesellschaft, 196I (Special publication No. 2 of the Stefan Zweig Gesellschaft; Ed. Erich Fitzbauer; With four Lithographs by Hans Fronius).

164. Gesammelte Erzählungen. Wien-Leipzig-Zürich: Reichner, 1936 ("Die Kette," "Kaleidoskop").

164a. "Die Gouvernante," Literatur für den Deutschunterricht. Erste Stufe. Ed. Bernard Rechtschaffen, Conrad P. Homberger and Victor Bobetsky. New York: American Book Co., 1964, pp. 14-29 [School ed.].

164b. "Im Schnee," Jii Al (1902) [Reprinted as the third Special Publication of the International Stefan Zweig Society. Wien: Im Verlag der Internationalen Stefan Zweig Gesellschaft, 1963; Drawings by Fritz Fischer].

165. Kaleidoskop. Wien-Leipzig-Zürich: Reichner, I936 (Contains: Tales: "Unvermutete Bekanntschaft mit einem Handwerk," "Leporella," "Die unsichtbare Sammlung," "Episode am Genfer See," "Buchmendel," "Angst"; Legends: "Rahel rechtet mit Gott," "Die Augen des ewigen Bruders," "Der begrabene Leuchter," "Die Legende der dritten Taube," "Die gleich-ungleichen Schwestern"; Sternstunden der Menschheit: "Die Weltminute von Waterloo," "Die Marienbader Elegie," "Die Entdeckung Eldorados," "Heroischer Augenblick," "Der Kampf um den Südpol," "Die Eroberung von Byzanz," "Georg Friedrich Händels Auferstehung").

166. Die Kette. Ring I und II. Leipzig: Insel, I 922 -1924.

(Erstes Erlebnis, I922, I 2.-I 5. Tsd. Amok, I922-1924, I.-32. Tsd.).

167. Die Kette. Ring I, II und III. Leipzig: Insel, 1925-1927 (Erstes Erlebnis, Amok, Verwirrung der Gefiuble).

168. - Wien-Leipzig-Zürich: Reichner, 1936.

169. Kleine Chronik. Leipzig: Insel, I 929 (Insel-Bücherei, No. 408; Contains: "Die unsichtbare Sammlung," "Episode am Genfer See," "Leporella," "Buchmendel"). I 929 - I. ed. ( I.-30. Tsd.)

1929 - 2., (31.-60. Tsd.)

1930-3. ", (6r.-100. Tsd.) 
170. Kleine Chronik. Drei Erzäblungen. Leipzig: Insel, I95 I (Without "Episode am Genfer See"; Epilogue by Fritz Adolf Hünich; i I r.-I 30 . Tsd.).

r7r. "Die Legende der dritten Taube," Aus Nah und Fern. Ed. Lore B. Foltin. Boston: Houghton and Mifflin, 1950, pp. 155-159 (School edition).

172. Legenden. Stockholm: Bermann-Fischer, 1945 (Contains: "Rahel rechtet mit Gott," "Die Augen des ewigen Bruders," "Der begrabene Leuchter," "Die Legende der dritten Taube," "Die gleich-ungleichen Schwestern"; Reprinted I948, 1959).

173. Legenden. Eine Auswabl. Ed. Oskar Maar. Frankfurt am Main: Fischer, r952 (FischerSchulausgaben; Contains: "Rahel rechtet mit Gott," "Die Augen des ewigen Bruders," "Die Legende der dritten Taube").

174. - Wien: Fischer, 1952.

175. Legenden. Eine Teilsammlung. Berlin-Darmstadt-Wien: Deutsche Buchgemeinschaft, I959 (For members only).

176. Die Liebe der Erika Ewald. Berlin: Egon Fleischel, 1904 (Contains: "Die Liebe der Erika Ewald," "Der Stern über dem Wald," "Die Wanderung," "Das Wunder des Lebens").

177. Meisternovellen. Eine Teilsammlung. Gütersloh: Bertelsmann Lesering, 1962 (For members only).

178. Menscben-Novellen. Stuttgart-Zürich-Salzburg: Europäischer Buchklub, I962 (Contains: Intro. by Wilhelm Schlösser, "Verwirrung der Gefühle," "Vierundzwanzig Stunden aus dem Leben einer Frau," "Leporella," "Episode am Genfer See," "Phantastische," "Der Zwang," "Sommernovellette," "Brennendes Geheimnis," "Schachnovelle," "Die unsichtbare Sammlung," Epilogue by Wolf R. Lang; For members only).

179. "Die Mondscheingasse," Grf, r. J., XI : Io (July, r914), 319-335.

180. Phantastische Nacht. Vier Erzäblungen. Frankfurt am Main-Hamburg: Fischer, 1954 (Fischer-Bücherei, No. 45; Reprinted 1955, 1956, 1957; Contains: "Phantastische Nacht," "Brief einer Unbekannten," "Vierundzwanzig Stunden aus dem Leben einer Frau," "Untergang eines Herzens").

I8I. "Phantastische Nacht," RoR, 2 (I929), 52-II9 (With five woodcuts by Otto R. Schatz; Cf. "Der Zwang" below).

182. Rabel recbtet mit Gott. Berlin: Aldus Druck, 1930.

183. - Der goldene Scbnitt. Grosse Erzäbler der Neuen Rundschau 1890-1960. Ed.Christoph Schwerin. Frankfurt am Main: Fischer, r960, pp. 347-358.

I84. - $\operatorname{In} A$ (I929), I I 2-I32.

185. - Neue deutsche Erzäbler (Stefan Zweig, Franz Werfel, Jakob Wassermann, Carl Zuckmayer, et al.). Berlin: Francke, 1930, pp. 393-410.

186. - NR, 38. J., I (Mar., I927), 260-273.

187. Schachnovelle. Buenos Aires: Pigmalion, 1942 (250 numbered copies).

I88. - Stockholm: Bermann-Fischer, I943 (Reprinted 1945, I95 I, I954).

189. - Wien: Bermann-Fischer, 1949 (With drawings by Hans Fronius).

190. - Frankfurt am Main: Fischer, I95 I (Reprinted 1957, 1959, 1960, 1961).

I91. - Ed. J. H. Schouten. Amsterdam: Meulenhoff, I950, (Meulenhoffs Sammlung deutscher Schriftsteller, No. 92; Reprinted 1952, I954, 1960).

192. - Ed. A. Werner. København: Hirschsprung, I950 (Deutsche Texte für das dänische Gymnasium, No. 4).

193. - Ed. Harry Zohn. New York: Norton, 1960 (School edition).

194. - Ed. Kristian Langlo and Margit Rogne. Oslo: Aschehoug, I95 I.

195. Schachnovelle und andere Erzäblungen. Wien: Buchgemeinschaft Donauland, 196r.

196. Scharlach. ÖR, XV : s (Apr.-June, 1908), 336-356, XV: 6 (Apr.-June, 1908), 41 5432.

197. Ungeduld des Herzens. Roman. Stockholm: Bermann - Fischer, I939. Amsterdam: Albert de Lange, 1939. New York-Toronto: Longmans, Green, Alliance Book Corp., I 939.

198. - Wien: Bermann-Fischer, 1949, Amsterdam: Querido, 949 (24.-34. Tsd.). 
199. - Wien: Büchergilde Gutenberg, 1953 (For members only).

200. - Frankfurt am Main: Fischer, I954 (Die Bücher der Neunzehn; Reprinted: 1955, 1958, 1963).

201. - Berlin-Darmstadt: Deutsche Buchgemeinschaft, 1956 (For members only). 202. - Zürich: Buchclub Ex Libris, 1957 (For members only).

203. - Stuttgart-Hamburg: Deutscher Bücherbund, 196r (For members only).

204. Die unsichtbare Sammlung. Eine Episode aus der deutschen Inflation. Berlin: Sonderdruck, n.p., 1927 (Hergestellt für die Mitglieder und Freunde des Berliner Bibliophilen-Abends beim Stiftungsfest am 8. Feb., 1927; 250 mit der Hand numerierte Exemplare).

205. - Maastricht: Halayon-Presse, 1933.

206. - Maastricht: Stols, 1933.

207. - Wien: Reichner, 1933.

208. - Amsterdam: Nederlandsche Vereniging voor Druk- en Boekkunst, I95 I (Illustrated by Harry Prenen).

209. "Die unsichtbare Sammlung," Auf böherer Warte. Ed. F. E. Coenen. New York: Holt, I94I (School edition).

2 10. - Helles und Dunkles. Ed. Roy Temple House and Johannes Malthaner. Boston: Ginn, 1948 (School edition).

211. - $\operatorname{In} A($ 1927), 91-108.

212. - $V Z$, May 31, 1925.

213. Verwirrung der Gefüble. Leipzig: Insel, 1927 (Contains: "Vierundzwanzig Stunden aus dem Leben einer Frau," "Untergang eines Herzens," "Verwirrung der Gefühle").

I 927 - I. ed. ( I.-20. Tsd.)

1927-2., (21.-30. Tsd.)

1927-3.", (31.-40. Tsd.)

1927 - 4. ", (41.-60. Tsd.)

1928 - 5. " (6r.-75. Tsd.)

1929-6. " (76.-85. Tsd.)

r 93 I - 7." (86.-90. Tsd.).

214. - Frankfurt am Main: Fischer, 1960.

215. Vier Novellen. Ed. (with notes and introduction) Harold Jensen. London (et al.): Harrap, I955 (Contains: "Buchmendel," "Episode am Genfer See," "Die unsichtbare Sammlung," "Unvermutete Bekanntschaft mit einem Handwerk"; School edition).

216. Vierundzwanzig Stunden aus dem Leben einer Frau. Gütersloh: Bertelsmann, 1958 (Kleine Bertelsmann-Lesering-Bibliothek, Vol. 14; For members only).

217. "Die Wanderung," NFP, Morgenblatt, April I I, I902, pp. I-2 (Reprinted in Die Liebe der Erika Ewald (1904)).

2I8. "Das Wunder des Lebens," Meister-Novellen neuerer Erzäbler. Vol. I. Ed. Richard Wenz. Leipzig: Hesse and Becker, 1926, pp. 95-204.

219. Der Zwang. Eine Novelle. Leipzig: Insel, I920 (With ro woodcuts by Frans Masereel). 220. "Der Zwang," RoR, 2 (I929), I-5 I (With Io woodcuts by Frans Masereel; Original title of this work according to the manuscript version was "Der Refractor," cf. Blätter, 3 (Oct., 1958), 7; Cf. also "Phantastische Nacht" above).

\section{ALBANIAN}

221. Njëzetekatër orë nga jeta e një gruaje ("Verwirrung der Gefühle"). Tr. Enver Fico. Tiranë: Ndërmarrja Shtetërore e Botimeve, 1958.

\section{BULGARIAN}

222. Amok. Roman. Tr. Stefan Pejkov. Pecatnica: "Stopansko Razvitie," n.d.(Translated 
from the French; Appeared in the series "Izbranie Sucinenija," Vol. IX, No. 5 of the "Ikonomija i Domakinstvo").

223. Dvadeset $i$ cetiri casa ot zivota na zenata ("Vierundzwanzig Stunden aus dem Leben einer Frau"). Tr. Metodi Vecerov. Sofija: Pravo, n.d. (Appeared in the "Library of Pearls of World Literature," No. 33).

224. Ocite na vecnija brat. Indiyskaja legenda ("Die Augen des ewigen Bruders"). Tr. S. Manolova. Sofija: Knigoizdatelstvo Vuzrazdane, r930.

\section{CATALAN}

225. Amok (sequit "Vint-i-quatre Hores de la Vida d'una Dona") ("Amok" and "Vierundzwanzig Stunden aus dem Leben einer Frau"). Tr. Ernest Martínez Ferrando. Badalona: Edicions Proa, 1929 (Appeared in the series "Biblioteca a tot Vent").

\section{$\mathrm{CZECH}$}

226. Dobrodruzstvi Zivota. Novely Vásne (Contains: "Erstes Erlebnis," “Amok", "Verwirrung der Gefühle"). Tr. Otto F. Babler, Rudolf Cerny, Egon Hostovsky. Praha: Nakladatelstvi Melantrich, I93 I.

227. Kaleidoskop. Tr. Jaroslava Votrubová Koutecká. Praha: Mladá Fronta, I9s 8.

228. Knibomol. ("Der Bücherwurm"). Tr. Luba and Rudolf Pelarovi. Praha: Cs. spisovatel, 1957.

229. Neviditelná Sbirka. Episoda z Inflace v Nemecku ("Die unsichtbare Sammlung"). Tr. Otto F. Babler. Olomouc: n.p., 1930.

230. Tri Novelly (Contains: "Putovani" ("Die Wanderung"), "Zazraky Zivota" ("Das Wunder des Lebens"), "Povidka za Soumraku" ("Geschichte in der Dämmerung”)). Tr. Otto Klein. Praha: Vydal J. Otto, I9r 2.

\section{DANISH}

231. Den begravede Lysestage ( $D e r$ begrabene Leucbter). Tr. Clara Hammerich. København: Jespersen and Pio, 1960.

232. Braendende Hemmelighed. ("Brennendes Geheimnis"). Tr. Jørgen Budtz-Jørgensen. København: Gyldendal, 1957 (3. ed.).

233. Den evige Broders Øjne. En Legende. ("Die Augen des ewigen Bruders"). Tr. Erik Nander. København: Hasselbalch, 1950 (4. ed., I959).

234. Eros. Tre erotiske Noveller. ("Angst," "Geschichte in der Dämmerung," "Die Frau und die Landschaft"). Tr. Clara Hammerich. København: Hirschsprung, 1953 (3. ed.).

235. En Fantastisk Nat ("Phantastische Nacht"). Tr. Helge Kjaergaard. København: Jespersen and Pio, 1958.

236. - København: Ti danske Forlaeggeres Bogklub, r960 (2. ed.).

237. Folelsernes Vildveje. Noveller. Tr. Erik Nander. Aalborg: Frede and Lauritzen, 1949.

238. - København: Jespersen and Pio. 1953 (3. ed.).

239. Et Hjertes Undergang ("Der Untergang eines Herzens"). Tr. Helge Kjaergaard. København: Jespersen and Pio, I957 (Sel. from Kaleidoskop; 2. ed., 1959).

240. Hjertets Utaalmodighed (Ungeduld des Herzens). Tr. Helge Kjaergaard. København: Jespersen and Pio, I949 (3. ed., I958).

241. Kaleidoskop. Udvalgte Noveller. Tr. Helge Kjaergaard. København: Jespersen and Pio, I950.

242. Legender (Legenden). Tr. Clara Hammerich. København: Jespersen and Pio, I95 I.

243. Skaknovelle (Scbachnovelle). Leporella. Tr. Harald Engberg. København: Aschehoug, r 948 (3. ed., r950; 5. ed., 1953; 6. ed., I955; 8. ed., I958). 
244. Sporg ikke ("Brennendes Geheimnis"). Tr. Jørgen Budtz-Jørgensen. København: n.p., 1936.

245. - København: Gyldendal, I95 I (Cf. new ed. above Braendende Hemmelighed).

\section{DUTCH}

246. Amok. Tr. Reinier P. Sterkenburg. Amsterdam: Van Ditmar, 1947.

247. Amok. Novellen van Hartstocbt. Tr. Reinier P. Sterkenburg. Zeist: Ploegsma, 1929.

248. De begraven Kandelaar. Een Legende (Der begrabene Leucbter). Amsterdam: de Lange, 1937.

249. Brandend Geheim. Vier Verhalen van Jeugdleven (Erstes Erlebnis). Tr. Mien Labberton. Zeist: Ploegsma, 1929.

250. - Amsterdam: Wereldbibliotheek, 1939 (2. and 3. ed.).

25 1. Deernis en Liefde (Ungeduld des Herzens). Tr. Guy Vanhamme. Hoogstraten (Belgium): Moderne Uitgeverij, I946.

252. Dwang ("Der Zwang"). Tr. Reinier P. Sterkenburg. 's-Gravenhage: Prometheus, I923 (Woodcuts by Frans Masereel).

253. De Legende van de derde Duif ("Die Legende von der dritten Taube"). Tr. Paul Huf. Amsterdam-Antwerpen: Wereldbibliotheek-Vereniging, I952 (3. ed., I953).

254. Het Onberaden Medelÿden (Ungeduld des Herzens). Tr. Reinier P. Sterkenburg. Amsterdam: de Lange, 1939.

255. De Oogen van den eeuwigen Broeder ("Die Augen des ewigen Bruders"). Tr. Reinier P. Sterkenburg. Huis ter Heide: De Tijdstroom, I925 (2. ed., I933).

256. Een Schaaknovelle (Schachnovelle). Tr. Paul Huf. Amsterdam: Keesing, I949.

\section{ESTONIAN}

257. Malenovell (Schachnovelle). Tr. N. Andresen. Tallin: Gaz.-zurn, 1957.

ESPERANTO

258. Brulanta Sekreto kai aliaj Rakontoj (Brennendes Gebeimnis und andere Erzäblungen). Tr. Kathe R. and Paul E. Schwerin.

Rickmansworth: Esperanto Publishing Co., 1949 (2. ed., I950).

\section{ENGLISH}

259. Amok. Tr. Eden and Cedar Paul. New York: Viking, I93 I.

260. - London: Cassell, I932.

261. "Amok," Doctors' Choice. Sixteen Stories about Doctors and Medicine (selected by famous physicians). Ed. Phyllis M. and Albert P. Blaustein. Intro. Walter C. Alvarez. New York: Funk and Wagnalls, 1957.

262. - Great German Short Novels and Stories. Ed. Bennett Alfred Cerf. New York: Modern Library, 1933.

263. - Panorama of Modern Literature Contributed by 31 Great Modern Writers. Intro. Christopher Morley. New York: Doubleday, I934.

264. Beware of Pity (Ungeduld des Herzens). Tr. Phyllis and Trevor Blewitt. New York: Viking, 1939; London: Cassell, I939 (Reprinted 1952, 1953, 1954).

265. "Book-Mendel" ("Buchmendel"), MJ, XX (July, I932), I I 8-1 22, I 98-206.

266. - Men at War. Best Short Stories of All Time. Intro. Ernest Hemingway. New York: Crown, 1942.

267. - Book of Contemporary Short Stories. Ed. Dorothy Brewster. New York: Macmillan, 1936. 
268. - Jewish Caravan. Great Stories of 25 Centuries. New York: Rinehart, 1935.

269. The Buried Candelabrum (Der begrabene Leuchter). Tr. Eden and Cedar Paul. Illus. Berthold Wolpe. New York: Viking, 1937.

270. - Oxford: Phaidon Press, I944.

271. The Burning Secret ("Brennendes Geheimnis"). New York: Scott and Seltzer, I9I9 (Author listed as Stephen Branch (pseud.) - cf. DLE, 22.J.: I4 (Apr. I5, 1920), 892-893 for Zweig's rejection of this translation and the use of a pseudonym as unauthorized).

272. - London: Allen and Unwin, I92 I.

273. Conflicts: Three Tales. (Contains: "Twentyfour Hours in the Life of a Woman" ("Vierundzwanzig Stunden aus dem Leben einer Frau"), "A Failing Heart" ("Untergang eines Herzens"), "Episode in the Early Life of Privy Councillor D." ("Verwirrung der Gefühle"). Tr. Eden and Cedar Paul. New York: Viking, I927.

274. - London: Allen and Unwin, 1928.

275. "Confusion of Sentiment" ("Verwirrung der Gefühle"), 2I Variations on a Theme. Ed. Donald Webster Cory (pseud.). New York: Greenberg, 1953.

276. Invisible Collection ("Die unsichtbare Sammlung"). Illus. Joseph Malay. New York: Pynson Printers, 1926 (First printing cf. VZ, May 3I, 1925).

277. "Invisible Collection," Art of Modern Fiction. Ed. Ray Benedict West and Robert Wooster Stallman. New York: Rinehart, 1949.

278. - Best of Modern European Literature - Heart of Europe. An Anthology of Creative Writing in Europe 1920-1940. Ed. Klaus Mann and Hermann Kesten. Intro. Dorothy Canfield Fisher. New York: L. B. Fischer, I943 (Reprinted 1945).

279. - Greatest Stories of all Times. Ed. William Somerset Maugham. Garden City (New York): Garden City Pub. Co., I943.

280. - Great Short Stories. Ed. Wilbur Lang Schramm. New York: Harcourt, 1950. 281. - Tellers of Tales; roo Short Stories from the United States, England, France, Russia and Germany. Ed. William Somerset Maugham. New York: Doubleday, 1939. 282. $G B$, XIX (June, 1934), 64I-649.

283. - $L A$, CCCXXVI: 4230 (Aug. I, I925), $255-262$.

284. - PCQ, XXVI (Dec., I 939), 425-443.

285. "Jupiter," Golden Book of Dog Stories. Ed. Era Zistel. Chicago: Ziff Davis, 1947.

286. - Famous Dog Stories. Ed. Page Cooper. New York: Doubleday, 1948.

287. - C, CXXII (July, I943), 30.

288. Kaleidoscope. I 3 Stories and Novellettes. Tr. Eden and Cedar Paul. New York: Viking, 1934; London: Cassell, I934 (Contains: "Book Mendel" ("Buchmendel"), "The Burning Secret" ("Brennendes Geheimnis"), "Fear" ("Angst"), "The Governess" ("Die Gouvernante"), "Impromptu Study of a Handicraft" ("Unvermutete Bekanntschaft mit einem Handwerk"), "The Invisible Collection" ("Die unsichtbare Sammlung"), "Leporella" ("Leporella"), "Letter from an Unknown Woman" ("Brief einer Unbekannten"), "Moonbeam Alley" ("Mondscheingasse"), "Rahel Arraigns God" ("Rahel rechtet mit Gott"), "The Runaway" ("Episode am Genfer See"), "Transfiguration" ("Phantastische Nacht"), "Virata" ("Die Augen des ewigen Bruders")).

289. Kaleidoscope I. Tr. Eden and Cedar Paul. London: Cassell, I949 (2. ed., I955).

290. Kaleidoscope II. Tr. Eden and Cedar Paul. London: Cassell, I95 I (2. ed., I959).

291. "Leporella," Crimes of Passion. Ed. Herbert J. Solomon. Intro. David Partridge (pseud.). New York: Garden City Books, 1947.

292. Letter from an Unknown Woman ("Brief einer Unbekannten"). Tr. Eden and Cedar Paul. New York: Viking, I932.

293. - London: Cassell, 1933.

294. "Moonbeam Alley," Tr. Eden and Cedar Paul. Stories of Scarlet Women. New York: Avon, 1955, pp. 54-70.

295. - A World of Great Stories. Ed. Hiram Haydn and John Cournos. New York: Crown, 1947, pp. 43 I-443. 
296. The Old-Book Peddler and Other Tales for Bibliophiles. Tr. Theodore W. Koch. Evanston (Ill.): Northwestern University Press, The Charles Deering Lib., I937 (Contains: "Books are the Gateway to the World" (Das Buch als Eingang zur Welt"), "The Old Book Peddler" ("Buchmendel"), "The Invisible Collection" ("Die unsichtbare Sammlung"), "Thanks to Books" ("Dank an die Bücher")). 297. Passion and Pain. Tr. Eden and Cedar Paul. London: Chapman and Hall, r 924; New York: Richards, 1925 (Contains: "Compulsion" ("Der Zwang"), "Fowler Snared" ("Sommernovellete"), "The Governess" ("Die Gouvernante”), "Letter from an Unknown Woman" ("Brief einer Unbekannten"), "The Runaway" ("Episode am Genfer See"), "Transfiguration" ("Phantastische Nacht"), "Virata" ("Die Augen des ewigen Bruders")).

298. The Royal Game. Tr. B. W. Huebsch. New York: Viking, I944; London: Cassell, I945 (Contains: "The Royal Game" ("Schachnovelle"), "Amok" (“Amok"), "Letter from an Unknown Woman" ("Brief einer Unbekannten")).

299. - New York: Viking, I96I (Compass Books, No. C99).

300. "The Royal Game," WHC, VII (Mar., I944), 22-23.

301. "The Runaway" ("Episode am Genfer See"), Adventures in Modern Literature. Ed. Ruth Matilda Stauffer, William Hayes Cunningham and Catherine J. Sullivan. New York: Harcourt, I95 I (3. ed.).

302. Stories and Legends. Tr. Eden and Cedar Paul and Constantine Fitz Gibbon. London: Cassell, I 955 (Contains: "24 Hours from the Life of a Woman" "Vierundzwanzig Stunden aus dem Leben einer Frau"), "Failing Heart" ("Der Untergang eines Herzens"), Episode in the Early Life of Privy Councillor D." ("Verwirrung der Gefühle"), "The Buried Candelabrum" ("Der begrabene Leuchter"), "The Legend of the Third Dove" ("Die Legende der dritten Taube"), "Dissimilar Doubles" ("Die Legende der gleichungleichen Schwestern")).

303. "Unseen Collection," Yisröl. The First Jewish Omnibus. Ed. Joseph Leftwich. New York: Heritage, I933 (Rev. ed. New York: Beechhurst Press, I952).

\section{FINNISH}

304. Malttamaton Sydän. Romaanie (Ungeduld des Herzens). Tr. Lauri Hirvensalo. Porvoo: Werner Söderström, I95 I (2.ed.).

305. Sbakkitarina. Pienoisromaani (Schacbnovelle). Tr. Aina Oksala. Jyväskylä: Gummerus, I95I.

\section{FRENCH}

306. Amok ou le Fou de Malaisie ("Amokläufer"). Tr. Alzir Hella and Olivier Bournac. Pref. Romain Rolland. Paris: A. Fayard, 1952 (Le Livre de Demain, No. 28).

307. - Dictionnaire des Oeuvres de tous les Temps et de tous les Pays. I (1952), 83. Paris: Laffont-Bompiani/Stock.

308. Amok ou le Fou de Malaisie ("Amokläufer"); Lettre d'une Inconnue ("Brief einer Unbekannten"); La Ruelle au Clair de Lune ("Die Mondscheingasse"). Tr. Alzir Hella and Olivier Bournac. Pref. Romain Rolland. Paris: Stock, 1930 (2. ed.); Bruxelles: Les Lettres latines, I930(2. ed.).

309. - Paris: Ferenczi et Fils, 1939 (Woodcuts by Michel Jacquot; Le Livre Moderne Illustré, No. 308).

310. - Paris: Delamain et Boutelleau, 1948 (97. ed.; Bibliothèque Germanique).

31 . Amok ou le Fou de Malaisie ("Amokläufer"); Lettre d'une Inconnue ("Brief einer Unbekannten"); Les Yeux du Frère Eternel ("Die Augen des ewigen Bruders"). Tr. Alzir Hella and Olivier Bournac. Pref. Romain Rolland. Paris: Stock, 1927 (Le Cabinet Cosmopolite, No. I3).

312. Brûlant Secret ("Brennendes Geheimnis"); Conte Crépusculaire ("Geschichte in der 
Dämmerung"); La Nuit Fantastique ("Phantastische Nacht"); Les Deux Jumelles ("Die gleich-ungleichen Schwestern"). Tr. Alzir Hella. Paris: Grasset, I945 (Romans étrangers).

313. - Bruxelles-Paris-Amsterdam: "La Concorde," 1947.

314. Le Chandelier Enterré. Légende (Der begrabene Leucbter). Tr. Alzir Hella. Paris: Grasset, I 937 (13. ed.; Romans étrangers).

315. La Confusion des Sentiments. Notes intimes du Professeur $R$. de D. ("Verwirrung der Gefühle"). Tr. Alzir Hella and Olivier Bournac. Paris: Stock, I929.

316. - Dictionnaire des Oeuvres de tous les Temps et de tous les Pays. I (I952), 509. Paris: Laffont-Bompiani/Stock.

317. Destruction d'un Caur ("Untergang eines Herzens"); La Gouvernante ("Die Gouvernante"); Le Jeu dangereux ("Sommernovellette"?). Tr. Alzir Hella and Olivier Bournac. Paris-Neuchâtel: Attinger, I93 I (Reprinted 1946).

318. Le Jouer d'Echecs (Schachnovelle). Tr. Jacqueline Des Gouttes. (Neuchâtel-Paris: Delachaux et Niestlé, n.d. (2. ed.).

319. La Peur ("Angst"). Tr. Alzir Hella. Paris: Grasset, 1935 (18. ed.; Romans étrangers).

320. - Tr. Alzir Hella and Manfred Schenker. Paris: Ferenczi and Fils, 1937 (Le Livre Moderne Illustré, No. 27I).

321. - N. I.: L'Amitié par le Livre, 1946 (Imprimé en Belgique).

322. "La Peur," $A P L$, CVI (Sept. Io, I 935), 238-24I (Résumé by M. Renier).

323. La Pitié dangereuse (Ungeduld des Herzens). Tr. Alzir Hella. Paris: Grasset, 1939.

324. - Paris: Club français du livre, I95 I.

325. La Ruelle au Clair de Lune ("Die Mondscheingasse"); La Gouvernante ("Die Gouvernante"). Tr. Alzir Hella and Olivier Bournac. Paris: Snell, I930 (Illustrated by Pierre Louchet).

326. Vingt-quatre Heures de la Vie d'une Femme ("Vierundzwanzig Stunden aus dem Leben einer Frau"). Tr. and Intro. Alzir Hella and Olivier Bournac. Paris-Neuchâtel: Attinger, I929 (Reprinted 1932, 1944, 1947).

327. - Paris: Fayard, I952 (Le Livre de Demain. Série étrangère, No. 36; Reprint 1953).

328. - Lausanne: La Guilde du Livre, I960 (La petite Ourse, No. 39).

329. - Lausanne: La Guilde du Livre, 1962 (Supplément au Bulletin de la Guilde," May, 1962 ; Frontispice Claudine Frochause).

330. "Vingt-quatre Heures de la Vie d'une Femme," Dictionnaire des Oeuvres de tous les Temps et de tous les Pays. IV (I954), 705. Paris: Laffont-Bompiani/Stock.

\section{GEORGIAN}

31. Amok (“Amokläufer”). Tr. Leli Dzapharidzis. Tphilisi: Phroma, I927.

332. Phiphi (“Angst”). Tr. Leli Dzapharidzis. Tphilisi: Phroma, I927.

GREEK

333. Abussos Aisthematon ("Verwirrung der Gefühle"). Tr. A. Trikolonios. Athenai: Kerameos, 1950 .

334. Amok ("Amokläufer"). Tr. S. Karagiannes. Athenai: Kerameos, I95 I.

335. - Tr. G. Meranaios. Athenai: Daremas, I954.

336. - Tr. T. Konstas. Athenai: Atlantis, 1958.

337. Amok ("Amokläufer"); To Gramma mias Agnostes ("Brief einer Unbekannten"). Tr. Al. Karrer. Athenai: Kerameos, 1948.

338. - Athenai: Gkobostes, I95 I.

339. Anypomoni Cardia ("Untergang eines Herzens"). Tr. M. Cranaki. Athenai: K. M., 1953.

340. Bradino Eidillio ("Phantastische Nacht"). Tr. Al. Karrer. Athenai: Kerameos, 1948 (Reprinted 1949). 
341. Eicossi Tesseres Thores Apo Ti Zoi Mias Gbynaicas ("Vierundzwanzig Stunden aus dem Leben einer Frau"). Tr. G. Semeriotes Athenai: Maris, 1948 (Reprinted 1954). 342. - Tr. L. Castanakis.Athenai: Govosti, n.d.

343. E Katastrophe mias Kardis ("Unterangang eines Herzens"). Tr. M. Loulis. Athenai: Kerameos, 1948.

344. E Katastrophe mias Kardis ("Untergang eines Herzens"); Sto Pheggarolousto Dromaki ("Die Mondscheingasse"). Tr. K. Meranaios and G. Semeriotes. Athenai: Maris, 1948.

345. Ena Axechasto Epeissodio ("Unerwartete Bekanntschaft mit einem Handwerk"). Tr. P. Vovolinis. Athenai: Keramefs, n.d.

346. - Tr. B. Papanastassiou. Athenai: Lagoussis-Chryssochou, 949.

347. Epikindune Sumponia (Ungeduld des Herzens). Tr. P. Spilotopoulos. Athenai: Govostis, 1949.

348. Ho Tan Xupnoun Ta Niata ("Phantastische Nacht"); He Gkoubernanta ("Die Gouvernante"); To Thanasimo Mustike ("Brennendes Geheimnis"). Tr. Al. Karrer. Athenai: Kerameos, 1949.

349. I Proti Apocalypsi (German original unknown). Tr. N. Constantinidis. Athenai: Loghotechniki, Ghonia, n.d.

350. Leporella ("Leporella"). Tr. A. Trikolonios. Athenai: Kerameos, I95 I.

351. Mantel (German original unknown). Tr. P. Papanastassiou. Athenai: Pelargos, 1950.

352. O Phobos ("Angst"). Tr. A. Trikolonios. Athenai: Kerameos, I95 I.

353. - Tr. Sp. Levantis. Athenai: Govostis, 1954.

354. Rachel ("Rahel rechtet mit Gott"). Tr. K. L. Meranaios. Athenai: Maris-Kordakis, I948.

355. Sugcbuse Aisthematon ("Verwirrung der Gefühle"). Tr. Al. Karrer. Athenai: Govostis, I949.

356. To Fovero Mystico ("Brennendes Geheimnis"). Tr. D. Dimitriou. Athenai: Loghotechniki, n.d.

357. To Thameno Cantileri ("Der begrabene Leuchter").Tr. G. Meranaios. Athenai: Daremas, I954.

358. To Nissi Me Tis Ghynaiko ("Die Frau und die Landschaft"). Tr. K. L. Meranaios. Athenai: Maris, 1948.

359. - Tr. P. Vovolinis. Athenai: Keramefs, n.d.

360. To Vassilico Paichnidi (Schachnovelle). Tr. A. Ghion. Athenai: Keramefs, n.d.

36r. Tres Omorphes Histories (German originals unknown). Tr. A. Trikolonios. Athenai: Kerameos, I949.

362. Virata ("Die Augen des ewigen Bruders"). Tr. K. Meranaios. Athenai: Maris, 1948.

\section{HEB REW}

363. Essim We-Arba Shoot Be-Hayeba Shel Isha. Sippurim (Tales, German originals unknown). Tr. A. Cohn, D. Kimhi et al. Tel Aviv: Masada, 1954.

364. Ha-R'Hov L'Or Ha-Yareab (Tales, German originals unknown). Tel Aviv: Masada, 1949.

365. Hisamer Min Ha-Rabamin (Ungeduld des Herzens). Tr. Elyaqim Weinberg. Tel Aviv: Masada, 1948 (Published in two volumes).

366. Kovshey Olam (German original unknown). Tr. Emil Feuerstein. Tel Aviv: Yavneh, I950.

367. Mishaq Ha-M'Lahim ("Schachnovelle," "Untergang eines Herzens," "Verwirrung der Gefühle"). Tr. Pesah Lipovetzki. Tel Aviv: Leihman-Pales, 1949.

\section{HUNGARIAN}

368. Amok. A Szenvedely Könyve (“Amokläufer”). Tr. L. Ujvary Lajos. Budapest: GeniusKiadas, 1929. 
369. Az Érzések Zïrzavara ("Verwirrung der Gefühle"). Tr. Kiss Dezsö. Budapest: Franklin-Társulat Kiadása, r928.

370. Égö Titok. Válogatott Elbeszélesek (Die Kette. Kaleidoskop (Extracts thereof)). Tr. Anna Dániel, Mme. Pal Fodor et al. Budapest: Európa, 1957.

37r. Elsö Élmény (Erstes Erlebnis). Tr. Dormándi László and Sandor Imre. Budapest: Pantheon, 1927.

372. Három Legenda (Three Legends - German originals unknown). Tr. Ferenc Góth. Budapest: Európa, 1957.

373. Huszonnégy Óra Egy Asszony Életéböl ("Vierundzwanzig Stunden aus dem Leben einer Frau"). Tr. Kiss Dezsö. Budapest: Lampel, I926.

374. A Láthatatlan Gyüjtemény ("Die unsichtbare Sammlung"). Tr. György Káldor. Budapest: Terra, 1957.

375. Rettegés Es Egyéb El-Beszélésék ("Angst" and Tales, German originals unknown). Tel Aviv: Alexander, 1952.

376. Rettegés. Regény (“Angst.Novelle”). Tr. Péter Bukovinszky. Budapest: Legszebb Könyvek Tára, I928.

377. Sakknovella (Scbacbnovelle). Tr. Péter János. Bukarest: A Stünta si Tehnica Folyóirat Kiadása, 1957.

378. - Tr. Iván Fónagy. Budapest: Európa, 1959.

\section{LANGUAGES OF INDIA}

BENGALI

379. Ajantiar Cbithi ("Brief einer Unbekannten"). Tr. Vidhayek Bhattacharya. Calcutta: Grantham, 1959.

380. Antarjvala ("Brennendes Geheimnis"). Tr. Shantiranjan Banerji. Calcutta: Sankar Sahitya Samsad, I955.

381. Godbulir Gan ("Amokläufer"). Tr. Shantiranjan Banerji. Calcutta: Calcutta Publishers, 1953 (Translation of the English edition).

382. Karuna Korona (Ungeduld des Herzens). Tr. Shantiranjan Banerji. Calcutta: Nababharati, 1960.

383. Priyatamesu ("Brief einer Unbekannten"). Tr. Shantiranjan Bandy-Opadhyay. Barrackpur: Sabita Dasgupta, 1957 (2. ed.; Translation from the English edition).

384 Priyatamesbu ("Brief einer Unbekannten"). Tr. Shantiranjan Banerji. Calcutta: Triveni Prakashan, 1960.

385. Rajasuya (Schacbnovelle). Tr. Shantiranjan Banerji. Calcutta: T. K. Banerji, n.d.

386. Sei Ascarya Rat ("Phantastische Nacht"). Tr. Shantiranjan Bandy-Opadhyay. Calcutta: Bengal Publishers, 1955 .

387. Setubandba ("Angst"). Tr. Shantiranjan Bandy-Opadhyay. Calcutta: Ghosh Brothers, 1956 ('Translation of the English edition).

388. Stefan Zweig'er Galpa Sangraba (Collected Tales, German originals unknown). Tr. Dipak Chaudhuri. Calcutta: Rupa, I960.

\section{HINDI}

389. Aparchita Ka Patra ("Brief einer Unbekannten"). Serialized in the New Delhi Weekly Saptabik Hindustan in 1958, exact dates unknown.

390. Bujadil (Ungeduld des Herzens). Tr. Shivadanasimha Chauhan and Vijaya Chauhan. Delhi: National Publishing House, n.d. (Contains an Introduction by the translators on Zweig and his works).

391. Shatranj Ka Khel (Schachnovelle and other tales, German originals thereof unknown). Tr. Shri S. C. Joshi. Girgaon, Bombay: Hindi Granth Ratnakar, I949.

392. Stefan Zweig Ki Maban Kabaniyan ("Novellen", German originals unknown). Tr. 
Shivadanasingli Chauhan and Vijaya Chauhan. Delhi: Ranjit, 1957 (Contains an Introduction by the translators).

393. Virata ("Die Augen des ewigen Bruders"). Tr. Yashpal Jain. New Delhi: Sasta Sahitya Mandal, 1958 (2. ed.).

OR I Y A

394. Aparichitara Patra ("Brief einer Unbekannten"). Tr. Lakshminarayana Mahanit. Cuttack: Lipikalaya, 1953 (Translation from the English edition).

MALA YALAM

395. Neela Thattavum Velutha Pookkalum ("Brief einer Unbekannten"). Tr. Mavelikkara Ascutan. Ernakulam: Sahitya Parishat, 1959.

\section{TELUGU}

396. Aparichitalekba ("Brief einer Unbekannten"). Tr. Abburi Chayadevi. Hyderabad: Sadhanaprakasan, 1956.

I R A N I A N

397. Namebe Yek Zane Nashenas ("Brief einer Unbekannten"). Tr. Hassan Ali Nasr. Tehran: n.p., 1958.

IT A LIAN

398. Adolescenza. Quattro Storia del Paese dell'Infanzia (Erstes Erlebnis. Vier Geschichten aus Kinderland). Tr. Marcella Dreyfus. Milano: Sperling and Kupfer, I933.

399. Amok (“Amokläufer"). Tr. Enrico Roçca. Milano: Sperling and Kupfer, I930.

400. Caleidoscopio (Kaleidoskop). Milano: Sperling and Kupfer, I945 (Contains: "Lettera di una Sconosciuta" ("Brief einer Unbekannten"), "Leporella" ("Leporella"), "Amok" ("Amokläufer"), "Le Oro Siderati"(from Sternstunden der Menschbeit)).

40r. Eventi e Raconti (Contains: "La Paura" ("Angst"), "Adolescenza" (Erstes Erlebnis)). Milano: Sperling and Kupfer, 1945 .

402. Leggende (Legenden). Tr. Anita Rho. Milano: Sperling and Kupfer, 1937 (Contains: "La Legenda della terza Colomba" ("Legende der dritten Taube"), "Il Candelabro Sepalto" ("Der begrabene Leuchter"), "Gli Occhi dell'eterno Fratello" ("Die Augen des ewigen Bruders"), "Rachele contende con Dio" ("Rahel techtet mit Gott”); Reprinted 1945).

403. Lettera di una Sconosciuta ("Brief einer Unbekannten"). Tr. Berta Burgio Ahrens. Milano: Sperling and Kupfer, 1932 (Reprinted 1937).

404. La Paura ("Angst"); Episodio sul Lago di Ginevra ("Episode am Genfer See"); Mel il Bibliofilo ("Buchmendel"); La Collezione Invisibile ("Die unsichtbare Sammlung"); Conoscenzi con un Mestiere ("Unvermutete Bekanntschaft mit einem Handwerk"). Milano: Sperling and Kupfer, 1938.

405. Sovvertimento dei Sensi ("Verwirrung der Gefühle"). Tr. Berta Burgio Ahrens. Milano: Corbaccio, 1931 .

406. Ventiquattro Ore della Vita di una Donna ("Vierundzwanzig Stunden aus dem Leben einer Frau"). Tr. Cristina Baseggio. Lettera di una Sconosciuta ("Brief einer Unbekannten"). Tr. Berta Burgio Ahrens. Leporella ("Leporella"). Tr. Berta Burgio Ahrens. Amok (“Amokläufer"). Tr. Enrico Rocca. Milano: Sperling and Kupfer, 1938. 
407. Aishu No Monte Carlo ("Vierundzwanzig Stunden aus dem Leben einer Frau"). Tr. Hikaru Tsuji. Tokyo: Kadokawa Shoten, I953.

408. Aiyoku No Umi ("Amokläufer et al.). Tr. Yoshitaka Kawasaki. Tokyo: Kawade Shobo, 1956 (Contains: "Amokläufer," "Brief einer Unbekannten," Fouché).

409. Aru Kokoro No Hametsu (Novellen). Tr. Yoshitaka Kawasaki. Tokyo: Kadokawa Shoten, I953 (Contains: "Untergang eines Herzens," "Brennendes Geheimnis," "Die unsichtbare Sammlung").

410. Irene - Fujin No Himitsu ("Angst" et al.). Tr. Yoshimoto Nishi. Tokyo: Kadokawa Shoten, 1957 (Contains: "Angst," "Schachnovelle," "Leporella," "Buchmendel").

4II. On'na No Niju-yo-Jikan ("Vierundzwanzig Stunden aus dem Leben einer Frau"). Tr. Kenji Takahashi. Tokyo: Shinchosha, I950.

4I2. Kokoro No Sboso (Ungeduld des Herzens). Tr. Kazuo Okubo. Tokyo: Keiyusha, I950. 413. Saisho No Taiken (Erstes Erlebnis). Tr. Yoshitaka Kawasaki. Tokyo: Hakusuisha, I953.

414. Ummei No Kake ("Schachnovelle"); Appaku ("Der Zwang"); Pari No Suri ("Unvermutete Bekanntschaft mit einem Handwerk”). Tr. Kazuo Okubo. Tokyo: Misuzu Shobo, I95 I (Nos. I and 3 translated from the French version).

415. Wasureji No Omokage ("Brief einer Unbekannten"). Tr. Kazuo Okubo. Tokyo: Mikasa Shobo, 1956.

\section{KOREAN}

416. Eoneu Yeoin Eui Isiba Sigan ("Verwirrung der Gefühle"). Tr. Choe Hyeog-sun. Seoul: Daedongdang, ig6o.

417. Gam'jeong Eui' Hon'ran ("Verwirrung der Gefühle"). Tr. Bag Changi. Seoul: Yang' mun'sa, 1959.

\section{LATVIAN}

4I8. Valija Brutäne ("Brennendes Geheimnis"). Riga: Gramatu Draugs, 1935 (Gramatu Drauga Romanu Serija, No. 74).

419. Vinas Vestule (German original unknown). Tr. B. Z. Riga: Mali, I929.

\section{LITHUANIAN}

420. Baime (“Angst”). Tr. K. Karnauskas. Kaunas: V. Remigijus, I929.

\section{NORWEGIAN}

421. Amokloper (“Amokläufer"); Brennende Hemmelighet ("Brennendes Geheimnis”). Tr. Barbara Ring. Oslo: Aschehoug, 1928.

422. Den evige Brors Øyne ("Die Augen des ewigen Bruders"). Tr. G. H. Carlsen. Berge : J. W. Eides Boktr., 1959.

423. Farspjarta ("Brennendes Geheimnis"). Tr. Severin Eskeland. Oslo: Olaf Norli, n.d. 424. Leporella ("Leporella"). Tr. Ursula Monsen. Oslo: Aschehoug, I956 (Also contains a translation of "Buchmendel").

425. Sjakknovelle (Schachnovelle). Oslo: Aschehoug, I95 I. 
426. Amok. Nowele Szalu (“Amokläufer”). Tr. Zofija Tadeuszowa Rittnerowa. LwowWarszawa: Panteon, 1925 (Introductory poem translated by Leon Koppens).

427. 24 Godziny z Zycia Kobiety $i$ inne Opowiadania ("Vierundzwanzig Stunden aus dem Leben einer Frau" and other tales). Tr. Izabella Czermakowa, Zofija Rittnerowa and Maria Wislowska. Warszawa: Panstw. Instytut Wydawn., 1957 (Reprinted I958).

428. 24 Godziny z Zycia Kobiety w Odmecie Uczuc ("Vierundzwanzig Stunden aus dem Leben einer Frau" and other tales). Tr. Melanja Wassermann. Warszawa: Instytut Wydawniczy "Renaissance," I927.

429. - Wien: n.p., I928.

430. Legendy (Legenden). Tr. Melanja Wassermann. Warszawa: Nakladem Tow. Wydawniczego "Roj", 1930 (Contains: Stefan Zweig - Biographische Notiz, "Rachela Prawuje Sie z Panem" ("Rahel rechtet mit Gott"), "Oczy Wiencznego Brata" ("Die Augen des ewigen Bruders"), "Legenda o Podobnych acz Roznych Blizniaczkach" ("Die Legende der gleich-ungleichen Schwestern"), "Legenda o Trzeciej Golebidy" ("Legende der dritten Taube"), "Wieza Babel" ("Der Turm zu Babel")).

43 I. Pierwsze Przezycie (Erstes Erlebnis). Tr. Melanja Wassermann. Warszawa: Towarzystwo Wydawnicze "Roj," I928.

432. - Tr. Maria Wislowska. Warszawa: Panstw. Instytut Wydawn., r959.

433. Wyborowe Dziela (Selected works; German originals unknown). Warszawa-KrakowWien: n.p., 1929.

\section{PORTUGUESE}

434. A Corrente. Novelas da Adolescencia, Novelas de Sofrimento (Erstes Erlebnis). Tr. Odilon Gallotti and Sylvio Aranha de Moura. Rio de Janeiro: Civilização Brasileira, 1960.

435. Amok. O Doids da Malacia ("Amokläufer"). Tr. Alice Ogando. Porto: Civilização, I956 (II. ed., I958).

436. As Três Paixôes. Trếs Novelas de Stefan Zweig (Contains: "Divida Traiamente Paga" ("Die spät bezahlte Schuld"), "Seria ele?" ("War er es?"), "A Partida de Xadrex" (Schacbnovelle)). Tr. Odilon Gallotti and Elias Davidovich. Rio de Janeiro: Guanabara, 1949 (Vol. XVI of Zweig's collected works; Novellen $I$ and 2 are not available in the German originals).

437. Caleidoscopio (Kaleidoskop). Tr. Alice Ogando. Porto: Civilização, 1943 (2. ed.; Reprinted I952, 1957).

438. O Candelabro Sagrado (Der begrabene Leucbter). Tr. Alice Ogando. Porto: Civilização, 1956 (4. ed.; 5. ed., 1958).

439. Confusão de Sentimentos ("Verwirrung der Gefühle"). Tr. Alice Ogando. Porto: Civilização, 1957 (8. ed.).

440. Um Coraf̧ão Destrog̣ado ("Untergang eines Herzens"). Tr. Campos Monteiro Filho. Porto: Civilização, I957 (8. ed.).

44I. O Medo (“Angst”). Tr. Alice Ogando. Porto: Civilização, 1956 (8. ed.; 9. ed., 1958). 442. Noite Fantástica ("Phantastische Nacht"). Tr. Alice Ogando. Porto: Civilização, I953 (6. ed.; 7. ed., 1958).

443. O Caso de um Coraf̧ão ("Untergang eines Herzens"); Noite Fantástica ("Phantastische Nacht"). Tr. Aurelio Pinheiro. Rio de Janeiro: Irmaõs Porgetti, I94r.

444. Um Segredo Ardente ("Brennendes Geheimnis"). Tr. Alice Ogando. Porto: Civilização, 1955 (7. ed.; 8. ed., 1958).

445. Vinte e quatro Horas de uma Mulher ("Vierundzwanzig Stunden aus dem Leben einer Frau"). Tr. Alice Ogando. Porto: Civilização, I955 (Io. ed.; II. ed., 1957; I 2. ed., 1959). 
446. Lis Ogls dal Frer Etern ("Die Augen des ewigen Bruders"). Tr. Clementina Gilli. Tusan-Stamp: Roth, 1948 (Chasa Paterna, No. 57).

\section{RUMANIAN}

447. Amok (“Amokläufer”). Tr. B. Madeleine. Bucureşti: Adeverul, 1929 (Biblioteca Dimineata, No. 107).

448. Jucatorul de Şah (Schachnovelle). Tr. J. Popper. Bucureşti: Editata de Revista Stiinta si Tehnica, 1957.

449. Leporella ("Leporella"). Tr. B. Madeleine. Bucureşti: Adeverul, n.d.

450. Ochii Fratelui Veşnic ("Die Augen des ewigen Bruders"). Tr. Joachim Botez. Bucureşti: Adeverul, n.d. (Lectura Biblioteca, No. 216).

45 I. Scrisoarea unei Necunoscute ("Brief einer Unbekannten"). Tr. Sarina Cassvan-Pas. Bucureşti: Adeverul, I929 (Biblioteca Dimineata, No. I19; A fragment of this Novelle appeared as No. 43 of the Lectura. Floarea Literaturilar Straine (Bucureşti: Adeverul) under the title "Adoratie" (Tr. F. Aderca); The same issue also contained "Strada Lunii" ("Die Mondscheingasse")).

452. Simturi Ratacite ("Verwirrung der Gefühle"). Tr. S. Paul. Bucureşti: Ciornei, I 929 (Contains: "Simturi Ratacite" ("Verwirrung der Gefühle"), "Donazeci si patru Ore din Viata unei Femei" ("Vierundzwanzig Stunden aus dem Leben einer Frau"); This latter Novelle also appeared in the Lectura. Floarea Literaturilar Straine, No. II (Bucureşti: Adeverul)).

453. Spaima. Romanul unui Adulter (“Angst”). Tr. E. Marghita. Bucureşti: Adeverul, 1928.

\section{RUSSIAN}

454. Amok. Novelly (Amok). Tr. D. M. Gorfinkel and I. B. Mandelstam. Leningrad: Vremja, I 96 (Contains: "Amokläufer" and "Brief einer Unbekannten"; 2 ed., 1928; 3. ed., 1929).

455. Dvadcat" chetyre Chasa iz Zhizni Zhenshchiny ("Vierundzwanzig Stunden aus dem Leben einer Frau"). Tr. L. Vol'fson. Moskva: Goslitizdat, I955.

456. Fantasticheskaya Noch. Novelly ("Phantastische Nacht" and other Novellen). Riga: Knigoizdatel'stvo Gramatu Draugs, I928 (Contains: "Geschichte in der Dämmerung," "Die Gouvernante," "Sommernovellette," "Phantastische Nacht," "Vierundzwanzig Stunden aus dem Leben einer Frau").

457. Gibel' Serdca. Novelly ("Untergang eines Herzens" and other Novellen). Riga: Knigoizdatel'stvo Gramatu Draugs, I927 (Contains: "Untergang eines Herzens," "Verwirrung der Gefühle," "Brennendes Geheimnis," "Brief einer Unbekannten").

458. Glaza Ubitogo ("Die Augen des ewigen Bruders"). Tr. L. N. Wsewolodskaja. n.l.; Izdatel'stvo Solnce, 1925 .

459. Nezrimaja Kollekcïja ("Die unsichtbare Sammlung"). Tr. P. S. Bernstein, I. E. Charodschinskaja. Leningrad: Vremja, 1928 (Contains: "Die unsichtbare Sammlung," "Leporella," "Der Zwang," "Episode am Genfer See," “Angst," "Das Geheimnis Byrons"; Each work also appeared individually; 2. ed., I 929).

46o. Novelly (Novellen; German originals unknown). Tr. P. S. Bernstein et al. Moskva: Goslitizdat, 1959 .

46r. Prichudy Serdca ("Vierundzwanzig Stunden aus dem Leben einer Frau"). Tr. O. Brosniowskaja, L. Weissenberg, W. Velskij, D. Gorfinkel, B. A. Sukkan and M. Zeiner. Leningrad: Biblioteka Vsemirnoj Literatury, I927.

462. Rokovyemgnovenija ("Sternstunden"). Tr. P. S. Bernstein, D. M. Gorfinkel, B. A. Sorgenfrei and M. Losinski. Leningrad: Vremja, 1928 (Contains: "Die Le- 
gende von den gleich-ungleichen Schwestern," "Die Augen des ewigen Bruders," "Episode in Lyon," Sternstunden der Menschbeit; Each work also appeared separately).

463. Smjatenie Cbusto ("Vierundzwanzig Stunden aus dem Leben einer Frau" and "Verwirrung der Gefühle"). Tr. P. S. Bernstein and S. Krasilschtschikov. Leningrad: Vremja, 1927 (3. ed., 1929).

464. Strach. Novella ("Angst"). Tr. I. E. Charodschinskaja. Leningrad: Vremja, I927 (2. ed.).

465. Vtoraja Kniga Amoka. Novelly ("Das zweite Buch des Amok"). Tr. I. B. Mandelstam. Leningrad: Atenej, I924 (Contains: "Phantastische Nacht," "Die Mondscheingasse")).

466. Zhguchaja Taüna. Pervye Perezhivanüa (Brennendes Geheimnis. Erstes Erlebnis). Tr. P. S. Bernštein, A. I. Kartuschanskaja. Leningrad: Vremja, I925 (Contains: "Brennendes Geheimnis," Geschichte in der Dämmerung," "Die Gouvernante," "Sommernovellette"; 2. ed., I928; 3. ed., I929).

SERBO-CROATIAN

467. Nestrpljivo Srce (Ungeduld des Herzens). Tr. Kacusa Maletin-Avakumovic. Novi Sad: Maticasrpska, 1956 (Reprinted 1960).

468. Novele (Novellen; German originals unknown). Tr. Vladan Jojkic, Radivoj Kovacevic and Milan Tokin. Novi Sad: Maticasrpska, I95 I.

469. Pismo Nepoznate Zene $i$ Druge Novele (Begegnungen mit Menschen, Büchern und Städten and Die Kette. Ein Novellenzyklus). Tr. Vlatko Saric. Zagreb: Zora, I957 (Reprinted 1959).

\section{SLOVINIAN}

470. Tri Novele o Ljubezni ("Drei Geschichten über Liebe”; German originals unknown). Tr. Herbert Grün. Ljubljana: Slovenski Knjizni Zavod, 1950.

\section{SPANISH}

471. Amok (Amok). Tr. Pedro Salazar Diaz. Buenos Aires: Tor, n.d. (Contains: "Amok" ("Amokläufer"), "Carta de una Desconocida" ("Brief einer Unbekannten"), "Una Noche Fantastica" ("Phantastische Nacht"), "La Mujer y el Paisaje" ("Die Frau und die Landschaft"), "La Callejuela a la Luz de la Luna" ("Die Mondscheingasse")).

472. - Tr. Koellen y Catalan. Madrid: Hoy, I93I.

473. Ardiente Secreto. Novela ("Brennendes Geheimnis"). Barcelona: Edita, 9952 (Illus. J. Narro).

474. Calidoscopio. Novelas (Kaleidoskop). Tr. José Lleonart. Buenos Aires-Barcelona: Juventud, 194I (Contains: "Conocimiento Casual de un Oficio" ("Unvermutete Bekanntschaft mit einem Handwerk"), "Leporella" ("Leporella"), "Miedo" ("Angst"), "Ardiente Secreto" ("Brennendes Geheimnis"), "Novela Veraniega" ("Sommernovellette"), "La Institutriz" ("Die Gouvernante"), "Buchmendel" ("Buchmendel"), "El Refugiado" ("Episode am Genfer See"), "La Colección Invisible" ("Die unsichtbare Sammlung"), "Raguel Litiga Dios" ("Rahel rechtet mit Gott"), "Noche Fantastica" ("Phantastische Nacht"), "La Calle del Caro de Luna" ("Die Mondscheingasse"), "Los Ojos del Hermano Eterno" ("Die Augen des ewigen Bruders"), "La Leyenda de la tercera Paloma" ("Die Legende der dritten Taube"), "Las Hermanas Iguales y Desiguales" ("Die Legende der gleich-ungleichen Schwestern"); Reprinted I945 (abridged), 1959, 1960, 1961). 
475. La Calle del Claro de Luna ("Die Mondscheingasse"); Leporella ("Leporella"). Barcelona: Juventud, 1953(Illus. Lorenzo Goñi).

476. El Candelabro Enterrado ("Der begrabene Leucbter"). Tr. Fernando Gutierrez and Diego Navarro. Barcelona: Lara, 1945.

477. - Barcelona: Germán Plaza, 1956 (Version Castellana).

478. - Barcelona: Janés, I957 (Club de los Lectores).

479. Carta de una Desconocida ("Brief einer Unbekannten"). Tr. Rafael Ballester Escalas. Barcelona: Imperia, 1949.

480. Carta de una Desconocida ("Brief einer Unbekannten"); Sendas Equivocas ("Verwirrung der Gefühle"). Tr. Rafael Ballester Escalas and J. Farrán y Mayoral. Barcelona: Matéu, 1956 (Col. La Puma, No. 4; Reprinted 1960).

48r. Un Caso de Conciencia (Ungeduld des Herzens). Tr. Alfredo Cahn. Cordoba (Argentina): Assandri, 1957.

482. Celos y Confusion de Sentimientos ("Verwirrung der Gefühle"). Tr. Alfredo Cahn. Buenos Aires: Anaconda, I942.

483. Impaciencia del Corazón (Ungeduld des Herzens). Tr. Alfredo Cahn. Barcelona: Luis de Caralt, 1960 (Col. Gigante).

484. - Buenos Aires: Claridad, I958 (2. ed.).

485. - Mexico: Drake, I943.

486. - Mexico: Diana, I960 (4. ed.).

487. "La Institutriz. Novela Corta" ("Die Gouvernante"), Tr. Octavio Méndez Pereira. Biblioteca Selecta (Panama), Feb., I946, pp. 33-55.

488. La Institutriz ("Die Gouvernante"); Bucbmendel ("Buchmendel"); El Refugio ("Episode am Genfer See"); La Colección Invisible ("Die unsichtbare Sammlung"). Barcelona: Juventud, I953.

489. Noche Fantástica ("Phantastische Nacht"). Barcelona: Juventud, I 953.

490. Novela Veraniega ("Sommernovellette"); Conocimiento Casual de un Oficio ("Unerwartete Bekanntschaft mit einem Handwerk"). Barcelona: Juventud, I953.

491. Los Ojos del Hermano Eterno ("Die Augen des ewigen Bruders"); Miedo ("Angst"). Tr. Mario Verdaguer. Barcelona: Apolo, 1953 (4. ed.; Biblioteca Freya).

492. - Barcelona: Juventud, I957 (Col. "Z," No. 25; 2. ed., I96I).

493. La Piedad Peligrosa (Ungeduld des Herzens). Tr. Aristides Camboa. Barcelona: HispanoAmericana de Ediciones, 1946 (Col. "Z," No. 25; 2. ed., I96I).

493. La Piedad Peligrosa (Ungeduld des Herzens). Tr. Aristides Gamboa. Barcelona: Hispano-Americana de Ediciones, 1946 (Col. Cumbre; Reprinted i955 (Club de los Lectores)).

494. - Barcelona: Germán Plaza, 1958 (Libros Plaza, Vol. 108).

495. Sendas Equivocadas ("Verwirrung der Gefühle"). Tr. J. Farran y Mayoral. Barcelona: Matéu, 1948 (Biblioteca Matéu, Vol. 2; Reprinted 1958).

496. Veinticuatro Horas de la Vida de una Mujer ("Vierundzwanzig Stunden aus dem Leben einer Frau"). Tr. Máximo Llorente. Prólogo de José Manuel Ripamonti. Buenos Aires: Tor, 1936.

497. Voluntad Prodigiosa (Schachnovelle). Barcelona: Edit. Cultural Ibérica, I960.

SWEDISH

498. Hjärtats Oro. Roman (Ungeduld des Herzens). Tr. Hugo Hultenberg. Stockholm: Skoglund, 1943.

499. Känslornas Irrväger. Tre Noveller ("Verwirrung der Gefühle" and two other Novellen; German originals unknown). Tr. Fanny von Wilamowitz-Moellendorff. Stockholm: Wahlström and Widstrand, 1927.

500. Kedjan (Die Kette); Amerigo (Amerigo). Tr. Hugo Hultenberg. Stockholm: Skoglund, I945.

5or. Den Outgrundliga Hemligheten. Romantiska och andra Berättelser (Kaleidoskop). Tr. Hugo Hultenberg. Stockholm: Skoglund, I954. 
502. Scback, Amok och andra Noveller ("Schachnovelle, Amok and other Novellen"). Tr Hugo Hultenberg. Stockholm: Skoglund, 1943.

TURKISH

503. Acimak (Ungeduld des Herzens). Tr. Samih Tiryakioglu. Istanbul: Varlik Yayinevi, I958.

504. Amok (Amok). Tr. Tahsin Yücel. Istanbul: Varlik Yayinevi, I954 (2. ed., 1958). 505. Bir Kalbin Ölümü ("Untergang eines Herzens"); Mïrebbiye ("Die Gouvernante"). Tr. Salah Birsel. Istanbul: Istanbul Yayinlari, I954.

506. Bir Kadmin Yirmi Dört Saati. Roman ("Vierundzwanzig Stunden aus dem Leben einer Frau"). Tr. Samih Tiryakioglu. Istanbul: Varlik Yayinevi, I957.

507. Meşbul Kadinin Mektuplari ("Brief einer Unbekannten"). Tr. Kemal Bekata. Ankara: Türkiye Matbaaclilik ve Gazetecilik, I950.

508. Oliunceye Kadar (Amok). Tr. Kemal Bekata. Ankara: Türkiye Matbaacilik ve Gazetecilik, I950.

509. Korku ("Angst"). Tr. Behçet Necatigil. Istanbul: Varlik Yayinevi, I96o.

510. Usta Isi Çeviren. Tr. Tahsin Yücel ("Meisterwerke"; German originals unknown). Istanbul: Varlik Yayinevi, I954.

\section{UKR AINIAN}

51 I. Amok (Amok). Tr. Vasil Bobynskyj. Kyiv: Zachidnja Ukraina, 1928.

51 2. Lyst Neznajomoi ("Brief einer Unbekannten"). Tr. Vasil Bobynskyj. Kyiv: Zachidnja Ukraina, n.d.

513. Novely (Novellen). Tr. Galina Jara. Kyiv: Sjajvo, n.d. (Contains: "Geschichte in der Dämmerung," "Brief einer Unbekannten," "Die Mondscheingasse," "Die Frau und die Landschaft," "Phantastische Nacht," "Sommernovellette"; Intro. Oswald Burckhardt).

514. Zbirnyk Tvariv (Novellen). Tr. Vasil Bobynskyj. Kyiv: Zachidnja Ukraina, 1929 (Contains: "Amokläufer," "Brief einer Unbekannten," "Verwirrung der Gefühle," "Die Mondscheingasse"; Intro. Dmitro Zagul).

\section{YIDDISH}

51 5. Am'oq. N'ow'eln fun 'a Leidnscha'ft (Amok. Novellen einer Leidenschaft). Tr. H. Br'aq' azsch. W'arsch'e: Tur'em, I929.

516. Di begrobene Menorah (Der begrabene Leuchter). Tr. Ch. Brakarz. Buenos Aires: n.p., I 942.

517. D'erwakung (Erstes Erlebnis). Tr. H. Br'aq'azsch. W'arsch'e: Bzsch'oza, 1926.

518. Der Plonter fun Gefiln ("Verwirrung der Gefühle"). Tr. Ch. Brakarz. Buenos Aires: n.p., 1942.

\section{ESSAYS}

519. Abschied von Rilke. Tübingen: Wunderlich, 1927 (Eine Rede öffentlich gesprochen beim Gedächtnisfeier Rilkes am 20. Feb., 1927 im Staatstheater zu München; Reprinted 1928).

520. - Tübingen-Stuttgart: Wunderlich, I946 (Reprinted I947, I952).

521. "Abschied von Rilke," Begegnungen, pp. 59-73 (An excerpt appeared in Von Juden in München. Ein Gedenkbuch. München: n.p., I958, p. 207).

522. Arturo Toscanini. Ein Bildnis. Wien-Leipzig-Zürich: Rechner, I 936 (This essay served originally as the Introduction to the book Arturo Toscanini. Ein Lebensbild. Wien: Reichner, I935 by Paul Stefan-Gruenfeldt). 
523. Ausgewäblte Prosa. Ed. H. Wolf. Amsterdam: Meulenhoff, 1930 (Meulenhoff's Sammlung deutscher Schriftsteller, No. 53; Contains: "Stefan Zweig," "Die Weltminute von Waterloo," "Die Entdeckung Eldorados," "Der Kampf um den Südpol," "Die Episode am Genfer see," "Die unsichtbare Sammlung," Joseph Foucbé, Chapter V; Reprinted 1935).

524. Ausgewählte Prosa. Ed. H. Wolf. Amsterdam: Meulenhoff, 1939 (Meulenhoff's Sammlung deutscher Schriftsteller, No. 66; Contains excerpts from Erasmus, Maria Stuart and Marie Antoinette).

525. Ausgewäblte Prosa. Ed. W. Kuiper. Amsterdam: Meulenhoff, 1950 (Meulenhoff's Sammlung deutscher Schriftsteller, No. 93; Contains: "Die Weltminute von Waterloo," "Der Kampf um den Südpol," and excerpts from Die Welt von gestern, Magellan and Marie Antoinette).

526. Begegnungen mit Menschen, Büchern, Städten. Wien-Leipzig-Zürich: Reichner, I 937 (Contains: Begegnungen mit Menschen: "Erinnerungen an Emile Verhaeren" (1916), "Abschied von Rilke" (I927), "Auguste Rodin" (I913), "Arturo Toscanini" (I935), "Erinnerungen an Theodor Herzl" (I929), "Rede zu Ehren Maxim Gorkis" (I928), "Geburtstagsbrief an Hermann Bahr" (I923), "Busoni" (n.d.), "Unvergessliches Erlebnis. Ein Tag bei Albert Schweitzer" (1932), "Der Dirigent. Gustav Mahler" (n.d.), "Bruno Walter" (1936), "Frans Masereel" (1923), "Die Stimme. Josef Kainz" (n.d.), "Abschied von John Drinkwater" (1937). Begegnungen mit der Zeit: "Die Monotonisierung der Welt" (1925), "Besuch bei den Milliarden" (1932); Worte Wäbrend des Krieges: "Die schlaflose Welt" (I9I4), "Bei den Sorglosen" (I9I6), "Berta von Suttner" (1917), "Das Herz Europas" (I917), "Das Feuer" (I918), "Das Haus der tausend Schicksale" (I937), "Die moralische Entgiftung Europas" (I932). Begegnungen mit Städten und Landschaften: "Die Stunde zwischen zwei Ozeanen" (I9I2), "Ypern" (I918), "Benares: Die Stadt der tausend Tempel" (n.d.), "Das schönste Grab der Welt" (I 228), "Der Rhythmus von New York" (I9I 2), "Salzburg. Die Stadt als Rahmen" (n.d.), "Kleine Reise nach Brasilien" (I936). Begegnungen mit Büchern: "Das Buch als Eingang zur Welt" (n.d.), "Marceline Desbordes-Valmore" (I920), "Ernest Renan" (I923), "Dante" (I92I), "Zu Goethes Gedichten" (I927), "Sainte-Beuve" (I925), "Anmerkung zu Joyces 'Ulysses'," (1928), "Hans Carossa" (1928), "Die unterirdischen Bücher Balzacs" (1920), "Arthur Rimbaud" (1907), "Sinn und Schönheit der Autographen" (1935), "Dank an die Bücher" (n.d.)).

527. - Berlin-Frankfurt am Main: Fischer, I955 (Reprinted I956).

528. Durch Zeiten und Welten. Intro. and sel. Erich Fitzbauer. Graz-Wien: Stiasny, 1961 (Stiasny Bücherei, No. 79; Contains: I. An der Schwelle der Neuzeit: "Grösse und Grenzen des Humanismus" (Aus Triumph und Tragik des Erasmus von Rotterdam), "Der grosse Augenblick" (Aus Magellan). II. "Das Antlitz Dostojewskis" (Aus Baumeister der Welt), "Tolstoi" (Aus Baumeister der Welt), "Nietzsches Doppelbildnis" (Aus Baumeister der Welt), "Der Dirigent. In memoriam Gustav Mahler" (Aus Begegnungen). III. Schicksale aus der Zeit: "Episode am Genfer See," "Die unsichtbare Sammlung." IV. Heimat Österreich: "Das Wien von gestern" (Aus Zeit und Welt), "Herbstwinter in Meran" (Aus Fabrten), "Salzburg: Die Stadt als Rahmen" (Aus Begegnungen). V. In der Fremde: "Sommer in Rio" (Aus Brasilien), "Abschiedsbrief (Petropolis, 22. II. 1942). VI. Lebenstafel. VII. Bibliographie: a) Werke Stefan Zweigs, b) Briefe Stefan Zweigs, c) Das wichtigste Schrifttum über Stefan Zweig in deutscher Sprache.

529. Erinnerungen an Emile Verbaeren. Wien: Christoph Reissers Söhne, I9 17.

530. - Im Selbstverlag des Verfassers. Leipzig: Spamerschen Buchdruckerei, I927.

531. - Salzburg: n.p., I927.

532. "Erinnerungen an Emile Verhaeren," Begegnungen, pp. 9-58.

533. Europäisches Erbe. Ed. Richard Friedenthal. Frankfurt am Main: Fischer, 1960 (Contains: "Montaigne," "Chateaubriand," "Jaurès," "Léon Bazalgette," 
"Edmond Jaloux," "Romain Rolland," "Pour Ramuz!," "Lafcadio Hearn," "Jens Peter Jacobsens 'Niels Lyhne'," "Rabindranath Tagores 'Sadhána'," "Das Drama in Tausendundeiner Nacht," "E.T.A. Hoffmann," "Gustav Mahlers Wiederkehr," "Arthur Schnitzler. Zum 6o. Geburtstag," "Jakob Wassermann," "Peter Rosegger," "Anton Kippenberg," "Vorbeigehen an einem unauffälligen Menschen - Otto Weininger," "Abschied von Alexander Moissi," "Walter Rathenau," "Rainer Maria Rilke," "Joseph Roth," "Die Tragik der Vergesslichkeit," "Ist die Geschichte gerecht?," "Der Turm zu Babel").

534. Fabrten. Landschaften und Städte. Leipzig-Wien-Zürich: Tal, I9r9 (Contains: "Die ferne Landschaft," "Herbstwinter in Meran," "Schöner Morgen. Bozner Berge," "Abendaquarelle aus Algier," "Nächte am Comersee," "Frühlingsfahrt durch die Provence," "Sonnenaufgang in Venedig," "Hydepark," "Stille Insel. Bretagne," "Frühling in Sevilla," "Alpenglühen an Zürichsee," "Die Stadt der tausend Tempel," "Tai Mahal," "Gwalier," "Stadt am See. Konstanz," "Der Rhythmus von New York," "Brügge," "Die Stunde zwischen zwei Ozeanen," "Der verlorene Himmel. Elegie der Heimkehr"; Volksausgabe, 1922).

535. Flïchtiger Spiegelblick. Leipzig: Insel, 1927 (Promotion material).

536. Das Herz Europas. Ein Besuch im Genfer Roten Kreuz. Zürich: Rascher, I918 (Cf. Begegnungen, pp. 194-207).

537. Reise nach Russland. Wien: Sonderdruck, 1928 (Cf. Zeit und Welt, pp. 203-245).

538. Sinn und Schönheit der Autographen. Wien: Sonderdruck, 1935.

539. Worte am Grabe Sigmund Freuds. London: Sonderdruck, I 939.

540. - Amsterdam: De Lange, 1939.

541. Zeit und Welt. Stockholm: Bermann-Fischer, 1943 (Contains: I. Menschen und Scbicksale: "Lord Byron. Das Schauspiel eines grossen Lebens" (1924), "Marcel Prousts tragischer Lebenslauf" (I925), "Hugo von Hofmannsthal" (I929), "Worte am Sarge Sigmund Freuds" (I939), "Mater Dolorosa. Die Briefe von Nietzsches Mutter an Overbeck" (1937), "Tolstoi als religiöser und sozialer Denker" (1937), "Irrfahrt und Ende Pierre Bonchamps. Die Tragödie Philippe Daudets"(I 924), "Legende und Wahrheit der Beatrice Cenci" (1926). II. Länder und Landschaften: "Die Gärten im Kriege" (I939), "Das Wien von gestern" (1940), "Dank an Brasilien" (1936), "Abendaquarelle aus Algier" (1908), "Brügge" (1904), "Die Kathedrale von Chartres" (I924), "Oxford" (I907), "Reise nach Russland" (1928). III. Zeit und Welt: "Das Geheimnis des künstlerischen Schaffens" (1938), "Geschichtsschreibung von morgen" (1939), "Der europäische Gedanke in seiner historischen Entwicklung" (1932), "I914 und heute"(1936), "Die Geschichte als Dichterin" (I939), "Thomas Mann: 'Lotte in Weimar"' (1939); Reprinted 1946).

\section{DANISH}

542. Møde Med en Svunden Tid. Tr. Karl Hornelund. København: Jespersen and Pio, 1958 (Excerpts from Begegnungen and Zeit und Welt).

\section{FRENCH}

543. Le Caur de l'Europe. Une Visite à la Croix-rouge Internationale de Genève ("Das Herz Europas"). Genève-Paris: Editions du Carmel, I918.

544. Derniers Messages (Zeit und Welt). Tr. Alzir Hella. Paris-Neuchatel: Attinger, I 949 (Contains: "L'Histoire de Demain" ("Geschichtsschreibung von morgen"), "La Pensée européenne dans son développement historique" ("Der europäische Gedanke in seiner historischen Entwicklung"), "La Vienne d'Hier" ("Das Wien von gestern"), "Le Secret de la Création artistique" ("Das Geheimnis des 
künstlerischen Schaffens"), "L'Histoire, cette poétesse" ("Die Geschichte als Dichterin"), "Hugo von Hofmannsthal" ("Hugo von Hofmannsthal"), "Tolstoi" ("Tolstoi als religiöser und sozialer Denker"), "Légende et Vérité de Béatrice Cenci" ("Legende und Wahrheit der Beatrice Cenci"), "Lord Byron" ("Lord Byron. Das Schauspiel eines grossen Lebens"), "Mater Dolorosa. Die Briefe Nietzsches Mutter an Overbeck"), "I914 et Maintenant" ("I9I4 und heute")).

545. Souvenirs et Rencontres (Begegnungen). Tr. Alzir Hella. Paris: Grasset, I95 I (Contains: "Prologue," "Rencontre avec Emile Verhaeren" ("Erinnerungen an Emile Verhaeren"), "Frans Masereel" ("Frans Masereel"), "Arturo Toscanini" ("Arturo Toscanini"), “Adieu à Rilke" (“Abschied von Rilke"), "Hans Carossa" ("Hans Carossa"), "Maxime Gorki" ("Rede zu Ehren Maxim Gorkis"), "Notes sur 'Ulysse' de Joyce" ("Anmerkungen zu Joyces 'Ulysses"),

"Arthur Rimbaud" ("Arthur Rimbaud"), "Notes sur Goethe" ("Zu Goethes Gedichten"), "Ernest Renan" ("Ernest Renan"), "Sainte-Beuve" ("SainteBeuve"), "Dante" ("Dante"), "Marceline Desbordes-Valmore" ("Marceline Desbordes-Valmore")).

546. Souvenirs sur Emile Verbaeren ("Erinnerungen an Emile Verhaeren"). Tr. Hendrik Coopman. Intro. Franz Hellens. Bruxelles: Kryn, I93 I.

GREEK

547. Anamnisseis Kai Synantisseis (Begegnungen). Tr. Ep. Cauris. Athenai: Moundjouridhis, 1956.

548. To Mustiko tes Kallitecbnikes Demiourgias ("Das Geheimnis des künstlerischen Schaffens"). Tr. L. Pavlidis. Athenai: Kerameos, r949.

549. Teleftaia Minymata (Zeit und Welt). Tr. N. Prionistis. Athenai: Atlas, I954.

ITALIAN

550. Arturo Toscanini ("Arturo Toscanini”). Tr. Lavinia Mazzucchetti. Milano: Officina Tipografica Gregoriana, 1935.

551. Incontri ed Amicizie (Excerpts from Begegnungen, Zeit und Welt and Marceline DesbordesValmore). Tr. Anita Limentani. (Intro. Lavinia Mazzucchetti. Milano-Verona: Mondadori, 1950.

JAPANESE

552. Asu no Rekishi (Excerpts from Zeit und Welt). Tr. Nobuo lizuka. Tokyo: Risô-sha, 1958.

553. Waga Shi Waga Tomo (Excerpts from Begegnungen and Zeit und Welt). Tr. Nobuo Iizuka. Tokyo: Risô-sha, I958).

PORTUGUESE

554. Encontros. Impressões sobre Livros e Escritores (Begegnungen). Tr. Maria Henrique Oswald. Porto: Civilização, I955 (3. ed.).

SPANISH

555. El Arcano de la Creación Artística ("Das Geheimnis des künstlerischen Schaffens"). Tr. Aristides Gregory and Alfredo Cahn. Buenos Aires: Espasa-Calpe Argentina, 1952. 
556. El Mundo Insomne. Ideas, Ciudades y Paisajes (Begegnungen). Tr. Sigfrido Krebs. Barcelona: Luis de Caralt, 1947 (Reprinted 1960 ).

557. Paises y Paisajes ("Länder und Landschaften" from Zeit und Welt). Tr. Tristán de la Rosa. Barcelona: Apolo, 1952 (Contains: "La Viena de ayer" ("Das Wien von gestern"), "Jardines durante la Guerra" ("Die Gärten im Kriege”), “Acuarela vespertina de Argel" (“Abendaquarelle aus Algier"), "Brujás" ("Brügge"), "La Catedral de Chartres" ("Die Kathedrale von Chartres"), "Oxford" ("Oxford"), "Viaje a Rusia" ("Reise nach Russland"), "Gracias al Brasil" ("Dank an Brasilien")).

558. Personas y Destinos ("Menschen und Schicksale" from Zeit und Welt). Tr. Tristán de la Rosa. Barcelona: Apolo, 1952 (Contains: "Lord Byron" ("Lord Byron. Das Schauspiel eines grossen Lebens"), "La trágica Vida de Marcel Proust" ("Marcel Prousts tragischer Lebenslauf"), "Hugo von Hofmannsthal" ("Hugo von Hofmannsthal"), "Palabras junto al Féretro de Sigmund Freud" ("Worte am Sarge Sigmund Freuds"), "Mater dolorosa - la Madre de Nietzsche" ("Mater Dolorosa. Die Briefe von Nietzsches Mutter an Overbeck"), "Tolstoi" ("Tolstoi als religiöser und sozialer Denker"), "Odisea y Muerte de Pierre Bonchamps" ("Irrfahrt und Ende Philippe Daudets"), "Leyenda y Verdad de Beatriz Cenci" ("Legende und Wahrheit der Beatrice Cenci")).

559. Tiempo y Mundo ("Zeit und Welt" from Zeit und Welt). Tr. Tristán de la Rosa. Barcelona: Apolo, 1953 (Contains: "El Misterio de la Creación artistica" ("Das Geheimnis des künstlerischen Schaffens"), "La Historia del Mañana" ("Geschichtsschreibung von morgen"), "El Desarrollo histórico del Pensamiento europeo" ("Der europäische Gedanke in seiner historischen Entwicklung"), "I9I4 y Hoy" ("I9I4 und heute"), "La Historia como Fuente de Poesia" ("Die Geschichte als Dichterin"), "Thomas Mann: Lotte in Weimar" ("Thomas Mann: 'Lotte in Weimar'”)).

560. Tiempo y Mundo. Impresiones y Ensayos. 1904-1940 (Zeit und Welt). Tr. Editorial Juventud. Texto revisado por el Richard Friedenthal. Barcelona: Juventud, I959 (Reprinted 1960).

\section{SWEDISH}

561. Möten med Människor, Böcker och Städer (Begegnungen mit Menschen, Büchern und Städten). Tr. Hugo Hultenberg. Stockholm: Skoglund, I 943.

562. Tiden och Världen. Samlade Essayer och Föredrag. 1904-1940 (Zeit und Welt). Tr. Hugo Hultenberg. Stockholm: Skoglund, I944.

\section{BIOGRAPHICAL STUDIES}

\section{VIIA. AMERIGO VESPUCCIO}

563. Amerigo, die Geschicbte eines bistorischen Irrtums. Stockholm: Bermann-Fischer, 1944. 564. - Frankfurt am Main: Fischer, 1954 (In the series "Fischers Schulausgaben moderner Autoren").

\section{ENGLISH}

565. Amerigo. A Comedy of Errors in History. Tr. Andrew St. James. New York: Viking, I 942 (Originally appeared serially in Blue Book under the title "The Mystery of America's Godfather"; Book edition contains an eight page facsimile of the I 504 Augsburg edition of the "Mundus Novus"). 
566. Americo Vespuci. I Historia mias bistorikis Planis. Athenai: Caravias, I953.

PORTUGUESE

567. Americo Vespúcio. Tr. José Francisco dos Santos. Lisbõa: Civilização, I956 (4. ed.).

RUSSIAN

568. Amerigo. Tr. L. P. Lezhneva. Moskva: Geografgiz, 1960 (1. ed. 96 pp.; 3. ed., 1960, I 59 pp.).

SPANISH

569. Américo Vespucio. Tr. Alfredo Cahn. Buenos Aires: Claridad, I942.

570. - Barcelona: Lara, 1946.

571. - Barcelona: Germán Plaza, 1958 (Col. "Quién fué).

572. - Madrid: Dédalo, I960.

SWEDISH

573. Amerigo. Kedjan (Die Kette). Tr. Hugo Hultenberg. Stockholm: Skoglund, I945.

VII B. BALZAC

574. Balzac. Der Roman seines Lebens. Epilogue Richard Friedenthal, ed. Stockholm: Bermann-Fischer, 1946 (Reprinted I947).

575. - Stockholm-Amsterdam-Wien: Bermann-Fischer, I950.

576. - Hamburg-Berlin: Deutsche Hausbücherei, I 953 (For members only).

577. - Frankfurt am Main: Fischer, I954.

578. - Zürich: Büchergilde Gutenberg, I957 (For members only; Reprinted 1959). Frankfurt am Main: Büchergilde Gutenberg.

579. - Leipzig: Insel, 1958 (Ed. for East Germany).

\section{BULGARIAN}

580. Romanát na Edin Zbivot Balzak. Tr. Dimitár Stoevski. Sofija: Nar. Kultura, 1960.

\section{CZECHOSLOVAKIAN}

58r. Balzac. Tr. Maria Klimová. Bratislava: Pravda, r949.

DANISH

582. Balzac. Hans Livs Roman. Tr. Clara Hammerich. København: Hirschsprung, 1949.

DUTCH

583. Balzac. De Roman van zün Leven. Tr. F. W. van Heerikhuizen. Amsterdam: De Lange, I950. 
ENGLISH

584. Balzac. Tr. William and Dorothy Rose. New York: Viking, I946 (Reprinted I948).

\section{FINNISH}

585. Balzac. Suuren Kirkailyan Elämä. Tr. Olli Nuorto. Jyväskylä: Gummerus, 1948. FRENCH

586. Balzac. Le Roman de sa Vie. Tr. Fernand Delmas. Paris: Michel, r950.

GREEK

587. Mpalzak. Tr. Ioannes Beratis. Athenai: Govostis, 1950.

HEBREW

588. Balzac. Tr. Uriel Shelah. Jerusalem: Karni, I952.

\section{HUNG ARIAN}

589. Balzac. Tr. Tamás Mátrai. Budapest: Bibliotheca, 1958.

IT A L I AN

590. Balzac. Il Romanzo della sua Vita. Tr. Lavinia Mazzucchetti. Milano-Verona: Mondadori, 1950.

$$
\text { JAPANESE }
$$

591. Balzac. Tr. Akira Mizuno. Tokyo: Hayakawa-Shobo, 1959.

PORTUGUESE

592. Balzac. O Romance da sua Vida. Tr. Mário José Domingues. Porto: Civilização, I95 I (Reprinted 1956, 1960).

SERBO-CROATIAN

593. Balzak. Tr. Nika Milićević. Sarajevo: Narodna Prosvjeta, I959 (Gama Biblioteka, No. 33I).

\section{SPANISH}

594. Balzac. Tr. Arístides Gamboa. Barcelona: Hispano-Americana de Ediciones, 1948 (Club de los Lectores; Reprinted I955).

595. - Tr. A. Gregory. Mexico: Cumbre, 1953. 
596. Balzac. Romanen om en Diktares Liv. Tr. Hugo Hultenberg. Stockholm: Skoglund, I947.

VII C. CALVIN

597. Castellio gegen Calvin oder ein Gewissen gegen die Gewalt. Wien-Leipzig-Zürich: Reichner, 1936.

598. - Berlin-Frankfurt am Main: Fischer, I954.

\section{DANISH}

599. Samvittighed mod Magt. Castellio mod Calvin. Tr. Aage Schiøttz-Christensen. København: Jespersen and Pio, 1959.

\section{DUTCH}

6oo. Strüdrond een Brandstapel. Castellio tegen Calvün. Tr. Reinier P. Sterkenburg. Amsterdam: De Lange, 1936.

\section{ENGLISH}

6or. The Right to Heresy. Castellio against Calvin. Tr. Eden and Cedar Paul. New York: Viking, 1936.

602. - Boston: Beacon Press, I95I.

603. - London: Cassell, I95 I.

\section{FRENCH}

604. Castellion contre Calvin ou Conscience contre Violence. Tr. Alzir Hella. Paris: Grasset, I946 (8. ed.; Cf. Nef., 3.a. 2 I (Aug., 1946), 42-54).

\section{ITALIAN}

6o5. Castillio contro Calvino. Una Coscienza contro la Forza. Tr. Albina Calendo. Napoli: Fiorentino, 1945.

PORTUGUESE

606. Castélio contra Calvino. Uma Consciencia contra a Violencia. Tr. Odillon Galloti. Porto: Civilização, 1957 (4. ed.).

SPANISH

607. Castelión contra Calvino. Tr. Ramón María Tenreiro. Barcelona: Juventud, n.d. 608. - Buenos Aires: Juventud, 1940.

\section{SWEDISH}

609. Vald och Rätt. Castellio contra Calvin. Tr. Hugo Hultenberg. Stockholm: Skoglund, 1942. 
610. Marceline Desbordes-Valmore. Das Lebensbild einer Dicbterin. Leipzig: Insel, I920 (With translations from the French by Gisela Etzel-Kühn; Zweig's introductory essay to this volume is reprinted in Begegnungen, pp. 318-372).

6I1. - Leipzig: Insel, 1927.

\section{FRENCH}

61 2. Marceline Desbordes-Valmore. Son Oeuvre. Tr. Alzir Hella and Olivier Bournac. Paris: La Nouvelle Critique, I928 (Document pour l'Histoire de la Littérature française; Reprinted 1945, 64 numbered copies).

POLISH

6r 3. Tragedja Kobiety. Powjeść o Marcelinie Desbordes-Valmore. Tr. R. Centnerszwerowa. Warszawa: Instytut Wydawniczy "Renaissance," r929.

\section{RUSSIAN}

614. Marselina Debord-Val'mor. Sud'ba Poetessy. Leningrad: Vremja, I930 (Vol. VIII of Zweig's collected works).

SPANISH

6I5. La Tragedia de una Vida. Marcelina Desbordes-Valmore. Tr. J. B. Thomas. Buenos Aires: Tor, 1936.

VII E. ERASMUS VON ROTTERDAM

6I6. Triumph und Tragik des Erasmus von Rotterdam. Wien-Leipzig-Zürich: Reichner, I 934 (Reprinted 1935).

617. - Frankfurt am Main: Fischer, 1950 (Reprinted 1958).

DANISH

618. Erasmus fra Rotterdam. En Biografi. Tr. Georg Rønberg. København: Jespersen and Pio, 1934 (Reprinted 1953).

DUTCH

619. Triomf en Tragiek van Erasmus van Rotterdam. Tr. Reinier P. Sterkenburg. MaastrichtBrussel: Stols, 1934.

620. - Amsterdam: Meulenhoff, r959.

ENGLISH

621. Erasmus of Rotterdam. Tr. Eden and Cedar Paul. New York: Viking, 934 (Reprinted as Viking Compass Book C-13, 1956, 1961). 
622. - Garden City (New York): Garden City Pub. Co., 1937.

623. - London: Cassell, 1934 (Reprinted with Calvin against Castellio. The Right to Heresy, I95 I).

FRENCH

624. Erasme. Grandeur et Décadence d'une Idée. Tr. Alzir Hella. Paris: Grasset, I935.

625. - Bruxelles: Editions du Frêne, 1945 (Illus. Alessandro Berretti).

\section{GREEK}

626. Erasmos. Tr. G. Mperates. Athenai: Govostis, I950.

\section{ITALIAN}

627. Erasmo da Rotterdam. Tr. Lavinia Mazzucchetti. Milano-Verona: Mondadori, 1935 (Reprinted 1937; Reprinted 1950 in the series "Biblioteca Moderna Mondadori," No. 137 with Introduction by the Translator).

\section{PORTUGUESE}

628. Triunfo e Infortúnio de Erasmo de Rotterdão. Tr. Alice Ogando. Porto: Civilização, I959 (6. ed.).

\section{SERBO-CROATIAN}

629. Erazmo Roterdamski. Tr. Nika Milićević. Sarajevo: Narodna Prosvjeta, I957. SPANISH

630. Triunfo y Tragedio de Erasmo de Rotterdam. Tr. Ramón M. Tenreiro. Barcelona: Juventud, I935 (Col. "Z," No. 73; Reprinted I95 I, I96I).

$$
\text { VIIF. JOSEPH FOUCHÉ }
$$

631. Joseph Fouché. Bildnis eines politischen Menschen. Leipzig: Insel, 1929 I $930-2$, ed. (21.-35. Tsd.)

1930-3. „, (36.-40. Tsd.)

1930 - 4. , (41.-48. Tsd.)

r 932 - 5., , (49.-53. Tsd.).

632. - Wien-Leipzig-Zürich: Reichner, 1936.

633. - Amsterdam-Stockholm: Bermann-Fischer, 1948 (Reprinted 1950).

634. - Zürich: Büchergilde Gutenberg, I95 I (For members only).

635. - Frankfurt am Main-Hamburg: Fischer, 1952 (Fischer Bücherei, No. 4).

636. - Berlin: Fischer, 1956 (Reprinted I960 (I6.-18. Tsd.)).

637. - Berlin-Darmstadt-Wien: Deutsche Buch-Gemeinschaft, 1960 (For members only).

\section{CZECHOSLOVAKIAN}

638. Josef Fouché. Sarlatan Europy. Román politickébo Clovéka. Tr. Vaclav Beneš-Sumavský. Praha: Vilimek, 1930. 


\section{DANISH}

639. Kamaeleonen. Romanbiografi om Joseph Fouché. Tr. Clara Hammerich. København: Hirschsprung, I950.

640. - København: Ti danske Forlaeggeres Bogklub, I960 (New ed.).

\section{DUTCH}

641. Joseph Fouché. De Roman van een Gevetenlooze. Tr. Reinier P. Sterkenburg. Utrecht: De Haan, I930.

642. - Amsterdam: Van Ditmar, 1947 (Reprinted Amsterdam-Antwerpen: Van Ditmar, I948).

643. - 's-Gravenhage: Succes, 1954.

ENGLISH

644. Joseph Fouché. Tr. Eden and Cedar Paul. New York: Viking, 1930.

645. - New York: Blue Ribbon Books, 1932.

646. - London: Cassell, 1930 (Reprinted 1934, 1948).

\section{FINNISH}

647. Poliisimestari Fouché. Elämänkuvaus. Tr. Martti Santavuori. Jyväskylä: Gummerus, I953.

\section{FRENCH}

648. Joseph Fouché. Un Ministre de la Police sous Napoléon. Tr. Alzir Hella and Olivier Bournac. Paris: Grasset, 1930 (Reprinted I931, I95 I, 1957, 1959).

649. - Paris: Flammarion, 1935.

650. - Bruxelles: Editions du Frêne, I946.

651. - Paris: Le Club du Meilleur Livre, 1957.

GREEK

652. Joseph Fouché. Tr. K. Meranaios. Athenai: O Cosmos tou Viveiou, I956.

\section{HEBREW}

653. Josef Fouché. Tr. Yizhak Hirschberg. Tel Aviv: Beker, I95 I.

654. - Tel Aviv: Ger'iney Zahav, 1958.

\section{HIN D I}

655. Raj Neta. Joseph Fouché. Tr. C. D. Pandey. Maliwada, Delhi: Shri Y. D. Sharma, Sharma, Sasta Sahitya Prakashan, n.d.

\section{HUNGARIAN}

656. A Rendörminiszter Fouché Élete. Tr. Szinnai Tivadar. Budapest: Pantheon-Kiadás, I930. 
657. Fouché. Tr. Lavinia Mazzucchetti. Milano: Mondadori, r930 (6. ed., 1937).

\section{JAPANESE}

658. Joseph Fouché. Tr. Teiji Takahashi and Hideo Akiyama. Tokyo: Iwanami Shoten, I95 I.

659. Joseph Fouché et al. Tr. Kôkichi et al. Tokyo: Kawade Shobô, 1956 (Besides Fouché

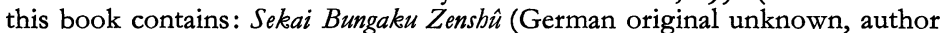
Hans Carossa), Malte no Sbuki (Rilke's Aufzeichnungen des Malte Laurids Brigge), Duine Nobika (Rilke's Duineser Elegien), Kamisama no Hanashi (Rilke's Geschicbten vom lieben Gott), Utisukushiki Madoi no Tosbi (Carossa's Das Jabr der schönen Täuschungen)).

NOR WEGIAN

66o. Joseph Fouché. Portrett av et politisk Menneske. Tr. Constance Wiel Schram. Oslo: Aschehoug, I930.

POLISH

66r. Józef Fouché. Powieść Biograficzna. Tr. R. Centnerszwerowa. Warszawa: Instytut Wydawniczy "Renaissance," n.d.

\section{PORTUGUESE}

662. José Fouché. Tr. Alice Ogando. Porto: Civilização, I956 (Reprinted 1960, 8. ed.).

RUSSIAN

663. Zhosef Fushe. Portret politicheskogo Dejatelja. Tr. P. S. Bernstein. Leningrad: Vremja, I93 I (Vol. IX of his collected works; Foreword by Prof. A. Kudrjavcev, 7-12).

SPAN ISH

664. Fouché. Retrato de un Politico. Tr. Máximo José Kahn and Miguel Pérez Ferrero. Madrid: Espasa-Calpe, r930.

665. Fouché, el Genio Tenebroso. Barcelona: Juventud, 1935 (Reprinted 1954, 1956, 1958; Col. "Z," Vol. 2 I - Grandes Biografias).

666. Joseph Fouché. Tr. Medeiros y Alburquerque. Rio de Janeiro: Guanabara, I949.

667. Fouché, Retrato de un Politico. Tr. Máximo José Kahn and Miguel Pérez Ferrero. Mexico City: Editorial Latino-Americana, 1957.

668. - Mexico City: Editora de Periódicos, I960 (Populibros La Prensa, No. 39).

\section{SWEDISH}

669. Fouché, Hertig d'Orrante. En Politikers Porträtt. Tr. Hugo Hultenberg. Stockholm: Norstedt and Söners, I930.

670. - Stockholm: Skoglund, I948. 
671. Magellan. Der Mann und seine Tat. Wien-Leipzig-Zürich: Reichner, 1938.

672. - Frankfurt am Main: Fischer, 1938 (Reprinted 1953, 1961).

673. - Zürich: Buch-Gemeinschaft "Ex Libris," 195 I (For members only).

\section{CZECHOSLOVAKIAN}

674. Magellan. Muž a Jeho Cin. Tr. Josef Nemeček. Epilogue, Vera Macháčková. Praha: NV, 1959 (In the Series "Khihovna Vojáka," No. 1 27).

\section{DANISH}

675. Jorden er Rund. Magellans Eventyrlige Bedrift. Tr. Clara Hammerich. København: Hirschsprung, I95 I.

ENGLISH

676. Conqueror of the Seas. The Story of Magellan. Tr. Eden and Cedar Paul. New York: Viking, 1938.

\section{ESTONIAN}

677. Magalbâes. Tr. Karin Reinla. Tallin: Estgosizdat, 1960.

FRENCH

678. Magellan. Tr. Alzir Hella. Paris: Grasset, 1938.

679. - Bruxelles: Editions du Frêne, 1946.

GREEK

680. Magellanos. Tr. G. Lampsa. Athenai: Bergadi, 1953.

681. - Athenai: K. M., 1956.

HEBREW

682. Magellan. Ha-ish u-foolo. Tr. Yizhak Hirschberg. Tel Aviv: Beker, I95 I.

\section{HUNGARIAN}

683. Magellan Ferdinand. A Föld elsö Körülhajózása. Tr. Zoltán Horváth. Budapest: Táncsics Kiado, 1957 (Reprinted 1958, 1960).

\section{ITALIAN}

684. Magellano. Tr. Lavinia Mazzucchetti. Milano-Verona: Mondadori, 1938. 
685. Magellan. Tr. Yoshitaka Kawasakai. Tokyo: Kawade Shobô, i956.

686. - Tr. Teiji Takahashi and Toshiak Feijii. Tokyo: Kadokawa Shoten, 1958.

MACEDONIAN

687. Magelan. Covek i Delo. Tr. Lazo Aleksov. Skopje: Kočo Racin, r956.

\section{MOLDAVIAN}

688. Epopeja luj Maželan. Tr. A. Gromov. Kišinev: Kartja Moldovenjaske, 1959.

POLISH

689. Magellan. Tr. Zofia Petersowa. Warszawa: Ksiạzka i Wiedza, I95I.

PORTUGUESE

690. Fernão de Magalhães. Tr. Maria de Castro Henriques Oswald. Porto: Civilização, I956 (8. ed.; 9. ed., I960).

\section{RUMANIAN}

691. Magellan. Omul si Fapta sa. Bucuresti: Editura Tineretului, 1956 (2. ed., 1959).

$$
\text { RUSSIAN }
$$

692. Podvig Magellana. Tr. A. S. Kulisher. Moskva: Gosud. Izd.-vo Geografichiskvi Literatury, 1956.

693. Magellan et al. Tr. A. P. Gelovani and S. N. Buačidze. Tbilisi: Codna, I959 (Also contains: Amerigo. Pobeg v Bessmertie. Bor'ba za Južnyj Poljus).

$$
\text { SERBO-CROATIAN }
$$

694. Magellan. Tr. Boško Petrović. Novi Sad: Matica Srpska, 1949.

695. - Sarajevo: Narodna Prosvjeta, I956.

\section{SLOVENIAN}

696. Magellan. Tr. Pavle Flerè. Ljubljana: Mladinska Knijiga, I956.

\section{SPANISH}

697. Magellanes. El Hombre y su Gesta. Tr. Alfredo Cahn. Buenos Aires: Claridad, 1942. 698. - Barcelona: Juventud, I954(Reprinted 1955, I957 in Col. “Z," No. 33).

699. Magellanes. La Aventura más Audaz de la Humanidad. Mexico City: Editora de Periódicos, 1959 (Populibros La Prensa, No. 33). 
700. Marie Antoinette. Bildnis eines mittleren Charakters. Leipzig: Insel, 1932.

701. - Wien: Reichner, 1936.

702. - Amsterdam: De Lange, 1939.

703. - Amsterdam: Querido, 1939.

704. - Wien: Bermann-Fischer, I948.

705. - Berlin: Suhrkamp, I949.

706. - Amsterdam: Bermann-Fischer and Querido, 1949.

707. - Wien: Büchergilde Gutenberg, I95 I (For members only).

708. - Zürich: Buchgemeinschaft "Ex Libris," I952 (For members only).

709. - Frankfurt am Main: Fischer, 1948 (Reprinted in Fischer in FrankfurtHamburg, I951, 1953, 1954, 1959, I961).

7ro. - Berlin-Darmstadt-Wien: Deutsche Buch-Gemeinschaft, I959 (For members only).

\section{DANISH}

71 I. Marie Antoinette. Et Gennemsnitsmenneskes Portraet. Tr. Kirstine Jespersen. København: Jespersen and Pio, 1933 (Reprinted 1951, 1952, 1953, 1954).

\section{DUTCH}

71 2. Marie Antoinette. Portret van een Middelmatig Karakter. Tr. G. J. Werumeus BuningEnsink. Amsterdam: De Lange, 1933 (Reprinted 1936, 1939, 1949, 1954, 1956).

\section{ENGLISH}

713. Marie Antoinette. The Portrait of an Average Woman. Tr. Eden and Cedar Paul. New York: Viking, 1933.

714. - Garden City (New York): Garden City Pub., Co., I935.

715. - London: Cassell, 1933 (Reprinted 1935, I952, 1953).

\section{FRENCH}

716. Marie Antoinette. Tr. Azlir Hella. Paris: Grasset, I933 (Reprinted 1934, 1952, 1958). 717. - Bruxelles: Editions du Frêne, I948.

718. - Bruxelles: Editions Biblis, 1954 (Col. "Figures de l'Histoire," No. 2. Sélection des Lettres).

719. - Paris: Club des Libraires de France, 1954.

720. - Paris: Librairie Générale Française, 1959 (Col. "Livre de Poche. Série historique").

\section{GREEK}

721. Maria Antouaneta. Tr. Ioannes Asteriadis. Athenai: Govostis, I950.

722. - Tr. J. Kouchtsoglou. Athenai: K.M., I953.

723. - Tr. I. Androulidakis. Athenai: Romantso, I954.

ITALIAN

724. Maria Antoinetta. Una Vita Involontariamente Eroica. Tr. Lavinia Mazzucchetti. Milano-Verona: Mondadori, 1933 (4. ed., I937). 


\section{JAPANESE}

725. Marie Antoinette. Tr. Teiji Takahashi and Hideo Akiyama. Tokyo: Mikasashobo, 1950(Reprinted I95 I, 1953).

726. - Tr. Hajime Yamashita. Tokyo: Kadokawa Shoten, 1958 (Reprinted 1959).

\section{LATVIAN}

727. Mar̈̈a Antuanete. Karalienea Tragiskas Dzwes Romas Tulkö̈is. Tr. Valdemara Karklins. Riga: Gramatu Draugs, 1933.

POLISH

728. Maria Antonina. Tr. Zofia Petersowa. Warszawa: J. Przeworski, 1948.

\section{PORTUGUESE}

729. Maria Antonieta. Tr. Alice Ogando. Porto: Civilização, I 958 (9. ed.).

\section{SPANISH}

730. Maria Antonieta. Mexico City: Editorial Drake, I 942.

731. Maria Antonieta. Una Vida Involuntariamente Heroica. Tr. Ramón María Tenreiro. Buenos Aires: Juventud, Argentina, I948.

732. - Barcelona: Juventud, I951 (Reprinted I953, 1954, 1956, I958, I961).

733. - Mexico City: Editorial Latino-Americana, I957.

SWEDISH

734. Marie Antoinette. En Olycklig Drattnings Historia. Tr. Erland Radberg. Stockholm: Forum, I946 (Reprinted I953, I959).

VIIJ. MARIA STUART

735. Maria Stuart. Wien-Leipzig-Zürich: Reichner, I935.

736. - Amsterdam: De Lange and Querido, 1939 (Forum Bücherei).

737. - Amsterdam-Wien: Bermann-Fischer and Querido, I949.

738. - Frankfurt am Main: Fischer, I95 I (Reprinted 1954).

739. - Wien: Österreichische Buchgemeinschaft, I 95 I (Vols. 68-69).

740. - Berlin-Darmstadt: Deutsche Buch Gemeinschaft, I954 (For members only).

74x. - Zürich: Buchclub "Ex Libris," I 958 (For members only).

742. - Frankfurt am Main-Hamburg: Fischer, I959 (Fischer Bücherei, No. 279).

743. - Düsseldorf: Deutscher Bücherbund, I959 (For members only).

744. - Stuttgart: Stuttgarter Hausbücherei, I959 (For members only).

\section{BULGARIAN}

745. Marj̈a Stjuart. Tr. Dimitar Stoevski. Sofija: Nar. Kultura, I957. 


\section{DANISH}

746. Maria Stuart. Tr. Georg Rønberg. København: Jespersen and Pio, 1936 (Reprinted 1953, 1954).

\section{DUTCH}

747. Maria Stuart. Tr. Reinier P. Sterkenburg. Amsterdam: De Lange, I935 (Reprinted 1950).

$$
\text { ENGLISH }
$$

748. Mary, the Queen of Scots. Tr. Eden and Cedar Paul. New York: Viking, I935.

749. - London: Cassell, 1935 (Reprinted 1950).

\section{FINNISH}

750. Maria Stuart. Elämäkerta Romaani. Tr. Elina Vaara Jyväskylä: Gummerus, 1938 (3. ed., 1955).

\section{FRENCH}

751. Marie Stuart. Tr. Alzir Hella. Paris: Grasset, 1936 (Reprinted 1958 in the series "Le Livre de Poche historique," Nos. 337-338).

752. - Bruxelles: Editions du Frêne, I947.

753. - Paris: Club des Libraires de France, I954.

\section{GREEK}

754. Maria Stouart. Tr. J. Asteriadis. Athenai: Govostis, I 948.

755. - Tr. G. Codjioulas. Athenai: Romantso, 1956.

HUNGARIAN

756. Stuart Maria. Tr. Ference Kelen, Zoltán Horváth and György Rónay. Budapest: Gondolat, 1957.

\section{R A N I AN}

757. Mari-Stuart. Tr. Farhad. Tehran: n.p., I958.

\section{ITALIAN}

758. Maria Stuarda. Tr. Lavinia Mazzucchetti. Milano-Verona: Mondadori, r936 (2. ed.).

$$
\text { JAPANESE }
$$

759. Maria Stuart. Tr. Teiji Takahashi and Yoshiyuki Nishi. Tokyo: Shinchô-sha, 1953.

$$
\text { POLISH }
$$

760. Maria Stuart. Tr. Maria Wislowska. Warszawa: Pánstw. Instytut Wydawn., I959. 
PORTUGUESE

76r. Maria Stuart. Tr. Alice Ogando. Porto: Civilização, I955 (7. ed.; 8. ed. 1957).

RUSSIAN

762. Marïa Stjuart. Tr. R. Gal'perinoj. Foreword, B. Suckova. Moskva: Izd. inostr. Lit., I959 (Reprinted 1960).

763. - Sverdlovsk: Kn. izd., I960.

SERBO-CROATIAN

764. Marija Stjuart. Tr. Nika Milicević. Sarajevo: Narodna Prosvjeta, 1956.

SPANISH

765. María Estuardo. Tr. Ramón María Tenreiro. Barcelona: Juventud, I95 8 (Reprinted 1959; Col. “Z," Vol. 40).

SWEDISH

766. Maria Stuart. Tr. Hugo Hultenberg. Stockholm: Skoglund, I 936 (Reprinted 1949).

VII K. FRANS MASEREEL

767. Frans Masereel.Der Mann und Bildner. Berlin: Juncker, 1923 (Written in conjunction with Arthur Holitscher; In the series "Graphiker unserer Zeit," Vol. I).

768. Frans Masereel. Dresden: Verlag der Kunst, 1959 (With contributions by Stefan Zweig, Pierre Vorms, Gerhard Pommeranz-Liedtke and a bibliography by Hanns-Conon von der Gabelentz).

VIIL. ROMAIN ROLLAND

769. Romain Rolland. Der Mann und das Werk. Frankfurt am Main: Rütter and Loening, I921 (Reprinted I923, 1926, 1929).

CHINESE

770. Lo-man Lo-lan. Tr. Yang Jen-piiän. Shanghai: Communist Press, I929 (Tr. from the English edition).

ENGLISH

771. Romain Rolland. The Man and bis Work. Tr. Eden and Cedar Paul. New York: Seltzer, I921.

772. - London: Allen and Unwin, I921. 


\section{FRENCH}

773. Romain Rolland. Sa Vie, son Oeuvre. Tr. Odette Richez. Bruxelles: Office de Publicité, I929.

774. - Neuchâtel: Editions de la Baconnière, 1929 (Reprinted 1936).

775. - Paris: Editions Pittoresques, 1929.

\section{GREE K}

776. Romain Rolland. Tr. N. Vrettacos. Athenai: Vivliecdhotiki, I954.

777. - Tr. Mina Zographon and C. Meranaios. Athenai: Ghalaxias, n.d.

\section{HUNG ARIAN}

778. Romain Rolland Élete. Négy Képpel. Tr. Lanyi Viktor. Budapest: A kultura könyvkiadó eś nyomda r.t. kiadása, 1923 (Contains only the section "Lebensbildnis" from the original).

\section{JAPANESE}

779. Roman Rooran. Tr. Riûtaro Hattori. Tokyo: Ars, 1925.

780. - Tr. Kazuo Okubo. Tokyo: Keiyû-sha, I95 I (Tr. from the French).

781. - Tokyo: Sôgen-sha, 1953.

782. Jônetsu no Hito Romain Rolland. Tr. Kazuo Okubo. Tokyo: Kadokawa Shoten, 1955.

RUSSIAN

783. Romen Rollan'. Ego Zhizn i Tvorchestvo. Tr. G. Henkel. Moskva-Petrograd: Gosudarstvennoc Izdatel'stvo, 1923.

SPANISH

784. Romain Rolland. El Hombre y su Obra. Tr. Alfredo Cahn. Buenos Aires: Claridad, I936 (Reprinted r942; Biblioteca de Obras Famosas, Vol. 74).

785. La Lucha contra el Mundo. Romain Rolland. Tr. G. Almagro Rodiera. Barcelona: A. G. Ivern, 1949 .

\section{YIDDISH}

786. R'om'en R'ol'an. Der M'entš 'un d'os W'erq. Tr. I. B'aše'wis. W'arse': Q'oop'er'atiw "Bik'er," I929 (2. ed.).

VII M. ÉMILE VERHAEREN

787. Emile Verbaeren. Leipzig: Insel, 1910 (2. ed., 1913).

ENGLISH

788. Emile Verhaeren. Tr. Jethro Bithell. Boston-New York: Houghton-Mifflin, I9I4. 789. - London-Edinburgh: Constable, I914. 
FRENCH

790. Emile Verbaeren. Sa Vie, son Oeuvre. Tr. Paul Morisse and Henri Chernet. Paris: Mercure de France, I9 Io.

\section{GREEK}

79r. Emile Verbaeren. Tr. Mina Zographon and C. Meranaios. Athenai: Podakis, I954.

SPANISH

792. Emile Verbaeren. Buenos Aires: Tor, 1942 (Also contains "Despedida de Rilke" (“Abschied von Rilke”)).

VIIN. PAUL VERLAINE

793. Paul Verlaine. Berlin-Leipzig: Schuster and Loeffler, I905 (In the series "Die Dichtung. Eine Sammlung von Monographien.” Ed. Paul Remer. Vol. XXX).

ENGLISH

794. Paul Verlaine. Tr. O. F. Theis. Boston-New York: Luce, I9I3.

795. - Dublin-W.C.: Maunsel, I913.

VIIO. BAUMEISTER DER WELT

796. Baumeister der Welt: Drei Meister - Der Kampf mit dem Dämon - Drei Dicbter ibres Lebens. Wien-Leipzig-Zürich: Reichner, 1935 (Reprinted 1936).

797. - Frankfurt am Main: Fischer, I95 I (Reprinted 1952, 1955, I956, 1958).

ENGLISH

798. Master Builders. Tr. Eden and Cedar Paul. New York: Viking, 1939.

SERBO-CROATIAN

799. Neimari Svijeta. Tr. Isak Samokovlija, Miodrag Petrović et al. Sarajevo: Veselin Masleša, I957.

TURKISH

800. Usta isi. Tr. Tahsin Uücel. Istanbul: Yayinevi, I954.

VII P. DREI DICHTER IHRES LEBENS

801. Drei Dicbter ibres Lebens: Casanova - Stendhal - Tolstoi. Leipzig: Insel, I928 (2. ed. I 928 (1 3.-20. Tsd.)).

802. - Frankfurt am Main: Fischer, 196I (Fischer-Bücherei, No. 42). 


\section{DANISH}

803. Liv og Digt. Tr. Clara Hammerich. København: Jespersen and Pio, 1957.

ENGLISH

804. Adepts in Self-Portraiture: Casanova - Stendhal - Tolstoy. Tr. Eden and Cedar Paul. New York: Viking, 1928.

805. - London: Allen and Unwin, r929.

806. - London: Cassell, I95 2 (Reprinted 1954)

FRENCH

807. Casanova. Tr. Alzir Hella and Olivier Bournac. Paris-Neuchâtel: Attinger, 1930 (9. ed.).

808. Tolstoi. Tr. Alzir Hella and Olivier Bournac. Paris: Attinger, 1928 (Reprinted 1946). 809. (Essai sur) Tolstoi. Tr. J. Angelloz. Paris: Corréa, i940.

810. Trois Poìtes de leur Vie. Introduction générale: Stendhal-Casanova - Tolstoi. Tr. Alzir Hella. Paris: Delamain et Boutelleau, 1937 (6. ed.; Reprinted 1950).

8II. - Paris: Stock, 1937/1938.

\section{GREEK}

81 2. Kasanobas. Tr. Mina Zographon and Kostas Meranaios. Athenai: Maris, 1950.

81 3. Stendhal. Tr. A. Pangalos. Athenai: Govostis, I954.

8r4. Tolstoj. Tr. K. Meranaios. Athenai: Nea Loghotechnia, 1956.

HUNGARIAN

81 5. Tolsztoj. Tr. Sajó Aladár. Budapest: Franklin-Társulat, 1928.

IT A L I AN

816. Tre Poeti della propria Vita. Tr. Enrico Rocca. Milano: Sperling and Kupfer, 1930 (Reprinted I931, 1945).

JAPANESE

817. Stendhal. Tr. Mizuho Aoyagi. Tokyo: Shinchô-sha, I95I.

81 8. Tolstoi. Tr. Teiji Takahashi. Tokyo: Kôbundô, 1950.

POLISH

819. Homo eroticus: Casanova. Tr. R. Centnerszwerowa. Wien: n.p., I93I.

RUSSIAN

820. Pevec svoej Zhizni. Lev Tolstoj. Tr. P. S. Bernstein. Leningrad: Vremja, I928 (2. ed., 1929; Contains the essays on Casanova and Stendhal as well as the Tolstoy 
study. It also contains a facsimile of the Foreword written by Zweig Sept. 14, I 928 while on a visit in Moscow. This forms volume VI of his collected works in Russian translation).

82 1. Velikaja Zhiznn. Lev Tolstoj. Tr. St. Wetkin. Leningrad: Izdatel'stvo Krasnaja Gazeta, 1928.

SPANISH

822. Tolstoi. Tr. Alfredo Gallard. n.l.: Proa, 1930.

823. - Buenos Aires: Tor, 1942.

824. Tolstoi. Tres Aspectos de su Vida. Tr. Joaquín Verdaguer. Barcelona: Apolo, i95 1. 825. - Barcelona: Germán Plaza, 1958 (Reprinted 1959).

\section{SWEDISH}

826. Liv som blev Dikt: Casanova-Stendhal-Tolstoj. Tr. Hugo Hultenberg. Stockholm: Skoglund, 1947 .

VII Q. DREI MEISTER

827. Drei Meister: Balzac-Dickens-Dostojewski. Leipzig: Insel, I920.

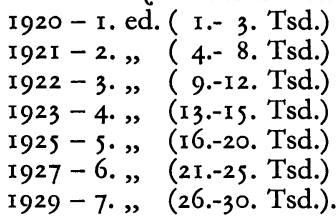

828. - Frankfurt am Main-Hamburg: Fischer, 1958 (Fischer Bücherei, No. 192).

\section{DANISH}

829. To Portraetter: Dostojevski og Nietzsche. Tr. Clara Hammerich. København: Jespersen and Pio, r955.

\section{ENGLISH}

830. Three Masters: Balzac-Dickens-Dostoeffsky. Tr. Eden and Cedar Paul. New York: Viking, 1930; London: Allen and Unwin, 1930.

FRENCH

831. Deux grands Romanciers du XIXe Siècle: Balzac-Dickens. Tr. Alzir Hella and Olivier Bournac. Paris: Simon Kra, I927.

832. Dostoievski. Tr. Henri Bloch. Paris: Rieder, I928-1929.

833. Trois Maîtres: Dostoievski-Balzac-Dickens. Tr. Henri Bloch and Alzir Hella. Paris: Grasset, 1949 (3. ed.).

\section{GREEK}

834. Balzac - Dickens. Tr. K. L. Meranaios. Athenai: Maris-Kordakis, 1948.

835. Pheontor Ntostogiebski. Tr. Yannis Beratis. Athenai: Govostis, I950. 
836. Három Mester. Balzac-Dickens-Dostojevskë. Tr. Térey Sándor. Budapest: FranklinTársulat, 1926 .

ITALIAN

837. Tre Maestri: Balzac-Dickens-Dostojevski. Tr. Berta Burgio Ahrens. Milano: Sperling and Kupfer, I 932 (Reprinted I938).

838. - Tr. Enrico Rocca. Milano: Sperling and Kupfer, 1945.

JAPANESE

839. Dostoevskii. Tr. Teiji Takahashi. Tokyo: Kôbundô, r95o.

PORTUGUESE

840. Tres Mestres. Tr. Alice Ogando. Porto: Civilização, r959 (5. ed.).

RUSSIAN

841. Tri Mastera: Bal'zak-Dikkens-Dostoevskë. Tr. G. A. Sukkau, B. A. Sorgenfrei, P. S. Bernstein. Leningrad: Vremja, I929(Vol. VII of collected works).

\section{SERBI A N}

842. Dostojevski. Tr. Miodrag Petrović. Beograd: Kńizarnica Svetlost, I93I.

843. Graditelji Svijeta: Balzak-Dickens-Dostojevski. Tr. Isak Samokovlija, Miodrag Petrović. Sarajevo: Veselin Masleša, I955.

SPANISH

844. Balzac, Honoré de. Eugenia Grandet. Biographical study of Balzac by Stefan Zweig. Madrid: Siglo XX, I 949.

845. Dickens, Charles. Oliverios Twist. Biographical study of Dickens by Stefan Zweig. Tr. E. L. V. Madrid: Siglo XX (José Ruiz Alonso), I949.

846. Dostoiewski. Barcelona: Juventud, 1959 (Col. “Z," No. 53).

847. Stendhal. Buenos Aires: Tor, n.d.

848. - Tr. Joaquín Verdaquer. Barcelona: Germán Plaza, I958 (Col. Quién fué).

849. Tres Maestros: Balzac-Dickens-Dostoiewski. Tr. and Intro. Wenceslas Roces Suárez. Madrid: Cenit, r929.

SWEDISH

850. Tre Mästre: Balzac-Dickens-Dostojevskij. Tr. Hugo Hultenberg. Stockholm: Skoglund, 1944 . 
85 1. Der Kampf mit dem Dämon: Hölderlin-Kleist-Nietzsche. Leipzig: Insel, I925

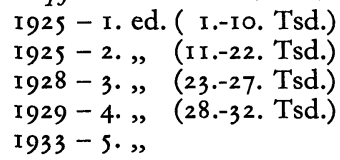

FRENCH

852. Le Combat avec le Démon: I - Hölderlin. Tr. Alzir Hella and Olivier Bournac. Paris: Stock, 1928.

853. Le Combat avec le Démon: II - Nietzsche. Tr. Alzir Hella and Olivier Bournac. Paris: Stock, I930.

854. Le Combat avec le Démon: Hölderlin-Kleist-Nietzsche. Tr. Alzir Hella. Paris: Stock, I 936.

855. - Paris: Delamain and Boutelleau, 1937.

856. - Bruxelles: Editions du Frêne, 1945.

\section{GREEK}

857. O Aghonas me to Daimona. Tr. G. Jiannacopoulos. Athenai: K.M., 1956.

858. Oi Dbianooumenoi Brosta sti Dhynami. Tr. G. Stavropoulos. Athenai: Loghotechniki Ghonia, 1956.

859. Frederic Nietzsche. Tr. K. L. Meranaios. Athenai: Maris, 1948.

860. Frederic Nietzsche. O Paidagogos tes Leuterias. Tr. P. Spiliotopoulos. Athenai: Govostis, 1948 .

IT A L I A N

861. La Lotta col Demone: Hölderlin-Kleist-Nietzsche. Tr. A. Overdorfer. Milano: Sperling and Kupfer, I930(Reprinted 1933, I945).

\section{J A P A NESE}

862. Masbin tono Tatakai. Tr. Hideo Akiyama. Tokyo: Kadokawa Shoten, 1958.

\section{PORTUGUESE}

863. Os Constructores do Mundo. O Combate com o Demónio: Hölderlin-Kleist-Nietzsche. Tr. Alice Ogando. Porto: Civilização, r955 (4. ed.; 5. ed., 1959).

\section{SPANISH}

864. La Lucha contra el Demonio: Hölderlin. Tr. Joaquín Verdaquer. Barcelona: Apolo, I95I.

865. - Barcelona: Germán Plaza, I959 (Col. Quién fué, Vol. 30).

866. La Lucha contra el Demonio: Kleist. Tr. Joaquín Verdaquer. Barcelona: Apolo, 1951. 867. - Barcelona: Germán Plaza, 959 (Col. Quién fué, Vol. 34).

868. La Lucha contra el Demonio: Nietzsche. Tr. Joaquín Verdaquer. Barcelona: Apolo, I95I. 
869. - Barcelona: Germán Plaza, I958 (Col. Quién fué, Vol. 8).

870. La Lucha contra el Demonio: Hölderlin-Kleist-Nietzsche. Tr. Joaquín Verdaquer. Barcelona: Apolo, 1934 (Reprinted I946).

87r. - Barcelona: Plaza and Janés, I96r.

VIIS. HEILUNG DURCH DEN GEIST

872. Die Heilung durch den Geist: Franz Anton Mesmer-Mary Baker-Eddy-Sigmund Freud. Leipzig: Insel, r931.

873. - Wien-Leipzig-Zürich: Reichner, I936.

874. - Frankfurt am Main-Wien: Fischer, I95 2.

DANISH

875. Sjaelens Laegedom. Tr. Clara Hammerich. København: Hirschsprung, 1936.

DUTCH

876. Genezing door den Geest: Sigmund Freud. Tr. Reinier P. Sterkenburg. Arnhem: Van Loghum Slaterus, 1932 (Kleine Bibliotheek van hedendaagsch Cultuursleven, No. 7).

ENGLISH

877. Mental Healers. Tr. Eden and Cedar Paul. New York: Ungar, I 932 (Reprinted 1962). 878. - London: Cassell, 1933.

879. - Garden City (New York): Garden City Pub. Co., I934.

FRENCH

880. La fantastique Existence de Mary Baker-Eddy. Tr. Alzir Hella and Juliette Pary. Paris: Stock, 1932 (9. ed.).

881. La Guérison par l'Esprit: Sigmund Freud. Tr. Alzir Hella and Juliette Pary. Paris: Stock, 1932.

882. La Guérison par l'Esprit: Franz Anton Mesmer-Mary Baker-Eddy-Sigmund Freud. Tr. Alzir Hella and Juliette Pary. Paris: Stock, 1934.

GREEK

883. Franz Anton Mesmer. Tr. G. Mperates. Athenai: Govostis, n.d.

884. Stokhasmoi kai Oramata. Tr. M. Lillis. Athenai: Prometheus, 1950.

IT ALIAN

885. L'Anima che guarisce. Tr. Lavinia Mazzucchetti. Milano: Sperling and Kupfer, I93 I (Reprinted 1937).

NORWEGIAN

886. Helbredelse ved Aand: Franz Anton Mesmer-Mary Baker-Eddy-Sigmund Freud. Tr. Constance Wiel Schram. Oslo: Aschehoug, I931. 
887. Mary Baker-Eddy. Tr. L. Belmont. Wien: n.p., I931.

\section{PORTUGUESE}

888. A Cura pelo Espirito: Franz Anton Mesmer-Mary Baker-Eddy. Tr. Alice Ogando. Porto: Civilização, 1957 (s. ed.).

\section{SPANISH}

889. La Curación por el Espiritu: Sigmund Freud. Tr. Francisco Payarols. Barcelona: Apolo, I934(Reprinted I949).

890. La Curación por el Espiritu: Mary Baker-Eddy. Tr. Francisco Payarols. Barcelona: Apolo, 195 I.

891. La Curación por el Espiritu: Mary Baker-Eddy-Sigmundo Freud. Tr. Alfredo Cahn. Buenos Aires: Espasa Calpe, I953.

892. Franz Anton Mesmer. Tr. Francisco Payarols. Barcelona: Germán Plaza, 1959 (Col. Quién fué, No. I 2).

893. Sigmund Freud. Tr. Francisco Payarols. Barcelona: Germán Plaza, I959 (Col. Quién fué, No. 24).

894. La Curación por el Espiritu. Tr. Francisco Payarols. Barcelona: Plaza and Janés, I96r.

\section{SWEDISH}

895. Själslig Läkekonst: Franz Anton Mesmer-Mary Baker-Eddy-Sigmund Freud. Tr. Hugo Hultenberg. Stockholm: Skoglund, i945.

\section{VIIT. STERNSTUNDEN DER MENSCHHEIT}

896. Georg Friedrich Händels Auferstehung. Eine historische Miniatur. Wien-Leipzig-Zürich: Reichner, 1937 (350 numbered copies).

897. Heroischer Augenblick. Dostojewski, Petersburg, Semenowskplatz. 22. Dez. I849. Leipzig: Staatliche Akademie für graphische Künste und Buchgewerbe, n.d. (25 numbered copies).

898. Kampf um den Sidpol. Kapitän Scott. 90. Breitengrad, 16. Jan. I912. Hannover: H. Nannen (Cf. Alpha Verlag), I 948 (Ill. Heinz Fehling; "Die bunten Hefte," No. I).

899. Sternstunden der Menschbeit. Drei historische Miniaturen. Ed. with notes by Dr. Robert Pick, Academy of Commerce, Vienna-London. London: G. Bell and Sons, 1930 (Special Intro. written by Stefan Zweig for this ed.; Contains: "Die Weltminute von Waterloo," "Die Entdeckung Eldorados," "Der Kampf um den Südpol").

900. Sternstunden der Menschbeit. Fünf historische Miniaturen. Leipzig: Insel, 1927 (Contains: "Die Weltminute von Waterloo," "Die Marienbader Elegie," "Die Entdeckung Eldorados," "Heroischer Augenblick," "Der Kampf um den Südpol"; School edition).

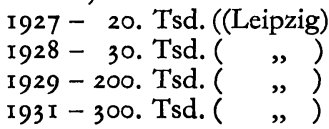


$1935-320$. Tsd. (Leipzig)

I 948 - 335. Tsd. ( \#)

I 949 - 355. Tsd. (Wiesbaden)

1950-365. Tsd. (Leipzig)

1950-375. Tsd. (Wiesbaden)

I95I - 395. Tsd.

I952 - 403. Tsd. ( ”, )

$1952-417$. Tsd. (Leipzig)

I $952-436$. Tsd. (Wiesbaden)

1953 - 456. Tsd. (Leipzig)

1957 - 553. Tsd. (Wiesbaden)

I96I - 6I5. Tsd. (Frankfurt am Main)

(The Leipzig editions appearing since 1952 do not include the foreword written by Zweig for the original edition).

90r. - Ed. with Intro., notes, German questions and vocabulary by Felix Wittner and Theodore Geissendörfer. New York: Prentice Hall, 193 I (School edition).

902. Sternstunden der Menschbeit. Zwölf bistorische Miniaturen. Stockholm: BermannFischer, 1945 (Contains: "Flucht in die Unsterblichkeit," "Die Eroberung von Byzanz," "Georg Friedrich Händels Auferstehung," "Das Genie einer Nacht," "Die Weltminute von Waterloo," "Die Marienbader Elegie," "Die Entdeckung Eldorados," "Heroischer Augenblick," "Das erste Wort über den Ozean," "Die Flucht zu Gott," "Der Kampf um den Südpol," "Der versiegelte Zug”). I943 - Stockholm: Bermann-Fischer (1.-9. ed.)

1947 - Stockholm: Bermann-Fischer (10.-16. ed.)

I 949 - Berlin-Frankfurt am Main: Fischer

1950 - Wien: Fischer

I95 I - Frankfurt am Main: Wien Büchergilde Gutenberg

I95 I - Frankfurt am Main: Fischer (Reprinted 1953, 1956, 1958).

\section{DANISH}

903. Stjernestunder. Tr. Clara Hammerich. København: Jespersen and Pio, r950 (Reprinted 1954).

\section{DUTCH}

904. Georg Friedrich Händel's Opstanding. Tr. Reinier P. Sterkenburg. Amsterdam: Wereldbibliotheek, 1939 (2. ed. Amsterdam-Antwerpen: Wereldbibliotheek, 1953). 905. Lotswendingen. Tr. Reinier P. Sterkenburg. Amsterdam: Wereldbibliotheek, I 939 (2. ed., I946; Contains: "Die Eroberung von Byzanz," "Flucht in die Unsterblichkeit," "Das Genie einer Nacht," "Der versiegelte Zug").

906. Noodlotsuren der Mensbeid. Vïf historische Miniaturen. Tr. Reinier P. Sterkenburg. Zeist: Ploegsma, r 93 I.

907. - Amsterdam: Wereldbibliotheek, I950 (2. ed.; 3. ed., 1951).

908. - Antwerpen: Wereldbibliotheek, I954 (4. ed.).

\section{ENGLISH}

909. Twelve Historical Miniatures. Tr. Eden and Cedar Paul. New York: Viking, I940 (Reprinted London: Cassell, 1955; Contains: "The Head Upon the Rostrum, Cicero's Death. Dec. 7, 43 BC," "The Conquest of Byzantium. May 29, 1453," "Flight into Immortality, Núnez de Balboa Discovers the Pacific Ocean. Sept. 25, I5 13," "The Lord Gave the Word, Handel's Messiah. Aug. 21, 1741," "The Decisive Hour at Waterloo. June I8, I815," "The Genius of 
one Night, the 'Marseillaise.' April 25, 1792," “The Marienbad Elegy Goethe's Last Love. Sept. 5, I823," "El Dorado - the Gold of California. Jan. I 2, I848," "The First Word Across the Atlantic - Cyrus Field's Cable. Aug. 5, 1858," "Quest of the South Pole - Scott Reaches the Pole. Jan. I6, I9I2," "The Sealed Train - Lenin Leaves Zürich. April 9, I917," "Wilson's Failure. Mar. 15, 1919").

\section{FIN N ISH}

910. Ibmiskunnan Täbtibetkiä. Yksitoista bistoriallista Pienoiskuvaa. Tr. J. A. Hollo. PorvooHelsinki: Söderström, 1953.

FRENCH

91 I. Les Heures étoilées de l'Humanité. Tr. Alzir Hella. Paris: Grasset, I928 (Reprinted 1939; Contains: "La Prise de Byzance," "La Fuite dans l'Immortalité," "La Résurrection de Georges Frédéric Haendel," "Le Génie d'une Nuit," "La Minute mondiale de Waterloo," "L’Elégie de Marienbad," "La Découverte de l'Eldorado," "Instant historique. Dostoiewsky, Saint Pétersbourg, Place Semenov 22.I 2.1849," "La Lutte pour le Pôle de Sud").

91 2. - Extracts ed. Fernand Delmas. Paris: Hachette, 1954.

\section{GREEK}

913. Oi Meghales Ores tis Antbropotitos. Tr. A. Karrer. Athenai: Rodaki-Pavlov, 1948. 914. - Tr. S. Patatzis. Athenai: n.p., n.d. (1956?).

\section{HEB REW}

915. Sheot Horat Goral. Tr. Edna Kornfeld. Tel Aviv: Gar'iney Zahav, 1957/58.

ITALI AN

916. Momenti Eccelsi. Cinque Miniature Storiche. Tr. Berta Burgio Ahrens. Milano: Sperling and Kupfer, 1935.

917. La Resurrezione di Händel. Tr. Marcella Gorra. Milano: Sperling and Kupfer, 1935.

\section{JAPANESE}

918. Lenin no Fûinressha ("Der versiegelte Zug"). Tr. Teiji Takahashi. Tokyo: Kôbundô, $195 \mathrm{I}$.

919. Ummei no Hoshi. Kagayaku Toki. Tr. Mayumi Haga. Tokyo: Kadokawa Shoten, 1956/57.

POLISH

920. Gwiazdy Ludzkości. Opowiadania biograficzne. Tr. Zofia Petersowa. Warszawa: Wiedza, 1948. 
921. Lupta pentru Polul Sud("Der Kampf um den Südpol”). Tr. Aurel B. Luca. Bucureşti: Adeverul, 1928.

\section{SPANISH}

922. "Balboa o la Fuga a la Immortalidad," BibS, II : 17 (May, 1947), 25-26.

923. El Genio de una Noche y otras Narraciones. Buenos Aires: Anacorda, 194I (Contains: "El Genio de una Noche," "El Tren precintado," "La Cabeza sobre la Tribuna," "El Fracaso de Wilson," "Fuga a la Immortalidad," "La Conquista de Bizancio," "La primera Palabra a través de Oceáno").

924. Momentos estelares de la Humanidad. Doce Miniatures históricas. Tr. Mario Verdaquer and Fernando Trías Beristain. Barcelona: Apolo, 1956 (Club de los Lectores, No. 33).

925. - Barcelona: Juventud, 1956 (Reprinted 1958; Col. “Z," Vol. 17).

926. Nuevos Momentos estelares de la Humanidad. Tr. Alfredo Cahn. Santiago de Chile: Ercilla, I940 (2. ed.; Contains: "Resurrección de Jorge Federico Händel," "El Genio de una Noche," "Fuga a la Immortalidad," "La Conquista de Bizancio," "La primera Palabra a través del Oceáno").

927. Voluntad prodigosa. Biografía de G. F. Händel. Barcelona: Cultural Ibérica, I960.

\section{SWEDISH}

928. Odödliga Ögonblick i Mänsklighetens Historia. Stockholm: Skoglund, 1947.

TUR KISH

929. Insanlik Taribinde Yildizin Parladigi Anlar. Tr. Burhan Arpad. Istanbul: Istanbul Matbaasi, I954(Reprinted I956).

\section{BRASILIEN}

930. Brasilien. Ein Land der Zukunft. Stockholm: Bermann-Fischer, 194I.

DUTCH

931. Brazilie, Het Land der Toekomst. Tr. Johan Winkler. Amsterdam: De Boer, 1953.

\section{ENGLISH}

932. Brazil, Land of the Future. Tr. Andrew St. James. New York: Viking, 194r. 933. - London-Toronto: Cassell, I942.

\section{FRENCH}

934. Le Brésil, Terre d'avenir. Tr. Jean Longeville. New York: Edition de la Maison française, 1942.

935. - Paris: Michel, I949. 
936. Brasilia, I Chora tous Mellontos. Tr. V. Rodapoulos. Athenai: Ecdhosseis, ıгs.

PORTUGUESE

937. Brasil, Pais do Futuro. Tr. Odilon Gallotti. Rio de Janeiro: Guanabara, r941.

938. - Porto: Civilização, 1959.

SPANISH

939. Brasil, Tierra del Futuro. Tr. Alfredo Cahn. Buenos Aires: Espasa Calpe Argentina, I94I (Reprinted I942, I943).

SWEDISH

940. Brasilien, Framtidslandet. Tr. Hugo Hultenberg. Stockholm: Skoglund, 1945.

\section{DIE WELT VON GESTERN}

941. Die Welt von gestern. Erinnerungen eines Europäers. Stockholm: Bermann-Fischer, 1944 942. - Leprinted 1947, I948).

943. - Frankfurt am Main: Suhrkamp, 1947.

944. - Wien: Bermann-Fischer, 1948.

945. - Berlin-Frankfurt am Main: Suhrkamp, 1949.

946. - Sel. and Intro. Jef. Jefsen. København: Schultz, 1949.

947. - Wien: Fischer, I952.

948. - Frankfurt am Main-Hamburg: G. B. Fischer, I953 (Grosse Romane der Zeit).

949. - Berlin-Frankfurt am Main: S. Fischer, I955 (Reprinted 1958).

950. - Zürich: Buchklub "Ex Libris," 1959 (For members only).

951. - Gütersloh: Bertelsmanns Lesering, I960 (For members only).

DANISH

952. Verden af $i$ Gaar. En Europaeers Erindringer. Tr. Helge Kjaergaard. København:

Jespersen and Pio, 1948 (Reprinted 1949, 1953, 1954).

DUTCH

953. De Wereld van gisteren. Herinneringen van een Europeaan. Tr. F. W. van Heerikhuizen. Amsterdam: De Lange, I948.

ENGLISH

954. The World of Yesterday. Tr. Eden and Cedar Paul. New York: Viking, I 943 (Zweig Bibliography, pp. 443-445).

955. - London: Cassell, 1953. 
956. Le Monde d'bier. Souvenirs d'un Européen. Tr. Jean Paul Zimmermann. Paris: Michel, I948 (Nouv. Sér., No. 23).

GREEK

957. O Chthessinoscosmos. Anamnisseis enos Evropaiou. Tr. M. Zographon and K. Meranaios. Athenai: Synchroni Loghotechnia, I956.

958. - Athenai: Dhiethneis Ecdhasseis, n.d. (1956?).

\section{ICELANDIC}

959. Veröld sem var. Sjálfsaevisaga. Tr. Halldór J. Jónsson and Ingólfur Pálmason. Reykjavík: Bókautgáfa Menningarjóds, 1958.

\section{NORWEGIAN}

960. Verden av $i$ gar. En Europeers Eindringer. Tr. Inger and Anders Hagerup. Oslo: Aschehoug, 1948.

$$
\text { POLISH }
$$

961. Swiat 2 Czorajszy. Tr. Maria Wislowska. Warszawa: Panstwowy Instytut Wydawniczy, 1958 .

\section{PORTUGUESE}

962. O Mundo de Ontem. Memórias de un Europeu. Tr. Manuel Rodrigues. Porto: Civilização, 1953 (3. ed., 1958).

963. O Mundo que eu vi. Minhas Memórias. Tr. Odilon Gallotti. Rio de Janeiro: Guanabara, I943.

$$
\text { SERBO-CROATIAN }
$$

964. Jučerašnji Svet. Seúanje Jednog Evropejca. Tr. Aleksandar Tišnia. Novi Sad: Matica Srpska, I952.

\section{SLOVENIAN}

965. VČerajšnji Svet. Spomeni Evropejca. Tr. Angela Vodè. Ljubljana: Cankarjeva Zalozba, 1958.

SPANISH

966. El Mundo de ayer. Autobiografia. Tr. Alfredo Cahn. Buenos Aires: Claridad, 1942. 967. - Barcelona: Hispano-Americana, 1947 (Reprinted 1955, 1956).

968. - Mexico: n.p., I949.

\section{SWEDISH}

969. Världen av i gar. En Europés Minnen. Tr. Hugo Hultenberg. Stockholm: Skoglund, I945. 
YIDDISH

970. Di Velt fun Nekbtn. Zikbroynes fun an Eyropeyer. Tr. Hayim Brakzgh. Buenos Aires: Yikdukh, 1959 .

\title{
X. CORRESPONDENCE IN BOOK-FORM
}

971. Briefwechsel: Stefan Zweig-Friderike Maria Zweig. 1912-1942. Bern: Scherz, I951.

\author{
ENGLISH
}

972. Stefan Zweig and Friderike Maria Zweig: Their Correspondence. Tr. Henry G. Alsberg and Erna MacArthur. New York: Hastings House, r954.

SPANISH

973. Correspondencia de Stefan y Friderike Maria Zweig (Seleccionada y Recopilada por Friderike Maria Zweig). Tr. Fernando Trías Beristain. Barcelona: AHR, I957.

974. Richard Strauss - Stefan Zweig. Briefwechsel. Frankfurt am Main: Fischer, I957.

975. Unbekannte Briefe aus der Emigration an eine Freundin. Ed. Gisella Selden-Goth. WienStuttgart-Basel: Hans Deutsch, 1963.

\section{A. LETTERS TO OR FROM ZWEIG IN BOOKS}

976. Alfred Kubin. Leben-Werk-Wirkung. Ed. Paul Raabe. Hamburg: Rowohlt, I 957 (Letters to Kubin: Oct. I5, I909 and April, 1937).

977. Briefwechsel: Rainer Maria Rilke-André Gide. I909-I926. Tr. Wolfgang A. Peters. Stuttgart: Deutsche Verlagsanstalt; Wiesbaden: Insel, 1957 (Includes letters from Zweig to Rolland and Rolland to Zweig in rgr6).

978. Correspondence: Rainer Maria Rilke-André Gide. 1909-1926. Intro. and Commentaires Renée Lang. Paris: Corrêa, I95 2 (Complétée par quelques lettres de Rilke et de Gide á divers correspondants, par des lettres de Romain Rolland, Stefan Zweig et Marie de Tour et Taxis et par des extraits du Journal de Gide).

979. Dehmel, Richard. Ausgewäblte Briefe. 1902-1920. 2 Vols. Berlin: Fischer, 1923 (Seven letters to Zweig between 1902 and 1917).

980. Erich Ebermayer. Buch der Freunde. Ed. Peer Baedeker and Karl Lemke.

Lohhof bei München: Lemke, 1960 (33 letters written by Zweig and Ebermayer between 1924 and 1939 ).

981. Freud, Sigmund. Briefe: 1873-1939. Frankfurt am Main: Fischer, 1960 (Eight letters from Freud to Zweig between 1908 and 1938).

982. Rolland, Romain. Journal des Années de Guerre. 1914-1919. Paris: Michel, 1952 (Includes innumerable letters and excerpts thereof written by Rolland and Zweig).

983. Starr, William Thomas. Romain Rolland and a World at War. Evanston (Ill.): Northwestern University Press, I956 (Includes some ten letters or parts thereof written by Rolland to Zweig).

984. Wildgans, Anton. Ein Leben in Briefen, Manuskripten und Bildern. Ed. Lilly Wildgans. 3 Vols. Wien: Frick, 1947 (Includes many letters from Wildgans to Zweig between I910 and 1930 and innumerable references to $Z$ weig in other letters). 


\section{XIB. LETTERS TO OR FROM ZWEIG IN PERIODICALS}

985. "Abschiedsbrief an die Freunde," Europäer, p. 43 (Photocopy of same p. 44; Cf. Leben-Werk, pp. I I6-II7 and Durch, p. I 20).

986. "Brief an die Redaktion," DLE, 22.J : 14 (April 15, 1920), 892-893 (Letter from Zweig to the effect that the English version of "Das brennende Geheimnis" published by Scott and Seltzer (New York, 1920) with Stephan Branch as its author was not authorized by him or by the Insel Verlag. Zweig decidedly rejects the use of an English version of his name merely to facilitate the sale of his book in America).

987. "Briefe Zweigs an Jean Schorer," Blätter, I3/14 (April, r962), 7 (Quote: "I7 Briefe und eine Karte von Stefan Zweig an Jean Schorer sind von Schorer der Zweig Gesellschaft überlassen. Es handelt sich um die gesamte Korrespondenz, die Zweigs Buch Castellio gegen Calvin. Ein Gewissen gegen die Gewalt betrifft...").

988. "Briefe Zweigs an verschiedene Korrespondenten," Spiegelungen, pp. 19-2 I, 74-77, 86-93 (Letters to Karl Emil Franzos, Karl Klammer, Kurt Frieberger, Rudolf Kayser, Alfred Wolfenstein, Felix Braun, Guido Fuchs).

989. "Cartas de Stefan Zweig a Jules Romain, I940-I942," Cuadernos Americanos (Mexico), Jan.-Feb., 1943, p. 52-72.

990. "Correspondencia inédita de Stefan Zweig," Correio da Manba (Rio de Janeiro), April 15, 1945 .

99x. Dehmel, Richard. "Two Letters to Stefan Zweig (Nov. I, I909 and Sept. 7, 1917)," Blätter, 8/10(Oct., 1960), 23-25.

992. "Lettre de Stefan Zweig à Charles Du Bos: xer septembre 1929," Cabiers Charles Du Bos (Société des Amis de Charles Du Bos), June, 1961, p. 35-36.

993. "Letter to the Editor," Adam, XV: I67-I68 (Feb.-Mar., I947) (This letter by Zweig to the editor of the periodical Adam concerns his article "The Mission of the Intellectuals" which appeared in this periodical, III : I 2 (Sept., I94I), 2).

994. McClain, William H. "Soviet Russia Through the Eyes of Stefan Zweig and Romain Rolland," MLN, LXIX : I (Jan., I954), I I-17 (Excerpts from the unpublished Zweig-Rolland correspondence to be found in the archives of the "Association des Amis de Romain Rolland" in Paris).

995. McClain, William and Harry Zohn. "Stefan Zweig and Romain Rolland: The Literary and Personal Relationship," Germ $R$, XXVIII : 4 (Dec., 1953), 26228I (Excerpts from the unpublished $Z_{w}$ eig-Rolland correspondence to be found in the "Association des Amis de Romain Rolland" archives in Paris).

996. Oechler, William F. "Reception of Emile Verhaeren in Germany. Some unpublished Letters of Stefan Zweig," MLN, LXII (Apr., I947), 226-234.

997. Romains, Jules. "Derniers Mois et dernières Lettres de Stefan Zweig," RP, 62. a. (Feb., I955), 3-23 (Letters of Zweig to Romains between I940 and Feb. 19, I942).

998. "Stefan Zweigs letzter Brief an Felix Braun," Blätter, 4/5 (Apr., I959), xo.

999. Zohn, Harry. "Stefan Zweig and Contemporary European Literature. In Memoriam Stefan Zweig," GLL, N.S., V : 3 (Apr., 1952), 202-2 I 2 (Excerpt from a letter of Zweig to Julius Bab on page 206).

Iоo०. Zohn, Harry. "Stefan Zweig's Last Years: Some Unpublished Letters," MfDU, XLVIII : 2 (Feb., I956), 73-77 (Excerpts from Zweig's correspondence with Mme. Selden-Goth from June, I935 to Christmas, I94I).

I00I. Zohn, Harry and Jean-Pierre Barricelli. "Music in Stefan Zweig's Last Years Some Unpublished Letters," JulR, III : 2 (Spring, 1956), 3-I I (Excerpts from Zweig's correspondence with Alfred Einstein and with Mme. Selden-Goth). 


\title{
XII. EXCERPTS OF ZWEIG'S WORKS APPEARING IN PERIODICALS, NEWSPAPERS AND BOOKS
}

\author{
ABSCHIED VON RILKE: \\ 1002. $D L$, XXIX : 6 (1926-1927), 346. \\ I003. Juden, p. 207. \\ 1004. $M N N$, No. 52, Feb. 22, 1927. \\ 1005. NFP, Feb. 20, 1927.
}

BALZAC:

I006. NLAS, 29.a.: I I84 (I950), I-2 ("Le Mariage de Balzac").

BRASILIEN. EIN LAND DER ZUKUNFT:

roo7. Journeys, pp. $38 \mathrm{I}-402$.

1008. LA, CCCLI (Jan., I937), 384-392 ("Brazilian Diary"; Tr. from the German by R. Norden).

1009. $P L$, Oct. I $7 / 2 \mathrm{I}, \mathrm{I} 936$.

roro. $R P$, 56.a.: 7 (July, 1949), 36-50 ("Brésil").

\section{CALVIN :}

IоI I. Nef, 3.a.: 21 (Aug., I946), 42-54 ("Castellion contre Calvin").

Ior 2. $P L$, April 5, I936 ("Sebastian Castellio: ein Gewissen gegen die Gewalt").

DREI DICHTER (Casanova):

ror3. DLW, IV : 2 (I929), 3 (“Casanova, homo eroticus").

ror4. In, IX : 2 (Spring, I928), I 20-1 25 ("Casanova").

ror 5. NFP, Jan. 21, I928 ("Casanova: die Heldenzeit der Abenteurer").

DREI DICHTER (Stendhal):

Ior6. $B d$, May 27, 1928 ("Stendhals Bildnis").

I017. BT, Mar. 25, I928 ("Stendhals Bildnis").

Iог 8. $D F R$, II (I929), 269-274 ("Lügenlust und Wahrheitsfreude bei Stendhal").

ror9. $D L W$, IV : I7 (I 929), 3 ("Stendhal als Psycholog").

I020. Isb, I : 1 2 (Oct., I954), 2I-23 ("Yalan söylemek zevki ve hakikat aşki: M. Henry Beyle Stendhal hakkinda" ("Les Plaisirs du Mensonge et l'Amour de la Vérité - à propos de Henry Beyle Stendhal"); Tr. in Turkish by Muzaffer Esen).

I021. $K Z$, Mar. 29, I928 ("Stendhal der Individualist").

1022. $M Z$, July 28, I928 ("Lügenlust und Wahrheitsfreude bei Stendhal"),

1023. NFP, Mar. I/4, I928 ("Stendhals Lebensbildnis").

I024. RHb, 46.a.: I I (I937-I938), 39I-4I 2 ("Poètes de leur Vie: Stendhal").

DREI DICHTER (Tolstoi):

I025. DLW, IV : I I (I929), 3 (“Tolstoi der Künstler”).

1026. HaN, Jan. 25, I929 ("In Tolstois Heim"). 
1027. In, IX : 4 (Fall, 1928), 249-260 ("Ein Tag aus dem Leben Tolstois").

1028. $K Z$, Nov. 8, 1927 ("Das Bildnis Tolstois").

1029. $L A$, CCCXXXIV (Jan. I, 1928), 56-62 ("Day from Tolstoi's Life").

1030. NFP, Nov. 19, I927 ("Das Bildnis Tolstois").

1031. NZZ, Nov. I6, 1927 (“Tolstois 'Rasputin',").

1032. SchM, Nov. 8, I927 ("Tolstoi: die Tragödie eines Gewissens").

1033. V, Nov. 20, I927 ("Tolstoi, Schtschegolew, Piscatur: 'Rasputin',").

DREI MEISTER (Dostojewski):

1034. $B T$, Feb. 23, 19r4 ("Dostojewski: der Sinn seines Schicksals").

1035. DWr, III (Feb., I9I4), 92-108 ("Dostojewski: die Tragödie seines Lebens").

I036. In, I : 4 (1920), I95-I97 ("Dostojewskis Antlitz").

1037. $O R, \mathrm{XL}: 3$ (July-Sept., I914), 199-203 ("Dostojewski: der Kampf um die Wahrheit").

ERASMUS:

1038. NFP, Dec. 30, 1933 ("Triumph und Tragik des Erasmus von Rotterdam").

1039. $R B$, LXXIII (Mar. I6, I 935), 194-1 98 ("Erasme et sa Mission").

1040. $R H$, 60.a., CLXXVI (1935), 98 ("Erasme").

I04I. $W u W, 6 . J$. (I95I), I8-I9 ("Dulce bellum inexpertiis - aus 'Erasmus von Rotterdam,").

FAHRTEN :

1042. $D g D$, 8.J.: 40 (1956), 5 ("Sevilla-Spaniens Lächeln").

1043. DZfS, VI : II4 (I923), 2 ("Frühling in Sevilla").

ro44. FuS, II (1928), 161 ("Die Kathedrale von Chartres").

1045. InA (1921), I30-1 32 ("Drei Landschaften: 'Alpenglühen am Zürichsee,'

'Taj Mahal', 'Schöner Morgen: Bozner Berge',”).

1046. NFP, Mar. 4, 1924 ("Die Kathedrale von Chartres").

FOUCHÉ :

1047. Cht, Jan. 3I, I93I.

I048. En, May I5, I93 I ("Une Évolution de Fouché").

1049. In, $\mathrm{X}: 4$ (Fall, I929), 269-273 ("Fouchés Votum").

1050. InA (1930), I 59-I68 ("Fouchés Kampf mit Robespierre").

I051. NFP, May 19, 1929 ("Joseph Fouché, Herzog von Otranto. Der Endkampf mit Napoleon").

I052. $R E I, \mathrm{X}$ (1930), I99-237 ("Fouché contre l'Empereur).

ro53. $R H b$, Oct. 4, 930 ("Fouché contre l'Empereur").

HEILUNG DURCH DEN GEIST:

1054. NR, XLII : 2 (I93I), 258-270 ("Die Heilung durch den Geist").

I055. $S M$, IV (1931), I 2I-I32 ("Die Heilung durch den Geist").

1056. $\quad S S A$, III : 10279. 
HEILUNG DURCH DEN GEIST (Mesmer):

I057. Hy, $\mathrm{X}$ (Feb., I932), I 30-1 34 (“Mesmerism minus Mesmer").

I058. In $A$ (I931), I I-I 8 ("Franz Anton Mesmer").

ro59. $K Z$, Feb. 5, 193 I ("Mesmers Nachfolger").

1060. NFP, July 13, I5, 17, 20, 27 and Aug. 3, 7, 8, 10, 17, 1930 ("Mesmer").

1061. $\quad V Z$, Feb. I7, I93 I ("Bildnis Franz Anton Mesmers").

\section{HEILUNG DURCH DEN GEIST (Mary Baker Eddy):}

I062. $A P L$, XCVIII (May-June, I932), 367-373, 4I 5-421, 459-466 ("La Vie merveilleuse de Mary Baker Eddy"; Tr. A. Hella and J. Pary).

1063. NR, XLI : I (1930), 610-641, 770-799 and XLI : 2 (1930), 14-60 ("Das Leben 1064. $R C$, 12.a.: 9/10 (1932-1933), I I-I7 ("La Vie merveilleuse de Mary Baker Eddy").

HEILUNG DURCH DEN GEIST (Freud):

1065. HK, Jan. 3I, I93 I ("Die Entdeckung der Hypnose").

1066. In, XII : 2 (Easter, I93 I), 94-IOS (“Abendlicher Blick ins Weite").

ro67. NFP, Dec. I0, 1930 ("Sigmund Freud als Charakter").

1068. NFP, Dec. 25, I930 ("Freuds Technik der Psychoanalyse").

ro69. NLZ, Jan. 31, I93 I ("Ein Bildnis Freuds").

1070. $P B$, III (193I), 5-I8 ("Sigmund Freud und die Situation der Jahrhundertwende").

1071. RA, 5.a. (1931), I089-1099; Cont. 6.a. (1932), 47-60 ("Sigmund Freud").

1072. $V Z$, Dec. 19, 1930 (“Ein Bildnis Freuds").

\section{KAMPF MIT DEM D ÄMON :}

1073. In, VI : 2 (Easter, 1925), I 23-136 ("Der Kampf mit dem Dämon: HölderlinKleist-Nietzsche"; This article serves as the Intro. to Zweig's book by the same name).

1074. RHb, 45.a.: 10 (1937-1938), 391-402 (“Le Combat avec le Démon”).

KAMPF MIT DEM D ÄMON (Hölderlin):

1075. $B U$, II (1927), 653-667 ("Hölderlin").

1076. DH, I (1924), I4 ("Die heilige Schar: Vorklang zu einem Hölderlin Bildnis").

I077. DL, 27.J.: 3 (I924), I3 I-I35 ("Hölderlin: Phaethon oder die Begeisterung").

ro78. $K t$, 38.J.: 7 (1925), 8-15 ("Hölderlin und Weimar").

1079. NA, CCLXV (May I, I929), 68-7I ("La santa Schiera"; Tr. from the German by G. Gentilli; Cf. $D H$ above).

го8०. NFP, Nov. I I, I924 ("Hölderlins Untergang").

ı081. PJ, CXCIX (I925), 26-46 ("Hölderlins drei gestaltiges Werk").

KAMPF MIT DEM D ÄMON (Kleist):

1082. $B B M$, June 24, I925, p. 2I ("Kleistens Lebensplan").

1083. DL, 26.J.: 10 (1924), $581-583$ ("Kleist der Erzähler").

1084. $D R a$ (1925-1926), 68 ("Heinrich von Kleist, der Gejagte"). 
1085. In $A$ (1925), I 18-133 ("Die Pathologie des Gefühls bei Kleist").

1086. $K t$, July, 1924, pp. 143-147 ("Das Drama zum Drama bei Kleist").

1087. $N F P-J b$ (1925), 33-40 ("Kleists Untergang”).

KAMPF MIT DEM D ̈̈MON (Nietzsche):

1088. $B B M$, Mar., 1925 ("Nietzsche als Erzieher zur Freiheit").

1089. GR, CXXXIV (1930), 200-207 ("Nietzsche et la Danse au dessus de l' Abîme").

1090. $L A, C C C X X V$ (Apr. I 8, 1925), I42-148 ("Nietzsche's Evensong").

ro9r. $L N L$, July 19, I930 ("L'Influence du Sud sur Nietzsche").

ro92. $R B, \mathrm{LXVIII}$ (Nov. I, 1930), 64I-646 ("Frédéric Nietzsche et la Maladie").

MAGELLAN :

1093. Journeys, pp. 54-67 ("Great Moments from 'Conqueror of the Seas: the Story of Magellan"').

1094. $R D, \mathrm{XXXII}$ (I938), I I I-I 27 ("Conqueror of the Seas: the Story of Magellan"; Tr. by Eden and Cedar Paul).

MARCELINE DESBORDES-VALMORE :

I095. DLE, XXII: 4 (Nov. I5, I9I9), 209-214 ("Die Dichterin Marceline DesbordesValmore").

1096. DLE, 23.J.: 24 (Sept. I5, I921) 1476-1479 (Franz Strunz, "Notre Dame des Pleurs - Marceline Desbordes-Valmore").

1097. In, VIII: 4 (Fall, I927), 282-285 ("Menschlichkeit").

1098. NFP, Nov. 7/9, 1918 ("Marceline Desbordes-Valmore. Das Lebensbild einer Dichterin").

MARIE ANTOINETTE:

1099. Crumbs, pp. 67-85 ("Marie Antoinette").

I I00. In, XIII : 4 (Fall, r932), I93-207 ("Königin des Rokoko").

I ror. In $A$ (1933), I 53-166 ("Ein Kind wird verheiratet").

I 102. NR, XLIII : I (Mar., I932), 300-324 ("War er es? war er es nicht? Fersen und Marie Antoinette").

I 103. NYT, 13 (April I, 1933), s ("Marie Antoinette. The Story of an Average Woman").

I 104. $\quad P B$, IV (1932), IOO-I I 2 ("Das eheliche Missgeschick Marie Antoinettes").

Iro5. $R B, C I$ (Nov. 3/17, I933), 498-503, 527-530, 556-560 ("Marie Antoinette"; Tr. Alzir Hella).

I 106. $R H b, 42 . a .:$ II (1936), 139-166, 285-313, 437-473 ("Marie Antoinette, Königin von Frankreich: Martyre de la Reine Marie- Antoinette").

STERNSTUNDEN :

I 107. Diktate, p. 74 ("Kapitän Scott"), p. 130 ("Der Kampf um die Erde").

I 108. $D M f C$, 8./9.J. (1929), 424-432 ("Die Entdeckung Eldorados. J. A. Suter, Kalifornien, Jan., I848").

I 109. DNSch, 20.J. (I959), 62-64, I02-IOS ("Georg Friedrich Händels Auferstehung"). 
I г г. $D u Z$, pp. 6r-69 ("Die Entdeckung Eldorados").

IIII. DWk, Ir.J. (I93I), I07-I Iо ("Die Entdeckung Eldorados").

I I 2. $E d D$, pp. I0-2I ("Der Kampf um den Südpol”).

I I 3. Geistesleben ("Die Weltminute von Waterloo").

II 14. $G B, \mathrm{XV}$ (April, 1932), 330-334 ("Goethe's Last Love Affair"; Tr. of "Die Marienbader Elegie" by Eden and Cedar Paul).

II I5. $G B, \mathrm{XV}$ (Feb.-Mar., I932), I I6-I24 ("Weltminute von Waterloo"), 23 I-237 ("Die Entdeckung Eldorados").

I I 16. In $A$ (1928), I08-г 27 ("Die Weltminute von Waterloo").

II 1 7. Karussell, pp. I47-I63 ("Der Kampf um den Südpol").

I I 7a. Negen Vuurmakers, pp. 124-134 (Äbbreviated form of "Der Kampf um den Südpol").

II 8. NFP, Jan. 28/29, I9I4 ("Kapitän Scotts letzte Fahrt").

III9. $P L$, Dec. 24, I 928 ("Die Weltminute von Waterloo").

I 120. $R B$, CXIII (Jan. Io, I939), I 2-I5 ("Le Messie de Haendel").

I I 21. $R d O, \mathrm{XXXII}$ (1930-1932), 27-35, 217-235 ("El Minuto universel de Waterloo. Napoléon, I8.6.1815").

I $22 . \quad R P$, CCLVII : 24 (Dec., I936), 789-808 ("Résurrection de Georges Haendel").

I 123. RP, 45.a.: 21 (Nov., I938), I 79-20I ("Prise de Byzance").

\section{VERHAEREN :}

I 1 24. In, I : 2 (Dec., 1919), 78-87 ("Verhaerens Sommer").

I 25 . In, VIII : I (Christmas, 1926), 69-73 ("Stunden mit Emile Verhaeren").

\section{WVG (WELT VON GESTERN):}

I 126. CJR, VII (April, 1944), 205 ("Die Welt von gestern").

I 1 27. $D d G$, pp. 52-54 ("Der junge Hofmannsthal").

I I 28. Hofmannsthal, pp. 63-64 (Excerpt from $W \nu G$, i955 ed., pp. 55-57 ("Die Schule im vorigen Jahrhundert")).

I 29. Merian, XVII : I ("Salzburg”) (Jan., I964), 57 (Cf. WvG, I 955 ed., pp. 269-270). II 29a. Spectrum, pp. 7-9 (Cf. $W v G$, I955 ed., pp. I3-I6).

II30. $Z d Z$, IV (I961), 29-32 (Cf. $W v G$, I955 ed., pp. I3-I6; $Z d Z$ also appeared in one volume form; The above quote is to be found therein on pages $432-436$ ).

\section{ARTICLES AND LECTURES BY ZWEIG}

I131. "Abendaquarella aus Algier" (1908) (Reprinted in Zeit und Welt, pp. 161-168). I132. "Abschied von Alexander Moissi," Lecture in Milan, June 5, I935 (Reprinted in Europäisches Erbe, pp. 227-23I).

Ir33. "Abschied von John Drinkwater" (r937) (Reprinted in Begegnungen, pp. I48-I5I). I x 34. "A la Mémoire d'Alfred Fried," $A L$, 3.a.: 6(June, 1921).

I135. "Alberta von Puttkammer," $D L E$, 8.J.: I 2 (Mar. I 5, I906), 836-84I, 846-849.

I I 36. "Alessandro Poerio. Ein Italiener bei Goethe," $N F P$, Sept. I 8, I9I 8.

I 137. "Alfred H. Fried," $N F P$, May 13, I921.

I 138. "Alfredo Canciani-Bildhauer," VFZM, 22.J.: 24 (1903).

I139. "A mes Frères français," $D$, II (I916-I9I 8), I 25 -I 28.

I I 40. "An den Genius der Verantwortlichkeit," $N R$, XXXVI : 6 (June, 1925), 624-626.

I I4I. "An Detlev von Liliencron," Liliencron (1904).

1 142. "An die Freunde in Fremdland," BT, Sept. I9, I914 (Reprinted in Mann-Werk, pp. $63-70)$. 
I 143. "An Karl Kraus," Kraus, pp. 38-39.

I 144. "Anmerkung zum 'Ulysses'," $N R$, XXXIX : ro (Oct., r928), 477-479 (Reprinted in Begegnungen, pp. 4I 8-42I).

I 45. "Anthologie: Aufmarsch der Jugend (Anthologie jüngster Prosa)," MP, Jan. 2, I929.

I 146. "Anton, Friend of all the World," $R D, \mathrm{XXXV}$ (Oct., I939), 69-72.

I 47. "Anton Kippenberg," $D B K$, X-XI (1924), 46-52 (Cf. also $F Z$, May 22, 1924; Kippenberg (1924); Europäisches Erbe, pp. 214-222).

I 148. "An Wilhelm Schmidtbonn," Schmidtbonn, pp. 9-1o.

r 149. "Arthur Rimbaud," $Z$, LVII (I907), 300-305 (Reprinted in In $A$ (1908), 84-96; Begegnungen, pp. 432-440; This article also serves as the Introduction to Arthur Rimbaud. Leben und Dichtung. Leipzig: Insel, r907).

I I 50. "Arthur Schnitzler - Narrateur," $R A$, 6.a. (1932), $387-398$.

I I I. "Arthur Schnitzler zu seinem 6osten Geburtstage," NR, 33.J.: I (1922), 510-513 (Reprinted in Europäiscbes Erbe, pp. 183-1 87).

I I 52. "Aufruf zur Geduld," DTb, I : I (Jan., I920), 7-Io.

I I 53. "Aus Romain Rollands Kindheit," $D R a$, I-I 7 (I924-1925), I97.

I I 54. "Autobiographische Skizze," DLE, XVII : 4 (Nov. I5, I9I4), 199-202.

I I55. "Das Autographensammeln," $V Z$, Sept. I4, I9 3.

I I 6. "Die Autographensammlung als Kunstwerk," $D B K$, II (I9I4), 44-50 (Reprinted in Spiegelungen, pp. 44-5 I).

I 157. "Balzacs Codices vom eleganten Leben," DLE, XIV :9 (Feb. I, I9I2), 6I3-6I6.

I 58. "Bei Albert Schweitzer," In, XIV : 2 (1933), 73-83 (Reprinted in Begegnungen, pp. II3-122; Schweitzer, pp. 7-19).

I 159 . "Bei den Franzosen in Kanada," FZ, Mar. 25, I9I I.

I I60. "Bei den Sorglosen" (I9r6) (Reprinted in Begegnungen, pp. I8I-186).

I 61. "Benares: Stadt der tausend Tempel," NFP, Mar. 23, r909 (Reprinted in Begegnungen, pp. 254-26r.)

I 162. "Ben Jonson," $N F P$, Nov. 4, I926 (Cf. also FTb (1927-1928), i 89).

I 163. "Ben Jonsons "Volpone' in Deutschland, " $L N N$, May 7, 1927.

I I64. "Berta von Suttner" (I9I7) (Reprinted in Begegnungen, pp. I87-194).

I 165. "Besuch bei den Milliarden" (1932) (Reprinted in Begegnungen, pp. I63-I 74).

I 166. "Bildnis Busonis," In, VI : I (1924), 54-56 (Reprinted in Begegnungen, pp. I I I-I I 2 ).

I167. "Brief an einen französischen Freund," Carossa (Dec. I5, I928), pp. I86-192 (Reprinted in Begegnungen, pp. 422-426).

I 68. "Brief an Romain Rolland," $B T$, Dec. 22, I9I 2 (This letter was reprinted in French translation as a preface to Rolland's open letter to Gerhardt Hauptmann in the JdG, Sept. 2, 1914).

I 69. "Brief eines deutschen Malers aus Italien," In A (1909), I 28-132.

I 170. "Brügge: das nördliche Venedig," $V F Z M, 23 . J .: 3$ (I902) (Altered version reprinted in Zeit und Welt, pp. I69-1 79).

I 7 I. "Bruno Walter: Kunst der Hingabe," Paul Stefan Gruenfeldt, Bruno Walter. Wien: Reichner, 1936 (Reprinted in Begegnungen, pp. 1 27-1 29).

I 172. "Das Buch als Eingang zur Welt," PL, Aug. Is, I93 I (Cf. also $S N T$, Nov. 28/29, I931; KT, Dec. 6, 1931; Begegnungen, pp. 309-3 I 7).

I 173. "Das Buch als Weltbild," Un, 45.J.: I (1928) (Cf. also $K H Z$, Mar. 22, I929).

I 174. "Bücherladen in London: Bermondsey Bookshop," FVb, III (1928), зоI.

I 175 . "Bücher und der Krieg," $D B K$, IV (Jul.-Dec., I9I 5), 37.

I 76. "Byroniana," FZ (Lit. Beil.), No. 602 (Cf. $D L E$, XXXII : 4 (I929-1930), 222; Cf. also "Max Brod," DLz, XVI (1929), 55-59).

I 1 77. "Camille Lemonnier," NFP, Oct. r 2, r902.

I I 78. "Cäsar und Napoleon," $N R$, XXXVI : 5 (May, 1925), 521-525.

I 1 79. "Eine Charakteristik von Paul Verlaine," $F Z$, 5 I (I905).

I 80. "Charles Baudelaire," $D D$, XXXII (Apr.-Sept., I902), 65-68 (Reprinted in MfL, LXXII (May I 5, 1903), 66-70).

I 8 I. "Charles Baudelaire und Paul Verlaine in deutschem Gewande," $W Z, 20178$ (I903). 
I I82. "Charles Dickens," $Z$, LXX (Jan.-June, I9ro), 249-264 (This article also serves as the Introduction to Dicken's Ausgewäblte Romane und Novellen. Leipzig: Insel, 1910; Appeared in English translation by Kenneth Burke in Di, LXXIV (Jan., I 923), I-24 (Picture of Zweig on page 16 by Henri le Fauconnier)).

I 1 83. "Charles Theodor H. de Coster: "Uilenspiegel,"” $M N N$, Dec. 29, I9ro.

I 184. "City Without a Heart: Chartres," Tr, LV (June, I930), 28-30 (Tr. from the German by F. Ladislas).

I 85 . "Constant Meunier," VFZM, 22.J.: 9 (1902).

I 186. "Crommelynck," NR, XIX : 4 (1908), I852-1853.

I 187. "Dank an die Bücher," $D B a$, I (I949), I (Reprinted in Begegnungen, pp. 449-450). I 188. "Dank an Romain Rolland," $P L$, Jan. 26, 1936.

I 189. "Dante," In, III : I (Oct., I92I), I-I3 (Reprinted in NFP, Sept. II, I92 I and Begegnungen, pp. $387-396)$.

I 190. "Demokratische Menge und ihre Dichter," Gwt, I4 (Jan.-June, I910).

I I91. "Denkwürdiger Tag - Zum Ioosten Geburtstag der 'Marienbader Elegie'," In, IV : 4 (1923), 243-253 (Reprinted in NFP, Sept. 2, 1923; Cf. also Sternstunden der Menschbeit).

I192. "Deutsche Bücherei," NFP, Sept. I4, I916.

I 193. "Ein deutscher Dichter: Hermann Hesse," KöT, Oct. 24, I9I 5.

I I94. "Le Dieu Pan," E, XVII (I930), 32 I-327.

I 195. "Das Drama Verhaerens," DSch, 39 (June-Dec., I910).

I 196. "Die drei Meyer-Sammlungen," $B T$, June 17, 1924.

I197. "Echte und falsche Autographen," ARsch, VII (1926), II 5.

I198. "Edmond Jaloux" (1931) (Reprinted in Europäisches Erbe, pp. 99-101).

I I99. "Effects of War on the Future of Writing," NYT, VI (July 28, I940), 2; VI (Aug. 25, I940), 2.

I 200. "Emil Ludwig at Fifty," $L A$, CCCXL (Apr., I93I), I63-167.

I 201. "Emil Ludwig zum sosten Geburtstag," $N F P$, Jan. 25, x931.

I 202. "Emile Verhaeren," DLE, V : r4 (Apr. 15, r903), 972-978.

I 203. "Emile Verhaeren - ein Dichter des Universums," $Z e$, Mar. 2, I910.

r 204. “E. M. Lilien,” MfL, LXXII : 44 (Nov., I 903), 439-446; LXXII : I6 (Dec., I903), 506-513 (This article serves as the Introduction to the book E. M. Lilien. Sein Werk. Berlin-Leipzig: Schuster and Loeffler, 1903).

I 205. "Ernst Lissauer," CVZ, XI (1932), 501.

1 206. "Ernst Renan," NFP, Feb. 25, 1923 (Reprinted in Begegnungen, pp. 373-386).

1207. Europa - America latino (Articles by Alcides Arquedas, E. Diez Canedo, Georges Duhamel, W. J. Entwistle, Joan Estelrich, F. de Fiqueiredo, P. HenriquezUrena, C. Ibarguren, Conde Keyserling, Emil Ludwig, Jacques Maritain, R. H. Mottram, Afranio Peixoto, Louis Piérard, Alfonso Reyes, Carlos Reyles, Jules Romains, Francisco Romero, B. Sanin Cano, Juan B. Teran, G. Ungaretti, Stefan Zweig). Tr. E. M. S. Danero. Buenos Aires: Comision Argentina de Cooperacion Intelectuel, 1937.

1 208. "Ex libris: Moderne Deutsche und ihre Künstler," $V F Z M$, 22.J.: 2 (1902).

1 209. "F. A. Brockhaus: Beschwerde gegen einen Verleger (Casanova betr.)," $B T$, June 29, 1926.

1 2 10. "Das Feuer" (1918) (Reprinted in Begegnungen, pp. 207-218).

I21 I. "Fishermen on the Seine," HM CLXXXII (Feb., I94I), 273-275.

I 2 I 2. "Flauberts Nachlass," $B T$, Jan. I I, I9 I (Cf. also DLE, XIII : I 2 (Mar. I 5, I9I I), 879-88r).

I 21 3. Fliichtiger Spiegelblick. Leipzig: Insel, 1927 (Promotion material).

1214. "Flug und Wort," $B T$, Aug. 23, 1913.

I 215 . "Frans Masereel," Masereel (Cf. the I923 article "Frans Masereel. Der Mann und Bildner" reprinted in Begegnungen, pp. I30-1 39).

I 2 16. "Franz Karl Ginskey zum Gosten Geburtstag des Dichters am 8.9.193 I," PL, Sept. 6, I93 I (Cf. also $O R$, 17.J. (June-Dec., I93 I), 845; Un, 49 (I93I)).

I 217. "Für Jacob Wassermann (by Thomas Mann, Stefan Zweig, Hermann Hesse and 
Alfred Döblin)," NR, XLIV : 3 (Mar., r933), 357-36I (Cf. also DL, XXXV : 8 (1932-1933), 468).

I 2 18. "Die Gärten im Kriege" (1939) (Reprinted in Zeit und Welt, pp. I 21-1 25).

1 219. "Galiziens Genesung," NFP, Aug. 31, 1915.

I 220. "Geburtstagsgruss an Schalom Asch," $D L W$, VI : 43 (1930), I.

I 221. "Dem Gedächtnis Jacob Julius Davids," DIDÖ (Feb., I906), I-3 (Cf. also $O R$, IX : 3 (Nov.-Dec., 1906), 21 7-219 and Blätter, 4/5 (April, 1959), 5-7).

1 222. "Die gefangenen Dinge: Brüsseler Weltausstellung," $N F P$, Aug. 17, 1910.

1 223. "Das Geheimnis der Beatrice Cenci," $M Z$, May I, I 927 (Cf. NFP, Dec. 2, I926 and Zeit und Welt, pp. I03-I I 5).

1224. "Das Geheimnis des künstlerischen Schaffens," Lecture given in America, 1938 (Reprinted in Zeit und Welt, pp. 25 1-274).

r 225. "Der geistige Aufbau der neuen Generation," NFP, Nov. 20, I 932.

I 226. "Die Geschichte als Dichterin,"A lecture destined to be delivered at the International P.E.N. Club in Stockholm, Sept., I 939 before the outbreak of World War II intervened (Reprinted in Zeit und Welt, pp. 337-360).

I227. "Geschichtsschreibung von morgen," Lecture in America before the outbreak of World War II (Reprinted in Zeit und Welt, pp. 275-298).

I 228. “G. Falke: Stadt mit den goldenen Türmen. Falke zu seinem Gosten Geburtstag," $B T$, Jan. 7, 1913.

I 229. "Ghetto Stimmungen," DLE, I0. J. (1908), I344-1349 (Contributions by St. Zweig, L. Adelt and P. Neuburger).

I 230. "Great Lesson from a Great Man: Rodin," CW, CLI (Aug., I940), 599-60I (Printed simultaneously in $R D$, XXXVII (Aug., I940), 26-28; Cf. also WE, 2.J.: 5 (1947-I 948), 48-50).

1231. "Gustav Mahlers Wiederkehr," NFP, April 25, 19I5 (Reprinted in Europäisches Erbe, pp. 172-182).

I232. "Gwalior: die indische Residenz," $B T$, Mar. 20, I909.

1 233. "Hans Carossa," $B T$, Nov. 8, I928 (Reprinted in Begegnungen, pp. 422-426).

1234. "Hans Franck," $M N N$, July 30, I929.

I 235. "Hartrott and Hitler," FW, IV (Dec., I942), 234-235.

1236. "Das Haus der tausend Schicksale" (London, 1937) (Reprinted in Begegnungen, pp. 219-222).

1237. "H. Benzmann," F 29 (1903).

I 238. "Heimkehr zu Goethe," NR, XXII : 9 (Sept., I921), 1007.

1239. "Heinrich Lammasch," $B T$, Jan. 20, I920.

1240. "Herbstwinter in Meran," Blätter, 6/7 (Oct., I959), I4-I 8 (First printing of this essay in Fabrten (1919; 2. ed. I922); Cf. Joseph Maurer, "Úber Zweigs Aufsatz 'Herbstwinter in Meran'," Blätter, 6/7 Oct., 1959), 18-19).

124r. "Hermann Bahr. Zu seinem Gosten Geburtstag," NFP, July 19, 1923 (Reprinted in Begegnungen, pp. I06-1 I0).

1 242. "Das Herz Europas. Ein Besuch im Genfer Roten Kreuz" (Dec., 1917) (Reprinted in Begegnungen, pp. 194-207).

1 243. "Hommage à Romain Rolland," Com, III : 3 I (Mar., 1936), 789-80I (Tribute to Rolland's 7oth birthday).

1244. "Honoré de Balzac," $H N$ (Beilage), is (I906) (Cf. also $Z$, LXIV (I908), 53-62, IOO-III).

1 245. "Hugo von Hofmannsthal. Gedächtnisrede zur Trauerfeier im Wiener Burgtheater" (1929) (Reprinted in Zeit und Welt, pp. 33-48).

1246. "Die indische Gefahr für England," NFP, July 1 3, 1909.

I 247. "Internationalismus oder Kosmopolitanismus," $D L W$, II : 27 (1929), I.

1248. "Irrfahrt und Ende Pierre Bonchamps'. Die Tragödie Daudets," NFP, Mar. 28, I 26 (Reprinted in Zeit und Welt, pp. 89-102).

I 249. "Isolde Weisshand," $N R, \mathrm{XX}: 3$ (1909), , 229-1 230.

I250. "Ist die Geschichte gerecht?" $D K, \mathrm{I}: 2$ (I952-I953), 3 (Cf. WaM, June 9, 1952; Reprinted in Blätter, 3 (Oct., 1958), 1-2; Europäisches Erbe, pp. 271-273). 
I251. "Jacob Wassermann," NR, 33.J.II: 8 (I9I 2), I I3 I-II45 (Reprinted in Europäisches Erbe, pp. 187-208).

I 252. "Jean Christophe," $L F$, I I (Mar., I913), 48I-543 (Articles and replies corcerning Rolland's novel Jean Christophe by Stefan Zweig, Georges Duhamel, Charles Lemonnier, Emile Verhaeren et al.).

1253. "Jeremias Gotthelf und Jean Paul," $B T$, May I4, I924.

1254. "Johannes Schlaf," DLE, IV : 20 (July, 1902), I377-1 388 (Also contains a Zweig portrait).

I 255. "Joseph Roth," Eulogy at funeral services in Paris, 1939 (Reprinted in Europäisches Erbe, pp. 251-264).

I 256. "Juden in einem allgemeinen politischen Leben," OS, I (April, I939), I03-105.

1 257. "Jüngste Generation der deutschen Lyriker," $D L W$, III : Io (I929), 4.

1258. "Karl Haushofer," $Z f G$, 26.J. (1955), 768-769.

1259. "Karl Loewe, der Dichter," NFP, Feb. 18, I91 2.

1260. "Die Kathedrale von Chartres" (1924) (Reprinted in Zeit und Welt, pp. 183-190). 1261. "Kleine Reise nach Brasilien" (Fall, I936) (Reprinted in Begegnungen, pp. 274-305).

I 262. "König der Juden," Herzl, pp. 55-57 (Cf. also "Erinnerungen an Theodor Herzl," Begegnungen, pp. 88-95).

1263. "Die Kunst des Briefes," Blätter, I5 (Jan., 1963), 8-9 (First printed as an epilogue to Otto Heuschele's Briefe aus Einsamkeiten, Berlin: n.p., I922).

1264. "Kunst und Wirklichkeit. Bermerkungen zum Drama 'Lord Byron kommt aus der Mode'," PTb (1930), 59 (Cf. DLz, XVI (1929), 55-;0 and DL, XXXII : 4 (1929-1930), 222).

1 265. "Laukhard der Landstörtzer," $N R, \mathrm{XIX}: 3$ (1908), I389-г 39 I.

1266. "Legende und Wahrheit der Beatrice Cenci," NFP, Dec. 2, I926 (Reprinted in Zeit und Welt, pp. 103-1 I5; Cf. also $M Z$, May I, 1927).

I267. "Die Lektion, die mir Auguste Rodin erteilt hat," WE, 2.J.: 5 (1947-1948), 48-50 (Cf. "Great Lesson from a Great Man: Rodin," CW, CLI (Aug., I 940), 599-60I and $R D$, XXXVII (Aug., I940), 26-28).

I 268. "Lemonniers 'L'Homme en Amour'," MfL, LXXII : 13 (Oct., I903), 407-409 (First printed as the Introduction to the German translation of this work. Tr. Paul Adler. Leipzig: Rothbarth, 1903).

1269. "Leo Feld, Gedächtnis eines Freundes," NFP, May I6, 1925.

1 270. "Léon Bazalgette" (1927) (Reprinted in Europäiscbes Erbe, pp. 95-98).

r271. "Lettres allemandes," $R E$, Jan., I929.

1272. "Os Livros subterrãneos de Balzac," Tr. Milton Araryo. Balzac. Historia dos treze... Rio de Janeiro, 1952 (Original version appeared as "Die unterirdischen Bücher Balzacs," In, II : 2 (1920), $71-77$; Reprinted in Begegnungen, pp. 427-43 I).

1273. "Lob des deutschen Verlegers," VZ, Nov. 9, 1912.

1 274. "Lord Byron," In, V : 3 (July, 1924), 147-156 (Reprinted in Zeit und Welt, 9-20).

1275. "The Lord gave the Word," Tr. Eden and Cedar Paul. $P R$, XXXVIII (Dec., I936), I 2-I 3 (Excerpt from the Paul translation of "Händel's Messiah" in Twelve Historical Miniatures. New York: Viking, 1940).

I 276. "Die Lyrik um Stefan George," $D L E$, VI : 3 (Nov. I, 1903), x69-172.

I 277. "Der lyrische Nachwuchs," VFZM, 22.J.: II (1903) (Eleven literary portraits).

1 278. "Marcel Prousts tragischer Lebenslauf," MfFL, 4 (I953), I, 6 (Cf. NFP, Sept. 27, I925; $P L$, Feb. 22, I931; $Z$ e, 9. J.: 3 (1954), 6; Entire article reprinted in Zeit und Welt, pp. 2I-3I).

I 279. "Mater Dolorosa. Die Briefe von Nietzsches Mutter an Overbeck" (I937) (Reprinted in Zeit und Welt, pp. 55-64).

I 280. "Max Brod," $D L z$, XVI (I929), $55-59$ (This article concerns Brod's drama Lord Byron kommt aus der Mode. Wien: Zsolnay, I929; Cf. also DL, XXXII : 4 (1929-1930), 222; PTb (1930), 59 and $W i, \mathrm{I} / \mathrm{II}$ (1929), 124).

1281. "Max Brod. Zu seinem 5osten Geburtstag," Brod (1934).

1 282. "Max Hermann-Neisse. Kleine Festrede an einen deutschen Dichter in der Emigration zum sosten Geburtstag," PL, May 3 1, 1936. 
I 283. "Max Hermann-Neisse zum Gedächtnis," $A u(N Y)$, Apr. 18, I94I (Reprinted as a postscript to Hermann-Neisse's book of poems Erinnerung und Exil. Zürich: Oprecht, 1946; Cf. also Blätter, I I/ I 2 (Oct., 1961), 13-15).

1 284. "Maxim Gorki zu seinem Gosten Geburtstag," $N F P$, Mar. 25, i 928 (Cf. also $V Q R$, s (Oct., r 929), 492-501 and Begegnungen, pp. 96-105).

I 285. "Meine Autographensammlung," $P$ h, III : 7 (I930), 279-289.

I 286. "The Mission of the Intellectuals," $A I R$, XIII : I52 (Sept., I94I), 2 (Cf. $A I R$, $\mathrm{XV}: 167 / 168$ (Feb.-Mar., 1947) for a reproduction of Zweig's letter to the Editor in Chief of the periodical concerning this article).

I 287. "Moissi im Gespräch," Moissi, pp. 56-57.

I 288. "Die Monotonisierung der Welt," NFP, Jan. 3 1, I925 (Reprinted in FuS, II (1928), 65-69 and Begegnungen, pp. I55-162).

1289. "Montaigne," NR, XI (1948), 257-265 (Entire article reprinted in Europäisches Erbe, pp. 7-8I).

I 290. "Die moralische Entgiftung Europas," Lecture before the Academia di Roma, 1932 (Reprinted in Begegnungen, pp. 223-236).

I 29I. "Moskauer Theater," HaN, Jan. I I, I 929.

1 292. "Musset und Baudelaire in deutscher UUbertragung," $F Z$, June 1, 1924.

I 293. "Nach einem Jahr - deutsch-französische Gesellschaft," $D F R$, II (I929), I-5.

I 294. "Das neue Belgien," $A Z, 43$ (1909).

I 295. "Neue Lyrik," $F$, VI : 3 (I904), 49-5 I.

I 296. "Neue Napoleon Manuskripte," $P h$., II (1929), 246.

I 297. "Das neue Pathos," DLE, XI : 24 (Sept. I 5, I909), I 70 I-I 707.

1298. "1914 und heute. Anlässlich des Romans von Roger Martin du Gard 'Eté 1914"” (1936) (Reprinted in Zeit und Welt, pp. 329-336).

I 299. "Nietzsche und der Freund," In $A$ (I9I9), I I I-I 23 (Originally printed in NFP, Dec. 21, I917).

1300. "Der Orient: Drama in Tausendundeiner Nacht," NFP, Jan. 30, I917.

r 301. "Oskar A. H. Schmitz," Schmitz (1923).

1302. "Ostende, Saisontage," VFZM, 22.J.: 2 (1902).

1303. "Oskar Břežina," $O R$, XIX : 6 (June, 1909), 444-450 (Discussion of the German version of Břežina's Hände, translated from the Czech by Emil Saudek. Wien: Moritz Frisch, 1908; Reprinted in Karel Ločak v Praze. Tr. Zdenek Doležil).

1304. "Oxford" (1907) (Reprinted in Zeit und Welt, pp. 193-202).

r 305 . "Paul Verlaine," MfL, LXXI : 40 (Oct. 4, 1902), 313-315.

I 306. "Peter Rosegger. Zum Tod des Dichters," Nz, June 26, I918 (Reprinted in Europäisches Erbe, pp. 209-213).

1307. "Le Poésie de Goethe," LNL, XX (Oct., I949), 6 (Cf. Begegnungen, pp. 397-405).

1308. "Pour Ramuz," Ramuz (1938) (Cf. Europäisches Erbe, pp. 1 22-1 26).

1309. "Ein Prager Dichter," PT, XXVII (I903), I4I (About Paul Leppin).

I310. "Profit from my Experience," $R D$, XXXIX (July, 194I), 39-43.

13 1 1. "Prolog und Epilog zu Shakespeares 'Sturm,' Quasi una Phantasia," In $A$ (1926), 68-75.

I 3 1 2. "Proust Himself," $A M$, CXLVI (Nov., 1930), 606-6ro (Cf. I 278).

1313. "Provisorisches über Rudolf Pannwitz," DLE, XXIV : I6 (May I5, I922), 982-984.

I 3 14. "Un pur Poète: Rainer Maria Rilke," $R E$ (Jan., I929), I 394-r401.

1315. "Rabindranath Tagores 'Sadhâna'. Ein zeitgemässes Gespräch," DLE, XXIV : I (Oct. I, I92 I), I-6 (Cf. Europäisches Erbe, pp. I48-1 56).

1316. "Rainer Maria Rilke," MNN, Feb. 22, I927 (Cf. "Abschied von Rilke" (1927), reprinted in Begegnungen, pp. 59-73; Cf. also DSlb, II (1946), I 70).

1317. "Rainer Maria Rilke," Lecture in London, 1936 (Reprinted in Europäisches Erbe, pp. 244-250).

13 18. "Rainer Maria Rilke in Paris," Erz, 2.J.: 7 (1948), 3-6.

I319. "Rathenau Reflexionen: der Kaufmann und der Künstler," NFP, Sept. 18, 1908.

1320. "Reconnaissance," Hommage, pp. 86-91. 
I321. "Reise nach Russland," $N F P$, Oct. 23, 26, 28, 1928 (Reprinted in $K H Z$, Nov. 29, I 928 and $Z$ eit und Welt, pp. 203-245).

1322. "R. H. Francé als Bildner," Francé, p. 35.

I323. "Der Rhythmus von New York," $S P$, Dec. 7, I9I3 (Reprinted in $D A$, Dec. 8, I9I 3 and Begegnungen, pp. 264-270).

1324. "Romain Rolland," $L J Z, 7$ (1926).

1325. "Romain Rolland," Lecture in Berlin, Meistersaal, Jan. 29, I926 (Reprinted in Europäisches Erbe, pp. 1 о2- I 21).

1326. "Romain Rolland after the War," Di, LXXVI (May, I924), 445-448.

1327. "Romain Rolland, el Hombre y la Obre," Ud, I : 3 (April, 1936), 5-6.

1328. "Romain Rolland und 'Jean Christophe'," NFP, Mar. 20, 1914, pp. I-4.

1329. "Romain Rolland und der Ruhm," NFP, Mar. 20, 1914.

I330. "Die Romantik der Bourgeoisie," In $A$ (I9I I), I I 2-I 23.

I331. "Rousseau, der Erzieher zu einer neuen Gesellschaft," $D A Z$, Dec. 8, I918 (Reprinted in $W Z$, Mar. 23, 192 I).

1332. "Rückkehr zum Märchen," NFP, Dec. 14, I913.

1333. "Sainte-Beuve," NFP, May 6, 1923 (This article also serves as the Intro. to the German version of Sainte-Beuve's Literarische Portraits aus dem Frankreich des $X V I I-X I X$. Jabrbunderts. Frankfurt am Main: Frankfurter Verlagsanstalt, 1923; Reprinted in Begegnungen, pp. 406-41 7.)

1334. "Salzburg: Die Stadt als Rahmen," Herz (I935) (Reprinted in Begegnungen, pp. $271-$ 273 und Blätter, 2 (July, I958), I-4).

1335. "Die Sammlung Morrison," VZ, Sept. 6, I917 (Reprinted in $D B K$, VI (1918), 73).

1336. "Die schlaflose Welt," NFP, Aug., 1914 (Reprinted in Begegnungen, pp. 175-180).

1337. "Schnitzler und die Jugend," $M$, May I5, I9I 2 (Schnitzler Geburtstagssondernummer, zu seinem 5osten Geburtstag) (Reprinted in Blätter, 13/14 (April, 1962), IO-I2).

I338. "Das schönste Grab der Welt: Tolstois Grab," Excerpt from 'Eine Russlandreise" (1928) (Reprinted in Begegnungen, pp. 262-263).

1339. "Die Schweiz als Hilfsland Europas," Do, II (I 18 8), 832-834).

1340. "Selbstanzeige: Gedichte von Paul Verlaine," $Z$, XL (Sept. 27, 1902), 536.

1341. "Shakespeare," FTb (1927-1928), I73.

1342. "Sigmund Freud, der Siebzigjährige," $D L W$, II : I9 (1929), I.

I343. "Sigmund Freuds 75 ster Geburtstag," $C V Z, \mathrm{X}$ (1931), 237.

I 344. "Sigmund Freud zum 8osten Geburtstag," $P L$, May 3, r 936.

I345. "Sinn und Schönheit der Autographen," $D N S c h$, I9. J. (I958), I I 8 (Original version was a lecture given at a book exhibition of the London Sunday Times (1935); Reprinted in Begegnungen (1937 ed.), pp. 469-476; Begegnungen (1955 ed.), pp. 44I-448; An excerpt of this lecture is used as an Intro. to the volume Lyrische Handschriften unserer Zeit (5o Gedichthandschriften deutscher Lyriker der Gegenwart). Ed. Hartfrid Voss. Ebenhausen bei München: Hartfrid Voss, I958, p. 5).

1346. "Soyka," NR, XXII : 2 (r9r I), I638-i640.

1347. "Stefan Georges Stellung im deutschen Geistesleben," DLE, IV : 28 (July I3, 1928) (Autobiographical notes from Walter Benjamin, Bert Brecht, Martin Buber, Willy Hellpach, Friedrich Muckermann, Josef Ponten, Franz Rosenzweig, Wilhelm Schäfer, Ruth Schaumann, P. Expeditus Schmidt, Oscar A. H. Schmitz, Ina Seidel. Reminiscenses from French contemporaries: Albert Saint-Paul, André Gide, Francis Vielé Griffin. Friedrich Sternthal: " $\mathrm{Zu}$ Georges Politeia." Reproduction of the manuscript of "Ein Angelico" with a dedication to Albert Saint-Paul and a picture of him in his youth. DLE, IV : 29 (July 20, I928) - further contributions by Frank Thiess, Paul Wiegler, Karl Wolfskehl, Stefan Zweig. Cf. also Max Rychner, "Zeitgenossen über Stefan George," NSchR, XXI : 34(1928), 561-563).

1348. "Stefan Zweig über Henri Barbusse. Nachwort zum Roman 'Die Schutzflehenden'," 
Blätter, 13/14 (April, 1962), I4-I (Cf. also the essay "Das Feuer," Begegnungen, pp. $207-218$ concerning Barbusse's antiwar novel 'Le Feu').

1349. "Standhals deutsche Wiederkehr," FZ, Dec. 6, I921.

r 350. "Die Stimme. In memoriam Josef Kainz," Begegnungen, pp. I 40-1 47.

r35I. "Die Stunde zwischen zwei Ozeanen - der Panama Kanal," NFP, July 6, igi I (Reprinted in Begegnungen, pp. 239-247).

1352. "Der Suez Kanal," NFP, Nov. 18, I914.

1353. "Das Tagebuch des jungen Flauberts," Pan (Jan.-June, I9I I), I8I-I 88, 226-234.

I354. "Das Tagebuch eines halbwüchsigen Mädchens," $A d P$ (I926), I40-I 45.

1355. "Thanks to Books," Tr. Theodore W. Koch. Lib, XXXV (Mar., I930), II 3-I 44 (Reprinted in Librarians, pp. 3-4; SRL, XLI (Feb. 8, 1958), 24; WLB, XXXII (Mar., I958), 477-478; Cf. also original "Dank an die Bücher," reprinted in Begegnungen, pp. 449-450).

1356. "Theresa Feodor Ries," VFZM, 21.J. :21 (1902).

1357. "Thomas Mann: 'Lotte in Weimar"” (1939) (Reprinted in Zeit und Welt, pp. 363366; Tr. into English in The Stature of Thomas Mann. Anthology of Critical Essays. Ed. Charles Neider, New York: New Directions, 1947; London: Owens, I95 I, pp. 188-190).

I357a. "Thomas Mann zum 5osten Geburtstag," BT, May 31, 1925, p. 5 (Messages of congratulations from Hermann Bahr, Rudolf Binding, Waldemar Bonsels, Herbert Eulenberg, Jakob Wassermann, Stefan Zweig, Alfons Paquet, Walter von Molo, Wilhelm von Scholz, Wilhelm Schäfer).

1358. "Tolstoi als religiöser und sozialer Denker" (1937) (Reprinted in Zeit und Welt, pp. 67-88).

1359. "Tolstois Lehre," Gewalt, pp. 227-232.

1360. "Die Tragik der Vergesslichkeit" (I919) (Reprinted in Europäisches Erbe, pp. 265-270).

1361. "Der Turm zu Babel," $V Z$, May 8, I916 (Reprinted in $P L$, Jan. 1, I930 and Europäisches Erbe, pp. 274-279; Appeared as "La Tour de Babel" in LC (AprilMay, 1916); This article was translated into English by Eden and Cedar Paul as "The Workers Dread Nought," cf. Charles Baudouin, Contemporary Studies. Tr. Eden and Cedar Paul. London: Allen and Unwin, 1924, p. 283).

I 362. "Überschätzung der Lebenden," $A R s c h, 7 . J .:$ VII (1926), I9.

1363. "Um Jaurès," NFP, Aug. 6, 1916 (Reprinted in $V$, Aug. 29, 1916 and Europäisches Erbe, pp. 85-94).

1364. "Die unterirdischen Bücher Balzacs," DBib, V (1916), 48-52 (Reprinted in In, II: 2 (Dec., 1920), 7I-77 and Begegnungen, pp. 427-43I).

1365. "Unvergessliches Erlebnis. Ein Tag bei Albert Schweitzer," Schweitzer, pp. 7-19 (Cf. In, XIV : 2 (1933), 73-83).

1366. "Verlaine," $D D i$, III (1905).

1367. "Die Vernachlässigten" (Antworten auf die Rundfrage von F. W. Bischoff, B. Diebold, H. Eulenberg, O. Flake, M. Hermann-Neisse, H. Hesse, H. Kasack, C. Köppen, Th. Mann, G. Pohl, E. M. Remarque, W. von Scholz, H. Sochaczewer, Stefan Zweig), $D L e$, III (r930), r-2 (Cf. also $D L$, XXXII : ro(1929-1930), 593).

1368. "Verse eines Gottsuchers," $D N$, XXIII (1905-1906), 57 I f.

1369. "Die Verstümmelten," $N R$, XXXIV : i I (Nov., I923), 1054-1055.

I 370. "Victor Hugo als Lyriker," MfL, LXXI : II (Mar. I 5, I902), 8 I-83.

I 37 I. "Vom österreichischen Dichter. Ein Wort zur Zeit," DLE, XVII : 5 (Dec., 1914), 263-265.

I 372. "Vom Sinn unseres Jahrhunderts," $A b z$, Sept. 6, 1932.

1373. "Von den Bäumen," Kt, 24.J. (June-Dec., I9I I), 42.

1374. "Vorbeigehen an einem unauffälligen Menschen - Otto Weininger," BT, Oct. 3, 1926 (Reprinted in Europäisches Erbe, pp. 223-226).

1375. "Walter Rathenau," Written immediately after his assassination on June 24, I 922 (Reprinted in Europäisches Erbe, pp. 232-243). 
1376. "Walt Whitman," NFP, May 28, 1919.

I 377. "Warum meine Bücher Erfolg hatten," $R v B, 29$ (I949), 20-2 I.

1378. "Warum nur Belgien, warum nicht auch Polen? Eine Frage an die Neutralen," NFP, Apr. 14, 1915.

I379. "Wedekind, der Unbürgerliche," Wedekind, pp. 242-244.

I 380. "Der Weg A.T. Wegners," BT, Dec. Is, r92 r.

I381. "Der Weg Hermann Hesses," NFP, Feb. 6, I923, pp. I-3.

1382. "Die Welt der Autographen," $D B K$, I2./13.J. (I927), 70-77 (Cf. $J D B, \mathrm{XII} /$ XIII (1925-1926), 70-77).

1383. "Where France Hoards Gold," LA, CCCXLII (May, I932), 214-220.

1384. "Das Wien von gestern," Lecture in Paris, 1940 (Reprinted in Zeit und Welt, pp. I 29-I 50).

1385. "Wilhelm Holzamer," MfL, LXXI : 38 (Sept. 20, 1902), 297-298.

1386. "Wille zur Universalität," Kippenberg, pp. I 54-16r.

1387. "Worte am Sarge Sigmund Freuds," Delivered at the Crematorium in London, Sept. 26, 1939 (Reprinted in Zeit und Welt, pp. 5 I-54).

I388. "Würdigung Ödön von Horvaths," $B d T G, 67$ (I954-1955).

1389. "Ypern," BT, Sept. I6, I928 (Reprinted in Begegnungen, pp. 248-253).

1390. "Zu Emil Luckas 5osten Geburtstag," $K Z$, May I 2 , I 927.

I39I. "Zum 70sten Geburtstag von Sigmund Freud," MNN, May 5, I926 (Reprinted in $\operatorname{AdP}(\mathrm{1} 937)$ ).

1392. "Zur Entstehung des 'Volpone'," $N Z Z$, Sept. 28, 1927.

1393. "Zur Geschichte des europäischen Gedankens," $A b z$, Jan. 29, I933 (Cf. Zeit und Welt, pp. 299-326).

1394. "Zur modernen Lyrik," KdöL, XVII (July-Dec., r9oI), 38-40.

I 395. "Zur Physiologie des dichterischen Schaffens. Ein Fragebogen," $D L W, 39$ (1928), $3-4 ; 40$ (I928), 3; 4I (1928), 3-4.

1396. "Zur Schutzfristfrage," BdBb, May 7, 1927.

1397. "Zutrauen zur Zukunft," Frau, pp. 7-17.

1398. "Zu Verhaerens Ioten Todestag," $D L W$, II : 48 (I926), 4.

1399. "Zwei Aphorismen," DD, XXVIII (Apr.-Sept., I900), 240.

I400. "Zwei Aphorismen," $D D$, XXVIII (Apr.-Sept., I900), 299.

\section{TRANSLATIONS BY ZWEIG}

I40I. Barbusse, Henri. Die Scbutzflehenden. Der Roman einer Vorkriegsjugend. ZürichLeipzig-Stuttgart: Rascher and Cie, 1932 (Tr. and epilogue by Zweig).

1402. Baudelaire, Charles. Die Blumen des Bösen. Berlin: Oesterheld, I92 I (Tr. of eight poems).

1403. - Gedichte in Vers und Prosa. Leipzig: Hermann Seemann Nachfolger, 1902 (Tr. with Camill Hoffmann; Introductory essay by Zweig entitled "Charles Baudelaire", pp. 7-20).

I404. - "Der Albatros" (poem), DD, XXX (Apr.-Sept., I90I), 246.

I 405. - "La Beauté" (poem), MfL, LXXII : b (May I 5, I903), 65.

1406. - "La Beauté" (poem), Weltdichtung, p. г6г.

I 407. - "Le Chat" (poem), MfL, LXXII : b (May I 5, I903), 65.

1408. - "Don Juan aux Enfers" (poem), MfL, LXXII : b (May I 5, I903), 65.

1409. - "Der Duft" (poem), DD, XXX (Apr.-Sept., I 901), 247.

I410. - "L'irreparable" (poem), $D D, \mathrm{XXX}$ (Apr.-Sept., I901), 289.

I4II. - "Harmonien" (poem), $D D, \mathrm{XXX}$ (Apr.-Sept., I901), 246.

I41 2. - "Herbstlied" (poem), $D D, \mathrm{XXX}$ (Apr.-Sept., I901), 248 (Reprinted in Spiegelungen, p. 32).

1413. - "Die Katze" (poem), DD, XXX (Apr.-Sept., I901), 247.

I414. - "Der Mensch und das Meer" (poem), $D D, \mathrm{XXX}$ (Apr.-Sept., 1901), 246. 
I4I5. - “Die Musik" (poem), DD, XXX (Apr.-Sept., I90I), 248.

1416. - "Noch heute" (poem), $D D, \operatorname{XXX}$ (Apr.-Sept., I90I), 246).

I417. - "Der Rahmen" (poem), DD, XXXI (Oct., I901 - Mar., I902), so.

I4I8. - "Die Riesin" (poem), $D D$, XXXI (Oct., I90I - Mar., I902), 50.

I419. - "Die Seele des Weins" (poem), DD, XXXI (Oct., I901 - Mar., 1902), 25.

I420. - "Semper eadem" (poem), DD, XXX (Apr.-Sept., r9or), 247.

I421. - “Spleen" (poem), DD, XXX (Apr.-Sept., r90I), 266.

1422. - "Was sagst Du heute..." (poem), $D D, \mathrm{XXX}$ (Apr.-Sept., 1901), 247.

1423. - "Der Wein des Einsamen" (poem), DD, XXX (Apr.-Sept., 190I), 248.

I424. - "Der Wiederkehrende" (poem), DD, XXX (Apr.-Sept., I 90I), 248.

1425. Browning, Elizabeth Barrett. "Blasse Liebe" (poem), DD, XXXI (Oct., I90I Mar., 1902), 96.

I426. Camoens. "Weh, wieviel Not und Fährnis auf dem Meere" (poem), Europäer, p. I3 and p. ${ }_{369}$ (Cf. also Leben-Werk, p. 89 and Spiegelungen, p. 102).

1427. Edman, Irwin. Ein Schimmer Licht im Dunkel. Stockholm: Bermann-Fischer, 1940 (Tr. by Stefan Zweig and Richard Friedenthal).

1428. Hugo, Victor. "Juninacht" (poem), DD, XXXI (Oct., I901 - Mar., I902), 254.

I 429. Keats, John. "Sonett" (poem), Spiegelungen, p. 34 (Zweig's rendition of Keats' last sonnet).

1430. Lemonnier, Camille. "Alte Leutchen," $Z e, 486$ (1904), 37-38, 47-48.

I431. de Lentino, Jacopo. "Sonett" (poem), $\operatorname{Fr} A$ (1923).

1432. Marx, Madeline. Weib. Roman. Basel: Rhein Verlag, I920 (Tr. by Stefan and Friderike Maria Zweig).

1433. Pirandello, Luigi. Man weiss nicht wie (Drama: Non si sa come). Wien: Reichner, 1935.

1434. Rolland, Romain. Clerambault. Gescbicbte eines freien Gewissens im Kriege. Frankfurt am Main: Rütten and Loening, I922 (Reissued in München: Kindler and Schiermeyer, 1960).

1435. - Den bingericbteten Völkern (Aux peuples assassinés). Zürich: Rascher, I91 8.

1436. - Die Zeit wird kommen (Le Temps viendra). Wien-Leizpig: Tal, 1919 (Reissued in Leipzig-Zürich: Rotapfel, I 92 I and Zürich: Hofmann, I 945).

1437. Russell, Archibald B. H. Die visionäre Kunstphilosophie des William Blake. Leipzig: Julius Zeitler, 1906.

1438. Silvestre, Armand. "Kennst Du...?" (poem), DD, XXVII (Oct., I899 - Mar., 1900), 220.

1439. - “Zärtliche Verse" (poem), $D D$, XXVII (Oct., I899 - Mar., I900), I 88.

1440. Suarès, André. Cressida. Wien: Tal, I920 (Tr. by Stefan Zweig and Erwin Rieger).

I44I. Symons, Arthur. "Die Schönheit spricht" (poem), Spiegelungen, p. 33.

r442. Verhaeren, Emile. Ausgewäblte Gedichte. Berlin: Schuster and Loeffler, 1904.

1443. - Ausgewäblte Gedichte. Leipzig: Insel, i 910 (Forms Vol. II of Zweig's translation of Verhaeren's “Ausgewählte Werke"; Reprinted I913, 1923).

1444. - Drei Dramen: Helenas Heimkebr - Philipp II - Das Kloster. Leipzig: Insel, I 910 (2. ed., I9I4; Vol. III of the "Ausgewählte Werke").

1445. - Helenas Heimkebr. Leipzig: Insel, I909 (Unpublished ms. of the original translation; Première Stuttgarter Hoftheater, Oct. I3, I910).

1446. - Helenas Heimkebr. Drama in Vier Akten. Leipzig: Reclams Universalbibliothek, 1928 (No. 6850).

1447. - Hymnen an das Leben. Leipzig: Insel, I9I I (Reprinted I912, I9I9, I93 I; Intro. by Zweig; Inselbücherei, No. 5 ).

1448. - Rembrandt, Leipzig: Insel, I912 (Reprinted 1918, I920, 1923).

1449. - Rubens. Leipzig: Insel, I913 (Reprinted 1917, 1920, 1922).

1450. - "Abendstimmung" (poem), $\operatorname{In} A$ (1914), I 28-1 30 .

I451. - "An meine Augen" (poem), In, VIII : I (Christmas, I926), 65-68.

1452. - "Die Arbeit" (poem), Lyrik, p. 537 (Translation of the poem by Zweig, Rilke and Stefan George).

1453. - "Der Baum" (poem), In $A$ (1910), г 8-21.

1454. - “Der Baum” (poem) $R R h$, 6.a.: 8/9 (May-June, 1926), 27-29. 


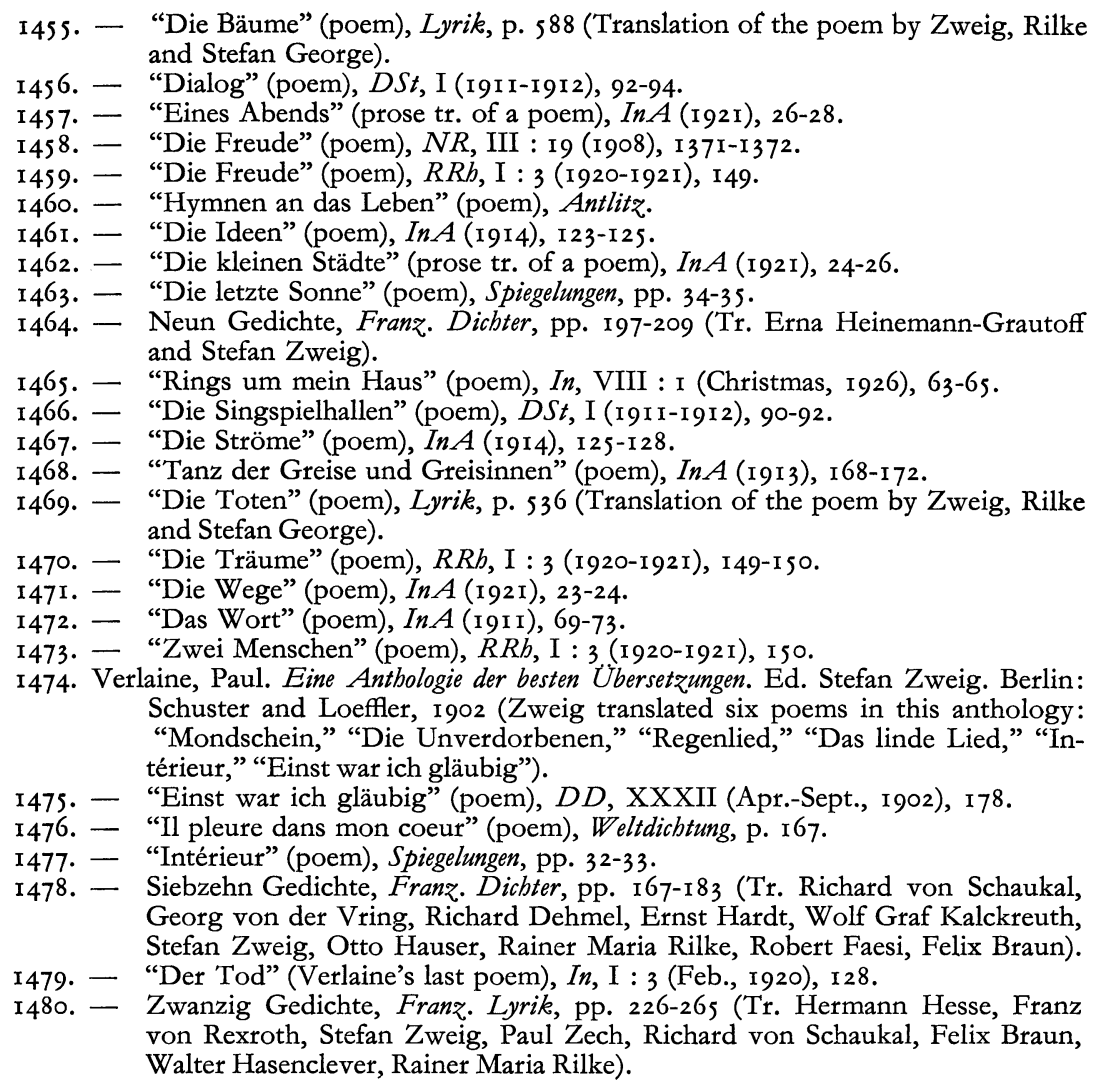

\section{FOREWORDS AND EPILOGUES WRITTEN BY ZWEIG}

I 48I. Ambrosi, Gustinus. "Trente ans de surdité," $R d V$, IX : 9 (Sept., 1935), I425-I 430 (Zweig wrote an Intro. for this article which was translated into French by L. Halberstam).

I482. Anderson, Erica. Die Welt Albert Schweitzers. Ein Photobuch. Berlin-Frankfurt am Main: Fischer, I955 (Foreword and picture titles by Zweig; Cf. "Unvergessliches Erlebnis: Ein Tag bei Albert Schweitzer," Begegnungen, pp. I I3-I 22. English version, The World of Albert Schweitzer, tr. Walter Hasenclever).

1483. Bahr, Hermann. Die schöne Frau. Novellen. Leipzig: Reclam, I924 (Epilogue by Zweig; Reclams Universal-Bibliothek, No. 645 I).

1484. Balzac, Honoré. Balzac. Sein Weltbild aus den Werken. Stuttgart: Lutz, 1908 (Intro. by Zweig; "Aus der Gedankenwelt grosser Geister," Vol. II. Gen. ed. Lothar Brieger-Wasservogel).

I485. Baum, Oskar. Nacbt ist umber. Erzäblung. Leipzig: Reclam, I929 (Epilogue by Zweig).

1486. Bianche, Renzo. Semblanza de Arturo Toscanini (Toscanini, intimo autógrafos de 
Wagner y Verdi). Montevideo: C. Garcia and Cía, 1940 (Foreword by Zweig; Cf. "Arturo Toscanini," Begegnungen, pp. 78-87).

1487. Bloch, Jean Richard. Vom Sinn unseres Jabrbunderts. Tr. from the French by Paul Amann. Berlin-Wien-Leipzig: P. Zsolnay, I932 (Intro. by Zweig).

1488. Brod, Max. Tycho Brabes Weg zu Gott. Berlin: Herbig, 1927 (Epilogue by Zweig; Reprinted Berlin: Herbig, I955).

1489. Casanova, Giacomo, Chevalier de Seingalt. Mémoires. Amsterdam: G. W. Breughel, 1950 (Foreword tr. into Dutch by C. J. Kelk (Cf. "Casanova," Drei Dichter ibres Lebens; Mémoires tr. into Dutch by J. A. Sandfort).

1490. Chateaubriand, François René Auguste Vicomte de. Romantische Erzäblungen. WienLeipzig-München: Rikola, I924 (Ed. with Intro. by Zweig).

1491. Dickens, Charles. Ausgewäblte Romane und Novellen. Vol. I-XII. Leipzig: Insel, 1910(Intro. by Zweig, "Charles Dickens," Vol. I, pp.v-xxx; Cf. later "Dickens" in Drei Meister).

1492. Dostojevsky, Feodor Mikailovich. La Voce sotterranea. Tr. from the Russian by Wanda Kruscinska and S. Catalano. Milano: Delia, r928 (Foreword by Zweig from his "Dostojewski," Drei Meister).

1493. Dostojewski, Fedor Michailowitsch. Sämtliche Romane und Novellen. Tr. from the Russian by H. Röhl and K. Nötzel. Vol. I-XXV. Leipzig: Insel, I92 I (Intro. by Zweig, Vol. I, pp. vii-cxxxvi; Cf. "Dostojewski," Drei Meister).

1494. Errante, Vincenzo. Lenau. Gescbicbte eines Märtyrers der Poesie. Mengen: H. Heine, 1949 (Intro. by Zweig in the form of a letter to Errante, Feb. 2, 1937).

1495. Ginzkey, Franz Karl. Brigitte und Regine und andere Dicbtungen. Leipzig: Reclam, I924 (Epilogue by Zweig; Reclam Universal-Bibliothek, No. 6453).

1496. - Franz Karl Ginzkey. Dem Dichter und Freunde zum sosten Geburtstag. Wien: Wiener Literarische Anstalt, I92 I (Epilogue by Zweig).

1497. Goethe, Johann Wolfgang von. Goethes Gedichte. Eine Auswabl. Leipzig: Reclam, 1927 (Ed. and Intro. by Zweig; This Intro. was reprinted in Begegnungen, pp. 397-405; Poems newly printed 1948, I949, 195 I, 1952, 1953, 1955).

1498. Goll, Claire. My Sentimental Zoo. Tr. from the French by May de Huyn. Mount Vernon, New York: Peter Pauper Press, 1942 (Intro. by Zweig).

1499. Gorki, Maxim. Erzäblungen. Tr. from the Russian by Arthur Luther. Leipzig: Insel, 193 I (Intro. by Zweig, pp. 5-16; Reprinted I948).

1 500. Hearn, Lafcadio. Das Japanbuch. Eine Auswabl aus Lafcadio Hearns Werken. Tr. from the English by Berta Franzos. Frankfurt am Main: Ruetten and Loening, I9 I I (Intro. by Zweig, pp. I-I2).

r gor. Hellens, Franz (pseud. for Friedrich van Ermengem). Bass-Bassina-Bulu. Roman. Berlin: Axel Juncker, 1922 (Intro. by Zweig, pp. I-6).

1 502. Herrmann-Neisse, Max. Erinnerung und Exil. Gedichte. Zürich: Oprecht, 1946 (Epilogue by Zweig).

1503. Heuschele, Otto. Briefe aus Einsamkeiten. Drei Kreise. Berlin: Juncker, I924 (Epilogue by Zweig entitled "Die Kunst des Briefes"; Reprinted in Blätter, is (Jan., I963), 8-9).

1504. Hoffmann, E. T. A. Princesse Brambilla. Tr. from the German by Alzir Hella and Olivier Bournac. Paris-Neuchâtel: Attinger, 1929 (In the series "Romantiques allemands," No. I; Intro. by Zweig).

I 505. Jacobsen, Jens Peter. Niels Lybne. Tr. from the Danish by Ottomar Enking. Leipzig: Paul List, I925 (Epilogue by Zweig; Reprinted 1948).

1 506. Latzko, Andreas. De Acbterboede. Tr. from the German by A. M. de Jong. Amsterdam: Wereldbibliotheek, 1946 (Intro. by Zweig tr. into Dutch by Nico van Suchtelen).

I507. - Le dernier Homme. Genève: Edition du Sablier, I920 (Intro. by Zweig; I I woodcuts by Franz Masereel).

1 508. Leftwich, Joseph. What Will Happen to the Jews? London: P. S. King and Sons, 1936 (Intro. by Zweig, pp. ix-xii; Further quotes from Zweig, pp. I43, 202).

1 509. Lemonnier, Camille. Die Liebe im Menscben. Tr. from the French by Paul Adler. 
Leipzig: Rothbarth, 1903 (Intro. by Zweig; I Ith ed. 1909; Reprinted Leipzig: Wigand, 1920).

1510. - Warum ich Männerkleider trug. Erlebnisse einer Frau. Tr. from the French by P. Cornelius. Berlin: Juncker, I910 (Intro. by Zweig).

I III. Leppin, Paul. Prager Rhapsodie. Erstes Buch. Prag: Stil Verlag, I938 (Intro. by Zweig).

I5x 2. (E.M.) Lilien. Sein Werk. Berlin-Leipzig: Schuster and Loeffler, 1903 (Intro. by Zweig, pp. 9-29).

I5 13. Lind, Emil. Albert Scbweitzer. Leven en Werk. Tr. from the German by Jan Poortenaar. Naarden: In den Toren, 1949 (Intro. by Zweig; 3. ed. 1955).

1514. Mann, Klaus and Willi Fehse. Anthologie jüngster Lyrik. Hamburg: Gebrüder Enoch, 1927 (Intro. by Zweig).

I5 I5. Mayer, Paul. Wunden und Wunder. Gedichte. Heidelberg: Saturn, 1913 (Intro. by Zweig).

I5 16. Mércereau, Alexander. Worte vor dem Leben. Tr. from the French by Paul Friedrich. Leipzig: Insel, I914 (Epilogue by Zweig, pp. 149-154).

I5 17. Mozart, Wolfgang Amadeus. Ein Brief von Wolfgang Amadeus Mozart an sein Augsburger Bäsle. Wien-Leipzig-Zürich: Reichner, I93 I (Intro. by Zweig).

I5 18. Prager, Hans. Die Weltanschaunng Dostojewskis. Hildesheim: Borgmeyer, 1925 (Intro. by Zweig).

I I I9. Relgis, Eugen. Muted Voices. Tr. from the Spanish by Rose Freeman-Ishill. Berkeley Heights, New Jersey: Oriole Press, 1938 (Intro. by Zweig; German version of this introduction reprinted in Blätter, 8/10(Oct., 1960), 25).

I 5 20. Renan, Ernest. Jugenderinnerungen. Tr. from the French by Hannah Szass. Frankfurt am Main: Frankfurter Verlags Anstalt, 1925 (Intro. by Zweig, pp. 5-27; Cf. $526)$.

I52 I. Rimbaud, Arthur. Gedichte. Tr. from the French by K. L. Ammer (pseud. for Karl Klammer). Wiesbaden: Insel, I 954 (Intro. by Zweig; Insel-Bücherei, No. 592; Cf. 526 ).

1522. - Leben und Dichtung. Tr. from the French by K. L. Ammer (pseud. for Karl Klammer). Leipzig: Insel, 1907 (Intro. by Zweig).

1523. Robakidse, Grigol. Das Scblangenbemd. Ein Roman des georgischen Volkes. Jena: Diederichs, I 928 (Intro. by Zweig).

15 24. Rolland, Romain. Liber amicorum Romain Rolland. Zürich-Leipzig: Rotapfel, I926 (Zweig was an editor and contributor to this work; Reprinted in French Paris: Michel, n.d.).

I525. Rose, William and G. Craig Houston. Rainer Maria Rilke. Aspects of bis Mind and Poetry. London: Sidgwick and Jackson, 1938 (Intro. by Zweig, pp. I-9).

I 526. Rousseau, Jean Jacques. Emil oder ïber die Erziebung. Postdam: Gustav Kiepenheuer, I919 (Intro. by Zweig, pp. 7-16).

I 527. Sainte-Beuve, Charles Augustin. Literarische Portraits aus dem Frankreich des XVII.$X I X$. Jabrbunderts. 2 Vol. Frankfurt am Main: Frankfurter Verlags Anstalt, I923 (Intro. by Zweig, Vol. I, pp. 5-25; Reprinted I925; Calw: Hatjè, 1947 (Reprinted 1949); Wien: Verkauf, I947 (Reprinted I949). St. Gallen: Zollikofer, I949).

1528. Soulie, Gaston. Plus jamais ça! Paris: Debresse, 1937 (Preface in the form of letters by Romain Rolland and Zweig).

I 529. Specht, Richard. Florestan Kestners Erfolg. Eine Erzäblung aus den Wiener Märztagen. Leipzig: Reclam, I929 (Epilogue by Zweig; Reclam Universal-Bibliothek, No. 7038-7039).

I 530. Stefan-Gruenfeldt, Paul. Arturo Toscanini. Tr. from the German by Eden and Cedar Paul. New York: Viking, 1936 (Intro. by Zweig; Reprinted New York: Blue Ribbon Books, 1938).

1531. - Arturo Toscanini. Ein Lebensbild. Wien: Reichner, 1936 (Intro. by Zweig; Reprinted as "Arturo Toscanini. Ein Bildnis" in Begegnungen, pp. 78-87).

I532. Stonehill, Charles Archibald, ed. The Jewish Contribution to Civilization. Birmingham, 
England: Frank Juckes Press, 1940 (Intro. by Zweig, pp. 5-7; Minor Zweig Bibliography, pp. 195-196).

1533. Tolstoy, Leo. De levende Gedachten van Tolstoi (verzameld door Stefan Zweig). Tr. Frank de Vries. Den Haag: Servire, I939 (Levende Gedachten. Eerste Reeks, No. 3).

1534. - (Stefan Zweig Presents the) Living Thoughts of Tolstoy. Greenwich, Conn.: Fawcett Publications, Inc., 1960 (In "The Living Thoughts Series - A Premier Book"; Tr. of the Tolstoy essay (1937) from the German by Barrows Mussey; Intro. essay, pp. 9-32).

I535. - Nusus Mukbtarob nun Tolstoy. Tr. Shukry Muhammed 'Ayyad. al-Qahirah: dar al-Qalam, I960 (Selected texts from Tolstoy; Intro. by Zweig).

1536. - Les Pages immortelles de Tolstoi. Paris: Corréa, I 939 (Intro. essay by Zweig, tr. into French by J. Angelloz).

1 537. Verhaeren, Emile. Hymnen an das Leben. Leipzig: Insel, I9 I I (Intro. and tr. by Zweig). 1538. Verlaine, Paul. Anthologie der besten Übertragungen der Gedicbte Verlaines. Berlin: Schuster and Loeffler, 1902 (Ed. and Intro. by Zweig with six Zweig translations; 2. ed. 1907; 3. ed. I91 I).

1539. - Gedicbte. Eine Auswabl der besten Übertragungen. Leipzig: Insel, 1927 (Sel. and epilogue by Zweig, pp. 69-70; Reprinted in 1929, 1938; Insel-Bücherei, No. 394).

1540. - Gesammelte Werke. Ed. Stefan Zweig. Leipzig: Insel, 1922 (Intro. to Vol. II, pp. 5-2I entitled "Paul Verlaines Leben"; Vol. I includes the following Zweig translations: "Mondschein," p. 45, "Die Unverdorbenen," p. 49, "Regenlied," p. 93, "Das linde Lied," p. I30, "Intérieur," p. 170, "Einst war ich gläubig..," p. $29 \mathrm{I})$.

1541. Voss, Hartfrid, ed. Lyrische Handscbriften unserer Zeit. Ebenhausen bei München: Voss, 1958 (Intro. by Zweig).

1542. Zodykow, Maxim. Stimme aus dem Dunkel. Berlin: Lehmann, I93 I (Intro. by Zweig).

\section{BOOK REVIEWS BY STEFAN ZWEIG}

1543. Bahr, Hermann, O Mensch. Roman. Berlin: Fischer, 1910 (Rev.: BT, Sept. 28, 1910).

1544. Baumberg, Antonie. Kleine Erzäblungen und Skizzen. Wien: Konegen, r902 (Rev.: DLE, 5.J.: Ir (Mar. I, I903), 744-748 (“Skizzen-und Novellenbücher")).

I 545. Blumenthal, Hermann. Der Weg der Jugend. Roman. Berlin: Marquardt, I907 and Knabenalter. Roman. Berlin: Marquardt, I908 (Rev.: DLE, IO.J.: I9 (Jul. I, I908), I 348).

1546. Buddha, Gotamo. Reden. German tr. Karl Eugen Neumann. München: Piper, I919 (Rev.: NFP, No. 19787, Sept. 26, 1919, pp. I-3 ("Erhabenes Vermächtnis")).

I547. Dahm, Paula. Gedichte. Dresden: Pierson, r902 (Rev.: DLE, 4.J.: I4 (April, I902), 974("Neue Frauenlyrik")).

I 548. D'Annunzio, Gabriele. Römische Elegien. German tr. Eugen Gugeia. Wien: Stern, I903 (Rev.: DLE, 5.J.: I8 (June I5, 1903), I 258).

1549. Dostoevskaja, Anna Grigorevna. Die Lebenserinnerungen der Gattin Dostojewskis. German tr. Dmitri Umanskij. Ed. René Fülöp-Miller and Friedrich Eckstein. München: Piper, I925 (Rev.: $D L$, XXVII : Io (June, 1925), 581-583).

1550. Duimchen, Theodor. Mittel und Wege. Berlin: Johannes Räde, I902 (Rev.: DLE, 5. J.: I I (Mar. I, I 903), 744-748 ("Skizzen- und Novellenbücher")).

155I. Eck, Miriam. Herbst. Berlin: Schuster and Löffler, I90I (Rev.: DLE, 4.J.: I4 (Apr., 1902), 972 ("Neue Frauenlyrik")).

1552. Eisenschitz, Fritz. Ja wir! Berlin: Jung-Deutschland Verlag, r901 (Rev.: DLE, 3.J.: I8 (June, I90I), I29I (“Neue Skizzen- und Novellenbücher")). 
I553. Finckl, Ludwig. Rosen. Stuttgart-Leipzig: Deutsche Verlagsanstalt, I906 (Rev.: $O R$, IX : 2 (Nov.-Dec., 1906), 147).

1554. Flakes, Otto. Ruland. Roman. Berlin: Fischer, 1922 and Die Simona. Roman. Berlin: Fischer, 1922 (Rev.: NR, XXXIV : 3 (Mar., 1923), 285-287).

I 555. Flaubert, Gustave. Herodias. German tr. Dr. Paul Adler. Leipzig: Seemann, 1903 (Rev.: DLE, 6.J.: 24 (Sept. I5, 1904), 1737-1738).

1556. - Ein scblichtes Herz. German tr. Ernst Hardt. Leipzig: Insel, I904 (Rev.: DLE, 6.J.: 24 (Sept. I5, I904), I737-1738).

1557. - Die Schule der Empfindsamkeit. German tr. Luise Wolf. Minden in Westfalen: Bruns, 1904 (Rev.: DLE, 7.J.: 24 (Sept. 15, 1905), 1793-1795).

1558. - Die Versuchung des beiligen Antonius. German tr. F. P. Greve. Minden in Westfalen: Bruns, 1905 (Rev.: DLE, 9.J.: 6(Dec. 15, 1906), 474-475).

I 559. Forbes-Mosse, Irene. Mezza voce. Berlin: Schuster and Löffler, I90r (Rev.: DLE, 4.J.: I4 (Apr., 1902), 972 ("Neue Frauenlyrik")).

I 560. Franck, Hans. Godiva. Ein Drama. München: Delphin, I919 (Rev.: DLE, 22.J.: I I (Mar. I, I920), 644-646 (Pp. 646-650 contain excerpts from the drama Godiva)).

156r. Frei, Leonore. Lebensflut. Gedichte. Berlin: Dümmler, I 899 (Rev.: DLE, II : 8 (Jan. I 5, 1900), 579-580).

1562. Gelber, A. IоOI Nacht, der Sinn der Erzäblungen der Scheberezade. Wien: Perles, 1917 (Rev.: Wien, 1917; Reprinted in Europäisches Erbe, pp. 157-168 ("Das Drama in Tausendundeiner Nacht")).

1563. Gerhardt-Amytor, Dagobert von. Röntgenstrablen. and Das Amselnest. Breslau: Schottländer, I902 (Rev.: DLE, 5.J.: I I (Mar. I, I903), 744-748 (“Skizzenund Novellenbücher")).

1564. Greiner, Leo. Das Tagebuch. Gedicbte. München-Leipzig: Georg Müller, Igo6 (Rev.: $O O R$, VIII : 102/103 (Aug.-Oct., I906), 420).

1565. Gundolf, Friedrich. Heinrich von Kleist. Berlin: Georg Bondi, 1922 (Rev.: FZ, Feb. 2, 1923).

I 566. Günther, Christian. Strophen. Sel. and ed. with Intro. by Wilhelm von Scholz. Leipzig: Diederichs, 1902 (Rev.: MfL, LXXI : 50 (Dec. 13, 1902), 398-399 ("Eine neue Ausgabe Christian Günthers")).

I 567. Guth, Alfred. Vom letzten Tag. Berlin: Hugo Steinitz, I901 (Rev.: DLE, 3.J.: I8 (June, I901), I 292 (“Neue Skizzenbücher")).

1568. Heijermans, Herman. Sabbath. German tr. R. Ruben. Pössneck in Th.: Bruno Feigenspann, 1904(Rev.: $D L E$, 7.J.: 8 (Jan. I5, 1905), 594).

1569. Heine, Heinrich. Deutscbland, ein Wintermärchen. Ed. Friedrich Hirth. Berlin: Lehmann, i9is (Rev.: DLE, XVIII : I3 (Apr. I, I916), 795-798 ("Eine Faksimileausgabe von Heines 'Deutschland, ein Wintermärchen"')).

1570. de Herédia, José Maria. Tropbäen. German tr. Emil von Gebsattet. München: Weber, I909 (Rev.: DLE, I3.J.: 3 (Nov. I, I910), 219).

1571. Hesse, Hermann. Blick ins Chaos. Bern: Seldwyla, r920 (Rev.: BN, July 27, 1922 ("Selbstbesinnungsschriften von Künstlern")).

1572. - Musik des Einsamen. Gedicbte. Heilbronn: Salzer, r915 (Rev.: BN, Feb. 28, I9Is, p. I).

1573. - Peter Camenzind. Berlin: Fischer, 1904 (Rev.: F, VI : 14 (1904), 270).

1574. Hochstetter, Sophie. Geduld. Roman. Berlin: P. Letto, 1904 and Er versprach ibr einst das Paradies. Novelle. Berlin: Gebr. Paetel, 1904 (Rev.: DLE, 8.J.: 2 (Oct. 15, 1905), (37-I39).

1575. Hofmannsthal, Hugo von. Elektra. Berlin: Fischer, 1903 (Rev.: MfL, LXXII : 17 (Dec., 1903), 528-530).

1576. Holzamer, Wilhelm. Der arme Lukas. Leipzig: Seemann, I903 (Rev.: DLE, 5. J.: I 2 (Mar. I5, 1903), 854-855).

1577. Hönigsberg, Margret. Rot und andere Gedicbte. Dresden-Leipzig: Pierson, 1899 (Rev.: DLE, I.J.: 23 (Sept. I, I899), I498-I499).

I578. Janitschek, Maria. Esclarmonde. Ibr Leben und Leiden. Stuttgart: Deutsche Verlagsanstalt, 1906 (Rev.: DLE, 9.J.: I (Oct. I, 1906), 67). 
I 579. Knorr, Josephine von. Gedichte. Berlin-Leipzig: Cotta, I90 I (Rev.: DLE, 4.J.: I4 (Apr., 1902), 97 (“Neue Frauenlyrik")).

I580. Kurowski, Ludwig. Menschenbilder. Wien: Selbstverlag, 1901 (Rev.: DLE, 3.J.: I 8 (June, I901), I 292 ("Neue Skizzenbücher")).

I 58 r. Laforgue, Jules. Sagenhafte Sinnspiele. German tr. Paul Wiegler. Stuttgart: Juncker, I 905 (Rev.: DLE, 7.J.: 23 (Sept. 1, 1905), 1716-1718).

1582. Lent, Gertrud. Im Sommer. Berlin: Harmonie, I903 Rev.: DLE, 5.J.: I I (Mar. I, I 903), 744-748 ("Skizzen- und Novellenbücher")).

1583. Liebish, Rudolf. Der zerbrocbene Krug und andere Geschichten. Dessau: Dünnhaupt, I903 (Rev.: DLE, 5.J.: I I (Mar. I, I903), 744-748 ("Neue Skizzen- und Novellenbücher")).

1584. Lucka, Emil. Dostojewski. Stuttgart-Berlin: Deutsche Verlagsanstalt, I924 (Rev.: DLE, XXVI : 7 (Apr.-Sept., 1924), 436).

1585. - Winland. Novellen und Legenden. Wien: Deutsch-Österreichischer, 191 2 (Rev.: DLE, 14.J.: 20 (July 15, I912), 1447-I448).

1586. Maeterlinck, Maurice. Gedichte. German tr. R. Ammer and Friedrich von OppelnBronikowski. Jena: Diederichs, 1906 (Rev.: DLE, 10.J.: 20 (July 15, 1908), I 464-1465).

I587. Mann, Thomas. Foreword to Rede und Antwort, dated München, Apr., I921; First book printing in Rede und Antwort. Berlin: Fischer, 1922 (Rev.: NR (Berlin), XXXII : 2 (I 121), I3 I 5-I 32I (“Thomas Manns 'Rede und Antwort").

1588. Meier-Gräfe, Julius. Dostojewski, der Dicbter. Berlin: Rowohlt, I926 (Rev.: DL, XXVIII : 8 (1925-I926), 461-462).

15 89. Mell, Max. Das bekränzte Jabr. Gedichte. Berlin: Juncker, I9I I (Rev.: NR, XXIII : I (I9I 2), 598-599 ("Schöne Verse")).

1590. Michel, Wilhelm. Friedrich Hölderlin. Weimar: E. Lichtenstein, 1925 (Rev.: $D L$, XXVIII : 4(1925-1926), 245).

I591. Moore, George. Aus toten Tagen. German tr. Max Meyerfeld. Berlin: Fleischel, I 907 (Rev.: DLE, I I.J. : 23 (Sept. I, 1909), 169).

1592. Netto, Hadrian Maria. Sibylle und der Papagei. Eine Salzburger Idylle. Dresden: Sibyllen-Verlag, I92 I (Rev.: DLE, 24.J.: 9 (Feb. I, I922), 56I).

1593. Orzeszko, Helene. Der starkere Simson und Anderes. Berlin: Siegfried Cronback, I903 (Rev.: DLE, 5.J.: I I (Mar. I, I903), 744-748 ("Skizzen- und Novellenbücher")).

I 594. Perfall, Anton von. Die Hexen von Nordeboog. München: Langen, 1902 (Rev.: DLE, 5.J.: I I (Mar. I, I903), 744-748 (“Skizzen- und Novellenbücher")).

I595. - Die Landstreicherin. Leipzig: Müller-Mann, I903 (Rev.: DLE, 5.J.: I I (Mar. I, 1903), 744-748 (“Skizzen- und Novellenbücher")).

1596. - Die Molscbule. München: Langen, I901 (Rev.: DLE, 5.J.: II (Mar. I, 1903), 744-748 ("Skizzen- und Novellenbücher")).

I 597. Pruschanski, N. Ein Blatt aus der Chronik unserer Stadt. Berlin: Cronbach, I 903 (Rev.: DLE, 6.J.: I9 (July I, 1904), I378-1 379).

1598. Rafael, L. (pseud. for Hedwig Kiesekamp). Abendgluten. Leipzig: Brietkopf and Härtel, I901 (Rev.: DLE, 4.J.: I4 (Apr., I902), 973 ("Neue Frauenlyrik")).

1599. Raff, Helene. Modellgeschichten. Berlin: Gebr. Pietel, I 902 (Rev.: DLE, 5.J.: I I (Mar. I, 1903), 744-748 ("Skizzen- und Novellenbücher")).

1600. Reinfels, Hans von. Flammen der Liebe. Leipzig: Pierson, i 900 (Rev.: DLE, 5.J.: I I (Mar. I, 1903), 744-748 ("Skizzen- und Novellenbücher")).

I601. Reischl, Friedrich and Luigi Kasimir. Das Buch von der schönen Stadt Salzburg. Wien: Hugo Heller, 1923 (Rev.: DLE, XXVI: 7 (Apr.-Sept., 1924), 437).

1602. Rice, Muriel. Von zwei Ufern. Gedicbte. German tr. Theodor Lessing. Göttingen: Hapke, 1909 (Rev.: DLE, I 2.J.: 9 (Feb. I, I910), 666-667).

1603. Rilke, Rainer Maria. Neue Gedichte. Leipzig: Insel, I90s (Rev.: DLE, i I.J.: 6 (Dec. I5, I 908), 416-418).

1604. Rössler, Arthur. Es gibt solche Menschen. München: August Schupp, r9or (Rev.: $D L E$, 3.J.: I 8 (June, I90I), I 29I ("Neue Skizzenbücher")). 
I605. Sachs, Erich. Ein Lebensmorgen. Berlin: Ebering, I900 (Rev.: DLE, 3.J.: I8 (June, I90I), I 292 ("Neue Skizzenbücher")).

I606. Salus, Hugo. Novellen des Lyrikers. Berlin: Fleischel, I903 (Rev.: DLE, 6.J.: 5 (Dec. I, 1903), 366-367).

1607. Samain, Albert. Gedichte. German tr. Lucy Abels. Berlin: Borngräber, I9I I (Rev.: DLE, I3.J.: I 2 (Mar. I5, I9II), 914-915).

1608. Schäfer, Wilhelm. Gottlieb Mangold. Berlin: Schuster and Loeffler, I901 (Rev.: $D L E$, 3.J.: I 8 (June, I 90I), I 29I (“Neue Skizzenbücher")).

I609. Schlaf, Johannes. Maeterlinck. Berlin: Marquardt, I 906 (Rev.: DLE, 9.J.: I I (Mar. I, 1907), 907).

I6ro. Schmitz, Oskar A. H. Haschisch. Frankfurt am Main: Südwestdeutscher Verlag, I 902 (Rev.: DLE, 5.J.: I I (Mar. I, I903), 744-748 ("Skizzen- und Novellenbücher")).

16r r. Schullern, Heinrich von. Neues Skizzenbuch. Linz: Österreichische Verlagsanstalt, I90I (Rev.: DLE, 3.J.: I 8 (June, I90I), I 290-I 29I (“Neue Skizzenbücher")).

161 2. Schwarz, Heinrich. Salzburg und das Salzkammergut. Wien: Anton Schroll, I926 (Rev.: $D L$, XXVIII : 7 (1925-1926), 443).

I6I3. Semmig, Jeanne Bertha. Enzio. Berlin: Georg Heinrich Meyer, I901 (Rev.: DLE, 4.J.: I4 (Apr., I902), 973 ("Neue Frauenlyrik")).

1614. Soederberg, Eduard. Gassenlieder. German tr. Max Bamberger. Strassburg: Josef Singer, 1902 (Rev.: DLE, 5.J.: 20 (July I 5, 1903), 1442).

16i g. Stendhal (pseud. for Henri Beyle). Bekenntnisse eines Egoisten. German tr. Artur Schurig. Jena: Diederichs, i 905 (Rev.: DLE, 8.J.: 22 (Aug. 15, 1906), 1623).

1616. Strassburger, Egon H. Firlefanz der Puppendoktor. Märchen. Berlin: Heinemann, I921 (Rev.: DLE, 24.J. : 6(Dec. I5, I921), 370-37I).

1617. Suttner, Bertha von. Die Waffen nieder. Dresden: Pierson, I889 (Rev.: NFP, June 2I, I9I 8, pp. I-4.

16r8. Theodor, Josef. Aus Tag und Traum. Breslau: Schottländer, r902 (Rev.: DLE, 5.J.: II (Mar. I, I903), 744-748 ("Skizzen- und Novellenbücher")).

I619. Thoma, Ludwig. Assessor Karlchen. München: Langen, I901 (Rev.: DLE, 3.J.: I 8 (June, I90I), I 290 ("Neue Skizzenbücher")).

1620. Verlaine, Paul. Gedichte. German tr. Otto Hauser. Berlin: Concordia, I 899 (Rev.: $D L E$, 2.J.: 23 (Sept. I, 1900), 166I).

1621. Wegner, Arnim T. Fünf Finger über Dir. Stuttgart: Deutsche Verlagsanstalt, 1930 (Rev.: $D L$, XXXII : 4(1929-I930), 235).

1622. Zech, Paul. Rimbaud. Querschnitt durch sein Leben und Werk. Leipzig: Wolkenwanderer, $1_{927}($ Rev.: $T b$, VIII : 6(1927), 225-227).

\section{XVIIA. ARTICLES AND BOOK REVIEWS OF WORKS BY STEFAN ZWEIG}

POETRY

Ausgewäblte Gedichte. Leipzig: Insel, I93 I (or later editions).

1623. $N L$, 33.J. (1931), 84 (J. Demmering).

1624. $R C C$, 36.a.: I (1936-1937), 553-557 (R. Pitrou).

"Der Bildner" (Rodin).

1625. DF, XXV : 640/648 (Jan., 1924), 49-52 (Karl Kraus).

Die früben Kränze. Leipzig: Insel, I906 (or later editions).

1626. $B b, 20(1907), 621-622$ (W. Unus). 
1627. $B T, 40$ (Paul Zech: "Zeitgeist") (Cf. DLE, 17.J.: 3 (Nov. I, 1914), 171-172, contains the poem "Die Dinge, die die Abende erzählen" from "Die Lieder des Abends").

1628. DLE, 9.J.: i4 (Apr. I5, 1907), ro89-1092 (Leo Greiner: "Neue Lyrik" 1629. Zweig's Frïbe Kränze et al.).

1630. $Z$, XVI (1908), I3 (Alberta von Puttkamer) (Cf. DLE, Iо.J.: 9 (Feb. I, I908), 652; DLE, IX (1906-1907), 109).

Die gesammelten Gedichte. Leipzig: Insel, 1924.

1631. DL, XXVII : I (Oct., I924 - Mar., 1925), 6-7 (Ernst Lissauer: “Zur Lyrik der Gegenwart" - Zweig's Gedichte et al.).

1632. $D L Z$, June 21, I924 (H. W. Keim) (Cf. $D L$, XXVI : 2 (Apr.-Sept., 1924), 1633. PJ, $\mathrm{CXCV}$ (1924), 303 (K. Busse).

Silberne Saiten. Berlin: Schuster and Löffler, 1901.

1634. DLE, III : 13 (Apr., I901), 937 (R. M. Werner).

DRAMAS

Das Haus am Meer. Ein Schauspiel. Leipzig: Insel, 1912.

1635. DLE, I5.J.: 9 (Feb. I, I913), 613-6I5 (Walter von Molo: “Die Persönlichkeit 1636. im Drama").

1637. PL, Oct. 27, I9I 2 (F. Salten: "Stefan Zweigs 'Das Haus am Meer,' ein Schau1638. $Z e$, Oct. 27, 191 2 (L. Feld: “Zweigs 'Haus am Meer”").

Jeremias. Leipzig: Insel, 1917 (or later editions).

1639. $A Z J$, LXXXIV (1920), I (J. B. Münz) (Cf. $D L E$, 22.J.: 1o (Feb. 15, 1920), 613).

1640. $B B C, \mathrm{XI}$ (1917), I7 (Ilse Reicke: "Historische Dichtung aus der Gegenwart")

164I. CV $\quad$, XIII : 40, I. Beibl. (Oct. 5, 1934), I-2 (Eva Reichmann-Jungmann: "Stefan Zweigs 'Jeremias.' Zur bevorstehenden Aufführung im Berliner Kulturbund").

1642. $C V Z$, XIII : 43, 3. Beibl. (1934), 2 (W. S. Matzdorff).

1643. $D D r$, I.J. (1918), 358 (H. Pankow).

1644. $D I Z, \operatorname{ros}(1920), 22-23$ (L. H. Wolf).

1645. DLE, 21.J.: 6 (Dec. 15, 1918), 353 (Theodor Hampe).

1646. DLE, 22.J.: 6 (Dec. I5, I919), 346-348 (Robert F. Arnold).

1647. DNE, IV (1920), 3 (P. Cornelius: "Stefan Zweig und sein 'Jeremias") (Cf.

DLE, 22.J.: I4 (Apr. I5, 1920), 865).

1648. $J e, \mathrm{~V}$ (1925) 559-575 (A. Blau).

1649. JR, XXXIV : 8I (1934), 3 ("Stefan Zweigs 'Jeremias'. Première im jüdischen

I650. KR, XXXIV: 84 (1934), 6 (R. Wischnitzer-Bernstein: "Bühnenlieder zum 'Jeremias"). 
1651. JR, XXXIV (1934), 644 (E. Simon: “Zeittheater in Palästina? Stefan Zweigs 1652. "Jeremias"'). $M$, IX (1918), 9 (Eugen Kilian) (Cf. DLE, 20.J.: I 8 (June 15, 1918), I ro9). 1653. NFP, Sept. 28, 1917 (Paul Zifferer) (Cf. DLE, 20.J.: 4 (Nov. I5, 1917), 2211654. NFP, Oct. I0, I919 ("Stefan Zweigs 'Jeremias,' das Trauerspiel der Pessimisten").

1655. NZZ, Sept. 2, 1917 (Paul Stefan) (Cf. DLE, 20.J.: 2 (Oct. 15, 1917), 103). 1656. $P b, 1918$, p. 273 (Fraedrich).

1657. $P L$, Mar. 9, I9I8 (F. Berau).

1658. $P T, 288$ (Friedrich Hirth) (Cf. DLE, 20.J.: 6 (Dec. I5, 1917), 348).

I659. $R T, \mathrm{I}$ (1921), 44-45 (Kurt Felix: "Über Zweigs 'Jeremias").

I660. Soz, S.J. (1919), 244-246.

1661. $V Z$, Oct. I3, I917 (Franz Servaes) (Cf. $D L E$, 20.J.: 4(Nov. I5, I917), 221-222)

1662. W-Z, June 13, 1918 (Eugen Kilian).

Jeremiah. Tr. Eden and Cedar Paul. New York: Viking, I922 (or later editions).

1663. BTr, Dec. 2, 1922, p. 3.

1664. $C W$, CXLVIII (March, 1939), 73 I.

1665. Cwl, XXIX (Feb. 17, 1939), 469 (Vernon Grenville).

I666. $L T$, June 29, 1934, p. 14; 4/5 Star Ed., June 29, I934, p. I4.

1667. $L T$, May 30,1950, p. 6.

1668. $N$, CXLVIII (Feb. I8, 1939), 212.

1669. NYHTB, Jan. I4, I923, p. I7 (A. D. Douglas).

1670. NYTBR, Dec. 24, 1922, p. 17 ("Drama for a Post-War World").

1671. SRL, VI (Sept. 28, 1929), I90 (Amelia von Ende: “A Zweig Drama").

1672. T, XXXIII (Feb. I3, 1939), 24.

r673. TAM, XXIII (Apr., I939), 248.

Das Lamm des Armen. Leipzig: Insel, 1929.

1674. DDr, N.F. II (1930), 272.

1675. $D L$, XXXII : 8 (1929-1930), 476 (W. Müller).

I676. DSL, 31.J. (1930), 264 (Hallener).

1677. Hw, VII (1930), 282-285 (H. Fischer: "Uraufführung von 'Das Lamm des Armen' in Prag").

1678. LNN, Sept. I8, I930 (E. Delpy: “Stefan Zweigs 'Das Lamm des Armen'; Erstaufführung in Leipzig").

Legende eines Lebens. Ein Kammerspiel. Leipzig: Insel, 1919 (or later editions).

1679. DLE, 21.J.: 10 (Feb. I5, 1919), 607-608 (Fritz Ph. Baader).

I680. DZ, Jan. 20, 1923 ("Stefan Zweigs 'Legende eines Lebens").

I681. $\quad H g$, III/IV (1923), 85 .

1682. HN, Dec. 27, 1919 (“Zweigs 'Legende eines Lebens' (Uraufführung)").

r683. PJ, CXCI (1923), 246-249 (H. Heynen).

I684. $W M$, CXXXIV (Mar., I 923), 96-97 (F. Düsel).

1685. $Z f B$, N.F. I1.J.: 2, Beibl. (1920), sז3.

Die schweigsame Frau. Komische Oper in 3 Aufzügen. Berlin: Adolph Fürstner, 1935 (or other editions).

1686. CVZ, XIII : 3 I, 2. Beibl. (Aug. 2, 1934), 2 (Hans Reisiger: "Richard Strauss und Stefan Zweig"). 
1687. LT, July 21, I934, p. II ; 4/5 Star Ed. July 21, 1934, p. II. ("Herr Stefan Zweig's (Germany) Libretto for Strauss-Opera").

1688. NA, CCLXV (May I, I 929), 53-67 (Enrico Rocca:"L'Opera di Stefan Zweig").

1689. $N K$, VIII : 48 (1957), 8 ("Stefan Zweig: Dzieje jednego libretta (Sprawa wystawienia opery R. Straussa 'Milcz ạca Kobieta')").

I690. NYT, July 18, I934, p. I I ("R. Strauss Attacked by Der Stürmer for Basing New Opera on Text Supplied by Zweig because He is Jewish").

1691. NYT, June I 2, 1935, p. 9 ("Zweig's Libretto for Opera 'The Silent Woman', by Strauss, Incurs Nazi Disfavor").

1692. NYT, June 24, I935, p. I 2 ("Preview of Opera 'The Silent Woman"').

1693. NYT, June 25, I935, p. Is ("Premiere of 'The Silent Woman").

r694. NYT, June 26, 1935, p. I9 ("Germany Hails Opera 'The Silent Woman").

Tersites. Ein Trauerspiel. Leipzig: Insel, 1907; 2. Auflage (veränd.), I919 (or later editions).

I695. $B T$, Nov. 27, 1908 ("Stefan Zweigs 'Thersites").

1696. $B W$, XI (Oct., I 908 - Mar., I 909), 300 (Edgar Pierson).

I697. $D L E$, ir.J. : I (Oct. I, I 908), 30-33 (L. Berg: "Die Tragödie des Neides").

I698. DM, 4.J. (Jan. 4, I909).

1699. $D W$, XI (1908), 25 (Josef Lamm) (Cf. $D L E$, i I.J.: 20 (July I 5, I908), I447).

1700. $\quad H K$, Feb. II, I909 (F. Hartmann).

1701. LNN, Jan. 20, I918 (E. Delpy: “Zweigs 'Tersites' (Erstaufführung)”).

1702. NFP, I5725 (Alberta von Puttkamer) (Cf. DLE, Io.J.: I8 (June I5, 1908), I 288).

1703. $\quad N z, 233$ (Cf. $D L E$, 10.J.: I 8 (June I5, I908), 1 288).

Der verwandelte Komödiant. Berlin: Felix Bloch Erben, I 912 (or later editions).

1704. $D B, 5$ (1947), Io (“Stefan Zweigs 'Der verwandelte Komödiant”").

I705. $D B$, Io (1947), i I ("Stefan Zweigs 'Der verwandelte Komödiant' aufgeführt in Augsburg").

1706. DLE, 14.J.: 17 (June I, 1912), 1226-1227 (Erich Freund).

1707. $D L E$, I6.J.: II (Mar. I, 1914), 907-909 (Moritz Necker).

Volpone. Eine lieblose Komödie. Bearbeitung von Stefan Zweig nach Ben Jonson. Potsdam: Kiepenheuer, 1926 (or later editions).

1708. $D Z$, Dec. 24, I926 (R. Biedrzynski: "Stefan Zweigs 'Volpone', Tanz ums Geld").

1709. JDSG, N.F. III/IV (1927), I 83-190 (Helene Richter: "Ben Jonsons 'Volpone' und sein Erneuerer Stefan Zweig").

1710. $M Z$, Nov. 28, 1926 (B. Guillemin: "Stefan Zweigs 'Volpone").

1711. NFP, Nov. 7, 1926 (Raoul Auernheimer: "Stefan Zweigs 'Volpone").

1712. $S$, 3.J.: 23 (1948), 7 (W. Lenning: "Zwei Dichter und ein Regisseur: Ben Jonson - Stefan Zweig - "Volpone")).

I713. TR, Dec. 24, I926 (G. Manz: "Stefan Zweigs 'Volpone"”).

Volpone. A Loveless Comedy in 3 Acts; by Ben Jonson, freely adapted by Stefan Zweig. Tr. Ruth Langner. London: Allen and Unwin, 1928 (or other editions).

I714. $B l$, XXIV (July, I928), 394 .

I715. Bm, LXVII : 4 (June, I928), 4I9-42I (Ernest Boyd: "Modernizing Ibsen and Ben Jonson"). 
1716. COS, Sept., I928, p. 100.

1717. $C W$, CXXVII (July, 1928), 340-343.

I718. $D i$, LXXXIV (June, I928), 528.

1719. $L T$, Nov. 9, 1926, p. I23.

I720. $L T$, Nov. 24, 1928, p. I1.

1721. NRep, LIV (Apr. 25, 1928), 295-296 (Stark Young).

I722. $N S N, \mathrm{XV}$ (Jan. 29, I938), I64-165.

1723. NYT, Apr. Io, I928, p. 32.

1724. NYT, Apr. 22, I928, p.9 (J. Brooks Atkinson: "Rare Ben. How Zweig's Adaptation of 'Volpone' Transforms Jonson's Text").

1725. $N Y T$, Apr. 22, 1928, p. 9 (R. G. Noyes).

1726. NYT, May 2, I928, p. I6 (L. Shubert Banton and T. Holburn).

1727. $N Y T$, June 27,1928, p. 29.

1728. $O$, CXLVIII (Apr. 25, 1928), 665.

1729. $P M B, \mathrm{XXXIII}$ (July, I928), 405 .

1730. $P M L A$, XLVI (June, I93 I), 605-607 (George W. Whiting: "Volpone, Herr von Fuchs and Les Héritiers Rabourdin").

1731. $S p$, CXLVIII (Jan. 30, 1932), I4I (P. Fleming).

I732. $S p$, CLX (Jan. 28, I938), I32.

1733. $S R L$, IV (Apr. 21, I928), 782 (J. M. Brown).

1734. TAM, XII (May, I928), 368 (Rosamond Gilder).

I735. $T A M$, XII (June, I928), 387 (J. M. Brown).

1736. $W L B, \mathrm{XXIV}$ (June, 1928), 189 .

Volpone, de Ben Jonson, adapté par Jules Romains, d'après Stefan Zweig. Paris, Fayard et $\mathrm{Cie}, \mathrm{I} 928$ (or later edition).

1737. $A P L, \mathrm{XCI}$ (Dec. I5, I928), 583 (Gérard Bauer).

1738. $E N$, Dec. I, 1928 (J. J. Bernard) (Cf. $D F R$, II (1928), 77).

1739. $I$, LXXXVI : 2 (Dec. 8, 1928), 704 .

I 740. JD, XXXVI : 2 (Aug. 30, 1929), 356-358 (H. Bidou).

1741. $M F$, CCVIII (Dec. I5, 1928), 647-653 (Critile).

1742. $M F$, CCLXXIX (Oct. I5, I937), 364-367.

1 743. NRF, N.S. XXXII (1929), I 26-I 28 (B. Crémieux).

\section{FICTION}

GERMAN

Amok. Leipzig: Insel, 1922.

1744. DLE, 25.J.: 19-20 (July I, 1923), 1036 (Hans Joachim Homann).

1745. NFP, Dec. 3, 1922, pp. 34-35 (Erwin Rieger: "Stefan Zweigs neues Novel-

1746. lenbuch: 'Amok'”). $\quad N R, 34$.J.: 2 (1923), 670 (Otto Zarek).

1 747. $N z$, Dec. 9, 1922 (Otto Zarek) (Cf. DLE, 25.J.: 9-10 (Feb. I, 1923), 526).

Angst. Leipzig: Insel-Reclam, I925.

I 748. $V K, 40 . J .:$ I (1925), 23 I (Karl Strecker). 
Die Augen des ewigen Bruders. Leipzig: Insel, 1922 (or later editions).

1749. $B B C$, No. 5 I9 (Cf. $D L E$, 25.J.: 6 (Dec. I 5, I922), 358 (Ernst Lissauer)).

1750. $N B L$ (Kunst), No. 376 (Cf. $D L E, 25$. J.: 23-24 (Sept. I, I923), I I 84 (Ernst Lissauer)).

1751. $N w, 6 . J .(1924), 28$ I.

Brennendes Geheimnis. Erzäblung. Leipzig: Insel, I914 (or later editions).

1752. SS (Lit. Beilage), 28.J. (1925), 28.

Erstes Erlebnis. Leipzig: Insel, r91 I (or later editions).

1753. Im, I : 2 (May, I912), 209-2I I (Dr. Theodor Reik).

I754. Ja, I-II (I9I 2-I9I3), I4I (H. Friedrich).

I755. $M F, \mathrm{XCVI}$ (I9I 2), 867 (Henri Albert).

1756. NG, IX : 7 (July I4, I913), 379-381 (Helene Stöcker: "Vier Geschichten aus Kinderland").

1757. $O R$, XXXII : I (July-Sept., I91 2), 82-83 (Ludwig Ullmann).

1758. $Z B$, N.F. IV : 2 (Oct.-Mar., I912-1913), 466-467 (C.N.).

Fragment einer Novelle. Ed. Erich Fitzbauer. Wien: Verlag der Internationalen StefanZweig-Gesellschaft, I96I (or later editions).

1759. Blätter, 13/14 (Apr., 1962), 4 (Robert Braun: “Vorgeahntes Schicksal”).

1760. $L T$, Oct. 27, 1961, p. $77^{2}$.

Die Liebe der Erika Ewald. Berlin: Egon Fleischel, 1904.

176r. DLE, 7.J.: 4 (Nov. I5, 1904), 291-292 (Hermann Hesse).

I 762. $F$, VI (I905), 5 I (Camill Hoffmann) (Cf. DLE, 7.J.: Io (Feb. 15, 1905), 712).

Verwirrung der Gefüble. Leipzig: Insel, 1927 (or other editions).

1763. $B A, \mathrm{I}: 4$ (Oct., 1927), 62 (Ernst Rose).

1764. $B B B$, VII (1926), 22 (Hans Franck) (Cf. $D L$, XXIX : 9 (1926-1927), 534).

1765. $C V Z$, 5.J. (1926), 667-668 (Ernst Lissauer).

1766. $D L$, XXIX : 3 (1926-1927), 175-176 (Anselma Heine).

1767. NBL, No. 495 (Leonhard Adelt) (Cf. $D L$, XXIX : 3 (1926-1927), I 57 ).

I768. NFP, Oct. 6, 1926 (Leonhard Adelt).

Vier Novellen. Ed. and Intro. Harold Jensen. London: Harrap, 1955.

1768a. GLL, N.S. IX : 3 (April, 1956), 231-232 (W. Schlegelmilch).

\section{DANISH}

Sporg ikke (Brennendes Geheimnis). Tr. Jørgen Budtz-Jørgensen. København: Jespersen and Pio, 1936.

1769. $B V$, I6.a. (1936-1937), 32. 
Amok. Tr. Eden and Cedar Paul. New York: Viking, I93 I.

I 770. $B T r$, July I 8, I931, p. I (W.E.H.).

1771. Fo, LXXXVI (Aug., I931), Iо.

1772. NRep, LXVII (July I 5, I931), 242.

1773. NYHTB, June 14, I93 I, p. 7 (Margaret C. Dawson: “A White Man Amuck").

1774. NYTBR, June I4, I93 I, p. 7 (Louis Kronenberger: "Zweig's Tale of Guilt").

1775. $P M B, \mathrm{XXXVI}$ (Oct., I931), 69.

1776. $S R$, July 26, I93I, p. 7.

1777. $S R L$, VII (July II, I931), 963.

Beware of Pity. Tr. Phyllis and Trevor Blewitt. New York: Viking, I939.

1778. $B l, \mathrm{XXXV}$ (Apr. I, 1939), 253.

I779. $B T r$, Mar. 25, 1939, p. I (O. E. Schoen-Rene).

1780. COS, June, r939, p. I 2.

1781. C Cwl, XXX (June 2, I939), I64-165 (P. T. Hartung).

1782. $L T$, May 5, 1939, p. 20 (J.S.).

1783. $L T$, May 6, 1939, p. 263.

1784. $M G$, May 5, 1939, p. 7 (Wilfred Gibson).

I 785. N, CXLVIII (Apr. I, I939), 38I (Maxwell Geismar: "Descent into Pity").

I 786. $N e$, XIII (1939), 43.

I787. $N S N$, XVII (May 20, I939), 786 (John Mair).

1788. $N Y$, XV (Mar. I8, I939), 85.

1789. NYHTB, Mar. 19, I939, p. Is (Alfred Kazin: "Stefan Zweig's Novel”).

1790. NYTBR, Mar. I9, 1939, p. 6 (Louis Kronenberger: "Stefan Zweig's Brilliant Novel").

1791. PIQ, Autumn, 1939, p. 29.

1792. $P W, \mathrm{CXXXV}$ (1939), 791 (A. Hackett).

1793. $S p$, CLXII (May I 2, I 939), 822 (Kate O'Brien).

1794. $S R$, Mar. 26, 1939, p. 7 (R. W. N.).

1795. SRL, XIX (Mar. 18, I939), I0 (George Stevens: "Code of a Gentleman").

I796. $T$, XXXIII (Mar. 27, 1939), 76.

I797. $W L B, \mathrm{XXXV}$ (Apr., I939), 66

The Buried Candelabrum. Tr. Eden and Cedar Paul. New York: Viking, 1937.

I798. $B l, \mathrm{XXXIV}$ (Dec. I, 1937), I30.

1799. ClW, XXXI (1938), I80.

I800. ILN, CXCII (1936-1937), 202.

1801. $L T$, Dec. II, 1937, p. 945 .

1802. NRep, XCIII (Dec. I 5, I 937), 179 (Harry Thornton Moore: "Light of Israel").

1803. NYHTB, Nov. 7, 1937, p. I4 (Alfred Kazin: "Parable for Wandering Jews").

1804. NYTBR, Oct. 17, I937, p. 8 (Harold Strauss: "Stefan Zweig's Legend of the Menorah").

I805. $\quad S R$, Oct. I7, 1937, p. 7 (F.G.B.).

1806. SRL, XVI : 25 (Oct. I6, 1937), 22 (S. V. Benét: "The Voice of Israel: The Buried Candelabrum").

I807. $T, \operatorname{XXX}($ Oct. 25, 1937), 8 o.

The Burning Secret. London: Allen and Unwin, I92I.

1808. $L T$, Apr. 7, 1921, 230 (L.S.). 
Conflicts. Three Tales. Tr. Eden and Cedar Paul. New York: Viking, 1927; London: Allen and Unwin, 1928.

I 809. $L A, C C C X X X I I I$ (Nov. I, 1927), 844 .

I810. $N$, CXXVI : 3262 (Jan. II, I928), 48-49 (Alter Brody: "Wanted, A Literary Tariff").

I8I I. $\quad$ NRep, LIII (Jan. 4, I928), 200-20I.

I81 2. $\quad N Y E P$, Oct. 29, 1927, p. 14 (Ruth Lechlitner).

1813. NYHTB, Sept. I8, I927, p. 5 (Babette Deutsch).

1814. NYTBR, Oct. 9, 1927, pp. 8, I4 (Harry Salpeter: “Three Powerful Stories").

1815. $N Y W$, Oct. 16, 1927, p. I0 (Harry Salpeter).

Kaleidoscope. Thirteen Stories and Novelletes. Tr. Eden and Cedar Paul. New York: Viking, I934; London: Cassell, 1934.

I816. $B l, \mathrm{XXX}$ (July, I934), 353.

I817. $B T r$, Apr. 21, 1934, p. I (F.M.).

1818. COS, July, 1934, p. I6.

I819. Fo, XCII (Jan., I934), v. (Edith H. Walton).

I820. $L T$, June 26, I934, p. 19.

1821. $L T$, July 1 2, 1934, p. 491.

1822. N, CXXXVIII (May 16, 1934), 571 (Florence Codman: "Middle Europe").

1823. NRep, LXXIX: 1022 (July 4, 1934), 216-217 (Barthold Fles: "Now as a Story Teller").

1824. NYHTB, Apr. I, 1934, p. 5 (F. L. Marsh: "Zweig, a Great European Story Teller").

1825. NYTBR, Apr. I, I934, p. 8 (Louis Kronenberger: "Brilliant Tales by Stefan Zweig").

1826. $S R$, May 1 3, 1934, p. 7 .

1827. $S R L, \mathrm{X}$ (Apr. 28, 1934), 661 ("Echoes of Events").

1828. $S p$, CLIII (July I3, I934), 64 (Graham Greene).

Letter from an Unknown Woman. Tr. Eden and Cedar Paul. New York: Viking, I932; London: Cassell, 1933.

I829. $\quad N, \operatorname{CXXXV}$ (July 20, 1932), 62.

I830. NSN, V (1933), 264.

1831. NYHTB, June 19, 1932, p. 3 (F. T. Marsh: "Unwavering Devotion").

I832. NYTBR, June 19, I932, p. 6 (Harold Strauss: "A Masterly Novelette by Stefan Zweig").

1833. $S R$, June 17,1932, p. 7.

I834. $S R L$, VIII (June I8, 1932), 79I (Wm. Rose Benét: "L'Inconnu").

Passion and Pain. Tr. Eden and Cedar Paul. New York: B. G. Richards, 1925; London: Chapman and Hill, I924.

I835. $D i$, LXXX (Mar. I, I926), 252 (Gilbert Seldes).

1836. $N, \mathrm{CXXII}(1926), 39$.

1837. NYHTB, Aug. I6, 1925, p. 5 (J. J. Smertenko).

1838. NYTBR, Apr. 26, 1925, p. 8.

1839. $N Y T B R$, June 6, 1925, p. 9 (Elizabeth Sanderson).

1840. $\quad N Y W$, May 3, 1925, p. 7. 
The Royal Game. (The Royal Game - Amok - Letter from an Unknown Woman). Tr. Eden and Cedar Paul. New York: Viking, 1944; London: Cassell, 1945.

1841. $B W k$, Apr. 16, 1944, p. 3 (Jack Conroy).

I842. $K$, XII (Jan. I, I944), 7.

1843. LJ, LXIX (Apr. I, I944), 304 (M. P. McKay).

I 844. N, CLVIII (May 6, I 944), 547 (Diana Trilling).

1845. $\quad N Y, \mathrm{XX}$ (Apr. I5, 1944), 83.

1846. NYHTB, Apr. 9, r944, p. 5 (F. H. Bullock).

1847. NYTBR, Apr. 9, 1944, p. 7 (Kenneth Fearing: "Chess Mad").

1848. $S R$, May I4, I944, p. 4 (L. S. Munn).

1849. SRL, XXVII(Feb. 26, I944), 5-7 (Christopher La Farge: "Say It With Fiction"; Zweig Portrait, p. 5).

FRENCH

Amok ou le Fou de Malaise. Tr. Alzir Hella and Olivier Bournac. Paris: Stock, I927.

1850. $R E L V$, 44.a. (1927), 32 I (Henri Loiseau).

La Confusion des Sentiments. Tr. Alzir Hella and Olivier Bournac. Paris: Stock, I929.

I851. NRF, N.S. XXXIV (1930), 585 (René Lalou).

Vingt-quatre Heures de la Vie d'une Femme. Tr. Alzir Hella and Olivier Bournac. Paris: Attinger, 1929.

1852. $B U$, II (1929), 79I (Daniel Rops).

IT A L I AN

Adolescenze (Erstes Erlebnis) Tr. Marcella Dreyfus. Milano: Sperling and Kupfer, I933 (or later editions).

1853. ICS, XVI (1933), 267.

1854. $R N$, 3. sér., XIX (1936-1937), 227.

Leggende. Tr. Anita Rho. Milano: Sperling and Kupfer, 1937.

1855. $N S B$, 4.a. (1938), 2 I.

Lettera d'una Sconosciuta ("Brief einer Unbekannten"). Leporella. Tr. Berta Burgio Ahrens. Milano: Sperling and Kupfer, 1932.

I856. ICS, XVI (1933), 55 .

La Paura ("Angst" et al.). Milano: Sperling and Kupfer, 1938.

I857. LI, 4.a.: 2 (1939), 796. 
Sovvertimento dei Sensi ("Verwirrung der Gefühle") Tr. Berta Burgio Ahrens, Milano: Corbaccio, 1931 .

1858. ICS, XV (1932), 2 I I (E. Palmieri).

POLISH

24 Godziny z Zycia Kobiety $i$ Inne Opowiadania ("24 Stunden aus dem Leben einer Frau"). Warszawa, 1957.

I859. TL, 213 (1957), 4 (Marceli Ranicki: “Dwaj Pisarze Niemieccy”).

ESSAYS

GERMAN

Abscbied von Rilke. Tübingen: Rainer Wunderlich, 1927 (or later editions).

I 860. $D T, 20 .-21$.J. (1929), 390 (Otto Jancke).

Arturo Toscanini - Ein Bildnis. Wien: Reichner, 1936.

I86r. $C V Z$, 15.J.: 8, 2. Beilage (1936), 2.

Erinnerungen an Emile Verbaeren. Wien: Christoph Reissers Söhne, 1917; Leipzig: Selbstverlag des Verfassers, 1927 (or other editions).

I862. $D$, 2.J. (1919), 265 .

1863. $J D B$, 7.J. (1920), ros.

Europäisches Erbe. Ed. Richard Friedenthal. Frankfurt am Main: Fischer, I960.

I864. LT, Jan. 6, I96I, p. 5.

Fabrten. Landschaften und Städte. Wien - Leipzig: Tal and Co., I9I9 (or later editions).

I 865. $B \ddot{u} B$, III (1923), 42 (E. Ackerknecht).

1866. DLE, 23.J.: Is (May I, I921), 953 (Wilhelm Schmidtbonn).

1867. DLE, 25.J.: 4 (Nov. I5, I922), 250 (Herbert Johann Holz).

1868. $Z B$, N.F., 1 2.J.: 2, Beilage (1920), 3 10.

Das Herz Europas. Zürich: Max Rascher, I91 8.

1869. DLE, 20.J.: 22 (Aug. I5, 1918), 1357-1360 (Kurt Martens: "Flugschriften über den Krieg").

1870. $I A R, 3 . \mathrm{J} .:$ 9-IO(I918), 7.

I 87 I. WL, II.J.: is (I918), 93-94 (O. Volkart).

Sinn und Schönheit der Autographen. Wien: Sonderdruck des Verfassers, I935.

I872. $D S, 2$.J. (1936), I45. 
Amerigo, A Comedy of Errors in History. Tr. Andrew St. James. New York: Viking, 1942.

I873. $A$, LXVI (Mar. 14, I942), 635 .

I874. $A H R$, XLVIII (Oct., I942), 86 (W. J. Wilson).

I875. $B l$, XXXVIII (May I, I942), 328.

1876. Cwl, XXXV (Apr. I0, I942), 628 (Max Fischer).

I877. $L J$, LXVII (Feb. I, I942), I3 I (G. E. Brown).

1878. N, CLIV (Feb. 21, I 942), 230 (Samuel Eliot Morrison: "Dr. Vespucci”).

1879. $N Y$, XVIII (Feb. 28, 1942), 60.

1880. NYHTB, Apr. 5, 1942, p. I9 (Henry Steele Connager: "Vespucci's New

188I. NYTBR, Mar. 8, 1942, p. 3 (Philip Ainsworth Means: "How America Received Its Name").

1882. $W L B, \mathrm{XXXVIII} \mathrm{(Apr.,} \mathrm{1942),} 68$.

\section{GERMAN}

Balzac. Der Roman seines Lebens. Stockholm: Bermann-Fischer, 1946 (or later editions). I883. NDL, 7.J.: I I (Nov., 1959), 45-46 (Kurt Böttcher: "Der Lebensroman des unerschöpflichen Balzac").

1884. W, 15.J.: 692 (1947), 5 (F. Bondy: "Balzac der Mächtige").

\section{ENGLISH}

Balzac. Tr. William and Dorothy Rose. New York: Viking, 1946 (or later editions).

I885. $B l$, XLIII (Jan. I5, I947), I 54 .

I886. $B M C N$, Nov., I 946 (Henry Seidel Canby).

1887. $B S$, VI (Dec. I, I946), I4I-I42 (Victor R. Yanitelli).

I 888. $B W k$, Nov. 24, I946, p. 3 (Jex Martin).

I 889. ILN, CCXII (Jan. 3 I, I 948), I 20 (John Squire: “A Great Creator Re-created").

I890. $L J$, LXXI (Nov. I, 1946), I 54I (F. E. Hirsch).

1891. NRep, CXV (Dec. 2, I 946), 730,732-733 (Harry Levin: "Grandeur and Decadence").

1892. NSN, XXXIV (Dec. 20, 1947), 493-494 (Raymond Mortimer).

1893. $N Y$, XXII (Nov. 23, 1946), 120 (Hamilton Basso).

1894. NYHTB, Nov. 17, 1946, p. 5 (Marvin Lowenthal).

1895. NYP, Dec. 9, I946, p. 3 I (Matthew Josephson: "Stefan Zweig's 'Balzac' and the Human Comedy").

1896. NYTBR, Nov. 24, I 946, p. I (Henri Peyre: “A Baffling, Superhuman Figure”).

1897. $S F C$, Dec. 26, 1946, p. I 2 (George Snell).

1898. Si, XXVI (Feb., I947), 52 (Boniface Buckley).

r899. SRL, XXIX (Dec. I4, I946), I3 (Robert Pick: “One of Literature's Immortals").

r900. T, XLVIII (Nov. 25, I946), II6 (“Posthumous Portrait”).

\section{PORTUGUESE}

Balzac. O Romance da sua Vida. Tr. Mario José Dominques. Porto: Civilização, I95 I. 1901. Oc, $\mathrm{XL}:$ I 54 (I95 I), 107-108 (J. de Castro Osorio). 
Castellio gegen Calvin; oder ein Gewissen gegen die Gewalt. Wien: Reichner, 1936 (or later editions).

1902. DSa, 2.J. (1947), 598-600 (Pierre Bertrand: "Das Problem der Toleranz oder Castellion gegen Calvin"; This article first appeared in French in the Tribune de Genève, Apr. 21, 1947; German translation by Harold Mason).

DUTCH

Strïd Rond een Brandstapel. Castellio tegen Calvïn. Tr. Reinier P. Sterkenburg. Amsterdam: Albert de Lange, 1936.

1903. Bks, 30.J. (1937), 469 .

\section{ENGLISH}

The Right to Heresy. Castellio Against Calvin. Tr. Eden and Cedar Paul. New York: Viking, 1936; London: Cassell, 1936.

1904. $B l$, XXXIII (Dec., I936), 106.

1905. CC, LIII (Dec. 2, I936), I6I6 (John T. McNeill: "Prophet versus Reformer").

1906. $C b m$, CLI (Jan. r, I937), I9 (A. L. Murray).

1907. $C b r$, II (1937), 332-335 (C. H. Moehlman).

I908. COS, Dec., 1936, p. 23.

1909. $\quad C W$, CXLIV (Jan., 1937), 499 (B.L.C.).

I910. $L T$, Nov. 7, 1936, p. 910.

I9I. $M G$, Oct. 30, 1936, p. 7 (George Jackson).

I912. $N$, CXLIII (Oct. 24, 1936), 488 (Alice Beal Parsons: “The Pitiless Christ”).

1913. NRep, LXXXIX (July 20, 1937), 362 (W. L. Sperry).

1914. NYHTB, Oct. 25, 1936, p. 7 (Reinhold Niebuhr: "On the Arrogance of Minor Prophets").

1915. NYTBR, Nov. I5, 1936, p. 9 (Lloyd W. Eshleman: "Stefan Zweig's New

Parable of the Right to Heresy").

1916. RaE, XXXIV (1936-1937), 235 (F. M. Powell).

1917. $S P$, CLVII (Dec. 25, 1936), r13 I (A. L. Rowse).

1918. $S R$, Nov. 22, 1936, p. 7 (F.G.B.).

I919. $S R L, \mathrm{XV}$ (Nov. 28, 1936), 10 (Garret Mattingly: "Humanist versus Dictator").

1920. Tab, CLXIX (Apr. 3, 1937), 485.

\section{FRENCH}

Castellion contre Calvin ou Conscience contre Violence. Tr. Alzir Hella. 8th ed. Paris: Grasset, I 936 (or later editions).

I921. $\quad L P$, May, I948.

1922. $M S$, June 10, I947.

1923. $P R O$, Sept. I5, 1947.

1924. Ré, Apr. I 2, I 947 (Roland de Pury).

I925. SC, Apr., I948.

1926. $\operatorname{TrG}$, Apr. 21, 1947 (Pierre Bertrand).

1927. VP, May 16, I947 (Jacques Courvoisier).

1928. $V P$, June 13,1947 . 
Marceline Desbordes-Valmore. Das Lebensbild einer Dicbterin. Leipzig: Insel, 1920 (or later editions).

1929. $B T$, Apr. 17, I921 (F. Michael).

1930. $D L, \mathrm{XXX}: 3$ (1927-I928), 182 (Georg Ransohoff).

I93I. $D L E$, 23.J. (1921), I475 (F. Strunz).

1932. DNS, XXX (1922), I94 (W. Friedmann).

I933. $\quad H l$, I8.J.: 2 (I922), 375-379 (F. Fuchs).

I934. LCfD, 72.J. (I92I), 52 I (O. Hachtman).

I935. LH, 64.J. (I927), 209 (L. Gausebeck-Dörper).

I936. PJ, CLXXXIV (1921), I32 (W. Heynen).

1937. $V V$, II (1922), 543 (Hermann Hesse).

I938. $W L$, XIV (I921), 936 (C. Seelig).

1939. $W M$, Sept., 1923, p. I05.

I940. $\quad Z B$, N.F., XIV : Lit. (1922), 193.

GERMAN

Triumph und Tragik des Erasmus von Rotterdam. Wien-Leipzig-Zürich: Herbert Reichner, 1934.

1941. $B I G, \mathrm{X}$ (1934), 50 (Badt-Strauss).

1942. $\quad C V Z$, 13.J.: 30, 3. Beibl. (Oct. 5, 1934), 3 (Julius Bab).

I943. $J R, 40$. J.: 20 (1935), 9 (Rosenkranz).

1944. $M o, \mathrm{X}$ (1935), 567 (Cohn).

1945. NFP, Nov. 4, 1934 .

D A N I H

Erasmus fra Rotterdam. Tr. G. Rønberg. København: Jespersen and Pio, I934 (or later editions).

1946. $B V$, 17.a. (1936-1937), 25.

\section{ENGLISH}

Erasmus of Rotterdam. Tr. Eden and Cedar Paul. New York: Viking, 1934; London:

Cassell, 1934 (or later editions).

1947. $A$, LII (Nov. Io, I934), in (H. H. Coulson: "Thomas More's Friend").

1948. $A R$, IV (Dec., I934), 246 (Charles F. Ronayne: "Two Portraits of Erasmus").

1949. $B l, \mathrm{XXXI}$ (Dec., I934), I 29.

I950. $\quad B T r$, Dec. I9, I934, p. 3.

1951. $\quad C C, \mathrm{LI}($ Dec. 19, 1934), 1626.

1952. $\quad C F, \mathrm{XV}$ (Mar., 1935), 236 (W. A. Breyfogle).

I953. $C w l, X X I$ (Jan. 25, I935), 379 (Paul Crowley).

r954. COS, Jan., i935, p. 3.

I955. $\quad C W$, CXL (Feb., I935), 622 (R.J.S.H.).

x956. Fo, XCIII (Feb., I935), vi (E. H. Walton).

I957. $J M H$, VII (1935), 365-366 (W. K. Ferguson). 
1958. $L M, \mathrm{XXXI}$ (1938), x80-181 (B. Dobrée).

I959. $L T$, Feb. I4, I935, p. 86.

I960. NRep, LXXXI (Jan. 23, 1934), 314

1961. NSN, VIII (Nov. 24, I934), 759 (K. John).

1962. NYHT, Nov. 3, 1934, p. 9 (Lewis Gannett).

1963. NYHTB, Nov. 4, 1934, p. 3 (Preserved Smith: "A Brains Truster of Reformation Days").

1964. NYTBR, Nov. 4, I934, p. 5 (Percy Hutchinson).

1965. $P I O$, Spring, r935, p. 34 .

1966. $S A Q, \mathrm{XXXIV}$ (1935), 445-448 (R. Giese).

I967. SeR, XLIII (1935), 236-240 (A. E. DuBois).

I968. $S p$, CLIII : Sup. 8, Nov. 23, I934 (A. L. Rowse).

1969. $S R$, Jan. 6, 1935, p. 7 (F.G.B.).

1970. $S R L, \mathrm{XI}$ (Dec. I, I934), 328 (H.T.C.).

1971. Th, XI (Sept., 1936), 301.

1972. $W L B, X X X I(J a n .$, 1935), I2.

FRENCH

Erasme. Grandeur et Décadence d'une Idée. Tr. Alazir Hella. Paris: Grasset, I935 (or later editions).

1973. $A P L$, CII (May 10, 1935), 564 (A. Lang).

r974. $C d S$, 22.a. (June, 1935), $517-518$.

I975. $E P C J, 72 . a .$, CCXXIV (1935), 858 (J. Lecler).

1976. $L L M, 33$. .. (1936-1937), 277.

1977. $M F, 46 . a .$, CCLXII (1936-1937), 423-425 (J. E. Spenlée).

I978. $O p, \mathrm{XXVIII}: 9$ (1937-1938), I4 (Y. Clogenson).

1979. $R d V$, 7.a. (1939), І $272-1273$.

1980. RELV, 53.a. (1936-1937), 365 (H. Chanchoy).

I981. $R H, 60 . a .$, CLXXVI (1935), 98 (H. Hauser).

1982. RHEF, XXII (I936-I937), 83-84 (P. Jourda).

1983. $R H P R$, I5.a. (1935), 379 (H. Strohl).

ITALIAN

Erasmo da Rotterdam. Tr. Lavinia Mazzucchetti. Milano-Verona: Mondadori, 1935 (or later editions).

1984. $B F$, 10.a. (1937-1938), 239-240 (E. Porena).

r985. $C M$, IX (1936-1937), I99 ff. (E. Cione).

GERMAN

Joseph Fouché. Bildnis eines politischen Menschen. Leipzig: Insel, I929 (or later editions).

1986. $A b l, \mathrm{~V}$ (1928-1929), 443 (O. Steinbrinck).

1987. $B B Z$, Kunst, No. 257 (Peter Hamecher: "Der Mann ohne Gewissen") (Cf.

$D L$, XXXII : 4 (1929-1930), 222).

1988. BT, Jan. 7, 1930 (Heinrich Eduard Jacob) (Cf. DL, XXXII : 6 (1929-1930),

1989. BüB, II.J.: I/3 (1931), I3 I (G. A. Narciss).

I990. $D A Z$, Oct. 23,1929 (Th. Böttiger). 


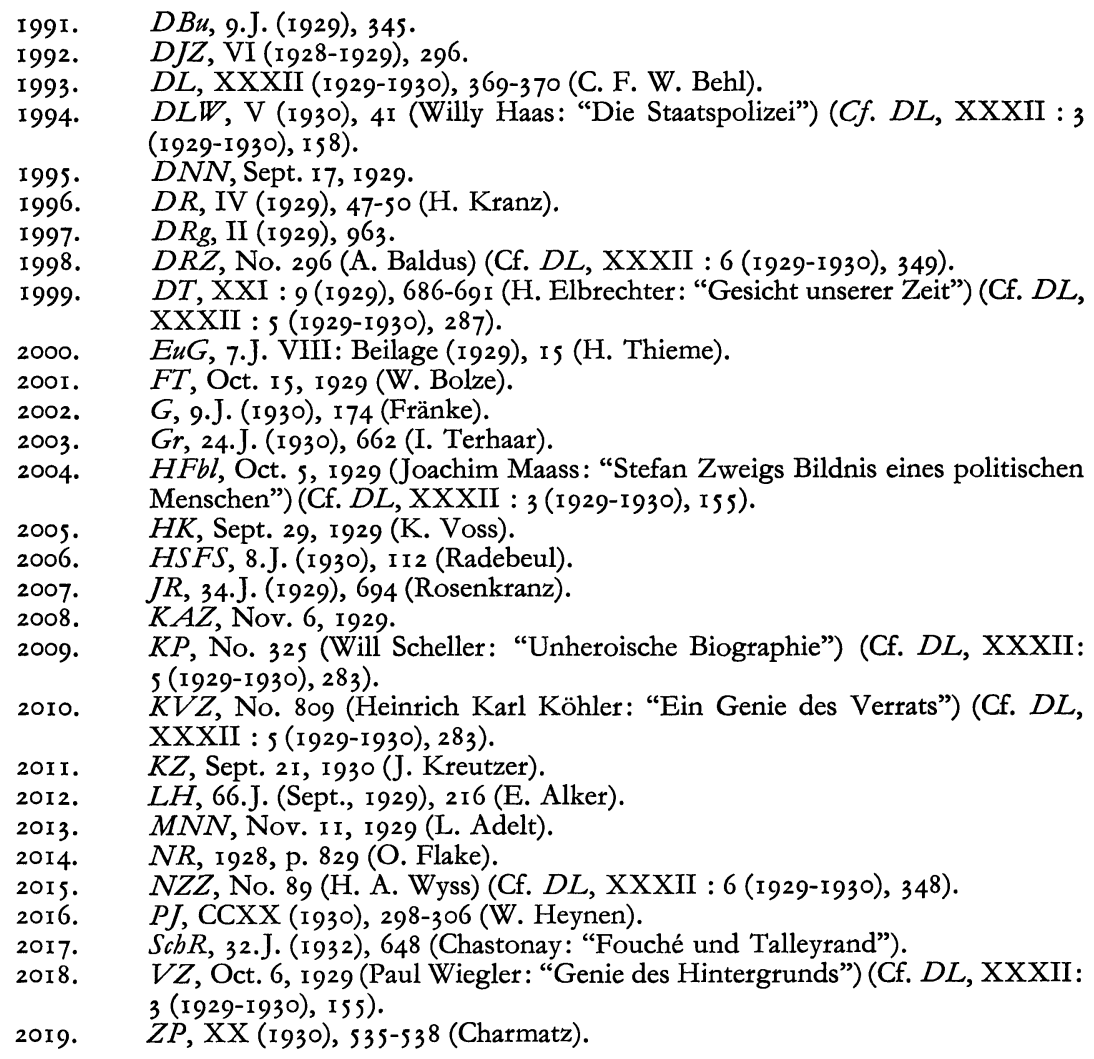

\section{ENGLISH}

Joseph Fouché. The Portrait of a Politician. Tr. Eden and Cedar Paul. New York: Viking, 1930; London: Cassell, 1930 (or later editions).

2020. $B A$, VI (1932), 30I (G. Mueller).

2021. $B l$, XXVII (Jan., 1931), 206.

2022. $B m, \mathrm{LXXI}$ (Aug., 1930), 555 (Margaret Wallace).

2023. $B T r$, Aug. 27, 1930, p. 2.

2024. HO, XXI : 5 (Dec., I930), 395 (C. R. Hall).

2025. LM, XXII (Oct., I930), 567 (Cl. Wilkinson).

2026. LT, Sept. 19, 1930, p. I5.

2027. N, CXXXI (Aug. 27, 1930), 226 (Eugene Loehrke: "Bloodless Biography").

2028. NAR, CCXXX (Oct., I930), 506 (Herschel Brickell: "A Stefan Zweig Biography").

2029. NRep, LXV (Dec. 31, 1930), 200.

2030. NS, XXXVI (Oct. I 8, I930), 58.

2031. $\quad N Y H T B$, Aug. 10, 1930, p. I (M. M. Colum).

2032. NYTBR, Aug. 17, 1930, p. 9 (Alexander Nazaroff: "Fouché, Whom Napoleon Called 'the perfect Traitor"'). 
2033. O, CLV (Aug. 13, 1930), 586 (Allen W. Porterfield: “The Season's Greatest Story").

2034. $\quad R R, \mathrm{LXXXII}$ (Sept., 1930), I 2.

2035. SRL, XXVIII (Nov., 1930), 273.

2036. SRL, VII (Aug. 9, I930), 36 (A. W. G. Randall).

\section{FRENCH}

Joseph Fouché. Tr. Alzir Hella and Olivier Bournac. Paris: Grasset, I930 (or later editions).

2037. $E, \operatorname{XXX}(1932), 634-635$ (P. Abraham).

2038. $G R, 35$. a. (Apr., I93I), 309-313 (J. Ernest-Charles).

2039. JD, XXXVII : I (Feb. 21, 1930), 318-320 (M. Muret: "Vie de Fouché").

2040. $P$, 3. sér. CLXXXI (193I), 207-208 (Geoffroy de Grandmaison).

204I. RB, LXIX : I3 (July 4, I93I), 403-407 (René Moulin: "Fouché, vu par Stefan Zweig").

2042. $R E H$, 99.a. (I932), 448 (L. Miran).

2043. $R E L V$, 47.a. (1930), 162-165 (C. Pitollet).

2044. $R P, 42 . a ., \mathrm{V}$ (I935), 458-462 (A. Albert-Petit: "Ministre de la police sous Napoléon: Fouché").

2045. Ts, Feb. 27, 1930 (A. Levinson).

\section{ITALIAN}

Fouché. Tr. Lavinia Mazzucchetti. Milano: Mondadori, 1930.

2046. $B F$, 5.a. (1930), 707-708 (G. Gabrielli).

2047. ICS, XIII (I930), 322 (E. Palmieri).

\section{SPANISH}

Foucbe, el Genio Tenebroso. Tr. de la Editorial. Barcelona: Juventud, 1935 (or later editions).

2048. U, I4.a. (1937-1938), 592 (J. M. Castro y Calvo).

\section{ENGLISH}

Conqueror of the Seas. The Story of Magellan. Tr. Eden and Cedar Paul. New York: Viking, r 938 (or later editions).

2049. $\quad A$, LVIII (Mar. 5, 1938), 526 (Mother Mary Lawrence).

2050. As, Mar., 1938, 203.

2051. $B A, \mathrm{XIV}$ (I940), 300 (Pieter H. Kollewijn).

2052. $B l, \mathrm{XXXIV}$ (Feb. Is, 1938), 226.

2053. $B T r$, Feb. 5, I938, p. I (Julius Adelberg).

2054. $C F$, XVIII (Aug., x938), I55 (Eleanor Godfrey).

2055. $C H$, XLVIII (Apr., I 938), 2 (N. B. Cousins: "Magellan's Odyssey").

2056. Cwl, XXVII (Feb. 25, 1938), 501 (Lloyd W. Eshleman).

2057. $\operatorname{COS}$, Mar., i 938, p. 6.

2058. GJ, XCII (1937-1938), 88.

2059. ILN, CXCII (1937-1938), 698 (J. Squire: "Magellan, Pioneer of the Pacific"). 2060. LT, Apr. I2, 1938, p. 9 ("Magellan, Pioneer of the Pacific"). 
206r. $L T$, Apr. 16, 1938, p. 261.

2062. $M G$, Apr. 22, 1938, p. 7 (R. Brown).

2063. N, CXLVI (Feb. 5, 1938), I58 (Alan Villiers: "Conqueror of the Seas").

2064. Ne, XI (1938), 29 ("Stefan Zweig Eulogizes the First Man Ever to Make a Round-the-World Cruise").

2065. NRep, XCIV (Feb. 16, 1938), 55 .

2066. NYHTB, Feb. 6, 1938, p. 3 (Charles J. Finger: "The Tale's the Thing; Here It's Nobly Told").

2067. NYTBR, Feb. 6, 1938, p. 3 (R. L. Duffus: "A Smooth-Sailing Narrative of Magellan and His Voyage").

2068. SE, II (Oct., 1938), 520.

2069. $S G M$, LIV (1937-1938), 254.

2070. $S R$, Feb. 6, I938, p. 72 (F. G. Bratton).

2071. SRL, XVII (Feb. 5, I938), 5 (Oliver La Farge).

2072. $T, \mathrm{XXXI}$ (Feb. 14, I938), 67 .

2073. $W L B, X X X I V(A p r ., 1938), 63$.

\section{IT ALI AN}

Magellano. Tr. Lavinia Mazzucchetti. Milano: Mondadori, 1938.

2074. $B F$, I3.a. (I 939), S I 2-5I4 (N. Naldoni-Centenari).

2075. NA, CD (Nov. I, r938), I 17 -1 20 (Raolo Girosi: "Vita e Cultura Italiana nel Mondo - Un Pilota Liqure di Magellano Dimenticato da Stefano Zweig").

2076. $R d C$, 13.a. (1938-r939), Ior3-rors (N. Nald ni-Centenari).

2077. $R M, 7$ r.a. (1937-1938), 133 .

POLISH

Magellan. Tr. Zofia Petersowa. Warszawa: Ksiạzka i Wiedza, 1957.

2078. NLW, 9(1957), 8 (Lucjan Wolanowski: "Przygoda która nazywa sie historia").

GERMAN

Marie Antoinette. Bildnis eines mittleren Charakters. Leipzig: Insel, 1932.

2079. $D L, \mathrm{XXXV}: 4$ (1932-1933), 240 (C. F. W. Behl).

2080. Fr, 4I.J. (1933), 6I (Spörri).

2081. $F Z$, Nov. 27, 1932.

2082. $K r$, I 93 I-1933, p. 250 (Benninghoff).

2083. $K Z$, Oct. 23, 1932 (O. Doderer).

2084. $K Z$, No. 43 (D. H. Sarnetzki)(Cf. $D L, \mathrm{XXXV}: 3$ (1932-1933), 149).

2085. $\quad L C f D$, July, 1933, p. 1043 (J. Hohlfeld).

2086. $\quad P L$, Nov. 4, I 932.

2087. VZ, No. 302 (Ernst Lissauer) (Cf. $D L, \mathrm{XXXV}: 4$ (1932-1933), 213).

DANISH

Marie Antoinette. Et Gennemsnitsmenneskers Portraet. Tr. Kristine Jespersen. København: Jespersen and Pio, 1933 (or later editions).

2088. BV, 16.a. (1936-1937), 73. 


\section{DUTCH}

Marie Antoinette. Portret van een Middelmatig Karakter. Tr. G. J. Werumeus Buning. Amsterdam: Albert de Lange, 1933.

2089. Bks, 27.J. (1933-1934), 184.

ENGLISH

Marie Antoinette. Portrait of an Average Woman. Tr. Eden and Cedar Paul. New York: Viking, 1933; London: Cassell, 1933.

2090. $B A$, VII (1933), 466 (H. H.).

2091. $B l$, XXIX (May, I933), 267.

2092. $B T r$, Apr. I9, 1933, p. 2 (J. W. Maury).

2093. $C D T$, Apr. 8, 1933, p. I 8 (Fanny Butcher).

2094. COS, Nov., 1933, p. 12.

2095. $\quad C w l, \mathrm{XX}($ May 4, 1934), 8.

2096. Fo, LXXXIX (June, I933), vii.

2097. $L T$, Feb. 2, 1933, p. 69.

2098. $M H$, XVIII (1934), 495-497 (B. Bunzel).

2099. N, CXXXVI (Apr. 26, I933), 478 (Catharine Young: "Portrait of Marie").

2 Ioo. $\quad N C$, CIX (I933), $\mathrm{xv}$ (J. Hayward).

2 гог. NRep, LXXIV (May I0, 1933), 371 (Leo Gershoy).

2102. NRep, LXXXIV (Sept. 25, 1935), 192 (Robert Morris Lovett: "A Pair of Queens").

2 103. $\quad N S N$, VI (Oct. I4, 1933), sup., xviii.

2104. NYEP, Apr. 8, 1933, p. 7 (William Soskin).

2105. NYHTB, Apr. 9, 1933, p. I (Albert Guerard: "Marie Antoinette's Romantic Life and Death").

2106. NYTBR, Apr. 2, 1933, p. 3 (Herbert Gorman).

2107. $\quad N Y T B R$, May 7, 1933, p. 5 .

2 108. $P A$, VI : Io (1932), 489 (No. 3943) (A. B. Herrig: "Das eheliche Missgeschick Marie Antoinettes," in $P B, \mathrm{IV}$ (1932), I00-I I 2).

2109. PIQ, Autumn, 1933, p. 34.

2I Iо. $\quad R R$, LXXXVII (May, I933), 4.

2III. $S G$, XXII (Oct., I933), 528.

2112. $S p$, CLI (Sept. 29, I933), 410 (James Laver).

2113. $S R, A$ pr. 23, 1933, p. 7 (R.R.C.).

2I14. SRL, IX (Apr. I, I933), 5I4 (Meade Minnigerode: "Portrait of a Queen").

2115. Th, IX (June, 1934), I42-144.

2116. $\quad W L B$, XXIX (May, 1933), г 37.

\section{FRENCH}

Marie Antoinette. Tr. Alzir Hella. Paris: Grasset, 1933.

2117. $A P L, \mathrm{CII}$ (May 25, 1934), 564 (A. Lang).

2118. $C d S$, 21.a. (1934), 250-25I (G. Petit).

2119. $J D, \mathrm{XL}: 2$ (July 28, I933), I49-I 52 (M. Muret).

21 20. $R A$, 7.a. (1931-1933), i 143.

21 21. $R B, \operatorname{LXXII}(1934)$, I 97.

2122. RELM, 5 r.a. (I934), $270-271$ (H. Loiseau).

2123. $R G, 25 . a$. (1934), 280 (L. Brun).

2124. $R Q H, 64 . a . \mathrm{CXXV}$ (1936-1937), II7-120. 
Maria Antoinetta. Tr. Lavinia Mazzucchetti. Milano: Mondadori, I933.

2125. ICS, XVI (1933), 82 (E. Palmieri).

2126. $L, 4$.a. (1933), I 32-134 (M. Corra).

2127. NA, CCCLXX (1933), I 27-129 (A. Pompeati).

\section{GERMAN}

Maria Stuart. Wien-Leipzig-Zürich: Herbert Reichner, 1935.

2128. $M O, \mathrm{XI}$ (1935), I36 (Silbergleist).

2129. $\quad N F P$, May 5, I935 (J. Gregor).

2130. $P L$, Apr. 25, 1935 (Otto Zarek).

\section{DANISH}

Maria Stuart. Tr. Georg Rønberg. København: Jespersen and Pio, I936.

2131. $\quad B V$, 19.a. (1936-1937), 44 .

\section{DUTCH}

Maria Stuart. Tr. Reinier P. Sterkenburg. Amsterdam: Albert de Lange, 1935.

2132. Bks, 29.J. (1936), 284-285.

2133. $G N$, I936, pp. 274-276 (Maart).

\section{ENGLISH}

The Queen of Scots. Tr. Eden and Cedar Paul. New York: Viking, 1935; London: Cassell' I935.

2134. $\quad A$, LIV (Oct. 19, 1935), 43 (Alfred G. Brickel: "The Catholic Queen”).

2135. $A B$, CLVI (Oct., 1935), ro (Carl Van Doren).

2136. $B l, \mathrm{XXXII}($ Oct., I935), 4I.

2137. $B T r$, Aug. 28, 1935, p. 2 (Julius Adelburg).

2138. CDT, Aug. 24, I935, p. 9 (Fanny Butcher).

2139. $C F, \mathrm{XV}$ (Nov., I935), 374 (E.G.).

2140. $\quad C H$, XLIII (Oct., I935), vii (John Chamberlin).

2141. $\quad C O S$, Aug., I935, p. 16.

2142. $\quad C W$, CXLII (Nov., I935), 245 (P.C.).

2143. Fo, XCIV (Nov., I935), vii (E. H. Walton).

2144. $L D, C X X$ (Sept. 21, I935), 24 (Sketch of Zweig included).

2145. $L M$, XXXII (Sept., I935), 505 (S. Spender).

2146. $L T$, Oct. 10, I935, p. 623.

2147. $L T$, Oct. 15, 1935, p. 20.

2148. $\quad M B, \mathrm{X}$ (1935), 365 .

2149. $\quad N$, CXLI (Sept. I I, I935), 306-307 (C. G. Stillman).

2150. NRep, LXXXIV (Sept. 25, 1935), I92 (Robert Morris Lovett: “A Pair of Queens"). 
2151. $\quad N S N, \mathrm{X}$ (Nov. 2, 1935), 652 (Renée Haynes).

2152. NYHTB, Aug. 25, 1935, p. 3 (Mary Lamberton Becker: "The Most Readable and the Most Reliable").

2153. NYTBR, Aug. 25, I935, p. I (Peter Monro Jack); Dec. I, I935, p. 42.

2154. $R R, \mathrm{XCII}$ (Oct., I935), 4 .

2155. Sp, CLV : 2 (Oct. I8, I935), 618 (A. L. Rowse).

2156. $S R$, Sept. 8, 1935, p. 7 (F.G.B.).

2157. SRL, XII (Aug. 3I, 1935), 6 (J. E. Neale: "The Daughter of Debate").

2158. Th, XII (Dec., I936), 694 (F. H. Reynolds).

2159. $\quad W L B, \mathrm{XXXI}(\mathrm{July}, \mathrm{1935)}, 84$.

2160. $Y R$, N.S. XXV (1936), 609-612 (C. Read).

\section{FINNISH}

Maria Stuart. Elämäkerta Romaani. Tr. Elina Vaara. Jyväskylä: Gummerus, I 938.

2161. $F k$, CXX (1938-1939), 61-66 (H. Hirn).

FRENCH

Marie Stuart. Tr. Alzir Hella. Paris: Grasset, 1936.

2162. $C d S$, 23.a. (June, 1936), si4-5I6.

2163. $E C, \mathrm{~V}$ (1936-1937), 693 (C. Leclère).

2164. EPCJ, 73.a. CCXXVII (1937-1938), 700 (J. Lecler).

2165. RELV, 55.a. (1937-1938), 410 (H. Loiseau).

2166. RH, CLXXX (1936-1937), 103-106 (R. Chauviré).

\section{ITALIAN}

Maria Stuarda. Tr. Lavinia Mazzucchetti. Milano: Mondadori, 1936.

2167. ICS, 19.a. (1936-1937), 149 (G. A. Andriulli).

2168. $\quad N S B$, 2.a. (1936), 54 .

2169. $R N, \mathrm{XXIV}$ (1936-1937), 57 (M. L. Fiumi).

SWEDISH

Maria Stuart. Tr. Hugo Hultenberg. Stockholm: Skoglund, I936.

$2170 . \quad H T$, LVI (1936), 432.

\section{GERMAN}

Romain Rolland. Der Mann und das Werk. Frankfurt am Main: Ruetten and Loening, I92 I (or later editions).

2171. $B g$, I0.J.: 7 (I921), 79 (F. Castelle).

2172. $B i b, 13 . J$. (1931), 1402 (O. Jonsen).

2173. $B T$, May 29, $192 \mathrm{I}$ (J. Chapire).

2I 74. Biih, II (192I), 95 (G. Morgenstern). 
2175. $D N Z$, 39.J.: I (I92I), 342 (I. Hift).

2176. $D T$, 13.J. (1921), 705 (E. Hoppe-Meyer).

2177. Gr, XX (1925-1926), 371-373.

2178. HFbl, Apr. I6, I921 (H. Meyer-Bonfey).

2179. $L G R P, 43 . J$. (1922), I87 (W. Friedmann).

2180. $L T b$, Dec. I, I92I (W. Friedmann).

2181. $L W$, 1926, p. 518 (Fr. Werner).

2182. NBKL, IV (1921), 5 (Martha Schiff).

2183. NFP, Mar. II, I923 (Ellen Key).

2184. $\quad N z$, Apr. 20, 192 I (Otto Zarek).

2185. $W A Z$, No. 12803 (CF. $D L E$, 23.J.: 10 (Feb. 15, 1921), 613).

2186. WL, XIV (1920), 740 (Hermann Hesse).

2187. $W L$, XIV (192r), 936 (C. Seelig).

2188. $Z F E U, \mathrm{XX}$ (1921), 274 (H. Engel).

2189. $Z F E U$, XXVI : I (1927), 6r (C. Appel).

\section{ENGLISH}

Romain Rolland. The Man and His Work. Tr. Eden and Cedar Paul. New York: Seltzer, I92I.

2190. $\quad B m$, LXIII (Oct., 1922), 36.

2191. $B T r$, Nov. 2, I921, p. 4 (E. F. Edgett).

2192. Di, LXXII (Jan., I922), 92 (Kenneth Burke).

2193. $F m$, IV (Nov. 30, 1921), 286.

2194. LT, Mar. 3, I921, p. I45 (L. S.).

2195. $\quad L T$, Dec. I5, 1921 (L. S.).

2196. $\quad N$, CXIII (Nov. 16, 1921), 571 (Gregory Zilboorg: “The Apostle of Peace").

2197. NRep, XXVIII (Oct. 19, I921), 222 (R. M. Lovett).

2198. $\quad N Y H T B$, Dec. 3, I921, p. 225 (Ernest Boyd).

2199. NYTBR, Dec. II, I921, p. I4 ("Romain Rolland, the Idealist").

2200. $O$, XLIX : I254 (Feb. II, I922), I I6-II7 (Newton Fuessle).

2201. $\quad R R$, LXIV (Dec., I921), 668.

FRENCH

Romain Rolland, sa Vie et son Oeuvre. Tr. O. Richey. Bruxelles: Office de Publicitè, I929.

2202. $\quad B U, \mathrm{I}$ (1930), 388-389 (E. Buenzod).

2203. $P$, CLXXXI (193 I), I99-200 (M. Citoleux).

SPANISH

Romain Rolland, el Hombre y su Obra. Tr. Alfredo Cahn. Buenos Aires: Claridad, I936.

2204.

$U d, \mathrm{I}: 3$ (Apr., I936), 5-6.

GERMAN

Emile Verbaeren. Leipzig: Insel, I910.

2205. DNS, XIX (I9II), 368-372 (L. Petry: "Stefan Zweig: Verhaeren. Verhaerens Gedichte"). 
2206. Gwt, 40 (I9II) (W. Wantoch: "Stefan Zweig: Verhaeren. Verhaerens Gedichte").

2207. NFP, Feb. I9, I9I I (F. Servaes).

2207a. RRh, 1.a.: 3 (Dec. I, 1920), r49-150 (Dr. H. Krell: “Emile Verhaeren").

2208. WR, r91 , p. 46 (E. W. Trojau: "Stefan Zweig: Verhaeren. Verhaerens Gedichte").

ENGLISH

Emile Verbaeren. Tr. Jethro Bithell. London-Edinburgh: Constable and Co., I914; New York: Houghton Mifflin Co., r9I4.

2209. $\quad A$, XII (Mar. I3, I915), 542.

2210. $\quad A n$, II (Nov. 28, I914), 559.

22I I. $B m$, XLVII (Dec., I9I4), 77-78 (Francis Bickley).

2212. $B T r$, Dec. I6, I914, p. 27.

2213. MLN, XXVII (Mar. 3, I9I 2), II7-1 I9 (A. Schinz: "Modern French Poets").

2214. $\quad N$, XCIX (Dec. 24, I914), 740.

2215. NYTBR, Jan. 3, I915, 2.

2216. $Y R$, N.S., V (I915), I97-198 (J. Erskine).

FRENCH

Emile Verbaeren, sa Vie, son Oeuvre. Tr. P. Morrise and H. Chernet. Paris: n.p., igro.

2217. $B M B$, I5.a. (I9II), 29 (F. Masoin).

\section{GERMAN}

Baumeister der Welt: Drei Meister - Der Kampf mit dem Dämon - Drei Dichter ibres Lebens. Wien-Leipzig-Zürich: Herbert Reichner, 1935.

2218. $\quad C V Z$, 14.J.: 50, 4. Beibl. (1935), 2.

ENGLISH

Master Builders. A Typology of the Spirit. Tr. Eden and Cedar Paul. New York: Viking, 1939.

2219. $B T r$, Nov. II, 1939, p. 4 (H. M. Jones).

2220. $B l, \mathrm{XXXVI}($ Nov. I 5, I939), irs.

2221. C Cwl, XXXI (Dec. I, I939), I 4I (Euphemia van Rensselaer-Wyatt).

2222. Fo, CII (Nov., I939), 230 (Mary M. Colum: “Artists at Work").

2223. JNMD, XCI (1939), 816.

2224. $N$, CXLIX (Nov. 25, I 939), 584 (James Orrick: “Trilogy of Trilogies").

2225. NY, XV (Sept. 23, I939), 78.

2226. NYHTB, Oct. 8, I939, p. 20 (Albert Guerard: “Three Times Three Men”).

2227. PIQ, Winter, 1940, p. 22.

2228. SRL, XXI (Nov. 25, 1939), ir (Paul Rosenfeld: “An Analysis of Literary Genius"). 
Drei Dichter ibres Lebens: Stendhal-Casanova-Tolstoi. Leipzig: Insel, 1928.

2229. $D G, 5$.J. (1929), 33 (F. Vogeler).

2230. $D L, \mathrm{XXX}$ : II (I927-1928), 676 (Felix Braun).

2231. LGRP, 50. J. (1929), 350-355 (Lerch).

\section{ENGLISH}

Adepts in Self-Portraiture: Casanova, Stendhal, Tolstoy. Tr. Eden and Cedar Paul. New York: Viking, 1928; London: Allen and Unwin, 1929.

2232. $A H, \mathrm{CXXII}: 20$ (Mar. 23, 1928), 707 (Franklin Gordon: "Stefan Zweig, Portrayer of Soul Conflicts").

2233. $B m$, LXIX (Mar., I 929), IOI (Lawrence S. Morris).

2234. $B T r$, Nov. I0, 1928, p. 6 (J. W. Maury).

2235. $C O S$, Dec., I928, p. I34.

2236. Di, LXXXVI (June, I929), 513-516 (Padraic Colum: "Studies in Personality").

2237. Fo, LXXXI (Jan. I, I929), vi.

2238. $L T$, Feb. 7, 1929, p. 93.

2239. N, CXXVIII (Apr. IO, I929), 43I-432 (Angus Burrell: "Men in Masks").

2240. NS, XXXII (Feb. I6, I929), 604 (R. A. Scott-James).

2241. NYEP, Nov. I0, 1928, p. 8 (Paul Eldridge).

2242. NYHTB, Aug. Io, I930, p. I (Mary M. Colum: "The Impulse to Biography").

2243. NYHTB, Nov. I8, I928, p. 4 (Eugen Löhrke: "Spiritual Surgery").

2244. NYTBR, Nov. I I, I928, p. 2 (Edwin Clark: "Three Great Egotists Portrayed by Stefan Zweig").

2245. NYW, Dec. I6, I928, p. Io (Harry Salpeter).

2246. $S L R$, XXVII (Feb., 1929), 59.

2247. $S R L, \mathrm{~V}$ (June I5, I929), III 7 .

2248. $Y R$, XIX (Sept., I929), I63-166 (Albert Fuillerat: “Stendhal's Genius for Self-Analysis").

\section{ITALI AN}

Tre Poeti della Propria Vita: Casanova-Stendhal-Tolstoi. Tr. Enrico Rocca. Milano: Sperling and Kupfer, 1930.

2249. IC, I2.a. (I93I), I4I-I46 (R. Poggioli).

2250. ICS, 14.a. (I93I), 148 .

225 I. L, 2.a. (Mar., I93 I), I3 I-I32 (Lavinia Mazzucchetti).

\section{DANISH}

Tolstoi - from Drei Dichter ibres Lebens (Danish version?).

2252. Fi, 25.a. (1943), 302.

\section{FRENCH}

Casanova. Tr. Alzir Hella and Olivier Bournac. Paris: Attinger, r930.

2253. $P, \operatorname{CLXXXI}($ I93I), I 2 I-I 22 (L. Mensch).

2254. $R M d$, CCI (1936-1937), 80. 
Tolstoi. Tr. Alzir Hella and Olivier Bournac. Paris: Attinger, 1928.

2255. $A P L, \mathrm{XCI}$ (Oct. I, 1928), 305-306 (Benjamin Crémieux).

2256. $B U$, II (1929), 906-907 (M. Beaufils).

2257. $L N E$, 3.a. (1929), 493 (R. Rey Alvarez).

2258. $M F, 40 . a$. CCXI (I929), 470-474 (A. Chevalley).

2259. $\quad M F$, CCXVI (I93I), 476-479 (J. E. Spenlée).

2260. $N R e, 4$.sér. XCIX (1930), I57.

226r. $R E L V$, 45.a. (1928), 460 (H. Loiseau).

\section{GERMAN}

Drei Meister: Balzac-Dickens-Dostojewski. Leipzig: Insel, I920.

2262. $B T$, Nov. 7,1920 (H. Sachaczewer).

2263. $B \ddot{u} B$, I (1921), 60 (Homann).

2264. $D L E$, 23.J.: 14 (Apr. I5, I921), 842-845 (H. W. Keim).

2265. $D N S$, July i 2, 1930, p. 444.

2266. $D T g$, Oct. 20, 1920 (H. Bieber).

2267. $K t$, June, 1921, pp. 166-168 (Fischer).

2268. LGRP, 44.J. (1923), 378 (A. Streuber).

2269. $L H$, 1921, p. 79 (Chr. Flaskamp).

2270. NFP, Sept. 16, 1920 (O. Walzel).

2271. $\quad N R$, I921, p. 669 (O. Flake).

2272. $N z$, Sept. I6, I920 (Otto Zarek: "Ein notwendiges Buch") (Cf. DLE, 25.J.: 3. (Nov. I, 1920), 162).

2273. $O R, L X V$ (1920), 282 (M. Pirker).

2274. $V V$, I (1919-1920), 8 I 7 (Hermann Hesse).

2275. $V Z$, Feb. I3, I92 I (Erich Marcus: "Der Romancier") (Cf. $D L E$, 23.J.: I 3 (Apr. I, I921), 806).

2276. $W Z V, 5$ (1923), 90-92 (H. Keyserling).

2277. $Z B$, N.F. I3.J.: I (I921), I8I.

\section{ENGLISH}

Three Masters: Balzac, Dickens, Dostoeffsky. Tr. Eden and Cedar Paul. New York: Viking, 1930; London: Allen and Unwin, 1930.

2278. $B l, \mathrm{XXVI}$ (July, 1930), 395 .

2279. $B m$, LXXI (June, I930), 338-339.

2280. $B T r$, June 28, 1930, p. 2.

228I. $C W$, CXXXII (Nov., 1930), 24I (J. J. R.).

2282. $L T$, June 26, 1930, p. 530 .

2283. NRep, LXIV (Sept. 3, 1930), 80 (Arthur Colton: “Three Novelists”).

2284. NS, XXXV (July I 2, I 930 ), 445 .

2285. NYHTB, Apr. 27, 1930, p. 6 (Angel Flores: "Masters of the Novel").

2286. NYS, Nov. 18, 1946, p. 2 I (Wm. McFee: "Balzac and Dostoevsky are Subjects of Biography by Zweig and Troyat").

2287. NYTBR, May Ix, I930, p. 2 (Louis Kronenberger).

2288. $\quad N Y W$, June r, 1930, p. 7.

2289. O, CLIV (Apr. 30, 1930), 709 (F.L.R.).

2290. $R X$, VI (July, 1930), 74-75 (S. P. Rudens).

2291. $S L R$, XXVIII (Sept., 1930), 217.

2292. SRL, CXLIX (June 28, 1930), 830. 
Deux Grands Romanciers du XIXe Siècle: Balzac-Dickens. Tr. Alzir Hella and Olivier Bournac. Paris: Simon Kra, 1927.

2293. $\quad N R F$, N.S. XXX (1928), 863 (R. Fernandez).

2294. $R A A, \mathrm{~V}$ (1928), 368-370 (E. Famière).

2295. RELV, 44.a. (1927), $32 \mathrm{I}$ (H. Loiseau).

2296. $R G$, 18.a. (1927), 373 (R. Pitrou).

Dostoievski. Tr. Henri Bloch. Paris: Rieder, I929.

2297. $B U$, II (I929), I4IS (E. Buenzod).

2298. $P$, 2. sér. CIX (I928-1929), I35 (P. Pisani).

2299. $R G$, i9.a. (1929), 345 (A. Fournier).

\section{IT A L I AN}

Tre Maestri: Balzac-Dickens-Dostojevski. Tr. Berta Ahrens. Milano: Sperling and Kupfer, 1932.

2300. IC, 14.a. (1933), 45-50 (M. Robertazzi).

2301. ICS, XVI (1933), I42 (E. Caprile).

2302. $L, 4 . a$. (1933), 252-254 (G. Zamboni).

2303. $N A$, CCCLXX (1933), I 29-I3I (A. Pompeati).

2304. $\quad R N$, 55.a. 3. ser., XVIII (1933), 156.

\section{GERMAN}

Der Kampf mit dem Dämon: Hölderlin-Kleist-Nietzsche. Leipzig: Insel, I925.

2305. $B T$, No. 350 (Erich Everte) (Cf. $D L$, XXVII : I2 (Apr.-Sept., 1925)).

2306. $B \ddot{u} B$, VI (1926), 2 I I (O. Bahrt).

2307. DBP, IV (I927), 453-458 (Zöckler; Article only on "Hölderlin").

2308. $D L$, XXVIII : I (Oct., 1925), 17-19 (Emil Lucka).

2309. $D V$, 2.J. (1929), 270-274 (J. Klein).

2310. $G O M$, Ir.J.: I (I925), 5 II (O. M. Fortana).

2311. $G r$, XIX (1924-1925), 493-494.

23I 2. $K A Z$, No. 34I (Erich Jenisch) (Cf. $D L, \mathrm{XXVII}:$ I 2 (Apr.-Sept., 1925)).

2313. $L N N$, July 3, I925 (K. Voss).

2314. LW, Sept., 1925, p. 740 (R. H. Grützmacher).

2315. $M M N$, XXVI (1927), 274 (Sudhoff).

2316. $M N N$, Aug. 21, I925 (Ernst Lissauer) (Cf. $D L$, XXVIII : I (1925-1926), 38).

2317. NFP, May 20, 1925 (H. Bahr).

2318. $\quad N G, 23 . J .:$ I (1927), I68 (H. Stöcke).

2319. $P N W$, 27.J. (1925), 53I (W. H. Becker).

2320. $R G, \mathrm{XVII}: 9$ (1926), 381-382 (L. Mis).

2321. $T R$, Aug. 30, I925 (Hanns Martin Elster) (Cf. $D L$, XXVIII : 2 (1925-1926), 99).

2322. $Z B$, N.F. 19.J. (1927), 272 (H. Bethge).

2323. $Z D, 40 . J$. (1926), 6r8-619 (J. Stern). 
Le Combat avec le Démon: Kleist-Hölderlin-Nietzsche. Tr. Alzir Hella. Paris: Stock, 1936. 2324. CdS, 24.a. (1936-1937), 452-455.

Le Combat avec le Démon: Frédéric Nietzsche. Tr. Alzir Hella and Olivier Bournac. Paris: V. Attinger, 1930.

2325. NRe, 4. sér, CXIII (1930), 240.

2326. NRF, N.S. XXXVII (1933), 960 (J. Wahl).

2327. RHb, 41.a.: 2 (1930), 350-355 (R. de Saint Jean).

Le Combat avec le Démon: Hölderlin. Tr. Alzir Hella and Olivier Bournac. Paris: V. Attinger, I 928.

2328. $B U, \mathrm{I}$ (1929), I 2I (M. Beaufils).

2329. NRF, N.S. XXXIV (1930), 574-577 (J. Decour).

2330. RELV, 45.a. (1928), 267-269 (H. Loiseau).

2331. $R M d$, CLXXXIV (I930), 284-288 (N. Ségur).

\section{ITALIAN}

La Lotta col Demone: Hölderlin-Kleist-Nietzsche. Tr. A. Overdorfer. Milano: Sperling and Kupfer, 1930 (or later editions).

2332. IC, 12.a. (193I), 285-289 (G. M. Tagliabue; Review only of "Nietzsche").

2333. ICS, 17.a. (1934), 86 (L. Tonelli).

2334. RdP, 30.a. (1936-1937), 279-28I (M. F. Canella; Review only of "Nietzsche").

2335. $R N, 3$. sér., $\mathrm{XX}(1936-1937), 227$.

\section{GERMAN}

Die Heilung durch den Geist. Franz Anton Mesmer-Mary Baker Eddy-Sigmund Freud. Leipzig: Insel, $193 \mathrm{r}$.

2336. $A \ddot{A b l}$, г931, p. гог.

2337. $B B Z$, Aug. 2, 1932 (F. Maraun).

2338. $B d$, Mar. II, I93I (M. Schnewlin).

2339. $B M$, VIII (I93I), 38, 75 (G. Feichtinger).

2340. Bng, V (193I), 3 (F. Humbel) (Cf. $D L$, XXXIII : I2 (Apr.-Sept., 1931), 701).

234I. $\quad B T$, Jan. 30, I93 I (P. Flamm) (Cf. DL, XXXIII : 7 (Apr.-Sept., I93 I), 399).

2342. $\quad C b W, 46 . J$. . (1932), 35 (Grabert).

2343. $\quad C V Z$, I0.J.: $\mathrm{XI}$ (I931), 237.

2344. DL, 33.J.: 8 (May, I93 I), $430-432$ (Lutz Weltmann) (On page 430 is also a caricature-drawing of Stefan Zweig by B. F. Dolbin).

2345. $D M W, 57 . J$. (I93 I), 857-859 (K. Birnbaum).

2346. $E A P$, II (I93 I-I932), 303 (A. Herzberg).

2347. Eck, VII (1933), 409-4I3 (Theodor Spoerri).

2348. $F d T, 8$.J. (1932), 220 (Haeberlin).

2349. Fu, XVIII (1932), 213 (J. Schottky).

2350. Gdl, 34.J. (I93I), 207 (Kurzrock). 
2351. $H F b l$, Feb. 28, 1931 .

2352. $H K$, Mar. 7, 193I (K. Voss).

2353. $K Z$, Mar. I5, I93I (Emil Lucka).

2354. $M M N, \mathrm{XXX}$ (I930), 202 (Haberling).

2355. $M M W, 78$.J. (193I), 925 (E. Bleuler).

2356. $N B$, 7.J. (1930-193 I), I85 (J. Peters).

2357. NBL, Kunst, No. 7 I (Leonhard Adelt) (Cf. $D L$, XXXIII : 7 (Apr.-Sept., 1931), 399).

2358. NFP, Feb. I, 1931 (Leonhard Adelt).

2359. NIW, 4.J.: 6 (I953), Io (Karl August Horst: "Zum Wiederlasen empfohlen:

Stefan Zweigs 'Heilung durch den Geist"').

2360. $\quad P N W$, 33.J. (1931), 347 (Bresler).

2361. ScbR, 31.J. (1931), 575 .

2362. $S M, I V($ I93I), I7I (Pryll).

2363. Um, 35.J. (1931), 686 (A. A. Friedländer).

2364. Wt, I3.J. (1931), rorg.

DANISH

Sjaelens Laegedom. Tr. Clara Hammerich. København: Hirschsprung, 1936.

2365. UfL, 95.a. (1937-1938), 1271.

DUTCH

Genezing door den Geest-Sigmund Freud. Tr. Reinier P. Sterkenburg. Arnhem: Van Loghum Slaterus, 1932.

2366. Bks, 26.J. (1932-1933), 252.

2367. DNG, 49.J. (Apr., I934), 459-460 (F. S. Bosman).

ENGLISH

Mental Healers: Franz Anton Mesmer-Mary Baker Eddy-Sigmund Freud. Tr. Eden and Cedar Paul. New York: F. Ungar, 1932; London: Cassell, I933.

2368. AJPH, XXII (June, 1932), 684 (Mazÿck P. Ravenel).

2369. $B l, \mathrm{XXVIII}$ (Apr., 1932), 348.

2370. $B m, \operatorname{LXXXV~(1933-1934),~} 458$.

2371. CC, XLIX (Apr. 13, I932), 483 (Paul Hutchinson: "Mesmer, Mrs. Eddy, Freud").

2372. $M H$, XVI (1932), I 28-133 (Cl. M. Hincks).

2373. N, CXXXIV (Apr. 27, 1932), 494 (Benjamin Ginzberg).

2374. $\quad$ NRep, LXX (Apr. 13, 1932), 250.

2375. NYEP, Feb. 13, 1932, p. 7 (Joseph Jastrow).

2376. NYHTB, Feb. 21, I932, p. 9 (Woodbridge Riley).

2377. NYTBR, Feb. 21, 1932, p. 9 (Herbert Gorman: "Seekers for the Power of Mind Over Matter").

2378. NYTBR, Feb. 13, 1932, p. 16

2379. $P A, \mathrm{~V}: 5$ (193 I), 242 (No. 233I) (A. B. Herrig: Review of "Sigmund Freud und die Situation der Jahrhundertwende," in $P B$, III (I93 I), 5-18).

2380. $P M B, \mathrm{XXXVII}($ Oct., I932), 61.

2381. $P Q, V I$ (1932), 7I7 (Brown). 
2382. $S R$, Feb. 28, I932, p. 7.

2383. SSR, VII (1942), 332 .

2384. Sy, LXVIII (Oct. I, I 932), 484 (George K. Pratt: "Healers Through the Mind").

2385. $W L B, X X V I I I$ (May, I932), 163.

\section{FRENCH}

La Guérison par l'Esprit. Tr. Alzir Hella and Juliette Parry. Paris: Stock, 932 (or later editions).

2386. CdS, I9.a. (1932), 221-222 (J. Audard; Review only of "Freud").

2387. CdS, 20.a. (I933), 296-298 (R. Baumgarten: "La fantastique Existence de Mary Baker Eddy").

2388. E, XXX (1932), 635-637 (P. Abraham; Review only of "Freud").

2389. $J D$, XXXIX : I (Feb. I 2, 1932), 234-236 (M. Muret).

2390. $L L M, 30 . a$. (1932), 565 .

2391. $M F, \operatorname{CCXXXIX~(1932),~179-18r~}$

2392. $M F$, 45.a., CCL (1934), 362-365 (P. Masson-Oursel; Review only of "Freud").

2393. $P$, 3.sér., CXC (1935), r61-163 (J. Ferrand).

2394. RdF, I2.a. (1932), I39-155 (L. Pierre-Quint; Review only of "Freud").

2395. RdF, I 2.a., IV (I932), 7 1 7-719 (P. Audiat: "Fantastique Existence de Mary Baker Eddy").

2396. $\quad R d V$, 7.a. (1939), 294 (P. Koruham-Europe).

2397. $R d V$, 9.a. (194I), 435 .

2398. RELV, 49.a. (I932), 22 I (H. Loiseau; Review only of "Freud").

2399. RELV, 52.a. (1936-1937), 26-27 (H. Loiseau).

2400. RHb, 4I.a.: 2 (1926-1930), 350 ("Freud, ou la Guérison par l'Esprit").

ITALIAN

L'Anima che guarisce. Franz Anton Mesmer - Mary Baker Eddy - Sigmund Freud. Tr. Lavinia Mazzucchetti. Milano: Sperling and Kupfer, I93 I.

2401. IC, 13.a. (1932), 340-347.

2402. ICS, XV (1932), 53 (E. Palmieri).

2403. L, 2.a. (I93I), I3 I-I 33 (Lavinia Mazzucchetti).

2404. L, 3.a. (Mar., I 932), I I 9-I 22 (Astolfo).

2405. $P e R$, 1934, pp. 26I-276 ("L'Anima che guarisce").

2406. $R N$, 54.a., 3. sér., XVI (1932), 3 ro.

SPANISH

La Curación por el Espiritu. Tr. Francisco Payarols. Barcelona: Apolo, 1934.

2407. $A U C$, 3. ser., I (1934), I32-133.

\section{GERMAN}

Sternstunden der Menschbeit. Ed. Felix Wittmer and Theodore Geissendörfer. New York: Prentice Hall, 193 I.

2408. $M f D U$, XXIII (1931), I56 (Straube). 


\section{DUTCH}

Noodlotsuren der Menscbbeid. Vïf bistorische Miniaturen. Tr. Reinier P. Sterkenburg. Zeist: J. Ploegsma, 1930; and, Lotswendingen. Tr. Reinier P. Sterkenburg. Amsterdam: Wereldbibliotheek, 1939.

2409. DNG, 54.J. (1938-1939), 196.

24ro. $\quad D W B, 3$ I.J. (I934), I53.

ENGLISH

The Tide of Fortune. Twelve Historical Miniatures. Tr. Eden and Cedar Paul. New York: Viking, 1940.

24Ir. $\quad A, \operatorname{LXIV}($ Nov. 30, 1940), 220.

24I 2. $A M$, Jan., I94I.

2413. $B l$, XXXVII (Jan. r, I94I), 172.

2414. $\quad C H F$, LII (Dec. 10, 1940), 3.

2415. C Cwl, XXXIII (Dec. 20, 1940), 235 (Euphemia van Rensselaer-Wyatt).

24I6. $L A$, CCCLIX (Dec., I940), 391.

2417. LJ, LXV (Nov. 15, 1940), 982 (J. B. Fogg).

2418. $L T$, Dec. 14, 1940, p. 627.

2419. $\quad N S N, \mathrm{XX}$ (Dec. 28, 1940), 688 (Flora Grierson).

2420. $\quad N Y$, XVI (Nov. 16, 1940), 100 (Clifton Fadiman).

2421. NYHTB, Nov. 24, I940, p. 9 (Albert Guerard: "Miniature Moments of Crisis in History").

2422. NYTBR, Dec. 8, 1940, p. 7 (Herbert Gorman: "Stefan Zweig's Notes On History").

2423. Sp, CLXVI : I (I94I), roo.

2424. $S R$, Jan. 5 , 194I, p. 7 (F. G. Bratton).

2425. SRL, XXIII (Nov. 23, 1940), Io (Wm. H. Chamberlin: "There is a Tide").

2426. T, XXXVI (Nov. 18, 1940), 90.

FRENCH

Les Heures Etoilées de l'Humanité. Tr. Alzir Hella. Paris: Grasset, I928.

2427. $R G$, 20.a. (1928-1929), I8 I-I83.

ITALIAN

Momenti Eccelsi. Cinque Miniature Storiche. Tr. Berta Burgio Ahrens; La Resurrezione di Händel. Tr. Marcella Gorra. Milano: Sperling and Kupfer, I935.

2428. $R N$, 3.sér., XXII (1936-1937), 435; XXIII (1936-1937), 78.

GERMAN

Brasilien, Land der Zukunft. Stockholm: Bermann-Fischer, 1942.

2429. $\quad C V, 20 . \mathrm{J}$. (1942), 73. 
Brazil, Land of the Future. Tr. Andrew St. James. New York: Viking, I94I.

2430. $A$, LXVI (Oct. 25, 194I), 77.

2431. $B A, \mathrm{XVI}: 4$ (Autumn, I942), 410-4I I (Edward Murry Clark).

2432. $\quad C b m, C L V$ (Nov. r, 1941), I8 (A. L. Murray).

2433. Cwl, XXXIV (Oct. 17, 1941), 617-61 8 (Philip Burnham).

2434. COS, Oct., I94I, p. 17.

2435. HLAS, r 941, p. 37 (James Granier).

2436. LJ, LXVI (Sept. I, I94I), 73 I (F. A. Boyle).

2437. Ne, XVIII (Sept. 29, I94I), pp. 50, 52.

2438. NRep, CV (Oct. 20, 194I), 517.

2439. $\quad N Y$, XVII (Oct. 4, I94I), 87.

2440. NYHTB, Oct. 5, I94I, p. 650 (Nicholas Roosevelt).

2441. NYTBR, Oct. 5, I941, p. 9 (Ernesto Montenegro); Oct. 19, I94I, p. 32 ; Nov. 9, I94I, p. 36.

2442. SoS, XXXIII (Jan., I942), 38 (Elizabeth Wilder).

2443. $S R$, Oct. 21, I94I, p. 8.

FRENCH

Le Brésil, Terre d'Avenir. New York: Edition de la Maison Française, 1942.

2444. $V d F$, Mar. I5, I942, p. 4 (Alceste (pseud.)).

\section{GERMAN}

Die Welt von gestern. Erinnerungen eines Europäers. Stockholm: Bermann-Fischer, 1944 (or later editions).

2445. $A u$, IV (1948), 817-819 (H. Uhlig).

2446. $\quad B \ddot{i} K$, II.J.: 2 (June I5, I962), 28 (B.K.).

2447. DPbl, LII (I952), 298-299 (Hans Jürgen Baden: "Stefan Zweig oder die Welt von gestern").

2448. Gwt, III : 22 (1948), 19-20 (Sieburg).

2449. HAR, I (1946-1947), 437-44I (E. Wolff).

2450. Uns, IV : Io (Oct., I949), I 222 (Carl Augstein).

ENGLISH

The World of Yesterday. Tr. Eden and Cedar Paul. New York: Viking, I943 (or later editions).

245 I. $\quad A$, LXIX (June 26, 1943), 329.

2452. $A M$, CLXXI (June, I943), I29.

2453. $B l$, XXXIX (May I5, 1943), 367 .

2454. $\quad C W$, CLVII (June, I943), 332.

2455. $C J R$, VII (Apr., I944), 205.

2456. Covl, XXXVIII (June 4, I 943), I 74 (Max Fischer).

2457. LJ, LXVIII (Apr., r 943), 288 (F. E. Hirsch).

2458. $L T$, Nov. 27, I943, p. 574 .

2459. $M G$, Dec. 29, 1943, p. 3 .

106 
2460. MJ, XXXI (Oct., I943), 307-314 (Hannah Arendt: "Portrait of a Period; Based on Zweig's Autobiography 'World of Yesterday"').

2461. $\quad N$, CLVI (May I 5, I 943), 7 I I-7I 2 (Hermann Kesten: "Citizen of the World"). 2462. NRep, CVIII (May 3, 1943), 600-602 (Irwin Edman: "World Not Without End").

2463. $\quad N Y$, XIX (Apr. 24, I943), 80.

2464. NYHTB, Apr. I8, I943, p. 4 (Virgilia Sapeiha).

2465. NYTBR, Apr. 18, 1943, p. 2 (J. W. Krutch: "Stefan Zweig's Two Worlds"). 2466. $S R$, May 2, 1943, p. 7 (Helen Lowe).

2467. SRL, XXVI (Apr. 24, 1943), 7 (Robert Pick: "The Storm Within a Storm"). 2468. WLB, XXXIX (June, I943), 84.

POLISH

Swiat w Czorajsyy. Tr. Maria Wislowska. Warszawa: Panstwowy Instytut Wydawniczy, 1958.

2469. $E a, \mathrm{I} 2(1958)$, 10.

\section{GERMAN}

Stefan Zweig-Friderike Zweig: Briefwechsel I91 2-1942. Bern: A. Scherz, I95 I.

2470. Gwt, VII (I952), I 50 (B. Reifenberg).

2471. $I P, \operatorname{IX}$ (1953), 232-233 (Lavinia Mazzucchetti).

2472. $D F u$, I (I93 I), I (Cf. $D L$, XXXIV : s (Oct., I93 I-Mar., 1932), 277).

\section{ENGLISH}

Stefan Zweig-Friderike Maria Zweig; Correspondence 1912-1942. Tr. and ed. Henry G. Alsberg, assisted by Erna MacArthur. New York: Hastings House Publishers, Inc., I954.

2473. $\quad C R$, I (Spring, I955), I 27-1 29 (Sherman Lewis).

2474. $\quad N Y, \mathrm{XXX}$ (Sept. 25, 1954), 142.

2475. NYHTB, July 27, I958, p. 2 ("Mail Pouch: Stefan Zweig and Friderike Maria Zweig").

2476. NYTBR, Nov. 14, 1954, p. 4 (René Fülöp-Miller).

2477. $S R$, Sept. 19, 1954, p. 9 (Richard McLaughlin).

2478. SRL, XXIX (Oct. I9, 1946), 20 (F. C. Weiskopf).

\section{GERMAN}

Richard Strauss - Stefan Zweig: Briefwechsel. Ed. W. Schuh. Frankfurt am Main: S. Fischer, I957.

2479. $B A, \mathrm{XXXII}: 4$ (Autumn, I958), 425-426 (Robert Breuer).

2480. $M A$, LXXVIII (Feb., I958), 236-237 (Robert Breuer: "Strauss - Zweig Correspondence Reflects Nazi Persecution").

248r. $M k g$, II.J. (1957-1958), 253-255 (E. H. M. von Asow).

2482. NYTBR, June 22, I958, p. 4 . 
2483. NZfM, CXIX (Mar., I958), I 3 I-136 (Heinz Joachim: "Europäisch und wahrhaft universal. Aus dem Briefwechsel von Richard Strauss und Stefan Zweig").

2484. $O \ddot{O M z}$, XII (Dec., 1957), 502.

2485. ScbMz, XCVII (Dec., 1957), 506-507.

2486. SoM, XXIII : 2 (I959), I92-194 (B. Cuckov: "R. Straus i St. Creig v ich Perepiske").

\section{B. REVIEWS OF BOOKS WITH FOREWORDS BY ZWEIG}

Anthologie jüngster Lyrik. Fr. Stefan Zweig. Ed. Klaus Mann und Willi Fehse, Hamburg: Gebrüder Enoch, 1927.

2487. Gr, 22.J. (1927), 189 (H. Bachmann).

Balzac. Sein Weltbild aus den Werken. Fr. Stefan Zweig. Ed. Lothar Brieger - Wasservogel. Stuttgart: R. Lutz, I908.

2488. $D L E$, Ir.J.: I4 (Apr. I5, I909), 994-995 (Franz Servaes).

2489. $\quad D L Z g$, p. II 94 .

Goethes Gedichte. Eine Auswabl. Fr. and ed. Stefan Zweig. Leipzig: Reclam, I927.

2490. $D F$, XXVIII: 75 1/756 (Feb., 1927), 87-89 (Karl Kraus).

(E.M.) Lilien. Sein Werk. Fr. Stefan Zweig. Berlin-Leipzig: Schuster and Loeffler, 1903. 2491. DLE, 7.J.: 13 (Apr. 1, 1905), 992-995 (Max Osborn).

Rainer Maria Rilke: Aspects of His Mind and Poetry. Fr. Stefan Zweig. Ed. Wm. Rose and G. Craig Houston. London: Sidgwick and Jackson, 1938.

2492. $\quad L M$, XXXVII (Apr., 1938), 649-650 (Edwin Muir).

Tolstoi. De Levende Gedacbten van Tolstoi. (Verzameld door Stefan Zweig). Tr. Frank de Vries. Den Haag: Servire, 1939 (Levende Gedachten: Eerste Reeks, No. 3).

2493. Bkg, XVIII (1940), 87 (C. A. Bouman).

Tolstoy. Stefan Zweig Presents the Living Thoughts of Tolstoy. New York: Longmans and Green, 1939.

2494. $B A, \mathrm{XIV}$ (Winter, I940), 92 (A.K.).

(Emile) Verbaerens Ausgewäblte Gedicbte. Tr. and ed. Stefan Zweig. Berlin: Schuster and Loeffler, 1904.

2495. $A Z$, No. 198, Beilage (K. H. Strobl).

108 
(Paul) Verlaine. Gesammelte Werke. Ed. Stefan Zweig. Leipzig: Insel, 1922.

2496. $B B C, 4$ II (I923) (E. Werner: "Die deutschen Verlaine - Úbersetzungen").

\section{XVIIC. REVIEWS OF WORKS ABOUT STEFAN ZWEIG}

Arens, Hanns, ed. Stefan Zweig. A Tribute to His Life and Work. Tr. from German by Christobel Fowler. London: W. H. Allen, I95 I.

2497. JLG, III (Apr. 20, I95 I), I-2 (“The World of Yesterday").

Bauer, Arnold. Stefan Zweig. Berlin: Colloquium, r96r (Köpfe des XX. Jahrhunderts, Band 2I).

2498. GQ, XXXVI : I (Jan., 1963), 75 (Randolph J. Klawiter).

Fitzbauer, Erich, ed. Stefan Zweig Spiegelungen einer schöpferischen Persönlicbkeit. Wien: Bergland, 1959.

2499. $G Q$, XXXIII : 4 (Nov., 1960), $381-383$ (George C. Schoolfield).

2500. $M f D U$, LIII (1961), 223-224 (Robert Rie).

250r. $M L J$, Mar., I961, p. 143 (Herbert Lederer).

Hünich, Fritz A. and Rieger, Erwin,eds. Bibliographie der Werke von Stefan Zweig. Leipzig: Insel, I93I.

2502. $J B B$, I8.-I9.J.: 104/107 (I93I), 274.

2503. NaQ, Jan. I6, I932, p. 44 (Otto F. Babler).

Rieger, Erwin. Stefan Zweig. Der Mann und das Werk. Berlin: Spaeth, I928.

2504. $D F a, \mathrm{XI}$ (1928), I (Manfred Sturmann) (Cf. $D L, \mathrm{XXXI}: 6$ (1928-I929),

2505. 345). XXXI : I (1928-1929), 5I (Felix Braun).

Zech, Paul. Stefan Zweig. Eine Gedenkschrift. Buenos Aires: Quadriga, I943.

2506. $B A$, XVIII : I (1944), 42-43 (Harold von Hofe).

Zweig, Friderike Maria. Stefan Zweig. Tr. Erna MacArthur. New York: Thomas Y. Crowell Co., 1946.

2507. $B l$, XLIII (Oct. I5, 1946), 52.

2508. Bwk, Sept. 29, I946, p. 3 (Emily Schossberger).

2509. C Cmy, III (Jan., I 947), 95-96 (H. Politzer).

25 10. Er, III : 9/10 (June Io, I 950), 346-347) (Genevieve Bianquis).

25I1. $K$, XIV (July I5, 1946), 34I.

25I2. LJ, LXXI (Sept. 15, I946), I 206 (F. E. Hirsch). 
2513. $\quad N Y, \mathrm{XXII}$ (Sept. 28, 1946), 102.

2514. NYHTB, Oct. 6, 1946, p. 26 (Virgilia Peterson).

2515. $\quad N Y T B R$, Sept. 29, 1946, p. 6 (Alfred Werner).

2516. $S F C$, Nov. 3, 1946, p. I2 (J. V.).

2517. $S R L$, XXIX (Oct. I9, I946), 20 (F. C. Weiskopf).

Zweig, Friderike Maria. Stefan Zweig, eine Bildbiograpbie. München: Kindler, I961.

2518. BüK, Ir.J.: 2 (June 15, 1962), 22 (Georg Böse).

2519. $\quad L T$, Oct. 27, 1961, p. 772.

\title{
XVIII. BOOKS AND ARTICLES ABOUT AND REFERENCES TO ZWEIG IN VARIOUS MONOGRAPHS
}

\author{
CZECHOSLOVAKIAN
}

2520. Hostovski, Egon. Padesát let Egona Hostovskébo. New York: Moravian Publishers House, 1958 (Liber amicorum to Hostovski in the Intro. to which Hostovski's blood relationship to Zweig is discussed).

DUTCH

252I. Kossmann, Alfred. "Het Noodlotsuur van Stefan Zweig," DeG, CXXI : 7 (July, I958), 302-3I 2.

ENGLISH

2522. Arendt, Hannah. The Origins of Totalitarianism. New York: Meridian Books, r960, pp. 50, 52, 332 (Fourth printing; Original printing with Harcourt, Brace and Co., I95 I).

2523. Arens, Hanns. "Stefan Zweig as a Collector of Manuscripts," $M s$, 9 (1957), 43-45. 25 24. Arens, Hanns, ed. Stefan Zweig. A Tribute to His Life and Work. Tr. from the German by Christobel Fowler. London: Allen, I95 I.

2525. Auernheimer. Raoul. "Stefan Zweig," Torch of Freedom. Ed. Emil Ludwig and Henry B. Kranz. New York: Rinehart, 1943, pp. 409-426 (First printing New York-Toronto: Ferrare and Rinehart, 1939).

2526. Barricelli, J. P. and Harry Zohn. "Music in Stefan Zweig's Last Years," JulR, III (Spring, I956), 3-I r.

2527. Bartlett, Robert Merrill. "Conscience of Europe. An Interview with Stefan Zweig," $W U$, VII (Mar., I93 I), 396-400.

2528. - "Spirit versus Force: Stefan Zweig,"They Dared to Live. New York: Association Press, 1937, pp. 46-50.

2529. Baudouin, Charles. Contemporary Studies. Tr. from the French by Eden and Cedar Paul. London: Allen and Unwin, 1924, pp. 87, 117, 270, 283.

2530. Belmore, H.W. Rilke's Craftsmanship. An Analysis of bis Poetic Style. Oxford, England: Basil Blackwell, I954, p. 226 (Concerning the influence of Rilke's Stundenbuch on Zweig's "Taj Mahal" (Cf. Fabrten); Cf. "The Effect of Rilke's Style on Contemporary Language and Literature, pp. $221-232$.

253 I. Bergler, Edmund, M.D. Homosexuality: Disease or Way of Life? New York: CrowellCollier, 1 962, Pp. I 14, I 22, I 32, I 33 (Discussion of and quotation from Zweig's biographical essay "Stendhal"; First printing with Hill and Wang, 1956). 
2532. Bithell, Jethro. Contemporary German Poetry. Sel. and tr. from the German by Jethro Bithell. London-New York: Scott, 1909, pp. 190-191 (Contains two Zweig poems: "The Dark Butterfly" and "Bruges").

2533. Bratton, Fred Gladstone. The Legacy of the Liberal Spirit. Men and Movements in the Making of Modern Thought. Boston: Beacon Press, 1960, pp. 44, 47 (Quotations from Zweig's Erasmus; First printing New York: Charles Scribner's Sons, 1943).

2534. Brittin, Norman A. "Stefan Zweig: Biographer and Teacher," SeR, XLVIII (Apr., 1940), $245-254$.

2535. Broadbent, Thomas L. Stefan Zweig: An American Bibliography. University of Utah, unpub., 1950.

2536. - "Stefan Zweig and his American Critics," $N B R M M L A$, IV : I I (I95 I), 2-4.

2537. Brockway, Wallace and Herbert Weinstock. Men of Music. Their Lives, Times and Achievements. Revised and enlarged ed. New York: Simon and Schuster, 1950, p. 57I (Concerning the libretto to the Strauss opera "Die schweigsame Frau").

2538. Burckhardt, Oswald. "Introduction" to Zweig's Novely. Sjajvo, Ukraine: n.p., n.d. 2539. Cahnman, Werner J. "Stefan Zweig in Salzburg," MJ, XXX (July, I942), I95-1 98. 2539a. "The Case of Stefan Zweig," JSp, VII : 6 (Apr., I942), 5.

2540. Cousins, Norman. "Stefan Zweig," $S R L$, XXV (Mar. 14, 1942), 2.

2541. Curtiss, Thomas Q. "Stefan Zweig," $B A$, XIII : 4 (Autumn, I939), 427-430.

2542. Da Cunha, Euclides. Rebellion in the Backlands. Tr. from the Portuguese by Livraria Francisco Alves. Chicago: University of Chicago Press, 1944, pp. iii-iv (Quotations from Zweig's Brasilien).

2543. Dali, Salvador. The Secret Life of Salvador Dali. Tr. from the Spanish by Haakon M. Chevalier. New York: Dial Press, 1942, pp. 23-25 (Dali, Zweig and Edward James visit Sigmund Freud in London).

2544. Dargan, E. Preston and Bernard Weinberg, eds. The Evolution of Balzac's 'Comédie bumaine'. Chicago: University of Chicago Press, 1942, pp. 427-430 (Appendix: Wells Chamberlin, "The Zweig Manuscript Proof of 'Une ténébreuse Affaire").

2545. Daviau, Donald G. "Stefan Zweig's Victors in Defeat," MfDU, LI : I (Jan., I959), I-I 2 .

2546. Ende, Amelia von. "Literary Vienna," Bm, XXXVIII (Oct., I9I3), I4I-I55 (p. 150, portrait of Zweig; p. I 53 , short discussion of Zweig).

2547. "European Writing Suffers - Summary of an Interview with Stefan Zweig," $P W$, CXXXV (Jan. 14, 1939), iro.

2548. Evensky, M. "Stefan Zweig," YCD (195 I), 70-77.

2549. Ewen, David. "Stefan Zweig Calls Anti-Semitism a Moldering Evil," $A H, \mathrm{CXXX}$ : 22 (Apr. I 5, 1932), 55 I.

2550. Feder, Ernst. "My Last Conversation with Stefan Zweig," BA, XVII (Jan., 1943), 2-9 (p. I, sketch of Zweig by Stan Hess).

2551. Flower, Sir Walter Newman. "Stefan Zweig," Just As It Happened. London: Cassell, 1950; New York: Morrow, 1950, pp. 176-180 (Also contains a portrait of Zweig).

2552. Freud, Sigmund. "Four-and-Twenty Hours in a Woman's Life," Collected Papers. Vol. V. London: Hogarth, I950, pp. 239-24I (Reprinted in "Dostoevsky and Parricide," Art and Psychoanalysis. Ed. William Phillips. New York: Criterion Books, I957, 3-2 I (Cf. esp. pp. I6-18)).

2552a. Gide, André. Journal. 1939-1949. Tr. from the French by Justin O’Brien. New York: Knopf, 1957, p. 318 (Ref. to Zweig's Calvin).

2553. - Journals. Vol. III. Tr. from the French by Justin O'Brien. New York: Knopf, I949, p. 4r.

2554. Gorki, Maxim. Preface to Zweig's Collected Works in Russian. Leningrad: Vremya, I928-1930 (Cf. "Collected Works" of this bibliography).

2555. Griffin, Jonathan. Review of Escape to Life (Klaus and Erika Mann. Boston: Houghton, I939), SRL, XIX : 26 (Apr. 22, I939), 3-4 (Zweig portrait, p. 3.). 
2556. Gunther John. Inside Europe Today. New York: Harper, 1961, p. I 80.

2557. Heilblut, Ivan. "Graves by the Roadside. In Memory of Stefan Zweig," MJ, XXX (July-Sept., 1942), 199-200.

2558. "H. G. Wells Raises a Storm in the P.E.N. Congress," LD, CXVI (July I 5, 1933), 17-18 (Discussion of a Nazi order banning certain German writers, Zweig among them. Portraits of Zweig, H. G. Wells, Thomas Mann, Erich Remarque, Leon Feuchtwanger, Ernst Toller and Harry S. Canby).

2559. "History of the Screen. Summary of an Interview with Stefan Zweig," NYTBR, Apr. 8, 1934, p. 4.

2560. "In Recollection of Stefan Zweig," $A I R, \mathrm{XV}:{ }_{1} 67 / 168$ (Feb.-Mar., 1947), 2 (Contains a letter from Zweig to the editor concerning Zweig's I94I article in Adam on H. G. Wells).

256r. Jones, Ernest, M. D. The Life and Work of Sigmund Freud. The Last Phase, 1919-1939. Vol. III. New York: Basic Books, I 957, f.m.

2562. Kaufmann, Walter. From Shakespeare to Existentialism. Garden City (New York): Doubleday, 1960, pp. 283, 336, 371, 445 (Anchor Book, A 213).

2563. Koegler, H. "Stefan Zweig," $M C$, CLI (Feb., I955), ror-102.

2564. Kracauer, Siegfried. From Caligari to Hitler. A Psychological History of the German Film. New York: Princeton University Press, I 947, p. I 9 r.

2565. Kris, Ernst. Psychoanalytic Explorations in Art. New York: International Universities Press, 1952, p. 24.

2566. Krutch, Joseph Wood. "Woe to Israel!" $N$, CXLVIII (Feb. I8, I939), 2 I 2.

2567. Leftwich, Joseph. "Stefan Zweig and the World of Yesterday," Ybk, III (I958), $8 \mathrm{I}-\mathrm{IO0}$.

2568. Lengyel, Emil. "German Emigré Literature," $B A$, XII (I938), 5-8.

2569. Liptzin, Solomon, "Stefan Zweig," Germany's Stepcbildren. Philadelphia: Jewish Publication Society of America, I944, pp. 2 I I-225.

2570. - "Young Vienna," PoL, XLVII : 4 (I94I), 337-346.

2571. Lucas, Frank Laurence. Tragedy: Serious Drama in Relation to Aristotle's 'Poetics'. Rev. ed. New York: Collier Books, 1962, p. 39 (Ref. to Beware of Pity (Ungeduld des Herzens); Originally appeared London: L. and Virginia Woolf, 1927 and New York: Harcourt, Brace, 1928).

2572. McClain, William H. "Romain Rolland and Russia," RRev, XXXIX : 2 (Apr., I948), I 22-I 29.

2573. - "Soviet Russia Through the Eyes of Stefan Zweig and Romain Rolland," $M L N$, LXIX : I (Jan., I954), I I-r 8.

2574. McClain, William H. and Harry Zohn. "Zweig and Rolland - Literary and Personal Relationship," GermR, XXVIII : 4 (I953), 262-28I.

2575. Mann, Klaus. "Stefan Zweig," Treasury of the Free World. Ed. B. Raeburn. New York: Arco, 1946, pp. $365-368$.

2576. - "Victims of Fascism," FW, II (Apr., 1942), 274-276.

2577. Marshall, Margaret. "Stefan Zweig," N, CLIV (Mar. 14, 1942), 3 I4.

2578. Mason, Eudoc. Rilke, Europe and the English-speaking World. Cambridge: Cambridge University Press, 196I, p. 200 (Quote from a conversation between Zweig, Emile Verhaeren, Romain Rolland and Rilke in 1913 on "the spiritual unity of Europe and the duty of the writer").

2579. Mathis, Alfred. "Stefan Zweig as Librettist and His Collaboration with Richard Strauss," MaL (1944), pp. I63-176, 226-245.

2580. Maurina, Zenta. A Prophet of the Soul: Fyodor Dostoievsky. Tr. from the Latvian by C. P. Finlayson. London: James Clarke, I939, p. 25.

2581. Mileck, Joseph. Hermann Hesse and His Critics. Chapel Hill, N.C.: University of North Carolina Press, 1958, pp. 7, 49, r63, 291.

2582. Morgan, Bayard Quincy. "Literature in Exil," $B A$, XVIII : 3 (1944), 23I234.

2583. Neider, C., ed. "Beloved Returns," Stature of Thomas Mann. New York: New Directions, I947, pp. 188-190. 
2584. Niles, Blair Rice. "Stefan Zweig the Author," Journeys in Time. New York: Coward McCann, I946, pp. 50-53.

2585. - "Stefan Zweig the Man," Journeys in Time. New York: Coward - McCann, I946, pp. 372-38I.

2586. Norman, Charles. Ezra Pound. New York: Macmillan, 1960, p. 309 (Letter of Pound to Zukofsky of Nov. 22, I93 I referring to lectures to be given by him, Zweig, et al. in Florence).

2587. Norden, R. "Brazilian Diary," $L A$, CCCLI(Jan., I937), 384-392.

2588. "On the War Effect on the Future of Writing. An Interview with Stefan Zweig," $N Y T$, July 28, 1940, p. 2.

2589. Oechler, William F. "Reception of Emile Verhaeren in Germany. Some Unpublished Letters of Stefan Zweig," MLN, LXII (Apr., I947), 226-234.

2590. Pick, Robert. "Stefan Zweig," $B M C N$, Nov., I946, pp. 4-5.

2591. - "The Storm Within a Storm," SRL, XXVI(Apr. 24, 1945), 7.

2592. Puner, Helen Walker. Freud, His Life and His Mind. New York: Dell, r959, p. 231 (Quote from Zweig's essay on Freud in Heilung durch den Geist).

2593. Putnam, Samuel. Marvelous Journey. A Survey of Four Centuries of Brazilian Writing. New York: Knopf, 1948, p. 203 (Quote from Zweig's Brasilien).

2594. Relgis, Eugen. "Stefan Zweig and Judaism," $J A, \mathrm{XI}:$ 10 (Oct., 1956), 4-9 (This article was translated into English by Dr. Harry Zohn from a chapter (slightly abridged) in Relgis' book Profetas y Poetas. Buenos Aires: Editorial Candelabro, 1955 ).

2595. Ring, O. T. "Life and Death of Stefan Zweig," NM, XLII : ro (Mar. 10, 1942), 22-23.

2596. Romains, Jules. Stefan Zweig, Great European. Tr. from the French by James Whitall. New York: Viking, I94I (A bibliography of the original works of Zweig, pp. 62-64).

2597. Rose, William. "German Literary Exiles in England," GLL, I (Apr., I948), 3. 2598. Ross, Nan. "Books in Germany," Sp CLXXVIII (May 16, I947), 554-555.

2599. Royce, William H. A Balzac Bibliography. Chicago: University of Chicago Press, I929, pp. $231,464$.

2600. Schoenberner, Franz. "Stefan Zweig and Ourselves," NRep, CVI (Mar. 9, 1942), 333.

2601. Schreiber, Georges. "Stefan Zweig," Portraits and Self-portraits. New York-Boston: Houghton Mifflin, I936, pp. I73-1 75 .

2602. Selden-Goth, Gisella. "Stefan Zweig, Lover of Music," $B A, \mathrm{XX}: 2$ (1946). I 49-I I.

2603. Sievers, W. David. Freud On Broadway. A History of Psychoanalysis and the American Drama. New York: Hermitage House, 1955, p. 256.

2604. Slochower, Harry. "In the Fascist Styx," No Voice Is Wholly Lost. New York: Creative Age Press, 1945, pp. 75-92.

2605. Slonim, Marc. Three Loves of Dostoevsky. New York: Rinehart, I955; Toronto: Clarke, Irwin, I955, p. 299 (Zweig's Three Masters listed in Slonim's bibliography as consulted but not specifically quoted).

2606. Slowacki, Juljusz. Mary Stuart. A Romantic Drama. Tr. from the Polish by Arthur Prudden Coleman and Marion Moore Coleman. Schenectady, N.Y.: Pub. by translators, 1937 (Foreword, pp. 3-4, by Arthur Prudden Coleman (May I, 1937), discusses Zweig's influence on Slowacki's stage interpretation of the character of Mary Stuart).

2607. Sperry, W. L. "Foreshadows of the Present," NRep, LXXXIX (Jan. 20, I937), 362 .

2608. Starr, William Thomas. A Critical Bibliography of the Published Writings of Romain Rolland. Evanston, Ill.: Northwestern University Press, r950, f.m.

2609. - Romain Rolland and a World at War. Evanston, Ill.: Northwestern University Press, I956, f.m.

26ro. Steinhauer, Harry. "Literary Survey of Republican Germany," $Q R, C I$ (Oct., I934), 315-326. 
26ri. Taylor, Edmund. The Fall of the Dynasties. The Collapse of the Old Order. 1905-1922. New York: Doubleday, 1963, pp. 18, 30, 393, 395, 396.

26r 2. Teller, Gertrude E. "Virata or the Eyes of the Undying Brother and Stefan Zweig's Thought," GermR, XXVII : I (I952), 3I-40.

26r3. Teller, Judd L. "Expatriate from the I9th Century," Cmy, XVIII (Dec., I954), 578-58 I.

2614. "The Tragic Case of Stefan Zweig," JSp, May, I943, pp. 9-i I.

26I 5. "Tribute to H. G. Wells at P.E.N. Club," NYT, Sept. I 5, I936, p. 27.

2616. Uhlman, Fred. The Making of an Englishman. London: Victor Gollancz, 1960, p. 217 (Ref. to Zweig's joining the "Free German League of Culture" in the spring of I939).

2617. Van Gelder, R. "The Future of Writing in a World at War. Summary of an Interview with Stefan Zweig," Writers and Writing. New York: Scribner, 1946, pp. 86-89.

26I 8. W.E.G. "Has Intelligence Failed?" CC, LI (Dec. 19, I934), 1626.

2619. "Werfel and Zweig," IM, LXXIII (Oct., 1945), 4I 8-42I.

2620. Werner, Alfred. "Pity for Stefan Zweig," ConW, IX : I4 (Apr. 10, I942), IO-I 3.

2621. Wilson, Ronald A. The Pre-War Biographies of Romain Rolland and Their Place in His Work and the Period. London: Oxford University Press, I939, f.m.

2622. Wilson, W. J. "Modern European History," $A H R$, XLVIII (Oct., I942), 86.

2623. Wittlin, A. "Stefan Zweig," ConR, CLXXV (1949), 62.

2624. Wollf, Charles. Journey into Chaos. Adventures and Experiences in Fifteen Countries of Europe. 2. ed. London-New York-Melbourne: Hutchinson, n.d., p. I9I (Concerning Wollf's last conversation with Zweig (London, Mar., 1938)).

2625. Wunderlich, Eva C., ed. "The Author and His Work," Intro. to the school ed. of Brennendes Geheimnis. New York: Farrer and Rinehart, 1938.

2626. - "Stefan Zweig," BET, Dec. ro, 1932.

2627. Yust, Walter, ed. Ten Eventful Years. 1937-1946. 4. Vol. Chicago-London-Toronto: Encyclopedia Britannica, Inc., 1947, Vol. I, p. 485 ; Vol. II, p. 464; Vol. III, p. 394.

2628. Zarek, Otto. "Recalling a Tragedy: Stefan Zweig," Je A, CVIII : s (Mar. I, I95 I), I. 2629. - "Stefan Zweig. A Jewish Tragedy," Life-Work, pp. I 78-i 9 I.

2630. Zilboorg, Gregory. Sigmund Freud. New York: Charles Scribner's Sons, I95 I, f.m. 2631. - Sigmund Freud. His Exploration of the Mind of Man. New York: Evergreen Books, I 960 , f.m. (Evergreen NE-239).

2632. Zohn, Harry, ed. Liber amicorum Friderike Maria Zweig. In Honor of Her Seventieth Birtbday. Dec. 4, I952. Foreword Dr. George N. Schuster. Stamford, Conn.: Dahl, I 952 (Cf. esp. Zweig Bibliography, pp. Io9-I I I).

2633. - "Stefan Zweig and Contemporary European Literature," GLL, V : 3 (1952), 202-2I 2.

2634. - "Stefan Zweig and Verhaeren. In Memoriam Stefan Zweig," MfDU, XLIII (Apr.-May, 195 I), 199-205.

2635. - "Stefan Zweig as a Collector of Manuscripts," GQ, XXV : 3 (May, I952), I 82-I9I.

2636. - "Stefan Zweig as Literary Mediator," $B A, \mathrm{XXVI}: 2$ (1952), I37-140.

2637. - "Stefan Zweig's Last Years. Some Unpublished Letters," MfDU, XLVIII : 2 (Feb., 1956), 73-77.

2638. Zohn, Harry and J. P. Barricelli. "Music in Stefan Zweig's Last Years. Some Unpublished Letters to Alfred Einstein and Mme. Gisella Selden-Goth," JulR, III (Spring, I 956), 3-I I.

2639. Zohn, Harry and William H. McClain. "Zweig and Rolland. Literary and Personal Relationship," GermR, XXVIII : 4 (I953), 262-28I.

2640. Zweig, Friderike Maria. "Joseph Roth and the Zweigs," $B A$, XVIII (Jan., 1944), 4-8.

2641. - Stefan Zweig. Tr. from the German by Erna McArthur. New York: Thomas Y. Crowell, 1946 (Reprinted London: W. H. Allen, 1948). 
2642. Angelloz, Jean François. Rainer Maria Rilke. L'Evolution spirituelle du Poète. Paris: Paul Hartmann, 1936, pp. $301,376$.

2643. Arcos, René. Romain Rolland. Paris: Mercure de France, r950, p. 7 "A la mémoire de Stefan Zweig," thereafter f.m.

2644. Baudoin, Charles. Hommage à Romain Rolland. Intro. Charles Baudoin. Genève: Editions du Mont Blanc, I945, m. (Article by Zweig, "Reconnaissance," Tr. from the German by Alzir Hella, pp. 86-9r).

2645. Beaulieu, Paul. "Réflexions sur le Suicide de Stefan Zweig," LNR, X (Aug., I942), 579-582.

2646. Béguin, Albert. Essais et Témoignages: Etapes d'une Pensée. Rencontres avec Albert Béguin. Neuchâtel: La Baconnière, 1957, p. 207 (Negative evaluation of Zweig's essays on Hölderlin and Kleist).

2647. Bon, Antoine. "Stefan Zweig et le Brésil," MF, CCCI : 1o09 (Sept. I, 1947), 73-78.

2648. Claudel, Paul and André Gide. Correspondance. 1899-1926. Intro. and Notes Robert Mallet. 5. ed. Paris: Gallimard, r 949, pp. $210,357$.

2649. Delarue, Henri. "Calvin, l'Histoire et Stefan Zweig," MS, Apr. 25, 1948 (Reprinted in Schorer, pp. 5-1 7).

2650. - "Histoire et Théologie font deux," VP, Feb. I8, I948 (Reprinted in Schorer, pp. (9-24).

265r. Delarue, Henri and Paul F. Geisendorf. Calvin, Stefan Zweig et M. Jean Schorer. Genève: Georg and Cie, I949.

2652. Décarreaux, Jean. "Le Message posthume de Stefan Zweig," $L M F$, XII (1948), 424-429.

2653. Demilly, Adolphe. "Sur une Mort," $V d F$, Mar. I 5, i942, p. I.

2654. Geisendorf, Paul F. "Calvin Dictateur ou M. Schorer Historien," LCP, Apr.-May, 1948 (Reprinted in Schorer, pp. 33-64).

2655. Gide, André. Journal 1939-1949. Tr. from the French by Justin O’Brien. New York: Knopf, 1957, p. 3 I8 (Ref. to Zweig's Calvin; Original version Journal 1939-1949. Souvenirs. Paris: Gallimard, 1954).

2656. Goffin, Robert. "Adieu à Stefan Zweig," $V d F$, Mar. I s, 1942, p. 4.

2657. Green, Julien. Journal. Vol. I-IV. Paris: Librairie Plon, I938-195 I, m.

2658. Hella, Alzir. "Avant-Propos," Derniers Messages. Tr. from the German by Alzir Hella. Paris: Attinger, I949, pp. 9-10.

2659. - "Avant-Propos," Vingt-quatre Heures de la Vie d'une Femme. Tr. from the German by Alzir Hella. Paris-Neuchâtel: Attinger, 1929.

2660. Hellens, Frans. "Avant-Propos," Souvenirs sur Emile Verbaeren. Tr. from the German by Hendrik Coopman. Bruxelles: Kryn, I93 I.

266r. Jouve, Pierre Jean. Romain Rolland Vivant. I9I4-1919. Paris: Librairie Paul Ollendorff, $1920, \mathrm{~m}$.

2662. Kadmi-Cohen. Nomades. Essai sur l'Ame Juive. Paris: Librairie Alcan, 1929 (Appraisals of Jewry by Jews and Gentiles).

2663. Kempf, Marcelle. Romain Rolland et l'Allemagne. Paris: Nouvelles Editions Debresse, I 962 , f.m.

2664. Lang, C.-L. Destin de l'Autriche. Paris: Société d'Editions Françaises et Internationales, 1945, pp. 10r, 103 (Zweig as a representative of the Freudian influence in literature).

2665. Lefevre, Frédéric. "Une Heure avec Stefan Zweig. Le Rôle de l'Intellectuel dans la Crise Actuelle," $L N L$, Jan. 23, 1932, p. I.

2666. Levy, Arthur. L'Idéalisme de Romain Rolland. Paris: Nizet, 1946, m.

2667. Mazor, Michel. Le Phénomène Nazi. Documents nazis commentés. Intro. Rémy Rouvre. Paris: Editions du Centre, 1957, pp. 163-173.

2668. Muret, Maurice. Le Désarroi de l'Esprit allemand. Lyon: Edition la plus grande France, I937, Pp. I I 2-I 49 . 
2669. - “M. Stefan Zweig," $R P$, 42.a.: V (Oct. 15, 1935), 864-889 (Reprinted in Le Désarroi del'Esprit allemand. Cf. above).

2670. Noth, Ernst Erich (pseud. for Paul Krantz). "Stefan Zweig et la France," PlV, Mar. 7, 1942, p. 6.

2671. Petijean, Armand. "Grands Hommes," Ven, IV : I 2 I (Feb. 25, I938), 4-5.

2672. Rilke, Rainer Maria and André Gide. Correspondance. I909-1926. Intro. and Commentaries Renée Lang. Paris: Corrêa, I95 2, f.m.

2673. Rolland, Romain. Cabiers Romain Rolland (Vol. II. Louis Gillet-Romain Rolland). Paris: Albin Michel, 1950, p. 267; (Vol. III. Romain Rolland-Richard Strauss). Paris: Albin Michel, I95I, pp. II, 172, 175; (Vol. XI. Lettres de Romain Rolland à Sofia Bertolini Guerrieri-Gonzaga). Paris: Albin Michel, 1960, pp. I67, 172, 230, 232, 340.

2674. - Journal des Années de Guerre. 1914-1919. Paris: Albin Michel, 1952 (Zweig is mentioned on some roo pages, cf. esp. pp. 348-350, I35 I-I362, I 473-1476).

2675. - "Préface," Amok. Tr. from the German by Alzir Hella and Olivier Bournac. Paris: Grasset, 1939.

2676. - "Vox Clamantis... 'Jeremias', Poème dramatique de Stefan Zweig," Les Précurseurs. Paris: Editions de l'Humanité, 1919, pp. I27-145 (This article originally appeared in Coe, Nov. 20, 1917).

2677. Romains, Jules. "Derniers Mois et dernières Lettres de Stefan Zweig," RP, 62.a.: 2 (Feb., 1955), 3-23.

2678. - "Souvenirs de mon Amitié avec Stefan Zweig," Lecture in the Französischer Institut (Wien), June I 3, I958 (Cf. Blätter, 3 (Oct., I958), 5).

2679. Stefan Zweig, Grand Européen. New York: N.Y. Editions de la Maison française, I 94I.

2680. - "Stefan Zweig, grand Européen," Saints de notre Calendrier. Paris: Flammarion, 1952, pp. I59-194.

2681. Schorer, Jean. "Calvin cet Inconnu," EeL, July, I947.

2682. - Jean Calvin et sa Dictature, d'après des Historiens anciens et modernes. Genève: Grivet, r948, f.m.

2683. Stern, Leopold. "Sur le Suicide de Stefan Zweig," Car, Aug. I9, I947.

2684. Valentin, Antonina. "Stefan Zweig," E, 25.a.: 22 (Oct., 1947), 48-67.

GERMAN

2685. Adelt, Leonhard. "Werk und Leben Stefan Zweigs," NFP, Aug. I 2, 1928, pp.27-28. 2686. Ahl, Herbert. "Stefan Zweig," Literarische Portraits. München-Wien: LangenMüller, 1962, pp. 235-244 (Also scattered references).

2687. Arendt, Hannah. "Juden in der Welt von gestern. Anlässlich Stefan Zweigs "The World of Yesterday. An Autobiography' (New York: Viking, I943)," Sechs Essays. Heidelberg: Lambert Schneider, I948, pp. I I 2-I 27.

2688. Arens, Hanns. "Dank an Stefan Zweig. Zum sosten Geburtstag," Un (I93I), 9 (Cf. $D L$, XXXIV : 5 (Oct., I93 I-Mar., I932), 277).

2689. - ed. Der grosse Europäer Stefan Zweig. München: Kindler, 1956. (Contents: Articles and Letters:

Hanns Arens, "Stefan Zweig," pp. 9-43

Richard Friedenthal, "Stefan Zweig und der humanitäre Gedanke," pp. 45-7I

Stefan Zweig, "Besuch bei Emile Verhaeren," pp. 72-76

Rainer Maria Rilke, "Briefe an Stefan Zweig," pp. 78-82

Stefan Zweig, "Abschied von Rilke," pp. 83-86

Romain Rolland, "Briefe an Stefan Zweig," pp. 87-91

Stefan Zweig, "Dank an Romain Rolland," pp. 92-95

Oskar Maurus Fontana, "Stefan Zweig und die Jungen," pp. 96-1oI

Hans Carossa, "Uneigennützige Förderung," pp. 102-103

Hans Reisiger, "Freundliches Erinnern," pp. I04-106

Alexander Lernet-Holenia, "Ich wollte, er lebte noch!" pp. I08-109 
Walter Bauer, "Stefan Zweig, Freund der Jugend," pp. I I O-I 27

Stefan Zweig, "Verse in ein Stammbuch," p. I 28

Anton Kippenberg, "Briefe an Stefan Zweig," pp. I 29-I 37

Hanns Arens, "Stefan Zweig und die Musik," pp. I39-I 49

Benarsi Das Chaturvedi, "Bekenntnis zu Stefan Zweig," pp. I50-I 52

Hans Reisiger, "Die Gedichte Stefan Zweigs," pp. I 53 -1 56

Stefan Zweig, "Ballade von einem Traum," pp. r $57-162$

Hermann Kesten, "Stefan Zweig, der Freund," pp. I63-179

Hanns Arens, "Der Sammler Stefan Zweig," pp. I80-1 89

Harry Zohn, "Stefan Zweig und Amerika," pp. 190-196

Irmgard Keun, "Stefan Zweig, der Emigrant," pp. I97-199

Klaus Mann, "Er war ein Verzweifelter," pp. 200-202

René Fülöp-Miller, "Erinnerungsblatt für Stefan Zweig," pp. 203-205

Joachim Maass, "Die letzte Begegnung," pp. 206-215

Friedrich Torberg, "Terzinen von der Flucht," pp. 2I 6-2I 7

Ernst Feder, "Stefan Zweigs letzte Tage," pp. 2 I 8-238

Richard Friedenthal, "Epistel an einen toten Freund," pp. 239-24I

Carl Zuckmayer, "Did you know Stefan Zweig?” pp. 242-249

Berthold Viertel, "Abschied von Stefan Zweig," pp. $250-260$

Albrecht Schaeffer, "Stefan Zweig zum Gedächtnis," pp. 26I-268

Franz Werfel, "Stefan Zweigs Tod," pp. 269-282

Friderike M. Zweig, "Antworten," pp. 283-294

Jules Romains, "Stefan Zweig, ein grosser Europäer," pp. 295-345

Richard Friedenthal, "Stefan Zweigs 'Montaigne"," pp. 347-350

Stefan Zweig, "Montaigne," pp. 35 I-368

Thomas Mann, "Stefan Zweig zum zehnten Todestag 1952," pp. 370-373

Contents: Facsimiles

Gedicht von Felix Braun, p. 27

Stefan Zweigs Abschiedsbrief, p. 44

Emile Verhaeren, Holzschnitt von Frans Masereel, p. 77

Notenblatt von Romain Rolland, p. 95

Brief Stefan Zweigs an Robert Faesi, p. 107

Bruno Walter, Gedenkblatt für Stefan Zweig, p. 138

Beethoven Noten, p. 140

Richard Strauss, Widmungsblatt für Stefan Zweig, p. 149

Karte von Frans Masereel, p. 176

Stefan Zweigs Brief-Signet, p. 202

Brief von Stefan Zweig an Ernst Feder, p. 227

Frans Masereel, Gedenkblatt für Stefan Zweig, p. 32 I

Gedenkblatt von Albert Schweitzer, p. 346

Contents: Pictures

Stefan Zweig, opp. p. 3

Stefan Zweig, 26 Jahre alt, opp. p. 32

Aufgang zum Hause Zweigs in Salzburg, opp. p. 33

Romain Rolland und Stefan Zweig, opp. p. 48

Stefan Zweig und Anton Kippenberg, opp. p. 49

Romain Rolland, opp. p. 80

Toscanini, Walter, Zweig, opp. p. 8I

Brief von Romain Rolland, opp. p. 96

Rainer Maria Rilke, opp. p. I 28

Anton Kippenberg, opp. p. I 29

Karte von Zweig, Rilke, Verhaeren, opp. p. I 44

Einbandzeichnung zu den Früben Kränzen, opp. p. 145

Korrekturblatt aus Castellio gegen Calvin, opp. p. 208

Manuskriptseite aus Werfels Rede, opp. p. 272

Manuskriptseite aus Castellio gegen Calvin, opp. p. 304 
Stefan Zweig, letzte Aufnahme, opp. p. 336

Stefan Zweig, letztes Gedicht, opp. p. 337

Stefan Zweigs Haus in Petropolis, opp. p. 352

Die Grabstätte in Petropolis, opp. p. 353).

2690. - "Ich besuche einen berühmten Dichter," KNN, Mar. 29, I93 I (Cf. $D L$, XXXIII : 9 (Apr.-Sept., I93 I), 508).

269r. - "Die letzten Jahre Stefan Zweigs," Wst, XXV (1956), i 5-19.

2692. - "Romain Rolland und Stefan Zweig," DBei, III : 5 (I949), 409-4I 5 (Contains two letters of Rolland to Zweig).

2693. - "Der Sammler Stefan Zweig," $D N Z g$, I 2 I (1949) (Reprinted in Imp, XI (I953), 199-205; NDH, 5 (1954), 394; WidZ, ro (Oct., 1961), 36-40).

2694. - "Stefan Zweig," Br, XVII (I930), 447-449 (Cf. variations of this essay in DeuR, LXXVII : 8 (Aug., 195I), 712-717; DL, XXX : 9 (I927-1928), 528529; KHZ, May 19, I930; Leben-Werk, pp. 7-I I 8; $S N T$, I 77 (1928); Wst, July 21, 1952, pp. 49-52; Europäer, pp. 9-43).

2695. - Stefan Zweig. Der Mensch im Werk. Wien: Krystall, 193 I.

2696. - ed. Stefan Zweig. Sein Leben - sein Werk. Esslingen: Bechtle, I949 (Contents: Articles and Letters:

Hanns Arens, "Stefan Zweig," pp. 7-I I9

Stefan Zweig, "Stammbuchverse," p. 70

Camoes-Zweig, "Gedicht," p. 89

Carl Zuckmayer, "Did you know Stefan Zweig?" pp. I 2 I-I 26

Hans Reisiger, "Freundliches Erinnern an Stefan Zweig," pp. I 28-I 29

Walter Bauer, "Stefan Zweig der Europäer," pp. I 30-I 45

Rainer Maria Rilke, "Zwei Briefe an Stefan Zweig," pp. I46-148

Alexander Lernet-Holenia, "Brief," p. 149

Ernst Feder, "Stefan Zweigs letzte Tage," pp. I50-165

René Fülöp-Miller, "Erinnerungsblatt für Stefan Zweig," pp. I67-I68

Romain Rolland, "Zwei Briefe an Stefan Zweig," pp. 169-172

Richard Friedenthal, "Epistel an einen toten Freund," pp. 173-1 76

Franz Werfel, "Stefan Zweigs Tod," pp. 177-1 87

Benarsi Das Chaturvedi, "Bekenntnis zu Stefan Zweig," pp. 188-189

Berthold Viertel, "Abschied von Stefan Zweig," pp. I9r-199

Richard Friedenthal, "Stefan Zweig und der humanitäre Gedanke," pp. 200-2 I 9 Contents: Pictures and Facsimiles:

Stefan Zweig, opp. p. 3

Romain Rolland und Stefan Zweig, opp. p. I4

Aufgang zum Hause Stefan Zweigs, opp. p. Is

Korrekturblatt Stefan Zweigs, opp. p. 48

Toscanini, Walter, Zweig, opp. p. 96

Anton Kippenberg und Stefan Zweig, opp. p. 97

Romain Rolland, opp. p. II 3

Manuskriptblatt von Stefan Zweig, opp. p. I44

Manuskriptblatt aus Franz Werfels Rede, opp. p. 176

Brief von Romain Rolland, opp. pp. 192-193

Stefan Zweigs Haus in Petropolis, opp. p. 208

Stefan Zweigs Grab in Petropolis, opp. p. 209

Stefan Zweigs Brief-Signet, p. 26

Brief Stefan Zweigs an Robert Faesi, p. 37

Emile Verhaeren, Holzschnitt von Frans Masereel, p. 55

Beethoven Noten, p. 66

Richard Strauss, Widmungsblatt für Stefan Zweig, p. 80

Karte von Frans Masereel, p. I I 2

Stefan Zweigs Abschiedsbrief, p. II7

Frans Masereel, Gedenkblatt für Stefan Zweig, p. I 27

Brief von Stefan Zweig an Ernst Feder, p. I57 
Bruno Walter, Gedenkblatt für Stefan Zweig, p. 166

Franz Werfels Namenszug, p. 187).

2697. - "Stefan Zweig und die Musik," NMz, 4.J.: 9 (1950), 249-253 (Reprinted in $A M o z, 5 . J$. (1958), 2-5; $D T p$, 23 (1952); $M u$, 6.J.: 2 (1952), 59-62 ; Vbii, 3.J.: 5 (1952), 80-82; Europäer, pp. I 39-1 49 .

2698. Bab, Julius. Die Chronik des deutschen Dramas, Vol. I-IV. Berlin: Oesterheld, I922, Vol. III, pp. 4I-45 (Discussion of Zweig's Haus am Meer); Vol. IV, pp. I4I-I 42 (Discussion of Zweig's Jeremias).

2699. - "Emile Verhaeren," NR, XXIII : 2 (July, 191 2), ro20-1028.

2700. - Richard Debmel. Die Geschichte eines Lebenswerkes. Leipzig: Haessel, 1926, p. 344.

2701. Belázs, Béla. Der Geist des Films. Halle, Saale: Wilhelm Knapp, 1930, pp. 67, 74 (Discussion of the film "Narkose" (I929), "based on a script by Balázs after a story by Stefan Zweig" (his "Brief einer Unbekannten"?)).

2702. Basil, Otto. "Stefan Zweig und Ben Jonson," Blätter, 4/5 (Apr., 1959), 8.

2703. Bauer, Arnold. Stefan Zweig. Berlin-Dahlem: Colloquium, I96I (In the series "Köpfe des XX. Jahrhunderts," Vol. XXI).

2704. Bauer, Walter. "Die Beschwörung Europas. Ein Blatt des Gedenkens für Stefan Zweig," Erz, I.J.: 8 (1947), Is (Reprinted in März, I.J.: 2 (I949), 49-5 I).

2705. - "Erinnerungen an einen Europäer," $K D$, 5.J. (195I), 1056-1060.

2706. "Der Europäer Stefan Zweig," Leben-Werk, pp.r 30-I 45.

2707. - "Stefan Zweig, Freund der Jugend," Europäer, pp. I I O-I 27.

2708. Baum, Vicki. Es war alles ganz anders. Berlin-Frankfurt am Main-Wien: Ullstein, I962, p. 246.

2709. Becker, Martha. "Emile Verhaeren. Nachträgliches zu seiner Würdigung," GRM, $X: 5 / 6$ (May-June, I922), I72-180.

2710. Beierle, A. "Stefan Zweig, Dichter und Dulder," $D Z$, 2.J.: 22 (1947), 3.

27 I. Benaroya, Mois. Stefan Zweig. Sofia: Hyperion, 1929.

2712. Berendsohn, Walter A. Die bumanistische Front. Einfübrung in die deutsche EmigrantenLiteratur. Erster Teil von 1933 bis zum Kriegsausbruch 1939. Zürich: Europa, I946, f.m.

2713. - "Warum ging Stefan Zweig in den Tod?" JüGbl, 4.J.: Io (1949), 6.

2714. Berger, Fritz. "Stefan Zweig als Deuter und Mahner," IF, IV : 5 (May, 1962), I6-I 7.

27I 5. Bertrand, P. "Das Problem der Toleranz. Castellio gegen Calvin oder Fanatismus," DSa, 2.J. (1947), 598-600.

2716. Bibliographie der Werke von Stefan Zweig, dem Dicbter zum sosten Geburtstag dargebracbt vom Insel-Verlag. Ed. Fritz Adolf Hünich and Erwin Rieger. Leipzig: Insel, I93 I (Reprinted in In, XIII : I (Christmas, I93 I), 17-30).

2717. Bieber, Hugo. "Deutscher und Jude," DLE, 24.J.: 6 (Dec. I5, I921), 31 3-321.

2718. Bierotte, Wolf. "Drei Dichter ihres Lebens'. Anmerkungen über die Sprache," DSL, XXIX : II (Nov., I928), 520-523.

2719. Bin Gorion, Emanuel. "Die Augen des ewigen Bruders," Ceterum, pp. 51-63 (Criticism à la stultitia).

2720. - 'Stefan Zweigs Novellen ('Brennendes Geheimnis,' 'Brief einer Unbekannten,' 'Verwirrung der Gefühle,' 'Untergang eines Herzens')," Ceterum, pp. 64-76 (Ludicrous criticism).

272 r. - “Über Stefan Zweigs Legendenstil," NZZ, Lit. Beil., No. I 264 (Cf. also DL, XXXI : I 2 (1928-1929), 714 ; Positively negative).

2722. Böttcher, Kurt. "Humanist auf verlorener Bastion," NDL, 4.J.: II (I956), 83-92. 2723. - "Der Lebensroman des unerschöpflichen Balzac," NDL, 7.J.: I I (I959), 45-46. 2724. Braun, Felix. "Ein Besuch bei Stefan Zweig," Spiegelungen, pp. 42-44 (Cf. Das Licht der Welt below).

2725. - "Brief an Stefan Zweig," Briefe in das Jenseits. Salzburg: O. Müller, I 952 (Reprinted in Blätter, 8/10 (Oct., I 960), 12).

2726. - "Erinnerungen an Salzburg," DeuR, LXXXIV (Dec., I958), I I 56-i I62.

2727. - "Das fremde Leben. Stefan Zweig" (poem), Das neue Leben. Neue Gedichte. Berlin: Reiss, 1912 (Reprinted in Blätter, 8/10 (Oct., 1960), 13). 
2728. - Das Licht der Welt. Geschichte eines Versuches, als Dichter zu leben. Wien: Herder, I949, f.m. (Cf. esp., pp. 457-46r ; Reprinted in Spiegelungen, pp. 42-44).

2729. - Das musische Land. Versucbe über Österreichs Landschaft und Dichtung. Innsbruck: Österreichische Verlagsanstalt, 1952, pp. I91-206.

2730. - "Stefan Zweig," Zeitgefährten. München: Nymphenburger Verlagshandlung, I963, pp. 59-77.

2731. - "Stefan Zweig," Stefan Zweig. Sonderpublikation der Funktionärblätter (London), Nov., 1943, pp. 2-6.

2732. - "Stefan Zweig und sein Dämon," $D P r, 943$ (I95 I).

2733. Braun, Robert. "Erinnerungen an Stefan Zweig," WieZ, 275 (1956) (Reprinted in Spiegelungen, pp. 78-85; BuL, 10 (Oct., 1962), I-3).

2734. Brod, Max. "Stefan Zweig zum Gedenken," Spiegelungen, p. 4I.

2735. - Streitbares Leben. Autobiographie. München: Kindler, 1960, f.m.

2736. Burchardt, Hansjürgen. "Die Autographen-Auktion bei Stargard vom I4ten November 1958", $B d B h$, I4.J.: I0I (I958), I 708-1 71 I.

2737. Burschell, Friedrich. "Stefan Zweigs Novellen," FZ, Lit. Beil., No. 64 (Cf. also DL, XXXVII : 8 (Apr.-Sept., I93 I), 449).

2738. Cahn, Alfredo. Stefan Zweig. Ein Nachruf. Buenos Aires: n.p., 1942.

2739. See Item 2539a.

2740. Chaturvedi, Benarsi Das. "Bekenntnis zu Stefan Zweig," Leben-Werk, pp. I88-189 (Reprinted in Europäer, pp. I 50-152).

274I. Csokor, Franz Theodor. "Ihr Leben. Für Stefan Zweig" (poem), Die Gewalten. Ein Band Balladen. Berlin: Juncker, I 9 I 2 (Reprinted in Blätter, 8/10 (Oct. 1960), I I).

2742. - "Stefan Zweig, der Freund," Spiegelungen, pp. I09-I IO (A photocopy of part of this essay is reprinted in Blätter, 8/10 (Oct., I960), 10).

2743. Dehorn, W. "Psychoanalyse und neuere Dichtung," GermR, VII (I932), 245-262, 330-358 (Cf. esp. pp. 330-339).

2744. Deissinger, Hans. "Kleine Erinnerung an Stefan Zweig," Blätter, 13/14 (Apr., I 962), 2-4.

2745. Dolbin, Benedikt F. Österreichische Profile. Ed. Oskar Maurus Fontana. München: Albert Langen-Georg Müller, 1959, p. 22 (Caricature sketch of Zweig).

2746. Drews, Richard and Alfred Kantorowitz, eds. Verboten und Verbannt. Deutsche Literatur - I 2 Jabre unterdrückt. Berlin-München: Ullstein-Kindler, I947, pp. $5,47,182,185$.

2747. Ebermayer, Erich. "Letzte Stunden mit Stefan Zweig," Pris, 19/20 (1948), 40.

2748. "Echo der Zeitungen zum sosten Geburtstag Stefan Zweigs," DL, XXXIV (1932), 267.

2749. Ecker, Karl. "Die Sammlung Stefan Zweigs in der Österreichischen Nationalbibliothek, Wien," Festschrift zum 25 jäbrigen Dienstjubiläum des General Direktors Josef Bick. Ed. Jasek Stummvoll. Wien: 1948, pp. 321-330.

2750. Eckhardt, Eduard. "Deutsche Bearbeitung älterer englischer Dramen," ES, LXVIII (1933), 195-208.

2751. Ehrentreich, Alfred. "Stefan Zweig und Henri Guilbeaux," $N p h Z$, 4.J. (I952), 263-265.

2752. Eloesser, Arthur. "Verhaerens Drama 'Das Kloster'," DLE, XIII : 3 (Nov. I, 1910), 208-209 (Discussion of the German premiere of Verhaeren's drama in Zweig's translation).

2753. Elster, Hanns Martin. Deutsche Dichterbandscbriften. Vol. XIII. Intro. Hanns Martin Elster. Dresden: Lehmann, I922 (Reproduction of the manuscript of Zweig's "Brief einer Unbekannten").

2754. - "Stefan Zweig," $B B B$, II : I38/139 (1923), I-3; II : 140 (1923), 2-3; II : I4I (1923), 2 (Cf. also $D L E, 25$.J.: 9/10 (Feb. 1, 1923), 532).

2755. Erkelenz, K. H. "Stefan Zweig," KZ, 650 (I93I) (Cf. also DL, XXXIV : s (Oct., I931 - Mar., 1932), 267).

2756. Faesi, Robert. "Stefan Zweig zu seinem sosten Geburtstag," NZZ, Nov. 29, 193 I (Cf. also $D L$, XXXIV : 5 (Oct., I93 I - Mar., 1932), 267). 
2757. Faktor, Emil. "Stefan Zweig zum sosten Geburtstag," $B B C, 553$ (I93 I) (Cf. also $D L$, XXXIV : s (Oct., I931 - Mar., 1932), 267).

2758. Feder, Ernst. "Die letzten Tage Stefan Zweigs," Begegnungen. Die Grossen der Welt im Zwiegespräch. Esslingen: Bechtle, 1950, pp. 197-210 (Reprinted in LebenWerk, pp. I 50-165; Europäer, pp. 218-238).

2759. Fedin, Konstantin. "Die Tragödie Stefan Zweigs," $A$, XII (1956), 959-96r.

2760. Fehse, Willi. "Erinnerungen an Stefan Zweig," $A u$, II : 2 (1946), I 230-1 233 (Reprinted in $L B$, II : 14 (Dec., 1947), I0-13).

276r. - "Das höchste Gut dieser Erde..." Spiegelungen, pp. 62-67.

2762. - "Stefan Zweig, Meister der Stimmungskunst," DSta, 2.J.: 25 (1947).

2763. Fitzbauer, Erich. "Einleitung," Durch Zeiten und Welten. Ed. Erich Fitzbauer. Graz-Wien: Stiasny, 1961, pp. 5-23 (Stiasny Bücherei, No. 79).

2764. - "Gedanken zu Klaus Manns 'Wendepunkt' mit dem Versuch einer Konfrontation des Verfassers mit Stefan Zweig. Im roten Todesjahr Klaus Manns," Blätter, 6/7 (Oct., 1959), I I-1 3 .

2765. - "Die 'Schachnovelle' als Film," Blätter, 8/10 (Oct., 1960), I7-19.

2766. - "Stefan Zweig als Dramatiker," DeuR, 86.J. (1960), 434-437.

2767. - Stefan Zweig (1881-1942). Ausstellung in der Residenz in Salzburg. 15. Juli-31. Aug., 196r. Ed. of the Catalogue Erich Fitzbauer. Salzburg: Salzburger Residenzgalerie, Salzburger Kulturvereinigung, 196r.

2768. - "Stefan Zweig, dichterisch-menschlicher Einklang," Spiegelungen, pp. 5-I 8.

2769. - ed. Stefan Zweig. Spiegelungen einer scböpferischen Persönlicbkeit. Wien: Bergland, I959.

\section{Contenst:}

Erich Fitzbauer, "Stefan Zweig, dichterisch-menschlicher Einklang," pp. 5-1 8.

Stefan Zweig, "Ein Brief," pp. I9-2I

Stefan Zweig, "Fünf Jugendgedichte," pp. 22-23

Harry Zohn, "Stefan Zweig und Emile Verhaeren," pp. 24-3 I

Stefan Zweig, "Fünf Nachdichtungen," pp. 32-35

Victor Fleischel, "Erinnerung an Stefan Zweig," pp. 36-40

Max Brod, "Stefan Zweig zum Gedenken," p. 41

Felix Braun, "Ein Besuch bei Stefan Zweig," pp. 42-43

Stefan Zweig, "Die Autographensammlung als Kunstwerk,", pp. 44-5 I

Joseph Gregor, "Stefan Zweig - Dank für zwei glückliche Jahrzehnte," pp. 52-55

Adelbert Muhr, "Bei Stefan Zweig auf dem Höhepunkt seines Lebens," pp. 56-6I

Willi Fehse, "Das höchste Gut dieser Erde...,", pp. 62-67

Lee van Dovski, "Mit Stefan Zweig in Paris," pp. 68-73

Stefan Zweig, "Drei Briefe," pp. 74-77

Robert Braun, "Erinnerung an Stefan Zweig," pp. 78-85

Stefan Zweig, "Fünf Briefe," pp. 86-93

Siegfried Freiberg, "Stefan Zweig und Brasilien," pp. 94-100

Heinrich Eduard Jacob, “Aus den Polizeiakten von Petropolis," pp. IoI-106

Ernst Waldinger, "Stefan Zweigs 'Feuilletonismus," pp. 107-108

Franz Theodor Csokor, "Stefan Zweig - der Freund," pp. I09-I Io

Ludwig Ullmann, "Stefan Zweig, der grosse Europäer," pp. I I I-I I6 Plates:

Stefan Zweig, Bronzebüste von Gustinus Ambrosi, opp. p. 3

Faksimile einer Karte Stefan Zweigs an Karl Klammer, p. 2I

Einbandzeichnung zu Zweigs erstem Buch, von Hugo Steiner-Prag, opp. p. 48

Teilansicht von Stefan Zweigs Haus am Kapuzinerberg in Salzburg, opp. p. 64

2770. - "Stefan Zweig und Salzburg," Blätter, 2 (July, 1958), 4-5.

2771. Fleischel, Victor. "Erinnerung an Stefan Zweig," Spiegelungen, pp. 36-40.

2772. - "Stefan Zweig," Stefan Zweig. Sonderpublikation der Funktionärblätter (London), Nov., 1943. 
2773. Foltin, Lore B. "Stefan Zweig," Intro. to "Die Legende der dritten Taube," Aus Nab und Fern. Boston: Houghton-Mifflin, 1950, pp. I 5 2-154 (School ed.).

2774. Freiberg, Siegfried. "Stefan Zweig und Brasilien," Spiegelungen, pp. 94-100.

2775. Freud, Sigmund. Briefe. I 873-1939. Sel. and ed. Ernst L. Freud. Frankfurt am Main: Fischer, I960, f.m.

2776. Friedenthal, Richard. "Epistel an einen toten Freund," Leben-Werk, pp. 173-176 (Reprinted in Europäer, pp. 239-24I).

2777. - "Nachwort," Angst. Novelle. Stuttgart: Reclam, 1954, pp. 69-74.

2778. - "Nachwort des Herausgebers," Balzac. Stockholm: Bermann-Fischer, 1946, pp. 569-574.

2779. - "Nachwort des Herausgebers," Zeit und Welt. Stockholm: Bermann-Fischer, 1944, pp. 369-373.

2780. - Stefan Zweig und der bumanitäre Gedanke. Esslingen: Bechtle, 1948 (Reprinted in Leben-Werk, pp. 200-219; Europäer, pp. 45-71).

2781. - "Stefan Zweigs literarischer Nachlass," Stefan Zweig. Sonderpublikation der Funktionärblätter (London), Nov., 1943, pp. I5-16.

2782. Friedrichs, Ernst. "Stefan Zweig zu seinem 45sten Geburtstag, "GS, No. 329 (Cf. also $D L$, XXIX : 5 (1926-1927), 279).

2783. Frischauer, Paul. "Stefan Zweig zum sosten Geburtstag," BT, No. 559 (Cf. also $D L$, XXXIV : s (Oct., I931 - Mar., 1932), 267).

2784. Fülöp-Miller, René. "Erinnerungsblatt für Stefan Zweig," Leben-Werk, pp. 167-168 (Reprinted in Europäer, pp. 203-205).

2785. "Gedenkabend zu Zweigs 8ostem Geburtstag in Wien," WieZ, Nov. 30, 1961 (Reprinted in Blätter, 13/14 (Apr., 1962), 9).

2786. Götzfried, Hansleo. Romain Rolland. 2. ed. Stuttgart: Engelhorns Nachf., I93 I, f.m.

2787. Green, Julien. Tagebiicher. Vol. I (1928-1945), Vol. II (1946-1950). Tr. from the French by Hanns Winter. Wien: Herold, 1952, f.m.

2788. Gregor, Joseph. "An Stefan Zweig - Eine Aussprache über Wertungen und Ziele, zum 28sten November, I93 I," In, XIII : I (Christmas, I93 I), I-IO.

2789. - "Stefan Zweig - Dank für zwei glückliche Jahrzehnte," Spiegelungen, pp. 52-55.

2790. Grisson, Alexandra Carola."Stefan Zweig," Postscript to the Reclam ed. of "Angst". Stuttgart: Reclam, I947, pp. 77-80.

2791. Grossberg, Mimi. "Gedenkabend für Stefan Zweig," Lecture given in the Austrian Forum (New York), Feb. 6, 1963 by Mme. Grossberg. Copyright by Mimi Grossberg. The following selections were read by Mr. Theodor Goetz:

I. "Stefan Zweigs letzter Abend" (taken from Ernst Feder's "Stefan Zweigs letzte Tage", cf. above).

2. One scene from Jeremias (highly abridged).

3. Excerpts from Zweig's "Besuch bei Emile Verhaeren".

4. Verhaeren's "Nun die Flimmer von Schnee auf unser Dach" in Zweig's translation.

5. A passage from Camoens' Lusiads in Zweig's translation.

6. The "Glockenmonolog" from Die schweigsame Frau.

7. Zweig's "Ballade von einem Traum" (abridged).

2792. Günther, Herbert. "Stefan Zweig und Frankreich," DGT, 4.J.: I (1949), 83-88.

2793. Günther, Werner. Weltinnenraum. Die Dichtung Rainer Maria Rilkes. 2. ed. Berlin: Erich Schmidt, 1952, p. 33I (Reference to Zweig's "Abschied von Rilke").

2794. Hellwig, Hans. Stefan Zweig. Ein Lebensbild. Lübeck: Wildner, 1948.

2795. Herrmann, W. "Arnold und Stefan Zweig: der gestürzte Olymp," $B B Z$, June 25, 1933.

2796. Herrmann-Neisse, Leni. "Erinnerung an Stefan Zweig," Blätter, I I/1 2 (Oct., 1961), Is.

2797. Hinterberger, Heinrich, ed. Repräsentative Original-Handschriften. Eine berïhmte Autographen-Sammlung. I. Teil. Katalog IX. Wien: Heinrich Hinterberger, n.d. (Cf. also Katalog XX: Interessante Autograpben aus zwei bekannten Sammlungen). 
2798. Hirth, Friedrich. "Deutsche Literatur in Frankreich," DL, XXX : 7 (1927-1928), 385-387.

2799. Hofmann, Else. "Besuch bei Stefan Zweig," FuT, July, I949, pp. 9-10.

2800. Holde, Artur. "Gedenkfeier des 'Aufbau' für Stefan Zweig," $A u(N Y)$, VIII : Iо (Mar. 6, 1942), 19-20.

2801. Homeyer, Fritz. Ein Leben für das Buch. Erinnerungen. Aschaffenburg: Pottloch, 1961, f.m.

2802. Hünich, Fritz Adolf. "Stefan Zweig zum sosten Geburtstag," $B d$, No. 555 (Cf. also $D L$, XXXIV : s (Oct., I93 I - Mar., I932), 267).

2803. Hünich, Fritz Adolf and Erwin Rieger, eds. Bibliographie der Werke von Stefan Zweig. Leipzig: Insel, I93 I (Reprinted in In, XIII (193 I), 17-30).

2804. Huppert, Hugo. "Der das Glück als Schuld erachtete... Zum 75 sten Wiederkehr von Stefan Zweigs Geburtstag," Wbï, XI (1956), I 534-I 540.

2805. "In Memoriam Stefan Zweigs," DWe, 5.J.: 8 (1950), 4.

2806. Ippisch, Gottfried. "Nachwort," Angst. Novelle. Wien: Humboldt, 947 (Kleine Humboldt Bibliothek, No. I04).

2807. Jacob, Heinrich Eduard. "Aus den Polizeiakten von Petropolis," Spiegelungen, pp. pp. Ior-ro6.

2808. - "Stefan Zweig - Zehn Jahre nach seinem Tode," NIW, 3.J.: 5 (I952), 8.

2809. Jacob, P. Walter. "Stefan Zweig als Dramatiker," Rampenlicht. Köpfe der Literatur und des Theaters. Buenos Aires: Editorial Cosmopolita, 1945, pp. 75-82.

28ro. Jacobi, Johannes and U. Seelmann-Eggebert. "Visionen der Entrechtung: Stefan Zweig und Georg Kaiser," $D Z$ e, 6.J.: 45 (195r), 3.

28I I. "Jahre der Reife Stefan Zweigs," $A W J D$, 5.J.: 40 (I950), 6.

28 1 2. J.D.L. "Stefan Zweig, ein gewissenhafter Dichter," $B d$, No. Io8 (Quote from same $D L, \mathrm{XXXV}: 8$ (1932-1933), 463).

2813. Kayser, Rudolf. "Max Brod, 'Streitbares Leben. Autobiographie'," Blätter, I I/ I 2 (Oct., I96I), 8.

2814. Kesten, Hermann. Dichter im Café. Wien-München-Basel: Kurt Desch, r959, f.m.

281 5. - Der Geist der Unrube. Literarische Streifzïge. Köln-Berlin: Kiepenhauer-Witsch, I959, f.m.

2816. - "Stefan Zweig," Meine Freunde die Poeten. Wien-München: Donau Verlag, 1953, pp. I I 4-1 26.

2817. - "Stefan Zweig," $W u W, \mathrm{~V}:$ I3 (Mar., 1950), 97-99.

2818. - "Stefan Zweig - Erinnerungen an den Freund," DMo, 5.J.: 50(1952), 225-228.

2819. Knevels, Wilhelm. "Stefan Zweig," GdG, LXVI : 4 (I930), I45-I 52.

2820. Kraus, Hans Johan. "Brasilien ehrt Stefan Zweig," NV zt, Mar. 14, 1942, p. 5.

2821. Kraus, Karl. "Pretiosen," DF, XXVIII : 726/729 (May, 1926), 55-56.

2822. - "Umsturz in der 'Neuen Freien Presse'," $D F$, XXVIII : 75 I/756 (Feb., 1927), 125-1 26.

2823. Kubin, Alfred. Alfred Kubin - Leben-Werk-Wirkung. Ed. Paul Raabe. Hamburg: Rowohlt, 1957, pp. 33, 53-54, 98 (Letters from Zweig to Kubin and reference to Kubin's illustrations for the I93 I edition of Jeremias, an edition that never appeared. These drawings are reprinted in Blätter, 8/10 (Oct., 1960), 26-27).

2824. Kuhn-Foelitz, August. "Begegnung in Salzburg. Gespräch mit Stefan Zweig," $M A Z$, s (r949).

2825. Kumming, Eugen. "Stefan Zweig - Weltbürger ohne Heimat," $A f d W$, 2.J. II: Io (1950), 5-I 2.

2826. Landmann, Georg Peter. Stefan George und sein Kreis. Eine Bibliographie. Hamburg: Ernst Hauswedell, i960, Nos. I78, $410 a, 574$.

2827. Lang, Wolf R. "Nachwort," Menscben. Novellen. Stuttgart-Zürich-Salzburg: Europäischer Buchklub, I962, pp. 405-408.

2828. - "Stefan Zweig. Umrisse seines Lebens," BuL, II (Nov., I962), I-3.

2829. Lange, Herbert. "Ein Bildnis Stefan Zweigs," OöN, Oct. 27, I947, p. 3.

2830. Lederer, Max. "Stefan Zweig," Rad, VI (I929), 2 (Cf. DL, XXXII : 3 (I929-1930), I58; DL, XXXII : 4(1929-I930), 226). 
2831. Lehrman, Charles. "Theodor Wolff und Stefan Zweig," $A u(N Y)$, May 13, 1949, p. 32.

2832. Lenteritz, G. "Das unerloschene Leuchten, Stefan Zweig," BdBh, II4.J. (I947), 17-25.

2833. Lerch, Eugen. Romain Rolland. München: Max Hueber, I 926, f.m.

2834. Lernet-Holenia, Alexander. "Aus einem Brief von Alexander Lernet-Holenia," Leben-Werk, p. 149 (Reprinted as "Ich wollte, er lebte uns nochl" in Europäer, pp. 108-109).

2835. L. H. "Stefan Zweig zum 65sten Geburtstag," ST, Nov. 29, 1946, p. 8.

2836. Lie, Robert. "Amerika und die österreichische Literatur," WidZ, 4 (Apr., r96r), $42-45$.

2837. Liess, Andreas. Joseph Marx. Leben und Werk. Graz: Sterische Verlagsanstalt, I 943 (Reference to Zweig's poem "Ein Drängen ist in meinem Herzen" (from Silberne Saiten) which Marx set to music in 1909).

2838. Lilge, Herbert. "Der Untergang eines Herzens. Nachruf auf Stefan Zweig," Bng, 6.J. (195 I), 3-9.

2839. Lissauer, Ernst. "Zu Stefan Zweigs sostem Geburtstag," CVZ, X : 47 (Nov. 20, I931), 537-538.

2840. Lothar, Rudolf. "Aus dem Engeren. Literaturbilder aus deutschen Einzelgauen," $D L E$, IV : 8 (Jan., I902), 509-524 (Cf. esp. p. 523).

284I. Lucka, Emil. "Stefan Zweig," DLE, XVII : 4(Nov. I5, I914), 193-I99.

2842. - "Stefan Zweig, der Menschengestalter," $D L z$, XX : 6 (1933), 89-93.

2843. Ludwig, Emil. "Stefan Zweig zum sosten Geburtstag," DLW, VII (1931), 47 (Cf. also DL, XXXIV : 5 (Oct., I93 I- Mar., 1932), 277).

2844. Lukács, Georg. "Der Kampf zwischen Liberalismus und Demokratie im Spiegel des historischen Romans der deutschen Antifaschisten," Essays ïber Realismus. Berlin: Aufbau, 1948. pp. 88-i 28 (2. enl. ed. reprinted as Probleme des Realismus. Berlin: Aufbau, 1955. The article about the historical novel appears pp. I 84-210).

2845. Maass, Joachim. "Neues von und über Stefan Zweig," HFbl, June 2, I928 (Cf. also $D L, \mathrm{XXX}:$ II (1927-1928), 654).

2846. Maderno, Alfred. "Stefan Zweig," $R T$, I (n.d.), 44-45.

2847. Mann, Thomas. "Stefan Zweig zum roten Todestag," Altes und Neues. Frankfurt am Main: Fischer, 1953, pp. 263-265.

2848. Marti, Hugo. "Der Europäer Stefan Zweig," $B d$, I I 3 (1933) (Cf. also $D L, \mathrm{XXXV}$ : 8 (1932-1933), 463).

2849. Maurer, Joseph, "Uber Zweigs Aufsatz 'Herbstwinter in Meran'," Blätter, 6/7 (Oct., 1959), 1 8-19.

2850. Marina, Zenta. Dostojewski. Menschengestalter und Gottsucher. 2. ed. Memmingen: Maximilian Dietrich, I960, pp. I 2, 23, 138.

295 I. Menter, L. "Stefan Zweig," $D W e$, 2.J.: 13 (1947), 9.

2852. Meyer-Benfey, Heinrich. "Stefan Zweig," HK, Lit. Beil. Oct. 28, 1923, pp. 505-506 (Cf. also $D L$, XXVI : 3 (Oct., I923 - Mar., 1924), I57).

2853. Michaelis, Edgar. "Rückblick auf Freud," Bng, V : 2 (Mar.-Apr., 1950), 76-83. 2854. "Millionenblutopfer und Ideenforschung. $\mathrm{Zu}$ den Artikeln des A.H. Fried und Stefan Zweig," $N Z Z$, Aug. I I, 1918.

2855. Mistral, Gabriela. "Un gran señor..." Blätter, 4/5 (Apr., 1959), Ir.

2856. Molo, Walter von. "Erinnerungen an Stefan Zweig," Blätter, 3 (Oct., 1958), 4. 2857. Muhr, Adelbert. "Bei Stefan Zweig auf dem Höhepunkt seines Lebens," Spiegelungen, pp. 56-6r.

2858. Mühsam, Paul. "Begegnung mit Stefan Zweig," Blätter, is (Jan., I963), Io.

2859. Müller-Einigen, Hans. Jugend in Wien. Erinnerungen an die scbönste Stadt Europas. Bern: Francke, 1945, f.m.

2860. Musil, Robert. Tagebiicher, Aphorismen, Essays und Reden. Ed. Adolf Frisé. Hamburg: Rowohlt, 1955, f.m. (Vol. II of Musil collected works; in general Musil rejects Zweig as superficial and as an "opportunist"). 
286I. Neumann, Robert. "Abschied von Stefan Zweig," tr. from the English by Stefanie Neumann. Blätter, I I/ I 2 (Oct., I 96I), I-4.

2862. Oetke, Herbert. "Stefan Zweig," $V u V$, 2. ser., 13 (1947), 22-3 I.

2863. Parandowski, Jan. "Erinnerungen an Stefan Zweig," WidZ, i I (Nov., I96r), 40-44.

2864. Pisk, Egon. "Stefan Zweigs Tod," WieZ, 277 (1949).

2865. Poss, A. "Ein Gedankenaustausch mit Jugendlichen über Stefan Zweigs Legende 'Die Augen des ewigen Bruders'," Mon, Lit. Beil. 43.J. (r930), r.

2866. Rainalter, Erwin H. "Nachwort," Angst. Novelle. Leipzig: Reclam, I925, pp. 72-76. 2867. Rauscher, Bert. "Stefan Zweig: Ein Gewissen gegen die Gewalt. Zum I sten Todestag von Stefan Zweig," Brï, I I. J.: 8 (1957), I-3.

2868. Reim, Paul. Probleme und Gestalten der österreichiscben Literatur. London: Verlag Jugend Voran (Junges Österreich), 1945, $\mathrm{m}$.

2869. Reishofer, Karl. "Wie erklären sich grosse Bucherfolge? Eine Analyse der literarischen Wirkung," BuI, V : 203 (June 9, I950), 278I-2782; V : 204 (June 16, I950), 2797-2798.

2870. Reisiger, Hans. "Freundliches Erinnern an Stefan Zweig," Leben-Werk, pp. I 28-1 29 (Reprinted in Europäer, pp. 104-107).

2871. Rieger, Erwin. "Stefan Zweig," Rad, VIII (I93 I), 8 (Cf. also DL, XXXIV : 5 (Oct., I93 I - Mar., I932), 277).

2872. - "Stefan Zweig als Dramatiker," $\mathrm{HFbl,} \mathrm{I5} 2$ (June 2, I928) (Cf. also $D L, \mathrm{XXX}$ : II (1927-1928), 654).

2873. - "Stefan Zweig als Essayist," $K Z$, Apr. 24, 1928 (Cf. $D L, \mathrm{XXX}$ : 9 (1927-1928), 528).

2874. - Stefan Zweig, der Mann und das Werk. Berlin: Spaeth, 1928 (Zweig bibliography, pp. 219-230).

2875. Rieger, Erwin and Fritz Adolf Hünich, eds. Bibliograpbie der Werke von Stefan Zweig. Leipzig: Insel, I93 I (Reprinted in In, XIII (193 I), I 7-30).

2876. Rieger, Harald. "Zwei Suchende: Siddhartha und Virata (Hermann Hesses 'Siddhartha' und Stefan Zweigs 'Die Augen des ewigen Bruders')," Gespr, 4 (Aug., I959), $8 \mathrm{f}$.

2877. Riemerschmid, Werner. "Salzburger Erinnerung. Aus einem Brief," Blätter, 6/7 (Oct., I959), 2.

2878. Rilke, Rainer Maria. Briefe (Vol. I. I 897-1914). Wiesbaden: Insel, I950, pp. I74-I 75.

2879. - Briefe aus den Jabren 1906 bis 1907. Leipzig: Insel, 1930, f.m.

2880. Rilke, Rainer Maria and André Gide. Briefwechsel. 1909-1926. Tr. from the French by Wolfgang A. Peters. Intro. and notes Renée Lang. Stuttgart: Deutsche Verlagsanstalt, I957, f.m. (Also contains a letter from Zweig to Rolland (Dec. 30, I915) and Rolland's response (Jan. 17, I916)).

2881. Rilke, Rainer Maria and Katharina Kippenberg. Briefwecbsel. Wiesbaden: Insel, r 954 , f.m.

2882. Rilke, Rainer Maria and Maria von Thurn und Taxis. Briefwechsel. Vol. I/II. Zürich: Niehause, Rokitansky and Insel, I95 I, Vol. I, p. 460.

2883. Ritzer, Walter. Rainer Maria Rilke - Bibliography. Wien: Kerry, I95 I, f.m.

2884. Rolland, Romain. "Zwei Briefe an Stefan Zweig (Mar. I5, I9I 5 , and May 4, I9I5)," Leben-Werk, pp. I69-1 72 (Reprinted in Europäer, pp. 87-9I).

2885. Rosenhaupt, Hans Wilhelm. Der deutsche Dicbter um die Jabrbundertwende und seine Abgelöstheit von der Gesellschaft. Bern-Leipzig: Paul Haupt, 1939, f.m. (Cf. esp. pp. I 45-I47).

2886. Rosenthal, Friedrich. "Jungwiener Novellistik," $O R$, XXXVIII : 2 (Jan.-Mar., I9I4), 90-103.

2887. Rosenzweig, Alfred. "Stefan Zweig als Operndichter," Stefan Zweig. Sonderpublikation der Funktionärblätter (London), Nov., I943, pp. I0-14.

2888. Schäke, Gerd. "Stefan Zweig - Dichter, Essayist, Schriftsteller - sein tragisches Ende," DWo, I.J.: 30(1946), 5 .

2889. Schaukal, Richard von. "Ein Abenteuer Henri Beyles und seine Verzweigung," DeuV, X : 9(1928), 680-683. 
2890. - "Deutsche Prosa auf Zeithöhe," DeuV, XII (I930), 55 I-552.

2891. - "Der Fall Stefan Zweigs. Beitrag zur Geschichte der Dummheit," DeuE, V : 7 (1931), 430-432 (Cf. also $D Z$, No. 170; $D L$, XXXIII : 12 (Apr.-Sept., I931), 696).

2892. - "Krönung Stefans des Grossen," DeuV, XII (I930), I I 3-I I 9.

2893. - "Stefan Zweig," $A P$ zt, Lit. Beil., 4 (I93I).

2894. Scheller, Will. "Über Stefan Zweig als Lyriker und Erzähler," $K r Z$ (Wissenschaft), 74 (1925) (Cf. DL, XXVII : 9 (Apr.-Sept., 1925), 537).

2895. Schlösser, Manfred, and Hans Rolf Ropertz, eds. Kein ding sei wo das wort gebricht. Stefan George zum Gedenken. Darmstadt: Agora, I96I (2. enl. ed.; orig. I958; Cf. $D L W$, IV : 29 (July 20, I928), 24).

2896. Schlösser, Wilhelm. "Geleitwort," Menschen. Novellen. Stuttgart-Zürich-Salzburg: Europäischer Buchklub, 1962, pp. 7-8.

2897. Schneider, Wilhelm, ed. "Stefan Zweig über Sprach- und Stillehre," Meister des Stils über Sprach-und Stillebre. Leipzig: Teubner, 1926, p. 137.

2898. Schömann, Milian. "Stefan Zweig. Ein Humanist in unserer Zeit," Pbilo, III : I/4 (1938), 347-358.

2899. Scholz, Wilhelm von. "Erinnerungen an Stefan Zweig," Blätter, 3 (Oct., 1958), 5. 2900. Seelig, Carl. "Stefan Zweig, ein europäisch gesinnter Schriftsteller," $W d A$, x.J.: 3 I (I950), 8.

2901. Selden-Goth, Gisella. "Stefan Zweig und die Musik," $A u(N Y)$, I : ro (Mar. 6, 1942).

2901a. Sieburg, Friedrich. "Stefan Zweigs Welt von gestern," Nur für Leser. Jabre und Bïcher. Stuttgart: Deutsche Verlags-Anstalt, 1955, pp. 42-45.

2902. Simenauer, Erich. Rainer Maria Rilke. Legende und Mythos. Bern: Paul Haupt, I953, f.m.

2903. "Die soziale Lage der deutschen Schriftsteller," Bb, 7/1 I (Jan.-June, I9I0).

2904. Spael, W. "Stefan Zweig," Germ, Jan. 20, 1923.

2905. Spanier, Max. "Stefan Zweig," BrsZ, No. 14 (Cf. $D L$, XXIX : 6 (1926-1927), 346).

2906. Specht, Richard. "Die Literatur der Gegenwart," Ewiges Österreich. Ed. Erwin Rieger. Wien: Manzscher Verlag, 1928, pp. 25-74.

2907. - Stefan Zweig. Versucb eines Bildnisses. Leipzig: Spamersche Buchdruckerei, 1927 (This essay was translated into Russian and forms the Intro. to Zweig's Collected Works in Russian, Leningrad: Vremya, 1928-1930).

2908. Stefan, Paul. "Zum Tode Stefan Zweigs," $N V z t$, Feb. 28, 1942, p. 3.

2909. "Stefan Zweig," BZ, Jan. 21, I 923.

2910. "Stefan Zweig," Büg, 6 (I95 I), 259.

291 1. "Stefan Zweig," NScbR, N.F., I6.J. (1948-1949), 574-576.

291 2. "Stefan Zweig," DWo, 3.J.: I8/19 (1948), 4.

2913. "Stefan Zweig, der Europäer," Bd, Mar. 8, 933.

2914. "Stefan Zweig, Künder des Friedens und eines geeinten Europas," Auf, 4.J. (1952), 515-516.

2915. "Stefan Zweig spricht über den europäischen Gedanken," BN, Mar. I5, I933. 2916. "Stefan Zweig wider Schieffenzahn," Die Standarte. Magdeburg: Frundsberg, 1930, p. 38r.

2917. "Stefan Zweig zum sosten Geburtstag," $D L$, XXXIV : 5 (I93I), 267.

2918. "Stefan Zweig zum sosten Geburtstag," $P L$, Nov. 27, I 93 I.

2919. "Stefan Zweig zum sosten Geburtstag," $P P$, No. 322 (Cf. $D L, \mathrm{XXXIV} \mathrm{:} 5$ (I93I), 267).

2920. "Stefan Zweig zum Gedächtnis," $A u(N Y)$, VIII : 9 (Feb. 27, 1942), I 5-16 (Brief contributions by Emil Ludwig, Paul Stefan, Bruno Frank, Hermann Kesten, Thomas Mann, Lion Feuchtwanger, Heinrich Mann, Walter Mehring, Alfred Polgar, Berthold Viertel, Lothar Wallerstein, Franz Werfel).

292 I. Storz, Gerhard. "Über die Wirklichkeit von Dichtung," $W W$ (Erstes Sonderheft), n.d.(ca. 1952), 94-103. 
2922. Strich, Christian, ed. "Anekdote um Stefan Zweig," Der Autorabend. Dicbteranekdoten von Rabelais bis Tbomas Mann. Zürich: Diogenes, 1953.

2923. Sturmann, M. "Stefan Zweig, ein jüdischer Dichter," BIG, I929, p. I04.

2924. - "Studie über Stefan Zweig," JR, 33.J. (1928).

2925. Thies, H. "Stefan Zweig," $\mathrm{HFbl}$, Jan. I0, 1927.

2926. Thommen-Girard, G. H. "Stefan Zweig als Autographen-Sammler," $D A n q$, ro.J. (I954), $205-208$.

2927. Tobias, J. "Stefan Zweig - zu seinem sosten Geburtstag," SLZt, Nov. 28, I93 I. 2928. Trebitsch, Siegfried. "Für Stefan Zweig (Gedicht zum 2osten Todestag Stefan Zweigs am. 22. Februar 1962)," Blätter, I3/14 (Apr., 1962), I.

2929. Trenner, Franz. Richard Strauss: Dokumente seines Lebens und Schaffens. München: Beck, 1954, pp. $228 \mathrm{ff}$. (Concerning the stormy history of the opera "Die schweigsame Frau").

2930. - “Zur Entstehung der 'Schweigsamen Frau'," BBSF (1962).

293 I. Trinius, B. "Stefan Zweig, zu seinem sosten Geburtstag," $D A Z$, Nov. 25, I 93 I (Cf. also $D L$, XXXIV : 5 (Oct., I93 I - Mar., 1932), 267).

2932. Truding, Lona. "Stefan Zweig und die Musik," $G$, XXXV (I956), 4I I-4I3.

2933. - "Stefan Zweig und die Musik," NZfM, CXIX (Mar., I958), I 36-1 39.

2934. "Über Sprach- und Stillehre," Meister des Stils über Sprach- und Stillebre. Ed. Wilhelm Schneider. Leipzig: Teubner, 1926, p. I37.

2935. "Über Stefan Zweig," RoR, 2 (1929), I 20.

2936. Ullmann, Ludwig. "Stefan Zweig, der grosse Europäer," Spiegelungen, pp. I I I-I I6.

2937. Ullrich, Hermann, ed. Salzburg. Bildnis einer Stadt. Essays, Briefe und Dokumente. Wien: Sexl, 1948 (Cf. Blätter, 8/10 (Oct., 1960), 7).

2938. - "Zum Geleit," Stefan Zweig. Sonderpublikation der Funktionärblätter (London), Nov., I943, pp. I-2.

2939. Van Dovski, Lee. "Mit Stefan Zweig in Paris," Spiegelungen, pp. 68-73.

2940. Van Gelder, Robert. "Stefan Zweig: Wie sich die Schriftstellerei in einer kriegerischen Welt entwickeln wird," Prominente plaudern. Tr. from the English by Gottfried Ippisch. Wien: Humboldt, I947, pp. 72-75.

2941. "Verwirrung der Gefühle - Todestag des Dichters Stefan Zweigs," BdBh, I I8.J. (I95I), II 3 .

2942. Viertel, Berthold. "Abschied von Stefan Zweig," Leben-Werk, pp. I9I-199 (Reprinted in Europäer, pp. 250-260).

2943. - "Das gestohlene Jahr," Blätter, 8/10(Oct., I960), I4-17 (Concerning the Zweig/ Viertel collaboration on the film by the same name).

2944. Vogelsang, Hans. "Stefan Zweig, ein Enthusiast des Dramas," WidZ, I 2 (Dec., 1961), 53-59.

2945. Waibler, Helmut. Hermann Hesse. Eine Bibliographie. Bern-München: Francke, 1962, Nos. P 1558-1561, L I 20, 907, 1926.

2946. Waldinger, Ernst. "Stefan Zweigs 'Feuilletonismus'," Spiegelungen, pp. 107-108.

2947. Walter, Bruno. Thema und Variationen. Erinnerungen und Gedanken. Stockholm: Bermann-Fischer, 1947 (Tr. into English by James A. Galstone. New York: Knopf, pp. 262, 307, 311).

2948. Weiss, Hansgerhard. Romain Rolland. Berlin-Leipzig: Volk und Wissen, I948, f.m. 2949. Wendel, Hermann. "Ein Vorläufer von Karl Marx. Auseinandersetzungen mit Stefan Zweig," $V$, No. 246 (Cf. $D L$, XXXII : 4 (1929-1930), 222).

2950. Werfel, Franz. "Stefan Zweigs Tod," Leben-Werk, pp. I77-187 (Reprinted in Europäer, pp. 269-282; Unsterblicher Genius. Deutsche Dicbter in Gedenken ibrer Freunde. Ed. Paul Schneider. Ebenhausen bei München: Hartfrid Voss, I959, pp. 317-323).

295 r. Werremeier. "Der Arzt in der Dichtung Stefan Zweigs und Hans Carossas," DNSch, r4.J. (1953), I75-176.

2952. Wilhelm, Paul. "Stefan Zweig," NWJ, 6829 (I9I 2) (Cf. DLE, I 5.J.: 5 (Dec. II, I9I 2), 328).

2953. Winternitz, Friderike Maria von. "Die Übersetzer und der Krieg," DLE, XVIII : 8 (Jan. I5, I916), 483-485. 
2954. Wittek, Suzan von. "Erinnerung an Stefan Zweig," OöN, Oct. 27, 1947, p. 3.

2955. Wittner, V. "Altösterreichische Abschiede." DLW, I (1946-1947), 300-304.

2956. Wolbe, Eugen. "Stefan Zweig als Autographensammler," $D K w$, XIII (Nov., I93 I), 72-73.

2957. Wolf, H. "Stefan Zweig," Zweigs Ausgewäblte Prosa. Ed. W. Kuiper. Amsterdam: Meulenhoff, $193 \mathrm{I}$.

2958. Wolff, Theodor. "Die Wohltat des Exils," $A u(N Y), \mathrm{XV}:$ i8 (May 6, 1949), 3.

2959. Zarek, Otto. "Stefan Zweig. Zu seinem sosten Geburtstage," NR, 42. J.: II (I93 I), 86r-862.

2960. Zech, Paul. "Erinnerungen an Stefan Zweig," Greifenalmanach auf das Jabr 1956, pp. 170-189.

2961. - "Stefan Zweig," BT, Oct. 5, 1914.

2962. - Stefan Zweig. Eine Gedenkschrift. Buenos Aires: Quadriga, 1943.

2963. Zohn, Harry. "Schulausgaben von Werken Stefan Zweigs," Blätter, 6/7 (Oct., I959), 20.

2964. - "Stefan Zweig," Wiener Juden in der deutschen Literatur. Tel Aviv: Olamenu, 1963, pp. 19-30 (Contains a Zweig portrait; p. 6, excerpts from Zweig's autobiography, cf. $W \nu G$, pp. 23, 31, 32, 33).

2965. - "Stefan Zweig," Wie sie es sehen. New York: Holt, I95 I, p. I 30 (School edition; "Die Augen des ewigen Bruders," pp. I 3 I-I 7 I).

2966. - "Stefan Zweigs Lebensabend," DeuR, 82.J. (I956), I I95-II 97.

2967. - "Stefan Zweig und Emile Verhaeren," Spiegelungen, pp. 24-32.

2968. - "Worte zum Gedächtnis Stefan Zweigs," Blätter, II/I 2 (Oct., I96I), IO-I 2.

2969. Zimmermann, Walter. "Stefan Zweigs 'Die Augen des ewigen Bruders'," Deutsche Prosadichtungen der Gegenwart. Interpretationen für Lebrende und Lernende. Düsseldorf: Pädagogischer Verlag Schwann, 1954-1956, pp. 8 I-98.

2970. Zuckmayer, Carl. "Did you know Stefan Zweig?" Leben-Werk, pp. I 2 I-1 26 (Reprinted in Europäer, pp. 242-249; NASG, I (I950), 25-28).

2971. - "Tage und Nächte," Mer, XVII : I ("Salzburg") (Jan., I964), 66-74 (Zuckmayer's remembrances of Salzburg and the people he knew there; picture of Zweig's house on the Kapuzinerberg, p. 74).

2972. "Zwei Dichter: Thomas Mann und Stefan Zweig," Colq, r.J.: 7 (I947), 23-24.

2973. Zweig, Arnold. "Biblische Stücke, Judenstücke," Juden auf der deutschen Bübne. Berlin: Welt-Verlag, 1928, pp. 257-267.

2974. Zweig, Friderike Maria. Stefan Zweig. Eine Bildbiographie. München: Kindler, I96I (Cf. Rev. of same by Georg Böse, BüK, I I.J.: 2 (June I 5, 1962), 22).

2974a. - Spiegelungen. Wien: Hans Deutsch Verlag, 1964 (Memoirs of Mrs. Zweig or, as she referred to them, "Fragmente meines Lebens").

2975. - Stefan Zweig wie ich ibn erlebte. Stockholm-New York: Neuer Verlag, 1947 (Reprinted Frankfurt am Main: Frankfurter Verlagsanstalt, 1947 and BerlinGrunewald: Herbig, 1948).

\section{HEBREW}

2976. Kastein, Josef (pseud. Julius Katzenstein). "Stefan Zweig," Midot Va'arakbim. Intro. Azrael Karlebach. Tel Aviv: Olympia, 1947.

\section{HIN D I}

2977. Chaturvedi, Benarsi Das. “Stefan Zweig," The Navyug(India), XVIII(Feb. 26, 1950), 9.

2978. Jain, Yashpal. "Author Who Ended His Life. Life and Work of Stefan Zweig," (In Hindi), Leader (Allahabad), Oct. I, I944.

2979. - "Stefan Zweig," Social Welfare, I944. 
2980. Burich, Enrico. "I due Zweig: Stefan e Arnold," $N A$, N.S., 4.a.: 2 (Mar.-Apr., 1956), I 22-1 27.

2981. De Lorenzo, Giuseppe. "Stefan Zweig ed il Buddhismo," $A A P$, N.S., II (19481949), 5 I-56.

2982. Mazzucchetti, Lavinia. "Introduzione," Incontri e Amicizie. Tr. from the German by Anita Limentani. Milano-Verona: Mondadori, I950, pp. 7-I 3.

2983. - "Richard Strauss e Stefan Zweig," $L A M$, 2.a.: 5 (Jan.-Mar., I959), 19-52. 2984. - "Ricordando Stefan Zweig," Novecento in Germania. Milano: Mondadori, 1959, pp. 266-272 (Cf. also pp. 55, 160, 220, 249-250; Reprinted in IP (1959)). 2985. Robertazzi, M. "Tre Maestri: Balzac-Dickens-Dostoievski," Poesia e Realta. Modena: Guandi, 1934, pp. 293-304.

2986. Settanni, E. "Freud visto da Zweig," Romanzi e Romanzieri d'oggi. Napoli: Guida, I933, pp. II-I7.

2987. Tilgher, Adriano. "Stefano Zweig," La Scena e la Vita. Nuovi Studi sul Teatro Contemporaneo. Roma: Libreria di Scienze e Lettere, 1925, pp. 206-2 I6.

2988. Zampa, Gregor. "Una Gloria che non ha resistada," CorS, May I9, ig6r, p. 3.

2989. Zweig, Friderike Maria. Stefan Zweig. Compagno della mia Vita. Tr. from the German by Ervino Pocar. Milano: Rizzoli, 1947 .

JAPANESE

2990. Iizuka, Nobuo. "Goethe und Stefan Zweig," GoeJb, II (1960), 98-I I 4.

2991. - "Stefan Zweig und der europäische Geist," DoiB, XVIII (I957), I2 I-I 29.

\section{NOR WEGIAN}

2992. Berendsohn, Walter A. "Hvorfor søkte Stefan Zweig døden?" Vin, VII (Sept., 1950), 533-537.

2993. Bredsdorff, Viggo. "Stefan Zweig som Dramatiker," Ed, LIII (I953), 257-274.

POLISH

2994. Nowaczynski, Adolf. "Clio again in Fashion," WiaL, Apr. 12, 1936.

2995. "Nowelistyka Stefana Zweiga," Two, 7 (1936), r 28-г 33.

2996. Wislowska, Maria. "Krótko o Stefanie Zweigu," $N L W$, I4 (1957), 4.

\section{PORTUGUESE}

2997. Azevedo, Raul de. Vida e Morte de Stefan Zweig. Rio de Janeiro: Brasil (“Aspectos”), I942.

2998. Fedèr, Ernesto. "Stefan Zweig," IBra, Mar., I942, pp. I6-17.

2999. - "Os últimos Dias de Stefan Zweig," Diálogos dos Grandes do Mundo. Rio de Janeiro: Dois Mundos Editora, 1944, pp. 185-200.

3000. Garcia, Antonio Augusto Apio. Algunos Aspectos da Vida e Obra de Stefan Zweig. Porto: n.p., r942.

300r. Lima, Claudio de Araujo. Ascenfao e Queda de Stefan Zweig. Rio de Janeiro: Olympio, I943.

3002. Navarro, Eugenio. "Stefan Zweig," Oc, II : 6 (Oct., I 938), 403-4I4.

3003. Souza, Claudio de. Os últimos Dias de Stefan Zweig. Rio de Janeiro: Valverde, I 942. 3004. Stern, Leopold. A Morte de Stefan Zweig. Rio de Janeiro: Civilização Brasiliera, 1942 (Pub. by the same company in French as La Mort de Stefan Zweig). 
3005. Rapoport, O. “Tvorcheskij Put' Stefana Zweigs," Yevreiskie Pisateli. Shanghai: n.p., I942, pp. $217-225$.

SPANISH

3006. Atias, Alejandro C. Ensayos: Goethe, José Asunción Silva, Stefan Zweig. Salto (Uruguay) Editado por Tipografia Mazzara, 1936.

3007. Arrieta, Rafael Alberto. "Stefan Zweig y las Letras francesas," Dickens y Sarmiento. Otros Estudios. Buenos Aires: El Ateneo, 1928, pp. I I I-I 20.

3008. Cahn, Alfredo. Literaturas Germánicas. Buenos Aires: Talleres Gráficos de la Companía General Fabril Financeira, I96r, $\mathrm{m}$.

3009. Feder, Ernest. "Stefan Zweig," ID, XXVIII (Feb., I952), 55-57.

30ro. - "Las últimas Conversaciones con Stefan Zweig," RdA, June, r945, pp. 20-25.

301 1. - "Los últimos Dias de Stefan Zweig," Encuentros. Rosario: Editorial Rosario, 1945, pp. 175-1 86.

30r 2. Franulic, Lenka, ed. "Stefan Zweig," Cien Autores Contemporaneos. Vol. II. Santiago de Chile: Ediciones Ercilla, I 94I, pp. 5 I I-523.

30r3. Fuente Gonzáles, Efrain de la. Stefan Zweig. Juan Tenorio. Ensayos. Santiago de Chile: Carabineros de Chile, 1946.

3014. Gandia, Enrique de. "Stefan Zweig y el Nombre de América," Ver, Mar. 3 1, 1947, pp. 263-27I (Contains Zweig portrait).

3015. Goldbaum, Wenzel. Stefan Zweig, su Vida, Obra y Muerte. Quito: Imprenta de la Universidad central, 1942 .

3016. Jarnés, Benjamín Millán. Stefan Zweig, Cumbre Apagada. Retrato. México, D.F.: Proa, 1942.

3017. Jarpa, Hugo Lazo. "Stefan Zweig y Benjamín Jarnés," Ate, LXXIII : 2 (Sept., 1943), 283-289.

3018. Moreno, Artemio. De Stefan Zweig a Jacques Maritain. Muerte y Resurrección del Espiritu. Buenos Aires: Claridad, 1943.

3019. Ortiz Oderigo, Alicia. Stefan Zweig. Un Hombre de Ayer. Buenos Aires: Nova, 1945.

3020. Osorio Lizarazo, J. "La Tragedia de Petrópolis," RdlI, Jan., I944, pp. 436-462. 3021. Relgis, Eugen. El Espíritu Activo. Buenos Aires: Humanidad, 1959, f.m. (Rev. ed.). 3022. - El Hombre Libre frente a la Barbarie Totalitaria. Un Caso de Conciencia: Romain Rolland. Montevideo: Anales de la Universidad, 1954. f.m.

3023. - Homenaje a Eugen Relgis en su 6o. Aniversario. Montevideo: Comité Nacional de Adhesión a la Candidatura de Eugen Relgis al Premio Nobel de la Paz, I955. m.

3024. - La Paz del Hombre. Montevideo: Humanidad, I96r, f.m.

3025. - Profetasy Poetas. Buenos Aires: Candelabro, 1955, f.m.

3026. - Stefan Zweig, Cazador de Almas. Entrevista y Paseo en Salzburgo. Post-mortem. Sus Huellas en Montevideo. Algunas Cartas. Montevideo: Humanidad, 1952.

3027. Romera, Antonio R. "Esquema de Stefan Zweig," Ate, XIX : 200 (Feb., 1942), $220-238$.

3028. Sanchez Tricado, José Luis. "Una Novela de Stefan Zweig," Stendhal y Otras Figuras. Buenos Aires: Republica Argentina (Imprenta López), 1943, pp. 8 5-92.

3029. Souza, Claudio de. Los últimos Dias de Stefan Zweig. Pref. André Maurois, pp. 10-16. Mexico, D.F.: Quetzal, I944.

3030. Tiempo, César (pseud.). "Stefan Zweig, el Poeta de su Vida," Bre, 4/5 (Jan., I960), I6-20.

3031. Wills Ricaurte, Gustavo. “Porque murio Stefan Zweig?” RdA, Oct., 1946, pp. $4 \mathrm{I}-48$.

3032. Zweig, Friderike Maria. Stefan Zweig. Tr. from the German by Alfredo Cahn. Barcelona: Hispano Americana de Ediciones, 1947. 
3033. Zagul, Dmitro. "Stefan Zweig," Zbirnyk Tvoriv. Kiev: n.p., I929 (Ukranian translation of some of Zweig's works).

YIDDISH

3034. Goldkorn, Yitzchak. "Stefan Zweig," Fun Welt-Kwal. Tel Aviv: Hamenorah, 1963, pp. $219-224$.

3035. Hameiri, Avigodor. Yirmiyabn. Jerusalem: n.p., I949 (Translation of Zweig's Jeremias with a critical essay about Zweig and his drama).

3036. Kreitner, R. L. "A Shu mit Stefan Zweig," DoA, II : 9 (May, r950), 2 I-22.

\section{LITERARY AND BIOGRAPHICAL PERSONALIA}

3037. $A H R$, XLVII (Apr., I942), 724-725 (Zweig portrait and obituary).

3038. Ate, XIX : 200 (Feb., 1942), 220-238 (Antonio R. Romera, "Esquema de Stefan Zweig").

3039. $A u$, XII (1956), 959-96r (Konstantin Fedin, "Die Tragödie Stefan Zweigs"). 3040. $A u(N Y)$, VIII : 9 (Feb. 27, 1942), I5-16 ("Stefan Zweig zum Gedächtnis").

3041. $A u(N Y)$, VIII : 10 (Mar. 6, 1942), 19-20 (Artur Holde, "Gedenkfeier des 'Aufbau' für Stefan Zweig").

3042. AWJD, 5.J.: 40 (I950), 6 ("Jahre der Reife Stefan Zweigs").

3043. $B A$, XIII : 4 (Autumn, I939), 427-430 (Thomas Q. Curtiss, "Stefan Zweig").

3044. $B B B$, II : 1 $38 / 139$ (1923), I-3 (Hanns Martin Elster, "Stefan Zweig").

3045. $B B C, 553$ (I93 I) (Emil Faktor, "Stefan Zweig zum sosten Geburtstag").

3046. $B d$, 555 (I93I) (Fritz Adolf Hünich, "Stefan Zweig zum sosten Geburtstag").

3047. $B d$, Mar. 8, I933 ("Stefan Zweig, der Europäer").

3048. BdBh, I I4.J. (I947), 17-25 (G. Lenteritz, "Das unerloschene Leuchten, Stefan Zweig").

3049. $B d B h$, I I 8.J. (I95 I), I I3 ("Verwirrung der Gefühle - Todestag des Dichters Stefan Zweig").

3050. Bm, XXXVIII (Oct., I93I), 150 (Zweig portrait and note).

305 r. BMCN, Nov., 1946, pp. 4-5 (Robert Pick, "Stefan Zweig").

3052. Bng, 6.J. (195I), 3-9 (Herbert Lilge, "Der Untergang eines Herzens. Nachruf auf Stefan Zweig").

3053. Br, XVII (1930), 447-449 (Hanns Arens, "Stefan Zweig").

3054. Bre, 4/5 (Jan., I960), 16-20 (César Tiempo, "Stefan Zweig, el Poeta de su Vida"). 3055. BT, Oct. 5, I9I4 (Paul Zech, "Stefan Zweig").

3056. $B T, 559$ (1931) (Paul Frischauer, "Stefan Zweig zum sosten Geburtstag").

3057. $B T r$, Dec. Io, I932, p. I ("Stefan Zweig").

3058. Büg, 6 (I951), 259 ("Stefan Zweig").

3059. BuL, II (Nov., I962), I-3 (Wolf R. Lang, "Stefan Zweig, Umrisse seines Lebens"). 306o. BZ, Jan. 21, I923 ("Stefan Zweig").

3061. Car, Aug. I9, I947 (Leopold Stern, "Sur le Suicide de Stefan Zweig").

3062. ConR, CLXXV (1949), 62 (A. Wittlin, "Stefan Zweig").

3063. ConW, IX : 4 (Apr. 10, I942), IO-I 3 (Alfred Werner, "Pity for Stefan Zweig").

3064. CorS, May I9, I961, p. 3 (Gregor Zampa. "Una Gloria che non ha resistada").

3065. CurBio. 1942, pp. 902-903 ("Stefan Zweig").

3066. CVZ, X : 47 (Nov. 20, I931), 537-538 (Ernst Lissauer, "Zu Stefan Zweigs sosten Geburtstag").

3067. $D A Z$, Nov. 25, I93 I (B. Trinius, "Stefan Zweig zu seinem sosten Geburtstag"). 
3068. $D e G$, CXXI : 7 (July, 1955), 302-312 (Alfred Kossmann, "Het Noodlotsuur van Stefan Zweig").

3069. DGT, 4.J.: I (1949), 83-88 (Herbert Günther, "Stefan Zweig und Frankreich"). 3070. DL, XXVII : 12 (Apr.-Sept., 1925), 746 (A. Busse, "Amerikanischer Brief," ref. to Passion and Pain).

307x. DL, XXVIII : 4 (1925-1926), 250 (Ref. to French translations of Zweig's works; Cf. also $V Z$, No. 522$)$.

3072. $D L$, XXVIII : 10 (I925-1926), 624 (Ref. to Japanese translations of Zweig's works Romain Rolland, "Die Augen des ewigen Bruders" and "Amok").

3073. $D L, \mathrm{XXXI}: 3$ (1928-1929), I74 (Ref. to the warm reception given to Zweig at the Tolstoy Jubilee and that a second collected works of Zweig was about to appear in Russian translation).

3074. DL, XXXI : ro (1928-1929), 602 (Hermann Sternbach, "Polnischer Brief," ref. to Polish translation of "Verwirrung der Gefühle").

3075. DL, XXXI : 10 (1928-1929), 617 (Ref. to Italian translation of Baumeister).

3076. DL, XXXIII : I (Oct., I930-Mar., I93 I), 58 (Ref. to English translation of Drei Meister).

3077. $D L$, XXXIII : io (Apr.-Sept., I931), 584 (Ref. to Swedish translation of Fouché). 3078. $D L$, XXXIV : s (1931), 267 ("Stefan Zweig zum sosten Geburtstag").

3079. DLE, 9. J.: 5 (Dec. I, I906), 363 (Ref. to translation of Zweig into Norwegian). 3080. DLE, 9.J.: I I (Mar. I, I907), 849-855 (S. Meisels, "Jüngjüdische Lyrik").

3081. DLE, XVII : 4 (1914), 193-199 (Emil Lucka, "Stefan Zweig").

3082. DLE, 17.J.: 24 (Sept., I9I5), I 5 I9 (Ref. to English translation of Zweig's Emile Verbaeren).

3083. DoA, II : 9 (May, I950), 2I-22 (R. L. Kreitner, "A Shu mit Stefan Zweig").

3084. DWe, 2.J.: I 3 (1947), 9 (L. Menter, "Stefan Zweig").

3085. DWo, 1.J.: 30 (1946), 5 (Gerd Schäke, "Stefan Zweig - Dichter, Essayist, Schriftsteller - sein tragisches Ende").

3086. $D W 0$, 3.J.: 18/19 (1948), 4 ("Stefan Zweig").

3087. DZe, 2.J.: 22 (1947), 3 (A. Beierle, "Stefan Zweig, Dichter und Dulder").

3088. E, 25.a.: 22 (Oct., 1947), 48-67 (Antonina Valentin, "Stefan Zweig").

3089. Europäer, pp. 21 8-238 (Ernst Feder, "Die letzten Tage Stefan Zweigs").

3090. Frw, Feb., 1942(?) (Salman Shneour, "Stefan Zweig").

3091. $G B, \mathrm{XV}$ (Feb., 1932), 190 ("Stefan Zweig").

3092. GdG, LXVI : 4 (I930), I45-I 52 (Wilhelm Knevels, "Stefan Zweig").

3093. GS, 329 (1926) (Ernst Friedrichs, "Stefan Zweig zu seinem 45sten Geburtstag").

3094. IBra, Mar., I942, pp. I6-I 7 (Ernesto Feder, "Stefan Zweig").

3095. IN, July, I960 ("Stefan Zweig Correspondence").

3096. JR, 33.J. (1928) (M. Sturmann, "Studie über Stefan Zweig").

3097. JSp, VII : 6 (Apr., I942), 5 ("The Case of Stefan Zweig").

3098. JSp, May, I943, pp. 9-I I ("The Tragic Case of Stefan Zweig").

3099. KZ, 444 (I92I) (Claims by Zweig that a Novelle entitled "Untreue" submitted to the $K Z$ in 1914 by Gräfin D. Stourza von Katschuro was in reality a slightly changed version of his "Angst": "Es liegt also literarischer Diebstahl unverschämtester Art vor"; Cf. also DLE, 23.J.: 21 (Aug., I921), 1342).

3100. KZ, 650 (1931) (K. H. Erkelenz, "Stefan Zweig").

3ror. $L D$, CXVI (July I5, I933), I7-18 ("H. G. Wells Raises a Storm in the P.E.N. Congress"; Contains a Zweig portrait).

3102. LD, CXX (Sept. 2I, I935), 24 (Rev. of Queen of Scots; Contains a Zweig portrait). 3103. Leader (Allahabad, India), Oct. I, I914 (Yashpal Jain, "Author Who Ended His Life. Life and Work of Stefan Zweig").

3104. $L M F$, XII (1948), 424-429 (Jean Décarreaux, "Le Message posthume de Stefan Zweig").

3105. LNR, X (Aug., I942), 579-582 (Paul Beaulieu, "Réflexions sur le Suicide de Stefan Zweig").

3106. $L T$, Feb. 24, 1942, p. 3 and Feb. 25, 1942, p. 7 ("Stefan Zweig's Death"). 
3107. $M A$, LXXIV (Oct., 1954), is (Portrait: "A Threesome at the Salzburg Festival of I934: Arturo Toscanini, Bruno Walter and Stefan Zweig").

3108. $M C$, CLI (Feb., I955), IOI-102 (H. Koegler, "Stefan Zweig").

3 109. N, CLIV (Mar. I4, 1942), 3 I4 (Margaret Marshall, "Stefan Zweig").

31 10. Navyug (India), XVIII (Feb. 26, 1950), 9 (Benarsi das Chaturvedi, "Stefan Zweig").

3III. Ne, I (Apr. 8, I933), 28.

3II2. Ne, XI(Feb. 7, I938), 29 (Portrait).

31 I3. Ne, XIII (Mar. 20, I939), 43 (Portrait).

31 14. Ne, XVII (Sept. 29, I94I), 52 (Portrait).

3II5. Ne, XIX (I942), 37 ("Stefan Zweig Epilogue").

3116. NFP, Aug. I2, I928, pp. 27-28 (Leonhard Adelt, "Werk und Leben Stefan Zweigs").

3I 7. NM, XLII : Io (Mar., I942), 22-23 (O. T. Ring, "Life and Death of Stefan Zweig"). 3I I 8. NR, 42.J. II (193 I), 86I-862 (Otto Zarek, "Stefan Zweig zu seinem sosten Geburtstag").

3119. NScbR, N.F., I6.J. (I948-1949), 574-576 ("Stefan Zweig").

31 20. $N V z t$, Feb. 28, I942, p. 3 (Paul Stefan, "Zum Tode Stefan Zweigs").

3 г 21. NV zt, Mar. 14, I942, p. 5 (Hans Johan Kraus, "Brasilien ehrt Stefan Zweig").

31 22. NYT, Apr. 23, I933, p. 22 ("German Students Purge Libraries").

3 I23. NYT, May I I, I933, p. I 2 ("Berlin Lukewarm to Book-Burning").

3 124. NYT, Nov. I, I933, p. I4 ("Nazis Raise Swastika on Vienna City Hall”).

3125. NYT, Nov. 2, 1933, p. I9 ("Zweig in London").

3126. NYT, Jan. 18, 1935, p. 7 ("Stefan Zweig Arrives in the United States - An Interview about His Plans").

31 27. NYT, Jan. 2, I939, p. 27 ("Zweig Says Unrest Hampers Writers").

31 28. NYT, Feb. 21, I939, p. 4 ("Institute Honors Stefan Zweig").

3129. NYT, Apr. 10, 1940, p. 2 ("Stefan Zweig Becomes a Briton").

3130. NYT, July 8, I940, p. 4 "“Stefan Zweig Arrives to Study the Americas"; Portrait of Stefan and Lotte Zweig).

3131. NYT, Feb. 24, 1942, pp. 1, 23 ("Stefan Zweig and Wife End Lives in Brazil").

3132. NYT, Feb. 25, 1942, p. 3 ("Brazil Pays Honor to Zweigs in Death").

3133. NYT, Feb. 25, 1942, p. I8 ("One of the Dispossessed - A Tribute to Stefan Zweig").

3134. NYT, Feb. 27, 1942, p. 5 ("Stefan Zweig's Will”).

3135. NYT, Mar. I, I942, p. 28 ("Memorial Meeting in Honor of Stefan Zweig").

3136. NYT, Feb. 23, 1943, p. I I ("Stefan Zweig Monument Unveiled by Jewish Colony in Petropolis").

3137. NYTBR, June 14, I93 I, p. 7 (Rev. of American ed. of Amok with Zweig portrait). 3138. NYTBR, Apr. 8, 1934, p. 4 (Article by Zweig: "History and the Schreen. On the Filming of Events of Bygone Times"; Ed. comment: MGM preparing to make film based on Zweig's Marie Antoinette).

3139. NYTBR, Aug. 25, 1940, p. 2 ("Letter in Honor of Stefan Zweig").

3140. NYTBR, Oct. 19, 1941, p. 32 ("About Brazil" - concerning the Ernesto Montenegro rev. of Zweig's Brasilien).

3141. NYTBR, Nov. 9, I94I, p. 36 (“The Word 'Decent"').

3142. NYTBR, Mar. I, I942, p. 6 (Austin Stevens, "Tribute to Stefan Zweig").

3143. NYTBR, Mar. 8, I942, p. 2 ("Speaking of Books").

3144. NZZ, Nov. 29, I93 I (Robert Faesi, "Stefan Zweig zu seinem sosten Geburtstag").

3145. OöN, Oct. 27, I947, p. 3 (Herbert Lange, "Ein Bildnis Stefan Zweigs").

3146. Philo, III : I/4 (1938), 347-358 (Milian Schömann, "Ein Humanist in unserer Zeit").

3147. $P L$, Nov. 27, I93 I "Stefan Zweig zum sosten Geburtstag").

3148. PP, No. 322 ("Stefan Zweig zum 5osten Geburtstag").

3149. Pris, 19/20 (1948), 40 (Erich Ebermayer, "Letzte Stunden mit Stefan Zweig").

3 I 50. PW, CXLI (Feb. 28, 1942), 950 ("Stefan Zweig").

3151. Rad, VI (1929), 2 (Max Lederer, "Stefan Zweig").

3152. Rad, VIII (I93 I), 8 (Erwin Rieger, "Stefan Zweig"). 
3153. $R D, \mathrm{XXXIX}$ (194I), 39-43 ("Stefan Zweig").

3154. RdlI, Jan. I944, pp. 436-462 (Osorio Lizarazo, "La Tragedia de Petrópolis").

3155. RP, 42.a.: 5 (Oct. I5, I935), 864-889 (Maurice Muret, "Stefan Zweig").

3156. $R P$, 62.a.: I2 (Feb., 1955), 3-23 (Jules Romains, "Derniers Mois et dernières Lettres de Stefan Zweig").

3157. $R R, \mathrm{LI}$ : 5 (May, I915), 620-62I ("Stefan Zweig: Austrian Poet, Critic and Dramatist").

3158. SLzt, Nov. 28, I93 I (J. Tobias, "Stefan Zweig zu seinem sosten Geburtstag"). 3159. Spiegelungen, pp. 68-73 (Lee Van Dorski, "Mit Stefan Zweig in Paris").

3160. SRL, VIII (June I8, I932), 791 (Rev. of "Letter from an Unknown Woman"; Portrait of Zweig).

316r. SRL, XVI (Oct. 16, 1937), 22, 26 (Stephen Vincent Benet, Rev. of "Buried Candelabrum").

3162. SRL, XVII (Feb. 5, 1938), I, 5 (Oliver La Farge, Rev. of Magellan; Portrait of Zweig).

3163. SRL, XIX (Apr. 22, 1939), 3 (Jonathan Griffin, Rev. of Escape to Life by Klaus and Erika Mann; Zweig portrait).

3164. SRL, XXI (Nov. 25, I939), I I (Paul Rosenfeld, Rev. of Master Builders; Portrait of Zweig from a drawing by George Schreiber).

3165. SRL, XXIII (Nov. 23, 1940), I0 (William H. Chamberlin, Rev. of Tide of Fortune; Zweig portrait).

3166. SRL, XXV (Mar. I4, I942), I 2 (Thomas Q. Cousins, "Stefan Zweig").

3167. SRL, XXVII (Feb. 26, I944), s (Christopher La Farge, "Say It With Fiction"; Zweig portrait).

3168. SRL, XLI (Feb. 8, 1958), 24.

3169. T, XXXII (July 25, I938), 30 ("Bad Boy" concerning the Richard Strauss-Stefan Zweig collaboration; Zweig portrait).

3170. $T$, XXXIX (Mar. 2, 1942), 53 (Zweig obituary).

3171. VdF, Mar. I5, 1942, p. I (Adolphe Demilly, "Sur une Mort").

$3172 . V d F$, Mar. 15, 1942, p. 4 (Robert Goffin, "Adieu à Stefan Zweig").

3173. Vin, VII (Sept., 1950), 533-537 (Walter A. Berendsohn, "Hvorfor søkte Stefan Zweig Døden?").

3174. $V u V$, 2. ser., I3 (I947), 22-3 I (Herbert Oetke, "Stefan Zweig").

3175. WieZ, 277 (1949) (Egon Pisk, "Stefan Zweigs 'Tod").

3176. $W L B, \mathrm{~V}$ (Sept., 1930), 8 (Rev. of Fouché; Biographical sketch and portrait of Zweig).

3177. WLB, XVI (Apr., I 942), 598 (Biographical sketch of Zweig).

3178. Wst, XXV (1950), I5-17 (Hanns Arens, "Die letzten Jahre Stefan Zweigs").

3179. $Y C D$, I95 I, pp. 70-77 (M. Evensky, "Stefan Zweig").

\section{UNPUBLISHED MASTERS' THESES AND DOCTORAL DISSERTATIONS}

3180. Bak, Olga. Stefan Zweig, sa Vie et son Oeuvre. Diss., Quebec (Laval University), 1950. 3181. Berry, Robert M. The French Symbolist Poets in Germany: Criticism and Translations. I870-1914. Diss., Harvard University, 1944.

3182. Böttcher, Kurt. Stefan Zweig und die Welt von gestern. Diss., Jena (Friedrich Schiller University), I95 I.

3183. Diamond, Helena. Protest gegen den Krieg als Hauptmotiv in den Dramen von 1917 bis I93I. Thesis, Hunter College, I933 (Zweig's Jeremias).

3 184. Eichbaum, Gerda. Die Krise der modernen Jugend im Spiegel der Dichtung. Diss., Giessen, I929.

3185. Firon, Erich. Stefan Zweig als Dramatiker, Diss., Wien, 1949.

31 86. Frese, Hans. Das Deutsche Buch in Amerika: Übersetzungen der Jabre 191 8-1935. Diss., Marburg, 1937. 
3187. Frets, Huberta. L'Elément germanique dans l'Oeuvre d'Emile Verbaeren. Diss., Paris, 1935 .

3188. Götzfried, Johannes L. Der beroische Idealismus bei Romain Rolland. Diss., BonnFreudenstadt, I929.

3189. Grebert, Ludwig. Paul Verlaine und seine deutschen Úbersetzer. Diss., Giessen, r923. 3190. Gschiel, Martha. Das dichterische Werk Stefan Zweigs. Diss., Wien, I 953.

319г. Kaemmer, Kurt. Die Versdichtungen Baudelaires und Verlaines in deutscher Sprache. Diss., Köln, I922.

3192. Kaempffer, Annemarie. Romain Rollands Frauengestalten. Diss., Jena, I93 I.

3193. Klawiter, Randolph J. Stefan Zweig's Novellen - An Analysis. Diss., University of Michigan, 1960.

3194. Lent, Ingrid. Das Novellenwerk Stefan Zweigs. Eine Stil- und Typenuntersucbung. Diss., München, 1956.

3195. Mertens, Gerhard M. Stefan Zweig's Biograpbical Writings as Studies of Human Types. Diss., University of Michigan, I950.

3196. Rosenberg, Justus. The German Translations of Paul Verlaine's Poetry. Diss., University of Cincinnati, 1950.

3197. Rosnovsky, Karl. Erinnerungsbïcher an das alte Österreich. Raoul Auernbeimer und Stefan Zweig. Diss., Wien, I95 I.

3198. Starr, William T. Romain Rolland's Internationalism. Diss., University of Oregon, 1938 .

3199. Thornton, Luanne. The Critical Reception in the United States of the Novellen of Stefan $Z$ weig. Thesis, Emory University (Georgia), $1_{9} 63$.

3200. Vanicek, Hilde. Der Einfluss der französischen Lyrik auf Anton Wildgans, Stefan Zweig und Felix Dörmann. Diss., Wien, I952.

3201. Wahrheit, Israel Albert. Jung-Wien as a Literary School. Schnitzler - Beer Hofmann Hofmannsthal. I 890-1914. Diss., University of Michigan, I940.

3202. Welter, M. L. Typus und Eros. Eine Untersucbung des Menschenbildes in den Novellen Stefan Zweigs. Diss., Freiburg i. Br., 1957.

3203. Werner, Bruno. Die deutschen Übersetzungen der Gedicbte Paul Verlaines. Diss., München, 1922.

3204. Wolff, Gerhart. Die Geschichte und ibre Künstler. Bewältigungen im Werk von Stefan Zweig. Ein Beitrag zum Problem geschichtlicher Wortkunst. Diss., Bonn, I958.

3205. Zohn, Harry. Stefan Zweig as Literary Mediator. Diss., Harvard University, I95 I.

\section{GENERAL REFERENCE WORKS}

3206. Autorenlexikon des $X X$. Jabrbunderts (Ed. Karl August Kutzbach). Bonn: Bouvier, I952, pp. 179-180.

3207. The Best Short Stories of 1926 (Ed. Edward J. O'Brien). New York: Dodd, Mead and Co., 1926, pp. 378, 464 .

3208. Bibliographia Philosopbica. 1934-r 945 . (Ed. G. A. De Brie). Vol. I/II. Bruxelles: Editiones Spectrum, 1950, f.m.

3209. Bibliographie der deutschen Literaturwissenschaft (Ed. Hanns W. Eppelsheimer). Vol. I/IV. Frankfurt am Main: Vittorio Klostermann, 1957-1961, Vol. I (1957), pp. 4I 7-4I 8; Vol. II (1958), pp. 8, 273, 293, 296; Vol. III (1960), p. I92; Vol. IV (I961), p. 236.

3210. Bibliographie deutscher Übersetzungen aus dem Französiscben. I700-1948 (Ed. Hans Fromm). Vol. I/VI. Baden-Baden: Verlag für Kunst und Wissenschaft, I950I953, f.m.

32 I I. Bibliographisches Handbuch des deutschen Schrifttums (Ed. Josef Körner). Bern: Francke, I 949 , p. 505 (3. ed.).

32 1 2. Bibliography Index. A Cumulative Bibliography of Bibliographies for 1943 (Ed. Joseph Bea). New York: H. W. Wilson, 1944, p. 243. 
3213. Bibliography of Critical and Biographical References for the Study of Contemporary French Literature (Ed. Douglas Alden, Gertrude Jasper, Robert Waterman). Vol. I/III. New York: Stechert-Hafner, I949-I954, Vol. I (1949), No. 4955; Vol. II (I95 I), No. 5 108; Vol. III (1954), No. I219I.

3214. The Bookman's Manual. A Guide to Literature (Ed. Bessie Graham). New York: R. R. Bowker, 194I (4. ed.; List of Zweig's books in English, by whom translated and when and where published).

321 5. Cassell's Encyclopaedia of Literature (Ed. S. H. Steinberg). London: Cassell, 1953, Vol. II, p. 2086.

3216. Chamber's Biographical Dictionary (Ed. J. O. Thorne). New York: St. Martin's Press, 1962, p. 1396.

3217. Collier's Encyclopedia (Charles P. Barry, Editor-in-Chief). Vol. I/XX with Index and Bibliography. New York: Collier and Son, I95 I ed., f.m.

321 8. Columbia Dictionary of Modern European Literature (Ed. Horatio Smith). New York: Columbia University Press, 1947, pp. 898-899 (Article on Zweig by William K. Pfeiler, University of Nebraska).

3219. The Columbia Encyclopedia in One Volume (Ed. William Bridgewater, Elizabeth Sherwood). New York: Columbia University Press, 1950, p. 2202 (2. ed.).

3220. Compton's Pictured Encyclopedia and Fact-Index. Vol. I/XV. Chicago: F. E. Compton, 1961, Vol. XV, p. 433.

3221. Current Biography. Who's News and Why. 1942. (Ed. Maxime Block). New York: H. W. Wilson, 1942, pp. 902-903 (Zweig obituary and short bibliography).

3222. Deutsche Dicbterbandschriften von 1400 bis 1900 . Ed. Wilhelm Frels). Leipzig: Hiersemann, 1934, p. 375 (Concerning Zweig's manuscript collection).

3223. Deutsche Geschicbte (Hubertus Prinz zu Löwenstein). Berlin: Haude und Spenersche Verlagshandlung, 1962 , p. 423.

3224. Deutsches Literaturlexikon (Ed. Wilhelm Kosch). Vol. I/II. Halle (Saale): Max Niemeyer, 1930, p. 3223.

3225. Deutsches Literatur-Lexikon. Biographisches und bibliographisches Handbuch (Ed. Wilhelm Kosch). Vol. I/IV. Bern: Francke, 1958, Vol. IV, pp. 3559-3560 (2. ed.).

3226. Diccionario de la Literatura (Ed. F. C. Sainz de Robles). Madrid: Aguilar, I950, p. 1652.

3227. Dictionnaire des Auteurs. Vol. I/II. Paris: Laffont/Bompiani, 1956, Vol. II, p. 729 (Article by Michel Mourre).

3228. Dictionnaire des Biographes (Ed. Pierre Crimal et al.). Vol. I/II. Paris: Presses Universitaires de France, I958, Vol. II, pp. I $562-1563$.

3229. Dictionnaire des Lettres (Ed. Pierre Clarac). Paris: Société d'Edition de Dictionnaires et Encyclopédies et Bompiani, 1961, p. 937.

3230. Dictionnaire des Oeuvres de tous les Temps et de tous les Pays. Vol. I/IV and Index. Paris: Laffont/Bompiani, 1955, Vol. I, p. 83 ("Amok"), p. 509 ("La Confusion des Sentiments"); Vol. III, p. 288 ("La Lutte avec le Démon"); Vol. IV, p. Is ("La Pitié dangereuse"), p. 705 ("Vingt-quatre Heures de la Vie d'une Femme").

323I. Dizionario Enciclopedico Italiano. Vol. I/XII. Roma: Instituto della Enciclopedia Italiana, 1961, Vol. XII, p. 1025.

3232. Dizionario Letterario Bompiani degli Autori di tutti Tempi e di tutte le Letterature. Vol. I/III. Milano: Bompiani, Vol. III, pp. 954-955.

3233. Dizionario Universale della Letteratura Contemporanea (Ed. Orlando Bernardi). Vol. I/IV. Milano: Mondadori, 1959, Vol. IV, pp. 1 259-1 26r.

3234. Enciclopedia Cattolica. Vol. I/XII. Città del Vaticano: Ente per l'Enciclopedia Cattolica e per il Libro Cattolico, I954, Vol. XII, pp. I834-1835 (Article by Sergio Lupi).

3235. Encilopedia de la Literatura (Ed. Benjamín Jarnés). Vol. I/VI. Mexico City: Editora Central, n.d., Vol. VI, pp. 428-430.

3236. Enciclopedia Italiana di Scienze, Lettere ed Arti. Vol. I/XXXVI and Index. Roma: Instituto della Enciclopedia Italiana, I939, f.m. (Cf. also Appendice. Vol. I/II (1938-1948), Vol. I, pp. 729, 1046; Vol. II, p. I I 44). 
3237. Enciclopedia Universal Illustrada - Europeo-Americana. Vol. I/X and Appendix. Bilbao-Madrid-Barcelona: Espasa-Calpe, 1933. Vol. X, p. I 326 (1930 ed., Vol. I/LXX. Vol. LXX, p. I 573 (Cf. also Supplemento 1942-r944, p. 309).

3238. Enciclopedia Vergara - Universal Didáctica Illustrada (Ed. Jaime Berenguer Amenós). Barcelona: Editorial Vergara, 196I, p. I546 (3 ed.).

3239. Encyclopedia Americana. Vol. I/XXX. New York-Chicago-Washington, D.C.: Americana Corp., I956, Vol. II, p. 639; Vol. XII, p. 589.

3240. Encyclopedia of Jewish Knowledge (Ed. Jacob De Haas). New York: Behrman's Jewish Book House, 1934, p. 66r.

3241. Encyclopédie des Citations (Ed. P. Dupré). Paris: Éditions de Trévise, 1959, Nos. 6174-6177 (Quotations from "Angst" and "Verwirrung der Gefühle" in French translation).

3242. Everyman's Encyclopedia. Vol. I/XII. New York: Macmillan, I95 I, Vol. XII, p. 853 (3. ed.).

3243. Der grosse Brockhaus. Handbuch des Wissens. Vol. I/XX. Leipzig: Brockhaus, 1935, Vol. XX, p. 74I (is. ed.; 16. ed., 1957, Vol. XII, p. 757).

3244. Der grosse Herder. Nachscblagewerk für Wissen und Leben. Vol. I/IX. Freiburg: Herder, I956, Vol. IX, p. I527.

3245. Grove's Dictionary of Music and Musicians (Ed. Eric Blom). London: Macmillan, 1954; New York: St. Martin's Press, I954, Vol. IX, Photogramme," 2. "Demuth - three songs with orchestra," 3. "Radó - songs with orchestra, translated from Verhaeren," 4. "Reger - two songs," 5. "Schibler - 'Augen des ewigen Bruders,' radio opera; 'Polyhem,' choral work," 6. "'Schweigsame Frau,' R. Strauss libretto," 7. "Seiber - 'Volpone,' translated from Ben Jonson," 8. "Szelényi - 'Virata,' oratorio," 9. "Toch - 'Heilige aus USA,,").

3246. The Guide to Catbolic Literature. I 888-1940 (Ed. Walter Romig). Part V. Detroit: Walter Romig, 1940, pp. 1 229-1 230.

3247. Harvard Guide to American History (Ed. Oscar Handlin et al.). Cambridge, Mass.: Harvard University Press, 1954, p. 205.

3248. Das kleine Lexikon der Weltliteratur (Ed. Hermann Pongs). Stuttgart: Union Verlag, 196r, p. 1757 (4. ed.).

3249. Kleines literarisches Lexikon (Ed. Wolfgang Kayser). Bern: Francke, I953, p. 580 (2. ed. rev.; Sammlung Dalp, Nos. I 5-1 7).

3250. Kleines literarisches Lexikon. Vol. II. Autorenlexikon des 20. Jabrbunderts (Ed. Horst Rüdiger). Bern-München: Francke, 1961, pp. 453-454 (3. ed.).

325 I. Kleines österreichisches Literatur-Lexikon (Ed. H. Giebisch et al.). Wien: Hollinek, 1948, pp. 536-537.

3252. Knaurs Buch vom Film (Ed. Rune Walderkranz, Arpe Verner). München: Droemersche Verlagsanstalt, i956, p. 468.

3253. Larousse du XXe Siècle (Ed. Paul Augé). Vol. I/VI. Paris: Libraire Larousse, I933, Vol. VI, p. I 445 .

3254. Lexikon der Weltiteratur (Ed. Heinz Kindermann, Margarete Dietrich). WienStuttgart: Humboldt, I95 I, pp. 90 I-902 (3. ed.).

3255. Lexikon der Weltliteratur (Ed. Gero von Wilpert). Stuttgart: Kröner, I963, p. I471.

3256. Lexikon der Weltliteratur im 20sten Jabrbundert. Vol. I/II. Freiburg-Basel-Wien: Herder, I96ז, f.m.

3257. The Lincoln Library of Essential Information. Buffalo, New York: The Frontier Press, 1959, p. 2010.

3258. Living Authors. A Book of Biographies (Ed. Tante Dilly). New York: H. W. Wilson, I931, pp. 461-462 (Contains Zweig portrait).

3259. Meyers Opernbuch. Leipzig: Bibliographisches Institut, 1935, pp. 438-439 (Otto Schumann, "Die schweigsame Frau").

3260. Das musikalische Theater. Ein Fübrer durch das Repertoire der Oper und Operette der Welt bis in die neueste Zeit (Ed. Francisco Curt Lange). Linz a. D.: Österreichischer Verlag für Belletristik und Wissenschaft, 1948.

3261. Pbilosophen-Lexikon. Handwörterbuch der Philosopbie nacb Personen (Ed. Werner 
Ziegenfuss, Gertrud Jung). Vol. I/II. Berlin: de Gruyter, I950, Vol. II, p. 957.

3262. Reallexikon der deutschen Literaturgeschichte (Ed. Paul Merker, Wolfgang Stammler). Vol. I/III. Berlin: de Gruyter, 1928-1929, Vol. III, pp. 394-402 (R. Leppla, "Übersetzungsliteratur").

3263. - Vol. I/IV. Berlin: de Gruyter, I93 I, f.m.

3264. Riemann Musik Lexikon (Ed. Willibald Gurlitt). Vol. I/III. Mainz: B. Schott's Söhne, 196r, Vol. II, pp. 742-745 (Cf. also the article "Richard Strauss").

3265. Der Romanfübrer. Teil III (Ed. Johannes Beer, Wilhelm Olbrich, Karl Weitzel). Vol. I/V. Stuttgart: Hiersemann, 1954, pp. 984-987 (Plot summaries of Ungeduld des Herzens, Erstes Erlebnis, "Amokläufer," "Verwirrung der Gefühle," Sternstunden der Menschbeit, Schacbnovelle).

3266. Salmonsens Konversations Leksikon (Ed. Johannes Brøndum-Nielsen, Palle Raunkjaer). Vol. I/XXV. København: Schultz, 1928, Vol. XXV, pp. 659-66o.

3267. Der Schauspielfiubrer (Ed. Joseph Gregor). Stuttgart: Hiersemann, I954, Vol. II, pp. $25-26$ ("Volpone"), t-p. 26-28 ("Die Flucht zu Gott").

3268. Schweizer Lexikon. Vol. I/VII. Zürich: Encyclios Verlag, 1948, VII, p. I671.

3269. 60 Years of Best Sellers. I895-1955 (Ed. Alice Payne Hackett). New York: R. R. Bowker, I956, pp. I55, I59-160.

3270. Standard Catalog for Public Libraries. Literature and Pbilology Section (Ed. Minnie Earl Sears). New York: W. W. Wilson, I93 I, p. 99.

3271. A Treasury of Jewish Quotations (Ed. Joseph L. Baron). New York: Crown, I956, f.m.

3272. Twentieth Century Authors (Ed. Stanley Kunitz, Howard Haycraft). New York: H. W. Wilson, 1942, pp. 1576-1577 (Contains a copy of Zweig's "Abschiedsbrief"; Cf. also Supplement I, I955, p. I I 23).

3273. The Universal Jewish Encyclopedia (Ed. Isaac Landman). Vol. I/X. New York: Universal Jewish Encyclopedia Co., 1943, Vol. X, p. 680 (Article by Emil Langyel; Also contains a Zweig bibliography).

3274. Who Was Who. 1941-1950. London: Adam and Charles Black, 1952, Vol. IV. p. I 277. 3275. Winkler Prins Encyclopaedia (Ed. E. De Bruyne, G. B. J. Hiltermann, H. R. Hoetink). Vol. I/XVIII. Amsterdam-Brussel: Elsevier, 1954, Vol. XVIII, p. 871.

3276. A World Bibliography of Bibliographies and of Bibliographical Catalogues, Calendars, Abstracts, Digests, Indexes and the like (Ed. Theodore Besterman). Vol. I/III. Genève: Societas Bibliographica, I955, Vol. III, col. 4408.

3277. The World Book Encyclopedia. Vol. I/XVIII. Chicago: Quarrie, 1947, Vol. XVIII, p. 9012.

3278. Writings on American History. 1902-1940. Washington, D.C.: American Historical Association, 1937, col. 894 .

\section{HISTORIES OF LITERATURE}

3279. Alker, Ernst. Geschicbte der deutschen Literatur von Goethes Tod bis zur Gegenwart. Stuttgart: Cotta, I950, Vol. II, pp. 38 I-382.

3280. Bennett, E. K. and H. M. Waidson. A History of the German Novelle. Cambridge: University Press, I961, pp. 272-273, 276 (2. ed.).

3281. Bertaux, Félix. A Panorama of German Literature from 1871 to I931. New York: McGraw Hill, 1935, pp. 176, 197-198, 277, 325.

3282. Bithell, Jethro, ed. Germany. A Companion to German Studies. London: Methuen, 1959, p. 366 (5. ed.).

3283. - Modern German Literature. 1880-1950. London: Methuen, 1959, f.m. (Cf. esp. pp. 384-385; 3. ed. rev.).

3284. Boesch, B. Deutsche Literaturgeschichte in Grundzïgen. Bern: Francke, 1946, pp. 33033 I (2. ed. Bern-München: Francke, 1946, pp. 386, 403). 
3285. Brand, Guido K. Werden und Wandlung. Berlin: K. Wolff, I933, pp. 45, 60, I 17. 3286. Castle, Eduard et al. Deutsch-österreichische Literaturgeschichte. Vol. I/IV. Wien: Frommer, I937, Vol. IV, pp. I884-I889 (Article "Stefan Zweig" by Erwin Rieger).

3287. Clark, Barrett H. and George Freedley, eds. A History of Modern Drama. New York: Appleton-Century-Crofts, 1947, pp. I44-I 45 .

3288. Diebold, Bernhard. Anarchie im Drama. Frankfurt am Main: Frankfurter Verlagsanstalt, I92I, pp. $43 \mathrm{I}-433$ (Discussion of Jeremias as a drama of pacifism).

3289. Duwe, Wilhelm. Deutsche Dichtung des 2osten Jabrbunderts. Vol. I/II. Zürich: Drell Füssli, 1962, f.m.

3290. Edschmid, Kasimir. Lebendiger Expressionismus. Auseinanderseztungen, Gestalten, Erinnerungen. Wien-München-Basel: Kurt Desch, 1961, pp. 22, 47, 199.

3291. Eloesser, Arthur. Die deutsche Literatur von der Romantik bis zur Gegenwart. Berlin: Bruno Cassirer, 193 I (Tr. into English by C. A. Phillips as Modern German Literature. New York: Knopf, 1933, p. 393 (2. ed.)).

3292. Franwallner, E., H. Giebisch and E. Heinzel. Die Welt-Literatur. Vol. I/II. Wien: Hollinek, 1953, f.m.

3293. Frenzel, Herbert A., ed. Daten deutscher Dichtung. Köln-Berlin: Kiepenheuer and Witsch, 1953, pp. 360, 368, 4I I, 41 5 ; Biography p. 344).

3294. Fricke, Gerhard et al. Geschichte der deutschen Dicbtung. Lübeck-Hamburg: Matthiesen, 1962, pp. 355-356 (9. ed.).

3295. Friedmann, Hermann and Otto Mann, eds. Deutsche Literatur im 2osten Jabrbundert. Heidelberg: Wolfgang Rothe, 1956, pp. 48, 131, 31 1, 324 (2. ed. rev.).

3296. Friedrich, Werner P. An Outline History of German Literature. New York: Barnes and Noble, 1948, pp. 226-227, 250 (College Outline Series; 5. printing, 1960).

3297. Fuchs,Albert. "Stefan Zweig," Moderne österreichische Dicbter.Wien: Globus-Verlag, I946, pp. 55-59.

3298. Garten, H. F. Modern German Drama. Fair Lawn, New Jersey: Essential Books, 1959, pp. I 23, I 25-I 26, 24I.

3299. Hieble, Jacob. An Outlibe of German Literature with Reading References to Cheap German Editions and English Translations. Ithaca: The Thrift Press, 1935, pp. 30-3I.

3300. Highet, Gilbert. A Clerk of Oxenford. Essays on Literature and Life. New York: Oxford University Press, 1954, p. 239.

330r. Horst, Karl August. Die deutsche Literatur der Gegenwart. München: Nymphenburger, 1957, pp. 64-65, 99, 209 (Zweig portrait p. 96).

3302. - Kritischer Fübrer durch die deutsche Literatur der Gegenwart. München: Nymphenburger, 1962 , f.m.

3303. Kindermann, Heinz. Wegweiser durch die moderne Literatur in Österreich. Innsbruck: Österreichische Verlagsanstalt, I954, p. I 2.

3304. Klein, Johannes. Geschicbte der deutschen Novelle von Goethe bis zur Gegenwart. Wiesbaden: Franz Steiner, 1956, pp. 502-5 I 2 (3. ed.).

3305. Körner, Josef. Bibliographisches Handbuch des deutschen Schrifttums. Bern: Francke, 1949, p. 505 (3. ed.).

3306. Krell, Leo and Leonhard Fiedler. Deutsche Literaturgeschichte. Bamberg: Buchners, I962, pp. 372, 400 (9. ed.).

3307. Kummer, Friedrich. Deutsche Literaturgescbicbte des Igten und 20sten Jabrbunderts. Vol. I/II. Dresden: Carl Reissner, 1924, Vol. II, p. 45 I.

3308. Kutscher, Artur. Stilkunde der deutschen Dichtung. Vol. I/II. Bremen-Horn: Dorn, I952, Vol. I, p. 33; Vol. II, p. 244.

3309. Laaths, Erwin. Geschichte der Weltliteratur. Eine Gesamtdarstellung. München: Knauer, 1953 , p. 727 (3. ed.).

33 Iо. Langer, Norbert. "Stefan Zweig," Dichter aus Österreich. Wien: Österreichischer Bundesverlag, I957, pp. I 22-I 27.

33 I I. Lennartz, Franz. Die Dichter unserer Zeit. Stuttgart: Kröner, 1952, pp. 571-573 (5. ed.). 
331 2. Lockemann, Fritz. Gestalt und Wandlungen der deutschen Novelle. Geschicbte einer literarischen Gattung im I9ten und 20sten Jabrbundert. München: Max Hueber, I957, pp. 364-366.

3313. Lueth, Paul E. H. Literatur als Geschicbte. Deutsche Dicbtung von I88, bis 1947. Vol. I/II. Wiesbaden: Limes, 1947, Vol. I, p. II I.

33 14. Maderno, Alfred. Die deutsch-österreichische Dichtung der Gegenwart. Leipzig: Gerstenberg, 1920, pp. 39, $175,260$.

331 5. Martini, Fritz. Deutsche Literaturgescbichte. Stuttgart: Kröner, I960, pp. 457, 48 5-486. 3316. Meyer, Richard M. Die deutsche Literatur des Igten und 2osten Jabrbunderts. Berlin: Bondi, 1921, p. 638 (Volksausgabe).

3317. Mittner, Ladislao. La Letteratura tedesca del Novecento. n.l.: Ein-audi Editore, 1960, p. 183 .

33 18. Mumbauer, Johannes. Die deutsche Dichtung der neuesten Zeit. Vol. I/II. Freiburg i.B.: Herder, I93 I, Vol. I, pp. 528-532.

3319. Nadler, Josef. Literaturgescbichte Ósterreichs. Salzburg: Otto Müller, 195 I, pp. 43 I433, 437 (2. ed.).

3320. Naumann, Hans. Die deutsche Dichtung der Gegenwart. Stuttgart: Metzler, 1924, p. 77.

3321. Pellegrini, Carlo. Storia della Letteratura e d'America. Vol. I/VI. Milano: Vallardi, 1958-1960 Vol. IV, pp. 371-373, 4I I, 470.

3322. Pongs, Hermann. Das Bild in der Dichtung. Vol. I/II. Marburg: Elwert, 1927, f.m.

3323. - Im Umbruch der Zeit. Das Romanschaffen der Gegenwart. Göttingen: Göttinger Verlagsanstalt, 1956, pp. I49-150, 154.

3324. Prampolini, Giacomo. Storia universale della Letteratura. Vol. I/VII. Torinese: Unione Tipografico Editrice, n.d.; Cf. esp. Vol. VI, pp. 496-498.

3325. Priestley, J. B. Literature and Western Man. New York: Harper, 1960, pp. 333, 336. 3326. Robertson, J. G. A History of German Literature. Edinburgh-London: William Blackwood, 1959, p. 563 (3. ed.; 4. ed. rev. Edna Purdie et al., New York: British Book, Centre, 1962, p. 563 ).

3327. Rocca, Enrico. Storia della Letteratura tedesca dal 1870 al 1933. Firenze: Samsoni, I950, f.m. (Cf. esp. pp. 285-304).

3328. Rose, Ernst. $A$ History of German Literature. New York: New York University Press, 1960, p. 291.

3329. Salzer, Anselm. Illustrierte Gescbichte der deutschen Literatur von den ältesten Zeiten bis zur Gegenwart. Vol. I/V. Regensburg: Josef Habbel, I931, Vol. IV, pp. 1666-1667, I 739; Vol. V, p. 2288.

3330. Samuel, Richard and Thomas R. Hinton. Expressionism in German Life and Literature and the Theater. 1910-1924. Cambridge: W. Heffer, 1939, f.m.

333r. Scherer, Wilhelm and Theodor Schultz. Geschichte der deutschen Literatur. Wien: Concordia, 1948, p. 695.

3332. Schmidt, Adalbert. Deutsche Dichtung in Österreich. Wien-Leipzig: Adolf Luser, 1935, pp. 24, 31, I23, 201.

3333. Schmitt, Fritz and Gerhard Fricke. Deutsche Literaturgeschicbte in Tabellen. Vol. I/III. Bonn: Athenäum, 1952, Vol. III, pp. 238, 283.

3334. Soergel, Alabert and Curt Hohoff. Dichtung und Dicbter der Zeit. Vol. I/II. Düsseldorf: August Bagel, 1961, Vol. I, p. 421 ; Vol. II, pp. 448-450(Reprinted 1963).

3335. Walzel, Oskar. Die deutsche Dichtung seit Goethes Tod. Berlin: Akademischer Verlag, I 920 , pp. 297, 43 I-432, 489, 49I (5. ed. I929, pp. I 5 I ff.).

3336. Weiskopf, Franz Karl. Unter fremden Himmeln. Ein Abriss der deutschen Literatur im Exil. 1933-1947. Berlin: Dietz, 1948, f.m.

3337. Wiegand, Julius. Geschicbte der deutschen Dicbtung. Köln: Schaaffstein, 1922, pp. $365-$ 39I (Excellent discussion of New Romanticism).

3338. Wiegler, Paul. Gescbicbte der deutschen Literatur. Vol. I/II. Berlin: Ullstein, 1930, Vol. II, pp. 821,858 .

3339. Wilpert, Gero von. Deutsche Literatur in Bildern. Stuttgart: Kröner, 1957, pp. 786, 812. 
Films Made from Zweig's Works (Cf. Knaurs Buch vom Film. Ed. Rune Walderkranz and Werner Arpe. München: Droemersche Verlagsanstalt, 1956, p. 468; Blätter, I (Apr., 1958), 8).

3340. "Amok," I927 (Russia; Directed by Konstantin Mardschanow).

3341. - I934 (France; Directed by Fedor Ozep).

3342. "Angst," I 928 (Germany; Directed by Hans Steinhoff).

3343. - I934 (France; Directed by Viktor Tourjansky).

3344. - I954 (Germany; Directed by Roberto Rosellini, starring Ingrid Bergmann).

3345. "Brennendes Geheimnis," I 933 (Germany; Directed by Robert Siodmak; Cf. also $N Y T$, Nov. 6, 1946, p. 30 ("M.G.M. Buys Film Rights to Story 'Burning Secret"')).

3346. "Brief einer Unbekannten" ("Narkose"), I 929 (Germany; Directed by Alfred Abel).

3347. - I943 (Finnland; Directed by Hannu Leminen, starring Helena Kara).

3348. - 1948 (USA; Directed by Max Ophüls, starring Joan Fontaine).

3349. "Das gestohlene Jahr" (Unpublished scenario with the collaboration of Berthold Viertel), I95 I (Austria; Directed by Wilfred Frass, starring Ewald Baseler).

3350. Das Haus am Meer (Drama), I 924 (Germany; Directed by Fritz Kaufmann, starring Asta Nielsen and Albert Steinrück).

335 I. Marie Antoinette (Biography), I938 (USA; Directed by W. S. van Dyke, starring Norma Shearer, Tyrone Power and John Barrymore).

3352. "Schachnovelle" (Film version written by Herbert Reinecker, Harold Medford and Gerd Oswald), 1960 (Austria; Starring Curd Jürgens; German premiere in Austria Sept. 2, I960 (Wien); English premiere Sept. I, I960; Cf. rev. in Die neue Film-Post, 7.J.: 4 (1960)).

3353. "Das Schicksal einer Nacht" (Sternstunden der Menscbbeit), I 927 (Germany; Directed by Erich Schönfelder, starring Erna Morena and Harry Liedtke).

3354. Ungeduld des Herzens (Novel), 1946 (England; English title Beware of Pity; Directed by Maurice Elvey, starring Lilli Palmer and Albert Lieven).

3355. "Vierundzwanzig Stunden aus dem Leben einer Frau," I 944 (Argentina; Directed by Carlos Borcosqu, starring Amelia Bence).

3356. - I952 (England; English title Twenty-four Hours in the Life of a Woman; Produced by Associated British Picture Corp.; Released in USA in 1953 as Affair in Monte Carlo by the Allied Artists Production; TV version written by John Mortimer in 1960, starring Ingrid Bergmann and Maximilian Schell).

Zweig's Works set to Music:

3357. Demuth, Norman. Three poems (1944).

3357 a. Ebenhöh, Horst. "Virata oder die Augen des ewigen Bruders" (Used as text basis for an oratorio in six scenes; Cf. Blätter, 8/10(Oct., I960), 21).

3357 b. Radó, Aladar. Four poems by Emile Verhaeren in Zweig's translation (I9I3).

3357 c. Reger, Max. Two poems ("Ein Drängen ist in meinem Herzen" and "Neue Fülle").

3358. Röntgen, Johannes. "Two poems from 'Musik der Jugend' (For soprano with instrumental accompaniment; Amsterdam: Alsbach, 1936).

3359. Schibler, Arnim. "Polyphem" (Cantata for Chorus, solo-tenor and two pianos; Opus 34; Zürich: Selbstverlag, 1952). Radio opera based on "Die Augen des ewigen Bruders."

3359a. Seiber, Matyas. Incidental music to Volpone.

3359 b. Strausz, Richard. Music to the text Die schweigsame Frau.

3359c. Szelényi, Istvan. Oratorio based on "Varata oder die Augen des ewigen Bruders".

3359 d. Toch, Ernst. Music to the text of "Die Heilige aus U.S.A." (1932). 
Recordings of Zweig's Works:

3360. "Brief einer Unbekannten," Read by Ruth Leuwerik. Deutsche Grammophon Gesellschaft, No. 440I I.

3361. "Novemberwind," (Original text by Emile Verhaeren in French, translated into German by Stefan Zweig). Shortened version read by Alexander Moissi. Monarch Record Gramophone, No. 041-023 (Cf. Blätter, I (Apr., 1958), 7).

3362. "Schachnovelle," Read by Curd Jürgens. Amadeo, No. AM-AVRS ror s.

3363. "Vierundzwanzig Stunden aus dem Leben einer Frau," Read by Lil Dagover. Deutsche Grammophon Gesellschaft, No. 44009.

3364. German tape entitled "Stefan Zweig" available at the German Consulate, Detroit, Michigan. Tape No. 358 ( $27 \mathrm{~min} .30 \mathrm{sec}$.).

3365. "Zweig im 'Osterreichischen Rundfunk'," Blätter, 8/10(Oct., 1960), 22.

\section{Further Reference Works to be Consulted:}

3366. Bürgin, Hans. Das Werk Thomas Manns. Eine Bibliographie (unter Mitarbeit von Walter A. Reichert und Erich Neumann). Frankfurt am Main: Fischer, 1959, Nos. I, Ioa; III, 4 ; V, 447.

3367. Garraty, John A. The Nature of Biography. New York: Knopf, 1957, pp. I 26, I 31. 3368. Jonas, Klaus W. Fifty Years of Thomas Mann Studies. A Bibliography of Criticism. Minneapolis: University of Minnesota Press, 1955, Nos. 760, 764, 1749, 2635. 3369. Reference to Zweig's Tolstoi essay in $B A$, III : 3 (July, 1929), 296-297 in H. G. Wendt's review of Philipp Witkop's Leo Tolstoi. Wittenberg: Ziemsen, 1928.

3370. The Dutch fragment from Jeremias (Cf. item roo) was originally chosen as the lustrum-play (7oth anniversary) of the Christian Philanthropic Institute at Doetinchem, Holland. The whole Jeremias was performed in 1938 as the lustrum-play (2nd anniversary) of the Vrijzinnig Christelijke Jeugd Centrale (in Dutch translation) at Ermelo, Holland. Translation and adaptation by Halbs C. Kool; Leader of the performance was the late Abraham van der Vies.

I have seen references to the following but bave not been able to trace them down:

3371. Articles about Zweig in the following:

Aufbau, I 2 (1946).

Der Bogen, 2.J.: I 3 (1946).

Das goldene Tor, 6 (1947).

Neue Auslese, 2.J.: 2 (1947).

Wiener Revue. Ein Jabr Wiener Theater, Folge 7/8 (1946).

\section{GERMAN MANUSCRIPTS IN ZWEIG'S MANUSCRIPT COLLECTION}

3372. Frels, Wilhelm. Deutsche Dicbterbandschriften von 1400 bis 1900 (Gesamtkatalog der eigenhändigen Handschriften deutscher Dichter in den Bibliotheken und Archiven Deutschlands, Österreichs, der Schweiz und der CSR). Leipzig: Verlag von Karl W. Hiersemann, 1934, p. 375.

Salzburg. Sammlung Stefan Zweig, Besitzer: Dr. Stefan Zweig, Kapuzinerberg 5. Nur Handschriften, keine Briefe. Aufnahme nach dem Bestand 1928 bis 1929.

I. Arndt, Ernst Moritz (1 769-1 860): 2 Gedichte (p. 10).

2. Arnim, Achim von (Ludwig Acbimo) (178I-1831): Gedicht "Der Leuchtturm," (p. I I). 
3. Arnim, Bettina v., geb. Brentano (1785-1859): I Gedicht "Petöfy dem Sonnengott," (p. I 3 ).

4. Bäuerle, Adolf Johann (1786-1859): Ms. e. Kritik (5 I/2 S.) (p. 16).

5. Bauernfeld, Eduard von (I802-1890): I Einakter "Der Zweifler" (1827) (p. 18 ).

6. Bierbaum, Otto Julius (1865-1910): 2 Gedichte (p. 25).

7. Blumauer, Joh. Alsys (1755-1798): Ein Bruchstuick der Aeneide (Buch 7, $55-88)($ p. 27).

8. Bodenstedt, Friedrich von (1819-1892): 2 Gedichte (p. 29).

9. Börne, Ludwig (I 786-1837): "Briefe aus Paris," Anfang (I/2 S.) u. I Sinnspruch (p. 30).

Iо. Brentano, Clemens (1778-1842): 5 Gedichte (p. 37).

I I. Chamisso, Adalbert von (1781-1 838): einige Gedichte (p. 46).

I2. Claudius, Matthias (1740-18I5): I Gedicht "Ihr Leute gross u. klein," (p. 48).

13. Cornelius, Peter (1824-1 874): I Gedicht an Liszt (p. 50).

14. Dehmel, Richard (1863-1920): 2 Gedichte (p. 55).

I5. Droste-Hülshoff, Annette Elizabeth Freiin von (1797-1848): 4 Gedichte $\left(2 \mathrm{~S} .8^{\circ}\right)($ p. 58$)$.

16. Eckermann, Johann Peter (1792-1854): 3 Gedichte (p. 62).

17. Eichendorff, Joseph Freiherr v. (1788-1857): mehrere Mss. (p. 63).

18. Fontane, Theodor (1819-1898): Entwurf u. Inhaltsskizze von "Cécile" mit 3 Briefen, das Gedicht "Der alte Derffling" mit Begleitbrief (p. 71).

19. Fouqué, Friedrich Heinrich Karl Baron de la Motte (1777-1843): einige Gedichte (p. 73).

20. Freiligrath, Ferdinand (1810-1876): 2 Gedichte (p. 76 ).

21. Geibel, Emanuel (I 8I 5-I 884): 2 Gedichte (p. 8I).

22. Gellert, Christian Fürchtegott (1715-1769): Ode auf den Tod Bernhard Christian Willes (p. 83).

23. Gleim, Johann Wilhelm Ludwig (1719-1803): I Gedicht an Jean Paul (1798) (p. 89).

24. Goethe, Johann Wolfgang von (1749-1832):

a. 52 Verse aus Faust II, Akt I (Plutusszene),

b. Brautlied aus d. Maskenzuge i $8 \mathrm{I}$ o,

c. 2 S. aus Vorarbeiten zu d. "Principes de philosophie zoologique" Tl. 2,

d. Gedichte: Eigenthum Im May (“Zwischen Weizen u. Korn")

An Amalie Wolf I 812

e. I Seite Sprüche aus d. Westöstl. Divan,

f. "Will einer sich gewöhnen" aus d. Zahmen Xenien V,

g. Die 2 letzten Strophen aus "An die verehrten I8 Frankfurter Festfreunde,"

h. Hochzeitsgedicht für Willemers "Reicher Blumen goldene Ranken" (I8I5),

i. 2 S. aus d. Jugend arbeiten 1759 (p. 96).

25. Gotthelf, Jeremias (wirklicher Name: Albert Bitzius) (1 797-г 854): Vorwort zu "Zeitgeist u. Bernergeist" (4 S.) (p. 98).

26. Gottsched, Johann Christoph (1700-1766): I Gedicht "Du bist des Phöbus Sohn" (173I) (p. 99).

27. Grabbe, Christian Dietrich (1 80I-1 836): ein Fragment aus "Don Juan u. Faust" (2. S) u. eines aus d. "Hermannsschlacht," erster Entwurf (8 S.) (p. 100).

28. Grillparzer, Franz (I 79I-1 872): 3 Gedichte: "Napoleon I821," "Vision," "Entsagung," (p. 104).

29. Groth, Klaus (1819-1899): Ms. "Vor de Gorn" (106 S.) mit vielen unveröffentlichten Gedichten (p. I06). 
30. Grün, Anastasius (wirklicher Name: Anton Alexander Graf von Auersperg) (1 806-1876): I Gedicht u.a. (p. 107).

31. Gryphius, Andreas (1616-1664): I Sonett "Was sindt wir Menschen doch" (1640) (p. 107).

32. Hagedorn, Friedrich von (1708-1754): I Promemoria über Voltaires Rechtgläubigkeit (p. I I I).

33. Haller, Albrecht von (1 708-1 777): I Gedicht an Gessner (p. I I 3).

34. Halm, Friedrich (wirklicher Name: Franz Joseph Eligius Reichsfreiherr von Münchhausen-Belling) (I 806-1871): I Gedicht "Beim Tode Lenaus," (p. II 3 ).

35. Hamann, Johann Georg (1730-1788): I Ms. von 8 S. (vgl. Klüber im Jahrbuch d. Goethe-Ges. I5. S. 89f.) (p. II4).

36. Hamerling, Robert (1830-I 889): I Gedicht (p. I I5).

37. Hammer-Purgstall, Joseph von (I774-1856): 3 Gedichte (p. II 5 ).

38. Hardenberg, Friedrich von (Deckname: Novalis) (1772-1 801 ):

a. Das Gedicht "Der Fremdling" (Anfang)

b. I Sonett an d. Bruder Karl (auf demselben Blatt noch ein Verzeichnis d. Werke J. Böhmes)

c. Trinklied u. Liebesgedicht (2. S).

d. Eingangsgedicht zum 2. Tl. des Ofterdingen (4 S.)

e. Fragment aus d. 2. Tl (I/2 S.)

f. I Gedichtfragment "Die Vermählung der Jahreszeiten" (2 S.) (p. I I6)

39. Hartleben, Otto Erich (I 864-1905): I Gedicht "Franzensfeste," (p. I I 8).

40. Hauff, Wilhelm (1802-1827):

I Gedicht "Serenade"

2 S. Notizen zu d. Skizzen aus Schwahen u. zum Fischerstechen (p. I I g).

41. Haug, Johann Christoph Friedrich (1761-1 829): I Gedicht an Hebel (p. I 20).

42. Hebbel, Friedrich (1813-1863): Novelle "Schnock" u. Schluss des Vorspiels der Niebelungen (p. 1 22).

43. Hebel, Johann Peter (1760-1826): 2 S. in $4^{\circ}$ mit Erzählungen (p. 122).

44. Heine, Heinrich (1797-1856): "Die Weber" in der ersten Fassung, I Gedicht aus d. Wintermärchen, erste Fassung, r/2 S. mit 3 Gedichten (p. 124).

45. Heinse, Wilhelm (1746-1803): I Gedichtfragment "In einem Tal am Fusse des Vesuv..." (p. 125).

46. Herder, Johann Gottfried (1 744-1 803): 2 Gedichte (p. 1 28).

47. Herwegh, Georg (1817-1875): I Gedicht "Den Reichstäglern," (p. 130).

48. Heyse, Paul (I830-19I4): Die Novelle "Einer von Hunderten" (45 S.) u. I Gedicht an Georg Scherer 1898 (p. 132).

49. Helle, Peter (1 854-1 904): 10 Gedichte (p. I 32 ).

50. Hölderlin, Friedrich (1770-1 843 ):

a. I6 Gedichte

b. Die Fragmente des Pindar 3-9 (früher in der Slg. der Frhrn v. Bernus)

c. Fragment aus d. Hyperion (6 vom gedruckten Text abweichende Briefe)

d. Die Abschrift Sinclairs von "Patmos," (p. I35).

51. Hölty, Ludwig Christoph Heinrich (1748-1776): I Gedicht "Aufmunterung zur Freude" u. "Maylied," (p. 135).

52. Hoffmann, Ernst Theodor Amadeus (1 776-1 833): "Kreislers musikalische Leiden," u. 2 S. aus d. Erzählung "Neueste Schicksale des Hundes Berganza."

53. Hoffmann von Fallersleben, August Heinrich (1798-1874): 3 Gedichte.

54. Jean Paul (d.i. Johann Paul Friedrich Richter) (1763-1825): 4 S. "Vom Verfasser der Auswahl aus des Teufels Papieren" u. Ms. der I. Fassung der "Rede des toten Christus vom Weltgebäude herab."

55. Immermann, Karl Lebrecht (1796-1840): 2 Sonette. 
56. Karschin, Anna Luisa (1722-1 791): 2 Gedichte.

57. Keller, Gottfried (1819-1890): "Feueridylle" (erste Fassung - I3 S. in gr. $-4^{\circ}$.

58. Kerner, Justinus (richtig Justinus Andreas Christian) (1 786-1 862): Gedicht "Der Wanderer in der Sägemühle" u. I Klexographie mit Gedicht I 854.

59. Kinkel, Johann Gottfried (I8I 5-I882): I Gedicht I 862 "Die Veteranen des Exils."

6o. Kleist, Ewald Christian von (1715-1759): 4 S. in $8^{\circ}$ mit I Gedicht "Einladung aufs Land" $u$. einigen Epigrammen.

6r. Kleist, Heinrich von (1777-I8I I): Fabel "Die Bedingung des Gärtners" u. 6 Gedichte: Sonett an d. Königin Louise (3. Fassung), An Friedrich Wilhelm III., Germania an ihre Kinder, an Kaiser Franz I., Kriegslied der Deutschen, An Wilhelmine von Zenge: "Nicht aus des Herzens Blossem Wunsche."

62. Klopstock, Friedrich Gottlieb (1724-1803): Ode "An die rheinischen Republikaner."

63. Körner, Karl Theodor (I79I-I8I3): Der Einakter "Die Blumen"(I S. in gr. $\left.2^{0}\right)$.

64. Kotzebue, August Friedrich Ferdinand von (1761-1819): Ms. des Lustspiels "Der Hagestolz u. die Körbe."

65. Laube, Heinrich (I 806-I 884): I S. mit d. Abschiedsworten am Grabe Grillparzers.

66. Lenau, Nikolaus (wirklicher Name: Nikolaus Franz Niembsch Edler von Strehlenau) (1802-1850): Das Widmungsgedicht zu den Albigensern, die Gedichte: Crucifix, Der Maskenball, Die Waldkapelle, sämtliche Schilflieder u. I Gedichtentwurf von 6 Versen.

67. Lenz, Jakob Michael Reinhold (1751-1 792): Gedicht "Ach, wo brennt sie himmlisch schön...".

68. Lessing, Gotthold Ephraim ( I 729- I 78I): I lat. Stammbuchblatt f. Seydel.

69. Leuthold, Heinrich (1827-1 879): I Gedichtentwurf.

70. Lichtenberg, Georg Christoph (1742-1 799): 2 S. Aphorismen u. Sentenzen.

71. Liliencron, Detlev Freiherr von (1844-1909): Ms. der Novelle "Die Mergelgrube" (39 S.), Prosagedicht "Schmetterlinge" ( $1 / 2$ S.), Korrekturbogen des Gedichts "Das verschüttete Dorf” mit Anmerkungen L's.

72. Lingg, Hermann von (1820-1905): 2 Gedichte.

73. Ludwig, Otto (1813-1865): Fragment e. Dramas.

74. Meyer, Conrad Ferdinand (I825-I898): I Gedicht "Lenz, wer kann Dir widerstehen!"

75. Mörike, Eduard (1 804-1 875): Gedicht, “Jedem das Seine”.

76. Müller, Wilhelm (1794-1 827): I Br. an Ludw. Tieck I4.Io.1826.

77. Nietzsche, Friedrich (1844-1900): Gedichtzyklus "Heimkehr" I 863 u. Novellenanfang "Euphorion," "Die Geburt des tragischen Gedankens" (Vorarbeit zur Geburt d. Tragödie), letzter Brief an Avenarius.

78. Opitz, Martin (I597-1639): I lat. Gedicht.

79. Pestalozzi, Johann Heinrich (I 746-I 827): I Fragment aus e. Roman(2. S.).

80. Pfeffel, Gottlieb Konrad (1736-1809): I Gedicht "Der schwarze Schwan."

81. Platen-Hallermünde, August Graf v. (I 796-1835): Gedicht "Gnome."

82. Raimund (eigentlich Raimann), Ferdinand (1790-1836) I I/2 Bl. zu "Der Bauer als Millionär," I Gedicht "An Gutenstein."

83. Ramler, Karl Wilhelm (1725-1798): 2 Gedichte "Amyntas" u. "Der zerbrochene Krug."

84. Reuter, Fritz (I810-1 874): Gedicht "Hei is dod."

85. Rilke, Rainer Maria (1875-1 926): Die I. Fassung d. "Weise von Liebe u. Tod des Cornets Chr. Rilke." s Gedichte: "Vor Ostern," "Die Greisin," "Das Bett," "Papageienpark," "Archäischer Torso Apollos." 
86. Rodenberg, Julius (183I-I9I4): I Gedicht.

87. Rosegger, Peter (1843-1918): Ms. der Novelle "Der Lachenmacher."

88. Rückert, Friedrich (I 788-1 866): Gedicht "Jusuff u. Suleika."

89. Saar, Ferdinand von (1833-1906): 2 Gedichte.

90. Scheffel, Joseph Viktor (seit 1 876: von) (1826-1 886): Gedicht "Waidmann u. Minne."

92. Schiller, Friedrich von (1759-1805):

2 Fragmente aus Phädra,

I aus Don Carlos,

7 Xenien,

2 S. mit Gedichtentwürfen.

92. Schlegel, August Wilhelm (1 767-1 845$)$ : 2 Gedichte "Auf die Taufe eines Negers" u. "Die veredelte Hexenzunft."

93. Schlegel, Friedrich (1772-1829): Aufsatz "Über die Aussichten der Kunst im österr. Kaiserstaat" (1812) (I6 S.).

94. Seidel, Heinrich (I 842-I 906): Ms. der Novelle "Eva."

95. Seume, Johann Gottfried (1763-1810): Elegie "An das deutsche Volk" (I 8 Io) u. das Ms. von "Mein Leben."

96. Stelzhamer, Franz (1802-1874): I Gedicht "Radikale Lieder. I."

97. Stolberg, Friedrich Leopold Graf zu (1750-1819): Gedicht "An Agnes" usw.

98. Storm, Theodor (1 817-I 888): Ergänzung d. Novelle "Im Sonnenschein" ( 12 S.) u. der Gedichtzyklus "Die rote Rose" (4 S.).

99. Strachwitz, Graf Moritz von (1822-1847): I Liebesgedicht an Harrie.

I00. Tieck, Ludwig (1773-1853): I S. zum Versdrama "Isodir u. Olga" u. I Buchbesprechung (I S.).

I0I. Uz, Johann Peter (1720-1 796): I Gedicht "Hagedorn" (1744).

102. Varnhagen von Ense, Karl August (I $785-1858): 4$ Gedichte.

103. Varnhagen von Ense, Rahel, geb. Levin (1 771 1-1 833): Eine philosophische Abhandlung 13.9.1820.

104. Vogl, Johann Nepomuk (1 802-1 866): 3 Gedichte.

ro5. Voss, Johann Heinrich (175 I-I 826): "Idylle," "Die Bleicherin."

106. Vulpius, Christian August (1762-1 827): Aufsatz von I r/2 S.

107. Wackenroder, Wilhelm Heinrich (1773-1798): 2 Entwürfe "Sprachbemerkungen über die Minnesinger" u. "Wörter u. Redensarten aus Hans Sachs."

ro8. Wagner, Richard (1813-1883): 8 S. aus "Siegfried," Akt I, Szene I, u. Festrede f. Friedrich Schneider, Dresden I 846 ( 3 S.).

ro9. Waiblinger, Friedrich Wilhelm (1804-1830): 2 Gedichte "Der Griechin Klage" u. "Die Griechin v. Korinth."

I 1о. Werner, Zacharias (1768-1823): Der 165. Gesang über Michelangelos jüngstes Gericht 18 IO ( $1 \mathrm{r} / 2 \mathrm{~S}$.).

II I. Wieland, Christoph Martin (I733-1813): I S. zur Horazübersetzung.

I I 2. Zschokke, Johann Heinrich Daniel (I $77^{1-1}$ 848): Aufsatz "Die Republik Krakau u. die Neutralität" (3 S.).

XXV. ARTICLES BY ZWEIG CONCERNING HIS

MANUSCRIP'T COLLECTION AND ARTICLES ABOUT ZWEIG AS A MANUSCRIPT COLLECTOR

3373. "Das Autographensammeln," $V Z$, Sept. I4, I913.

3374. "Die Autographensammlung als Kunstwerk," $D B K$, II (1914), 44-50 (Reprinted in Spiegelungen, pp. 44-5 I).

3375. "Die drei Meyer-Sammlungen," BT, June 17, I924 (Arthur Meyer, Cornelius Meyer, R. M. Meyer). 
3376. "Echte und falsche Autographen," $A$ Rsch, VII (1926), i 15.

3377. "Meine Autographensammlung," $P h$, III : 7 (1930), 279-289.

3378. Mozart, Wolfgang Amadeus. Ein Brief von Wolfgang Amadeus Mozart an sein Augsburger Bäsle. Wien-Leipzig-Zürich: Reichner, 193 I (Intro. by Zweig with a four page facsimile of Mozart's letter and a transcription of it).

3379. "Neue Napoleon Manuskripte," $P h$, II (1929), 246.

3380. "Die Sammlung Morrison," $V Z$, Sept. 6, I9I7 (Cf. $D B K$, VI (1918), 73).

3381. "Sinn und Schönheit der Autographen," DNSch, 19.J. (1958), I 8 (Cf. also Begegnungen (1937 ed.), pp. 469-476; Begıgnungen (1955 ed.), pp. 44I-448; Excerpts of this essay are used as the Intro. to Lyrische Handschrift unserer Zeit. Ed. Hartfrid Voss. Ebenhausen bei München: Hartfrid Voss, 1958, p. 5).

3382. "Die Welt der Autographen," $D B K$, I 2./13.J. (1927), 70-77.

3383. Arens, Hanns. "Der Sammler Stefan Zweig," $D N Z$, I 2I (1949) (Reprinted in Imp, XI (1953), I99-205; NDH, s (1954), 394; WidZ, 10 (Oct., 1961), 36-40).

3384. - "Stefan Zweig as a Collector of Manuscripts," $M s, 9$ (1957), 43-45.

3385. Blätter, 6/7 (Oct., I959), 9 ("Zweigs Sammlung von Autographenkatalogen noch geschlossen vorhanden im Privatbesitz von Herrn Heinrich Eisemann").

3386. Burchardt, Hansjürgen. "Die Autographen-Auktion bei Stargard vom I4. November I958," BdBh, I4.J.: I0 (1958), I 708-17 1 I.

3387. Claudel, Paul and André Gide. Correspondance. I 899-1926. Pref. and Notes Robert Mallet. 5. ed. Paris: Gallimard, I949, pp. 2 I0, 357 (Ref. to Zweig's attempt to purchase Claudel's L'Annonce faite à Marie).

3388. Dargan, E. Preston and Bernard Weinberg, eds. The Evolution of Balzac's 'Comedie bumaine'. Chicago: University of Chicago Press, 1942, pp. 427-430 (Appendix: Wells Chamberlin, "The Zweig Manuscript Proof of 'Une ténébreuse Affaire"”).

3389. Ecker, Karl. "Die Sammlung Stefan Zweigs in der Österreichischen Nationalbibliothek - Wien," Festschrift zum 25 jäbrigen Dienstjubiläum des General Direktors Josef Bick. Ed. Jasek Stummvoll. Wien, I948, pp. 32 I-330 ("Stefan Zweig Autographen:

a. In der Handschriftensammlung: I7 Autographen verschiedener Art (Briefe, Postkarten, Widmungen, teilweise nur Unterschriften)

b. In der Theatersammlung: Die schweigsame Frau, Oper für Richard Strauss").

3390. Hintenberger, Heinrich, ed. Repräsentative Original-Handschriften. Eine berübmte Autographen-Sammlung. I. Teil. Katalog IX. Wien: Heinrich Hintenberger, n.d. (Cf. also Katalog XX: Interessante Autographen aus zwei bekannten Sammlungen).

3391. Thommem-Girard, G. H. "Stefan Zweig als Autographen-Sammler," $D A n q$, 10.J. (1954), 205-208.

3392. Wolbe, Eugen. "Stefan Zweig als Autographensammler," $D K w$, XIII (Nov., 193I), 72-73.

3393. Zohn, Harry. "Stefan Zweig as a Collector of Manuscripts," CQ, XXV : 3 (May, I952), I 82-I91.

\section{ADDENDA}

3394. Arens, Hanns. "Erinnerungen an Stefan Zweig. Aufgezeichnet aus Anlass seines 80. Geburtstags," Welt und Wort, XVI (1961), 334-336, 340.

3395. Barthel, L. F., ed. Das war Binding. Ein Buch der Erinnerung. Wien-Berlin-Stuttgart, 1955 (Contributions by Thomas Seidenfaden, Karl Rauch, O. Jancke, Tilla Fleischel, Stefan Zweig, Richard Scheibe, Willi Fehse, et al.).

3396. Berenson, Bernard. Sunset and Twilight. From the Diaries of 1947-1958. Intro. by Iris Origo. Epilogue by Nicky Mariano. New York: Harcourt, Brace and World, r963, p. 9 (Reference to Zweig's Balzac).

3397. Butler, E. M. The Tyranny of Greece over Germany. Boston: Beacon Press, 1958, pp. 2 I 4n, 333 (Reference to Zweig's Der Kampf mit dem Dämon). 
3398. Courts, Gerd. Das Problem des unterliegenden Helden in den Dramen Stefan Zweigs. Köln, 1962 (Unpub. Doctoral Dissertation).

3399. Faesi, Robert. "Erinnerung an Stefan Zweig. Zu seinem 20. Todestag," Schweizer Monatshefte (Zürich), XLI (1961-1962), 1301-1310.

3400. Grossberg, Mimi. "Joseph Roth, sein Schicksal und sein Werk," Bulletin des Leo Baeck Instituts (Tel-Aviv), VII (1964), 76-94.

340I. Huder, Walther. "Stefan Zweig," Sinn und Form. Beiträge zur Literatur (Berlin), XIV (1962), 135 -1 40.

3402. Kesten, Hermann. Hermann Kesten. Ein Bucb der Freunde. Zum 60. Geburtstag. München: Desch; Köln: Kiepenheuer and Witsch. Frankfurt am Main: Büchergilde Gutenberg, I960 (Contributions by Heinrich Mann, Joseph Roth, Stefan Zweig, Alfred Döblin, Thomas Mann et al.).

3403. - "Stefan Zweig," Meine Freunde, die Poeten. München: Kindler, 1959, pp. 141154 (Cf. No. 2816).

3404. Kohn, Hans. Karl Kraus - Artbur Schnitzler - Otto Weininger. Aus dem Jidischen Wien der Jabrbundertwende. Tübingen: Mohr, 1962, pp. 14, 30-31, 68-69.

3405. Langer, N. "Stefan Zweig," Dichter aus Osterreich (Wien), 2.F. (1957), I 22-1 27.

3406. Prater, Donald A. "Stefan Zweig and England," German Life and Letters, XVI (I962), I-I3.

3407. Schramm, Werner. Stefan Zweig. Essay. Itzehoe: Selbstverlag, r961.

3408. Zweig, Stefan. "Abschiedsrede (In memoriam Joseph Roth)," Österreichische Post (Paris), July I, 1939 (Reprinted in Sie trugen Österreich mit sich in die Welt. Ed. Arthur Breycha-Vauthier. Wien: Weltbund der Osterreicher im Ausland, 1962, pp. $126-130)$.

3409. - "An Arthur Schnitzler: Kundgebungen österreichischer Zeitgenossen," Moderne Welt, III: I 2 (?), ro-Is (Contributions by Felix Salten, Anton Wildgans, Stefan Zweig et al.).

34ro. - "Das Geheimnis des künstlerischen Schaffens," Wort und Tat (Mainz-Weisenan), 9 (1948), 7-2I (Cf. No. I 224).

34I I. - "Die Geschichte als Dichterin," Erbe und Zukunft, I (1946), 54-64 (Cf. No. 1 226).

34I2. - "Persönliche Erinnerungen an Arthur Schnitzler," Literarische Welt, VII (?), 45 (Contributions by Hermann Bahr, Felix Salten, Stefan Zweig).

3413. - Der Turm zu Babel. Wien: Im Verlag der Internationalen Stefan-ZweigGesellschaft, 1964 (4. Sonderpublikation der Stefan Zweig Gesellschaft; Cf. No. 1361).

3414. - Die Welt von gestern. Erinnerungen eines Europäers. Frankfurt am Main-Berlin: G. B. Fischer, 1962 . 


\section{NOTES}

1 These words, attributed to Marguerite of Austria, are used as the motto to the last chapter of Zweig's Calvin (1954 ed.), p. 262.

2 Kurt Böttcher, "Humanist auf verlorener Bastion, NDL, XI (Nov., 1956), p. 83.

${ }^{3}$ Stefan Zweig, $W v G$, pp. 17-23. Zweig's autbiography first appeared in English as: The World of Yesterday. New York City: Viking, 1943; the following year it appeared in German with the Berman-Fischer Verlag.

${ }^{4}$ Friderike M. Zweig, $S Z$, pp. 9-I 2.

${ }^{5}$ Ibid., pp. I 8-22.

'S. Zweig, $W \nu G$, pp. 23-69.

'For Zweig's evaluation and appreciation of Rimbaud, Verlaine, Rilke and Hofmannsthal compare the following:

a. Stefan Zweig, "Arthur Rimbaud" (1907), Begegnungen, pp. 432-44I.

b. S. Zweig, Verlaine.

c. S. Zweig, "Abschied von Rilke" (1927), Begegnungen, pp. 59-74.

d. S. Zweig, "Hugo von Hofmannsthal. Gedächtnisrede zur Trauerfeier im Wiener Burgtheater," Zeit und Welt, pp. 33-49.

8 S. Zweig, "Erinnerung an Theodor Herzl" (1929), Begegnungen, pp. 88-96.

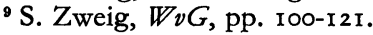

${ }^{10}$ For succinct, penetrating appraisals of this characteristic of Zweig see:

a. Willi Fehse, "Das höchste Gut dieser Erde...," Spiegelungen, pp. 64-65.

b. Romain Rolland, Preface to the French edition of Zweig's Amok (Paris: Ferenczi and Fils, I939), pp. I I-I 2. The Preface first appeared in Nov., I926 in the first edition of the text cited above.

${ }^{11} \mathrm{~K}$. Böttcher, op.cit., pp. 84-85.

${ }^{12} \mathrm{~S}$. Zweig, $W \nu G$, p. 1 20; compare the same sentiment expressed in a letter Zweig had received 32 years previously from Richard Dehmel, which in its latter part anticipates some of Zweig's own editorial problems:

\section{Lieber Herr Zweig!}

Blankensee, I.I r.9.

Es ist in jeder Hinsicht bewundernswert, wie Sie für Verhaeren eintreten; und Sie werden sehen, dass Sie dadurch unmerklich auch für Ihren eignen Weg schrittfester geworden sind. Es ist ja vollkommen einerlei, an was für Aufgaben man seine Kräfte setzt, wenn's nur zu höchsten Aufgaben und nach besten Kräften geschieht. Auch ich werde noch einige Zeit für den höchsten Wert eines andern arbeiten müssen: die Säuberung des Liliencronschen Gedächtnisbildes von dem wohlgemeinten, aber übelbeschaffenen Firnis der vulgären Reklame wird mich noch mindestens ein halbes Jahr lang beschäftigen (Sichtung des Briefnachlasses u. dergl.). Ich halte die Zeit nicht für verloren an mir selber; denn man wird sich bei solcher Arbeit klarer über seelische Allgemeinwerte, als wenn man sich immer bloss mit seinen eignen Spielen und Zielen befasst. Úbrigens war es vielleicht eine Dummheit von mir, die Poesie meiner letzten Jahre unter die Titel meiner früheren Bücher miteinzuschachteln; infolgedessen merken die meisten Leute garnicht, was mir wesentlich Neues zugewachsen ist. Aber das sagt 
mir wohl bloss das Bewusstsein meiner persönlichen Entwickelung, also die liebe Eitelkeit; schliesslich kommt es doch nur darauf an, was als sachliches Werk von uns übrig bleibt.

Mit allen guten Wünchen Ihr Dehmel.

Richard Dehmel, Ausgewäblte Briefe. 1902-1920. Berlin: Fischer, 1923, No. 573, p. I89. ${ }^{13} \mathrm{~S}$. Zweig, "Erinnerungen an Emile Verhaeren" (1916), Begegnungen, pp. 9-59; cf. WvG, pp. II G-I II.

${ }^{14}$ For a vibrant, loving description of Paris compare S. Zweig, $W v G$, pp. 1 26-1 28.

${ }^{15}$ For Zweig's work on Desbordes-Valmore cf:

a. Marceline Desbordes-Valmore.

b. "Marceline Desbordes-Valmore," Begegnungen, pp. 31 8-373.

${ }^{16} \mathrm{~S}$. Zweig, "Auguste Rodin" (I913), Begegnungen, pp. 74-78.

${ }^{17} \mathrm{~S}$. Zweig, $W \nu G$, pp. 122-148.

18 Ibid., pp. I48-I 5 I.

19 Zweig's essay "Die Autographensammlung als Kunstwerk" (of which the original manuscript is at present in the Manuscript Collection of the Wiener Stadtbibliothek) was first published in the $D B K$, II (1914), 44-50; reprinted in Spiegelungen, pp. 44-52; "Sinn und Schönheit der Autographen," Begegnungen, pp. 44I-449.

${ }^{20}$ S. Zweig, "Benares: die Stadt der tausend Tempel," Begegnungen, pp. 254-262; reprinted from Fabrten.

${ }^{21} \mathrm{~S}$. Zweig, $W v G$, pp. 171-173.

22 S. Zweig, "Der Rythmus von New York" (I912), Begegnungen, pp. 264-27r ; reprinted from Fabrten.

${ }^{23} \mathrm{~S}$. Zweig, $W v G$, pp. 299-308.

${ }^{24}$ S. Zweig, "Die Stunde zwischen zwei Ozeanen" (I912), Begegnungen, pp. 239-248; reprinted from Fabrten.

${ }^{25} \mathrm{~S}$. Zweig, $W v G$, p. I98.

${ }^{26} \mathrm{~S}$. Zweig, "Das schönste Grab der Welt" (1928), Begegnungen, pp. 262-264; "Tolstoi als religiöser und sozialer Denker," Zeit und Welt, pp. 65-89; "Reise nach Russland," Zeit und Welt, pp. 203-249.

${ }^{27}$ S. Zweig, $W v G$, pp. 299-308.

${ }^{28} \mathrm{Cf}$. footnote $\mathrm{I} 3$.

${ }^{29}$ For a far too effervescent, though undoubtedly sincere appraisal of Rolland see: Rolland.

${ }^{30} \mathrm{~S}$. Zweig, $W \nu G$, pp. 222-224.

31 Ibid., p. 220.

32 Ibid., pp. 224-225.

${ }^{33}$ Donald G. Daviau, "Stefan Zweig's Victors in Defeat," MfDU, LI : I (Jan., I959), pp. I-I 2.

${ }^{34}$ S. Zweig, Begegnungen, pp. 207-219; cf. WvG, pp. 277-278.

${ }^{35} \mathrm{~S}$. Zweig, $W \nu G$, pp. $227-234$.

${ }^{36}$ Ibid., pp. $255-256$.

${ }^{37}$ Ibid., pp. 246-253.

s8 S. Zweig, "Der Turm zu Babel," $V Z$, May 8, 1916; reprinted as "La tour de Babel," $L C$, April-May, I916.

${ }^{30}$ A small number of Zweig's articles written during the First World War have recently been reprinted and are collected in his Begegnungen. See:
a. "Die schlaflose Welt" (I914), pp. 175-181.
b. "Bei den Sorglosen" (1916), pp. 181-187.
c. "Bertha von Suttner" (1917), pp. 187-194.
d. "Das Herz Europas" (1917), pp. 194-207.
e. "Das Feuer" (1918), pp. 207-219.

${ }^{40}$ In his article "Humanist auf verlorener Bastion" (see footnote 2), Kurt Böttcher makes several interesting observations, which, though easily enough misinterpreted, even 
detrimentally so, do have more than a mere grain of truth in them. His argument runs somewhat as follows: After the war Zweig sought to promote international understanding, justice and the abolition of force through a spiritual reformation, a transformation of human consciousness, completely misunderstanding, no, ignoring the proletarian movement which was steadily forcing itself to the fore. Although his ideas found almost no sympathy in an era of revolutionary ideals and revenge, he remained true to these ideals of serving the realm of the spirit and “... hierin liegt das grosse (politische!) Verdienst seines Schaffens" (p. 87). Around I918 Zweig sought to actively mould public opinion, and when this attempt failed (a fact which he doesn't mention in his autobiography) he was faced with an important decision: should he aid the propagation of the new social ideas which advocated removal of economic inequality of states, imperialism, misery of the lower classes and oppression of minorities, ideas which he decidedly championed? When Barbusse, after his tour of Russia openly advocated Communism, believing democracies incapable of accomplishing international brotherhood, attempted to turn their international peace group "Clarté" into a classmachine for the spread of Communism, Zweig withdrew his support. "Wieder hatten wir im Kampf um die geistige Freiheit versagt aus zu grosser Liebe zur eigenen Freiheit und Unabhängigkeit" (S. Zweig, $W \nu G$, p. 278). Böttcher interprets this sentence as a self-remonstrance on Zweig's part for not having openly advocated Communism and for not having given up his personal wealth. Thus to avoid an unequivocal decision Zweig fled again into the realm of spirit (“... die blosse Auseinandersetzung mit dem Menschen als Individuum." Böttcher, p. 88), reasserting his former inclination that society can only be understood if we first have grasped the mental and the emotional essence of the individual. Böttcher sees this individualism in combination with philosophic relativism as the basis upon which Zweig based his defense of the worth of the individual, or should we say "individuals", individuals who need one another, that is society, ergo Communism.

Böttcher's article is based on extracts from his Doctoral Dissertation, "Stefan Zweig und 'die Welt von Gestern'," which was written in 195 I at the Friedrich-SchillerUniversität, Jena.

${ }^{41}$ Mrs. Zweig's book, $S Z$, is a pertinent accout of Zweig's life as an individual, his life with her, and an exceptionally penetrating discussion of his works. Upon reading the work, however, one is left with a strange feeling that she showed somewhat too much heroism with respect to Stefan's escapade in his 5 os and was far too tolerant with respect to his moodiness. G. Bianquis in her review of this work ( $E r$, III (June 10, I959), 347) brings this fact out rather too bluntly, reducing Zweig's actions to mere selfishness.

${ }^{42}$ F. M. Zweig, $S Z$, pp. 125-1 28.

${ }^{43}$ S. Zweig, $W \nu G$, pp. 264-265.

${ }^{44}$ S. Zweig, "Salzburg: die Stadt als Rahmen," Begegnungen, pp. 271-274; reprinted in Blätter, 2 (July, I958), I-4.

${ }^{45} \mathrm{~S}$. Zweig, "Arturo Toscanini" (1935), Begegnungen, pp. 78-88.

${ }^{46}$ S. Zweig, "Bruno Walter: Kunst der Hingabe" (1936), Begegnungen, pp. г 27-1 30.

${ }^{47}$ S. Zweig, "Geburtstagsbrief an Hermann Bahr" (1923), Begegnungen, pp. ro6-I I I.

${ }^{48}$ S. Zweig, "Unvergesslisches Erlebnis. Ein Tag bei Albert Schweitzer"(I932), Begegnungen, pp. I 13-1 23.

${ }_{49}$ S. Zweig, "Der Dirigent (Gustav Mahler)," Begegnungen, pp. I23-г 27.

${ }^{50} \mathrm{~S}$. Zweig, "Frans Masereel" (1923), Begegnungen, pp. I30-140.

${ }^{51} \mathrm{~S}$. Zweig, $W \nu G$, pp. 315-317.

${ }_{52}$ S. Zweig, "Die moralische Entgiftung Europas" (1932), Begegnungen, pp. 223-239; "Der europäische Gedanke in seiner historischen Entwicklung," Zeit und Welt, pp. 299-327.

${ }^{53}$ Olga Bak, "Stefan Zweig, sa vie et son oeuvre," (Unpub. diss., Laval Université,. Quebec, 1950), pp. 67-68.

${ }^{54}$ For a summary bibliography of Zweig's major works consult Blätter, I (April, 1958), 5-7 and "Bibliographie der Werke von Stefan Zweig," In, XIII (193 I), 17-30.

${ }^{55}$ Otto Zarek, "Stefan Zweig zum sosten Geburtstag," NR, XLII : 2 (Dec., I93I), 86I863.

${ }^{56}$ S. Zweig, $W \nu G$, pp. 334-343. 
57 Walter A. Berendsohn, Die bumanistische Front; Einfübrung in die deutsche EmigrantenLiteratur. Erster Teil von 1933 bis zum Kriegsausbruch 1939. Zürich: Europa, 1946.

${ }^{58} \mathrm{~S}$. Zweig, $W \nu G$, p. 345 .

${ }^{50}$ Mary Lamberton Becker, "The Most Readable and the Most Reliable," NYHTB, Aug. 25, 1935, p. 3.

${ }^{\circ 0}$ Werner J. Cahnmann, "Stefan Zweig at Salzburg," MJ, XXX (July, 1942), 195-198.

${ }^{61}$ F. M. Zweig, $S Z$, pp. 364-388.

${ }^{62} \mathrm{~S}$. Zweig, $W \nu G$, pp. 359-362.

${ }^{63} \mathrm{~S}$. Zweig, "Dank an Brasilien," Zeit und Welt, pp. I I-1 59; "Kleine Reise nach Brasilien" (1936), Begegnungen, pp. 274-309; Brasilien.

${ }^{64}$ S. Zweig, "Geschichtsschreibung von Morgen," Zeit und Welt, pp. 275-299.

${ }^{65}$ To demonstrate that Zweig realized these ideals in his own life was the purpose of an article by Normal A. Brittin entitled "Stefan Zweig: Biographer and Teacher," SeR, XLVIII (April, 1949), 245-254; that Zweig himself was not always the "eternal idealist" is obvious from much of his correspondence. To this effect compare the letters written by Zweig to Alfred Wolfenstein and Felix Braun reprinted in Spiegelungen, pp. 86-88.

B S. Zweig, Heilung durch den Geist; "Worte am Sarge Sigmund Freuds," Zeit und Welt, pp. 49-55.

${ }^{67}$ F. M. Zweig, $S Z$, pp. 402-433.

${ }^{68} \mathrm{~S}$. Zweig, Balzac. This work was completed and published posthumously by Dr. Richard Friedenthal, the executor of Zweig's literary estate; cf. "Die unterirdischen Bücher Balzacs" (1920), Begegnungen, pp. 427-432; Drei Meister.

- For a photographic reprint of Zweig's last declaration see Hanns Arens, "Stefan Zweig," Europäer, p. 44; the printed text of the letter is found on the preceding page.

${ }^{70}$ F. M. Zweig, $S Z$, pp. 416-424.

${ }^{71} \mathrm{~S}$. Zweig, $W \nu G$, pp. 7-10.

72 F. M. Zweig, $S Z$, pp. 356-363.

${ }^{73}$ Raoul Auernheimer, "Stefan Zweig," The Torch of Freedom. Ed. Emil Ludwig and Henry B. Kranz. New York City: Farrar and Rinehart, I943, pp. 409-426.

${ }^{74}$ Paul Beaulieu, "Reflexions sur la suicide de Stefan Zweig," LNR (Montreal, Canada), $\mathrm{X}$ (Aug., 1942), 579-582; Thomas Mann, "Stefan Zweig zum ro. Todestag," Altes und Neues. Frankfurt am Main: S. Fischer, 1943, 263-265; Franz Schoenberner, "Stefan Zweig and Ourselves," NRep, CVI (March, 1942), 333.

${ }^{75}$ In his article "Erinnerungen an Stefan Zweig" (Spiegelungen, pp. 78-85) Robert Braun maintains that he believes Zweig was more than passively interested in conversion to Christianity, indeed to Roman Catholicism, following the example of some of the leading German literary Jews, among them Franz Werfel. Had he (Braun) only dared be more forceful Zweig would possibly have chosen the only possible line of salvation left open to men of humanistic idealism - religion.

Vielleicht wäre Stefan Zweig damals geneigter gewesen denn je, meinen Schritt zum religiösen Bekenntnis, der dieser Einsicht gefolgt war, nicht allein zu tolerieren, sondern auch als eine Möglichkeit zu begreifen, die nicht allein für den fremden Anderen annehmbar blieb. Unsere Feinden konnten uns ja alles absprechen, Heimat, Besitz, Freiheit, ja das Recht zu leben, nur das Bekenntnis zum Kreuz nicht. Noch nie war es verbunden mit jahrtausende altem Schicksal so an uns herangetreten wie unter dem Schatten Hitlers. Hätte ich damals gewagt, Stefan Zweig dies in Zusammenhang mit der politischen Situation zu bekennen, hätte er vielleicht aufgehorcht. Es hätte freilich auch sein können, dass er, ähnlich wie Franz Werfel, gerade unter dem Druck der Verfolgung sich weiter denn je von einer Anerkennung dessen entfernt hätte, was mir die einzige Rettung schien. Ich erwähnte jedoch kein Wort davon. Es war nich seine Schuld, sondern allein die menige, dass ich es nicht wagte, die Rede auf das mir Wichtigste zu bringen, und es so - durch Zuhören geheimhielt. p.78. This of course may be true but judging strictly from Zweig's own writings there seems nothing to substantiate such a view.

${ }^{76} \mathrm{~K}$. Böttcher, op. cit., p. 92.

"Antoine Bon, "Stefan Zweig et le Brésil," MdF, CCCI (Sept. I, 1947), 78. 


\section{INDEX}

Note: Roman numerals refer to pages in the Introduction, Arabic numbers in parentheses refer to the foot-notes in the Introduction, Arabic numbers without parentheses refer to the entries in the body of the text.

\section{WORKS BY STEFAN ZWEIG: BIOGRAPHICAL STUDIES}

Amerigo Vespuccio, 9-I I, 563-573, 693, I 873-1 882

Balzac, XXXVI, XXXVII, (68), 9-II, 574-596, 1006, 1883-1901, 2723, 2778

Baumeister der Welt, 528, 796-800, 22182228, 3075, 3164, 3195

Brasilien, XXXVII, (63), 526-528, 541, 557, 930-940, 1007-гого, 2429-2444, 2542, 2593, 2769, 2774, 3140

Briefwechsel; Stefan Zweig-Friderike Maria Zweig, 971-973, 2470-2478

Briefwechsel: Richard Strauss-Stefan Zweig, 974, 2479-2486

Calvin, XXXV, XXXVI, (I), 597-609, 987, IOII-IOI 2, I902, I928, 2529, 2649-265I, 2654, 2681-2682, 2689, 27 I

Correspondence, 971-1001, 2689, 2696, 2769,2823

(Marceline) Desbordes-Valmore, (I 5), 8-9, 610-615, 1095-1098, 1929-1940

Drei Dicbter ibres Lebens, XXXIV, 8, IO-II, 8OI-826, IOI3-I033, I489, 2218-226I

Drei Meister, XXXIII, (68), 8, ro, 827850 , I034-1037, I49I-I493, $2218-$ 2228, 2262-2304, 2605, 2985, 3076

Erasmus von Rotterdam, XXXV, 524,528 , 616-630, I038-104I, I941-I985, 2533

(Josepb) Fouché, XXXIV, 8-9, 523, 63I-
670, 1047-1053, I986-2048, 3077, 3 I 76

Die Heilung durch den Geist, XXXIV, (66), I I, 872-895, 1054-1072, 23362407, 2592

Der Kampf mit dem Dämon, XXXIII, 8, I I, 85 I-87I, 1073-ro92, 22 I 8-2228, $2305-2335,3230$

Magellan, 9-10, 525, 528, 671-699, 1093I094, 2049-2078, 3 I 62

Maria Stuart, XXXIV, 9, 524, 735-766, 2132-21 70, 2606, 3102

Marie Antoinette, XXXIV, I0, 524-525, 700-734, I099-I106, 2090-2I27, 3 I $38,335 \mathrm{I}$

(Frans) Masereel, 767-768, I 215

(Romain) Rolland, XXXIII, (29), 9, 769786, 2 I 7 I-2204

Sternstunden der Menschbeit, XXXIV, 8-9, 165, 400, 462, 523, 525, 896-929, I 107-I I 23, I I II, I 275, 2408, 2428, $3165,3265,3353$

Unbekannte Briefe aus der Emigration an eine Freundin, 975

(Emile) Verbaeren, 9, 787-792, I I 24-II25, 2205-22I 7, 3082

(Paul) Verlaine, (7), 793-795

Die Welt von gestern, XXXVI, (3), (6), (9), (1 2-I4), (1 7-1 8), (2 I), (23), (25), (27), $(30-32),(34-37),(40),(43),(51),(56)$, (58), (62), (7I), 525, 94 I-970, I I 26II $30,2445-2469,2687,2964,3182$ 
Dramas in general, 94-135, I635-1743, 2698, 2766, 2809, 2872, 2944, 2993, 3185

Die Flucbt zu Gott, 94-95, 3267

Das Haus am Meer, XXIX, 96, 1635$1638,2698,3350$

Jeremias, XXXII, I0, 97-1 I1 , 1639-1673, $2676,2698,2791,2823,3035,3288$, 3370

Das Lamm des Armen, I I 2-I I4, I674-I678
Legende eines Lebens, II 5-I I6, 1679, I685 Quiproquo, II 7

Die schweigsame Frau, XXXIV, I18-120, I686-I694, 2537, 2791, 2929-2930, $3245,3259,3389$

Tersites, XXVIII, I $21,1695-1703$

Der verwandelte Komödiant, XXIX, I22I 24, I 704-1 707

Volpone, $\mathrm{x} 25-135, \mathrm{I}_{1} 63, \mathrm{1} 392, \mathrm{1} 708-\mathrm{r} 743$, 3245,3267
Essays: Alphabetical list of essays appearing in monographs, periodicals, and newspapers, I1 32-1400, 2873

\section{Essays in book form:}

Begegnungen, (7), (8), (13), (15), (16), (19), (20), (22), (24), (26), (34), (39), (44-50), (52), (63), (68), 9, 23, 30, $469,521,526-528,542,545$, 547, $55 \mathrm{I}, 553-554,556,56 \mathrm{I}, \mathrm{II} 33$, II 44 , II49, I I 58, II60-II6I, II64-II67, II 7I-II 72, II 87, I189, I 206, I I10, X21 5 , I $233,1236,1241-1242,1261-$ I 262, I 272, I 284, I 288, I 290, I 307 , 1316, 1323, 1333-1334, 1336, I338, I345, I 348, I $350-1351,1355,1364$, I389, I482, I 486, I497, I 53 I, 2982, $338 \mathrm{I}$

Durch Zeiten und Welten, 528, 985, 2763

Europäisches Erbe, 533, II 32, I I 47, I I I I, I 198, I 23I, I 250-I 25I, I 255, I 270, 1289, 1306, 1308, 1315, 1317, 1325, 1360-136r, I363, I374-1375, I 562, I 864

Fabrten, (20), (22), (24), 528, 534, 10421046, I 240, 1865-1868, 2530

Zeit und Welt, (7), (26), (52), (63), (64), (66), 528, 537, 541-542, 544, 549, 55I-553, 557-560, 562, II 31 , II 70 , I21 8, X 223-1224, I 226-I 227, I 245, I 248, I 260, I 266, I 274, I 278-I 279 , I 298, I 304, I 32 I, I $357-1358,1384$, I387, I393, 2658, 2779

Essays which appeared separately and in collections:
“Abendaquarelle aus Algier," 534, 54I, 557, I I 3 I

"Abschiedsbrief an die Freunde im Ausland," XXXI

"Abschiedsbrief Zweigs (Petropolis, 22 Feb., I942)," XXXVI, (69), 528, 2689, 3272

"Abschied von Alexander Moissi," 533, I 32

"Abschied von John Drinkwater," 526527, I I 33

"Abschied von Rilke," (7), 519-52 I, 526527, 545, 1002-1005, 1316, 1860, 2689, 2793

"Alpenglühen an Zürichsee," 534, 1045

"Anmerkung zu Joyces 'Ulysses'," 526527,545, I I 44

"Anton Kippenberg," 533, I 147

"Arthur Rimbaud,"(7), 5 26-5 27, 545, I I 49

"Arthur Schnitzler zum Gosten Geburtstag," 533, II 5 I

"Arturo Toscanini. Ein Bildnis," (45), 522, 526-527, 545, 550, 1486, I531, I86I

“Auguste Rodin," (16), 23, 526-527

"Die Autographensammlung als Kunstwerk," (I9), I I 56

"Balzac," 526- $527,827-828,830-83 \mathrm{I}$, $833-838,840-84 \mathrm{I}, 843-844,849-850$

"Bei den Sorglosen," (39), 526-527, I 160

"Benares: Die Stadt der tausend Tempel, (20), 526-527, 534, I I6I

"Berta von Suttner," (39), 526- 27, I I 64

"Besuch bei den Milliarden," 526-527, II65

"Bildnis Busonis," 526-527, I 166

"Brief an einen französischen Freund," II 67

"Bruno Walter: Kunst der Hingabe," (46), 526-527, II 7 I 
"Das Buch als Eingang zur Welt," 296, 526-527, I 172

"Casanova," Io, 801-807, 810-8I 2, 8 I6, 8 19, 826, ror3-1015, I 209, I489

"Chateaubriand," 533

"Dank an Brasilien," (63), 54I, 557

"Dank an die Bücher," 296, 526-527, I 187,1355

"Dante," 526-527, 545, I I 89

"Charles Dickens," 9-10, 827-828, 830$83 \mathrm{I}, 840-84 \mathrm{I}, 843,845,849-850$, I 49 I

"Dostojewski," XXX, ıо, 528, 827-830, $832-833,836-843,846,849-850$, I034-I037, I 492-I 493

"Das Drama in Tausendundeiner Nacht," 533,1562

"Edmond Jaloux," 533

"Die Entdeckung Eldorados," I65, 523 , 899-903, 909, 9 I I, II 08, IIIO-IIII, I I 5

"Erinnerungen an Emile Verhaeren," XXXI, (1 3), 526-527, 529-532, 545$546,1862-1863,2660,2689,2791$

"Erinnerungen an Theodor Herzl," (8), 526-527, I 262

"Ernest Renan," 526-527, 545, I 206

"Die Eroberung von Byzanz," 902-903, 905, 909, $91 \mathrm{I}, 923,926$

"Das erste Wort über den Ozean," 902$903,909,923,926$

"E.T.A. Hoffmann," 533

"Der europäische Gedanke in seiner historischen Entwicklung," (52), 54I, 544, 559, I 393

"Die ferne Landschaft," 534

"Das Feuer," (39), 526-527, I 2 го, I 348

"Flucht in die Unsterblichkeit," 902-903, 905, 909, 91 I, 922-923, 926

"Flüchtiger Spiegelblick," 535, I 2 I 3 ,

"Frans Masereel," (50), 5 26-527, 545, I 2 I s

“Franz Anton Mesmer," 9, 872-875, $877-879,882-883,885-886,888,892$, 894-895, I057-106I

"Frühling in Sevilla," 528, 534, I043,

"Frühlingsfahrt durch die Provence," 534

"Die Gärten im Kriege," 541, 557

"Geburtstagsbrief an Hermann Bahr," (47), 526-527, I 24I

"Dem Gedächtnis Jacob Julius Davids," I22 I

"Das Geheimnis Byrons," 459, 54I, 544, 558

"Das Geheimnis der Beatrice Cenci," 54I, $544,558,1223$

"Das Geheimnis des künstlerischen
Schaffens," 541, 544, 548, 555, 559, I 224

"Das Genie einer Nacht," 462, 902-903, 905, 909, 91 I, 923-926, 3353

"Georg Friedrich Händels Auferstehung," I65, 896, 902-904, 909, 9I r, 91 7, 926-927, I I09, I I 20, I I 22, I 275,2428

"Die Geschichte als Dichterin," 541, 544, 559, I 226

"Geschichtsschreibung von morgen," (64), 54I, 544, 559, I 227

"Gustav Mahlers Wiederkehr," 533, I 23 I

"Gwalier," 534, 1232

"Hans Carossa," 526-527, 545, 659, I 233

"Das Haus der tausend Schicksale," 526527,1236

s'The Head Upon the Rostrum, Cicero's Death," 909, 923

"Herbstwinter in Meran," 528, 534, I 240, 2849

"Heroischer Augenblick (Dostojewski)," I65, 900-903, 9 I I

"Das Herz Europas," XXXII, (39), 526$527,536,543,1242,1869-1871$

"(Friedrich) Hölderlin," 9, 85 I-852, 854$856,861-865,870-871$, 1073, 1075I08I, 2646

"Hugo von Hofmannsthal," (7), 54I, 544, 558, I I 27-I I 28, I 245

"Hydepark," 534

"Irrfahrt und Ende Pierre Bonchamps. Die Tragödie Phillipe Daudets," $54 \mathrm{I}, 558, \mathrm{I} 248$

"Ist die Geschichte gerecht?" 533, I 250 "Jakob Wassermann," I85, 533, I 25 I

"Jens Peter Jacobsens 'Niels Lyhne'," 533

"Joseph Roth," 533, I 255

"Der Kampf um den Südpol (Kapitän Scott)," x65, 523, 525, 898-903, 909, 9II, 92I, I I07, II I 2, I I I 7-I I I 8

"Die Kathedrale von Chartres," 54I, 557, ro44, r046, I 260

"Kleine Reise nach Brasilien," (63), $526-$ 527, I 26I

"(Heinrich von) Kleist," 9, 85 1, 853-856, $861,863,866-867,870-871$, 1073, I082-1087, 2646

"Die Kunst des Briefes," I 263, I 503

"Lafcadio Hearn," 533

"Legende und Wahrheit der Beatrice Cenci," 541, 544, 558, I 266

"Léon Bazalgette," 533, I 270

"Lord Byron. Das Schauspiel eines grossen Lebens," 459, 54I, 544, 558, I 264, r 274 
"Marceline Desbordes-Valmore," (I s), 526-527, 545, ro95-1098

"Marcel Prousts tragischer Lebenslauf," 54 I, 558, I 278, I 3 I 2

“Die Marienbader Elegie," I65, 526-527, $545,900-903,909,9$ I I, I I I 4

"Mary Baker Eddy," 872-875, 877-880, $882,885-888,890-891,894-895$, $1062-1064$

"Mater Dolorosa. Die Briefe von Nietzsches Mutter an Overbeck," 54I, 544,558, I 279

"Max Hermann-Neisse zum Gedächtnis," $\mathrm{I}_{28} 3$

"Die Monotonisierung der Welt," 526527,1288

"Montaigne," 533, I 289, 2689

"Die moralische Entgiftung Europas," (52), 526-527, I290

“(Friedrich) Nietzsche," 528, 54I, 544, $558,829,85 \mathrm{I}, 853-856,859-86 \mathrm{I}$, $863,868-87$ I, I073, I088-I092, I 299

"r9r4 und heute," 541, 544, 559, r 298

"Oxford," 54I, 557, I304

"Peter Rosegger," 533, 1306

"Pour Ramuz!" 533, I308

"Rabindranath Tagores 'Sadhánà," 533 , I3IS

“Rainer Maria Rilke," 533, I314, I316I 3 I 8

"Rede zu Ehren Maxim Gorkis," 526$527,545,1284$

"Reise nach Russland," (26), 537, 54I, 557,1321

"Der Rhythmus von New York," (22), 526-527, 534, I323

"Romain Rolland," 533, I 325

"Sainte-Beuve," 526-527, 545, I 333

"Salzburg. Die Stadt als Rahmen," (44), 526-527, 528, I 334

"Die schlaflose Welt," (39), 526-527, 1336

"Schnitzler und die Jugend," I 337

"Schöner Morgen. Bozner Berge," 534, 1045

"Das schönste Grab der Welt," (26), s 26527,1338

“Sevilla: Spaniens Lächeln," 528, 534, ro42
"Sigmund Freud," 539-54I, 558, 872-879, $881-882,885-886,889,891,893-895$, 1065-1072

"Sinn und Schönheit der Autographen," (I9), 526-527, 538, I345, I 872

"Stefan Zweig über Henri Barbusse," 1348

"Stendhal," 9-10, 801-806, 8I0-8 I I, 8I 3, 8 I6-8 I 7, 826, 847-848, ror6-I024, 2531

"Die Stunde zwischen zwei Ozeanen (Panama Kanal)," XXX, (24), s26$527,534,1351$

"Tai Mahal," 534, I045, 2530

"Thomas Manns 'Lotte in Weimar!," $541,559,1357$

"Tolstoi als religiöser und sozialer Denker," (26), 541, 544, 558, 1025-1033, 1358

"Die Tragik der Vergesslichkeit," 553, I360

"Der Turm zu Babel," (38), 430, 533, I36I

"Um Jaurès," 533, 1363

"Die unterirdischen Bücher Balzacs," (68), 526-527, I 272, I 364

"Unvergessliches Erlebnis: Ein Tag bei Albert Schweitzer," (48), 526-527, I I $58,1365,1482$

"Der verlorene Himmel. Elegie der Heimkehr," 534

"Der versiegelte Zug," 902-903, 905, 909, 918, 923-925

"Vorbeigehen an einem unauffälligen Menschen: Otto Weiniger," 533, I 374

"Walter Rathenau," 533, I319, I375

"Die Weltminute von Waterloo," I65, $523,525,899-903,909,9 \mathrm{II}$, IO5 II053, III3, III5-III6, III9, II2I

"Das Wien von gestern," 528, 54I, 544, 557,1384

"Wilson's Failure," 909, 923

"Worte am Grabe Sigmund Freuds," (66), 539-541, 558, I387

"Ypern," 526-527, I 389

"Zu Goethes Gedichten," s26- 27, s45, I 307, I 497
Poetry in general, I 2-93, I623-1634, 2689, 2696, 2769, 2791, 2837, 2894

Ausgewäblte Gedichte, I 2-13, 1623-1624

"Der Bildner: Rodin," I625
"Brügge," 25-26, 534, 54I, 557, I I 70, 2532

"Der Dirigent: Gustav Mahler," (49), 30, ร26- 528, I 23 I 
Die früben Kränze, XXVIII, 14, 29, 1626I 630,2689

Die gesammelten Gedichte, 15, 23, 1631I633

"Heroischer Augenblick (Dostojewski)," $3 \mathrm{I}, 60,165,897,900-903$, 91 I
Silberne Saiten, XXVIII, 16, 1634, 2837 "Sommernächte am Komersee," 75, 534 "Sonnenaufgang in Venedig," 77, 534 "Die Stadt am See: Konstanz," 28, 534 "Stille Insel: Bretagne," 8I, 534

"Die Stimme: Josef Kainz," 526, I350

\section{PROSE FICTION}

Prose Fiction in general, I 744-I 859, 2894, 2995, 3193-3194, 3199, 3202, 3304, 33 I 2

Amok, XXXIII, (10), 8-9, I36-137, I66I 68, 222, 225-226, 246-247, 259-260, $426,454,465,471-472$, 504, 508, 511, 515, 1744-1747, 1770-1 777 , I $850,2675,3072,3137$.

"Der Amokläufer," (I0), I36-137, I48, 222, 225-226, 246-247, 259-263, 298-299, 306-3 I I, 33 I, 334-338, 368, $38 \mathrm{I}, 399-400,406,408,42 \mathrm{I}, 426$, $435,447,454,47^{1-472}$, 502, 504, 508, 5II, 5I4-5I5, I846-1849, 2675, $3072,3230,3265,3340-334 \mathrm{I}$

"Angst," XXXIII, 9-10, I38-14I, I 53, 165, 234, 288-290, 319-322, 332, $352-353,375-376,387,401,404$, 4Io, 420, 44I, 453, 459, 464, 474, 49I-492, 509, I748, I857, 2777, 2790, 2806, 2866, 324I, 3342-3344

"Die Augen des ewigen Bruders," Io, I42-I47, I65, I73, 224, 233, 255, 288-290, 297, 311, 362, 393, 402, 422, 430, 446, 450, 458, 462, 474, 49I-492, I749-175I, 26I2, 27I9, $2865,2876,2965,2969,3072,3245$, 3357

"Der begrabene Leuchter," I0, I65, I 72, 231, 248, 269-270, 302, 314, 357, 402, 438, 476-478, 516, I 798-1807, $3 \mathrm{I} 6 \mathrm{I}$

“Brennendes Geheimnis," XXXIV, 8-ro, I II-I 54, I62, I78, 232, 244-245, 249-250, $258, \quad 271-272, \quad 288-290$, $356,380,409,418,421,423,444$, $457,466,473-474,1752,1769,1808$, $2625,2720,3345$

"Brief einer Unbekannten," I0, I36-I37, I48, I 55, I80, 288-290, 292-293, 297-299, 308-3 II, 337-338, 379, $383-384,389,394-397,400,403$, $406,408,415,45 \mathrm{I}, 454,457,47 \mathrm{I}-$ $472,479-480,507,5$ I 2-514, I $829-$ I 834, I 84I-I 849, I 856, 270I, 2720, $2753,3160,3346-3348,3360$
"Buchmendel (Der Bücherwurm)," XXXII, I0, I37, I48, I56, I65, I69I 7O, 2I , 228, 265-268, 288-290, 296, 404, 410, 424, 474, 488

"Episode am Genfer See," XXXII, ro, I37, I48, I 57-16I, I65, I69-170, I $78,215,288-290,297,301,404$, $459,474,488,523,528$

Erstes Erlebnis, XXIX, 8, I62, 164, 166I68, 226, 249-250, 37I, 398, 40I, $413,431-432,434,466,517,1753-$ I 758, I $853-1854,3265$

Fragment einer Novelle, $\mathrm{I}_{3}, 1759-1760$

"Die Frau und die Landschaft," I 36, I 53 , 234, 358-359, 471-472, 5 I 3

"Geschichte in der Dämmerung," 9, I62, 230, 234, 3I2-3I3, 456, 466, 5I3

"Das gestohlene Jahr," 2943, 3349

"Die Gouvernante," 9-IO, I53, I62, 164a, 288-290, 297, 317, 325, 348, $456,466,474,487-488$, 505

"Im Schnee," I64b

"Jupiter," 285-287

Kaleidoskop, 10, I64-165, 227, 239, 24I, 288-290, 370, 400, 437, 474, s01, I 8 I 6-I 828

Die Kette, I64, I66-168, 370, 469, 500

Kleine Chronik, I69, I 70

"Die Legende der dritten Taube," I65, I $7 x-174,253,302,402,430,474,2773$

"Die Legende der gleich-ungleichen Schwestern," I 72, 302, 3 I 2-3 I3, 430, 462, 474

Legenden, 9, I65, I72-175, 242, 402, 430, I $855,272 \mathrm{I}$

"Leporella," I0, I48, I 53, I65, I69-170, $178,243,288-290,291,350,400$, 406, 410, 424, 449, 459, 474, 475, I 856

Die Liebe der Erika Ewald, XXVIII, I 76, I 76 工 -1762

Menschen. Novellen, I 78, 2827

"Die Mondscheingasse," Iо, I36, I48, I 53, 179, 288-290, 294-295, 308-3 10, $325,344,465,47^{1-472}, 474,475$, $513-514$ 
"Phantastische Nacht," Iо, I 36-1 37, I 48, I 78, I 80-I 8 I, 235-236, 288-290, 297, 3I2-3 I3, 340, 348, 386, 442-443, $456,465,47$ I-472, 474, 489, 5I3

"Rahel rechtet mit Gott," I65, I72, I 74, I 82-I 86, 288-290, 354, 402, 430, 474

"Schachnovelle," XXXVI, Io, I 78, I87I95, 243, 256-257, 298-300, 305, $318,360,367,377-378,385,391$, $410,414,425,536,448,497,502$, I $84 I-1849,2765,3265,3352,3362$

"Scharlach," I96

"Sommernovellette," Io, I62, I 78, 297, $317,456,466,474,490,513$

"Die spät bezahlte Schuld," 436

"Der Stern über dem Wald," I 76

Ungeduld des Herzens, 9-10, 197-203, 240, $25 \mathrm{I}, 254,264,304,323-324,347$, $365,382,390,412,467,481,483-$ $486,493-494,498,503$, I $778-1797$, 2571, 3230, 3265, 3354

"Die unsichtbare Sammlung," 8, I0, I 37 , 148, 165, 169-170, 178, 204-21 2 , $215,229,27^{6-284}, 288-290,296$, $303,374,404,409,459,474,488$, 523,528

"Untergang eines Herzens," I53, I 80,
213-214, 239, 273-274, 302, 317, $339,343-344,367,409,440,443$, $457,505,2720$

"Unvermutete Bekanntschaft mit einem Handwerk," I0, I 53, I65, 215,288 290, 345-346, 404, 414, 474, 490

"Verwirrung der Gefühle," XXXIII, 8, Io, $137,148,167-168,178,180$, 213-214, 22I, 226, 273-275, 302, $315-316,333,355,367,369,405$, 416-4I7, 439, 452, 457, 480, 482, $495,499,514,518,1763-1768,1851$, I $858,2720,3074,3230,3241,3265$

Vier Novellen, I $768 \mathrm{a}$

"Vierundzwanzig Stunden aus dem Leben einer Frau," I0, 137, 178, 213-214, $216,223,225,273-274,302,326-$ $330,341-342,373,406,407,4 \mathrm{II}$, $427-429,445,452,455-456,46 \mathrm{r}$, $463,496,506,1852,1859,2552$, $2659,3230,3355-3356,3362$

"Die Wanderung," 176, 21 7, 230

"War er es?" 436

"Das Wunder des Lebens," I 76, 2 I 8, 230

"Der Zwang," XXXII, XXXIII, I 78, 219-220, 252, 297, 414, 459

\section{MONOGRAPHS ABOUT STEFAN ZWEIG}

Algunos Aspectos da Vida e Obra de Stefan Zweig (Apio Garcia), 3000

Ascençcao e Queda de Stefan Zweig (Araujo Lima), 3001

Bibliographie der Werke von Stefan Zweig (Eds. E. Rieger and F. Hünich), (54), 2502-2503, 27I 6, 2803, 2875

Blätter, (44), (54), 987, 99 , 998, I $22 \mathrm{I}$, I 240,1250, I 264, I 283, I 334, I 337 , $1348, x 503,1519,1759,2678,2702$, $2725,2727,2742,2744,2764-2765$, $2770,2785,2796,2813,2823,2849$, $2855-2856,2858,2861,2877,2899$, 2928, 2943, 2963, 2968, 3340-3356, $3357,3361,3365,3385$

De Stefan Zweig a Jacques Maritain. Muerte

y Resurrección del Espiritu (Moreno), 3018

Ensayos: Goetbe, José Asunción Silva, Stefan Zweig(Arias), 3006

Europäer, (69), 22, 44, 58, 73, 89, 985, I426, 2689, 2694, 2697, 2707, 2740, $2758,2776,2780,2784,2834,2870$, 2884, 2942, 2970, 3089

Leben-Werk, 58, 89, 985, 1426, 2629,
2694, 2696, 2706, 2740, 2758, 2776, 2780, 2784, 2834, 2870, 2884, 2942, 2950, 2970

Mann-Werk, 1 I 42, 2504-2505, 2874 $A$ Morte de Stefan Zweig (Stern), 3004

Spiegelungen, (10), (19), (65), (75), 32, 42, $79,90,92,988$, I 1 56, 1426, 1429, I44I, I463, I477, 2499-250I, 2724, $2728,2733-2734,2742,2761,2768-$ $2769,2771,2774,2789,2807,2857$, 2936, 2939, 2946, 2967, 3159, 3374

Stefan Zweig (Bauer), 2498, 2703

Stefan Zweig (Benaroya), 27 I I

Stefan Zweig (F. M. Zweig), 2507-25I7, 2641

Stefan Zweig. A Tribute to His Life and Work (Ed. H. Arens), 2497, 2524

Stefan Zweig. An American Bibliograpby (Broadbent), 2535-2536

Stefan Zweig. Ausstellung in der Residenz in Salzburg (1961), 2767

Stefan Zweig, Cazador de Almas (Relgis), 3026

Stefan Zweig, Cumbre Apagada (Jarnés), 3016 
Stefan Zweig. Ein Lebensbild (H. Hellwig), 2794

Stefan Zweig. Ein Nachruf (A. Cahn), 2738

Stefan Zweig. Eine Bildbiographie (F. M. Zweig), 25 1 8-2519, 2974

Stefan Zweig. Eine Gedenkschrift (P. Zech), 2506, 2962

Stefan Zweig, grand Européen (Romains), 2679-2680

Stefan Zweig, Great European (Romains), 2596

Stefan Zweig. Juan Tenario. Ensayos (Fuente Gonzáles), 301 3

Stefan Zweig. Der Mensch im Werk ( $\mathrm{H}$. Arens), 2695

Stefan Zweig. Sonderpublikation der Funk- tionärblätter, 273 I, 2772, 278 1, 2887, 2938

Stefan Zweig, su Vida, Obra y Muerte (Goldbaum), 3015

Stefan Zweig und der bumanitäre Gedanke (R. Friedenthal), 2780

Stefan Zweig. Un Hombre de Ayer (Ortiz Oderigo), 3019

Stefan Zweig. Versuch eines Bildnisses (R. Specht), 2907

$S Z$, (4-5), (4I-42), (6r), (67), (70), (72), 2975, 2989, 3032

Os últimos Dias de Stefan Zweig (Souza), 3003,3029

Vida e Morte de Stefan Zweig (Azevedo), 2997

\section{MONOGRAPHS IN GENERAL}

(Excluding works by or about Stefan Zweig, general reference works (Cf. 3206-3278) and histories of literature (Cf. 3279-3339)):

\author{
Abendgluten, I 598 \\ De Achterboede, I 506 \\ Adventures in Modern Literature, $30 \mathrm{I}$ \\ $A L A, 25,3$ I, 85 \\ Albert Schweitzer. Leven en Werk, I 513 \\ Alfred Kubin. Leben - Werk - Wirkung, 976, \\ 2823 \\ Altes und Neues, (74), 2847 \\ Das Amselnest, I5 63 \\ L'Annonce faite à Marie, 2648, 3387 \\ Eine Antologie der besten U'bersetzungen \\ (Verlaine), 1474, I538 \\ Anthologie jüngster Lyrik, I 5 I4, 2487 \\ Antlitz, I460 \\ Der arme Lukas, I 576 \\ Art and Psychoanalysis, 2552 \\ Artbur Rimbaud. Leben und Dichtung, I 149 \\ Art of Modern Fiction, 277 \\ Arturo Toscanini, I530-I 531 \\ Assessor Karlchen, I6I9 \\ L'Aube, XXXI \\ Au-dessus de la mêlée, XXXI \\ Auf böberer Warte, 209 \\ Ausgewäblte Briefe (R. Dehmel), (1 2), 979 \\ Ausgewäblte Gedicbte (E. Verhaeren), 1442- \\ I 443, 2495 \\ Ausgewäblte Romane und Novellen (C. \\ Dickens), I 491, I I 82 \\ Aus Nab und Fern, I7I, 2773 \\ Aus Tag und Traum, I6I 8 \\ Aus toten Fragen, I 59I \\ Aus unserer Zeit, I 58 \\ Der Autorabend. Dicbteranekdoten von
}

Rabelais bis Thomas Mann, 2922

Aux peuples assassinés, 1435

A Balzac-Bibliography, 2599

Balzac. Historia dos treze, I 272

Balzac. Sein Weltbild aus den Werken, 1484, 2488-2489

Bass-Bassina-Bulu, I 501

Begegnungen. Die Grossen der Welt im Zwiegespräch, 2758

Bekenntnisse eines Egoisten, I6I

Das bekränzte Jabr, I 589

La Belgique sanglante, XXXI

Best of Modern European Literature, 278

Ein Blatt aus der Cbronik unserer Stadt, I 597

Blick ins Chaos, $157 \mathrm{I}$

Die Blumen des Bösen, 1402

Book of Contemporary Short Stories, 267

Briefe aus den Jabren 1906 bis 1907 (Rilke), 2879

Briefe aus Einsamkeiten, $\mathrm{I}_{26} \mathrm{C}_{3}, \mathrm{I}_{5} \mathrm{O}$

Briefe : 1873-1939 (S. Freud), 981, 2775

Briefe: 1897-1914 (Rilke), 2878

Briefe in das Jenseits, 2725

Ein Brief von Mozart an sein Ausburger Bäsele, I $517,337^{8}$

Briefwechsel (Rilke-Gide), 977-978, 2880

Briefwechsel (Rilke-Kippenberg), 288 I

Briefwechsel (Rilke-Marie von Thurn und Taxis), 2882

Brigitte und Regine und andere Dicbtungen, 1495

Brod, I 28I 
Das Buch von der schönen Stadt Salzburg, 1601

Cabiers de la Quinzaine, XXXI

Cabiers Romain Rolland, 2673

Carossa, $\mathrm{II} \mathrm{67}$

Ceterum, 2719-2720

CGP , 25, 27, 2532

Die Chronik des deutschen Dramas, 2698

Cien Autores Contemporaneos, 3012

Clerambault, 1434

Collected Papers (S. Freud), 2552

Contemporary Studies (C. Baudouin), $136 \mathrm{I}$, 2529

Correspondance. 1899-1926 (Claudel-Gide), 2648,3387

Correspondance. 1909-1926 (Rilke-Gide), 2672

Cressida, 1440

Crimes of Passion, 291

A Critical Bibliography of the Published Writings of Romain Rolland, 2608

Crumbs, rog9

$D d G$, I 127

Le dernier Homme, 1507

Le Désarroi de l'Esprit allemand, 26682669

Destin de l'Autriche, 2664

Deutsche Dicbterbandschriften, 155, 2753

Deutsche Dicbterbandscbriften von 1400 bis 1900, 3222, 3372

Der deutsche Dicbter um die Jabrbundertwende und seine Abgelöstheit von der Gesellschaft, 2885

Deutsche Prosadichtungen der Gegenwart, 2969

Deutschland, ein Wintermärchen, 1569

Diálogos dos Grandes do Mundo, 2999

Dicbter im Café, 2814

Dickens y Sarmiento, 3007

Dictionnaire des Oeuvres de tous les Temps et de tous les Pays, 307, 316, 330

Diktate, I 107

Doctoral Dissertations and Masters' Theses on Zweig, 31 80-3205

Doctors' Choice. Sixteen Stories about Doctors and Medicine, 26r

Dostojewsski(Lucka), I 584

Dostojewski, der Dicbter, I 588

Dostojewski. Menschengestalter und Gottsucher, 2850

Drei Dramen: Helenas Heimkebr - Pbilip II - Das Kloster, 1444

$D u Z$, IIIO

$E d D$, I 22

Elektra, 1575

Emil oder über die Erziebung, I 526
E. M. Lilien. Sein Werk, 1 204, 2491

Encuentros, 30I I

Enzio, 1613

Erich Ebermayer. Buch der Freunde, 980.

Erinnerung und Exil, I 283, I 501

Er versprach ibr einst das Paradies, 1574

Erzäblungen (Gorki), I 499

Escape to Life, 2555, 3163

Exclarmonde. Ibr Leben und Leiden, 1578

Es gibt solche Menschen, 1604

El Espiritu Activo, $302 \mathrm{I}$

Essais et Témoignages, 2646

Essays über Realismus, 2844

Es war alles ganz anders, 2708

Eugenia Grandet, 844

Europa-America latino, 1207

The Evolution of Balzac's 'Comédie bumaine', 2544, 3388

Ewiges Osterreich, 2906

Ezra Pound, 2586

The Fall of the Dynasties, 26I I

Famous Dog Stories, 286

Festscbrift-Josef Bick, 2749, 3389

Le Feu, XXXII

Fifty Years of Thomas Mann Studies, 3368

Firefanz der Puppendoktor, I6IG

Flammen der Liebe, 1600

Florestan Kestners Erfolg, I 529

Flücbtiger Spiegelblick, I 213

Francé, 1322

Franz Karl Ginzky. Dem Dichter und Freunde zum sosten Geburtstag, 1496

Französische Dichter, 1464, 1478, 1480

Frau, 1397

Freud, His Life and His Mind, 2592

Freud On Broadway. A History of Psychoanalysis and the American Drama, 2603

Friedrich Hölderlin, 1590

From Caligari to Hitler. A Psychological History of the German Film, 2664

From Shakespeare to Existentialism, 2562

Fünf Finger über Dir, I62I

Fun Welt-Kwal, 3034

Gassenlieder, 16I4

Gedicbte (Dahm), 1547

Gedicbte (Knorr), I 579

Gedichte (Maeterlinck), I 586

Gedichte (Rimbaud), I 2 I

Gedicbte (Samain), 1607

Gedichte (Verlaine), I539, I620

Gedicbte in Vers und Prosa (Baudelaire), 1403

Geduld. Roman, 1574

Der Geist der Unrube, 2815

Der Geist des Films, $270 \mathrm{I}$ 
Geistesleben, I I 13

General Reference Works, 3206-3278

Germany's Stepchildren, 2569

Gesammelte Werke (Verlaine), I 540, 2496

Gespr., 2876

Gewalt, I 359

Die Gewalten (Csokor), 274I

Godiva. Drama, I 560

Goethes Gedichte. Eine Auswabl, 1497, 2490

Golden Book of Dog Stories, 285

Der goldene Schnitt. Grosse Erzäbler der Neuen Rundscban 1890-1960, I83

$A$ Golden Treasury of Jewish Literature, 102 Greatest Stories of All Times, 279

Great German Sbort Novels and Stories, 262

Great Short Stories, 280

Hände, 1303

Haschisch, I6 10

Heinrich von Kleist, I 65

Helenas Heimkebr, I444-1 446

Helles und Dunkles, 2 Io

Herbst, I 55 I

Hermann Hesse and His Critics, 258I

Hermann Hesse. Eine Bibliographie, 2945

Herodias, I555

Herz, I 334

Herzl, 1262

Die Hexen von Nordeboog, I 594

Den bingerichteten Völkern, I 435

Histories of Literature, 3279-3339

Hofmannstbal, I 128

El Hombre Libre frente a la Barbarie Totalitaria, 3022

Homenaje a Eugen Relgis, 3023

Hommage, I 320,2644

L'Homme en Amour, I 268

Homosexuliaty: Disease or Way of Life, 2531

Die bumanistische Front, (57), 2712

Hymnen an das Leben, 1447, I460, I537

L'Idealisme de Romain Rolland, 2666

Im Sommer, 1582

Inside Europe Today, 2556

Interessante Autographen aus zwei bekannten Sammlungen, 2797,3390

Das Japan Buch, I 500

Ja wirl, 1552

Jean Calvin et sa Dictature, 2682

Jean Christophe, $\mathrm{x} 252,1328$

Jewish Caravan. Great Stories of 25 Centuries, 268

The Jewish Contribution to Civilization, I 532

Joseph Marx. Leben und Werk, 2837

Journal (Green), 2657

Journal des Années de Guerre(Rolland), 982, 2674
Journal. 1939-1949 (Gide), 2552a, 2655

Journals (Gide), 2553

Journey into Chaos, 2624

Journeys, 1007, 1093, 2584-2585

Juden, 1003

Juden auf der deutschen Bühne, 2973

Jugenderinnerungen (Renan), I 520

Jugend in Wien, 2859

Just As It Happened, $255 \mathrm{I}$

Karussell, I I I 7

Kein ding sei wo das wort gebricht. Stefan George zum Gedenken, 2895.

Kippenberg, I 147, 1386

Kleine Erzäblungen und Skizzen (Baumberg), I 54

Knabenalter. Roman, I 545

Kraus, I I 43

Die Landstreicherin, I595

Ein Leben für das Buch, 2801

Ein Leben in Briefen, Manuskripten und Bildern, 984

Die Lebenserinnerungen der Gattin Dostojewskis, I 549

Lebensflut. Gedichte (Frei), I 56I

Ein Lebensmorgen, I605

Leben und Dichtung (Rimbaud), I 52

The Legacy of the Liberal Spirit, 2533

Lenau. Geschichte eines Märtyrers der Poesie, I 494

Leo Tolstoi, 3369

De levende Gedachten van Tolstoi, I 533, 2493

Liber amicorum Friderike Maria Zweig, 2632

Liber amicorum Romain Rolland, 1524

Librarians, 1355

Das Licht der Welt, 2724, 2728

Die Liebe im Menschen, I 509

The Life and Work of Sigmund Freud, $256 \mathrm{I}$

Liliencron, 43, I I 4I

Literarische Portraits, 2686

Literarische Portraits aus dem Frankreich des XVII-XIX Jabrbunderts, I333, I 527

Literatur für den Deutschunterricht. Erste Stufe, I64a

Literaturas Germánicas, 3008

Lord Byron kommt aus der Mode, I280

Lyrik, 1452, 1455, 1469

Lyriscbe Handschriften unserer Zeit, 1345, I $54 \mathrm{I}, 338 \mathrm{I}$

Maeterlinck, 1609

The Making of an Englishman, 2616

Man weiss nicht wie (Non si sa come), I433

Marvelous Journey. A Survey of Four Centuries of Brazilian Writing, 2593

Mary Stuart. A Romantic Drama, 2606 
Masters' Theses and Doctoral Dissertations, 3 180-3205

Meine Freunde die Poeten, 281 6

Meister des Stils über Sprach- und Stillebre, 2897, 2934

Meister-Novellen neuerer Erzäbler, 218

Mémoires (Casanova), 1489

Men at War. Best Short Stories of All Times, 266

Men of Music. Their Lives, Times and Acbievements, 2537

Menschenbilder, I580

Mezza voce, 1559

Midot Va'arakbim, 2976

Mittel und Wege, 1550

Modellgeschicbten, 1599

Moissi, 1287

Die Molschule, 1596

Musik des Einsamen, 1572

Das musische Land, 2729

Muted Voices, I5 I9

My Sentimental Zoo, 1498

Nacbt ist umber, 1485

The Nature of Biography, 3367

Negen Vuurmakers, i I 7 a

Neue deutsche Erzäbler, I85

Neue Gedichte (Rilke), 1603

Das neue Leben, 2727

Neues Skizzenbuch, 16I I

Niels Lybne, I 505

Nomades. Essai sur l'Ame juive, 2662

Novecento in Germania, 2984

Novellen des Lyrikers, 1606

No Voice Is Wholly Lost, 2604

Nur für Leser. Jabre und Biicher, 2901 a

Nusus Mukbtaroh nun Tolstoy, I535

Oliver Twist, 845

$O$ Mensch, I543

I00I Nacht, der Sinn der Erzäblungen der Scheberezade, 1562

The Origins of Totalitarianism, 2522

Österreichische Profile, 2745

Padesát let Egona Hostovskébo, 2520

Les Pages immortelles de Tolstoi, I 536

Panorama of Modern Literature, 263

La Paz del Hombre, 3024

Peter Camenzind, I 573

Le Phénomène Nazi, 2667

Plus jamais ca!, I 528

Poesia e Realta, 2985

Portraits and Self-Portraits, $260 \mathrm{I}$

Prager Rhapsodie, I5 II

Les Précurseurs, 2676

The Pre-War Biographies of Romain Rolland and Their Place in His Work and the Period, 2621
Princesse Brambilla, I504

Probleme des Realismus, 2844

Probleme und Gestalten der österreichischen Literatur, 2868

Profetas y Poetas, 2594, 3025

Prominente plaudern, 2940

A Prophet of the Soul: Fyodor Dostoievsky, 2580

Psychoanalytic Explorations in Art, 2565

Rainer Maria Rilke. Aspects of His Mind and Poetry, I 525,2492

Rainer Maria Rilke - Bibliography, 2883

Rainer Maria Rilke. Legende und Mytbos, 2902

Rainer Maria Rilke. L'Evolution spirituelle du Poìte, 2642

Rampenlicht, 2809

Rebellion in the Backlands, 2542

Reden (Buddha), I546

Rede und Antwort, I58I

Reference Works and Histories of Literature, 3206-3339

Rembrandt, 1448

Repräsentative Original-Handschriften, 2797, 3390

Richard Debmel, 2700

Richard Strauss. Dokumente eines Lebens und Scbaffens, 2929

Rilke, Europe and the English-speaking World, 2578

Rilke's Craftsmanship. An Analysis of His Poetic Style, 2530

Rimbaud. Querschnitt durch sein Leben und Werk, 1622

Romain Rolland (Arcos), 2643

Romain Rolland (Lerch), 2833

Romain Rolland (Weiss), 2948

Romain Rolland and a World at War, 983 , 2609

Romain Rolland et l'Allemagne, ${ }_{266}$

Romain Rolland Vivant, 266I

Romantiscbe Erzäblungen, 1490

Romanzi e Romanzieri d'oggi, 2986

Römische Elegien, I548

Röntgenstrablen, I563

Rosen, I553

Rot und andere Gedicbte, 1577

Rubens, I 449

Ruland, I 554

Sabbath, I568

Sagenhafte Sinnspiele, I58 I

Salzburg. Bildnis einer Stadt, 2937

Salzburg und das Salzkammergut, I6 2

Saints de notre Calendrier, 2680

Sämtliche Romane und Novellen (Dostojewski), 1493 
La Scena e la Vita, 2987

Ein Scbimmer Licht im Dunkel, 1427

Das Scblangenbemd, I 23

Ein schlichtes Herz, I556

Scbmidtbonn, I 48

Schmitz, I301

Die scböne Frau. Novellen, 1483

Schorer, 2649-265 I, 2654

Die Schule der Empfindsamkeit, I557

Die Scbutz flehenden, 1348, 1401

Scbiveitzer, II58, I365

Sechs Essays (Arendt), 2687

The Secret Life of Salvador Dali, 2543

Semblanza de Arturo Toscanini, I486

Sibylle und der Papagei, I 592

Sigmund Freud (Zilboorg), 2630

Sigmund Freud. His Exploration of the Mind of Man, $263 \mathrm{I}$

Die Simona, 1554

Spectrum, I I 29a

Speigelungen (F. M. Zweig), 2974a

Die Standarte, 2916

Der starkere Simson und Anderes, 1593

The Stature of Thomas Mann. Antbology of Critical Essays, I 357, 2583

Stefan George und sein Kreis. Eine Bibliographie, 2826

Stefan Zweig Presents the Living Thoughts of Tolstoy, 1 534, 2494

Stendhal y Otras Figuras, 3028

Stimme aus dem Dunkel, I542

Stories of Scarlet Women, 294

Streitbares Leben, 2735, 2813

Stropben (Günther), I 566

Stundenbuch (Rilke), 2530

Tag, 21, 53, 56

Tagebiicher (Green), 2787

Tagebiicher, Aphorismen, Essays und Reden, 2860

Das Tagebuch. Gedichte (Greiner), I564

Tellers of Tales, $28 \mathrm{I}$

The Tempest (Shakespeare: Sturm), I 3 II

Le Temps viendra (Die Zeit wird kommen), 1436

Une ténébreuse Affaire, 2544

Ten Eventful Years. 1937-1946, 2627

Thema und Variationen, 2947

They Dared to Live, 2528

Three Loves of Dostoevsky, 2605
(Maria von) Thurm und Taxis, 2882

Torch of Freedom, (73), 2525

Tragedy: Serious Drama in Relation to Aristotle's 'Poetics', 2571

Treasury of the Free World, 2575

Trophäen, 1570

Twenty Best European Plays on the American Stage, I 31

21 Variations on a Theme, 275

Tycho Brabes Weg zu Gott, 1488

Unsterblicher Genius, 2950

Verboten und Verbannt, 2746

Die Versuchung des beiligen Antonius, 1558

Die visionäre Kunstphilosophie des William Blake, 1437

La Voce sotterranea, 1492

Vom letzten Tag, 1567

Vom Sinn unseres Jabrbunderts, 1487

Von zwei Ufern, 1602

Die Waffen nieder, XXXII, 1617

Warum ich Manneskleider Trug, I5 I0

Wedekind, 1379

Der Weg der Jugend. Roman, I545

Weib, 1432

Die Welt Albert Schweitzers, I482

Die Weltanschaunng Dostojewskis, I 518

Weltdichtung, 1406, 1476

Weltinnenraum. Die Dichtung Rainer Maria Rilkes, 2793

Das Werk Thomas Manns, 3366

What Will Happen to the Jews? I 508

Wiener Juden in der deutschen Literatur, 2964

Wie sie es sehen, 147, 2965

Winland. Novellen und Legenden, I 85

A World of Great Stories, 295

Worte vor dem Leben, I5 16

Writers and Writing, 267

Wunden und Wunder. Gedichte, I I IS

Yevreiskie Pisateli, 3005

Yisröel. The First Jewish Omnibus, 303

Zbirnyk Tvoriv, 3033

$Z d Z$, 1130

Zeitgefäbrten, 2730

Die Zeit wird kommen (Le Temps viendra), 1436

Der zerbrochene Krug und andere Geschichten, 1583

Zweigs Ausgewäblte Prosa, 2957

PERIODICALS AND NEWSPAPERS

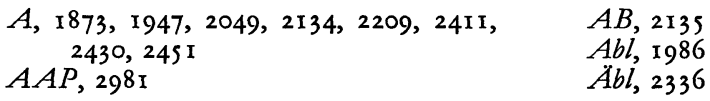


Abz, 1372, I393

Adam, 993

AdP, 1354, r391

$A D V, 60 a$

$A f d W, 2825$

$A H, 2232,2549$

$A H R, \mathrm{1874,2622,} 2037$

$A I R, \mathrm{1286}, 2560$

AJPH, 2368

$A L$, I 134

$A M$, I3I 2, 241 2, 2452

AMoz, 2697

An, 22 го

$A P L, 1062,1737,1973,2117,2255$

$A P z t, 2893$

$A R, \mathrm{r} 948$

$A R s c h, 1197,1362,3376$

As, 2050

Ate, $3017,3027,3038$

$A u, 2445,2759,2760,3039,337$ I

$A U C, 2407$

Auf, 2914

$A u(N Y), 1283,2800,283 \mathrm{I}, 2901,2920$, 2958, 3040-304I

$A W J D, 281 \mathrm{I}, 3042$

$A Z$, I 294, 2495

$A Z J, \mathrm{r} 639$

$B A, \mathrm{I}_{763}, 2020,205 \mathrm{I}, 2090,243 \mathrm{I}, 2479$, 2494, 2506, 254I, 2550, 2568, 2582 , $2602,2636,2640,3043,3369$

$B b, 1626,2903$

$B B B, 1764,2754,3044$

$B B C, 1640,1749,2337,2496,2757,3045$

$B B M, 1082,1088$

$B B S F, 2930$

$B B Z, 1987,2795$

$B d$, 1016, 2338, 2802, 281 2, 2848, 2913, 3046-3047

$B d B h$, I 396, 2736, 2832, 294I, 3048-3049, 3386

$B d T G, 1388$

$B E T, 2626$

$B F$, 1984, 2046, 2074

$B g, 2171$

$B i b, 2172$

$B I G$, 194I, 2923

$B \mathrm{~kg}, 2493$

$B k s$, 1903, 2089, 2132,2366

$B l$, I $714,1778,1798,1816,1875,1885$, 1904, 1949, 2021, 2052, 2091, 2130 ,

$B M, 2339$

$2136,2220,2369,2413,2453,2507$

$B m$, I $715,2022,2190,2211,2233,2279$, $2370,2546,3050$

$B M B, 2217$

$B M e N, 1886,2590,3051$
$B N$, I57I-I 572, 2915

Bng, 2340, 2838, 2853, 3052

Der Bogen, 337 I

$B r, 2694,3053$

Bre, 3030, 3054

Brs $Z, 2905$

Brï, 2867

$B S, 1887$

$B T, \mathrm{XXXI}$, I01 7, 1034, I 142, I 168, I 196, I 209, I 2 I 2, I 214, I 228, I 232, I 233, I 239, I 253,1357 a, I 374, I380, I 389 , I $543,1627,1695,1929,1988,2173$, 2262, 2305, 234I, 2783, 2961, 30553056,3375

$B T r, 1663,1770,1779,1817,1950,2023$, 2053, 2092, 2137, 2191, 2212, 2219, $2234,2280,3057$

$B U, 1075,1852,2202,2256,2297,2328$

$B \ddot{B} B, 1865,1989,2263,2306$

Bïg, 2910, 3058

Büh, 2 I 74

BuI, 2869

$B \ddot{u ̈ K}, 2446,2518,2974$

$B u L, 2733,2828,3059$

$B V$, I 769, I946, 2088, 2 I 3 I

$B W, 1696$

$B w k, 184 \mathrm{I}, \mathrm{r} 888,2508$

$B Z, 2909,3060$

Cabiers Charles Du Bos, 992

Car, 2683, 306I

$C C$, 1905, 1951, 2371, 261 8

$C d S, 1974,2118,2162,2324,2386-2387$

$C D T, 2093,2138$

$C F, 1952,2054,2139$

$C H, 2055,2140$

CHF, 24I 4

Cbm, 1906, 2432

Cbr, 1907

Cht, 1047

$C b w, 2342$

$C J R, 1126,2455$

ClW, 1799

$C M, 1985$

Cmy, 2509, 2613

Coe, 2676

Colq, 2972

Com, I 243

ConR, 2623, 3062

ConW, 2620, 3063

Correio da Manba, 990

CorS, 2988, 3064

COS, г716, 1780, 1818, 1908, 1954, 2057, 2094, 2 I 4I, 2235, 2434

$C R, 2473$

Cuadernos Americanos, 989

CurBio, 3065

I 64 
$C V, 2429$

$C V Z, \mathrm{1} 205, \mathrm{1} 343, \mathrm{I} 64 \mathrm{I}-1642, \mathrm{1} 686, \mathrm{1} 765$, I 86I, 1942, 2218, 2343,2839, 3066

$C W, \mathrm{1230}, \mathrm{1267}, \mathrm{1664}, \mathrm{1717}, \mathrm{1909}$, 1955, 2 I 42, 228 I, 2454

Cwl, 1665, 1781, 1876, 1953, 2056, 2095, 222 I, 24I $, 2433,2456$

$D, \mathrm{XXXII,}$ I 139 , 1862

$D A, 1323$

$D$ Ang, 2926, 339r

$D A Z$, I331, 1990, 2931, 3067

$D B, 1704-1705$

$D B a$, I 187

$D B e i, 2692$

$D B i b,{ }_{3} 64$

$D B K$, (19), I I47, I I 56, I I 75, I335, I 382 , 3374,3382

$D B P, 2307$

$D B u$, I991

DD, I8-20, 24, 33, 35-36, 40-4I, 45, 48$52,54-56,59,61-64,66,68-71,76$, $78,80,82,84,86,88,9$ I-92, I I 80, I399-1400, I404, I409-I425, I428, I 438-1 439, I 475

$D D i, 1366$

$D D r, 1643,1674$

$D e B, 93$

$D e G, 2521,3068$

DeuE, 289 I

DeuR, 2694, 2726, 2766, 2966

DeuV, 2889-2990, 2892

$D F, 2490,282$ I-2822

$D F a, 2504$

$D F R$, 1018, I 293, 1738

$D F u, 2472$

$D G, 2229$

$D g D, 1042$

$D G T, 2792,3069,3371$

$D H, 1076,1079$

$D i$ I I 82, I 326, I 718, I 835,2 I92, 2236

$D I D O ̈$, I 221

$D I Z, \mathrm{I} 644$

$D J Z$, I992

$D K, \mathrm{x} 250$

$D K w, 2956,3392$

$D L$, 1002, 1077, 1083, I $217,1264,1280$, I 367, I 549 , I 588, I 590, I 61 2, I621, I631, I675, I766, 1930, 1987-1988, 1993-1994, 1998-1999, 2004, 20092010, 2015, 2018, 2079, 2084, 2087 , 2230, 2278, 2305, 2308, 23 I 2, 23I 6 , 232I, 2340-234I, 2344, 2357, 2505, 2688, 2690, 2694, 2717, 2721, 2737 , $2748,2755-2757,2782-2783,2798$, 2802, 28I 2, 2830, 2843, 2845, 2848, 2852, 287I-2873, 289I, 2894, 2905,
2917, 2919, 2931, 2949, 3070-3078 $D L E, 29,986,1095-1096$, I I35, I154, I I 57 , I I 76, I 202, I 2 I 2, I 229, I 254, I 276, I 297, I3 13, I $315,1347,137$ I, I 544-I 545, I 547-I 548, I 550-I 552, I555-I558, I559-I561, I563, I567I 570, I574, I 576-1586, I 591-I6II, I6I3-I6I6, I6I8-1620, I628, I630, 1634-1635, I639-1640, $1645-1647$, 1652-1653, I655, I658, I661, I679, 1697, I699, 1 702-1703, 1 706-1707, 1744, I 747, 1749-1750, 1 761-1 762, I 866-1867, 1869, I931, 2264, 2272, 2275, 2488, 249I, 2752, 2754, 2840-

DLe, I 367 284I, 2952-2953, 3079-3082, 3099

$D L W$, IO13, 1019, I025, I 220, 1 247, I 257, I 342, I 395, I 398, I994, 2843 , 2895, 2955

$D L Z, 1632$

$D L z$, I I 76, I 264, I 280, 2842

$D L Z g, 2489$

$D M$, I 698

$D M f C$, I 108

$D M o, 28$ I 8

$D M W, 2345$

$D N$, г 368

$D N E, 1647$

$D N G, 2367,2409$

$D N N$, I995

$D N S$, 1932, 2205, 2265

DNSch, I 109, 1345, 295 ז, 338 I

$D N Z, 2175$

$D N Z g, 2693,3383$

Do, 1339

Do $A, 3036,3083$

$D o i B, 299$ I

$D P b l, 2447$

$D P r, 2733$

$D R, \mathrm{I} 996$

$D R a, 1084$, II 53

$D R g, 1997$

$D R Z$, 1998

$D S, \mathrm{x} 872$

$D S a, 1902,2715$

$D S c h$, I 195

$D S L, 1676,27 \times 8$

DSlb, 1316

DSt, 1456,1466

DSTa, 2762

$D T$, 1860, 1999, 2176

$D T b$, I 52

$D T g, 2266$

$D T p, 2697$

$D V, 2309$

$D W$, I 699 
$D W B, 2410$

$D W e, 2805,285$ I, 3084

$D W k$, I I I I

$D W 0,2888,2912,3085-3086$

$D W r, 1035$

$D Z, 1680,1708$

$D Z e, 2710,2810,3087$

$D Z f S, \mathrm{I} 043$

$E$, II94, 2027, 2388, 2684, 3088

$E a, 2469$

$E A P, 2346$

$E C, 2 \mathrm{I}_{3}$

Eck, 2347

$E d, 2993$

EeL, 268I

$E N, 1738$

En, 1048

EPCJ, I975, 2164

Er, (4I), 25 IO

Erasmus, cf. Er

Erz, 1318, 2704

$E S, 2750$

$E u G, 2000$

$F$, 1 237, 1 295, I 573, 1762

FdT, 2348

La Feuille, XXXII

$F i, 2252$

$F k, 216$ I

$F m, 2193$

Fo, 1771, 1819, 1956, 2096, 2143,2222 , 2237

Fr, 2080

$\operatorname{Fr} A$, I43 I

Frw, 3090

$F T, 2001$

$F T b, 1162,1341$

Fu, 2349

FuS, 1044, 1288

$F u T, 2799$

$F V b$, I 74

$F W, 2576$

$F Z$, I 147, I I 59, I I 76, I I 79, I 292, І 349, I 565,208 I

$G, 2002,2932$

$G B$, I I I4-I I I , 309 I

$G d G, 2819,3092$

$G d l, 2350$

$\mathrm{Ge}, 17,37$

Germ, 2904

GermR, 995, 2574, 261 2, 2639, 2743

GJ, 2058

GLL, 999, 178a, 2597, 2633

$G N, 2$ I33

GoeJb, 2990

$G O M, 23$ I0

$G Q, 2498-2499,2635,3393$
$G R, 1089$

Gr, 2003, 2038, 2177,23 I I, 2487

Greifenalmanach (1956), 2960

GRM, 2709

$G S, 2782,3093$

Gwt, I 190, 2206, 2448, 2470

$H a N$, 1026, 1291

$H A R, 2449$

HFbl, 2004, $2178,2351,2845,2872,2925$

$H g, \mathrm{I} 68 \mathrm{I}$

$H K, 1065,1700,2005,2352,2852$

$\mathrm{Hl}, \mathrm{1} 933$

$H L A S, 2435$

$H M$, I 2 I I

$H N, 1244,1682$

$H O, 2024$

HSFS, 2006

$H T, 2170$

$H w, 1677$

$H y, \operatorname{Ios} 7$

$I, 1739$

$I A R, 1870$

IBra, 1998, 3094

IC, 2249, 2300, 2332, 240I

ICS, I 853, I $856,1858,2047,2125,2167$,

ID, 3009 2250, 2301, 2333, 2402

$I F, 2714$

$I L N$, I $800,1889,2059$

$I M, 2619$

Im, 1753

$\operatorname{Imp}, 2693,3383$

$I N, 3095$

In, (54), 23, 34, 47, 57, 67, I014, 1027, 1036, 1049, 1066, 1097, I 100, I I 24I I 25, I I 58, I I66, I I 89, I I 91 , I 272, 1 274, I364-1365, I45 I, 1465, I479, $2716,2788,2803,2875$

In $A, 30,46,60,72,74,87,1045$, 1050, 1058, I085, I IOI, I II6, I I49, I I69, I299, I $311,1330,1450,1453$, I457, I 46I-I 462, I 467-I 468, I 47 I-I 472

$I P, 247 \mathrm{I}, 2984$

Isb, 1020

$J A, 2594$

$J a, 1754$

$J B B, 2502$

$J D, 1740,2039,2119,2389$

$J D B, 1_{3} 82,1_{86}$

JdG, I 168

JDSG, 1709

$\mathrm{Je}, \mathrm{1} 648$

Je A, 2628

JLG, 2497

$J M H, 1957$

$J N M D, 2223$

I66 
$J R, \mathrm{x} 649-165 \mathrm{x}, \mathrm{1943,} 2007,2924,3096$ $J S p, 2539 a, 2614,3097-3098$

JüGbl, 2713

$J U L R, 1001,2526,2638$

$K$, 1842,25 I

$K A Z, 2008,2312$

$K D, 2705$

$K d \ddot{L} L, 1394$

$K H Z$, I I 73, 1321, 2694

$K N N, 2690$

$K \ddot{0} T$, I 193

$K P, 2009$

$K r, 2082$

$K r Z, 2894$

$K T$, I 172, 1373

$K t, 1078,1086,2267$

$K V Z, 2010$

$K Z$, 102I, 1028, 1059, I390, 20I I, 2083$2084,2353,2755,2873,3099-3100$

$L, 2126,225 \mathrm{I}, 2302,2403-2404$

$L A, 1008,1029,1090,1200,1383,1809$, 2416,2587

$L A M, 2983$

La Nouvelle Relève, cf. LNR

$L B, 2760$

$L C$, (38), I 36 I

LCfD, 1934, 2085

$L D, 2$ I 44, 2558, 3 IOI-3102

Leader (Allahabad, India), 2978, 3 I03

Le Carmel, cf. LC

$L F, 1252$

LGRP, $2179,223 \mathrm{I}, 2268$

$L H$, 1935, 2012, 2269

$L I$, I 857

Lib, 1355

$L J$, I843, I877, I890, 24I 7, 2436, 2457, 2512

$L J Z$, 1324

$L L M, 1976,2390$

$L M, 1958,2025,2145,2492$

$L M F, 2652,3104$

$L N E, 2257$

$L N L$, 1091, I 307, 2665

$L N N$, I163, 1678, 1701, 2313

$L N R,(74), 2645,3$ Ios

$L P$, I $92 \mathrm{I}$

$L T$, I666-1667, I687, I $719-1720,1760$, 1782-1 783, I801, $1808,1820-1821$, 1864, 1910, 1959, 2026, 2060-2061, 2097, $2146-2147,2$ I94-2195, 2238, 2282, 2418, 2458, 2519, 3106

$L T b, 2180$

$L W, 218 \mathrm{I}, 23 \mathrm{I} 4$

$M$, 1337, 1652

$M A, 2480,3107$

MaL, 2579
März, 2704

$M A Z, 2824$

$M B, 2148$

$M C, 2563,3108$

Mer, I I 29, 297 I

$M F,(77)$, I 74I-1 742, I 755, I 977, 22582259, 239I-2392, 2647

$M f D U$, (33), rooo, 2408, 2500, 2545, 2634,2637

$M f F L, 1278$

$M f L$, I I 80, I 204, I 268, I 305, I 370 , I 385 , 1405 , I 407-1408, I 566, I 575

$M G, 1784$, 191 I, 2062, 2459

$M H, 2098,2372$

$M J,(60), 2460,2539,2557$

$M \mathrm{~kg}, 248 \mathrm{I}$

$M L J, 2501$

$M L N, 994,996,2213,2573,2589$

$M M N, 23$ I, 2354

$M M W, 2355$

$M N N$, I004, I 183, I 234, I316, I391, $1636,2013,2316$.

$M O, 2128$

Mo, 1944

Mon, 2865

Monatsheft, cf. $M f D U$

$M P$, I 145

$M S, 1922,2649$

$M s, 2523,3384$

$M u, 2697$

$M Z, 1022,1223,1266,1710$

$N$, I668, I 785 , I810, I822, I 829, I 836 , I 844, I 878 , I $912,2027,2063,2099$, 2149, 2196, 2214, 2224, 2239, 2373, $2461,2566,2577,3109$

$N A, 1079,1688,2075,2127,2303,2980$

$\mathrm{NaQ}, 2503$

$N A R, 2028$

$N A S G, 2970,3371$

Navyug (India), 2977, 3110

$N B, 2356$

$N B K L, 2182$

$N B L, 1750,1767,2357$

$N B R M M L A, 2536$

$N C, 2$ roo

$N D H, 2693,3383$

$N D L,(2), 1883,2722-2723$

$N e, 1786,2064,2437,3$ III-3IIS

Nef, гог г

Die neue Film-Post, 3352

NFP, XXVII, I005, Ior 5, 1023, 1030, 1038, 1046, 105 I, 1060, 1067-1068, I080, 1098, III 8, I I 36-II37, I I6III62, II77, II89, II9I-II92, I 201, I 206, I II9, I 222-I223, I225, I 23 I, I 24I, I 246, I 248, I 259, I 266, I 269, 
I 278, I 284, I 288, I 299, I 300, I 3 I 9 , I321, I328-1329, I 332-1333, 1336, I35I-I352, I363, I376, I378, I38I, I 546, I6I7, 1653-1654, I 702, I 711 , I 745, I 768, 1945, 2 I 29, 2 I83, 2207, $2270,2317,2358,2685,2822,3116$ $N F P-J b, 1087$

$N G, 1756,2318$

$N K, 1689$

$N L$, I623

$N L A S, 1006$

NLW, 2078, 2996

$N I W, 2359,2808$

$N L Z, 1069$

$N M, 2595,3117$

$N M z, 2697$

$N p h Z, 2751$

$N R, \mathrm{XXXIV},(55), 22,1054,1063,1102$, I I40, I I 44, I I I I, I I 78, I I 86, I 2 I 7 , I 238, I 249, I 25 I, I 265, I 289, I 346, 1 369,1458 , I 554, I 587 , I 589 , 1746, 2014, 227I, 2699, 2959, 3 I I 8

$N R e, 2260,2325$

NRep, (74), I $72 \mathrm{I}$, I 772, I802, I8I I, 1823, 1891, 1913, 1960, 2029, 2065, 2101-2102, 21 50, $2197,2283,2374$, $2438,2462,2600,2607$

$N R F, 1743,1851,2293,2326,2329$

$N S, 2030,2240,2284$

$N S B, 1855,2168$

$N S c h R$, I 347, 291 I, 3II9

$N S N$, I 722, 1787, 1830, 1892, 1961, 2 I03, 2I II, 2419

NVzt, 2820, 2908, 3 I 20-3 I 2 I

$N w, x 751$

$N W J, 2952$

$N Y$, I 788, I 845, I 879, I $893,2225,2420$, $2439,2463,2474,25$ I $3_{3}$

NYEP, 1812, 2104, 224I, 2375

$N Y H T$, 1962

NYHTB, (59), I669, 1789, I803, 1813, I824, I83 I, I837, I846, I880, I894, 1914, 1963, 2031, 2066, 2105,2152 , $2198,2226,2242-2243,2285,2376$, 242I, 2440, 2464, 2475, 2514

$N Y P, 1895$

$N Y S, 2286$

$N Y T$, г103, г199, I690-1694, г 723-г 727 , $2588,2615,3122-3136,3345$

$N Y T B R$, 1670, I773-1774, 1790, I 804, I814, I825, I832, I838-I839, I847, I881, 1896, 1915, 1964, 2032, 2067 , 2106-2107, 2153, 2199, 2215,2244 , 2287, 2377-2378, 2422, 244I, 2465, $2476,2482,2515,2559,3137-3143$

$N Y W$, r8I , r840, 2245, 2288
$N z$, 1 306, 1703, 1 747, 2184,2272

$N Z f M, 2483,2933$

$N Z Z$, 1031, 1392, 1655, 2015, 2721, 2756, 2854,3144

$O, 1728,2033,2200,2289$

Oc, I901, 3002

$O M z, 2484$

$O \bar{o} N, 2829,2954,3145$

$\mathrm{Op}, \mathrm{1} 97^{8}$

$O R, 26,28,39,77,81,1037,1216,1303$,

OS, 1256 I $553,1564,1757,2273,2886$

$P, 2040,2203,2253,2298,2393$

$P A, 2108,2379$

Pan, 1353

$P B$, 1070, I104, 2379

$P b, 1656$

$P e R, 2405$

$P b$, I 285, I 296, 3377, 3379

Philo, 2898, 3146

PIO, 1965

PIQ, 1791, 2109, 2227

PJ, 1081, 1633, 1683, 1936, 2016

$P L$, 1009, I01 2, I I19, I I 72, I I88, I 216 , I 278, I 344, I361, I637, I657, 2086, 29I 8,3 I 47

PlV, 2670

$P M B, 1729,1775,2380$

$P M L A, 1730$

$P N W, 2319,2360$

PoL, 2570

$P P, 2919,3148$

$P Q, 2381$

$P R, \mathrm{I} 275$

Pris, 2747, 3149

PRo, 1923

$P T, 1309,1658$

$P T b, 1264,1280$

$P W, 1792,2547,3150$

$Q R, 2610$

$R A$, I071, I I50, 2 I 20

$R A A, 2294$

Rad, 2830, 287I, 3ISI-3I52

$R a E$, I9I6

$R B$, 1039, I092, I I05, I I 20, 204I, 2 I 2 I

$R C, 1064$

$R C C, 1624$

$R D$, ro94, I146, I230, I267, 1310, 3153

$R d A$, 3010, 3031

$R d C, 2076$

$R d F, 2394^{-2395}$

$R d l I, 3020,3154$

$R d O$, I I 2 I

$R d P, 2334$

$R d V, 1481,1979,2396-2397$

$R E$, I 27I, I3I4 
$R e ́, 1924$

REH, 2042

$R E I, \operatorname{105} 2$

$R E L M, 2$ I 22

$R E L V$, 1850, 1980, 2043, 2165, 226I, 2295, 2330, 2398-2399

$R G, 2123,2296,2299,2320,2427$

$R H$, 1040, 1981, 2 I 66

$R H b, 2327,2400$

$R H E F, 1982$

$R H G$, 1024, I053, 1074, I 106

$R H P R, \mathrm{I}^{283}$

$R M, 2077$

$R M d, 2254,233 I$

$R N, 1854,2169,2304,2335,2406,2428$

RoR, 2935

$R P$, 997, IoIo, I I 22-II 23, 2044, 2669, $2677,3155-3156$

$R Q H, 2124$

$R R, 2034,2$ I IO, 2I 54, 2201, 3 I 57

$R \operatorname{Rev}, 2572$

$R R h, \mathrm{I} 454, \mathrm{I} 459, \mathrm{I} 470, \mathrm{I} 473,2207 \mathrm{a}$

$R T, 1659,2846$

$R v B, \mathrm{I} 377$

$R X, 2290$

$S$, I712

$S A Q, 1966$

$S C, 1925$

SchM, 1032

ScbMr, 2485

ScbR, 2017, 2361

$S E, 2068$

SeR, (65), 1967, 2534

Sewanee Review, cf. SeR

$S F C$, I 897,2516

$S G, 2$ I I I

SGM, 2069

Si, 1898

$S L R, 2035,2246,2291$

SLzt, 2927, 3158

$S M, 1055,2362$

$S N T$, I 772

Social Welfare (India), 2979

SoM, 2486

SoS, 2442

Soz, I660

$S P$, I 323

Sp, I731-1732, I 793, I828, I917, г968, 2 II $2,2155,2423,2598$

$S R, \mathrm{I} 776, \mathrm{I} 794, \mathrm{I} 805, \mathrm{I} 826, \mathrm{I} 833, \mathrm{I} 848$, 1918, 1969, 2070, 2113, 2156, 2382, 2424, 2443, 2466, 2477

$S R L, 1355, \mathrm{I} 733, \mathrm{I} 777, \mathrm{I} 806, \mathrm{I} 827, \mathrm{I} 834$, 1849, I 899, 1919, 1970, 2036, 2071, 2292, 2425, 2467, 2478, 25 I 7, 2540, 2555, 259 I, 3 I 60-3 168
$S S, 1752$

$S S A, 1056$

$S S R, 2383$

$S T, 2835$

Sy, 2384

$T$, 1672, I796, I 807, I900, 2072, 2426, $3169-3170$

$T a b, \mathbf{1 9 2 0}$

$T A M, \mathrm{I} 673, \mathrm{I} 734-\mathrm{I} 735$

$T b, 1622$

$T h$, I97I, 2 II 5,2 I 58

$T L, 1859$

$T R, 1713,2321$

$\operatorname{Tr}$, I 184

$\operatorname{Tr} G, 1926$

Tribune de Genève, 1902

$T s, 2045$

Two, 2995

$U, 2048$

$U d, 1327,2204$

UfL, 2365

$U m, 2363$

Un, I173, I 216,2688

Uns, 2450

$V, 1033,1363,2949$

Vbü, 2697

$V d F, 2444,2653,2656,3171-3172$

$V e n, 2671$

Ver, 30I4

$V F Z M$, I I38, II 70, I I 85, I 208, I 277, 1302,1356

Vin, 2992, 3173

$V K, 1748$

$V P, 1927-1928,2650$

$V Q R, \mathrm{I} 284$

$V u V, 2862,3174$

$V V, 1937,2274$

$V Z$, (38), 1061, 1072, I 155, 1273, I 335 , 136I, 166I, 2018, 2087, 2275, 3373, 3380

W, 1884

WaM, I 250

$W A Z, 2$ I 85

Wbï, 2804

$W d A, 2900$

WE, I230, 1267

$W i$, 280

WiaL, 2994

$W i d Z, 2693,2836,2863,2944,3383$

Wiener Revue, $337 \mathrm{I}$

WieZ, 2733, 2785, 2864, 3175

$W L$, 1871, 1938, 2187

$W L B$, I 736, I 797, I882, I972, 2073, 2 I I6, $2159,2385,2468,3176-3177$

$W M, 1684,1939$

$W R, 2208$ 


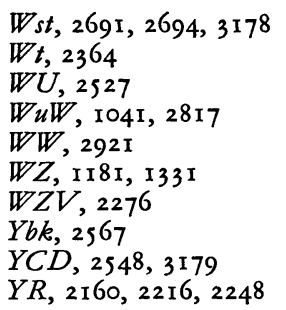

$Z, 38,65,83$, I I 49, I I 82, I 244, І 340, I629-1630

$Z B, 175^{8,} \mathrm{1} 868, \mathrm{1940}, 2277,2322$

$Z D, 2323$

$Z e, \mathrm{I} 203, \mathrm{I} 278,1430, \mathrm{I}_{3} 8$

$Z f B, 1685$

$Z F E U, 2$ 188-2189

$Z f G$, г 258

$Z P, 2019$

\section{TRANSLATORS AND EDITORS}

Abels, Lucy (Tr.), 1607

Adler, Paul (Tr.), I 268, 1509, I 555

Ahrens, Berta Burgio (Tr.), 403, 405-406, $837,916,1856,1858,2300-2304$, 2428

Akiyama, Hideo (Tr.), 658, 725, 862

Aladár, Sajó (Tr.), 8 I 5

Alden, Douglas (Ed.), 32 I 3

Aleksov, Lazo (Tr.), 687

Almagro Rodiera, G. (Tr.), 785

Alsberg, Henry G. (Tr.), 972, 2473-2478

Alves, Livraria Francisco (Tr.), $254^{2}$

Amann, Paul (Tr.), 1487

Ammer, K. L. (Cf. K. Klammer, Tr.), 988, I 52 I-I 522,2769

Ammer, R. (Tr.), I 586

Andresen, N. ('Tr.), 257

Androulidakis, I. (Tr.), 723

Angelloz, J. (Tr.), 809, I 536

Aoyagi, Mizuho ('Tr.), 817

Araryo, Milton (Tr.), 1272

Arens, Hanns (Ed.), (69), 2497, 2524

Arpad, Burhan (Tr.), 929

Arpe, Werner (Ed.), 3252, 3340-3356

Ascutan, Mavelikkara (Tr.), 395

Asteriadis, Ioannes (Tr.), $72 \mathrm{I}, 754$

Augé, Paul (Ed.), 3253

Babler, Otto F. (Tr.), 226, 229

Baedeker, Peer (Ed.), 980

Ballester Escalas, Rafael (Tr.), 479-48o

Bamberger, Max (Tr.), I6I 4

Bandy-Opadhyay, Shantiranjan (Tr.), 383 , 386-387

Banerji, Shantiranjan ('Tr.), 380-382, 384385

Baron, Joseph L. (Ed.), 327 I

Barry, Charles P. (Ed.), 3217

Baseggio, Cristina (Tr.), 406

B'ase'wis, I. ('Tr.), 786

Bea, Joseph (Ed.), 32 I 2

Bedford, Herbert (Tr.), I 9

Beer, Johannes (Ed.), 3265
Bekata, Kemal (Tr.), 507-508

Belmont, L. (Tr.), 887

Beneš-Sumavsky, Vaclav (Tr.), 638

Beratis, Ioannes (Tr.), 587, 835

Bernardi, Orlando (Ed.), 3233

Berenguer Amenós, Jaime (Ed.), 3238

Bernstein, P. (Tr.), 4, 459-460, 462-463, $466,663,820,84 \mathrm{r}$

Besterman, Theodore (Ed.), 3276

Bhattacharya, Vidhayek (Tr.), 379

Birsel, Salah (Tr.), sos

Bithell, Jethro (Tr.), 788-789, 2209-22 I6, 2532, 3282-3283

Blaustein, Phyllis M. and Albert P. (Eds. and Trs.), $26 \mathrm{I}$

Blewitt, Phyllis and Trevor (Trs.), 264, I 778-1 797

Bloch, Henri (Tr.), 832-833, 2297-2299

Block, Maxime (Ed.), 322 I

Blom, Eric (Ed.), 3245

Bobetsky, Victor (Ed.), I64a

Bobynskyj, Vasil (Tr.), 5 I I-ร I 2, 5 I 4

Boon, Josef (Tr.), I I

Botez, Joachim (Tr.), 450

Boudouin, Louis Charles ( $\mathrm{Tr}$.), Ios

Bournac, Olivier (Tr.), 306, 308, 3 II, $315,317,325-326,612,648-651$, $807-808,831,852-853$, I 504, I850I852, 2037-2045, 2253-226I, 22932296, $2325-2331,2675$

Br'aq'azs, Hayim (Cf. Ch. Brakarz, Tr.), I I I, 5 I 5-5 I 8, 970

Brewster, Dorothy (Ed.), 267

Bridgewater, William (Ed.), 32 I 9

Brieger-Wasservogel, Lothar (Ed.), I484, 2488-2489

Brøndum-Nielsen, Johannes (Ed.), 3266

Brosniowskaja, O. (Tr.), 46r

Buacidze, S. (Tr.), 693

Budtz-Jørgensen, Jørgen (Tr.), 232, 244, 1769

Bukovinszky, Peter (Tr.), 376 
Burke, Kenneth ('Tr.), I 182, 2 I92

Cahn, Alfredo (Tr.), II, I09-IIo, II4, $48 \mathrm{I}-483,555,569,697,784,89 \mathrm{I}$, $926,939,966,2204,3032$

Calendo, Albina (Tr.), 605

Carlsen, G. H. (Tr.), 422

Carvalho, Cándido de (Tr.), Io8

Cassvan-Pas, Sarina (Tr.), 45 I

Castanakis, L. (Tr.), 342

Castle, Eduard (Ed.), 3286

Castro Henriques Oswald, Maria de (Tr.), 554,690

Catalano, S. (Tr.), I 492

Cauris, E. (Tr.), 547

Centnerszwerowa, R. (Tr.), 6r3, 66r, 8 I9

Cerf, Bennett (Ed.), 262

Cerny, Rudolf (Tr.), 226

Changi, Bag (Tr.), 4I 7

Charodschinskaja, I. E. (Tr.), 459, 464

Chaudhuri, Dipak (Tr.), 388

Chauhan, Shivadanasimha and Vijaya (Trs.), 390, 392

Chayadevi, Abburi (Tr.), 396

Chernet, Henri (Tr.), 790, 2217

Chevalier, Haakon (Tr.), 2543

Clarac, Pierre (Ed.), 3229

Clark, Barrett H. (Ed.), 3287

Codjioulas, G. (Tr.), 755

Coenen, F. E. (Ed.), 209

Cohn, A. (Tr.), 363

Coleman, Arthur and Marion (Trs.), 2606

Constantinidis, N. (Tr.), 349

Cooper, Page (Ed.), 286

Coopman, Hendrik (Tr.), 546

Cornelius, P. (Tr.), I 5 Io, I 647

Cory, Donald W. (Ed.), 275

Cournos, John (Ed.), 295

Cranaki, M. (Tr.), 339

Cunningham, William (Ed.), 30I

Czermakowa, Izabella (Tr.), 427

Dániel, Anna (Tr.), 370

Dargan, E. Preston (Ed.), 2544, 3388

De Brie, G. A. (Ed.), 3208

De Bruyne, E. (Ed.), 3275

De Haas, Jacob (Ed.), 3240

Delmas, Fernand (Tr. and Ed.), 586, 912

Des Gouttes, Jacqueline (Tr.), 3 I 8

Dezsö, Kiss (Tr.), 369, 373

Dietrich, Margarete (Ed.), 3254

Dilly, Tante (Ed.), 3258

Dimitriou, D. (Tr.), 356

Dolezil, Zdenek (Tr.), I 303

Dominques, Mário José (Tr.), 592

Drews, Richard (Ed.), 2746

Dreyfus, Marcella (Tr.), 398, I853-1 854

Dupré, P. (Ed.), 324I
Dzapharidzis, Leli (Tr.), 331-332

Eckstein, Friedrich (Ed.), I 549

Elster, Hanns Martin (Ed.), I55

Engberg, Harold (Tr.), 243

Enking, Ottomar (Tr.), I 505

Eppelsheimer, Hanns (Ed.), 3209

Esen, Muzaffer (Tr.), I020

Eskeland, Severin (Tr.), 423

Etzel-Kühn, Gisela (Tr.), 6ro-6I I

Farhad (Tr.), 757

Farrán y Mayoral, J. (Tr.), 480, 495

Fehse, Willi (Ed.), I 5 I 4,2487

Feijii, Toshiak (Tr.), 686

Feuerstein, Emil ('Tr.), 366

Fico, Enver (Tr.), 22 I

Finlayson, C. P. (Tr.), 2580

Fitzbauer, Erich (Ed.), (10), I63, 528 , 1759-1 760, 2499-2501, 2763, 27672769

Fitz Gibbon, Constantine (Tr.), 302

Flerè, Pavle (Tr.), 696

Fodor, Pal (Tr.), 370

Foltin, Lore B. (Ed.), I $7 \mathrm{I}$

Fónagy, Iván ('Tr.), 378

Fontana, Oskar M. (Ed.), 2745

Fowler, Christobel (Tr.), 2497, 2524

Franulic, Lenka (Ed.), 3012

Franwaller, E. (Ed.), 3292

Franzos, Birta (Tr.), I 500

Freedley, George (Ed.), 3287

Freeman-Ishill, Rose (Tr.), I 5 I9

Frels, Wilhelm (Ed.), 3222, 3372

Frenzel, Herbert (Ed.), 3293

Friedenthal, Richard (Ed.), (68), 533, 560, 574-579, I 864

Friedmann, Hermann (Ed.), 3295

Friedrich, Paul (Tr.), I5 I6

Frisé, Adolf (Ed.), 2860

Fromm, Hans (Ed.), 3210

Fülöp-Miller, René (Ed.), I 549

Gallard, Alfredo (Tr.), 822

Gallotti, Odilon (Tr.), 434, 606, 937-938, 963

Gal'perinoj, R. (Tr.), $76_{2-763}$

Galstone, James (Tr.), 2947

Ganboa, Arístides(Tr.), I I, 493, 594

Gebsattet, Emil von (Tr.), 1570

Geissendörfer, Theodore (Ed.), 901, 2408

Gelovani, A.P. (Tr.), 693

Gentilli, G. (Tr.), I079

Ghion, A. (Tr.), 360

Giebisch, H. (Ed.), 3251, 3292

Gilli, Clementina (Tr.), 446

Gorfinkel, D. M. (Tr.), 454, 56r-562

Gorra, Marcella (Tr.), 91 7, 2428

Góth, Ferenc (Tr.), 372 
Graham, Bessie (Ed.), 3214

Gregor, Joseph (Ed.), 3267

Greogory, Aristides (Tr.), 555, 595

Greve, F. P. (Tr.), I 558

Grimal, Pierre (Ed.), 3228

Gromov, A. (Tr.), 688

Grün, Herbert (Tr.), 470

Gugeia, Eugen (Tr.), 1548

Gurlitt, Willibald (Ed.), 3264

Gutierrez, Fernando (Tr.), 476

Haantjes, J. (Tr.), 100

Hackett, Alice Payne (Ed.), 3269

Haga, Mayumi (Tr.), 919

Hagerup, Inger and Anders (Trs.), 960

Halberstam, L. (Tr.), I48 I

Ha-meiri, Avigdor (Tr.), 106

Hammerich, Clara (Tr.), 23I, 234, 242, $582,639-640,675,803,829,875$, 903,2365

Handlin, Oscar (Ed.), 3247

Hardt, Ernst (Tr.), I 556

Hasenclever, Walter (Tr.), I482

Hattori, Riútaro (Tr.), 779

Hauser, Otto (Tr.), I 620

Haycraft, Howard (Ed.), 3272

Haydn, Hiram (Ed.), 295

van Heerikhuizen, F. W. (Tr.), 953

Heinemann-Grautoff, Erna (Tr.), 1464

Heinzel, E. (Ed.), 3292

Hella, Alzir (Tr.), I I 3, 306, 308, 3 I I-3 1 2, 314-315, 317, 319-320, 323, 325-326, $544-545,604,612,624-625,648-$ $651,678-679,716-720,751-753$, 807-808, 8I0-8I I, 83I, 833, 852$856,880-882$, 9II-9I 2, IO62, I5O4, I850-I852, I92I-1928, 1973-1983, 2037-2045, 2II7-2124, 2644, 26582659, 2675

Henkel, G. (Tr.), 783

Hiltermann, G. B. J. (Ed.), 3275

Hirschberg, Yizhak (Tr.), 653-654, 682

Hirth, Friedrich (Ed.), I 569

Hirvensalo, Lauri (Tr.), 304

Hoetink, H. R. (Ed.), 3275

Hollo, J. A. (Tr.), 910

Homberger, Conrad (Ed.), I64a

Hornelund, Karl (Tr.), 542

Horvath, Zoltan (Tr.), 683, 756

Hostovsky, Egon (Tr.), 226

House, Roy Temple (Ed.), 2 IO

Houston, G. Craig (Ed.), 1525,2492

Huebsch, B. W. (Tr.), 298

Huf, Paul (Tr.), 253, 256

Hultenberg, Hugo (Tr.), 498, 500-502, 561-562, 573, 596, 609, 669-670, $766,826,850,895,940,969,2170$
Hünich, Fritz A. (Ed.), 2502-2503, 2803, 2875

de Huyn, May (Tr.), I 498

Hyeog-sun, Choe (Tr.), 416

Iizuka, Nobuo (Tr.), $552-553,2990-2991$

Imre, Sandor (Tr.), $37 \mathrm{I}$

Ippisch, Gottfried (Tr.), I4I, 2806, 2940

Jain, Yashpal (Tr.), 393

János, Péter (Tr.), 377

Jara, Galina (Tr.), 513

Jarnés, Benjamín (Ed.), 3235

Jasper, Gertrude (Ed.), 3213

Jen-piiän, Yang (Tr.), 770

Jensen, Harold (Ed.), 21 5, $1768 \mathrm{a}$

Jespersen, Kirstine (Tr.), 7 I I , 2088

Jiannacopoulos, G. (Tr.), 857

Jojkić, Vladan (Tr.), 468

de Jong, A. M. (Tr.), I 506

Jónsson, Halldór, J. (Tr.), 959

Joshi, Shri (Tr.), 39I

Jung, Gertrud (Ed.), 326r

Kahn, Máximo José (Tr.), 664, 667-668

Káldor, György (Tr.), 374

Kantorowitz, Alfred (Ed.), 2746

Karagiannes, S. (Tr.), 334

Karklins, Valdemara (Tr.), 727

Karnauskas, K. (Tr.), 420

Karrer, Al. (Tr.), 337, 340, 348, 355, 913

Kartuschanskaja, A. (Tr.), 466

Kawasaki, Yoshitaka (Tr.), 408-409, 4I3, 685

Kayser, Wolfgang (Ed.), 3249

Kelen, Ference (Tr.), 756

Kelk, C. J. (Tr.), r489

Kimhi, D. (Tr.), 363

Kindermann, Heinz (Ed.), 3254

Kjaergaard, Helge (Tr.), 235, 239-24I, 952

Klammer, Karl (Cf. K. L. Ammer, Tr.), 988, I $521-1522$

Klein, Otto (Tr.), 230

Klimová, Maria (Tr.), 581

Koch, Theodore W. (Tr.), 296, 1355

Koelen y Catalan (Tr.), 472

Kokichi (Tr.), 659

Konstas, T. (Tr.), 336

Kool, Halbs C. (Tr.), 3370

Koppens, Leon (Tr.), 426

Körner, Josef (Ed.), 32 I I

Kornfeld, Edna (Tr.), 915

Kosch, Wilhelm (Ed.), 3224-3225

Kouchtsoglou, J. (Tr.), 722

Koutecká, Jaroslava Votrubová(Tr.), 227

Kovacević, Radivoj (Tr.), 468

Kranz, Henry B. (Ed.), (73), 2525

Krasilschtschikov, S.(Tr.), 463 
Krebs, Sigfrido (Tr.), 556

Kruscinska, Wanda (Tr.), I 492

Kuiper, W. (Ed.), I49, 2957

Kulisher, A. S. (Tr.), 692

Kunitz, Stanley (Ed.), 3272

Kutzbach, Karl August (Ed.), 3206

Labberton, Mien (Tr.), 249

Ladislas, F. (Tr.), I 184

Lajos, L. Ujvary (Tr.), 368

Lampsa, G. (Tr.), 680

Landman, Isaac (Ed.), 3273

Lange, Francisco Curt (Ed.), 3260

Langlo, Kristian (Ed.), I 94

Langner, Ruth (Tr.), I29, I714-1736

Lanyi, Viktor (Tr.), 778

Lászlo, Dormandi (Tr.), 37I

Lemke, Karl (Ed.), 980

Lessing, Theodor (Tr.), 1602

Levantis, S. (Tr.), 353

Lezhneva, L. P. (Tr.), 568

Lillis, M. (Tr.), 884

Limentani, Anita (Tr.), 55 , 2982

Lipovetzki, Pesah (Tr.), 367

Lleonart, José (Tr.), 474

Llorente, Máximo (Tr.), 496

Longeville, Jean (Tr.), 934-935

Loran, I. and C. (Eds.), I 58

Losinski, M. (Tr.), 462

Loulis, M. (Tr.), 343

Luca, Aurel (Tr.), 92 I

Ludwig, Emil (Ed.), (73), 2525

Luther, Arthur (Tr.), 1499

Maar, Oskar (Ed.), I 73

MacArthur, Erna (Tr.), 972, 2473-2478, 2507-2517, 264I

Madeleine, B. (Tr.), 447, 449

Mahanit, Lakshminarayana (Tr.), 394

Maletin-Avakumović, Kaćusa (Tr.), 467

Malthaner, Johannes (Ed.), 2 10

Mandelstam, I. B. (Tr.), I I6, 544, 465

Mann, Klaus(Ed.), 278, I 5 I4, 2487, 2555 , $2575-2576$

Mann, Otto (Ed.), 3295

Manolova, S. (Tr.), 224

Marghita, E. (Tr.), 453

Martínez-Ferrando, Ernest (Tr.), 225

Mátrai, Tamás (Tr.), 589

Maugham, William S. (Ed.), 279, 28I

Mazzucchetti, Lavinia (Tr.), 2, $550-55$ I, $590,627,657,684,724,758,885$, 1984-1985, 2046-2047, 2074-2077, 2I25-2127, 2 I67-2I69, 240I-2406

Medeiros y Alburquerque (Tr.), 666

Méndez Pereira, Octavio (Tr.), 487

Meranaios, Kosta (Tr.), 335, 344, 354,
$357-358,362,652,777,791,812$, $8 \mathrm{I}_{4}, 834,859,957$

Merker, Paul (Ed.), 3262-3263

Meyerfeld, Max (Tr.), I 59I

Milićević, Nika (Tr.), 593, 629, 764

Mizuno, Akira (Tr.), 59I

Monsen, Ursula (Tr.), 424

Monteiro, Campos (Tr.), 440

Morisse, Paul (Tr.), 790, 2217

Moura, Sylvio Aranhade (Tr.), 434

Mperates, G. (Tr.), 626, 883

Mussey, Barrows (Tr.), 1534

Nander, Erik (Tr.), 233, 237

Nasr, Hassan Ali (Tr.), 397

Navarro, Diego (Tr.), 476

Necatigil, Behçet (Tr.), 509

Neider, Charles (Ed.), 1357, 2583

Nemecek, Josef (Tr.), 674

Neumann, Karl E. (Tr.), I 546

Neumann, Stefanie (Tr.), 286I

Nishi, Yoshimoto (Tr.), 410, 759

Norden, R. (Tr.), 1008

Nötzel, K. (Tr.), I 493

Nuorto, Olli (Tr.), 585

O'Brien, Edward (Ed.), 3207

O'Brien, Justin (Tr.), $2552 \mathrm{a}-2553$

Ogando, Alice (Tr.), 435, 437-439, 44I$442,444-445,628,662,729,761$, $840,863,888$

Oksala, Aina (Tr.), 305

Okubo, Kazuo (Tr.), 4I 2, 414-4I5, 780, 782

Olbrich, Wilhelm (Ed.), 3265

Oppeln-Bronikowski, Friedrich von (Tr.), I 586

Overdorfer, A. (Tr.), 86I, 2332-2335

Pálmason, Ingólfur (Tr.), 959

Pandey, C. D. (Tr.), 655

Pangalos, A. (Tr.), 8I 3

Papanastassiou, P. (Tr.), 346, 35 I

Parry, Juliette (Tr.), 880-882, 1062, 23862400

Patatzis, S. (Tr.), 9I4

Paul, Eden and Cedar (Trs.), r03, 259260, 269, 273-274, 288-290, 292294, 297, 302, 60I-603, 62I-623, 644-646, 676, 713-715, 748-749, 771-772, 798, 804-806, 830, 877879, 909, 954-955, I094, III 4, I 275, I36I, I530, I663-1673, I770-1777, I 798-I807, I 809-1849, I904-I920, I947-1972, 2020-2036, 2049-2073, 2090-2II6, 2134-2160, 2190-2201, 2219-2228，2232-2248，2278-2292, 2368-2385, 24II-2426, 245 I-2468, 2529 
Paul, S. (Tr.), 452

Pavlidis, L. (Tr.), 548

Payarols, Francisco (Tr.), Ir, 889-890, 892-894, 2407

Pejkov, Stefan (Tr.), 222

Pelarovi, Luba and Rudolf (Trs.), 228

Pérez Ferrero, Miguel (Tr.), 664, 667-668

Peters, Wolfgang (Tr.), 977, 2880

Petersowa, Zofia (Tr.), 689, 728, 920, 2078

Petrović, Bosko (Tr.), 694

Petrović, Miodrag (Tr.), 799, 842-843

Phelps, Leland (Ed.), I58

Phillips, G. A. (Tr.), 3291

Phillips, William (Ed.), 2552

Pinheiro, Aurelio (Tr.), 3, 443

Pocar, Ervino (Tr.), 2989

Poortenaar, Jan (Tr.), I 5 I 3

Pongs, Hermann (Ed.), 3248

Popper, J. (Tr.), 448

Precioso, Artemio (Tr.), I 35

Prionistis, N. (Tr.), 549

Raabe, Paul (Ed.), 976, 2823

Radberg, Erland (Tr.), 734

Raeburn, B. (Ed.), 2575

Raunkjaer, Palle (Ed.), 3266

Rechtschaffen, Bernard (Ed.), I64a

Reinla, Karin (Tr.), 677

Remer, Paul (Ed.), 793

Rho, Anita (Tr.), 402, 1855

Richez, Odette (Tr.), 773-775, 2202-2203

Rieger, Erwin (Ed.), 2502-2503, 2803, 2875

Ring, Barbara (Tr.), $42 \mathrm{I}$

Ripamonti, José Manuel (Tr.), 496

Rittnerowa, Zofia (Tr.), 426-427

Rocca, Enrico (Tr.), 399, 406, 816, 838, 2249-225I

Roces Suárez, Wenceslas (Tr.), 849

Rodapoulos, V. (Tr.), 936

Rodrigues, Manuel (Tr.), 962

Rogne, Margit (Ed.), 194

Röhl, H. (Tr.), I 493

Romig, Walter (Ed.), 3246

Rónay, Gyórgy (Tr.), 756

Rønberg, Georg (Tr.), 618, 746, 1946, 2I3I

Ropertz, Hans Rolf (Ed.), 2895

Rosa, Tristan de la (Tr.), 557-559

Rose, William and Dorothy (Trs. and Eds.), 584, 1885-1900, I 25, 2492,

Ruben, R. (Tr.), I 568

Rüdiger, Horst (Ed.), 3250

Ruiz Alonso, José (Ed.), 845

St. James, Andrew (Tr.), 565, 932-933, I873-I 882, 2430-2443
Sainz de Robles, F. C. (Ed.), 3226

Salazar Diaz, Pedro (Tr.), 47I

Samokovlija, Isak (Tr.), 799, 843

Sánchez Guerra, Rafael (Tr.), I35

Sandfort, J. A. (Tr.), I489

Sándor, Térey (Tr.), 836

Santavuori, Martti (Tr.), 647

Santos, José Francisco dos (Tr.), 567

Sarić, Vlatko (Tr.), 469

Saudek, Emil (Tr.), I 303

Schanzer, Ottone (Tr.), I 20

Schenker, Manfred (Tr.), 320

Schiøttz-Christensen, Aage (Tr.), 599

Schlösser, Manfred (Ed.), 2895

Schneider, Paul (Ed.), 2950

Schneider, Wilhelm (Ed.), 2897, 2934

Scholz, Wilhelm von (Ed.), I 566

Schouten, J. H. (Ed.), I9I

Schram, Constance Wiel (Tr.), 660, 886

Schramm, Wilbur Lang (Ed.), 280

Schuh, W. (Ed.), 2479-2486

Schwrig, Artur (Tr.), I6Is

Schwarz, Leo W. (Ed.), IO2

Schwerin, Christoph (Ed.), I 83

Schwerin, Kathe R. and Paul E. (Trs.), 258

Sears, Minnie (Ed.), 3270

Selden-Goth, Gisella (Ed.), 975, roooIOOI, 2602

Semeriotes, G. (Tr.), 34r, 344

Shelah, Uriel (Tr.), 588

Sherwood, Elizabeth (Ed.), 3219

Skith, Horatio (Ed.), 3218

Solomon, Herbert (Ed.), 29I

Sorgenfrei, B. A. (Tr.), 462

Sorgenfrei, P. S. (Tr.), $84 \mathrm{I}$

Spilotopoulos, P. (Tr.), 347, 860

Stallman, Robert Wooster (Ed.), 277

Stammler, Wolfgang (Ed.), 3262-3263

Stauffer, Ruth (Ed.), 30I

Stavropoulos, G. (Tr.), 858

Steinberg, S. H. (Ed.), 3215

Sterkenburg, Reinier P. (Tr.), 246-247, 252, 254-255, 600, 619-620, 64I-643, $747,876,904-908,1903,2132-2133$, 2366-2367, 2409-2410

Stoevski, Dimitár (Tr.), 580, 745

Stonehill, Charles A. (Ed.), I 532

Strich, Christian (Ed.), 2922

Stummvoll, Jasek (Ed.), 2749, 3389

van Suchtden, Nico (Tr.), I 506

Sukkau, B. A. (Tr.), 46r, $84 \mathrm{r}$

Sullivan, Catherine (Ed.), 301

Szass, Hannah (Tr.), I 520

Takahashi, Kenji (Tr.), 4I I, 658, 686, $725,759,818,839,918$ 
Tenreiro, Ramón Maria (Tr.), 607, 630, 731,765

Theis, O. F. (Tr.), 794

Thomas, J. B. (Tr.), 615

Thorne, J. O. (Ed.), 3216

Tiryakioglu, Samih (Tr.), 503, 506

Tisnia, Aleksandar (Tr.), 964

Tivadar, Szinnai (Tr.), 656

Tokin, Milan (Tr.), 468

Trias Beristain, Fernando (Tr.), 924-925, 973

Trikolonios, A. (Tr.), 333, 350, 352, 36r

Tsuji, Hikaru (Tr.), 407

Ullrich, Hermann (Ed.), 2937-2938

Umanskij, Dmitri (Tr.), I 549

Uücel, Tahsin (Tr.), 800

Vaara, Elina (Tr.), 750, 216r

Vanhamme, Guy ('Tr.), 25 I

van Heerikhuizen, F. W. (Tr.), 583

Vecerov, Metodi (Tr.), 223

Velskij, W. (Tr.), 46I

Verdaquer, Joaquin (Tr.), I I, 824, 848, 864-871

Verdaquer, Mario (Tr.), 49I, 924-925

Verner, Arpe (Ed.), 3252

Vodè, Angela (Tr.), 965

Vol'fson, L. (Tr.), 455

Voss, Hartfrid (Ed.), I 54I, 338 I

Vovolinis, P. (Tr.), 345, 359

de Vries, Frank (Tr.), I 533, 2493

Vrettacos, N. (Tr.), 776

Wahl, Anders de (Tr.), I 24

Walderkranz, Rune(Ed.), 325 2, 3340-3356

Wassermann, Melanie (Tr.), I07, 428, 430-43I

Waterman, Robert (Ed.), 3213

Weinberg, Bernhard (Ed.), 2544, 3388
Weinberg, Elyaqim (Tr.), 365

Weissenberg, L. (Tr.), $46 \mathrm{I}$

Weitzel, Karl (Ed.), 3265

Wenz, Richard (Ed.), 218

Werner, A. (Ed.), 192

Werumeus Buning-Ensink, G. J. (Tr.), 712, 2089

Wery, L. (Ed.), 99

West, Roy B. (Ed.), 277

Wetkin, S. (Tr.), 82 I

Whitall, James (Tr.), 2596

Wiegler, Paul (Tr.), I $58 \mathrm{I}$

Wilamowitz-Moellendorf, Fanny von (Tr.), 499

Wildgans, Anton (Ed.), 984

Wilpert, Gero von (Ed.), 3255

Winklerm, Johan (Tr.), 93 I

Winter, Hanns (Tr.), 2787

Wislowska, Maria (Tr.), 427, 432, 760, 961, 2469

Wittner, Felix (Ed.), 901, 2408

Wolf, H. (Ed.), 523-524

Wolf, Luise (Tr.), I 557

Wsewolodskaja, L. N. (Tr.), 458

Wunderlich, Eva (Ed.), I 52, $2625-2626$

Yamashita, Hajime (Tr.), 726

Yücel, Tahsin (Tr.), 505, 510

Yust, Walter (Ed.), 2627

Zeiner, M. (Tr.), $46 \mathrm{I}$

Ziegenfuss, Werner (Ed.), 326I

Zimmermann, Jean Paul (Tr.), 956

Zistel, Era (Ed.), 285

Zographon, Mina (Tr.), 777, 791, 81 2, 957

Zohn, Harry (Tr.), 2594, 2632

Zweig, Stefan (Ed. and Tr.), I40I-1480, I 490 , I 497, I 524, I 527, I $537-1540$, 2490, 2495-2496, 2752, $336 \mathrm{r}$

PROPER NAMES

Abel, Alfred, 3346

Abraham, P., 2037, 2388

Ackerknecht, E., I865

Adelberg, Julius, 2053, 2137

Adelt, Leonhard, 1229, 2013, 2357-2358, 2685,3116

Ahl, Herbert, 2686

Albert, Henri, 1755

Albert-Petit, A., 2044

Alceste (Pseud.), 2444

Alker, Ernst, 201 2, 3279

Alvarez, Walter, 26I

Ambrosi, Gustinus, I48 I, 2769

Amerigo Vespuccio, 9-1 I, 563-573, 693, I $873-1882$
Ammer, K. L. (Cf. K. Klammer), 988, I $21-1522,2769$

Anderson, Erica, 1482

Andriulli, G. A., 2167

Angelloz, Jean François, 2642

Apio Garcia, Antonio Augusto, 3000

Appel, C., 2189

Araujo Lima, Claudio de, 3001

Arcos, René, 2643

Arendt, Hannah, 2460, 2522, 2687

Arens, Hanns, (69), 2497, $2523-2524,2688-$ $2697,3053,3178,3383-3384,3394$

Arias, Alejandro, C. 3006

Arndt, Ernst Moritz, 3372

Arnim, Achim von, 3372 
Arnold, Robert F., I646

Arquedas, Alcides, I 207

Arrieta, Rafael Alberto, 3007

Asch, Schalom, 1220

Asow, E. H. M. von, 248 I

Astolfo, ?, 2404

Atkinson, J. Brooks, 1724

Audard, J., 2386

Audiat, P., 2395

Auernheimer, Raoul, (73), I7II, 2525, 3197

Augstein, Carl, 2450

Azevedo, Raul de, 2997

Baader, Fritz P., I 679

Bab, Julius, 999, 2698-2700

Babler, Otto F., 2503

Bachmann, H., 2487

Baden, Hans Jürgen, 2447

Badt-Strauss, ?, I94I

Bahr, Hermann, XXVII, XXXIII, (47), 526-527, I24I, I35 Ia, I483, I 543, 2317,3412

Bahrt, O., 2306

Bak, Olga, (53), 3180

Balboa, Vasco, 902-903, 905, 909, 9זі, 922-923, 926

Baldus, A., 1998

Balzac, Honoré, XXXVI, XXXVII, (68), 8-II, 526-527, 574-596, 827$828,830-83 \mathrm{I}, 833-838,840-84 \mathrm{I}$, 849-850, I006, 1034-1037, II57, 1244, I 272, I364, I484, I883-190I, 2218-2228, 2262-2296, 2300-2304, $2488-2489,2544,2599,2723,2778$, 2985,3388

Banton, L. Shubert, 1726

Barbusse, Henri, XXXII, (40), 526-527, I 2 IO, I 348, I 40 I

Barricelli, Jean-Pierre, I00I, 2526, 2638

Barrymore, John, $335 \mathrm{I}$

Bartlett, Robert Merrill, 2527-2528

Baseler, Ewald, 3349

Basil, Otto, 2702

Basso, Hamilton, 1893

Baudelaire, Charles, XXVIII, I I80-I I 8 I, I 292, I 402-I 424, 3 I 91

Baudouin, Charles, 1361, 25 29, 2644

Bauer, Arnold, 2498, 2703

Bauer, Gerard, 1737

Bauer, Walter, 2689, 2696, 2704-2707

Bäuerle, Adolf Johann, 3372

Bauerfeld, Eduard von, 3372

Baum, Oskar, 1485

Baum, Vicki, 2708

Baumberg, Antonie, I 544

Baumgarten, R., 2387
Bazalgette, Léon, XXIX, 533, 1270

Beaufils, M., 2256, 2328

Beaulieu, Paul, XXXVII, (74), 2645, 3 IO

Becker, Martha, 2709

Becker, Mary Lamberton, (59), 2152

Becker, W. H., 2319

Beer-Hofmann, Richard, XXXIV, 3207

Beethoven, Ludwig van, XXXI, 2689, 2696

Béguin, Albert, 2646

Behl, C. F. W., 1993, 2079

Behrend, Erich, I6I

Beierle, A., 2710, 3087

Belázs, Béla, 270 r

Belmore, H. W., 2530

Benaroya, Mois, $271 \mathrm{I}$

Bence, Amelia, 3355

Benét, S. V., 1806, 3161

Benjamin, Walter, 1347

Bennett, E. K., 3280

Benninghoff, ?, 2082

Benzmann, H., I 237

Berendsohn, Walter A., (57), 271 2-2713, 2992, 3173

Berg, L., I697

Berger, Fritz, 2714

Bergler, Edmund, 253 I

Bergmann, Ingrid, 3344, 3356

Bernard, J. J., 1738

Berretti, Alessandro, 625

Berry, Robert M., 318I

Bertaux, Félix, 328I

Bertrand, Pierre, I902, 1926, 2715

Bethge, H., 2322

Beyle, Henri, cf. Stendhal

Bianche, Renzo, 1486

Bianquis, Geneviève, (4I), 2510

Bick, Josef, 2749, 3389

Bickley, Francis, 22 I I

Bidou, H., 1740

Bieber, H., 2266, 2717

Biedrzynski, R., 1708

Bierbaum, Otto J., 3372

Bierotte, Wolf, 2718

Binding, Rudolf, $1357 \mathrm{a}, 3395$

Bin Gorion, Emanuel, 2719-272I

Birnbaum, K., 2345

Bischoff, F. W., 1367

Bithell, Jethro, 788-789, 2209-22 16, 2532, 3282-3283

Blake, William, XXIX

Blau, A., 1648

Bleuler, E., 2355

Bloch, Jean Richard, 1487

Blumauer, Johann Alsys, $337^{2}$

Blumenthal, Hermann, i 545 
Bodenstedt, Friedrich von, 3372

Boesch, B., 3284

Bolze, W., 200I

Bon, Antoine, (77), 2647

Bondy, F., I 884

Bonsels, Waldemar, $1357 \mathrm{a}$

Borcosqu, Carlos, 3355

Börne, Ludwig, 3372

Böse, Georg, 25 I 9,2974

Bosman, F. S., 2367

Böttcher, Kurt, (2), (10), (40), (76), I883, 2722-2723, 3182

Böttiger, Theodor, 1990

Bouman, C. A., 2493

Boyd, Ernest, I 71 5, 2198

Boyle, F. A., 2436

Branch, Stephan, 986

Brand, Guido K., 3285

Brandes, Georg, XXVIII

Bratton, Fred G., 2070, 2424, 2533

Braun, Felix, (65), 988, 998, 1478, 1480, 2230, 2505, 2689, 2724-2732, 2769

Braun, Robert, (75), 1759, 2733, 2679

Brecht, Berthold, XXXIV, I 347

Bredsdorff, Viggo, 2993

Brentano, Clemens, $337^{2}$

Bresler, ?, 2360

Breuer, Robert, 2479-2480

Breyfogle, W. A., I95 2

Brezina, Oskar, I303

Brickel, Alfred G., 21 34

Brickell, Herschel, 2028

Brittin, Norman A., (65), 2534

Broadbent, Thomas L., 2535-2536

Brockhaus, F. A., I 209

Brockway, Wallace, 2537

Brod, Max, XXXIV, I176, I264, I280I 28 I $, 1488,2734-2735,2769,28$ I $_{3}$

Brody, Alter, I8IO

Brown, ?, 238I

Brown, G. E., I 877

Brown, J. M., I733, I 735

Brown, R., 2062

Browning, Elizabeth Barrett, I 425

Brun, L., 2 I 23

Buber, Martin, I347

Buckly, Boniface, 1898

Buddha, Gotamo, 1546, 2981

Buenzod, E., 2202, 2297

Bullock, F. H., 1846

Bunzel, B., 2098

Burchardt, Hansjürgen, 2736, 3386

Bruckhardt, Oswald, 513, 2538

Bürgin, Hans, 3366

Burich, Enrico, 2980

Burke, Kenneth, I I 82, 2192
Burnham, Philip, 2433

Burrell, Angus, 2239

Burschell, Friedrich, 2737

Busoni, Ferruccio, 526- 527

Busse, K., I633, 3070

Butcher, Fanny, 2093, 21 38

Byron, George Gordon, Lord, 459, 54I, 544,558, I I 76, I 264, I 274, I 280

Caesar, Julius, II 78

Cahn, Alfredo, 2738, 3008 (Cf. also Cahn as translator)

Cahnman, Werner, J., (60), 2539

Caligari, 2564

Calvin, Jean, XXXV, XXXVI, (I), 597609 , 987, IOII-IOI 2, I9O2-I928, 2649-265I, 2654, 2681-2682, 2715

Camoens, Luis Vaz de, I426, 2696, 2791

Canby, Henry Seidel, 1886, 2558

Canciani, Alfredo, 1138

Canella, M. F., 2334

Caprile, E., 2301

Carossa, Hans, 526-527, 545, 659, II67, 1233, 2689, 295 I

Casanova, Giacomo, Chevalier de Seingalt, 10, 801-807, 810-81 2, 816, 819, 826 , IOI3-IOI5, I209, I489, $2218-$ 225I, 2253-2254

Castelle, F., 217I

Castellio, Sebastian, XXXV, XXXVI, (I), 597-609, 987, IOIr-1012, I902I928, 2715

Castro Osorio, J. de, I90I

Castro y Calvo, J. M., 2048

Cena, Giobanni, XXVIII

Cenci, Beatrice, 54I, 544, 558, I 223, I 266

Chamberlin, John, 2 I 40

Chamberlin, Wells, 2544, 3388

Chamberlin, William H., 2425, 3165

Chamisso, Adalbert von, $337^{2}$

Chanchoy, H., I980

Chapire, J., 2173

Charmatz, ?, 2019

Chastonay, ?, 2017

Chateaubriand, François René, Vicomte de, 533,1490

Chaturvedi, Benarsi Das, 2689, 2696, 2740, 2977, 3 I IO

Chauviré, R., 2166

Chevalley, A., 2258

Cicero, 909, 923

Cione, E., I985

Citoleux, M., 2203

Clark, Edward Murry, 243 I

Clark, Edwin, 2244

Claudel, Paul, 2648, 3387

Claudius, Matthias, $337^{2}$ 
Clogenson, Y., 1978

Codman, Florence, 1822

Cohn, ?, 1944

Coleman, Arthur P., 2606

Colton, Arthur, 2283

Colum, Mary M., 2031, 2222, 2242

Colum, Padraic, 2236

Connager, Henry Steele, 1880

Conroy, Jack, I84I

Cornelius, Peter, 3372

Corra, M., 21 26

Coster, Charles Theodor H. de, I 183

Coulson, H. H., I947

Courvoisier, Jacques, 1927

Cousins, Norman B., 2055, 2540

Cousins, Thomas Q., 3166

Crémieux, Benjamin, I 743, 2255

Critile, ?, I74I

Crommelynck, F., I 186

Crowley, Paul, 1953

Csokor, Franz Theodor, 2741-2742, 2769

Cuckov, B., 2486

Curtiss, Thomas Q., 254I, 3043

$\mathrm{Da}$ Cunha, Euclides, $254^{2}$

Dagover, Lil, 3363

Dahm, Paula, I 547

Dali, Salvador, 2543

D'Annunzio, Gabriele, I 548

Dante, 526-527, 545, I I 89

Daudet, Philippe, 54I, 558, I 248

Daviau, Donald G., (33), 2545

David, Jacob Julius, I 221

Dawson, Margaret, 1773

Debusy, Claude, XXVII

Décarreaux, Jean, 2652, 3 104

Decour, J., 2329

Dehmel, Richard, (12), 979, 991, 1478, 2700,3372

Dehorn, W., 2743

Deissinger, Hans, 2744

Delarue, Henri, 2649-265 I

De Lorenzo, Giuseppe, 298 I

Delpy, E., 1678, 1701

Demilly, Adolphe, 2653, 3171

Demmering, J., I623

Demuth, Norman, 3245, 3357

Desbordes-Valmore, Marceline, XXIX, (is), 8-9, 526-527, 545, 6ro-6rs, 1095-1098, 1929-I 940

Deutsch, Babette, $18 \mathrm{I}_{3}$

Diamond, Helena, 3183

Dickens, Charles, 8-10, 827-828, 830-83 r, 833-838, 840-84I, 843, 845, 849-850, I034-1037, II 82 , I $491,2219-2228$, 2262-2296, 2300-2304, 2985, 3007

Diebold, Bernard, 1367,3288
Diez Canedo, E., I 207

Döblin, Alfred, 1217

Dobrée, B., r 958

Doderer, O., 2083

Dolbin, B. F., 2344, 2745

Dörmann, Felix, 3200

Dostoevskaja, Anna Grigorevna, I 549

Dostoersky, Feodor, XXVII, XXX, 8 , I0, $31,60,165,528,827-830,832-$ $833,836-843,846,849-850,897$, 900-903, 9 I I, I034-1037, I 492-I493, I 549, I 584, I $588,2218-2228,2262-$ 2292, 2297-2304, 2552, 2580, 2605, 2985

Douglas, A. D., 1669

Dinkwater, John, 526-527

Droste-Hülshoff, Annette Elizabeth, Freiin von, 3372

Du Bois, A. E., 1967

Du Bos, Charles, 992

Duffus, R. L., 2067

Duhamel, Georges, XXIX, XXXIII, I 207, I 252

Duimchen, Theodor, I550

Düsel, F., 1684

Duwe, Wilhelm, 3289

Ebenhöh, Horst, $3357^{\mathrm{a}}$

Ebermayer, Erich, 980, 2747, 3149

Eck, Miriam, I55 I

Ecker, Karl, 2749, 3389

Eckermann, Johann Peter, 3372

Eckhardt, Eduard, 2750

Eddy, Mary Baker, XXXIV, (66), II, $872-875,877-880,882,885-888$, 890-89I, 894-895, 1054-1056, 1062$1064,2336-2365,2368-2407$

Edgett, E. F., $219 \mathrm{I}$

Edman, Irwin, 1427, 2462

Edschmid, Kasimir, 3290

Ehrenstein, Alfred, $275 \mathrm{I}$

Eichbaum, Gerda, 3 I 84

Eichendorff, Joseph, Freiherr von, 3372

Einstein, Albert, XXXIII

Einstein, Alfred, IOOI, 2638

Eisemann, Heinrich, 3385

Eisenschitz, Fritz, I 552

Elbrechter, H., I999

Elchinger, R., $\mathrm{I}_{3} 6$

Eldridge, Paul, 224I

Eloesser, Arthur, 2752, $329 \mathrm{I}$

Elster, Hanns Martin, I55, 2321, 27532754, 3044

Elvey, Maurice, 3354

Ende, Amelia von, I671, 2546

Engel, H., 2 I 88

Entwistle, W. J., I 207 
Erasmus of Rotterdam, XXXV, 524, 528, 616-630, 1038-104I, I94I-I 985, 2533

Erkelenz, K. H., 2755, 3100

Ermengem, Friedrich van (Cf. F. Hellens), 546, I 501,2660

Ernest-Charles, J., 2038

Errante, Vincenzo, I494

Erskine, John, 2216

Eshleman, Lloyd W., 1915, 2056

Estelrich, Joan, I 207

Eulenberg, Herbert, 1357 a, ${ }_{13} 67$

Evensky, M., 2548, 3179

Everte, Erich, 2305

Ewen, David, 2549

Fadiman, Clifton, 2420

Faesi, Robert, 1478, 2689, 2696, 2756, $2769,3144,3399$

Faktor, Emil, 2757, 3045

Falke, G., I 228

Famière, E., 2294

le Fauconnier, Henri, I 182

Fearing, Kenneth, 1847

Feder, Ernst, 2550, 2689, 2696, 2758, 279I, 2998-2999, 3009-30II, 3089, 3094

Fedin, Konstantin, 2759, 3039

Fehse, Willi, (I0), I I I4, 2760-2762, 3395

Feichtinger, G., 2339

Feld, Leo, 1 269, I638

Felix, Kurt, 1659

Ferguson, W. K., I957

Fernandez, R., 2293

Ferrand, J., 2393

Feuchtwanger, Leon, 2558, 2920

Fiedler, Leonhard, 3306

Field, Cyrus W., 902-903, 909, 923, 926

Finckl, Ludwig, I553

Finger, Charles J., 2066

de Fiqueredo, F., I 207

Firon, Erich, 3185

Fischer, ?, 2267

Fischer, Dorothy Canfield, 278

Fischer, Fritz, I64b

Fischer, H., I 677

Fischer, Max, 1876,2456

Fitzbauer, Erich, (I0), 163, 528, I7591760, 2499-2501, 2763-2770

Fiumi, M. L., 2169

Flake, Otto, 1367, 1554, 2014, $227 \mathrm{I}$

Flamm, P., 234I

Flaskamp, Charles, 2269

Flaubert, Gustave, I 212 , I353, I 555-1558

Fleischel, Victor, 2769, 2771-2772

Fleming, P., I $73 \mathrm{I}$

Fles, Barthold, 1823
Flores, Angel, 2285

Flower, Sir Walter Newman, $255 \mathrm{I}$

Fogg, J. B., 24I 7

Foltin, Lore B., 2773

Fontaine, Joan, 3348

Fontana, Oskar Maurus, 2689, 2745

Fontane, Theodor, 3372

Forbes-Mosse, Irene, I 559

Fortana, O. M., 23 I0

Fouché, Joseph, XXXIV, 8-9, 523, 631670, 1047-1053, 1986-2048,

Fouqué, Friedrich H., 3372

Fournier, A., 2299

Fraedrich, ?, 1656

Francé, R. H., I 322

Franck, Hans, I 244, I 560

Frank, Bruno, 2920

Frank, Leonhard, XXXII

Fränke, ?, 2002

Franzos, Karl Emil, 988

Frass, Wilfred, 3349

Frei, Lenore, I 561

Freiberg, Siegfried, 2769, 2774

Freiligrath, Ferdinand, 3372

Frese, Hans, 3186

Frets, Huberta, 3187

Freud, Ernst L., 2775

Freud, Sigmund, XXXIV, XXXV, (66), II, 539-54I, 558, 872-879, 88I-882, 889, 891, 893-895, 1065-1072, I 342I344, I354, I387, I391, 2336-2407, $2543,2552,2592,2603,2630-2631$, 2664, 2775, 2853, 2986

Freund, Erich, 1706

Fricke, Gerhard, 3294, 3333

Frieberger, Kurt, 988

Fried, Alfred H., I I 34, I I37, 2854

Friedell, Egon, XXVI

Friedenthal, Richard, (68), 533, $560,574-$ $579,1427,2689,2696,2776-278$ I

Friedländer, A. A., 2363

Friedmann, W., 1932, 2180

Friedrich, H., I754

Friedrich, Werner P., 3296

Friedrichs, Ernst, 2782, 3093

Frischauer, Paul, 2783, 3056

Frochause, Claudine, 329

Fronius, Hans, 163 , 189

Fuchs, Albert, 3297

Fuchs, F., I933

Fuchs, Guido, 988

Fuente Gonzáles, Efrain de la, 30r 3

Fuessle, Newton, 2200

Fuillerat, Albert, 2248

Fülöp-Miller, René, 2476, 2689, 2696, 2784 
Cabelentz, Hanns Conon von der, 768

Gabrielli, G., 2046

Gandia, Enrique de, 3014

Gannett, Lewis, 1962

Garraty, John A., 3367

Garten, H. F., 3298

Gausebeck-Dörper, L., I935

Geibel, Emanual, 3372

Geisendorf, Paul F., 265 I, 2654

Geismar, Maxwell, I785

Geissendörfer, Theodore, 901, 2408

Gelber, A., 1562

Gellert, Christian F., 3372

George, Stefan, I 276, I347, I452, I455, $1469,2826,2895$

Gerhardt-Amytor, Dagobert von, $156_{3}$

Gershoy, Leo, 2 IOI

Gibson, Wilfred, 1784

Gide, André, 977-978, 1347, $25522-2553$, $2648,2655,2672,2880,3387$

Giese, R., 1966

Gilder, Rosamund, 1734

Gillet, Louis, 2673

Ginskey, Franz Karl, I 216, 1495-1496

Ginzberg, Benjamin, 2373

Girosi, Raolo, 2075

Gleim, Johann W. L., 3372

Godfrey, Eleanor, 2054

Goethe, Johann Wolfgang von, 165 , 526-527, 545, 900-903, 909, 911, II14, II36, I191, I 238, I307, I497, 2490, 2990, 3006, 3372

Goffin, Robert, 2656, 3172

Goldbaum, Wenzel, 3015

Goldkorn, Yitzchak, 3034

Goll, Claire, I 498

Gordon, Franklin, 2232

Gorki, Maxim, XXXIII, 8, 526, 545, I 284, I 499, 2554

Gorman, Herbert, $2106,2377,2422$

Gotthelf, Jeremias, I 253, 3372

Gottsched, Johann Cgr., 3372

Götzfried, Hansleo, 2786, 3188

Grabbe, Christian D., 3372

Grabert, ?, 2342

Grandmaison, Geoffroy de, 2040

Granier, James, 2435

Grebert, Ludwig, 3189

Green, Julien, 2657, 2787

Greene, Graham, 1828

Gregor, Joseph, 21 29, 2769, 2788-2789, 3267

Greiner, Leo, 1564,1628

Grenville, Vernon, 1665

Grierson, Flora, 2419

Griffin, Francis Vielé, I 347
Griffin, Jonathan, 2555, 3163

Grillparzer, Franz, 3372

Grisson, Alexandra Carola, 140, 2790

Grossberg, Mimi, 279r, 3400

Groth, Klaus, 3372

Grün, Anastasius, 3372

Grützmacher, R. H., 2314

Gryphius, Andreas, 3372

Gschiel, Martha, 3190

Guerard, Albert, $2105,2226,2421$

Guerrieri-Gonzaga, Sofia B., 2673

Guilbeaux, Henri, 275 I

Guillemin, B., 1710

Gundolf, Friedrich, I565

Günther, Christian, I 566

Günther, Herbert, 2792, 3069

Günther, John, 2556

Günther, Werner, 2793

Guth, Alfred, 1567

Haas, Willy, I994

Haberling, ?, 2354

Hachtman, O., I 934

Hackett, A., I 792

Haeberlin, ?, 2348

Hagedorn, Friedrich von, 3372

Hall, C. R., 2024

Hallener, ?, 1676

Haller, Albrecht von, 3372

Halm, Friedrich, 3372

Hamann, Johann Georg, 3372

Hamecher, Peter, 1987

Hameiri, Avigodor, 3035

Hamerling, Robert, 3372

Hammer-Purgstall, Joseph von, 3372

Hampe, Theodor, 1645

Händel, Georg Friedrich, 165, 896, 902904, 909, 911, 917, 926-927, Iro9, II 20 , I I 22, I 275,2428

Hardt, Ernst, 1478

Hartleben, Otto E., 3372

Hartmann, F., 1700

Hartrott, 1235

Hartung, P. T., I78I

Hasenclever, Walter, XXVI

Hauff, Wilhelm, 3372

Haug, Johann Christian, 3372

Hauptmann, Gerhardt, XXVII, I 68

Hauser, H., 1981

Hauser, Otto, 1478

Haushofer, Karl, I 258

Haynes, Renée, 2 I 5 I

Hayward, J., 2100

Hearn, Lafcadio, 533, 1500

Hebbel, Friedrich, 3372

Hebel, Johann Peter, 3372

Heijermans, Hermann, I 568 
Heilblut, Ivan, 2557

Heine, Heinrich, 1569,3372

Heinse, Wilhelm, 3372

Hella, Alzir (Cf. Hella as translator), 2658-2659

Helle, Peter, 3372

Hellens, Franz, Cf. F. van Ermengem

Hellpach, Willy, I 347

Hellwig, Hans, 2794

Hemmingway, Ernest, 266

Henriquez-Urena, P., 1207

Herder, Johann G., 3372

de Herédia, José Maria, I $57^{\circ}$

Hermann-Neisse, Max. I 283, I367, 1502

Herrig, A. B., 2108,2379

Hermann-Neisse, Leni, 2796

Herrsmann, W., 2795

Herwegh, Georg, 3372

Herzberg, A., 2346

Herzl, Theodor, XXVII, (8), 526-527, I 262

Hess, Stan, 2550

Hesse, Hermann, I I 22, I $217, I_{3} 67$, I 381 , 1480, I571-1573, 1761, 1937, 2186, 2274, 2581, 2876, 2945

Heuschele, Otto, 1263 , I 503

Heynen, H., 1683

Heynen, W., 1936, 2016

Heyse, Paul, 3372

Hieble, Jacob, 3299

Hift, I., 2 I 75

Highet, Gilbert, 3300

Hille, Peter, XXVIII

Hincks, Cl. M., 2372

Hinterberger, Heinrich, 2797, 3390

Hinton, Thomas R., 3330

Hirn, H., 216I

Hirsch, F. E., I890, 2457, 2512

Hirth, Friedrich, 1658,2798

Hitler, Adolf, XXXV, I 235,2564

Hochstetter, Sophie, I574

Hofe, Harold von, 2506

Hoffmann, Camill, 1403,1762

Hoffmann, E. T. A., 533, I 504, 3372

Hoffmann von Fallersleben, August Heinrich, 3372

Hofmann, Else, 2799

Hofmannsthal, Hugo von, XXVII, XXXIV, (7), 54I, 544, 558, II 27 Ir 28, I 245, I 575,3201

Hohlfeld, J., 2085

Hohoff, Curt, 3334

Holburn, T., I 726

Holde, Artur, 2800, 304I

Hölderlin, Friedrich, XXXIII, 9, $85 \mathrm{I}-$ $852,854-856,86 \mathrm{I}-865,870-87 \mathrm{I}$,
1073, 1075-1081, I 590, 22 I 8-2228, 2305-2324, 2328-2335, 3372

Holitscher, Arthur, 767

Hölty, Ludwig Chr., 3372

Holz, Herbert Johann, I867

Holzamer, Wilhelm, 1385 , 1576

Homann, Hans Joachim, I 744, 2263

Homeyer, Fritz, 280I

Hönigsberg, Margret, 1577

Hoppe-Meyer, H., $217^{8}$

Hornfeld, A. G., I 16

Horst, Karl August, 2359, 3301-3302

Horvaths, Ödön von, I 388

Hostovski, Egon, 2520

Hugo, Victor, 1370,1428

Humbel, F., 2340

Hünich, Fritz Adolf, (54), 170, 2716, 2802-2803, 2875, 3046

Huppert, Hugo, 2804

Hutchinson, Paul, $237 \mathrm{x}$

Hutchinson, Percy, 1964

Ibarguren, C., I 207

Iizuka, Nobuo, 552-553, 2990-2991

Immermann, Karl L., 3372

Ippisch, Gottfried, I4I, 2806, 2940

Jack, Peter Monro, 2153

Jackson, George, I9I I

Jacob, Henrich Eduard, I988, 2769, 2807-2808

Jacob, P. Walter, 2809

Jacobi, Johannes, 28 10

Jacobsens, Jens Peter, 533, 1505

Jacquot, Michel, 309

Jain, Yashpal, 2978-2979, 3103

Jaloux, Edmond, 533, I 98

James, Edward, 2543

Jacke, Otto, I 860

Janitschek, Maria, I 578

Jarnés, Benjamin Millán, 3016-3017, 3235

Jarpa, Hugo Lazo, 3017

Jastrow, Joseph, 2375

Jaurès, Jean Leon, $533, \mathrm{I}_{3} 63$

Jean Paul (Johann P. Richter), I 253, 3372

Jefsen, Jes. 946

Jenisch, Erich, 23 I 2

Joachim, Heinz, 2483

John, K., I96r

Jonas, Klaus W., 3368

Jones, Ernest, $256 \mathrm{I}$

Jones, H. M., 2219

Jonsen, O., 2172

Jonson, Ben, II8-120, I25-I35, I162I I63, I 708-I 743, 2702, 3245

Josephson, Matthew, I895

Jourda, P., I982 
Jouve, Pierre Jean, 266I

Joyce, James, 526-527, 545, I 144

Jürgens, Curd, 3352, 3362

Kadmi-Cohen, 2662

Kaemmer, Kurt, 3191

Kaempffer, Annemarie, 3192

Kafka, Franz, XXXIV

Kainz, Josef, 526 , I 350

Kaiser, Georg, 28 ro

Kalclreuth, Wolf Graf, 1478

Kara, Helena, 3347

Karschin, Anna Luisa, 3372

Kasack, H., ${ }_{13} 67$

Kasimir, Luigi, I60I

Kastein, Josef (Pseud. for Julius Katzenstein), 2976

Kaufmann, Firtz, 3350

Kaufmann, Walter, 2562

Kayser, Rudolf, 988, 28I 3

Kazin, Alfred, 1789, 1803

Keats, John, 1429

Keim, H. W., I632, 2264

Keller, Gottfried, 3372

Kempf, Marcelle, 2663

Kerner, Justinus, 3372

Kesten, Hermann, 278, 246I, 2689, 28I42818, 2920, 3402-3403

Keun, Irmgard, 2689

Key, Ellen, XXVIII, 2183

Keyserling, Conde, 1207

Keyserling, H., 2276

Kiesekamp, Hedwig (Cf. L. Rafael), 1598

Kilian, Eugen, 1652 , 1662

Kindermann, Heinz, 3303

Kinkel, Johann, G., 3372

Kippenberg, Anton, 89, 533, I 147, I386, 2689,2696

Kippenberg, Katharina, 288 I

Klammer, Karl, Cf. K. L. Ammer

Klawiter, Randolph, J., 2498, 3193

Klein, Johannes, 2309, 3304

Kleist, Ewald Christian von, 3372

Kleist, Heinrich von, XXXIII, 9, 851 , 853-856, 861, 863, 866-867, 870-87x, 1073, $1082-1087,1565,2218-2228$, 2305-2324, 2332-2335, 3372

Klopstock, Friedrich G., 3372

Knvels, Wilhelm, 2819, 3092

Knorr, Josephine von, 1579

Koegler, H., 2563, 3108

Köhler, Heinrci Karl, 2010

Kollewijn, Pieter H., $205 \mathrm{I}$

Köppen, C., ${ }^{3} 367$

Körner, Josef, 3305

Körner, Karl T., 3372

Koruham-Europe, P., 2396
Kossmann, Alfred, 2521, 3068

Kotzebue, August F. von, 3372

Kracauer, Siegfried, 2564

Kranz, H., I 996

Krantz, Paul (Cf. E. E. Noth), 2670

Kraus, Hans Johan, 2820, 3121

Kraus, Karl, I I 43, I625, 2490, 282 I-2822

Kreitner, R.L., 3036, 3083

Krell, H., 2207a

Krell, Leo, 3306

Kreutzer, J., $201 \mathrm{I}$

Kris, Ernst, 2565

Kronenberger, Louis, 1774, I790, I825, 2287

Krutch, Joseph Wood, 2465, 2566

Kubin, Alfred, 976, 2823

Kudrjavcev, A., 663

Kuhn-Foelitz, August, 2824

Kummer, Friedrich, 3307

Kumming, Eugen, 2825

Kurowski, Ludwig, I580

Kurzrock, ?, 2350

Kutscher, Artur, 3308

Laaths, Erwin, 3309

La Farge, Christopher, 1849, 2071, 3167

La Farge, Oliver, 3162

Laforgue, Jules, I58 1

Lalou, René, I85I

Lamm, Josef, I 699

Lammasch, Heinrich, I 239

Landmann, Georg Peter, 2826

Lang, A., I973, 2117

Lang, C.-L., 2664

Lang, Renée, 977-978, 2672, 2880

Lang, Wolf, R., I 78, 2827-2828, 3059

Lange, Herbert, 2829, 3145

Langer, Norbert, 3310,3405

Langyel, Emil, 3273

Latzko, Andreas, I 506-1 507

Laube, Heinrich, 3372

Laver, James, 2 I 12

Lawrence, Mother Mary, 2049

Lechlitner, Ruth, I8 12

Lecler, J., I975, 2164

Leclère, C., $2 \mathrm{IG}_{3}$

Lederer, Herbert, 250 I

Lederer, Max, 2830, 3 ISI

Lefevre, Frédéric, 2665

Leftwich, Joseph, 303, 1508, 2567

Lehrman, Charles, $283 \mathrm{I}$

Leminen, Hannu, 3347

Lemonnier, Camille, XXVIII, I I 77, I 252, I 268, 1 430, I 509-1 5 IO

Lenau, Nikolaus, 3372

Lengyel, Emil, 2568 
Lenin, Nikolai, 902-903, 905, 909, 918, 923-925

Lennartz, Franz, 33 II

Lenning, W., I 712

Lent, Gertrud, I 582

Lent, Ingrid, 3194

Lenteritz, G., 2832, 3048

de Lentiono, Jacopo, I 43 I

Lenz, Jakob M., 3372

Leppin, Paul, I309, I5 II

Leppla, R., 3262

Lerch, Eugen, 2231, 2833

Lernet-Holenia, Alexander, I17, 2689, 2696,2834

Lessing, Gotthold Ephraim, 3372

Leuthold, Heinrich, 3372

Leuwerik, Ruth, 3360

Levin, Harry, I89 I

Levinson, A., 2045

Levy, Arthur, 2666

Lewis, Sherman, 2473

Lichtenberg, Georg Christoph, 3372

Lie, Robert, 2836

Liebish, Rudolf, 1583

Liedtke, Harry, 3353

Liess, Andreas, 2837

Lieven, Albert, 3354

Lilge, Herbert, 2838, 3052

Lilien, E. M., I 204, I 5 I 2, 249 I

Liliencron, Detlev von, I I4I, 3372

Lind, Emil, I 5 I 3

Lingg, Hermann von, 3372

Liptzin, Solomon, $2569-2570$

Lissauer, Enst, I205, I631, 1749-1750, $2087,2316,2839,3066$

Lockemann, Fritz, 3312

Loehrke, Eugene (Eugen Lörhke), 2027, 2243

Loewe, Karl, I 259

Loiseau, Henti, 1850, 2122 , 2165, 226r, 2295, 2330, 2398-2399

Lothar, Rudolf, $284^{\circ}$

Lovett, Robert Morris, 2102, 2150, 2I 97

Lowe, Helen, 2466

Löwenstein, Hubertus Prinz zu, 3223

Lowenthal, Marvin, 1894

Lucas, Frank Laurence, $257 \mathrm{I}$

Lucka, Emil, I390, I584-1585, 2308, $2353,284 \mathrm{I}-2842,308 \mathrm{I}$

Ludwig, Emil, XXXIII, (73), I 200-1 201, I 207, 2525, 2843, 2920

Ludwig, Otto, 3372

Lueth, Paul E. H., 33 I 3

Lukács, Georg, 2844

Lupi, Sergio, 3234
McClain, William H., 994-995, 25722574, 2639

McFee, William, 2286

McKay, M. P., I 843

McLaughiln, Richard, 2477

McNeill, John, T., I905

Maart, ?, 2 I 33

Maass, Joachim, 2004, 2689, 2845

Machácková, Vera, 674

Maderno, Alfred, 2846, 3314

Maeterlinck, Maurice, I 586, 1609

Magellan, Ferdinand, 9-10, $525,528,671-$ 699, 1093-1094, 2049-2078

Mahler, Gustav, XXXIII, (49), 30, 526$528,533, \mathrm{I} 23 \mathrm{I}$

Mair, John, 1787

Malay, Joseph, 276

Mallet, Robert, 2648, 3387

Mann, Erika, 2555, $3 \mathrm{IG}_{3}$

Mann, Heinrich, 2920, 3402

Mann, Klaus, 278, I5 14, 2487, 2555, $2575-2576,2689,2764,3163$

Mann, Thomas, XXXIII, XXXIV, (74), 541, 559, I217, 1357-1357a, 1367, I $587,2558,2583,2689,2847,2920$, $2972,3366,3368,3402$

Manz, G., $17 \times 3$

Maraun, F., 2337

Marcus, Erich, 2275

Mardschanow, Konstantin, $334^{\circ}$

Margenstern, G., 2 I 74

Marguerite of Austria, (I)

Maria Stuart, XXXIV, 9, 524, 735-766, 2132-2170, 2606, 3102

Marie Antoinette, XXXIV, I0, 524-525, 700-734, I099-1106, 2090-21 27, $3138,335 \mathrm{I}$

Maritain, Jacques, 1207

Marsh, F. L., I824, I83I

Marshall, Margaret, 2577, 3109

Martens, Kurt, 1869

Marti, Hugo, 2848

Martin, Jex, I 888

Martin du Gard, Roger, 541, 544, 559, I 298

Martini, Fritz, 3315

Marx, Joseph, 2837

Marx, Karl, 2949

Marx, Madeline, 1432

Masereel, Frans, XXXII, XXXIII, (50), 219-220, 252, 526-527, 545, 767-768, I $215,1507,2689,2696$

Masoin, F., 2217

Mason, Eudoc, 2578

Masson-Oursel, P., 2392

Mathis, Alfred, 2579 
Matkowsky, Adalbert, 6oa

Mattingly, Garret, I9I9

Matzdorff, W. S., I 642

Maurer, Joseph, I 240, 2849

Marina, Zenta, 2580, 2850

Maurois, André, 3029

Maury, J. W., 2092, 2234

Mayer, Paul, I5IS

Mazor, Michel, 2667

Mazzucchetti, Lavinia (Cf. L. Mazzucchetti as translator), 225 I, 2403, 247I, 2982-2984

Means, Philip A., I 88I

Medford, Harold, 3352

Mehring, Wlater, 2920

Meier-Cräfe, Julius, I 588

Meisels, S., 3080

Mell, Max, 1589

Mensch, L., 2253

Menter, L., $285 \mathrm{I}, 3084$

Mércereau, Alexander, I 16

Mertens, Gerhard M., 3195

Mesmer, Franz Anton, XXXIV, (66), 9, II $872-875,877-879,882-888$, $892,894-895$, I054-1072, 2336-2365, 2368-2407

Meunier, Constant, II 85

Meyer, Arthur, 3375

Meyer, Conrad F., 3372

Meyer, Cornelius, 3375

Meyer, Richard, 3316, 3375

Meyer-Benfey, Heinrich, 2852

Michael, F., I929

Michaelis, Edgar, 2853

Michel, Wilhelm, $159^{\circ}$

Mileck, Joseph, $258 \mathrm{I}$

Minnigerode, Meade, 2114

Miran, L., 2042

Mis, L., 2320

Mistral, Gabriela, 2855

Mittner, Ladislo, 3317

Moehlman, C. H., I 907

Moissi, Alexander, 533, I 287, 336r

Molo, Walter von, I357a, I635, 2856

Montaigne, Michel, XXXVI, XXXVIII, 533, I 289, 2689

Montenegro, Ernesto, 244I

Moore, George, I59I

Moore, Harry, T. I802

Morena, Erna, 3353

Moreno, Artemio, 3018

Morgan, Bayard Q., 2582

Mörike, Eduard, 3372

Morley, Christopher, 263

Morris, Lawrence S., 2233

Morrison, Samuel E., 1878
Mortimer, John, 3356

Mortimer, Raymond, I 892

Mottram, R. H., 1207

Moulin, René, 204I

Mourre, Michel, 3227

Mozart, Wolfgang Amadeus, XXXIII, I 517,3378

Muckermann, Friedrich, 1347

Mueller, G., 2020

Muhr, Adelbert, 2769, 2857

Mühsam, Paul, 2858

Muir, Edwin, 2492

Müller, Wilhelm, I675, 3372

Müller-Einigen, Hans, 2859

Mumbauer, Johannes, 3318

Munn, L. S., 1848

Münz, J. B., I639

Muret, Maurice, 2039, 2119, 2389, 26682669,3155

Murray, A. L., I906, 2432

Musil, Robert, 2860

Musset, Alfred de, I 292

Nadler, Josef, 3319

Naldoni-Centenari, N., 2074, 2077

Napoleon Bonaparte, 165, 523, 525, 899903, 909, 9II, IOSI-I053, III3, IIIS-III6, III9, II21, II 78, I296, 2032, 2044, 3379

Narciss, G. A., 1989

Naumann, Hans, 3320

Navarro, Eugenio, 3002

Nazaroff, Alexander, 2032

Neale, J. E., 2157

Necker, Moritz, 1707

Netto, Hadrian Maria, 1592

Neuburger, P., I 229

Neumann, Erich, 3366

Neumann, Robert, 286r

Niebuhr, Reinhold, I9I4

Nielsen, Asta, 3350

Nietzsche, Friedrich, XXVII, XXXIII, $528,54 \mathrm{I}, 544,588,829,851,853-$ $856,859-86$ I, 863, 868-87I, 1073, I088-1092, I 279, 2218-2228, 2305$2327,2332-2335,3372$

Niles, Blair Rice, $2584^{-25} 85$

Nobel, Alfred, XXXII

Norden, R., 2587

Norman, Charles, 2586

Noth, Ernst E. (Cf. Paul Krantz), 2670

Novalis (Friedrich von Hardenberg), 3372

Nowaczynski, Adolf, 2994

Noyes, R. G., I725

O'Brien, Kate, 1793

Oechler, William F., 996, 2589 
Oetke, Herbert, 2862, 3174

Ophüls, Max, 3348

Opitz, Martin, 3372

Orrick, James, 2224

Oritz Oderigo, Alicia, 3019

Orzeszko, Helene, 1593

Osborn, Max, 249I

Osorio Lizarazo, J., 3020, 3154

Oswald, Gerd, 3352

Overbeck, Franz, 54I, 544, 558, I 279

Ozep, Fedor, 3341

Palmer, Lilli, 3354

Palmieri, E., 2047, 21 25, 2402

Pankow, H., I643

Pannwi $z$, Rudolf, $\mathrm{I}_{3} \mathrm{I}_{3}$

Paquet, Alfons, I $357^{\mathrm{a}}$

Parandowski, Jan, 2863

Parsons, Alice Beal, 191 2

Partridge, David, 291

Peixoto, Afrancio, 1207

Pellegrini, Carlo, $332 \mathrm{I}$

P.E.N. Club, XXXV, 1226, 2558, 26I5

Perfall, Anton von, I594-1596

Pestalozzi, Johann H., $337^{2}$

Peters, J., 2356

Peterson, Virgilia, 2514

Petijean, Armand, $267 \mathrm{I}$

Petit, G., 2 I 18

Petry, L., 2205

Peyre, Henri, 1896

Pfeffel, Gottlieb K., 3372

Pfeiler, William K., 3218

Pick, Robert, 899, r 899, 2467, 2590-259I, 3051

Piérard, Louis, 1207

Pierre-Quint, L., 2394

Pierson, Edgar, 1696

Pirandello, Luigi, 1433

Pirker, M., 2273

Pisani, P., 2298

Piscatur, 1033

Pisk, Egon, 2864, 3175

Pitollet, C., 2043

Pitrou, R., 1624, 2296

Platen-Hallermünde, August Graf von, 3372

Poerio, Alessandro, I 136

Poggioli, R., 2249

Pohl, G., I 367

Polgar, Alfred, 2920

Politzer, H., 2509

Pommeranz-Liedtke, Gerhard, 768

Pompeati, A., 21 27, 2303

Pongs, Hermann, 3322-3323

Ponten, Josef, 1347

Porena, E., I 984
Porterfield, Allen W., 2033

Poss, A., 2865

Pound, Ezra, 2586

Powell, F-M., I916

Power, Tyrone, $335 \mathrm{I}$

Prager, Hans, I 18

Prampolini, Giacomo, 3324

Pratt, George K., 2384

Priestley, J. B., 3325

Proust, Marcel, 54I, 558, I 278, I 3 I 2

Pruschanski, N., I 597

Pryll, ?, 2362

Puner, Helen Walker, 2592

Purdie, Edna, 3326

Pury, Roland de, I924

Putnam, Samuel, 2593

Puttkammer, Alberta von, I135, I6291630,1702

Radebeul, ?, 2006

Radó, Aladar, 3245, 3357b

Rafael, L. (Cf. Hedwig Kiesekamp), i 598

Raff, Helene, I 599

Raimund, F., 3372

Rainalter, Erwin H., 139, 2866

Ramler, Karl W., 3372

Ramuz, Charles Ferdinand, 533, 1308

Randall, A. W. G., 2036

Ranicki, Marceli, I859

Ranschoff, Georg, I930

Rapoport, O., 3005

Rasputin, I03 I, I033

Rathenau, Walter, XXXIII, 533, I319, I 375

Rauscher, Bert, 2867

Ravel, Maurice, XXXIII

Ravenel, Mazyck P., 2368

Read, C., 2160

Reger, Max, 3245, 3357c

Reichert, Walter, A., 3366

Reichmann-Jungmann, Eva, I64I

Reicke, Ilse, 1640

Reifenberg, B., $247^{\circ}$

Reik, Theodor, 1753

Reim, Paul, 2868

Reinecker, Herbert, 3352

Reinfels, Hans von, I600

Reinhardt, Max, XXXIII

Reischl, Friedrich, IGOI

Reishofer, Karl, 2869

Reisiger, Hans, I686, 2689, 2696, 2870

Relgis, Eugen, I $519,2594,3021-3026$

Remarque, Erich Maria, XXXIV, I367, 2558

Rembrandt, van Rijn, 1448

Renan, Ernest, 526-\$27, 545, I 206, I 520

Renier, M., 322 
Renoir, Auguste, XXIX

Rensselaer-Wyatt, Euphemia van, 222I, 24I5

Reuter, Fritz, 3372

Rexroth, Franz von, I 480

Rey Alvarez, R., 2257

Reyes, Alfonso, 1207

Reyles, Carlos, 1207

Reynolds, F. H., 2 I 58

Rice, Muriel, 1602

Richter, Helene, I 709

Richter, Johann Paul F. (Jean Paul), I 253, 3372

Rie, Robert, 2500

Rieger, Erwin, (54), 1440, 1745, 25022505, 2716, 2803, 2871-2875, 2906, 3152,3286

Rieger, Harald, 2876

Riemerschmid, Werner, 2877

Ries, Theresa Feodor, 1356

Riley, Woodbridge, 2376

Rilke, Rainer Maria, XXVII, (7), 519$521,526,533,545,659,792,977-$ 978, 1002-1005, I3I4, I3I6-1318, I $452,1455,1469,1478$, I 480 , I 525 , I603, 2492, 2530, 2578, 2642, 2672, 2689, 2696, 2793, 2878-2883, 2902, 3372

Rimbaud, Arthur, XXVII, (7), 526-527, 545, II 49, I $521-1522,1622$

Ring, O. T., 2595, 3117

Ritzer, Walter, 2883

Robakidse, Grigol, 1523

Robertazzi, M., 2300, 2985

Robertson, J. G., 3326

Robespierre, Maximilien François, I050

Rocca, Enrico, I688, 3327

Roces Suátèz, Wenceslas, 849

Rodenberg, Julius, 3372

Rodin, Auguste, XXIX, (I6), 23, 526$527,1230,1267,1625$

Rolland, Romain, XXX, XXXI, XXXII XXXIII, (10), (29), 9, 306, 308, 3 I I, $533,769-786,977-978,982-983$, 994-995, II 53 , II 68, II 88, I 243 , I 252, I320, I324-I329, I 434-I436, I $524,1528,2171-2204,2572-2574$, $2578,2608-2609,2621,2639,2643-$ 2644, 266I, 2663, 2666, 2673-2676, 2689, 2692, 2696, 2786, 2833, 2880, $2884,3022,3072,3188,3192,3198$

Romain, Jules, I32, 989, 997, I 207 , I737$1743,2596,2677-2680,2689,3156$

Romera, Antonio R., 3027, 3038

Romero, Francisco, I 207

Ronayne, Charles, F., I948
Röntgen, Johannes, 3358

Roosevelt, Nicholas, $244^{\circ}$

Rops, Daniel, r852

Rose, Ernst, 3328

Rose, William, 584, I525, I 885-I900, 2492, 2597

Rosegger, Peter, 533, I 306, 3372

Rosellini, Roberto, 3344

Rosenberg, Justus, 3196

Rosenfeld, Paul, 2228, 3164

Rosenhaupt, Hans Wilhelm, 2885

Rosenkranz, ?, 1943, 2007

Rosenthal, Friedrich, 2886

Rosenzweig, Alfred, 2887

Rosenzweig, Franz, I 347

Rosnovsky, Karl, 3197

Ross, Nan, 2598

Rössler, Arthur, I604

Roth, Joseph, 533, 1255, 2640, 3400, 3402,3408

Rouget de Lisle, Claude Joseph, 902-903, 905, 909, 91 I, 923-926

Rousseau, Jean Jacques, I331, I 526

Rouvre, Rèmy, ${ }^{2667}$

Rowse, A. L., 1917, 1968, 2155

Royce, William H., 2599

Rubens, Peter Paul, I 449

Rückert, Friedrich, 3372

Rudens, S. P., 2290

Russell, Archibald B. H., I437

Rychner, Max, I 347

Saar, Ferdinand von, 3372

Sachaczewer, H., 2262

Sachs, Erich, I605

Sainte-Beuve, Charles Augustin, 526-527, 545, I 333, I 527

Saint Jean, R. de, 2327

Saint Paul, Albert, 1347

Salpeter, Harry, I8I4-18I 5, 2245

Salten, Felix, I637, 3409, 341 2

Salus, Hugo, 1606

Salzburg (City of), XXXIII, XXXIV, XXXVI, (44), (60), 526-528, II 29 , I334, I60I, 1612, 2539, 2726, 2770 , 2824, 2877, 2937, 297 I

Salzer, Anselm, 3329

Samain, Albert, 1607

Samuel, Richard, $333^{\circ}$

Sanchez Tricado, José Luis, 3028

Sanderson, Elizabeth, 1839

Sanin Cano, B., I 207

Sapieha, Virhilia, 2464

Sarnetzki, D. H., 2084

Schaeffer, Albrecht, 2689

Schäfer, Wilhelm, 1347, 1357a, 1608

Schäke, Gerd, 2888, 3085 
Schatz, Otto R., I 8 I

Schaukal, Richard von, I478, I480, 28892893

Schaumann, Ruth, 1347

Scheffel, Joseph, 3372

Schell, Maximilian, 3356

Scheller, Will, 2009, 2894

Scherer, Wilhelm, 333 I

Schibler, Armin, 3245, 3359

Schieffenzah, ?, 2916

Schiff, Martha, 2182

Schiller, Friedrich von, 3372

Schinz, A., 2213

Schlaf, Johannes, I 254, I609

Schlegel, August W., 3372

Schlegel, Friedrich, 3372

Schlegelmilch, W., $1768 \mathrm{a}$

Schlösser, Wilhelm, 178

Schmidt, Adalbert, 3332

Schmidt, P. Expeditus, I 347

Schmidtbonn, Wilhelm, I I48, I 866

Schmitt, Fritz, 3333

Schmitz, Oskar A. H., I301, 1347, I6ro

Schnewlin, M., 2338

Schnitzler, Arthur, XXVII, XXXIV, 533 , I I SO-I I I, I337, 3201, 3404, 3412

Schoenberner, Franz (74), 2600

Schoen-Rene, O. E., 1779

Scholz, Wilhelm von, 1357 a, 1367,1566 , 2899

Schömann, Milian, 2898, 3146

Schönfelder, Erich, 3353

Schoolfield, George C., 2499

Schorer, Jean, 978, 2649-265I, 2654, 268I-2682

Schossberger, Emily, 2508

Schottky, J., 2349

Schreiber, George, 2601, 3164

Schtschegolew, ?, I033

Schullern, Heinrich von, I6I I

Schultz, Theodor, 3331

Schumann, Otto, 3259

Schuster, George N., 2632

Schwarz, Heinrich, I6r 2

Schweitzer, Albert, XXXIII, (48), 526527, I I 58, I $365,1_{482}$, I I I3, 2689

Scott-James, R. A., 2240

Scott, Robert Falcon, $165,523,525,898-$ 903, 909, 9I I, 92 I, I r 07, III 2, I I I 7I I 18

Seelig, Carl, 1938, 2187,2900

Seelmann, Eggebert, U., 28 Io

Ségur, N., $233 \mathrm{I}$

Seiber, Matyas, 3245, 3359a

Seidel, Heinrich, $337^{2}$

Seidel, Ina, 1347
Selden-Goth, Gisela, 975, I000-I00I, 2602, 2638, 2901

Seldes, Gilbert, 1835

Semmig, Jeanne Bertha, 1613

Servaes, Franz, I661, 2207, 2488

Settanni, E., 2986

Seume, Johann, 3372

Shakespeare, William, I3 II, I34I

Shearer, Norma, $335 \mathrm{I}$

Shneour, Salman, 3090

Shukry, Muhammed 'Ayyad, I 535

Sieburg, Friedrich, 2448, 290 Ia

Sievers, W. David, 2603

Silbergeist, ?, 2 I 28

Silva, José Asunción, 3006

Silvestre, Armand, I 438-1439

Simenauer, Erich, 2902

Simon, E., I65 I

Siodmak, Robert, 3345

Slochower, Harry, 2604

Slonim, Marc, 2605

Slowacki, Juljusz, 2606

Smertenko, ?, 1837

Smith, Preserved, 1963

Snell, George, I 897

Sochaczewer, $\mathrm{H}$., ${ }^{1} 367$

Soederberg, Eduard, I6I4

Soergel, Albert, 3334

Soldevilla, Carlos, io

Soskin, William, 2 104

Soulie, Gaston, I 28

Souza, Claudio de, 3003, 3029

Soyka, ?, I 346

Speal, W., 2904

Spanier, Max, 2905

Specht, Richard, 8, I 29, 2906-2907

Spender, S., 2145

Spenlée, J. E., 1977, 2259

Sperry, W. L., 1913, 2607

Spörri, Theodor, 2080, 2347

Squire, John, I 889, 2059

Starr, William Thomas, 983, 2608-2609, 3198

Steersma, J. G., 146

Stefan, Paul, I655, 2908, 2920, 3120

Stefan-Gruenfeldt, Paul, 522 , I 17 I, I $530-$ IS3I

Steinbrinck, O., 1986

Steiner, Rudolf, XXVIII

Steiner-Prag, Hugo, 2769

Steinhauer, Harry, 26 ro

Steinhoff, Hans, $334^{2}$

Steinrück, Albert, 3350

Stelzhauer, Franz, 3372

Stendhal (Pseud. for Henri Beyle), 9-Io,

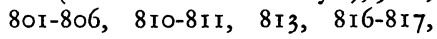


$826,847-848$, Iо16-1024, I 349 , I6I5, 22I8-225I, 2289, 3028

Stern, J., 2323

Stern, Leopold, 2683, 3004, 3061

Sternbach, Hermann, 3074

Sternthal, Friedrich, 1347

Stevens, Austin, 3142

Stevens, George, I 795

Stillman, C. G., 2 I 49

Stöcke, H., 23 I 8

Stöcker, Helene, 1756

Stolberg, Friedrich L. Graf zu, 3372

Storm, Theodor, 3372

Storz, Gerhard, 2921

Stourza von Katschuro, Gräfin D., 3099

Strachwitz, Graf Moritz von, $337^{2}$

Strassburger, Egon H., 1616

Straube, ?, 2408

Strauss, Harold, I 804, I 832

Strauss, Richard, XXVII, XXXIII, XXXIV, I 18, 874, 1686-1694, 2479$2486,2537,2579,2673,2689,2696$, 2929, 2983, $3169,3245,3264,3359 b$

Strecker, Karl, I 748

Streuber, A., 2268

Strobl, K. H., 2495

Strohl, H., 1983

Strunz, Franz, I096, I93 I

Sturmann, Manfred, 2504, 2923-2924, 3096

Suarès, André, 1440

Suckova, B., 762-763

Suhoff, ?, 23 Is

Sutter, John A., 165, 523, 899-903, 909, 9II, II08, IIIO-IIII, IIIS

Suttner, Berta von, XXXII, 526- 527 , I 164,1617

Symons, Arthur, I44I

Szelényi, István, 3245, $3359 \mathrm{C}$

Tagliabue, G. M., 2332

Tagore, Rabindranath, 533, I3 Is

Taine, Hyypolyte, XXIX

Talleyrand, -Périgord, Charles Maurice de, 2017

Taylor, Edmund, 26I I

Teller, Gertrude, E., 26 r 2

Teller, Judd L., 26r 3

Teran, Juan B., I 207

Terhaar, I., 2003

Theodor, Josef, I6I 8

Thieme, H., 2000

Thies, H., 2925

Thiess, Frank, 1347

Thoma, Ludwig, I6rg

Thommen-Girard, G. H., 2926, 3391

Thornton, Luanne, 3 I99
Tieck, Ludwig, 3372

Tiempo, César, 3030, 3054

Tilgher, Adriano, 2987

Tobias, J., 2927, 3158

Toch, Ernst, 3245, 3359d

Toller, Ernst, XXVI, 2558

Tolstoy, Leo, XXX, (26), 10, 94-95, 526528 , 54I, 544, 558, 80I-806, 808$8 \mathrm{II}, 8 \mathrm{I} 4-8 \mathrm{IG}, 8 \mathrm{I} 8,820-826,902-$ 903, 1025-1033, 1338, I 358-r359, I533-1536, $2218-2252,2255-2261$, 2493-2494, 3073, 3369

Tonelli, L., 2333

Torberg, Friedrich, 2689

Toscanini, Arturo, XXXIII, (45), 522, 526, 545, 550, 1486, 2689, 2696, 3107

Tourjansky, Viktor, 3343

Trebitsch, Siegfried, 2928

Trenner, Franz, 2929-2930

Trilling, Diana, 1844

Trinius, B., 293 I, 3067

Trojau, E. W., 2208

Troyat, ?, 2286

Truding, Lona, 2932-2933

Tucholsky, Kurt, XXVI

Uhlig, H., 2445

Uhlman, Fred, 2616

Ullmann, Ludwig, $1757,2769,2936$

Ullrich, Hermann, 2937-2938

Ungaretti, G., 1207

Uz, Johann Peter, 3372

Valentin, Antonina, 2684, 3088

Valéry, Paul, XXIX

Van der Stappen, Charles, XXVIII, XX

Van der Vies, Abrahem, $337^{\circ}$

Van Doren, Carl, 2 135

Van Dovski, Lee, 2769, 2939, 3159

Van Dyke, W. S., $335 \mathrm{I}$

Van Gelder, Robert, 2617, 2940

Vanicek, Hilde, 3200

Van Lerberghe, Charles, XXVIII

Varnhagen von Ense, Karl August, 3372

Verdi, Guiseppi, 1486

Verhaeren, Emile, XXVIII, XXIX, $\mathrm{XXX}, \mathrm{XXXI}$, (I3), 9, 526-527, 529-532, 545-546, 787-792, 996, I I 24-I I 25 , I I 95, I 202-I 203, I 252, I 398, I 442-I 473, I 537, 2205-22 I 7, 2495, 2578, 2589, 2634, 2660, 2689, 2696, 2699, 2709, 2752, 2769, 2791, $3082,3187,3205,3245,3361$

Verlaine, Paul, XXVII, XXVIII, (7), 793-795, II 79, II 8I, I305, I340, I366, I 474-1480, I 538-I 540, I 620, 2496, 3189, 3191, 3196, 3203, 3205 
Viertel, Berthold, 2689, 2696, 2920, 29422943, 3349

Villiers, Alan, 2063

Vogeler, F., 2229

Vogelsang, Hans, 2944

Vogl, Johann N., 3372

Volkart, O., I 87 I

Vorms, Pierre, 768

Voss, Johann H., 3372

Voss, K., 2005, 2313, 2352

Vring, Georg von der, 1478

Vulpius, Christiana, 3372

Wackenroder, Wilhelm H., 3372

Wagner, Richard, I486, 3372

Wahl, J., 2326

Wahrheit, Israel Albert, 3201

Waibler, Helmut, 2945

Weiblinger, Friedrich Wilhelm, 3372

Waidson, H.M., 3280

Waldinger, Ernst, 2769, 2946

Wallace, Margaret, 2022

Wallerstein, Lothar, 2920

Walter, Bruno, XXXIII, (46), 526-527, I I 7I, 2689, 2696, 2947, 3167

Walton, Edith H., I819, 1956, 2143

Walzel, Oskar, 2270, 3335

Wantoch, W., 2206

Wassermann, Jakob, I 85, 533 , I 217 , I 25 I, $1357 \mathrm{a}$

Wedekind, Frank, 1379

Wegner, Arnim T., I380, I62I

Weiniger, Otto, 533, 3404

Weinstock, Herbert, 2537

Weiskopf, Franz Karl, 2478, 2517, 3336

Weiss, E., XXVI

Weiss, Hansgerhard, 2948

Weisshand, Isolde, I 249

Wells, H. G., 2558, 2560, 261 5, 3 IOI

Welter, M. L., 3202

Weltmann, Lutz, 2344

Wendel, Hermann, 2949

Wendt, H. G., 3369

Werfel, Franz, XXXII, XXXIII, (75), I85, 2619, 2689, 2696, 2920, 2950

Werner, Alfred, 25r5, 2620, 3063

Werner, Bruno, 3203

Werner, E., 2496

Werner, Friedrich, $218 \mathrm{I}$

Werner, R. M., I634

Werner, Zacharias, 3372

Werremeier, ?, $295 \mathrm{I}$

Whiting, George W., 1730

Whitman, Walt, 1376

Wiegand, Julius, 3337

Wieland, Christoph Martin, $337^{2}$

Wiegler, Paul, 1347, 2018, 3338
Wilder, Elizabeth, 2442

Wildgans, Anton, 984, 3200, 3409

Wilhelm, Paul, 2952

Wilkinson, Cl., 2025

Wills Ricaurte, Gustavo, $303 \mathrm{I}$

Wilpert, Gero von, 3339

Wilson, Ronald A., 2621

Wilson, W. J., I814, 2622

Wilson, Woodrow, 909

Winternitz, Felix von, XXXII

Winternitz, Friderike Maria von, Cf. F. M. Zweig

Wischnitzer-Bernstein, R., I650

Wislowska, Maria, (Cf. M. Wislowska also as translator), 2996

Witkop, Philipp, 3360

Wittlin, A., 2623, 3062

Wittek, Suzan von, 2954

Wittner, V., 2956

Wolanowski, Lucjan, 2078

Wolbe, Eugen, 2956, 3392

Wolf, H., 2957

Wolf, L. H., I644

Wolfenstein, Alfred, (65), 988

Wolff, E., 2449

Wolff, Gerhart, 3204

Wolff, Theodor, 283 I, 2958

Wolfskehl, Karl, I 347

Wolf, Charles, 2624

Wolpe, Berthold, I5O

Wunderlich, Eva, I 52, 2625-2626

Wyss, H. A., 20I5

Yanitelli, Victor R., I887

Young, Catharine, 2099

Young, Stark, 1721

Zagul, Dmitro, 514, 3033

Zamboni, G., 2302

Zampa, Gregor, 2988, 3064

Zarek, Otto, XXXIV, (55), 1746-1747, 2I 30, 2 I 84, 2272, 2628-2629, 2959, 3 I I 8

Zeich, Paul, 29, I480, I622, I627, 2506, 2960-2962, 3055

Zifferer, Paul, 1653

Zilboorg, Gregory, 2196, 2630-263 I

Zimmermann, Walter, 2969

Zöckeler, ?, 2307

Zodykow, Maxim, I 542

Zohn, Harry, 147, 193, 995, 999-1001, $2526,2574,2594,2632-2639,2689$, $2769,2963-2968,3205,3393$

Zola, Emile, XXVII

Zschokke, Johann H., 3372

Zuckmayer, Carl, 185, 2689, 2696, 2970$297 \mathrm{I}$

Zukofsky, ?, 2586 
Zweig, Arnold, 2795, 2973, 2980

Zweig, Frederike Maria, XXXII, XXXIII, XXXIV, XXXV, XXXVI, (5), (6), (4I), (42), (6I), (67), II, 971-973, 1432, 2470-2478,
2507-25I9, 2640-264I, 2689, 2953, 2974-2975, 2989, 3032

Zweig, Lotte, XXVI, XXXV, XXXVI, 3130-3132

Zweig, Moritz, XXVI

\section{ADDENDA}

Barthel, L. F., 3395

Berenson, Bernard, 3396

Butler, E. M., 3397

Courts, Gerd, 3398

Döblin, Alfred, 3402

Fleischel, Tilla, 3395

Huder, Walther, 340 I

Jancke, O., 3395
Kohn, Hans, 3404

Mariano, Nicky, 3396

Origo, Iris, 3396

Prater, Donald A., 3406

Rauch, Karl, 3395

Scheibe, Richard, 3395

Schramm, Werner, 3407

Seidenfaden, Thomas, 3395 



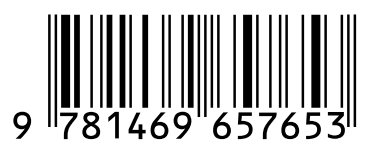

